Furopean

Surgical Research
Eur Surg Res 2006;38:129-312

DOI: 10.1159/000093831

The abstracts are only available online, free of charge, under www.karger.com/doi/10.1159/000093831

\title{
19th National Congress of the Italian Polyspecialist Society of Young Surgeons (IPSYS)
}

June 21-23, 2006, Bologna, Italy

\section{Abstracts}

Guest Editors

Fausto Catena, Bologna, Italy

Luca Ansaloni, Bologna, Italy 


\section{Contents}

General Surgery 131

Thoracic Surgery $\quad 175$

Vascular Surgery $\quad 190$

Obesity Surgery $\quad 207$

Cardiac Surgery $\quad 209$

Laparoscopic Surgery $\quad 214$

Transplantation $\quad 219$

Urology $\quad 229$

Experimental Surgery $\quad 234$

Day Surgery $\quad 238$

Surgical Oncology $\quad 240$

Breast Surgery $\quad 250$

Emergency Surgery $\quad 251$

Gynaecology $\quad 259$

Orthopaedic $\quad 260$

Maxillofacial Surgery $\quad 265$

Phlebological Surgery $\quad 273$

Plastic Surgery $\quad 274$

Endoscopic Surgery $\quad 280$

Endocrine Surgery $\quad 282$

Neurosurgery $\quad 287$

Orl Surgery $\quad 290$

Pediatric Surgery $\quad 296$

Oculistic Surgery $\quad 300$

Anaesthesiologist $\quad 301$

Author Index $\quad 304$ 


\section{Furopean Surgical Research}

\section{General Surgery}

\section{1}

\section{New 'Biological' Markers to Identify Early Colorectal Neoplasms}

C. Casolino, M. Annecchiarico, R. D'Avino, A. Palumbo, T. Pellegrino, N. Della Volpe

Dip. Di Chirurgia Generale e Tecnologie Avanzate, Univeristà Federico II Napoli

Introduction: This study regards the research, in the patients affected by colorectal pathologies, of the Streptococcus bovis, a 'nonenterococcus organism' included into the group ' $\mathrm{D}$ ' of the streptococci.

Methods: We have enrolled 523 patients: 298 of them were affected by colorectal pathology and the remaining 225 represented the 'case control'. The patients with colic pathology have been divided into 4 groups. All these patients have been undergone to the same Research Protocol in order to isolate $S$. bovis (in biological materials).

Results: In our study, S. bovis has been isolated in 127/523 patients coming to be examined for colorectal pathologies. 102 of them were the patients included into the groups from the I to the IV. From the analysis of the above mentioned study, the prevalence of the infection from $S$. bovis in the patients with carcinoma of colon-rectum results significantly higher than the prevalence of the population of control.

Discussion: The easy isolation and identification of $S$. bovis from the biological materials, led us to consider this bacterium reliable as a new marker for the colorectal neoplasms. So we can conclude that the isolation of the $S$. bovis in the biological materials must be always considered really important, because the data obtained from the Test of $\chi^{2}$ can allow us to say that the statement 'the $S$. bovis is a marker of the neoplasms of the colon-rectal' has more of $99 \%$ of the probabilities to be true. Our declared purpose is that identification of $S$. bovis in patients can support the early diagnosis of the colorectal neoplasms.

2

\section{The Endometriosis of the Superficial Levels}

M. Annecchiarico, C. Casolino, F. Maione, C. Bonuso, N. Della Volpe, T. Pellegrino

Dip. Di Chirurgia Generale e Tecnologie Avanzate, Univeristà Federico II Napoli

Introduction: The endometriosis is one of the most common gynaecological pathologies in the world, even if it is little known. The incidence of endometriosis is about $10 \%$ of the women in fertile age. Everybody knows that in the vast majority of cases there are few or no symptoms at all.

Methods: This study was conducted from June 2003 to December 2005 on 6 cases of coetaneous endometriosis. Five patients out of six presented a tumefaction, of variable size, on the superficial levels. The sixth patient presented a tumefaction corresponding to the point of the inflexion of the needle of the amniocentesis. The histological examination confirmed the diagnosis of endometriosis in all the 6 cases without any atypical cellular signs.

Results: From our study the necessity emerges to turn a greater attention in carrying out operations on the genital sphere that could have as consequences an endometriosis from surgical system, and the necessity not to undervalue the possible presence of endometriosis in patients with positive anamnesis for such participations, in which there are nodules on superficial plans or on scars

Discussion: Even today, despite the routine use of advanced diagnostic and surgical procedures, the endometriosis foci are often identified only accidentally because of the insufficient symptomatology. The aim of our study is to stress on the diagnostic difficulties of this pathology, but above all the crescent spread of the coetaneous endometriosis and/or on scars of the surgical wounds or on points of inflexion of needles of the amniocentesis.

3

\section{Intraductal Papillary-Mucinous Tumors (IPMT) of the Pancreas: Clinico-Pathological Correlations}

G.L. Baiocchi, M. Gardani, F. Gheza, K. Cerea,

G.F. Calandra, T. Piardi, G.A.M. Tiberio, S.M. Giulini

Clinica Chirurgica, Brescia

Introduction: IPMT are cystic tumours of the pancreas presenting clinicopathological features distinct from ductal cell carcinoma.

Methods: 8 resected IPMT (M/F 4/4, mean age 68.9, range 57-81), presenting acute pancreatitis in 7 cases. Diagnosis was mainly based on MNR and ERCP, while CT failed in 3/5 patients. 5 cases were classified as main duct and 3 as branch duct tumour. 7 cases were considered at high-risk and 1 at low-risk of malignancy.

Results: 1 duodenopancreatectomy, 2 total- and 5 distal-pancreatectomies were done. In $2 / 6$ cases the frozen examination of the Wirsung margin changed the operative strategy. 2 pancreatic fistulas and 1 gastric haemorrhage were managed conservatively. Histological examination showed invasive carcinoma in 2 patients, in situ ca. in 1 patient and displasia in 5 patients, 2 of which also showed PANin. The preoperative judgement of malignancy and multifocality was correct in $42.8 \%$ and $66.6 \%$ of the cases. At a mean follow-up of 22.6 months (range 2-43), all patients are disease free.

\begin{tabular}{ll}
\hline KARGER & (c) 2006 S. Karger AG, Basel \\
0014-312X/06/0383-0131/\$23.50 \\
$\begin{array}{l}\text { Fax +4161306 1234 } \\
\text { E-Mail karger@karger.ch } \\
\text { www.karger.com }\end{array}$ & $\begin{array}{l}\text { Accessible online at: } \\
\text { www.karger.com/esr }\end{array}$
\end{tabular}


Discussion: The surgical management of IPMT is based upon preoperative suspect of malignancy and definition of the extension of pancreas involvement; both these data are difficult to obtain from the available diagnostic tools. However, IPMT is a pancreatic neoplasia with a very good prognosis after resection, even in case of malignancy; so, the surgical indication must be largely considered.

\section{4}

\section{Appendicitis Mimed Omental Necrosis and Vice Versa. Case Report}

L. Battaglia, R. Bottura, P. Franzini, P. Giannini, G. Nella, C. Pansoni, P. Taliente, E. Trinca, P.A. Spelzini

Azienda Ospedaliera Valtellina Valchiavenna,

S.C. Chirurgia Generale, Presidio di Sondalo

Introduction: Omental infarction is an uncommon cause of acute abdominal pain in adults.

Methods: A 47-year-old woman presented to the emergency department complaining of right lower abdominal pain of $48 \mathrm{~h}$ duration along with high fever and nausea. Physical examination revealed normal vital signs and a tender abdomen in the right lower quadrant with guarding and rebound tenderness. Results of laboratory studies included a white blood count of $12,560 / \mathrm{mm}^{3}$ and a CPR of 173 . Due to the clinical presentation and blood test results, an acute appendicitis was suspected and the patient was taken to OT.

Results: An explorative laparotomy, through a pararectal incision, revealed a segmental necrosis of the distal part of the omentum associated with appendicitis. Appendicectomy and resection of the necrotic omentum were performed. Histopathologic examination revealed congestion, haemmorrage, fat necrosis of the omentum and acute appendicitis

Discussion: Omental infarction often mimics acute appendicitis preoperatively, we emphasize the importance of considering omental infarction in the differential diagnosis of right iliac fossa syndrome even if in this experience the two pathologies were synchronous and mimicked each other.

5

\section{Analysis of Outcome and Prognostic Factors in Liver Resection for HCC}

\section{E. Benzoni, A. Favero, A. Cojutti, L. Rossit, D. Lorenzin, G.L. Adani, A. Zompicchiatti, U. Baccarani, F. Bresadola, A. Uzzau \\ Department of Surgery, University of Udine, School of Medicine, Italy}

Introduction: The aim of this study is to examine the factors associated with improved prognosis in $\mathrm{HCC}$ after liver resection.

Methods: From September 1989 to March 2005, 134 consecutive patients had liver resection for HCC on cirrhosis at our department. We performed 54 major liver resections and 80 limited resections. All patients enrolled in the study were followed-up three times during the first year after resection and twice the next years.

Results: In-hospital mortality rate was $7.4 \%$, about $50 \%$ of these cases were Child-Pugh B patients. Morbidity rate was $47.7 \%$, caused by the rising of ascites, hepatic insufficiency, biliary fistula, hepatic abscess, hemoperitoneum and pleural effusion. Overall survival resulted to be influenced by etiology $(p=0.03)$, underlying liver disease, in particular Child A vs. BC ( $p=0.04)$, EndmondsonSteiner grading $(\mathrm{p}=0.01)$, and the associated variables Grading $1-2+$ Child A vs. Grading 3-4 + Child BC $(p=0.03)$, the absence of a capsule $(\mathrm{p}=0.004)$, the presence of more than one lesion $(p=0.02)$, the measure of lesion more than $5 \mathrm{~cm}(\mathrm{p}=0.04)$, Pringle maneuver length more than $20 \mathrm{~min}(\mathrm{p}=0.03)$, the amount of resected liver volume more than $50 \%$ of total liver volume $(\mathrm{p}=0.03)$, and the relapse of HCC $(\mathrm{p}=0.01)$.

Conclusion: A better preoperative selection of patients, based on a defined panel of criteria could improve survival, reduce postoperative complications and recurrence's rate after liver resection.

\section{6}

\section{Improvements in Liver Resective Surgery: Analysis on an Oncological Population}

\section{E. Benzoni, A. Cojutti, A. Favero, L. Rossit, \\ A. Zompicchiatti, D. Lorenzin, G.L. Adani, U. Baccarani, F. Bresadola, A. Uzzau}

Department of Surgery, University of Udine, School of Medicine, Italy

Introduction: Herein we analyzed improvements obtained on lowering postoperative morbidity on the ground of data derived from a single center surgical population and we draw a learning curve in liver surgery.

Methods: From 1989 to 2005, 287 consecutive patients had liver resection for either HCC or liver metastasis. We performed 22 major hepatectomy, 20 left hepatectomy, 14 trisegmentectomy, 77 bisegmentectomy and/or left lobectomy, 74 segmentectomy and 80 wedge resection.

Results: In-hospital mortality rate was $4.5 \%$, about $7 \%$ in $\mathrm{HCC}$ cases and $2.6 \%$ in liver metastasis. Morbidity rate was $47.7 \%$. Pringle maneuver length more than $20 \mathrm{~min}(\mathrm{p}=0.001)$, the type of liver resection procedure: major hepatectomy $(p=0.02)$ and the request of an amount of blood transfusion over $600 \mathrm{cc}(\mathrm{p}=0.04)$ are responsible of the rising of complication, according to the results of multivariate analysis. The learning curve, derived from a logarithmic regression model, showed that we achieved a reduction of development of: pleural effusion $(\mathrm{r}=0.85)$, transitory impairment liver function $(\mathrm{r}=0.81)$, biliary leakage $(\mathrm{r}=0.80)$, liver abscess $(\mathrm{r}=0.79)$, and haemoperitoneum $(\mathrm{r}=0.69)$.

Conclusion: Every liver resection should be plan after intraoperative ultrasonography, anatomical surgical procedure like segmentectomy should be preferred instead of wedge resection; the use of modern devices, like Argon Beam and Ligasure ${ }^{\circledR}$ dissector reduce the incidence of both intraoperative, postoperative bleeding and biliary leakage. 
7

\section{Pancreatic Resection for Oncological Pathologies: Outcome and Prognostic Factors}

\section{E. Benzoni, L. Rossit, A. Favero, A. Cojutti, D. Lorenzin, E. Saccomano, U. Baccarani, G. Adani, F. Bresadola, S. Intini \\ Department of Surgery, University of Udine, School of Medicine, Italy}

Introduction: The aim of this study was to determine the long term survival of patients with pancreatic cancer who underwent surgical at our Department of Surgery.

Methods: From 1989 to 2005, 137 consecutive patients were followed at our department. Staging was as follows: Ia 3.6\%, Ib 16\%, IIa $13.9 \%$, IIb $48.9 \%$, III $6.6 \%$, IV $11 \%$. We performed 76 pancreaticoduodenectomy, 26 distal pancreasectomy and 35 total pancreasetomy.

Results: In Hospital mortality rate was $4.37 \%$. The mean survival after surgery was $27.7 \pm 26.93$ months, and mean disease free survival after surgery was $15.7 \pm 2.93$ months . The 1-, 3-, 5-, 7- and 9 -year survival rates for all 137 patients were $63.9 \%, 33.7 \%, 21.17 \%$, $12.7 \%$ and $10.2 \%$ respectively. In multivariate analyses survival was influenced by: age $>70$ years $(p=0.0087)$, T stage $(2, p=0.0018$, $3, \mathrm{p}=0.0018$ and $4, \mathrm{p}=0.0364), \mathrm{N}$ status $(1, \mathrm{p}=0.00969)$, presence of metastases ( $p=0.005)$, Stage $(3, p=0.000834$ and $4, p=0.00035)$, type of surgical procedure: pancreaticoduodenectomy $(p=0.003602)$ or distal pancreasectomy $(\mathrm{p}=0.0044958)$, the arising of postoperative complications: leakage $(p=0.034)$ and haemoperitoneum $(p=0.036)$ and the development of metastases during follow up $(\mathrm{p}=0.007723)$.

Conclusion: Even if pancreatic cancer has a dismal prognosis with pancreatic resection offering the only chance for cure. Improved morbidity and mortality rates of pancreatic resection have led to renewed interest in the outcomes of patients who undergo surgical resection both for radical resection intention (stage I and II) and for palliative intention (stage III and IV).

8

\section{Postoperative Complications Linked to Resective Surgery for Liver Tumor}

E. Benzoni, A. Cojutti, A. Favero, L. Rossit,

A. Zompicchiatti, D. Lorenzin, G.L. Adani,

U. Baccarani, F. Bresadola, A. Uzzau

Department of Surgery, University of Udine, School of Medicine, Italy

Introduction: Herein we analyzed causes and foreseeable risk factors linked to postoperative morbidity on the ground of data derived from a single center surgical population.

Methods: From 1989 to 2005, 287 consecutive patients had liver resection for either Hcc or liver metastasis at our department. We performed 22 major hepatectomy, 20 left hepatectomy, 14 trisegmentectomy, 77 bisegmentectomy and/or left lobectomy, 74 segmentectomy and 80 wedge resection.
Results: In-hospital mortality rate was $4.5 \%$. Morbidity rate was $47.7 \%$, caused by the rising of ascites (10\%), hepatic insufficiency $(19 \%)$, biliary fistula $(6 \%)$, hepatic abscess $(25 \%)$, hemoperitoneum $(10 \%)$ and pleural effusion $(30 \%)$. The variables responsible of the rising of complication are: Pringle maneuver $>20^{\prime}(p=0.001)$, the type of liver resection procedure: major hepatectomy $(p=0.02)$, left hepatectomy $(p=0.04)$, trisegmentectomy $(p=0.04)$, bisegmentectomy and/or left lobectomy $(\mathrm{p}=0.04)$; and the request of an amount of blood transfusion $>600 \mathrm{cc}(\mathrm{p}=0.04)$; also both liver dysfunction, in particular Child A vs. B and C $(\mathrm{p}=0.01)$ and histopathological grading $(\mathrm{p}=0.01)$ are associated with an high rate of post-surgical complication in HCC cases.

Conclusion: Complications arisen during postoperative time, although could be treated, delay patient's recovery and resumption of liver function; the evaluation of causes and foreseeable risk factors linked to postoperative morbidity during the planning of surgical treatment should play the same role as other factors weighted in the selection of patient eligible to liver resection.

\section{9}

The Role of Pancreatic Leakage in Rising of Complications Linked to Pancreatic Surgery

E. Benzoni, L. Rossit, A. Favero, A. Cojutti, D. Lorenzin, E. Saccomano, U. Baccarani, G.L. Adani, A. Uzzau,

F. Bresadola, S. Intini

Department of Surgery, University of Udine, School of Medicine, Italy

Introduction: An analysis of the role of pancreatic leakage in rising of complications linked to pancreatic surgery.

Methods: From '89 to '05, 137 consecutive patients underwent surgical procedure for pancreatic cancer at our Department. We performed 76 pancreaticoduodenectomy (PD) and 26 distal pancreasectomy (DP); we recorded results of only 102 patients who underwent PD or DP. The surgical reconstruction after PD was as follows: 11 manual non-absorbable stitches closure of the main duct, 24 closure of the main duct with linear stapler, 17 temporary occlusion of the main duct with neoprene glue and 24 duct-to-mucosa anastomosis.

Results: Morbidity rate was $60 \%$, caused by: pancreatic leakage (48\%) haemorrhagic complication (10\%) and infectious complication $(15 \%)$. At the multivariate analysis complications were linked to: age $>70$ years $(\mathrm{p}=0.0139), \mathrm{T}_{3}(\mathrm{p}=0.031)$ and $\mathrm{N}_{2}(\mathrm{p}=0.000001)$, surgical procedure $(\mathrm{PD}, \mathrm{p}=0.0018)$ and pancreatic residual treatment (duct-to-mucosa anastomosis $\mathrm{p}=0.003$ and stapler closure $\mathrm{p}=0.002$ ). Haemorragic complication, biliary anastomosis leakage and infectious complication were consequences of pancreatic leakage (all $\mathrm{p}=0.025$ ).

Conclusion: On the ground of our data we believe that manual non-absorbable stitches closure of the main duct and temporary occlusion of the main duct with neoprene glue should be avoided in the reconstructive phase. 
10

\section{Laparoscopic Assisted Duodenopancreatectomy}

\author{
P. Bisagni, E. Orsenigo, S. Di Palo, M. Carlucci, \\ C. Staudacher \\ Osp San Raffaele, Milano
}

Background: Laparoscopy has been suggested in treatment of abdominal tumours such as colorectal cancer. Recently, laparoscopic surgery has been proposed for gastric and pancreatic tumours. In the video we describe a laparoscopic assisted duodenopancreatectomy.

Methods: A 75 years old woman has been investigated for abdominal pain. Ultrasound and CT showed $8 \mathrm{~cm}$ lesion within the pancreatic head. Pneumoperitoneum was established using Hasson's method. No peritoneal seeding was found. 4 additional ports were inserted, gastrocolic ligament divided, and pancreas exposed. Laparoscopic cholecystectomy was performed and the hepatic duct sectioned $3 \mathrm{~cm}$ above the sup pancreatic border. The Kocher maneuver was performed. Gastroduodenal artery was divided. The duodenum was resected distal to pylorus. The jejunum was resected $10 \mathrm{~cm}$ beyond Treitz. The pancreas was divided using harmonic scalpel and cleared from mesenteric artery and vein. Common hepatic and splenic artery were dissected and node dissection performed. The specimen has been removed through a 5-7 cm incision. Therefore, a pancreatico-, hepaticand duodeno-jejunostomy have been performed through the incision.

Results: Laparoscopic duodenopancreatectomy has been successfully performed. Operate time: $420 \mathrm{~min}$. Pathology revealed mucinous cystoadenoma of the pancreas.

Conclusions: Laparoscopic assisted duodenopancreatectomy is feasible.

11

\section{Abdominal Neoplasms and Intestinal Neurofibromas Associated to Von Recklinghausen's Disease}

\author{
A. Polistena1, G. Cavallaro1, F. Fornari' ${ }^{1}$ M. Ruperto ${ }^{1}$, \\ S. Calvieri², G. De Toma ${ }^{1}$ \\ 'Department of Surgery 'P. Valdoni', Policlinico Umberto I, \\ University of Rome 'La Sapienza', 'Department of \\ Cutaneous Diseases and Plastic Surgery, Policlinico \\ Umberto I, University of Rome 'La Sapienza', Italy
}

Introduction: Type 1 Neurofibromatosis (NF1), known as Von Recklinghausen's disease, is a common autosomal dominant disorder, related to dysfunction of p21-ras oncogene. Variety in clinical manifestation represents one of the most important clinical issues in NF1. Gastrointestinal involvement is rare and is usually in the form of neurofibromas and leyomiomas in the bowel wall. Involvement of mesentery, retroperitoneum and liver is very rare.

Methods: Records of 464 patients in follow-up for NF1 have been reviewed. Among them, four cases of NF1 were treated for associated abdominal neoplasms and gastrointestinal neurofibromas.

Results: All Patients showed small intestine neurofibromas associated to sub-clinical obstruction. In one case neurofibromas were as well located in the mediastinum and a large one in the retroperitoneum. In another case left adreanal pheochromocytoma was identified and adrenalectomy was performed. In the third patient a neurofibroma was identified in the mesorectum with adhesions to the utherus. In the last patient huge GIST arising from the stomach was present with clinical evidence of abdominal mass. All Patients underwent surgery with excision of the associated tumors and intestinal resection for the occluding neurofibromas.

Discussion: Variability in clinical presentation of NF1 with possible manifestation of severe systemic benign and malignant disease requires strict follow-up and since the lack of genetic patterns of phenotypic expression, programs of specific screening in order to obtain precocious diagnosis of extra-cutaneous disorders.

\section{2}

\section{Surgical Strategies in the Treatment of Primary Lumbar Hernias}

\author{
A. Sadighi, G. Cavallaro, G. De Toma, A. Cavallaro \\ Department of Surgery 'P. Valdoni', Policlinico Umberto I, \\ University of Rome 'La Sapienza', Italy
}

Background: Primary lumbar hernias arise through posterolateral abdominal wall defects, named inferior lumbar triangle (Petit's triangle) and superior lumbar triangle (Grynfelt's triangle). Most of lumbar hernias described in literature are secondary to trauma or previous surgery, while primary lumbar hernia are rare. There are 2 possible surgical approaches to these hernias. The anterior approach with lumbar incision (with direct suture or with use of mesh), and the laparoscopic (transabdominal or totally extraperitoneal) approach.

Methods: The Authors present a serie of 9 surgical procedures for primary lumbar hernia performed using polypropylene or PTFE mesh placed in the extraperitoneal space, and review all the literature concerning the treatment of primary lumbar hernias.

Results: Many different surgical procedures are described to treat primary lumbar hernia: direct repair, use of rotational or onlay flaps, use of synthetic mesh via the anterior approach or the laparoscopic (transabdominal or extraperitoneal) approach. Of these, the procedures performed by use of mesh seem to achieve better results in terms of effectiveness, hospital stay and return to normal life activities.

Conclusions: Repair of primary lumbar hernias can be successfully carried out by anterior approach with use of synthetic mesh; this method of repair is easy, safe and effective. Laparoscopic approach, as affective as the anterior approach, is more expensive and need general anesthesia; this method could be preferable to the open approach in case of larger hernias with involvement of visceral structures. 
13

\section{The Light Reflection Rheography in a Pre and Post-Operatory Study on Patient with Superficial CVI}

\author{
A. Comandi, F. Berna, M. Bucalossi, L. Gazzabin, \\ S. Mancini, G. Botta \\ Chirurgia Generale e Specialità Chirurgiche, Siena
}

Introduction: The LRR is a non-invasive technique that can detect local changes of the flow and of the volume of the blood through the skin of the lower limbs. This technique permits the evaluation and the registration of the Refilling Time. So we can study a condition of reflux in the main superficial trunks or an alteration of the muscle pump efficency.

Methods: The aim of the study is to determ if the surgical treatment improves the muscle pump efficency by increasing the RT. Beside we compared I and II class stocking to evaluate their effecency.

Results: We studied 60 pacients with superficial CVI. Every patient was visited and examinated by Duplex scan. The LRR was performed before and after 1 week from the operation, and classificated on the results of RT. The kind of operations are: 36 LSV stripping, 7 CHIVA II, 17 laser treatments.

Discussion: The LRR permits the observation of the muscular pump efficency and permits a comparison between the stocking with different kind of compression. The results obtained give us the dimostration that the surgical way improves the muscular pump efficency, and stocking were a support for the patient who was waiting for operation.

Conclusion: The LRR is a valid instrument for the diagnosis of CVI, because demostrating the quantity of the reflux and it is usefull for suggesting to the patient the kind of stocking that he needs.

\section{4 \\ Endovenous Laser Treatment of Chronic Venous Insufficiency of the Lower Limbs}

\author{
F. Berna, S. Mancini, A. Comandi, M. Bucalossi, \\ L. Gazzabin, F.M. Consiglio, G. Botta \\ Chirurgia Generale e Specialità Chirurgiche, Siena
}

Introduction: The IVC is an evolutive disease, most frequently in female patients. The surgical therapy is indicated for advanced stages of illness. Recently are available new less invasive techniques: for example the endovenous laser procedure that use laser energy.

Materials and Methods: In our Centro di Flebologia of Siena from December 2001 to December 2005 we have treated 120 LSV with endovenous laser procedure. Our casuistry is composed of 118 patients $(80 \% \mathrm{~F}, 20 \% \mathrm{M})$, average age 54 years, 64 left legs $(53 \%), 56$ right legs $(47 \%)$. The surgical $(95 \%)$ or percotaneus $(5 \%)$ entry point to LSV is under the knee in most of cases.

Results: Well tolerated by all patients, no scar to the groin, no skin burns, no paresthesias, no thrombogenic complications, transitory bruises, surgical and recovery time reduction. Complete obliteration of the vein in $90 \%$ of the vessels treated.
Discussion: At the end of our study we consider endovenous laser procedure as an important device for the treatment of the varicouse veins for the patiens and also for the surgeon.
15

\section{Intrascrotal Hernia of the Ureter}

R. Stano, D. de Tullio, D. Pellegrini, F. Messina,

S. Occhionorelli, G. Ferrocci, G.N. Cavallesco, G. Azzena

Department of General and Thoracic Surgery, Istituto di Clinica Chirurgica, S. Anna Universitary Hospital, Ferrara, Italy

Introduction: Scrotal ureteral hernia is an unusual event with possible serious surgical complications. A case of scrotal para-peritoneal ureteral hernia with acute renal failure is reported.

Methods: A left inguinoscrotal hernia associated to re-acutization of chronic renal failure with left hidronephrosis and oligo-anuria was found in a man, aged 79-year-old affected by right kidney atrophia.

Results: A retrograde pyelogram showed an ureter kinking. An ureter stenting improved the renal failure; CT scan with contrast revealed an inguinoscrotal sac with ileal handles and stented ureter. An umbelical-pubic laparotomic access showed a hernia containing ileal segments slided in the left scrotum, with the ureter fall back.

The hernial content was replaced in the abdomen, and the ureter in its anatomic locus. The hernial opening was closed using a pre-peritoneal polypropilene mesh. A partial improvement of the kidney function occurred in the early postoperative period; 7 days after surgery the patient was discharged without complications.

Discussion: Ureteral hernia is a rare, often misdiagnosed condition which affects male obese patients, aged 50-60 years old. The preoperative identification is really important in order to avoid possible damages to the ureter during the hernia repairing. The lack of symptoms is related to an increased surgical risk.

\section{6 \\ Necrotizing Fasciitis, a Real Surgical Emergency}

D. de Tullio, D. Pellegrini, R. Stano, L. Baiano, G. Resta, F. Messina, S. Occhionorelli, G. Azzena

Department of Surgical, Surgical Clinic Institute, Anesthesiological, Radiological Sciences, University of Ferrara, Italy

Introduction: Necrotizing Fasciitis (NF) is a rare infection of the soft tissues. A case of postoperative NF of the thigh in a perforation of the rectum due to a trauma is reported.

Methods: A 37 years old female patient, referred to our institution for a deep perineal wound due to a work incident, was found affected with a $4 \mathrm{~cm}$ laceration of the rectum posterior wall. An intestinal suture to close the perforation, multiple washes to clean up peritoneum and retro-peritoneal planes and a temporary colostomy to protect the intestinal suture were performed. 3 days after surgery fever 
with spikes occurred; a large abscess involving the thigh lateral surface, with crepitus, oedema and rash, was found. A second look revealed a large zone of necrosis involving subcutaneous and fascial tissues. Wide removal of the involved tissues till the nearer sane areas was performed.

Results: Final histology confirmed the NF diagnosis. 20 days after surgery our patient was discharged on the mend in good conditions and referred to our outpatient clinic; 4 months after the wound was found healed.

Discussion: Capillary thrombosis due to interleukins activated by bacteria introduced by traumas could be the 'primum movens' of the necrotic events which will involve fascia and subcutaneous tissues. NF mortality rate $(>75 \%$ for patients with delays in diagnosis/inadequate therapy) depends by early recognition and aggressive surgical debridement.

\section{7}

\section{The 'Suture Hook' Technique for Biportal Thoracoscopic Sympathectomy}

F. Messina, D. de Tullio, P. Maniscalco, D. Pellegrini, R. Stano, M. Santini, G. Cavallesco, G. Azzena

Department of Surgical, Surgical Clinic Institute, Anesthesiological, Radiological Sciences University of Ferrara, Italy

Introduction: Primary hyperhidrosis is a distressing condition characterized by excessive sweating of unknown cause, that affects $0.6-1 \%$ of the population involving the hands, feet, axillae and not frequently, face, trunk, and scalp. Conservative treatment is often unsuccessful. The treatment of choice for palmar hyperhidrosis is surgery. The aim of our study is to purpose a novel surgical thoracoscopic technique for the treatment of palmar hyperhidrosis, the 'Suture Hook Technique'.

Methods: A case of a 31 years old male, referred to our institution by neurologist after the patient had refused the treatment with botulinum toxine, is reported.

Results: The patient was discharged in the first postoperative day, without any complication. Follow-up data, obtained with a detailed standard questionnaire by telephone interview at 6-12 months, revealed the success of surgical procedure and the absence of compensatory sweating.

Discussion: The dual-port approach provided excellent visualization of the sympathetic chain up to the first rib. Suture hook technique moreover, gives us the opportunity to identify the correct level of proximal and distal section of T2-T3 sympathetic chain, avoiding sympathetic chain retraction after the first scissor's cut and making easier the uptaking of the resected ganglia. The surgical-time procedure is therefore reduced.
18

\section{Thyroid Microcarcinoma: An Actual Surgical Problem}

F. Messina, D. de Tullio, D. Pellegrini, R. Stano,

A. Colognesi, L. Possamai, S. Occhionorelli, G. Azzena

Department of Surgical, Anaesthesiological and

Radiological Sciences, Surgical Clinic Institute,

University of Ferrara, Italy

Introduction: The goal of this study was to evaluate the clinical behaviour of papillary microcarcinoma (PMA), by describing its histological features and our standard therapeutic strategy for this disease.

Methods: A retrospective chart review between January 2001 and February 2006 at our institution, identified 262 patients who underwent surgical therapy for thyroid disease. 55 patients for which histology demonstrated the presence of a PMC were selected to evaluate the role of surgical therapy.

Results: Final histopathology identified in 75 patients the presence of a thyroid carcinoma. Papillary thyroid microcarcinoma occurred in 55 patients. The preoperative indication to surgical therapy was in 16 cases multinodular goiter and in 39 cases thyroid suspicious nodule. A total thyroidectomy was performed in 44 patients; 11 patients underwent hemithyroidectomy. In all the patients who underwent to hemithyroidectomy, a completion to a total thyroidectomy was required. The final histology of the opposite lobe identified in 5 cases the presence of a single PMC focus.

Discussion: Patients affected with PMC, could present PMC occult foci also in the opposite lobe. Therefore in such selected cases a radical surgery can guarantee a correct oncologic follow-up avoiding that multifocal carcinoma localizations could be responsible of an early recurrence of the disease.

\section{9}

\section{Effect of Electrostimulation on Sphincter Function in Faecal Incontinence}

\section{R.R. Rago, M. De Luca, A. Castelli, F. Leopardi \\ I Divisione Clinicizzata di Chirurgia Generale, Ospedale Civile Maggiore, Verona}

Introduction: The aim of this prospective study was to analyse the outcome of electrostimulation of the pelvic floor in patients with faecal incontinence, as conservative treatment options.

Methods: 12 patient were treated by electrostimulation, $(\mathrm{M} / \mathrm{F}=5 / 7)$, range $55-79$ years mean age 68 years. All patients were treated with the following protocol: intermittent impulse with an electrical intensity varying from patient to patient. The treatment was performed during a 12 weeks session, three times a week for $30 \mathrm{~min}$ each.

Results: The results were evaluated prospectively by clinical classification using a Wexner continence score and anal manometry before and after 8 weeks of treatment. The training with anal electrostimulation increases anal squeeze and resting pressures, thus leading to an improvement in clinical incontinence symptoms. 
Conclusions: The anal electrostimulation should be the first choice in the therapy of faecal incontinence. A training period of 3 months seems to be sufficient.

\section{0}

\section{Surgical Treatment of Ascending Saphenous Thrombophlebitis}

L. Gazzabin, M. Bucalossi, S. Mancini, A. Comandi,

F. Berna, G. Botta, A. Gazzabin

Chirurgia Generale Terza, Siena

Introduction: Generally acute thrombophlebitis of the superficial veins of the lower limbs is considered as a common and not dangerous pathology; but sometimes it may evolve in a clinic pattern of extreme seriousness. Thrombotic process may spread from the superficial venous system to the deep one and then lead to a pulmonary embolism; this occours expecially in the thromboflebitis involving S-F junction, through a process called 'Ascending'. The best examination (100\% accuracy) is Duplex Scan, useful to define: distance, extent of the thrombus from the S-F junction and if it is 'floating' in CFV. We can treat this type of pathology in two ways: conservative and surgical.

Methods: From January 2001 to December 2005 we treated 236 patients $(70 \mathrm{M}-166 \mathrm{~F})$, middle age about 61 years old who presented acute ascending saphenous thronboplebitis. We performed saphenofemoral disconnection in 111 patients, and in 4 with 'floating' thrombus we associated femoral thrombectomy. The other patients was treated with a conservative method; EBPM and elastic stocking (II Kcl).

Results: Surgical treatment was performed in local anestesia without any pre-, post-operative complications; the patients came back home $24 \mathrm{~h}$ after the operation.

Discussion: In our experience surgical S-F disconnection results the best method to prevent trombo-embolic complications in ascending thromboplebitis involving the S-F junction.

\section{1}

\section{Surgical Management of Acute Cholecystitis: Our Experience}

\section{B. Genco, A. Castelli, I. Tomasi, M. De Luca, R.R. Rago, G. Borsellino \\ I Divisione Clinicizzata di Chirurgia Generale, Ospedale Civile Maggiore, Verona}

Background: Urgent laparoscopic cholecystectomy (LC) is recommended for acute cholecystitis (AC). However systemic associated diseases, severity grade of cholecystitis and association with common bile duct (CBD) stones either complicated and not make this approach questionable since greater conversion and morbidity rates have been reported.

Methods: We report a prospective study the aim of which was to evaluate a regimen treatment of acute gallstone cholecystitis (AGC). LC has been the first line treatment, however when associated diseases and/or severe cholecystitis were present a percutaneous cholecystostomy (PC) has been first performed in emergency setting while patient with $\mathrm{CBD}$ stones have been treated intra-operatively by the way of combined endoscopic and laparoscopic sphincterotomy for bile duct clearing.

Results: Study consist of $180 \mathrm{pz}$; male and 92 female with mean age of 62 years (range 22-93). Simple AC has been observed in $124 \mathrm{pz}$, complicated cholecystitis in $56 \mathrm{pz}$. LC was performed in $117 \mathrm{pz}$ with 5 conversion and a laparotomic cholecystectomy in $3 \mathrm{pz}$. PC was performed in $40 \mathrm{pz}$ with followed cholecystectomy in $32 \mathrm{pz}$, a combined endoscopic and surgical procedure for CBD clearance in $16 \mathrm{pz}$. No statistical differences has been observed in morbility and post-operative stay between patients with simple or complicated cholecystitis.

Conclusion: Urgent LC may be proposed in cases of AGC, however selective approach by the way of PC and intra-operative clearing of CBD make it possible to reduce increment of hospital stay, morbility and morbidity associated with such conditions.

\section{2 \\ Haemorrhoid Treatment with Rubber Band Ligation: Our Experience and Review of the Literature}

M. De Luca, A. Castelli, R.R. Rago, B. Genco, F. Leopardi

I Divisione Clinicizzata di Chirurgia Generale, Ospedale Civile Maggiore, Verona

Introduction: Haemorrhoidal disease is related to many predisposing factors, such as familiarity and excessive straining on defecation, constipation and diarrhoea. Nowadays both medical and surgical treatments are available according to the severity of the disease; among the surgical options, non exeretic procedures like rubber band ligation are widely used for symptomatic first- and second-degree haemorrhoids.

Objective: To value the efficacy and morbidity of rubber band ligation treatment of first- and second-degree haemorrhoids through the analysis of our personal experience and review of data of the literature.

Methods: From January 1995 to January 2005 in our Department, we treated 934 patients with a mean age of 43.7 years (M/F:1/1.41) affected by symptomatic first- and second-degree haemorrhoids with rubber band ligation for a total of 3,148 procedures ( 3 per patient).

Results: At the end of each session, we valued its efficacy through the execution of anoscopy and by collecting anamnestic data about symptoms resolution, morbidity and disease relapse. All our results were compared with those present in the literature.

Conclusion: According to our experience, the treatment of firstand second-degree haemorrhoids with rubber band ligation can be considered an effective treatment option not only for its feasibility in ambulatory settings, but also for its low cost and low morbidity. 
23

\section{Bulking Agents for Treatment of Fecal Incontinence: The Colorectal Eporediensis Centre Experience with Coaptite ${ }^{\circledR}$}

\author{
I. Giani, F. Marino, A. Realis Luc, G. Clerico, E. Ganio, \\ M. Trompetto \\ Dipartimento di Area Critica Medico Chirurgica, Università \\ degli Studi di Firenze. PAT CHIR 1-AOUC Careggi, Firenze
}

Introduction: Bulking agents for the treatment of fecal incontinence seem to be a good choice for selected patients. The goal of this study is to show the real efficacy of synthetic calcium hydroxylapatite Coaptite ${ }^{\circledR}$ as a bulking agents feasible for passive fecal incontinence in adults, for a period of 12 months of follow up.

Methods: Ten patients (nine women and a man) affected by passive fecal incontinence from September 2004 to February 2005 have been treated with injectable Coaptite implant. All patients have been selected by clinical history, physical examination and anorectal physiological evaluation, including anal endosonography and anal manometry. At 3,6 and 12 months after the procedure all patients have been assessed using FISS, FIQL and manometry.

Surgical Procedure: Under local anesthesia, $2.5 \mathrm{~cm}$ from the anal verge, directed by an anally placed digit, four injections of $1 \mathrm{ml}$ each have been distributed on the anal ring.

Results: $80 \%$ of the patients showed a significative reduction in FISS from 87.5 (10.1) to 13 (14.9) $\mathrm{p}<0.001$ up to 12 months followup, an improvement of the global FIQL and the manometric measurements showed an increasing of the anal canal resting pressure, without incidence of sepsis.

Conclusion: The injectable Coaptite is an effective treatment for passive fecal incontinence. The results of our study suggest that the perianal use of synthetic calcium hydroxylapatite is safe and with a good impact on quality of life.

\section{4}

\section{Acute Appendicitis?}

\section{Grassi, G. Bongini, S. Civitelli, G. Tanzini \\ General Department Surgery, Hospital S. Maria Le Scotte, University of the Studies of Siena}

Introduction: A subject, 60 aa, C.V., fever $38^{\circ} \mathrm{C}$, it is given shelter to and comes submitted to intervention of emicolectomia $\mathrm{dx}$ for acute appendicitis. It arrives in our department after $15 \mathrm{gg} \mathrm{p}$. or for sepsis generalized.

Methods: Comes submitted to a laparotomia esplorativa: rafia on the anastomosi and ileostomia. I give shelter to in intensive therapy. To the suspension of the sedazione the patient appears tasty, opens the alone eyes after intense stimulation, presence of fascicolazioni and clonie also facciali.

Results: The RMN puts down for a damage evocative encefalico of an encefalite of Wernike. Moves spontaneously but not contattabile né reacts to the stimuli after 15 days from the I awaken, presents hemodynamic building. The encefalite of W. has an incidence of $0.9 \%$ $\mathrm{d}$ is bound to a deficit of tiamina, in literature little cases of such pathology are described and to the moment were not pointed out cases of such awkward encefalite to an asepsi of abdominal orginine. It affirms, how it is shown in literature, that the development of the sepsis and the to last of a septic painting for about 30 days the cause was that given beginning to the development dell' encefalite.

Discussion: The surgical choice of the surgical second intervention has pointed out taken possession for the seat of the infection, for the presence of deiscenza and for the clinical conditions of the patient.

\section{5}

\section{GISTS}

I. Grassi, J. Pagiataki, A. Chessa, S. Civitelli, G. Tanzini

General Department Surgery, Hospital S. Maria Le Scotte, University of the Studies of Siena

Introduction: The gold-standard for the differential diagnosis is the positività for $\mathrm{CD} 34$ and $\mathrm{CD} 117$ and the negatività for the markers of the woven muscular smooth and of the neural cells. It hits the male (4:1), the age to the diagnosis, superior to 50 aa.

Methods: They are 5 the obstinate cases: stomach 3; fine 2, 1 duodeno, like in literature. Four of the ours you are patient were given shelter to of urgency for hemorrhage (3) and intestinal blockage (two). The main factors prognostici I am: the dimensions of the tumor and the indicator mitotico. In our experience, an alone neoplasia, of inferior dimensions to the centimeter, had a high indicator mitotico.

Results: The contribution proliferating was low but, in 4 cases, the diameter of the neoplasia was superior to $5 \mathrm{~cm}$. The patients were submitted to surgical resezione of the neoplasia, without linfadenectomia. The renewal of illness, enter two years from the treatment, in the $40-60 \%$.

Discussion: Therapy of the illness metastatica is medical: antibody monoclonale, STI571. The association of the GIST with at least a neoplasia epiteliale in two patients: in a case is been about an adenocarcinoma sincrono of the sigma, in the patient with pregresso carcinoma of the endometrio, in the other of a carcinoma of the kidney diagnosed to distance of you are months from the removal of the GIST.

\section{6}

\section{The Coronary Intestinal}

\section{Grassi, A. Chessa, S. Civitelli, G. Tanzini}

General Department Surgery, U. O. General Surgery I, Hospital S. Maria Le Scotte, University of the Studies of Siena

Introduction: I study it driver retrospective in the last 10 years and limit to subjects operated in which the ischemia was verified to the laparotomia or to the examination istologico.

Methods: $67 \mathrm{pz}$ (Middle $74.52 \pm 11.53$ aa). Eight with peritonite from profound perforation in the stroke ischemia. To the first evaluation, 6 already had been submitted to RCP and 24 were in state 
of shock or calculable evil clinician because calmed, intubati or doing not collaborate. 32 were in the immediate post-operating of var interventions. Risul: the seat of the ischemia was in the territory of the hook in 38 cases and in that of the hook in 22: between the two groups do not exist significant differences care the age, the factors of risk and the mortality post-operating.

Results: In 40 patients the ischemia is demonstrated during the hospitalization in another department: with respect to those arrived at our observation in first petition, you are had more often had an episode of low throw, but the seat of the ischemia, so like the other factors of risk and the mortality were superimposable.

Discussion: In the critical patients, particularly, the intestinal ischemia is able more often to be unacknowledged or malinterpretation and represents, finally, a significant risk of death.

27

\section{Splenomegalia: Houses Report}

I. Grassi, G. Bongini, S. Civitelli, G. Tanzini

General Department Surgery, U.O. General Surgery, Hospital S. Maria Le Scotte, University of the Studies of Siena

Introduction: A subject (M) and, of 35 aa, C.A., performed in 2002 an abdominal ultrasonography for epigastralgia.

Methods: The ultrasonography documented the presence of a neoformazione splenica of the dimensions of $32 \mathrm{~mm}$. November 2005 is emphasized a volumetric development of such formation. Later on Tac-Abdomen documented a solid injury, ipodensa, of $5 \mathrm{~cm}$ in middle region; besides documented in the superior III of the area splenica a small nodule ipodensa of $8 \mathrm{~mm}$, that never had been signalled in some examination I diagnose.

Results: In conformity to such report the patient a surgical intervention of splenectomy was submitted. The examination istologico documented a vascular neoplasia to coastal cells. The you istologici and immunoistochimici orientate verse emangioendotelioma to coastal cells. Immunoistochimica: HHV8, mibi, CD31, CD8, CD1a, lisosima. Macroscopically: Spleen of $400 \mathrm{~g}$ with neoformazione of the diameter of $3 \mathrm{~cm}$ of colored brown.

Discusssion: In the patients with neoformazione splenica, like in literature, come studied with instrumental examinations what ultrasonography, tomography, to the moment in which some anatomical alterations or clinical note themselves there is the indication to the surgical therapy. The surgical choice has pointed out taken possession for the seat of the injury for the presence of splenomegalia and for the clinical conditions of the patient.
28

\section{Diagnosis of Carcinoma in Multinodular Goiter by Electromagnetic Interactions. Preliminary Results}

\author{
A.M. Lucisano, N. Innaro, F. Pata, R. Sacco \\ General Surgery, 'Magna Graecia' University, \\ Catanzaro, Italy
}

Introduction: In the valuation of multinodular goiter (MNG), finding a malignant neoplasia is often an unexpected result of histological diagnosis. TRIMprob is a portable system for non-invasive diagnosis, that utilizes the different electromagnetic properties between healthy and pathological tissues, producing a low power magnetic field that interacts with molecular structure of tissues. The interference levels are detected by a receiver device and are elaborated with a software in a graph consisting in 3 bands easy interpretable. The objective of our study is to assess TRIMprob utility in a preoperative diagnosis of carcinoma in patients with MNG.

Methods: Between January 2005 and March 2006 we've screened with TRIMprob 50 patients with clinical diagnosis of MNG, then operated on total thyroidectomy; then we've compared the TRIMprob response with histological diagnosis.

Results: The TRIMprob results suggested 46 cases compatible with non-malignant goiter and 4 suspected cancers. The final histological diagnosis confirmed these results with 46 MNG and 4 papillary carcinomas. Sensibility, specificity and accuracy resulted to be $100 \%$.

Discussion: From these preliminary results, TRIMprob seems to be a method with high accuracy in detection of suspected carcinomas in MNG. If these results will be confirm, new prospects could be open in diagnosis of thyroid pathologies.

29

\section{Post-Operative Pain in the Milligan-Morgan (M-M) Technique vs. Laser (Surgical Fiber) vs. Stapled Circumferential Mucosectomy (L). The Authors' Experience}

\author{
F. Pata, F. Ferrari, A. Carpino, M. Cannistrà, R. Sacco \\ General Surgery, 'Magna Graecia' University, Catanzaro, \\ Italy
}

Introduction: The objective of this study is to assess the progression of post-operative pain following hemorrhoidectomy respecting different techniques.

Methods: From January 2001 to May 2003, 60 patients were classified and grouped according to the PATE 2000. Group 1, 15 patients presented circumferential prolapses, P4E4, treated according to the Milligan-Morgan (M-M) with laser. Group 2, 15 patients affected by circumferential prolapses, P4E4, treated according to the Longo tachnique (L). Group 3, 15 patients presented triple prolapses, P3E3, treated according to the Milligan-Morgan (M-M) with laser. Group 4, 15 patients presented circumferential prolapses, P4E4, treated according to the original Milligan-Morgan (M-M) technique. 
Results: In hemorrhoids classified as P4E4, the pain was better controlled in the Longo's Group: after $48 \mathrm{~h}$ the V.N.S. media was 2.3 (r.2-3) with $\mathrm{p}=0.0473$, in contrast to the Group 1, which was 3.3 (r.2-5) and Group 4, which was 3.9. In hemorrhoids P3E3, it's noticed that after $48 \mathrm{~h}$ the V.N.S. media was 2.8 (r.2-4) with $\mathrm{p}=0.0473$ : this result was the same as Longo's group.

Discussion: The post-operative pain is better controlled in hemorrhoids classified as P4E4 with Longo's technique. In hemorrhoids classified P3E3, the use of Milligan-Morgan with its variants, seems to be more advantageous for it's less expensive and because pain control is the same.

\section{0}

\section{Italian DRG in Abdominal Surgery. Is This an Efficient System?}

\author{
F. Pata, R. Cardona, R. Sacco \\ General Surgery, 'Magna Graecia' University, \\ Catanzaro, Italy
}

Introduction: DRG (Diagnosis-Related-Groups) System in Italy was introduced in the 1990s in order to reduce hospitalization times and sanitary costs. The purpose of this study is evaluating DRG system in abdominal surgery for showing its real efficacy.

Methods: The most frequent operations in Abdominal Surgery in 2004 were selected (colecistectomy, appendicectomy, inguinal hernioplasty, hemorrhoidectomy). DRG estimates of each operation were analysed according to different parameters: real cost, presence of complications, open vs. laparoscopic approach.

Results: Many disputable estimates were discovered. Colecistectomy DRG estimate (€2,811.81) is the same in open or laparoscopic approach, also associated with endoscopic sphincterectomy.

Open or laparoscopic appendicectomy cost $(€ 2,239.87)$ is evaluated all the same.

Even, estimate of the DRG cost of haemorrhoidectomy $(€ 1,800)$ doesn't consider higher costs with the use of laser, Ultracision, Ligasure in Milligan-Morgan technique or in stapled circumferential mucosectomy (Longo Technique).

Discussion: DRG system in Italy even if has been reduced hospitalization times, doesn't evaluate correctly a lot of procedures and it runs the risk of conditioning negatively the choice of procedure. It's necessary a periodical revision of DRG estimates in Abdominal Surgery, according to new procedures and indications of surgeons' associations.
31

\section{Surgical Indication for Distal Multifocal EGC}

C. Marfisi, P. Morgagni, D. Garcea, A. Avanzolini, D. Bettini,

A. Gardini, A. Lucchi, G. Vittimberga, F. Bazzocchi,

G. Castellini, A. Lattuneddu, D. Morgagni, V. Orlandi,

A. Zaccaroni

Dipartimento di Chirurgia Generale, U.O. Chirurgia Gastro-Endocrina-Mininvasiva, Ospedale MorgagniPierantoni Forlì

Introduction: Multifocality in Early gastric cancer (EGC) is quite common although the frequency varies from 3.4 to $13.3 \%$. Multiple EGC (mEGC) can cause problems of correct diagnosis with the risk to overtreat the patients.

Methods: We analyzed clinicopathologic characteristic of 31 mEGC over a period from 1976 to 2005 and the outcome of patients submitted to surgical treatment. All patients with an EGC localized at the upper third of the stomach were submitted to a total gastrectomy (GT), while subtotal gastrectomy (GST) was performed for lesions sited at the lower third. In any case surgical procedures were completed by D2 lymphadenectomy. No patients presented infiltration of the surgical line section and nobody was subjected to complementary treatment.

Results: A total of 519 patients with EGC underwent gastric resection in the examined period; 31 were diagnosed as EGC with multiple lesions according to Moertel criteria. 24 GST and 7 GT were performed. Multifocality was not considered as a negative prognostic factor or associated to lymph node metastasis. Five years survival is $80 \%$. Five patients recurred with distant metastasis: 2 submitted to GT had local recurrences but none operated on with GST recurred on the stump.

Discussion: GST is a justified treatment in distal mEGC. It's fundamental a careful examination of the entire stomach and a constant endoscopic follow-up.

\section{2}

\section{Permanent Sacral Nerve Stimulation for Treatment of Chronic Constipation}

\section{F. Marino, I. Giani, G. Clerico, A.R. Luc, E. Novelli, M. Trompetto, E. Ganio \\ Colorectal Eporediensis Centre, Gruppo Sanitario \\ Policlinico di Monza, Clinica S.Rita, Vercelli, Italy}

Introduction: Permanent Sacral Nerve Stimulation (SNS) has been proposed to improve bowel habits in patients with chronic constipation that have not responded to conservative treatments.

Methods: 9 patients ( 8 females), mean age 51 (range 28-79 years) affected by chronic constipation ( 7 obstructed defecation, 1 slow transit and 1 miscellaneous) submitted to permanent implant of SNS were followed up. Mean follow up was of 27.2 months (range 2-68 months). Changes from baseline in Straining score (SC) and Cleveland Clinic constipation score (CCS) were evaluated at 24 months of follow up.

Results: All patients had derived significant benefit from SNS: mean SC value improved from 14.9 (4-29) to $1.3(0-4), p=0.0008$ and mean CCS value decreased from $10.7(2-18)$ to $1.3(0-4)$, 
$p=0.0004$. One implant was removed due to the beginning of recurrent cystitis while another device after becoming infected after 26 and 23 months, respectively.

Discussion: Despite infection appears to be the main potential complication, SNS seems to be a safe, effective and durable treatment for patients with intractable chronic constipation. However, further studies with larger number of patients and longer follow up are required.

\section{3}

One-day Thyroid Surgery: Retrospective Analysis of Safety and Patient Satisfaction on a Consecutive Series of 1,571 Cases in a Three-year Period

\author{
G. Materazzi ${ }^{1}$, G.L. Dionigi ${ }^{2}$, P. Berti ${ }^{1}$, M. Puccini ${ }^{1}$, \\ G. Frustaci ${ }^{1}$, P. Miccoli ${ }^{1}$ \\ ${ }^{1}$ Department of Surgery, University of Pisa, Pisa, \\ University of Insubria, Varese, Italy
}

Background: Short-stay thyroid surgery ( $<24 \mathrm{~h}$ hospital stay) is becoming increasingly popular but some potentially lethal complications are considered strong arguments against shortening hospitalization after thyroidectomy.

Method: The authors reviewed the data of 1,571 patients undergoing one day thyroid surgery in a 3-year period to determine safety and patient satisfaction.

Results: There were 1,244 females and 327 males. Mean age was 43 years. $65 \%$ of the operations were performed for benign disease. Total thyroidectomy was performed in 1,119 patients $(71 \%)$, hemithyroidectomy in $450(29 \%)$, isthmusectomy in 2. Patient satisfaction was analyzed by a questionnaire for $90 \%$ of patients, and the post-discharge surgical recovery scale for 150 randomly selected patients. Morbidity occurred in 162 patients $(10.3 \%)$. Complications were transient hypocalcemia in 112 patients $(10.1 \%$ of 1,119$)$ and permanent hypoparathyroidism in 3 patients $(0.2 \%$ of 1,119$)$. Transient monolateral recurrent nerve paralysis occurred in 10 patients $(0.6 \%$ of 1,571$)$, transient bilateral recurrent nerve palsy in 3, definitive monolateral recurrent palsy in $4(0.2 \%)$, postoperative bleeding requiring re-intervention in $10(0.6 \%)$

Conclusion: Our results confirm that one-day surgery for thyroid disease is safe, effective, and more acceptable for patients and relatives if compared to thyroidectomy performed with standard hospitalization ( $48 \mathrm{~h})$.

34

\section{Immunostimulation to Reduce Recurrences After Surgery for Anal Condyloma Acuminatum}

S. Cornaglia, M. Mistrangelo, M. Pizzio, A. Mussa

Sezione di Chirurgia Oncologica dell'Università degli Studi di Torino, Dir. Prof. A. Mussa, Ospedale Molinette, Torino

Introduction: Anogenital warts are the most common sexually transmitted disease seen in surgical practice, found in up to $1.7 \%$ of the population. They cause emotional distress for its marked tendency to recurrence. Immunosuppression is an important risk factor.

Patients and Methods: At the Oncological Surgical Department 57 patients were submitted to surgical excision of perianal and endoanal condylomata and were immunostimulated, for 20 days with a natural product. We use a product containing Echinacea, Uncaria, Tabebuja, Papaya, grapefruit and Andrographis. This group was compared with a group operated without any immunostimulation. Control group were 262 patients. HIV+ patients were excluded from the study.

Results: Follow up was performed for 6 months after surgery. After 1 month we observed recurrences in $4.84 \%$ of study group vs. $15.74 \%$ in control group ( $\chi^{2} 0.0231$, confirmed with Fisher's test $)$; while after 6 months recurrences were $4.44 \%$ in study group vs. $11.75 \%$ in control one $\left(\chi^{2} 0.1439\right)$. Total amount of recurrences were $8.06 \%$ in study group, vs. $23.84 \%$ in control group $\chi^{2}$ of 0.0053 and a Fisher's test Pr of 0.0025 .

Conclusions: Considering these results, immunostimulation in patients submitted to surgery for anal condylomata is useful to reduce recurrences. The use of a natural product guarantee good results without the risk of side effects.

35

\section{Traumatic Lesions of the Diaphragm. Experience from a General and Thoracic Surgery Unit}

\author{
A.F. Bravo, G.L. Baiocchi, M. Benvenuti, D. Benetti, \\ G.L. Pariscenti, M. Cantu, G.A.M. Tiberio, S.M. Giulini \\ Clinica chirurgica, Brescia
}

Introduction: The aim of this retrospective work is to investigate the treatment modalities and the results of traumatic diaphragmatic rupture through the analysis of two different series observed in a General (group 1) or a Thoracic (group 2) Surgery Unit.

Methods: We reviewed 77 patients operated on for diaphragmatic injuries between 1980 and 2005 (36 vs. 41). Sixty-eight patients were observed immediately after the trauma, while 9 had a delayed presentation (2-216 months). In the acute presentation group, trauma was blunt in $83 \%$ of the cases and penetrating in $17 \%$; associated lesions were observed in $87 \%$ of cases. Surgical approach was dictated by the life-threatening lesions. The clinical data of the two groups of patients are compared.

Results: In the patients with acute presentation, hemorrhagic shock was more frequent in group 1 (47\% vs. 16\%); respiratory distress in group $2(33 \%$ vs. 55\%). Associated lesions were mainly abdominal in group $1(80 \%$ vs. $40 \%)$ and pulmonary in group $2(7 \%$ vs. $66 \%)$. The delayed presentation was more frequent in group $1(17 \%$ vs. $7 \%$ ), and requiring emergency surgery. In the two groups mortality and morbility were $22 \%$ vs. $20 \%$, and $53 \%$ vs. $76 \%$, respectively.

Discussion: When the patient is observed in the acute phase, the dominating and life-threatening lesions dictate the surgical approach. The surgical exploration is mandatory whenever a diaphragmatic rupture is suspected. 
36

Pre-Operative and Post-Operative Peristaltic Activity Evaluation in Achalasic Patients: 24-hour Esophageal Ambulatory Manometry Study

L. Monaco, G. Izzo, A. Cosenza, A. Renzi, L. Vicenzo,

F. Torelli, A. Basciotti, A. Brillantino, M. Schettino,

F. La Mura, N. Di Martino

VIII Chirurgia Generale e Fisiopatologia Gastroenterologica, Seconda Università degli Studi di Napoli

Introduction: The aim of this work was to evaluate, using a 24 hour esophageal manometry, the motor patterns of the esophageal achalasia before and after Heller's myotomy and Nissen's fundoplication with more accuracy comparing to the stationary manometry. We also wanted to stress any correlation between clinical results after treatment and the observed modifications of the motor activity.

Methods: 25 achalasic patients underwent a 24 hour esophageal manometry using a portable manometric system connected to a probe with 4 solid state pressure microtransducers. Then they all had a laparoscopic surgical treatment consisting in Heller's myotomy and Nissen fundoplication and a further 24 hour manometry during the follow-up.

Results: We observed a statistically significant increase of the amplitude of contractions and an increase, but not statistically significant, of the frequency and the duration of contractions. Furthermore the study of peristalsis showed an increase of peristaltic activity in total and complete sequences and a decrement of dropped and interrupted peristaltic sequences.

Discussion: Our data show the presence of a residual peristaltic activity in $31.2 \%$ of patients and complete sequences were observed in $20 \%$ of the achalasic esophageal bodies. We also found a significant qualitative and quantitative improvement of the esophageal motor activity after treatment, particularly for the peristaltic sequences and the capacity of the esophagus to produce post-deglutitive sequences. This seem to confirm the positive effect of the HM and, on the other side, the absence of any effect of the NF on the esophageal transit.

37

\section{Multimodal Strategies in the Treatment of Advanced Gastric Cancer: Preliminary} Results

G. Mura, A. Vagliasindi, M. Framarini, D. Cavaliere, R. Bertelli, F. Tauceri, R. Galassi ${ }^{1}$, F. Matteucci ${ }^{1}$, C. Milandri ${ }^{2}$, P. Rosetti ${ }^{2}$, L. Saragoni ${ }^{3}$, G.M. Verdecchia

UO Chirurgia e Terapie Oncologiche Avanzate

${ }^{1}$ Medicina Nucleare, 2 UO Oncologia, ${ }^{3}$ Anatomia Patologica e Citodiagnostica, Forlì

Introduction: Gastric cancer in Western countries is often diagnosed in an advanced stage, with poor prognosis. Advanced gastric cancers are nowadays increasingly approached with multimodal strategies.
Methods and Results: Five patients with clinical diagnosis of T3-T4, confirmed by laparoscopy, without extra-abdominal metastasis, submitted to neoadjuvant chemotherapy (PELF), are enrolled in present study. All patients were positive at baseline FDG-PET evaluation and 'metabolic responders' (positive response to chemotherapy) at mid treatment FDG-PET evaluation. 3 of them, nowadays, completed the therapy and 28-35 days after the end of therapy underwent D2-D3 gastrectomy and complete debulking of the neoplasia (R0), followed by hyperthermic peritoneal chemo-perfusion (HPCP) with Cisplatin plus Mitomycin. Patients began per os realimentation at a medium time of 15 days; mean hospital-stay was 28 days. All patients are alive and disease-free. There were two cases of subtotal regression of the neoplastic mass (grade 1 - Becker classification) and one case of partial regression (grade 2).

Discussion: Neoadjuvant chemotherapy could improve both resectability and survival. HPCP could reduce the local and peritoneal recurrences. Our preliminary data confirm the role of FDG-PET in differentiating responsive and not-responsive patients during preoperative therapy.

38

\section{Unusual Multiple Complications of Acute Biliary Pancreatitis: A Case Report}

A. Vagliasindi, R. Bertelli, G. Mura, M. Framarini,

D. Cavaliere, G. Solfrini, F. Tauceri, G.M. Verdecchia

UO di Chirurgia e Terapie Oncologiche Avanzate.

Ospedale G.B. Morgagni, L Pierantoni di Forlì

Introduction: Acute pancreatitis is a complication of biliary lithiasis.

Methods and Results: We report the case of a 62 years old male patient who was referred to a medical centre for treatment of acute biliary pancreatitis. A conservative treatment was applied with benefit. The patient was discharged and cholecystectomy programmed. After 2 months the patient represented with acute cholecystitis. The CT scan described a big pseudocyst involving pancreas' body and tail and another smallest one in pancreas'head. Cholecystectomy was performed with drainage of the bigger cyst by cystojejunostomy. The postoperative course was characterized by stenosis of the choledocic duct due to compression by the cefalo-pancreatic pseudocyst; the patient underwent endoscopic sphincterectomy and positioning of an endo biliary prosthesis with resolution of the symptomatology. After 4 months the patient was referred to our hospital with recurrent episodes of acute bleeding by gastric varices secondary to splenic vein thrombosis. Splenectomy was executed and the postoperative course was uneventful. The patient is still well and alive.

Discussion: Biliary pancreatitis is a disease with potential high morbidity. The current recommendation is for cholecystectomy to be performed during the same hospital admission or as soon as possible after resolution of the pancreatitis. 
39

\section{Predictive Factors of Conversion of the Laparoscopic Cholecystectomy}

\author{
T.P. Valentino, C. Santacroce, A. Fersini, N. Tartaglia, \\ F. Samele, A. Ambrosi, V. Neri \\ Department of Surgical Sciences, Division of General \\ Surgery, Polyclinic of Foggia, University of Foggia, \\ Foggia, Italy
}

Introduction: Still today, in the trials of laparoscopic cholecystectomies (LC) are noticed conversions to the open procedure, even if with an unimportant incidence. The aim of the study is to find the factors that conditioned the conversions.

Patients and Methods: In the period 1997-2004 were treated 315 patients with cholelithiasis and underwent to a LC, in the Department of Surgical Sciences of the University of Foggia; mean age 54 years, 120 males, 195 females; the diagnosis was: 188 patients with simple cholelithiasis, 69 with acute cholecystitis started from 7 days in mean, 57 with acute biliary pancreatitis, 1 cholelithiasis in hepatic cirrhosis. In 6 patients there was conversion to the open procedure: 5 males, 1 female, mean age 60 years, mean BMI of 30.8 .

Results: The considered parameters were: sex, age, gallbladder acute phlogosis (from 8 days), ultrasonographic thickness of the gallbladder wall (more than $6 \mathrm{~mm}$ ), portal hypertension from cirrhosis. Five converted patients had acute cholecystitis $(83 \%)$, five were of male gender $(83 \%)$, four had BMI more than $30(66 \%)$, and one patient had portal hypertension (16\%).

Conclusions: The analysis of our casuistic has shown that the following data are cause of conversion: the previous phlogosis (causes the sclerosis of the gallbladder infudibular-neck-cystic region and of the Calot's triangle, than it modifies the anatomical relations among the structures), male gender, BMI more than 30 (abundance of adipose tissue in the hepatic-duodenal ligament and of the infundibulus), and the portal hypertension.

40

\section{Timing of the Endoscopic Sphincterotomy (ES) and of the Videolaparocholecystectomy (VLC) in the Acute Biliary Pancreatitis}

C. Santacroce, T.P. Valentino, A. Fersini, N. Tartaglia, F. Samele, A. Ambrosi, V. Neri

Department of Surgical Sciences, Division of General Surgery, Polyclinic of Foggia, University of Foggia, Foggia, Italy

Introduction: There is not uniformity in the literature about the timing of execution of the endoscopic sphincterotomy (ES) and later the videolaparocholecystectomy (VLC) in course of acute biliary pancreatitis (ABP): the aim of the study was to suggest the optimal timing.

Patients and Methods: In the period September 1997-November 2004, 67 patients were treated for ABP. Fifteen cases were severe ABP and 52 were mild ABP. In 55 patients an ES was executed within 48-72 h; in 12 cases the ES was delayed of 10 days. After 8-10 days, 62 patients had a VLC; 5 patients had an open cholecystectomy.
Results: The coledochal stone was removed in 32 cases (47.7\%); in the last 35 patients $(52.3 \%)$ the biliary sand or sludge was removed. Immediate results: 1 case $(1.5 \%)$ of pancreatitis reacutizzation, 6 cases $(9.8 \%)$ increase of the lypase and amylase, 2 (2.9\%) duodenal perforations. The cholecystectomy, laparoscopic and open, did not have relevant complications. Later in time we have registered the develop of 3 postnecrotic pancreatic pseudocysts, treated with surgical therapy.

Conclusions: The ES in course of severe and mild ABP has the double goal to clean the principal biliary duct by the stones, and moreover to remove the papillar obstacle because of stenosis, biliary sand or sludge. In our experience, the golden therapeutic timing foresees the ES within 48-72 $\mathrm{h}$ from the beginning of the symptomatology and the VLC within $8-10$ days: this time is necessary to establish the absence the progression of the acute pancreatitis.

41

\section{Duration of Perioperative Prophylaxis of Thromboembolism in High-Risk Oncological Patients}

F. Rizzuti, F. Attene, F. Scognamillo, C. Pala, A. Xidas, M. Trignano

Ist. Patologia Chirurgica, Sassari

Introduction: The rate of Venous Thromboembolism (VTE) in neoplastic patients undergoing surgical procedure is bigger then in non neoplastic patients undergoing surgery. Different published studies show increase of VTE between 15th and 30th day post-operative. The aim of our study was evaluate the ideal duration of prophylaxis in selected high-risk patients with cancer of gastrointestinal tract.

Methods: From November 2003 to February 2006 we enrolled 90 patients with gastrointestinal tract cancer, with one or more of the following risk factor: age $>60$, obesity, congenital or acquired thrombophylia, chronic venous insufficiency, previous thromboembolic events. Patients were divided in two homogeneous arms. Both groups undergone prophylaxis with compression stockings, eraly mobilization, calcic nadroparin 0.3 UI once daily, for 15 days the first arm, for 30 days the second. All patients have a color doppler-US in the 5 th, 20th and 30th post-operative day.

Results: Four of 90 patients (4.4\%) developed a VTE diagnosed at third control. Three of the 4 in the first arm and one in the second. None pulmonary embolism.

Discussion: We notice that extended out of hospital Low Molecular Weigh Heparine prophylaxis until the 30th post-operative day, in patients undergone surgery fot gastrointestinal tract cancer, may reduce the incidence of clinically important thrombolic events. 
42

\section{Acute Cecal Necrosis: Report of a Case}

M. Grande, A. Crocoli, G.M. Attinà, C. Nigro, A.M. Farinon

General Surgery Chair, Università degli Studi di Roma, 'Tor Vergata'

Introduction: Acute cecal necrosis is a rare entity which may develop secondary from several causes. Pathogenesis could be related either to occlusive or non occlusive causes.

Methods and Results: A 37 years old caucasian female was admitted to the 'Tor Vergata' Hospital with fever, right and epigastric abdominal pain of sudden onset, nausea and vomiting. The patient had a story of several surgical operations. Physical examination: abdominal distension, tenderness, especially on right side. Blumberg and Giordano were positive. Peristalsis was absent. CT showed aerobilia, distension of intestinal handles. No abdominal or pelvic abscess was evident. Explorative laparotomy was performed. Cecal necrosis with a clear demarcation between the necrotic tract of the colon and the other part of this was discovered. Ileocecal resection with mechanical anastomosis was performed. Histopathology revealed cecal mucosal necrosis, acute transparietal flogosis, diffuse vessel thrombosis. Patient was dismissed from hospital in 7th p.o. day in quite good general condition.

Discussion: Cecal ischemia should be included in the differential diagnosis when patient presents acute right lower quadrant pain, particularly if the patient is elderly or has predisposing risk factors. Intestinal ischemic necrosis, especially of the cecum, rarely occurs in the absence of diffuse vascular disease; even if in our case we don't have evidence of such pathology. If the premium movements of the vessel thrombosis may be ascribed by improper use of oral contraceptives or analgesics, or alternatively at the post traumatic osteonecrosis is not verified.

\section{3}

Comparison of Laparoscopic Colectomy with 'Open' Surgery in the Treatment of Colonic Diverticulitis

G. Bissolotti, P. Bisagni, E. Orsenigo, S. Di Palo, A. Tamburini, C. Staudacher

Department of Surgery, San Raffaele Scientific Institute, University Vita-Salute, Milano

Background: Sigmoid colectomy for diverticulitis can be technically challenging because of severe inflammation. In order to evaluate the results of minimally invasive surgery in the treatment of colonic diverticulitis, we have retrospectively analysed our database, comparing the results of the laparoscopic approach (LPS) with open surgery (LPT).

Materials and Methods: We retrospectively reviewed our database from 1993 to December 2005 and compared the intraoperative and postoperative data of patients who underwent laparoscopic or conventional open surgery for diverticulitis. A total of 203 patients (median age: 63 years) have been submitted to colonic resection for diverticulitis [LPS: $\mathrm{n}=73 \quad(36 \%)$; LPT: $\mathrm{n}=130 \quad(64 \%)$ ].
Uncomplicated diverticulitis was the most common indication for surgery (79\%). Complicated patients (with abscess, fistulas, etc) were $41(21 \%)$. In 194 patients (96\%) a sigmoid colectomy and in $7(3.5 \%)$ a left hemicolectomy has been performed; in only 2 cases $(0.5 \%)$ a right hemicolectomy has been performed.

Results: The mean operating time was 210 and $160 \mathrm{~min}$ ( $p=0.045)$, blood loss 185 and $217 \mathrm{~min}(\mathrm{p}=\mathrm{NS})$ and mean time of hospital stay 8 and 11 days in LPS and LPT group $(\mathrm{p}=0.040)$, respectively. Conversion rate for the laparoscopic group was $6.5 \%$. Morbidity rate was similar (20 and $21 \%$ in LPS and LPT group).

Conclusions: Laparoscopic colectomy appears to be a reliable treatment for diverticular disease.

44

\section{Laparoscopic Excision of Gastrointestinal Stromal Tumour of the Duodenum}

\section{G. Bissolotti, E. Orsenigo, S. Di Palo, C. Staudacher}

Department of Surgery, San Raffaele Scientific Institute, University Vita-Salute, Milano

Background: Benign duodenal tumours are rare and less common than malignant tumours. They comprise a wide variety of pathologies such as gastrointestinal stromal tumour (GIST), schwannoma etc. A duodenal location is rare. Therapy consists in the radical excision of the tumour.

Methods: We therefore report a case of a gastrointestinal stromal tumour measuring $2.5 \mathrm{~cm}$ in diameter, arising from the lateral wall of the second portion of the duodenum about $2 \mathrm{~cm}$ below the papilla of Vater. Because of this rare location, a very invasive procedure (duodenopancreatectomy) might have been required for tumour resection. We avoided this operation and implemented an alternative solution.

Results: A laparoscopic resection of the duodenal tumour was successfully completed. Operating time was $200 \mathrm{~min}$ and blood loss $50 \mathrm{ml}$. The post-operative course was uneventful. Histopathological examination revealed that the duodenal submucosal tumor consisted of spindle cells, and immunohistochemical analysis revealed positive tumor staining for CD117, CD34 S-100. Based on these findings, the tumour was diagnosed as a GIST of low-grade malignancy, classified as the muscular type.

Conclusions: Despite laparoscopic surgery requires more complex technique than open surgery and acquisition of advanced laparoscopic skills depending on prior laparoscopic experience, laparoscopic excision should be considered as a valid treatment for duodenal GIST. 


\section{5 \\ Primary Malignant Melanoma of the Rectum: Case Report}

W. Bugiantella, E. Cavazzoni, D. Severini, E. Carbone, A. Contine, N. Gullà

Department of Surgery, Section of General and Emergency Surgery, University of Perugia, Santa Maria della Misericordia Hospital, Perugia, Italy

Introduction: Primary Malignant Melanoma (PMM) arising from the digestive, respiratory or genitourinary tract is extremely rare. Rectal PMM accounts for $0.4-1.6 \%$ of all melanomas; it is usually advanced at initial presentation with a poor prognosis and a 5-year survival below $10 \%$.

Methods: A 79 years old man presenting change in bowel habit underwent a colonoscopy showing a $1.5 \mathrm{~cm}$ mass in the posterior wall of the rectum, $2 \mathrm{~cm}$ above the pectinea line. Bioptic examination revealed amelanotic malignant melanoma. Total body CT, 18FDGPET CT, dermatologic, ocular and ORL examination showed no evidence of other tumour sites or metastasis. An abdomino-perineal resection (APR) with total mesorectal excision was performed without dissection of the internal and external iliac nodes.

Results: PMM is a rare aggressive malignancy associated to an high rate of local recurrences and metastasis. Initial symptoms are often non-specific (mild rectal bleeding, anal pain or change in bowel habits) being easily confused with benign anal disorders. Radical surgical approach (APR) with or without intra-abdominal pelvic lymph node dissection is generally advocated, however some series suggest that wide local excision followed by radiation therapy may have similar survival outcomes. Moreover prophylactic lymph node dissection has not proven to have any prognostic benefit.

Discussion: Early diagnosis of rectal PMM would improve the survival of the disease. Treatment recommendations are not standardized and tend to be individualized. Despite extended resections, poor survival rates indicate that new approaches should be investigated.

46

\section{Surgical Experience with Zeus Robotic System: Critical Aspects and Perspectives}

\author{
G. Fracastoro, G. Borzellino, A. Castelli
}

$I^{\wedge}$ Divisione Clinicizzata di Chirurgia Generale-Direttore

Prof. C. Cordiano, Ospedale Civile Maggiore di Verona

Objective: Actually mini invasive surgery can relay on complex informatic systems (Computer Assisted Surgery) like robotics, telementoring and telepresence. Zeus and da Vinci robotic surgical system are used in more than 120 centres worldwide and their applications in general surgery have been widely applied. We report our experience with Zeus robotic surgical system.

Methods: In our Department between December 2003 and March 2005, after a training with sperimental procedures, 28 operations (22 cholecystectomies, 4 anti-reflux surgery and 2 left colonic resections) were performed.
Results: All the operations were carried out with the robotic assistance; the mean operative time, except for the setting time, was the same as for the laparoscopic technique. No intra- or post-operative complications occured.

Discussion: According to our experience, robotic surgical systems allow a better comfort for the surgeon, but don't reduce the mean operation time or the mean post-operative hospital stay. Moreover, the high cost of the robot and the disposable material, the absence of force feed-back, the size and complexity of the robotic system, don't still allow a routine use of these systems.

\section{7 \\ D2 Lymph Node Dissection in Gastric Cancer Surgery}

S. Celotti, G.B. Steffano, P. Lagoussis, P. Veronesi,

A. Saccone, A. Vignati, R. Pozzi, G. Puletti, M. Mauri

Department of General Surgery, Istituto Policlinico San

Donato, via Morandi n³0 20097 San Donato Milanese

Introduction: D2 LND, proposed as standard treatment in gastric cancer (GC) resection, still remains controversial.

Methods: From 2001 to 2006, 41 patients had a GC resection. D1 group had a N1 LND (22 patients) and D2 group had a N1+N2 LND (19 patients). Disease staging was respectively in D1 and D2 group: stage I 5 and 2, II 5 and 5, III 5 and 7, IV 5 and 5. We had 2 EGC in D1 group. Total gastrectomy was performed in 12 and 17 cases and a subtotal gastrectomy in 10 and 2 cases in D1 and D2 group respectively.

Results: Average number of resected nodes was 17.9 in D1 and 37.5 in D2 group. We had 1 splenectomy for i.o. bleeding in both groups ( $4.5 \%$ in $\mathrm{D} 1,5.3 \%$ in D2). We had post-operative complications in 6 patients in D1 group (27.3\%) and in 5 patients in D2 group (26.3\%). Post-operative mortality was $4.5 \%$ (1 patient in D1 group). The average follow-up was 17.85 months (1-53) and 8 patients were lost at follow up. In D1 group 10 patients (71.4\%) are still alive ( 9 disease free) and 4 died for disease progression. In D2 group 10 patients (55.5\%) are still alive and disease free and 8 patients died for disease progression.

Discussion: Our opinion is that D2 LND for GC resection allows a better staging without increased intra and post-operative complications.

\section{8}

\section{Gastro Intestinal Stromal Tumors: The Experience in a General Surgical Unit} A. Chessa, N. Di Mari ${ }^{1}$, I. Grassi, C. Vindigni ${ }^{1}$, S. Civitelli,
G. Tanzini
Department of Surgery, I General Surgery Division, University of Siena, 'Department of Pathology, University of Siena

Introduction: GISTs the most common mesenchymal lesions of the gastrointestinal tract ( $<1 \%$ of all neoplasm's), preferentially 
located in the stomach and the small intestine, originate from interstitial cells of Cajal. Clinical presentation is variable, from vague complaints to major bleeding, abdominal mass, occlusion so GISTs are definitively detected on pathological features and immunohistochemical staining $(\mathrm{CD} 117+)$. Surgical resection is the mainstay of treatment; 5 years survival from 20 to $88 \%$ depends on grading and completeness of surgical resection. Imatinib mesylate, provides a target therapy, an encouraging option for high risk GISTs.

Methods: We reviewed medical records of patients treated for GISTs in our institution.

Results: From 1995 to 2005,10 patients $(8 \mathrm{M} / 2 \mathrm{~F}$ mean age 66.2 range 40-86 years) underwent complete surgical resection for GISTs. The most frequent presentations were gastrointestinal bleeding and intestinal occlusion. Stomach was the most common site of localization. Mean tumor size was $4.5 \mathrm{~cm}$ (range $2-19 \mathrm{~cm}$ ); in 5 cases the diameter was $<5 \mathrm{~cm}, 5-10 \mathrm{~cm}$ in 3 cases, $>10 \mathrm{~cm}$ in 2 cases. According to Fletcher classification, GISTs were at very low-low risk in 3 cases, at intermediate-high risk in 7 cases. Association with other tumors occurred in 3 cases. 5 years survival rate was $80 \%$.

Discussion: GISTs'prognosis in aggressive forms, enhances follow up value and new therapy chance.

\section{9 \\ Crohn's Disease-Associated Carcinoma: The Malignant Potential of an Inflammatory Bowel Disease}

\section{A. Chessa, A. Fontani, I. Grassi, J. Martellucci, S. Civitelli, G. Tanzini \\ Department of Surgery, I General Surgery Division, University of Siena}

Introduction: Different studies have reported an increased risk for intestinal cancer in patients with Crohn's disease although it occurs in less than $1 \%$ of cases.

Methods: We report the cases of 2 patients treated for a colon cancer with a sinchronous Crohn's disease detected on the operating table and confirmed on pathological specimen.

Results: A 70 years old man, with no signs of intestinal occlusion presents with fever. A pancolonoscopy reveales a right colon cancer. At laparotomy an adenocarcinoma of the ascending colon (pT4N0M0/G2) involving the distal ileal loop is confirmed. Both intestinal tracts presents with inflammation suggesting a Crohn's disease than reported by histhologic examination. After chemotherapy this patient is alive without disease at 24 months. A 68 years old man presents mucous diarrhea, anemia and weight loss. An abdominal CT detects an ileo sigmoid fistulization that seems to involve the bladder. At laparotomy are performed a left colectomy, an ileal resection and an upper bladder resection. The pathologic report deels with an ileocolic Crohn's disease with a sigmoid adenocarcinoma on enterocolic fistula (pT4N0M0/G3). After 18 months from chemotherapy anastomotic cancer recurrence with peritoneal and pelvic seeding indicate palliative surgery.

Discussion: A lot of evidences in Literature suggest the advisability of surveillance for Crohn's disease because the long duration of IBD seems the main risk factor for transformation; IBD symptoms may obscure malignancy and delay diagnosis. In our cases is probably overimposed cancer to reveal IBD and to make the surgical treatment mandatory.

\section{0}

\section{Laparoscopic Cholecystectomy in Day Surgery: A Three-Year Experience}

S. Cuccomarino, C. Monticoni, M. Agresti, F. Aprà, F. Finelli, A. Jannaci, V. Margarita, P. Milone, M. Ponsetto, A. Ravera, P. Raviola, S. Sottimano, C. Ferraris

Introduction: Day Surgery Laparoscopic Cholecystectomy (DSLC) is a standardised procedure since over 15 years. Nevertheless, it's not widely spread in Italian hospitals. We report our data about the DSLC between September 2002 and March 2006.

Methods: In this period, we performed 185 DSLC; $140(75.5 \%)$ were female, and $45(24.3 \%)$ male. Females were $50.4 \pm 15.3$ years old, males $55.37 \pm 14.37$ years old. All surgeons in our department are fully skilled in performing this operation. We performed standard surgical technique, with pneumoperitoneum at $12 \mathrm{~mm} \mathrm{Hg}$ and 4 trocarts. In 2 cases, we contextually performed an intraoperative cholangiography. At the end of surgery, we always positioned a subhepatic drainage through the right flank trocart; this drainage was always removed before discharge. Patients did stay in hospital the night of surgery, and then were discharged next morning.

Results: There were 2 conversions to open cholecystectomy, for severe cholecysto-pericholecystitis and for a big mold stone. In these cases, the patients did prolong their hospital stay. Other causes of prolonged hospitalization were 2 biliary leakages (one for abnormal hepatobiliary duct from VI hepatic segment to the gallbladder, not recognized at surgery) and 3 cases of persistent postoperative pain. We never performed a reoperation.

Discussion: DSLC is a standardized, safe and economic procedure. The postoperative control of complications is fully allowed by the subhepatic drainage. An adequate intra and postoperative anaesthesiologic assistance and a complete ability of the surgical team allow the routinary performing of this methodic in our Hospital.

\section{1}

\section{Need for Process Monitoring and EBM Founded Risk Management in Surgery: Mapping before Remodelling}

M. D'Ambra, F. Rubba, A. Della Vecchia, S. Di Lillo P. Cuccaro, D. Capone, M.V. Scafarto, S. Panico, M. Triassi, G.P. Ferulano

Scuola di Dottorato in Management Sanitario, Università Federico II Napoli

Introduction: Reliability is defined as failure-free operation over time. In health care, this definition connects to several aims for the health care system, particularly effectiveness (where failure can result from not applying evidence), timeliness (where failure results from not taking action in the required time), and patient-centeredness 
(where failure results from not complying with patients' values and preferences). Reliability is measured as 'Number of actions that achieve the intended result/Total number of actions taken' and failure rate calculated as 1 - Reliability, or 'unreliability,' as an index. Albeit it represents a strategic and trendy methodology, the remodelling of the care pathways per se may be cost-expensive and not always crucially determinant.

Methods: We found the prevalent General Surgery procedures among the University Hospital Federico II in-patients and we tried to map the risk of converting patient from laparoscopic to open cholecystectomy procedure after a systematic reviewing of the literature and retrieving evidence in different evidence-based resources; we also assess evidence based risk factors that predispose patients to the conversion to open procedures.

Results: We found an increment of the risk of conversion 0.50/1.20 when Risk factors are present. We identified as Risk factors: Diabetes, Fever, high Total Bilirubinemia, ALT and ALP and high White Blood Cell count.

Conclusions: Using EBM Methods for risk mapping can focalize the dimension of the problem representing an useful and efficient preliminary tool before taking any decision of care pathways remodelling.

\section{2}

Postoperative Delirium after Elective and Emergency Surgery: Analysis and Checking of Risk Factors
A. De Cataldis', L. Ansaloni², A. Potalivo"3, R. Chattat, M. Agulli, V. Moscaritolo ${ }^{3}$, L. Mazzoni ${ }^{4}$, S. Pivi ${ }^{3}$,
A. Guglielminetti ${ }^{3}$, L. Piancastelli ${ }^{3}$, P. Mascitti ${ }^{3}$, S. Blandino ${ }^{3}$,
S. Cassetti ${ }^{3}$, V. Altimari ${ }^{3}$, M.R. Melotti ${ }^{3}$, M. Taffurelli'
${ }^{1}$ Department of General Surgery, 'S. Orsola-Malpighi' City Hospital, ${ }^{2}$ Department of Emergency Surgery, 'S. Orsola-
Malpighi' City Hospital, 'Department of Anaesthesiology,
'S. Orsola-Malpighi' City Hospital, ${ }^{4}$ Department of
Psychology, University of Bologna, Bologna, Italy

Background: Delirium is defined as an acute disorder of attention and cognition and may be associated with increased morbidity and mortality, length and patient care costs. A lot of factors can be involved in the onset of delirium. In elderly patients, delirium is often an early indicator of patho-physiological disturbances.

Aims: Re-evaluate the typical risk factors mentioned in literature and in a pilot study performed by some Authors of the present and add others such as genetic and inflammatory ones.

Methods: Using a case/control design, we recruited all patients aged $>65$ years operated in our Emergency Unit from 16 May 2005 to 16 March 2006; an informed consent was obtained. Exclusion criteria were: presence of severe psychiatric disorders or severe sensory deprivation. Our exposures are the following: ASA, Pain (SVS; VAS), Blood gas analysis, Residence, pharmacological therapy, Body temperature, Arterial pressure, Heart frequency, Breath frequency, $\mathrm{Na}, \mathrm{K}$, Creatinine, Glicemia, Albumin, Hct, White blood cells, Glasgow Coma Scale (GCS), Cognitive state (SPMSQ), Functional state (ADL, IADL), Psychological Distress (HADS), Cumulative Illness Rating Scale (CIRS), Hypotension, Blood loss, Quantity of red cells and plasma transfusions, Visual VAS / SVS (timing: I-II-III-VI postoperative day), Blood count evaluation and Saturation, Postoperative analgesia, Presence of malignant tumoral disease, APACHE Score II. To detect delirium after surgical intervention the Confusion Assessment Method (CAM) and the Delirium Rating Scale (DRS) were used. For statistical analysis SPSS package was used.

Results: 358 patients were included; 2 died during hospital stay. Delirium was detected in (truncated at 250 words).

53

\section{Endo Anal Ultrasound is Useful for the Diagnosis of Persistent Pain Syndrome After Circular Stapled Haemorroidectomy? Our Experience of Three Cases}

\author{
C. Delconte, I. Righi, A. Giori, F. Fiore, C. Corsi \\ Unità operativa di Chirurgia Generale I, Ospedale San \\ Paolo Milano
}

Introduction: Haemorroihoids are one of the most common disorders of the anal canal; stapled mucosectomy has been developed as an alternative to conventional haemorrhoidectomy. Several studies showed that time of recovery and pain were significantly less after stapled haemorrhoidectomy. Nevertheless, other kind of complications, such as postdefecation pain, persistent pain, fecal urgency were noted in $1-4 \%$ of patients. In particular, persistent pain has an aetiology and a treatment unclear. This report describes diagnosis and management of 3 clinical cases of persistent pain after stapled haemorrhoidectomy.

Description: 3 patients underwent surgical treatment for symptomatic prolapsed haemorrhoids in different hospitals, where were performed stapled haemorrhoidectomy using the PPH set. They came to our observation ( 3 months, 6 months and 2 weeks after surgery) for new-onset cronic perianal pain. Our assessment consisted of clinical review and endoanal ultrasound. Digital examination revealed sphincter normotonicity. The transanal ultrasonography showed an intact sphincter complex in all cases; in 2 cases a pseudodiverticolus of rectum just at the suture line, in 1 case a trombosis of haemorroidal veins. In 1 case we performed a diverticolotomy. The others underwent a conservative medical treatment.

Discussion: In case of persistent pain post $\mathrm{PPH}$, because of its unclear ethiology, endoanal ultrasound is useful for the diagnosis. 
54

\section{Emergency Surgery for Colorectal Cancer: Thirty-years Trend-Analysis}

R. Nascimbeni, G. Pata, I. Ngassa, F. Di Fabio, E. Valloncini, E. Di Betta, B. Salerni

Cattedra di Chirurgia Generale, Università degli Studi di Brescia

Background: A wide group of colorectal cancer patients seeks first medical attention due to conditions requiring emergency surgery, such as bowel obstruction or perforation. To this aim, the present study compared two series of colorectal cancer patients undergoing emergency surgery, prospectively collected during two decades.

Methods: Data on patients undergoing emergency surgery for colorectal cancer were prospectively collected from 1975 to 1984 , and from 1995 to 2004, respectively, in a single unit of general surgery. The study compared frequency of emergencies, patient and tumour variables, operative mortality and 5-years overall and cancer-related survivals.

Results: During the $1975-84$ decade 81 patients out of 513 patients operated for colorectal cancer underwent emergency surgery (15.8\%), whereas 41 out of 471 patients $(8.7 \%)$ underwent emergency surgery during the 1995-2004 decade. The difference was statistically significant $(p=0.005)$. Analysis of baseline characteristics of emergency cases showed no differences between the two decades in age at presentation and gender of patients, and in site, stage and grade of primary tumour. During 1995-2004, a higher rate of patients underwent radical surgery $(p=0.0002)$, and among those treated with curative intent a higher rate of patients had a resection procedure at first approach $(p=0.0015)$ and an adjuvant treatment afterwards $(\mathrm{p}=0.0002)$. Operative mortality after emergency surgery was $13.6 \%$ in the first decade (1995-2004), and 4.9\% in the second one (1995-2004): the difference was not statistically significant. Kaplan-Meyer survival estimates showed significant improvements in both overall and cancer-related 5-years survivals after emergency surgery from 1975-84 to 1995-2004 ( $\mathrm{p}=($ truncated at 250 words).

\section{5}

Primary Neoplasms of the Small Bowel. The Clinico-Diagnostic and Surgical Therapy Considerations: The Clinical Cases and a Retrospective Study of Your Experience

C. Dobrinja, M. Roseano, A. Turoldo, G. Liguori

Clinica Chirurgica, Trieste

Introduction: Primary tumors of the small bowel is a rare and constitute about $2 \%$ of gastrointestinal neoplasms.

Purpose: The authors reviewed our surgical experience of 20 patients treated at the Surgical Clinic of the Università degli Studi di Trieste, of Trieste during a 5 years period from January 2000 to December 2005. In the current study, the records of 20 patients with small bowel carcinoma were reviewed retrospectively for the presentation, treatment modalities, and outcome.
Materials and Methods: There were 20 patients: $6(30 \%)$ men and $14(70 \%)$ women an the median age of the patients was 70.2 years (range, 43-87). Tumors originated in the ileum in $12(60 \%)$ patients, the duodenum in $5(25 \%)$ patients, the jejunum in $2(10 \%)$ patients, and in the Meckel diverticulum in $1(5 \%)$ patient.

Results: Adenocarcinoma was the most common (30\%) followed by carcinoid (25\%), lymphoma (20\%), neuroendocrine tumors $(15 \%)$ and gastrointestinal stromal tumors (GIST) (10\%). The distribution of the malignancies showed preponderance in the ileum $(60 \%)$, in the duodenum (25\%), in the jejunum (10\%) and in the Meckel diverticulum (5\%). The most common presentation was weight loss (45\%) followed by abdominal pain (40\%), obstruction (40\%), anemia and gastrointestinal bleeding (30\%). A correct preoperative diagnosis was made in only $35 \%$ of the patients. All the 20 tumors were resected: in 17 patients $(85 \%)$ the resection were considered as curative. Survival analysis was by the Kaplan-Meier method. The median overall survival rate was $50 \%$.

Conclusions: Performing an oncologic surgery resulted in (truncated at 250 words).

56

\section{Sentinel Node in Treatment of Breast Cancer: Analysis of Personal Experience of 527 Cases}

E. Procaccini, R. Ruggiero, G. Docimo, F. lovino,

F. Procaccini, R. De Luca, E. Irlandese, F.L. Schiavo

Department of Surgical, Second University of Naples, Italy

Introduction: Knowing the state of the axillary lymph nodes is essential to the stage prognostic aims and it represents a guideline for the adjuvant systemic therapy in the breast cancer. Despite the correlation with the morbidity, the complete axillary dissection was indispensable in the treatment of the breast cancer for a long time. The biopsy of the sentinel lymph node ( $\mathrm{SN}$ ) proved so reliable in the evaluation of the parameter $\mathrm{N}$ that it induced to limit the axillary dissection just to the SN biopsy (in case of negativity).

Methods: Our experience was composed of different phases. In the first one (September 1997-January 1998) we enlisted 50 patients for the learning curve of the searching methodic for SN. In the second phase (February 1998-March 2001) we enlisted 256 patients between 27 and 81 years old who were affected with infiltrative breast cancer, with $\mathrm{T}$ less than $3 \mathrm{~cm}$, clinical N0. After the extemporaneous examination, if the $\mathrm{SN}$ was negative the protocol would expect the limitation of the axillary dissection just to the first level. In the third phase (April 2001-March 2006) we enlisted 221 patients with T less than $3 \mathrm{~cm}$. In this case, the protocol expected the axillary dissection just to the non metastatic-SN.

Results: The preoperative identification of the $\mathrm{SN}$ happened in $98.6 \%$ of the cases, submitted to radio-tracing peritumoral and intradermic injection of radionuclide tracer. The intraoperative identification was reached in $99 \%$ of the cases $(90 \%$ with radioguided surgery, $10 \%$ with blue dye). 


\section{7 \\ Post-Operative Peritonitis Due to Anastomotic Dehiscence After Colonic Resection: Risk Factors}

\author{
L. Docimo, L. Sparavigna, A. Di Muria, P. Papagno, \\ I. Verde, R. De Luca, R. Ruggiero, G. Docimo \\ Department of Surgical, Second University of Naples, Italy
}

Introduction: Intraperitoneal sepsis due to anastomotic leakage significantly affects the outcomes of intestinal surgery. The aim of this review is to examine retrospectively general and local risk factors involved and their prognostic value.

Methods: Between April 1995 and April 2005, 367 patients underwent elective $(217=59 \%)$ or emergency $(150=41 \%)$ primary colonic resection for benignant $(77=21 \%)$ or malignant $(290=79 \%)$ disease. We performed 124 right colon resections with immediate anastomoses (primary resection), 65 (52.4\%) emergency and 59 (47.6\%) elective; 171 left colon primary resections, $73(42.7 \%)$ emergency and $98(57.3 \%)$ elective; 72 rectal primary resections, 12 $(16.7 \%)$ emergency and $60(83.3 \%)$ elective. We considered stapled or manual anastomoses, protective stomas and medical comorbidities.

Results: Perioperative mortality rate was $6.6 \%$ for emergency and $3.6 \%$ for elective procedures. The leak rate was $8.7 \%(32 / 367)$, $13.3 \%$ for emergency and $5.5 \%$ for elective procedures. Fistula affected 7/124 (5.6\%) ileo-colic, 13/171 (7.6\%) colo-colic and 12/72 $(16.6 \%)$ colo-rectal anastomoses. 21 dehiscences were treated conservatively ( 3 of these were reoperated), while 11 , severe and all located in left colon, underwent a Hartmann's procedure, with a perioperative mortality rate of $35.7 \%$.

Discussion: In our experience the site of colonic anastomoses represents the risk factor more strictly related to anastomotic leak rate, while other technical factors seem weakly associated to leakage; medical comorbidities are found in anastomotic fistulas in a significantly high percentage of cases $(65.6 \%)$.

\section{8}

\section{Surgical Treatment of Obstructed Defecation in Rectocele. Our Experience}

U. Brancaccio, A. Palazzo, F. Stanzione, M.G. Esposito,

S. Celsi, G. Conzo, A. Livrea

IV Div. of Gen. Surg. and Endocrinosurg,

Second University of Naples, Italy

Introduction: Anterior rectocele $(\mathrm{R})$ represents a rectal hernia in the posterior vaginal wall, associated to rectal prolapse, that may cause discomfort, pain and syndrome of obstructed defecation (OD) ( $>4 \mathrm{~cm}$ diameter). In the surgical repair of $\mathrm{R}$ a variety of approaches have been utilized: transanal, transvaginal, transabdominal. Longo's techinique, STARR - stapled transanal rectal resection - represents an innovative, safe and simple operation in the treatment of rectocele allowing a surgical treatment of rectal prolapse associated.

Material and Methods: 36 women, mean age 45 (range 30-60) years, were investigated in our surgical division from January 2002 to December 2005. All patients complain of rectal OD in terms of excessive straining, incomplete evacuation, and need for digitally assist evacuation $(10 \mathrm{pz})$. Faecal incontinence (FI) was present in 3 patients, in 4 patients a cystocele with urinary incontinence was associated. The preoperative diagnostic work-up included anorectal manometry, defecografy, rectal sigmoidoscopy associated to pelvic dynamic RMI in such cases. The patients were submitted to STARR according to Longo's techinique, by a double stapler (PPH 01.33, Ethicon $^{\circledR}$ ). In 4 cases a Burch operation was associated, in one case after isteroannessectomy; in 3 cases an anterior spincteroplasty - in one case associated to overlapping of externals phincter - for FI was performed. Postoperative course was uneventfull. Every patient showed a significant improvement of preoperative symptomatology.

Conclusion: Anterior $\mathrm{R}$ is a frequent cause of $\mathrm{OD}$, associated to mucosal rectal prolapse. STARR according to Longo's technique represents an interesting surgical option, allowing good medium-long term results.

\section{9}

\section{Inguinal Hernia Repair: 457 Cases in One-day Surgery. The Experience of an District Hospital} F. Pastena, P. Falco, F. Lojodice, D. Loffredo, D. Del Ciampo,
N. Causa, D. D'Antonio, G.G. Manco, A. Marvas

U.O.C. Chirurgia, Ischia, NA

Introduction: Inguinal hernia repair can be performed in many methods. Actually a basic type of treatment is tension free technique with use of many types of meshes. Advantages of this surgery are low recurrence, short hospital stay and a quick postoperative recovery with improved quality and good economic results. Complications most often observed are chronic pain, wound infections, inguinal and scrotal ecchimosis.

Aims: Analysis of our experience on 457 inguinal hernia repair in one day surgery between January 2004 and February 2006. The last $15(3.2 \%)$ were performed with use of Tissucol ${ }^{\circledR}$ to fix the mesh.

Material and Methods: 457 operations for inguinal hernia were performed between January 2004 and February 2006. The age of patients ranged from 16 to 91 years (mean age 64.8). In $402(87.9 \%)$ was performed elective surgery, in $55(12 \%)$ surgery was performed in emergency. The Lichtenstein technique was used in in 442 cases. The last 15 operations were performed with use of Tissucol to fix the mesh on posterior wall. All operations had loco-regional anaesthesia and short-term profilaxis with ceftriaxone.

Results: Early post-operative morbidity rate was $2.8 \%$ (13 patients) concerning specific complications. Surgery wasn't necessary. The mean hospital stay was $22 \mathrm{~h}$. The follow up performed on 389 patients showed no recurrences, but demonstrated chronic mild pain in 8 patients. 
60

\section{Is Antibiotic Prophylaxis Necessary in Inguinal Hernia Surgery?}

P. Falco, F. Pastena, D. Loffredo, F. Lojodice, D. Del Ciampo,

N. Causa, G.G. Manco, A. Evangelista, G. Cioffi,

A. Marvaso

U.O.C. Chirurgia, Ischia, NA

Introduction: Postoperative wound infections (WI) are mostly due to bacterial contamination during surgery. Infection are correlated with surgical risk, age, and performance status of the patients. The overall incidence of WI is estimated between 3\% and $4 \%$ in general and emergency surgery hospitals.

Materials and Methods: Analysis of 457 inguinal hernia repair with prosthetic material were performed in correlation to the incidence of wound infections in the period between January 2004 and February 2006. The technique used was the Lichtenstein one in loco-regional anaesthesia, in one-day surgery. Only in $15(3.2 \%)$ cases we used Fibrin Glue (Tissucol ${ }^{\circledR}$ ) to fix the mesh. 55 (12\%) operations were performed in emergency. We performed short-term profilaxis with Ceftiaxone one shot 2 gr. Ev. before surgery in all cases.

Results: The overall infection rate was $2 \%, 9$ patients, of which only one $0.2 \%$ underwent elective surgery. The median age of patients with WI was 68 years range 48-87. No infection were observed in Tissucol group.

Conclusions: Antibiotic short-term prophylaxis and the use of Tissucol may be useful in inguinal hernia surgery to reduce the incidence of WI specially if performed in emergency and in old aged patients. More investigations will be necessary to assess results.

61

\section{Liver Resection for Colorectal Cancer Metastases: Our Experience}

\section{F. Feroci, F. Cianchi, F. Perigli, A. Ottaviano, P. Bechi, C. Cortesini \\ Dipartimento Area Critica Medico-Chirurgica Università degli Studi di Firenze}

Introduction: Liver resection is still considered as the treatment of choice for colorectal cancer metastases. The aims of the present retrospective study were to: (1) identify possible prognostic factors after surgery in order to optimize patient treatment, (2) compare our results with those reported by previously published studies.

Methods: From 1986 to 2002, 92 liver resections were performed in 82 patients (41 were operated on for single metachronous, 26 for multiple metachronous metastases and 15 patients were operated on for synchronous ones). No adjuvant scheduled protocols were used. Thirty-five patients with not resected metastases were considered as control group for survival comparison.

Results: Overall survival median time after surgery was 28 months (3-221). Survival median time was 30 months (range 3-221) and 13 months (range 3-70) after resection of metachronous and synchronous metastases, respectively. Survival of these two groups was significantly higher than that of the control group $(\mathrm{p}<0.001)$.
Moreover, survival median time was 36 months (range 15-221) after resection of single metachronous metastases and 19 months (range 3-64) after resection of multiple metachronous ones.

Discussion: This study confirmed the effectiveness of liver resection for colorectal cancer metastases. Two survival-related factors after surgery seem to emerge: time of liver resection (vs.) and number of metastases. Interestingly, both living patients and long survivor ones (more than 5 years) were all resected for single lesion.

\section{2 \\ Distance Between Anal Sphincter and Low Rectal Cancer before and after Preoperative Therapy by Endorectal Ultrasound}

M. Gavioli, L. Losi, F. lacchetta, G. Luppi, A. Schiavone, A. Mauro, M.C. Gallo, A. Rinaldi, G. Natalini

Divisione di Chirurgia III, Azienda Policlinico, Modena

Introduction: For the lower rectal cancer the distance from the anal sphincter is the most important element to decide between an abdomino-perineal resection (APR) and a sphincter-saving surgery. Long-course preoperative therapies can improve this distance. The aim of our work is evaluate the frequency and the quantitative importance of this improvement.

Methods and Results: The distance between the lower pole of the tumor and the upper edge of the anal sphincter was measured in millimeters by linear endorectal ultrasound (Aloka UST 664) before and 8 weeks after preoperative radio chemotherapy ( 50 Gy in 25 daily fractions for 5 weeks with concomitant $5 \mathrm{~F}-\mathrm{U}$ ) in 92 locally advanced cancers (uT3/T4 N0/N + M0) of the lower rectum $(<6 \mathrm{~cm})$. No modifications in the distance underwent in 44 patients $(47.8 \%)$, a decrease in $5(5.4 \%)$, an increase in 43 cases $(46.7 \%)$. The median increase was $0.7 \mathrm{~cm}(0.1-2.5 \mathrm{~cm})$. The improvement in the distance after preoperative therapy changed the intended APR to a colo-anal anastomosis in $12 / 43$ patients and facilitated the sphincter saving surgery in the others.

Conclusions: Preoperative therapy increased the distance of the low rectal tumor from the anal sphincter until the near $50 \%$ of cases in our experience, and can make possible or facilitate an anal preserving operation. The linear endorectal ultrasound is an effective tool to measure this distance.

63

\section{To Clean A.M.S. Houses Report}

1. Grassi, A. Chessa, S. Civitelli, G. Tanzini

General Department of Surgery, University of the Studies of Siena, Hospital S. Maria Le Scotte

Introduction: A subject, 85 years, P. TO. F. arrives in department in state of schok. Ultrasonography of the negative abdomen for abdominal pathology.

Methods: The Tac abdomen and I study radiological with means of contrast of the pots documented obstruction A.M.S. basic TO to 
such report was submitted to surgical intervention of: To clean A.M.S. and to cut small intestine and to do an ano with the small intestine. The examination pathological examination documented: IMA of the intestine.

Results: In the patients with injury of the spleen, like in literature, come I continue with instrumental examinations what ultrasonography, tomography to the moment in which some anatomical alterations or clinical note themselves there is the indication to the therapy surgery. The surgical choice has revealed taken possession for the seat of the obstruction and for the critical conditions clinics of the patient.

Discussion: In the greater part of the patients it is nearly impossible to appraise the exact moment of the beginning of the symptomatology that, however, went back likely to different first days. In our experience the intestinal ischemia high note is confirmed weigh down from high mortality, likely for it mattering delay I diagnose bound to the fact that beyond the half of the patients developed the ischemia during the hospitalization in other department, above all in intensive therapy, or in the post-operating, either arrived at the operating table in state of shock or, however, after maneuvers rianimatorie advanced.

\section{4}

\section{Cystic Non-functioning Endocrine Tumour of} the Pancreas: A Diagnostic Pitfall

\section{Iusco, F. Tallarico, F. Martuzzi, F. Lerro, S. Selleri, A. Lazzari, G. Landolfo, L. Mastrangelo, F. Biolchini, E. Jovine \\ Dipartimento Chirurgico, Chirurgia A, Ospedale Maggiore, Largo B. Nigrisoli 2, 40133 Bologna}

Introduction: Among endocrine tumours of the pancreas the cystic ones are rare and often represent a diagnostic problem. An unusual variation of these kind of tumours is the non-functioning type.

Patients and Methods: We present a case of a 52 years old patient with cystic non-functioning tumour of the pancreas that manifests itself with dyspepsia. Intraoperative finding showed a $7.5 \mathrm{~cm}$ lesions in the uncinate process that infiltrated the superior mesenteric vein and its first jejunal branches. The patients was treated with a pylorus preserving duodenopancreatectomy and resection of the superior mesenteric vein with mesenteric portal reconstruction by interposition of an homologous iliac vein graft and an end-to-side anastomosis among the first jejunal branch and the graft.

Discussion: Only 70 cases have been described in literature and in almost all cases the diagnosis was made at post-operative pathological examination since they were almost always asymptomatic and current diagnostic image technique are not able to differentiate these lesions among the other cystic ones. We discuss the major diagnostic and therapeutic problems concerning this kind of tumours.

\section{5 \\ Modified Stapler Trans-Anal Rectal Resection (STARR) to Treat Obstructed Defecation Syndrome}

\author{
D. Izzo, A. Renzi, G. Izzo, N. Di Martino \\ UCP, Clinica Santo Stefano, Napoli; Chirurgia Generale, \\ Seconda Universita' degli Studi di Napoli
}

Introduction: Obstructed defecation syndrome (ODS) represents a very common clinical problem. The aim of the study was to evaluate the efficacy and safety of stapler trans-anal rectal resection (STARR) in patients with ODS.

Methods: All the patients underwent STARR for ODS caused by RI a/o RE, were prospectively introduced into a database. All the patients underwent anorectal manometry (ARM) and cinedefecography (CD). The grade of ODS was assessed using a new obstructed defecation syndrome score (ODS-S). All the patients with an ODS-S \pm 12 and RI a/o RE, were enrolled. Patients were followed-up clinically and by ARM and CD at 3 months. Furthermore a 6-month follow-up was obtained by a telephone/mail questionnaire.

Results: One-hundred-seventeen, $102(87.1 \%)$ females, mean age $48.7 \pm 13.3$ years, underwent STARR between June 2002 and January 2004. Preoperatively, ODS-S was $14.3 \pm 0.9$. RI was present in 96 $(85.7 \%)$ and $\mathrm{RE}(36 \pm 14 \mathrm{~mm})$ in $105(93.7 \%)$ patients. No major postoperative complications occurred. The length of hospital stay was $21 \pm 7.1 \mathrm{~h}$ RI disappeared in $86(76.7 \%)$ and RE in $89(79.4 \%)$ patients. At 6 month follow-up ODS-S was $5.8 \pm 1.7(\mathrm{p}<0.0001)$. Successful outcome was achieved in $102(91.0 \%)$ patients.

Discussion: STARR seems to be an effective and safe procedure to treat ODS. Longer follow-up and larger number of patients are needed to confirm these results.

66

The Surgical Treatment of Transverse Colon Cancer: Controversial Technical Findings

L. Marinocosentino, A. Bellotti, A. Belardi, B. Battaglia,

S. Ghinassi, S. Mancini, L. Leone, M. Catarci, G.B. Grassi

Department of General and Oncological Surgery, A.C.O. 'S. Filippo Neri', Rome, Italy

Introduction: Transverse colon neoplasm represents 5-10\% of all colon cancers. The surgical treatment depends on the site of primary tumor (proximal, medium or distal transverse colon) and presence of associated synchronous neoplastic colic lesions (5\% of cases). Most controversial technical findings (extent of resection for curative surgery, type of anastomosis, lymphadenectomy, en bloc removal of attached organ) exist about the type of surgical resection, especially for the tumors of the middle third (potential difficulty in achieving a tension-free anastomosis with adequate bloody supply, as the marginal artery of Drummond is sacrificed).

Methods: From 1995 to 2005, 54 (4.1\%) out of 1,310 consecutive patients, submitted to surgery for colorectal cancer, were affected by neoplasm of transverse colon. They were 25 women and 29 men (average age 66.7 years); 21 primary tumors were in the right third, 
12 in the medium, 21 in the left. In 2 cases an exact localization of the tumor was obtained by a preoperative tattoo and in 3 cases by an intraoperative colonoscopy. Three out of 14 associated colic polyps were synchronous tumors.

Results: Most of tumors in the proximal third of transverse colon were managed with an extended right hemicolectomy (18 patients); in 5 cases a trasverse colectomy was performed in the middle third, and a segmental resection in 7 cases of distal neoplasms. An adequate lymphadenectomy was obtained in $96.3 \%$ of the cases. In the distal third an en bloc removal of attached organ was more frequent (5 cases). A mechanical anastomosis was favorite in (truncated at 250 words).

67

\section{Early Treatment of Bile Duct Injury After Laparoscopic Cholecystectomy Could Improve Liver Failure. An Experimental Study in Rats}

\author{
G. Lissidini ${ }^{1}$, P. Portincasa ${ }^{2}$, L. Nacchiero ${ }^{1}$, M. Testini ${ }^{1}$ \\ 'Section of General Surgery 'A. De Blasi'. Department for \\ the Application in Surgery of Innovative Technologies, \\ 2Section of Internal Medicine 'A. Murri'. Department of \\ Internal Medicine and Public Medicine, University of Bari, \\ Italy
}

Introduction: Laparoscopic cholecystectomy (LC) is considered to be the 'gold standard' in surgical management of symptomatic cholecystolithiasis. Bile duct injury (BDI) resulting in post-hepatic obstruction continues to be one of the most serious complication of LC. Early detection and treatment of BDI is important to prevent extrahepatic cholestasis that may result in liver failure. Aim of this study is to investigate if oxidative stress can be early detected in experimental model of short-term cholestasis induced by bile duct ligation (BDL).

Materials and Methods: Wistar rats underwent proximal BDL $(\mathrm{n}=12)$. Controls underwent a sham operation consisting of exposing the common bile duct $(\mathrm{n}=12)$. Animals were sacrificed at days 1, 3, 7 and 10 after BDL. Livers were removed for histological studies and biochemical analysis, measuring total serum levels of oxidized glutathione (GSH, GSSG) and protein sulphydril (P-SH).

Results: While there were no major changes in liver tissue in the control group, we observed bile duct dilatation, inflammatory modifications, cell degeneration and mitochondrial damage in the study group. Particularly, we observed that GSH and P-SH levels declined and GSSG increased earlier in mitochondria than cytosol. Data were analyzed for statistical significance by ANOVA analysis of variance followed by the post-hoc test of comparison.

Conclusion: Short-term cholestasis is able to affect mitochondrial redox and protein redox status at an early stage, progressing to rapid liver damage. We emphasize early detection of iatrogenic BDI to ensure appropriate management and optimal patient outcome.
68

\section{ACTH Secreting Pheochromocytoma: A Clinic Case}

\section{F. Pata, R. Cardona, V. Orsini, G. Vescia, D. Voci,}

A.M. Lucisano, R. Sacco

General Surgery, 'Magna Graecia' University, Catanzaro, Italy

Introduction: Operative management of pheochromocytoma dictates resection of the involved adrenal and exploration-resection of the contralateral gland if enlarged. We describe an exception to this rule.

Methods: We report a case of patient with ACTH-secreting pheochromocytoma with Cushing's syndrome. The standard laboratory data showed hyperglycaemia and hypokaliemia with high levels of potassium excretion. ACTH, Cortisol, $17 \mathrm{OH}-\mathrm{Corticosteroids,}$ cathecolamines increased and 24 hours urinary values of free cortisol $(3,634 \mathrm{ug} / 24 \mathrm{~h})$ and the vanilymandelic acid levels increased. The ACTH levels were not changed neither by low or high-dose dexamethasone suppression test. Head Tc scan was negative for a pituitary lesion; Abdominal computed tomography scans revealed a left unilateral adrenal mass and a slight increase of the contralateral adrenal. The surgical procedure was left adrenalectomy only.

Results: After surgery levels of plasma ACTH, Cortisol, $17 \mathrm{OH}-$ corticosteroids, cathecolamines and urinary free cortisol, glycemia and kaliemia become normal and clinical symptomatology relapsed.

Discussion: ACTH-secreting pheochromocitoma is the exception to the rule: unilateral adrenalectomy is curative and the contralateral hyperplastic adrenal can be preserved. The goal of this approach is the resolution of both the endocrine syndrome and the preserving of long-term adrenal function.

69

\section{Allograft in Leg Ulcer Treatment}

S. Mancini, M. Bucalossi, L. Gazzabin, F. Berna, A. Comandi, S. Mancini

Chir. Gen. $3^{\circ}-$ Centro Flebologico, Siena

Introduction: We present our experience of six years with allograft in the treatment of leg ulcers.

Methods: We performed allograft skin from April 2000 up to now taken from the local 'Skin Bank'. We treated 507 ulcers, of which $138(27.2 \%)$ considered 'resistent' (present $>6$ months) and no responder at the normal topical medication. The mean age of 184 women was 72.2 years (18-98), while about 78 men was 67.8 years (34-91). The etiopathogenic classification demonstrated that the principal cause both in normal and in resistant ulcers was mixed, than venous, traumatic, arterial etc. We performed 2,198 allograft using 481 times derma, 828 times thin glycerolised skin, 439 times thigh glycerolised and 450 times crioconserved. In 43 patients we performed an autologous graft.

Results: In the 'resistent' group $48.5 \%$ of the ulcers healed vs. $69.1 \%$ of 'normal', $9.4 \%$ showed an evident improvement vs. $7 \%$ of 'normal', and $35.5 \%$ dropped out vs. $18.5 \%$ of 'normal'. At 3 months healed the $41.5 \%$ of 'normal' ulcers healed vs. $29.2 \%$ of the 'resistant'. 
No graft rejection was registered, while in $1.6 \%$ graft infection. The analgesic action was evaluated with the visual-analogical scale (VAS): in $18.6 \%$ pain disappeared in a week, in $57.3 \%$ in a month, a reduction to $3.1+2.4$ in $18.6 \%$, while no result in $5.5 \%$.

Discussion: Allograft skin demonstrated to be very efficacy in every kind of ul (truncated at 250 words).

\section{0}

\section{Platelet Gel in Leg Ulcer Treatment}

S. Mancini, M. Bucalossi, L. Gazzabin, F. Berna,

A. Comandi, S. Mancini

Chir. Gen. $3^{\circ}$ - Centro Flebologico, Siena

Introduction: We study the effect of platelet gel in leg ulcers treatment.

Methods: Many growth factors are involved in tissue repair process, but in chronic wounds is demonstrated a considerable reduction, especially platelet derived. In the CET of our hospital is possible to produce platelet gel derived from autologous and homologous blood too. From November 2003 up to now we treated with platelet gel 64 ulcers of 40 patients ( 29 women and 11 men) with mean age of 73 years old. A group of 45 wounds were no responder at the treatment with allograft or advanced medication, while 19 were started the therapy with gel. The etiopathogenesis was multiple. We performed a weekly medication with gel and a control after 3 days. In all cases except one, we used homologous gel platelet.

Results: We considered the result only after 4 consecutive medication with gel. Based on this we obtain a healing in 29 wounds (15 treated only with gel), while 1 is in closure, 3 at the start and 31 ulcers showed a clear improvement. About these lasts 3 are still in treatment with gel, 6 ulcers with allograft or advanced medication, 6 patients died ( 8 ulcers) and 6 underwent to a recovery for decay of general conditions (14 ulcers). No host reaction was notice.

Discussion: In our experience platelet gel demonstrated to be efficacious in the reactivation of healing process and to conduce to the healing too (truncated at 250 words).

\section{1}

\section{Stapled Transanal Rectal Resection (STARR)} in the Outlet Obstruction Syndrome: Preliminary Experience on $\mathbf{5 0}$ Patients

\author{
P. Mariani, G. Arrigoni, R. Cadoni, G. Quartierini, \\ E. De Nicola, G. Perrone \\ Chirurgia Generale, Ospedale Bolognini, Seriate, BG
}

Introduction: Obstructed defecation syndrome is a frequent condition in the female population; ODS is characterized for the impossibility to obtain a physiological evacuation, due to rectocele and rectal prolapse-intussusception. The aim of the present study was to evaluate the clinical outcome in a consecutive series of 50 patients, underwent a STARR procedure for ODS.
Methods: From January 2005 to March 2006, 50 patients affected by ODS were enrolled in the study. The series is composed by 48 females and 2 males; the mean age 58 years (range 28-65). All patients were studied with proctoscopy, colonscopy, dynamic defecography and ano-rectal manometry; the female series was studied with gynaecological examination too. At defecography all the females have anterior rectocele (II-III degree), in the $83 \%$ associated with ano-rectal intussusception. In the male series, defecography demonstrated rectal intussusception. Twenty patients $(42 \%)$ underwent surgery with general anesthesia, thirty-eight (58\%) under spinal anaesthesia. In all patients STARR procedure according to Longo technique was performed.

Results: The mean surgical time was $50 \mathrm{~min}$ (range 38-70); the mean hospital stay was 3.5 days. Post-operative early surgical complication was bleeding from the stapling line in one patients and required surgical hemostasis. None of the patients presented significant postoperative pain (mean VAS score $2 \pm 1$ ); faecal urgency was present in twenty-five patients $(50 \%)$ for the first two weeks. All the patients, after a median follow-up (1-15 months), presented significant improvement of the symptoms; none showed stenosis or fistulas. Pathological analysis of specimens demonstrated full-thickness rectal wall resection.

Conclusions: The STARR is (truncated at 250 words).

\section{2 \\ VATS in the Management of Solitary Pulmonary Nodules}

\author{
F. Martini, F. Apicella, S. Spini, P. Cappellini, L. Maggi \\ Chirurgia Generale, Università di Firenze
}

Introduction: Solitary pulmonary nodules (SPNs) are increasingly detected because of the extensive use of chest X-rays and CT scans. New imaging techniques and nuclear medicine have provided more information about SPNs, but surgical removal is still the most sensitive and specific way to establish a definitive diagnosis.

Methods: 22 patients were treated by VATS because of a SPN at our unit.

Results: A definitive pathologic diagnosis was achieved in all cases. In 3 cases only a diagnostic biopsy was performed. Among the remaining 19 patients we had: 9 bronchogenic carcinomas, 7 metastasis ( 6 from colon cr and one from kidney $\mathrm{cr}$ ), 1 carcinoid, 1 condroma, 1 abscess. The patients with lung cancer were treated by 4 atypical resections, 4 wedge resections and 1 standard lobectomy with mediastinal lymphadenectomy. Atypical resection or wedge resection were reserved to high risk patients aiming to confirm the diagnosis and to debulk the tumor. We had no intraoperative complications and no perioperative deaths.

Conclusions: VATS represents the approach of choice for both diagnosis and treatment of SPNs, especially if they have a high risk of malignancy, and offers the benefit of lower perioperative morbidity and decreased length of hospital stay. In selected patients atypical resection or even anatomic lobectomy for lung cancer are technically feasible by VATS. 
73

\section{Bochdalek Hernia in the Adult: Case Report and Review of the Literature}

\section{F. Martini, F. Apicella, S. Spini, E. Solfanelli, P. Cappellini \\ Chirurgia Generale, Università di Firenze}

Introduction: The Bochdalek hernia can be diagnosed incidentally in adult patients, so rarely that to date a few more than 100 cases have been described in the literature, on the left side in $90 \%$.

Methods: We present the case of a healthy 28 year old man who presented with an acute onset of left flank pain. CT scan showed the herniation of part of the colon into the left thoracic cavity through the postero-lateral part of the diaphragm. After exploration by VATS it was decided to convert to antero-lateral thoracotomy to lyse the strong adhesions in correspondence of the hilum in order to reduce the colon and the greater omentum in the abdominal cavity. The diaphragmatic defect was corrected with the insertion of a synthetic dual mesh plus direct suture.

Results: The patient recovered uneventfully and was discharged from the hospital on the 13th postoperative day. Two months after he is asymptomatic.

Discussion: The surgical approach has been thoroughly discussed: both thoracic and abdominal approaches have been described in the literature. In this case we preferred the thoracic via because it enables a direct observation of the herniated viscera and allows an easier lysis of the adhesions with the hilum located in the postero-lateral region of the diaphragm. As the choice of procedure. VATS is advantageous over thoracotomy for surgical exploration and intervention.

74

\section{Pancreatic Resection in the Elderly: A Single Center Experience}

R. Ballarin, M. Masetti, F. Di Benedetto, R. Montalti, N. De Ruvo, A. Romano, G. Guerrini, G.L. Rompianesi, M. Spaggiari, G.E. Gerunda

Liver and Multivisceral Transplant Center, University of Modena

Introduction: We analyzed retrospectively the last two years of surgical activity to assess the perioperative morbidity and mortality rates of major pancreatic surgery in patients who are 65 years of age or older compare to younger patients.

Methods: Patient medical records were reviewed retrospectively. Major pancreatic resection was a radical pancreaticoduodenectomy, distal pancreatectomy, or total pancreatectomy. Minor complications were those that required only minimal intervention and were not life-threatening. Significant complications were those that necessitated a major procedure, close monitoring, were potentially life threatening, or delayed discharge.

Results: Among the 52 patients in the study, 22 (42\%) were 65 years or older. Median ASA classifications between the two groups were not significantly different. Nine $(17.3 \%)$ patients $(56 \%$ of the elderly and $44 \%$ of the young) had one or more major complications. Patients who underwent radical pancreaticoduodenectomy were more likely to have a postoperative complication than those who underwent distal pancreatectomy $(\mathrm{p}<0.005)$. Cardiac complications were more frequent among older patients. Five elderly patients $(22.7 \%)$ and one young patient $(3.3 \% ; \mathrm{p}<0.005)$ had atrial fibrillation. Older patients had a greater likelihood of intraabdominal abscesses, pneumonia, but these differences were not significant. Although the elderly tended to have a greater mean length of stay in the ICU than the young, these differences were not significant.

Discussion: Older patients are more likely to require an ICU stay, suffer a cardiac complication, and experience compromised nutritional and functional status after major pancreatic resection. Major pancreatic surgery can be done safely in elderly.

75

\section{Laparoscopic Treatment of Ventral Hernias}

P.A. Riccio, R. Linguerri, G.P. Mingolla, P.M. Ravanello

Ospedale S.M.della Scaletta, Imola

Introduction: The laparoscopic technique is the preferred approach to the treatment of incisional hernias in our unit. We report a retrospective analysis of preliminary data of our experience.

Methods: Since March 2005 to April 2005, 41 patients (26 woman and 15 man) underwent laparoscopic treatment of ventral hernias. Mean age was 62 years. Only 2 cases (4.9\%) were primary defect of abdominal wall. In postsurgical hernias 9 patient $(23 \%)$ had 2 or more defects. Three access ports were inserted; the prosthesis used was a Composix E/X mesh (Bard) in 10 cases, Proceed (Ethicon) in 31 cases introduced through a $12 \mathrm{~mm}$ trocar. The prosthesis was fixed with helicoidal tacks without sutures. An external compressive pad was used to prevent the development of seromas.

Results: Mean operative time was $120 \mathrm{~min}$. Only one procedure was converted to open surgery. In 1 patient a small bowel enterotomy was recognized and repaired without removing prosthesis. Minor complications included seromas in 3 patients, prolonged abdominal wall pain in 1 patient. Major complication that required relaparotomy occurred in 2 cases (4.8\%): 1 post-operative bleeding from a port site, 1 small bowel perforation that manifested with peritonitis signs on five post-operative day. Mean hospital stay was 4 days. Although follow up is limited no recurrences developed.

Discussion: Laparoscopic repair of ventral hernias is safe and feasible. Minimal bowel injury can be safely repaired laparoscopically. Major complications can occur and must be promptly recognized. Laparoscopic access considerably shortens hospital stay. 19th National Congress of the Italian Polyspecialist Society of Young Surgeons (IPSYS) 
76

\section{Reexpansion Pulmonary Edema: A Case Report}

G.P. Mingolla, P.A. Riccio, R. Linguerri, C. Tuci, G. Borioni, P.M. Pavanello

Ospedale S.M.della Scaletta, Imola

Introduction: Reexpansion pulmonary edema is a rare complication which develops after toracostomy tube placement for treatment of pneumothorax.

Methods: We report a case of reexpansion pulmonary edema treated in our unit. Onset was immediate and dramatic with a clinical picture that resembled pulmonary edema in congestive heart failure. Only a prompt treatment with intubation permitted the improvement of the respiratory distress.

Results: A 25 year old man presented to the emergency with a history of dispnea on exertion and mild cough for 8 days after a chest pain that had rapidly resolved spontaneously. Clinical examination and chest radiography confirmed a total pneumothorax of the left lung. Triage vital signs were stable with a pulse oximeter reading of $96 \%$; the patient seemed comfortable and was no in respiratory distress. The patient was transeferred to the surgical floor and a 24 French thoracostomy tube was placed in the operating theatre with monitored anesthesia care. A gush of air of air was noted entering pleural cavity. The tube was connected to a pleurevac device without suction. Soon after the patient's condition deteriorated. He began to cough more vigorously with a large amount of frothy sputum production. The pulse oximetry reading falled to $72 \mathrm{~mm} \mathrm{Hg}$ and the respiratory distress worsened rapidly. The anaesthesist intubated the patient. An urgent chest X-ray showed a good reexpansion with a pulmunary edema on the left. The patient was subsequently admitted to the intensive care unit. After $48 \mathrm{~h}$ of mechanical ventilation the patient was extubated and transferred to (truncated at 250 words).

\section{7}

Colorectal Laparoscopic Surgery: Experience with 110 Cases

\author{
G.P. Mingolla, P.A. Riccio, R. Linguerri, G. Borioni, \\ P.M. Pavanello \\ Ospedale S.M.della Scaletta, Imola
}

Introduction: The clinical outcome of randomized trial of laparoscopic versus open colectomy for cancer recently validated the efficacy of laparoscopy for colon cancer. Laparoscopic approach to colorectal diseases presents more technical difficulties and larger learning curve than open surgery. We report a retrospective analysis of our initial experience.

Methods: Since 2004 to April 2006, 110 patients underwent laparoscopic colectomy in our unit. Patients were assessed for operative indications, type of resection, operative time, conversion, complications and hospital stay.

Results: Mean age was 68.8 (range 48-81). The types of resection were right colectomy: 35 , left colectomy: 55, rectal resection: 19 and abdominal-perineal resection of the rectum: 1. The conversion rate was $12 \%$. The mean operative time was $252 \mathrm{~min}$ for the first 40 procedure and 226 for the last. The mean hospital stay was 8.7 days. The most frequent minor surgical complications were wound infection $(8.7 \%)$ and ileus $(3.7 \%)$. Major complication requiring re-operation were: post-operative bleeding (3.6\%), anastomotic leak $(1.8 \%)$ and intestinal obstruction $(2.7 \%)$.

Discussion: Our experience confirms the benefits of the laparoscopic approach including a shorter hospital stay, decreased post operative pain, faster ileus resolution and improved cosmesis when compared to conventional surgery.

78

\section{Surgical Treatment of Anal Condyloma}

V. Costamagna, M. Mistrangelo, S. Delmonte,

S. Rondoletti, V. Ghisetti, A. Mussa

Sezione di Chirurgia Oncologica dell'Università degli Studi di Torino, Ospedale Molinette, Torino

Introduction: Anogenital warts are among the most common sexually transmitted diseases seen in surgical practice (upto $1.7 \%$ of the population). They are not usually a serious problem, but it causes emotional distress to the patient and the physician for its marked tendency to recurrence.

Materials and Methods: At our Department 643 patients (409 $\mathrm{M}$ and $234 \mathrm{~F}$ ) were visited between October 1999 and August 2005. Mean age was 34 years. $68.1 \%$ were heterosexual; $22.5 \%$ homosexual and $9.4 \%$ bisexual. Between the onset of symptoms and diagnosis elapsed 8 months. $30.1 \%$ of patients were submitted to previous treatments. 9.7\% were $\mathrm{HIV}+$; $3.1 \% \mathrm{HCV}+$; $6.7 \% \mathrm{HBV}+$. Localization: perianal in $93.9 \%$; endoanal in $62.5 \%$; genital in $35.8 \%$ and others in $3.4 \%$. 506 patients were submitted to surgery; $18.6 \%$ in several times for the extension of disease.

Results: No mortality was observed. Morbility: bleeding in $0.6 \%$; stenosis in $0.6 \%$ unhealing scar in $2.9 \%$ and others in $1 \%$. Recurrence after 1 month was observed in 71 patients (14.3\%) $(1.9 \%$ of drop out). 425 patients were followed for 6 months with further 31 recurrences $(7.3 \%)$. Total recurrence rate was $21.6 \%$. We performed HPV tipization in 135 patients: 30 were HR or LR/HR.

Conclusions: A meticoluos surgical procedure and an accurate follow up are mandatory in the treatment of anogenital warts. They permit a reduction of recurrence and of sexual transmission.

\section{9}

\section{Our Experience in Treatment of Hydatid - Cysts}

M. Kaci, E. Celiku, A. Llukaci, E. Cobani, A. Bodeci, A. Dibra

I Surgical Clinic, Hospital University Center 'Mother

Teresa', Tirana Albania

Liver is the most common site of infection and several methods of surgery have been described to treat this common disease. In this prospective study have been treated 126 patients ( 52 males, 74 females). 
The average age: 44.09 years old

The confines age: $10-75$ years old

For males. . . . . . . . The average age: 42.4 years old (17-67)

For females . . . . . . . . The average age: 46.6 years old $(10-75)$

Statistical analysis by Fisher-students results with a significance

$\mathrm{t}=1.43$

We underwent surgery from:

Liver. . . . . . . . . . . . . . . . . . . . . . . 115 cases

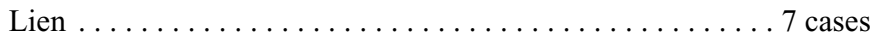

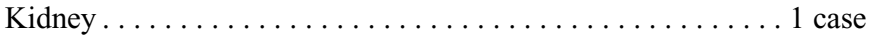

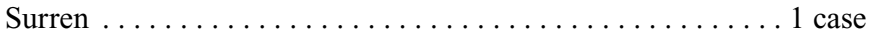

Pelvis (abdominal wall) . . . . . . . . . . . . . 1 case

Ovar........................... 1 case

Liver 115 cases $(91.27 \%)$ :

Lobus hepatic dexter . . . . . . . . . . . . . 81 cases

Lobus hepatic sinister . . . . . . . . . . . . . 31 cases

Bifocal localization .................. 3 cases

Our patients were treated with:

- Liver. . . . . . . Drain of cyst and cover the cavity with omentum. (involve of choledochus ........... choledochothomy/KEHR)

- Spleen ........ spleen-ectomy

- Kidney . . . . . . . nephr-ectomy

- Surren . . . . . . . surren-ectomy

- Ovar. . . . . . . . ovar-ectomy

At the present study, we planned to evaluate the rate of $\mathrm{CE}$ in Albanian area between 1990 and 2003 and we will propose our solutions.

\section{0}

\section{Three Cases with Morbid Gauscher of One Family - Casuistic}

M. Kaci, E. Celiku, A. Llukaci, E. Cobani, A. Bodeci, A. Dibra

I Surgical Clinic, Hospital University Center 'Mother

Teresa', Tirana Albania

The disease Gauscher is a disorder of greasy metabolism, that consist in an exaggerated depositing of cerebrosidas in the cells of the reticule-endothelial system, preferig hepar, lien, lympho-nodules and the osseous skeleton.

Through these our 3 cases (by one family) we want to draw the attention of all physicians, clinicians to take into consideration and to think these disease if in the give of diagnoses and even for the differential diagnose.

Complains that present the three of our patients:

1. Gravitation, on the left hypochondria because of spleenomegalia (lien arrived up to the spinal iliac anterior superior). This was the common symptom that forced/obligated the patients to be presented at the doctor.

2. The three were presenting hemorrhagic phenomena (epistaxis and gingivorrhagy) and only one of them osseoum pain.

3. The three patients have paleness of derma especially of face, hand and the feet.

4. The white pigment in yellow of derma, which is described by the special author such as derma of Goose and found in $45-75 \%$ of cases (used literature ...)

The Gauscher cells were observed in myelography of the three our patients taken under study.

In the beginning the patients were treated with symptomatic and after such a treatment were a spleen-ectomy for 2 reasons:

1. In order to avoid this affects of measure by spleeno-megalia.

2. Hemorrhagic phenomena.

In the beginning we were content of the symptomatic treatment and later when it began to appear the hemorrhagic phenomena and the spleen achieved enormous dimensions were perform the (truncated at 250 words).

\section{1}

\section{Debridement of Necrotic Fibrinous Chronic Ulcers with Ultrasound Equipment}

R.D. Campa, I. Simonelli, S. Manfredelli, A.G. Nasti, M. Bezzi, P. Pastore

IV Clinica Chirurgica, Policlinico Umberto I, Roma

Introduction: The treatment of necrotic-fibrinous chronic ulcers with ultrasound equipment is based on a cavitation process, a mechanical effect that causes wound washing, fibrinolysis and destruction of bacteria and fungi. We used the ultrasound equipment Sönoca 180 orig. Soring to evaluate the healing of chronic wound in our Outpatient Unit.

Methods and Materials: Between September 2005 and February 2006 we treated 40 patients in our Outpatient Unit affected by chronic ulcers. Twenty-two of them had ischemic ulcers, 5 venous ulcers, 4 diabetic foot, 9 pressure ulcers. The wounds have been treated with an ultrasound equipment (Soring Sonoca 180), that use low-frequency ultrasound and $\mathrm{NaCl}$ solution to enhance the cavitation effect. After each treatment the wound were covered with advanced dressing.

Results: We obtained a complete wound cleansing, reduction of the infection and a sufficient local bleeding. We observed also a mild reduction of the pain after 3-5 applications. Ulcers debridement by ultrasound system reduce the diameter of the wound with less applications than with the use of sole advanced dressing.

Conclusion: The use of ultrasounds for the curettage of chronic ulcers is demonstrated more effective than whichever methodical one up to now applied why it reduces the number of medications and therefore the pain, it is for the bactericidal action that for the modifications of the local microatmosphere and moreover stimulates the formation of the granulation woven one.

82

\section{Chronic Advanced Treatment of Chronic Wounds}

S. Manfredelli, I. Simonelli, R.D. Campa, A. G. Nasti, M. Bezzi, P. Pastore

IV Clinica Chirurgica, Policlinico Umberto I, Roma

Introduction: When using advanced medications we can't obtain a complete repair of the chronic wounds, we use the TissueTech Auto- 
graft System, an autologous dermal substitute - Hyalograft 3D - and an autologous epidermal replacement, Laserskin autograft. Each includes a matrix of a Hyaluronic acid ester to promote cellular migration and graft take.

Materials and Methods: We have treated in our outpatients department 70 cases of chronic wounds, answering to all indispensable requirement for the application of this treatment, 20 cases of venous ulcers; 15 cases of vascular ulcers; 10 diabetic foot ulcers; 10 cases post flebite-thrombus syndrome and 15 cases of pressure ulcers with the application of tissue-engineering. Every patient has been subordinate to a biopsy to level of the inner portion of the thigh of approximately $2 \mathrm{~cm}^{2}$ of skin.

Results: We have found a good cicatrization of the wounds without recidive in 67 patients; in 3 of them the biopsy has not turned out suitable to the cellular cultivation.

Conclusions: With the application of Tissue Tech Autograft System we have carried our patients to a complete health of the chronic wounds in 4-8 weeks in approximately $95 \%$ of the cases, obtaining optimal compliance from part of the patients, a drastic reduction of the times and the number of medications and consequently of the costs.

83

Treatment of Gunshot Wounds to the Colon: Experience in a Rural Hospital During the Civil War in Somalia

\author{
A.G. Nasti, S. Manfredelli, R. Campa, A.M. Angelici \\ IV Clinica Chirurgica, Policlinico Umberto I, Roma
}

Introduction: In the last few decades there has been a clear tendency in civilian practice towards primary repair of gunshot wounds to the colon, resulting in a substantial decrease of the number of colostomies performed for this type of injury.

Materials and Methods: The series described here comprises 24 patients with gunshot wounds to the colon treated at the hospital of Jowar in the Middle Shebelle region of Somalia between 1999 and 2001. All injuries were caused by war arms firing high-velocity projectiles.

Results: In 18 patients surgery consisted of resection and immediate anastomosis, while in the remaining 6 patients colostomies were performed including five loop colostomies and one terminal colostomy. The sepsis-related mortality was $25 \%(6 / 24)$.

Conclusions: Our experience had a peculiar setting, namely that of a civil war in a developing country. In this kind of 'difficult situation' there probably exists no ideal procedure and the decision taken at the operating table are influenced by various factors that are often related to subjective assessment.
84

\section{Subtotal Colectomy with Antiperistaltic Ceco-Rectal Anastomosis in the Treatment of Slow-Transit Constipation: Long-Term Impact on Quality of Life}

\author{
A. Palladino, L. Sarli, L. Roncoroni \\ Department of Surgical Sciences, Parma University \\ Medical School, Parma, Italy
}

Purpose: The aim of the study is to evaluate the effectiveness of subtotal colectomy with cecorectal anastomosis (SCCA) in the treatment of slow-transit constipation, not just in terms of symptom resolution, but more in general, as overall impact on patients' quality of life.

Methods: Between 1991 and 2005, 43 patients underwent SCCA at our Institution, 22 of whom for slow transit constipation. They have been submitted to a 50 item telephonic questionnaire, including GIQLI, the Wexner constipation and incontinence scale, the willingness to repeat the procedure. Questionnaire data and other parameters such as age, sex, length of follow-up, complications and length of hospital stay has been analyzed and statistically matched, in order to evaluate qualitative differences related to pathology (constipation vs. non constipation) and surgical approach (laparotomy vs. video-laparo-assisted).

Results: A total of total of 29 patients have been submitted to the questionnaire, 17 of whom affected by slow-transit constipation (STC), 12 affected by other kinds of colonic disease (NSTC). We had no procedure-related deaths (mortality: $0 \%$ ) finding 2 complications in STC group (9.1\%), one of whom requiring reintervention. The GIQLI mean score for STC group has been $115.47 \pm 20.89$ (mean score for healthy people $125.8 \pm 13$ ), while, for Wexner constipation scale, the mean score passed from a preoperative value of 20.93 to a postoperative of 2.75. Statistical analysis revealed significative correlation between GIQLI and urgency and abdominal pain, while abdominal pain correlates significantly with pathology (STC). A hight rate of patients ( $88.24 \%$ in STC) expressed the willingness to repeat the procedure in the same preperative conditions.

Conclusions: Comparing our results to the most homogeneous literature datas, SCCA does not seem to be inferior to subtotal colectomy with ileo-rectal anastomosis in terms of therapeutic effectiveness, postoperative mortality and morbility, overall impact on quality of life. The opportunity (truncated at 250 words).

85

Artificial Neural Network and Expert System in Breast Cancer Treatment (ANNESBC)

D. Parmeggiani, M. Siciliano', F. Sibilio², A. Chiacchio ${ }^{2}$, R. Cerqua ${ }^{2}$, G. Cimmino, M. Perrotta

General and Laparoscopic Surgical Unit, Frattaminore Hospital, ${ }^{1}$ Engineering Department of University of Naples Federico II, ${ }^{2}$ Administrative Department of Sanitary Direction A.S.L.Na3, Naples

Introduction: We've tried to develop methodologies and algorithms for a system that uses Artificial Neural Network (ANN), to 
improve the interpretation of the breast imaging, and to create an autonomous (Expert) system software like instrument of diagnostic integration for the physicians.

Materials and Methods: Patients Fat risk for breast cancer $>40$ years, since J-2006, were screened in 2 arms (double blind), a) traditional diagnostic group ( $15 \mathrm{pz}), \mathrm{b})$ integrated diagnostic group (15 pz). After an anamnesis schedule, we've selected patients needing for diagnostic exam, then the acquired images (in b group) were inserted in a tool-software, based on ANN, proposing a new classification. The extrapolated information teach the Expert System, that planned on the acquired anamnesis and diagnostic variables, gives a support for therapeutical strategy.

Results: In few months in b group, we've recognize 4 patients that underwent prophylactic surgery, 3 were bilateral and 1 monolateral subcutaneous skin ad nipple sparing mastectomy with an immediate reconstructive sub muscular breast expander implantation. We found $1-\mathrm{T} 1$ mic infiltrating ductal carcinoma, multifocal and multicentric and with contextual ductal in situ carcinomas bilaterally and $1 \mathrm{~T} 1 \mathrm{a}$ ductal infiltrating carcinoma of the axillary prolungaments of UEQ of left breast.

Discussion and Conclusion: This is only a preliminary study and for the poor casistic is not jet possible to arise conclusions but the objective is: to produce an informed tool (Experienced System), that integrated with our neural system, able to identify and to classify a greater number of neoplasias in initial phase.

86

\section{Abdominal Compartment Syndrome: Case Report}

\author{
D. Parmeggiani, G. Cimmino, D. Cerbone1, G. Rugiero², \\ G. Barbato ${ }^{2}$, M. Perrotta \\ General and Laparoscopic Surgical Unit, Frattaminore \\ Hospital, 'U.O.C. General Surgery, P.O. Card. Ascalesi, \\ ASL NA 1, ${ }^{2}$ U.O.C. General Syrgery, University of \\ Catanzaro
}

Introduction: The incidence of ACS (Abdominal Compartment Syndrome) is debated, in the abdominal major traumatism, especially multi traumatism is around $14-15 \%$ and arise even to $34 \%$ in aorticsurgery for traumatic rupture of aneurism, The high Intra Abdominal Pressure (IAP) produce injury on cardio-pulmonary, renal, muscular and finally on Central Nervous System physiology with a characteristic high morbidity and mortality syndrome.

Materials and Methods: We divide IAP in 4 degree: I $10-$ $15 \mathrm{~mm} \mathrm{Hg}$, II Over $15 \mathrm{~mm} \mathrm{Hg}$, III 20-25 $\mathrm{mm} \mathrm{Hg}$ - begins an important multi organs hypo-perfusion, IV Over $30 \mathrm{~mm} \mathrm{Hg}$ the prognosis 'quod vitam' become serious. We describe a case of a male patient (68 years), cirrhotic and ascitis from chronic viral $\mathrm{C}$ correlated hepatitis, with a severe abdominal traumatism, liver and spleen ruptures.

Results: The patient underwent traditional emergency surgery with an hepatic atypical resection of the 6th and partially of the 7th segments and splenectomy and after 4 days developed a multi organs failure with a retroperitoneal (misdiagnosed haematoma), acute, IV degree IAP that we've treated with a new operation, treating the haematoma and using a pure pork a-cellular collagen mesh $\left(\right.$ Permacol $\left.^{\circledR}\right)$ to close the wound, leaving enough space between fascia extremities, to solve the IAP ( $<10 \mathrm{~mm} \mathrm{Hg}$ in 2 nd day).
Discussion and Conclusions: This kind of innovative mesh, most used for enormous incisional hernias, can be completely in contact with intestinal part, because doesn't produce any adherence; at the same time has consistence of a muscular fascia with a complete inert and safety.

87

The Biliary Tract Injuries: Two Cases Report

G. Cimmino, D. Parmeggiani, D. Cerbone ${ }^{1}$, G. Rugiero², G. Barbato ${ }^{2}$, M. Perrotta

General and Laparoscopic Surgical Unit, Frattaminore Hospital, ${ }^{1}$ Ascalesi Hospital, Naples; ${ }^{2}$ General Surgery, University of Catanzaro

Introduction: Biliary tract injuries (BTI) represents the most serious and potentially life-threatening complication of cholecystectomy. During lap cholecystectomies (LC), the prevalence of bile duct injuries has been estimated at only $0.5-1 \%$.

Materials and Methods: Case 1: In 2004, 45 years old, male, affected by symptomatic cholelithiasis. The patient was planned for LC; during dissection of Calot's triangle a partial resection of common biliary duct was made. Case 2: In 2005, 60 years old, female, affected by cholecystitis with gallstones. LC was performed after $36 \mathrm{~h}$, during operation, common biliar duct was misidentified, for subverted anatomy caused by the flogosis. The coledochos was clipped, and the patient presented jaundice after three days after operation. The colangiography recognize the stop.

Results: Case 1: Immediately the lesion was evident and sheltered in laparoscopy, suturing with a spin reabsorbable. The postoperative outcome was good and the patient was dismissed after three days. At the last follow-up the cholangiography didn't show stricture or leakage. Case 2: Therefore a reoperation in 15th day was needed and laparotomic Roux-en-Y hepatico-jejunostomy was performed. The patient was dismissed in 22nd day, with a Kehr drainage. After 40 days the drainage was removed and last follow-up evidenced a good condition.

Discussion and Conclusions: The most common cause of BTI is the failure to recognize the anatomy of the triangle of Calot. This is attributed to factors inherent to inadequate training of the surgeon and to local anatomical risk factors. The laparoscopic 'learning curve' of the surgeon is the most important (truncated at 250 words). 19th National Congress of the Italian Polyspecialist Society of Young Surgeons (IPSYS) 


\section{8 \\ Common Bile Duct Lithiasis: Role of Magnetic Resonance Cholangiopancreatography (MRCP) and Transcystic Drainage \\ P. Sperlongano, D. Parmeggiani, L. Del Viscovo', D. Pisaniello, I. Sordelli, A. Apicella², N. Avenia, \\ A. Piatto, A. Barbarisi, U. Parmeggiani \\ Department of Anaesthesiological, Surgical and Emergency Science, V Division of General Surgery, Second University of Naples, 'Department of Experimental and Clinical Medicine 'F. Magrassi-A. Lanzara'. Section of Radiodiagnostics. Second University of Naples, P.zza Miraglia, Naples, Italy; \\ ${ }^{2}$ Department of Hystopathology}

Introduction: The aims of this study were to evaluate the efficacy of MRCP (Magnetic Resonance Cholangiopancreatography) in detecting stones in patients presenting risk factors for CBD (Common Bile Duct) lithiasis, but negative ultrasonography (US). The Authors (AA.) also suggest the use of a transcystic drainage placed during VLC (videolaparocholecystectomy) and to perform a post-operative cholangiography through it, to detect possible missed stones and improve the sensibility of the technique.

Materials and Methods: From January 2002 to May 2006, 404 patients underwent VLC for cholelithiasis. Among these, 48 (11.7\%) presented risk factors for CBD lithiasis, too. Only 15 (31\%) patients had stones confirmed by US. Thus, the other 33 patients underwent MRCP, which revealed stones in 7 of them (21.2\%). The lasting 26 of 33 patients had a drainage inserted through the cystic duct during VLC and a cholangiography performed three days after surgery, which confirmed the MRC data.

Results: In the Authors' experience, the sensibility and specificity of MRCP in detecting CBD stones are, respectively, 88.8 and $100 \%$, with positive and negative predictive values, respectively of 100 and $96.5 \%$.

Discussion and Conclusions: The Authors suggest MRCP as an effective tool to detect CBD stones missed at ultrasonography in patients with risk factors and recommend an improvement of its utilization, in order to avoid the risks of ERCP, which should be reserved for therapeutic by US. Any enlargement of CBD.

89

Utility of the Sub-Hepatc Drainage in Bile Spillage Following VLC. Clinical Experience

P. Sperlongano, D. Parmeggiani, D. Pisaniello, I. Sordelli, A. Apicella ${ }^{1}$, N. Avenia, A. Piatto, A. Barbarisi, U. Parmeggiani

Department of Anaesthesiological, Surgical and

Emergency Sciences, V Division of General Surgery,

${ }^{1}$ Department of Histopathology

Introduction: In this study, the AA. report their experience with two case of bile spillage subsequent VLC among a series of 326 patients who underwent such procedure.
Methods: Our series include 326, unselected, patients symptomatic for gallstones. The first $40 \mathrm{VLC}$ were performed without the placement of the sub-hepatic drainage. Actually we routinary place it and we remove it after $48 \mathrm{~h}$. Case report 1: F 29 years old. VLC for gallstone and, since the first p.o. day, presented jaundice and bile leak through the sub-hepatic drain without signs of perithonism. The ERCP evidenced a stone in the right hepatic duct. The papillosphyncterotomy was then achived with removal of the stone. Recovery. Case report 2: F 45 years old. VLC for gallstones, with any placement of sub-hepatic drain. She lamented pain to the upper abdominal quadrants and the left shoulder, which persisted until the 3rd p.o. day. The US showed a collection close to the spleen and spreading to the Douglas cavity. We performed a US-guided drainage which resulted in bile. Recovery.

Results: The only intra-operative complication was a persistent bleeding from the gallbladder bed which required the conversion to laparotomy $(0.3 \%)$, post-operative complication were: bile spillages $(0.6 \%)$; gallbladder rupture during the extraction with subsequent loss of bile and stones in abdomen (1.9\%), transient sub-scapular pain (4\%), subcutaneous incisional emphysema $(0.6 \%)$, infection of the umbelical wound (1.8\%). Mean operative time was $60^{\prime}(40-180)$. The conversion to the laparotomy was necessary in 5 patients $(1.5 \%)$, (truncated at 250 words).

\section{0}

\section{Rubber-Band Ligation in a Proctologic Unit}

G. Cimmino, D. Parmeggiani, D. Cerbone1, F. Cammisa, M. Perrotta

Proctologic Unit of General and Videolaparoscopic Surgical Department, Frattaminore Hospital, Naples; ${ }^{1}$ Proctologic Unit, General Surgery ASL Napoli 1 Ascalesi Hospital

Introduction: The conservative and mini-invasive treatment of haemorrhoids, anal thrombosis, anal fistulas and anal fissures is very important.

Methods: We report our experience in two proctologic unit in the last two years, for treatment of haemorrhoidal disease. The main indication were the haemorrhoids of second degree. Normally we put 1 or 2 banding in the jack-knife position. We use disposable anoscope, $23 \mathrm{~mm}$ of diameter connected at cold light. There is no need of any kind of anaesthesia. The procedure is repeated in 10-14 days intervals, once or more times. The control examination is done after 2 months after the last banding. We have treated 185 patients (100 women and 85 men) in two years (January 2004 to December 2005). 15 of them had to be operated urgently. Were administrated 350 banding.

Results: About pain, $45 \%$ have moderate pain 1.2 days, $14 \% 3$ or more days. $37 \%$ have strange feeling in anal region several hours to several days. 3\% have severe pain 1.3 days. Slight bleeding has been observed in 68 patients $(36 \%)$. Severe bleeding, which caused special control was at 39 patients $(21 \%)$. They were treated by surgical suture, 7 patients had to be observed in the hospital. We observed no death, no pelvic or retroperitoneal sepsis.

Discussion: We consider the rubber-banding as the best and most safe semi-invasive method of the treatment of the second degree 
haemorrhoids. Step by step treatment of haemorrhoids is, on one hand, time consuming, but on the other hand the best and (truncated at 250 words).

\section{1}

\section{Schwannoma's of the Brachial Plexus}

P. Sperlongano, D. Parmeggiani, D. Pisaniello, I. Sordelli, P. Biondi', A. Apicella², N. Avenia, A. Piatto, G. Colella',

A. Barbarisi, U. Parmeggiani

Department of Anaesthesiological, Surgical and Emergency Sciences, ${ }^{1}$ Department of Head and Neck Surgery, ${ }^{2}$ Department of Histopathology

Introduction: Schwannomas are benign solitary tumors arising from the nerve sheath of a Schwann cell. We present a case of bulky Schwannoma arising from the brachial plexus. This primary tumor is an unusual cause of cervical mass.

Materials and Methods: A 38-year-old man presented with a slow-growing left-sided upperclavear mass and complained paresthesia of the third and forth finger of he hand so as forearm weakness. Physical examination revealed shooting pain on palpation and forearm flexing inability (Tine's sign). A neck and sopraclavear CT scan revealed a $5.5 \times 4.5 \mathrm{~cm}$ enhancing oval solid mass, well-delineated, situated in the left upperclavear fossa. MRI revealed relatively low signal intensity on T1-weighted images and high signal intensity on T2-weighted images.

Results: The tumor was resected with microsurgical techniques. During the operation, care was taken to preserve the nerve function. The histologic examination showed a benign schwannoma. During the follow-up pain nor signs of malfuncion were recorded.

Discussion and Conclusions: Those tumors of the brachial plexus belongs to neurilemmomas, normally those kind of cervical benign mass can easily be misunderstood by young surgeons.

\section{2}

\section{Antibiotic Prophylaxis for Patients} Undergoing Breast Surgery: Is it Worthy?

\section{E. Perego, S. De Fina, C. Franciosi, L. Degrate, C. Nobili, F. Romano, F. Uggeri \\ Ospedale San Gerardo, Chirurgia Generale, Monza}

Introduction: Even breast surgery is considered a clean procedure, the reported rate of wound infections varies from 5 and $30 \%$. Identify the situations with a particularly high risk of wound infection after breast surgery is desirable because, aside from the advantages obtained by preventing such complications, wound infection delays the beginning of adjuvant regimens within an optimum time after surgery.

Methods: Between November 1, 2005 and April 30, 2006, all breast surgeries carried out at our division were recorded. Patients were operated on by the same surgical team and managed by a standard protocol of care. 62 breast surgeries were performed divided in 13 mastectomies, 26 quadrantectomies and 23 open biopsies. 8 procedures were reoperations after a primary diagnostic biopsy performed from 7 to 14 day before. Immediate breast reconstruction was performed in 3 cases. Antibiotic prophylaxis was administered in selected cases: a single preoperative dose of cefalosporin ( 1 or $2 \mathrm{~g}$ intravenously) in patients with $\mathrm{BMI}>27$, in patients who underwent previous open surgery and in case of immediate breast reconstruction. A 5-7 daytherapy was performed only if an expander or definitive prosthesis was inserted. The wounds were reviewed during each outpatient visit with a final phone-review 4 weeks after surgery. Wound infection was defined as the presence of either pus or a serous discharge that contained pathogenic organisms. Axillary seroma was not concerned.

Results: No infections were reported in the study period. Discussion: Antibiotic prophylaxis in breast surgery should be performed just in selected patients with (truncated at 250 words).

\section{3}

\section{Meigs Syndrome: Cases Report}

\author{
E. Piazzese, V. Pacilé, G. Faro, F. Fiumara, A. Bonsignore, \\ L.G. Angiò \\ Università degli Studi di Messina, Scuola di \\ Specializzazione in Chirurgia Generale I
}

Introduction: Meigs Syndrome (MS) is characterized by benign ovarian tumor (BOT), ascite and hydrothorax with characters of transuded, reappeared after aspiration and negative cytologic examination; disprotidemias coexist, increase CA125, general decadence; the removal of ovarian tumor is decisive. MS interests $1 \%$ of patients with benign ovarian tumor and has a peak of incidence between the 50 and the 60 years of age.

Patients and Methods: We report 2 cases of MS treated between 2000 and 2005. Both patients presented tension and abdominalgia, dyspnea, cough, edemas to the inferior limbs, contraction of diuresis, precarious general condition, hydrothorax, ascites and ovarian tumor (to ultrasoound and TC), reduction of albumin, increase of CA125. The cytological examination of thoracic and abdominal liquid resulted negative for tumoral cells. The patients have been submitted to laparoscopy and to removal of ovarian tumor. The histological examination confirmed the diagnosis of BOT (fibromas and tecomas).

Results: The postoperative course has been regular. The followup (max 24 months) has shown resolution of hydrotorax and ascites, with CA125 normalization.

Conclusion: The preoperative diagnosis of MS results complex above all for the difficulty to identify the nature of ovarian tumor; the laparoscopy allows to exploit the action dirimente of the extemporaneous histological examination and to proceed to the alone removal of ovarian tumor. 


\section{4}

\section{Spontaneous Hematoma of Abdomen Recti Muscles: Cases Report}

\author{
E. Piazzese, V. Pacilè, G. Sfuncia, A. Bonsignore, \\ F. Fiumara, L.G. Angiò \\ Università degli Studi di Messina, Scuola di \\ Specializzazione Chirurgia Generale I
}

Introduction: Spontaneous hematoma of abdomen recti muscles (SHARM) is consequence of vascular or muscular lesion to the action of one or more eziologic factors in partnership, among which the respiratory affections with cough (56-78\%), coagulopaties (22-65\%), preceding laparotomies, cardiovascular pathologies, female sex and multiple pregnancies.

Patients and Methods: Among 1998 and 2005 we have hospitalized 9 patients for SHARM; all were in anticoagulant therapy, presented abdominalgia, palpable mass, anemia and coagulative alteration. All have been submitted to Rx abdomen, ultrasoound and in 7 cases to TC. Initial therapy has been preservative, but in one case for severe amenia and onset emodynamic instability, proceeded to evacuation of clots, washing of hollow, haemostasis and positioning of drain in residual space.

Results: The rate of mortality results void. The morbility has been $11 \%$ (venous thrombosis). Middle hospitalization is 11 days.

Conclusion: Our experience, even though numerically limited, confirmation the data presents in literature, both for that it concerns the clinical presentation, the diagnostic procedure essentially submitted to wall ultrasoound (sensibility 70-90\%) and to TC in the least clear cases (sensibility 100\%). Consolidated is the treatment of SHARM, initially giving precedence to a conservative attitude (adopted in the $80 \%$ of the cases) and only in case of negative evolution to that surgical.

\section{5}

\section{Secondary Intestinal Occlusion to Adhesions Syndrome in Patient with Family Mediterranean Fever: Case Report}

\author{
E. Piazzese, V. Pacilè, F. Aricò, A. Bonsignore, F. Fiumara, \\ L.G. Angiò \\ Università degli Studi di Messina, Scuola di \\ Specializzazione in Chirurgia Generale I
}

Introduction: Intestinal adhesions in $10-20 \%$ are manifested in patient that have ever suffered surgical interventions or abdominal traumas and are due to inflammation of abdominal organs or peritoneum, as in the case of Family Mediterranean Fever (FMF).

Case Report: MG (male, 64 years) with recurrent acute arthritises, has been hospitalized for fever and intestinal occlusion. Excluded BID, for occlusion persistence, we have performed exploratorative laparoscopy, during which have been cut numerous adherences that obstructed the ileum and are been performed biopsies on the serous intestinal and on the mesentere that appeared bloodshot. The clinical data, the intraoperative and histological finds, the positiveness of ANA dosing address the diagnosis toward a FMF, for which the patient has been treated with colchicina.
Conclusion: The abdominal manifestation, in peritonitic form, can often represent the first sign of the FMF making to perform in $30-40 \%$ of cases useless surgical interventions. Repeated peritoneal inflammations can form adhesions that obstruct the ileum in 3\% of cases. For such considerations and for our case report can conclude that in presence of obstructed patients, ever operated, with recurrent abdominal sierositis, kind if young people, are had to also advance suspicion of adhesions syndrome from FMF: this can allow to optimize the diagnostic run avoiding useless interventions.

\section{6 \\ Plastics of Reinforcement Second Chevrel for the Treatment of Recti Abdominis Muscles Diastasis: Proposal for Technique's Variation

E. Piazzese, V. Pacilè, A. Calopresti, A. Bonsignore,
F. Fiumara, L.G. Angiò
Università degli Studi di Messina, Scuola di
Specializzazione Chirurgia Generale I

Introduction: Diastasis of recti abdominis muscles (DRAM), differs from the incisional hernia for the absence of miofascialis plain solution and adherences. The operating indications are represented by important functional throngs and aesthetics kind in young women.

Patients and Methods: 11 patients with DRAM ( 8 xifo-pubic, 3 xifo-umbilical) have been treated through direct plastics of linea alba according to Welty-Eudel's technique and plastics of reinforcement according to Chevrel's technique (PRC) as from us modified.

Results: Mortality and morbility have been void. After 2 years, the reparation acquits to functions of containment and aesthetics, without stiffening of abdominal wall neither respiratory limitation.

Conclusion: The gotten results induce us to express a favorable judgment on the technical variation of PRC, whose moments qualificantis are: lodging of mesh below the side edges of anterior scabbard of recti abdominis muscles, to exploit, even if only partially, the advantages of suture for apposition (Pascal's principle); his anchorage to the solid lines side dawns and to the inside borders of mottos side edges and to the underlying plan, to guarantee stability, to prevent its folding and to annul the dead spaces responsible of sieromas and infections. For his rational the easy procedure is set as alternative to the methodic ones till now you suggest for care of DRAM.

\section{7}

\section{Acute Occlusion of Small Bowel Secondary} to Benign Pathology: Personal Experience

\author{
A. Bonsignore, E. Piazzese, V. Pacilè, P. Viggiani, \\ F. Fiumara, L.G. Angiò \\ Università degli Studi di Messina, Scuola di \\ Specializzazione in Chirurgia Generale I
}

Introduction: Acute occlusions (AO) of small bowel (SB) secondary to benign pathology are responsible of $10-20 \%$ hospitalizations 
in patient with acute abdomen and of 300 operations every year. The most frequent causes are represented by adhesions syndrome and hernias.

Patients and Methods: From 1996 to 2005, 119 patients have been treated in urgency for AO caused by following benign pathologies of SB: hernias strangulation (61), adhesions syndrome (34), Crohn's disease (9), biliar ileus (4), Meckel's diverticula (3) and other pathologies (8).

Results: In all cases have been performed laboratory examinations and abdomen radiography; frequently we have also employed ultrasoound (65\%) and TC abdomen (34\%). We have gotten the preoperative eziologic diagnosis alone in $59 \%$ of cases. We have adopted endoscopic treatment in 1\% of cases, laparoscopic in 5\%, laparotomic in $94 \%$. Mortality has been of $4 \%$ and morbility $29 \%$, with a postoperative middle hospitalization of 7 days.

Conclusion: In benign AO of SB the behavior of surgeon, appraised the clinical condition of patient, has to allow a rapid diagnosis and a timely treatment with the purpose to improve the prognosis. In the impossibility to formulate a preoperative eziological diagnosis, as often happens, the surgeron has to contemplate to precocious treatment, also through surgical intervention, to reduce to least one risk of further evolutionary complication.

\section{8}

Sequential Prophylaxis with Levofloxacyn for Prosthesic Repair of Inguinal Hernia with PHS, in 'One Day Surgery'

V. Pacilè, E. Piazzese, A. Calopresti, F. Fiumara,

A. Bonsignore, L.G. Angiò

Università degli Studi di Messina, Scuola di

Specializzazione Chirurgia Generale I

Introduction: Prosthesic repair of inguinal hernia (PRIH), even though imputable to 'clean surgery', is susceptible to exogenous contamination; requires therefore of antibacterial prophylaxis with purpose to prevent the septic complication of surgical site, that can frustrate the social-economic advantages.

Patients and Methods: Between 2003 and 2005, 58 males (age middle 59 years) have been submitted in 'one day surgery', to PRIH with PHS (Prolene Hernia System) and sequential prophylaxis with levofloxacyn (SPL) according to the following scheme: $500 \mathrm{~g} \mathrm{ev}$ $30 \mathrm{~min}$ before surgery and $500 \mathrm{mg}$ os die in 7 following days.

Results: Evaluation of surgical site has not underlined septic complication; only in 3 cases (5\%) light wound inflammation is found. In any patient signs of intolerance are manifested local and systemic pharmacology; 2 patients $(3.5 \%)$ have presented nausea and diarrhea of light entity; in 1 patient increase of values of liver indices is observed, quickly regressed to the therapy suspension.

Conclusion: Satisfactory results gotten with the levofloxacyn under the profile of the clinical effectiveness of prophylaxis and tolerability induce to standardize its employment in PRIH. The choice is crossed by the peculiar pharmacological characteristics of molecule, from the elevated compliance of patient, above all if operated in 'one day surgery', and from the advantageous relationship costs/benefits.
99

\section{Incisional Hernia Repair with Prosthesis: Personal Experience}

\author{
F. Fiumara, E. Piazzese, V. Pacilè, A. Costantino, \\ A. Bonsignore, L.G. Angiò \\ Università degli Studi di Messina, Scuola di \\ Specializzazione Chirurgia Generale I
}

Introduction: In last 20 years the surgery of incisional hernia (IH) has suffered a notable evolution, passing from a therapeutic approach that used the direct suture (DS) to a based on the employment of bio-material [Polypropilene (PP), Polytethrafluoroethylene expanded (PTFE-e), Bard Composix (BC), etc] variedly positioned to closing of wall defect to level suprafascial (SF), intraparietal (IP) or endoperitoneal (EP).

Patients and Methods: Of 125 patients operated for IH, 96 (76.8\%) have been treated through prosthetic techniques:

- Miofascial DS + PP's mesh SF (15)

- PP's mesh IP (28)

- PTFE-e's mesh IP (22)

- BC's mesh IP (7)

- PTFE-e's mesh EP (8)

- BC's mesh EP (10)

- PTFE-e's mesh EP + PP's mesh IP (6)

Results: In immediate postoperative course, in absence of mortality and systemic morbility, we have recorded 23 local complications (23.9\%). In distance follow-up (to 2 years) we have noticed 3 complications (3.1\%). We have achieved a suit success in $96.9 \%$ of cases.

Conclusion: The results gotten in the treatment of HI are satisfactory and they induce us to affirm the effectiveness of wall prosthetization in to guarantee solid reconstruction, without the risk of an abdominal and lasting visceral contention iperpression, practically in absence of recurrence; nevertheless, doesn't resolve the alterations of respiratory dynamics and is not lacking from complication, rarely of such entity to be asked for a second look.

\section{0}

\section{Treatment of Colorectal Anastomotic Dehiscence by Metallic Covered Endoprothesis}

\section{Riccardo \\ I Clinica Chirurgica-Policlinico Universitario G. Martino, Messina}

Treatment of colorectal anastomotic dehiscence by metallic covered/plated endoprothesis One of the first cause of morbidity in colorectal surgery is anastomotic dehiscence. Such complication, in severe cases, can be responsible of a stercoracea peritonitis because of the spreading of intestinal contents in the abdominal cavity. Anastomotic dehiscence may be due to many causes: local factors such as poor anastomotic vascularization; inadequate bowel preparation; incorrect surgical techniques and general factors such as age of the patient, obesity, smoking or alcohol. The diagnosis is relative easy, in particular for the symptomatic forms. Contrast enema and colonoscopy are effective and 
low cost procedures. In the literature it is still debated the best way of treatment and prevention of this complication. Many studies reported that parental nutrition can reduce the incidence of anastomotic dehiscence. Instead of diverting stoma didn't prevent dehiscence, but reduced the risk of peritonitis. Moreover use of stapler for low rectal anastomosis seems to avoid the occurrence of a dehiscence. When a stercoracea peritonitis develops as consequence of an anastomotic dehiscence, an immediate surgical treatment is required. Use of intestinal endoprothesis as alternative treatment option for such complication has been reported in experimental studies. We report two cases of intestinal dehiscences treated successfully by positioning of metallic covered endoprothesis.

101

\section{Use of Totally Implanted Venous Access Device: Notes of Technique and Our Experience}

\section{A. Rinaldi, M.C. Gallo, A. Schiavone, T.D. Usò, M. Gavioli, A. Mauro, G. Natalini \\ Divisione di Chirurgia III, Azienda Policlinico, Universitaria di Modena}

Introduction: Totally implanted venous access device (TIVAD) is constituted of a catheter inserted into the central venous system connected with a reservoir located in the subcutaneous tissue. The surgical technique consists of surgical isolation of a peripheral vein, like the cephalic vein $(\mathrm{CV})$ and advance the catheter up to the cavoatrial junction. We describe our experience on TIVAD using the surgical technique.

Methods: Between January 2003 and February 2006, 259 ports have been implanted at our Unit. We treated 121 women, 138 men; mean age 61 (range 22-93). We performed an incision at the level of the deltoid-pectoral groove, dissected the $\mathrm{CV}$ and incanulated. The catheter is inserted through the vein up to the cavoatrial junction under fluoroscopy. The catheter is connected to the reservoir placed in a subcutaneous pocket fixed to the fascia of the pectoralis muscle.

Results: No intra-perioperative complications occurred. Regarding late complications we experienced: $11(4 \%)$ infections of subcutaneous pocket, for all of them was necessary to remove the port; $6(2 \%)$ hematoma in the implant site, 2 cases required surgical evacuation; 2 (1\%) kinking port reservoir.

Conclusion: Our study confirms the safety of TIVAD. The limit to the surgical approach is represented by anatomical variation of the $\mathrm{CV}$. In this cases we dissect the external jugular vein (40 cases, $15 \%$ ). The percutaneous subclavia access represents our last option (12 cases, $4 \%$ ). The advantages of percoutaneous technique are essentially linked to the reduced surgical time. The disadvantages are increased rate of pneumothorax (1-6\%). The surgical technique increased operative (truncated at 250 words).
102

\section{Sentinel Lymph Node Micrometastasis in Breast Cancer}

A. Rinaldi, M.C. Gallo, A. Schiavone, S. Guaitoli,

M. Gavioli, A. Mauro, G. Natalini

Divisione di Chirurgia III, Azienda Policlinico, Universitaria di Modena

Introduction: Axillary node dissection in patients with early breast cancer, is often not necessary and has high morbidity (15-30\%), sentinel lymph node biopsy (SLNB) is a valid alternative in the staging of breast cancer, for its less morbidity and accuracy. Currently the standard of care is to perform SLNB in patients with small tumors and clinically negative axilla. With dectetion micrometastasis in sentinel lymph node (SLN) the new question of performing or not axillary dissection arises in these patients.

Methods: We reported our experience with SLNB and micrometastasis. All SLN were evaluated by frozen section and immunohistochemistry. Micrometastasis was defined as tumor deposit between 0.2 and $2 \mathrm{~mm}$ classified as $\mathrm{pN} 1 \mathrm{mi}$ and isolated tumor cells no larger $0.2 \mathrm{~mm}$ classified as pN0 (6th edition of AJCC Cancer staging).

Results: Between February 2004 and December 2005, 214 patients (T1-T2 $<3 \mathrm{~cm} \mathrm{N0}$ ) underwent quadrantectomy or wide resection and SLNB (median age 52.5 range 31-74). 144 patients had SLN negative (67\%), 38 patients (18\%) had SLN positive for macrometastasis and 32 patients $(15 \%)$ had SLN positive for micrometastasis. In these 32 patients with micrometastasis we found $28(87.5 \%) \mathrm{pN} 1 \mathrm{mi}$ (in 4 patients other lymph node were positive at axillary dissection) and 4 $(12.5 \%) \mathrm{pN} 0$.

Conclusion: Actually the role of isolated cells and small micrometastasis remains controversial. For our experience, the number of cases with micrometastasis was small, so firm conclusions cannot be drawn. We agree that out with clinical trials, complete axillary dissection should be performed on all patients with micrometastasis in SLN.

103

RFA and TACE vs. RFA in Hepatocellular Carcinoma in Cirrhotic Patients: Local Therapeutic Effects and Patients Outcome

A. Ruzzenente ${ }^{1}$, M. D'Onofrio ${ }^{2}$, S. Pachera ${ }^{1}$,
T. Campagnaro ${ }^{1}$, M. Fontana ${ }^{1}$, A. Guglielmi ${ }^{1}$

${ }^{1}$ Department of Surgery and Gastroenterology, University of Verona, ${ }^{2}$ Department of Radiology, University of Verona, Italy

Introduction: RFA is effective for small HCCs $(<3 \mathrm{~cm})$ but results for larger lesions $(>4 \mathrm{~cm})$ are less satisfactory. The aim of our study is evaluate efficacy of combination therapy of TACE and RFA compared to RFA in $\mathrm{HCC}>4 \mathrm{~cm}$.

Methods: 68 cirrhotic patients with 84 unresectable $\mathrm{HCC}$ were treated during the study period, lesions were $>4 \mathrm{~cm}$ and $<6 \mathrm{~cm} .23$ patients were submitted to combined treatment with TACE before to RFA (group A); 61\% were Child A and 39\% Child B; 57.8\% had 
unifocal and $42.2 \%$ multifocal tumour. 47 patients were treated with only RFA (group B); $56.8 \%$ were Child A and $43.2 \%$ were Child B; $43.5 \%$ had unifocal tumour and $56.5 \%$ multifocal.

Results: Complete tumour necrosis after the first treatment was achieved in $82 \%$ of lesions in group A and in $66 \%$ in group B $(\mathrm{p}=0.15)$; after single and/or multiple treatments complete necrosis was achieved in $87 \%$ and $83 \%(p=0.6)$. Number of treatments to achieve complete necrosis was significant lower in group A $(\mathrm{p}<0.05)$ (range 1-4). After a mean follow-up of 23.7 months local recurrence occurred in $18.5 \%$ in group A and $20 \%$ in group B $(\mathrm{p}=0.87)$. Median survival for patients of group A and B was 20 and 25 months, respectively ( $\log$ rank test $\mathrm{p}=0.41$ ).

Discussion: RFA and TACE reduce number of session for complete necrosis but it do not increase complete necrosis rate, frequency of local recurrences and survival.

\section{4}

\section{Survival of Cirrhotic Patients with HCC After RFA: Comparison of 7 Staging Systems}

\author{
A. Ruzzenente, S. Pachera, A. Valdegamberi, \\ M. Fontana, T. Campagnaro, A. Guglielmi \\ Department of Surgery and Gastroenterology and \\ Radiology, University of Verona, Italy
}

Introduction: RFA can improve survival in patients with HCC not eligible for surgery. Many staging systems have been proposed but there is no convincing evidence that one system is better than others. The aim of this study is to identify which system allows better prognostic evaluation in cirrhotic patients with HCC submitted to RFA.

Methods: We retrospectively analyzed 112 patients with HCC and liver cirrhosis treated with percutaneous RFA from 1998 to 2005. Before treatment all the patients were classified according to 7 different stadiation systems: TNM, BCLC, CLIP, GRETCH, CUPI, JIS. The follow-up was performed with serum AFP dosage, CT or MRI evaluation. Factors related to survival and performance staging system were analysed by univariate and multivariated analysis.

Results: Univariated analysis shows that factors related to survival were tumour type of growth, serum level AFP greater than $20 \mathrm{ng} / \mathrm{dl}$ and complete response to RFA. In a multivariated analysis, Child-Pugh score, serum level of AFP, and the response to treatment were significant predictors for survival. Among different staging system CLIP score showed best values of homogeneity and monotonicity.

Discussion: CLIP resulted the statistically better stadiation system in staging patients with HCC after RFA. Other systems such as JIS and BCLC, nevertheless, appeared effective in stratifying these patients.

\section{5}

\section{Minimally Invasive Management of Obstructive Colonic Malignancy}

\section{R. Sampietro, E. Orsenigo, S. Di Palo, C. Socci, C. Staudacher}

Department of Surgery, San Raffaele Scientific Institute, University Vita-Salute, Milano

Background: Left colonic malignancies may manifest themselves as an emergency with an acute obstruction. Surgical management is controversial. Colonic resection with end colostomy, subtotal colectomy or intraoperative colonic lavage with primary anastomosis are the most frequent treatments. Recently, endoscopic self-expanding metal stenting (SEMS) procedure has been advocated as a possible choice to relive obstruction either for palliative purpose or as a 'bridge to surgery'.

Materials and Methods: We present a video showing the minimally invasive management of a left colonic obstruction due to a malignancy treated by emergency endoscopic stenting and after 10 days a 3-port laparoscopic left colectomy was successfully performed.

Results: The presence of the metallic stent did not compromise the surgical procedure at all. The patient was discharged after 7 days with no postoperative complications.

Conclusions: Sequential endo-laparoscopic management might be evaluated as treatment of choice for occlusive coloni malignancies. The procedure could be a valid alternative to traditional emergency surgery.

\section{6}

\section{Laparoscopic Excision of Gastric GIST}

\author{
N. Osman, E. Orsenigo, M. Frasson, S. Di Palo, \\ C. Staudacher
}

Department of Surgery, San Raffaele Scientific Institute, University Vita-Salute, Milan

Aim: Laparoscopic approach is now an accepted treatment in small Gastrointestinal Stromal Tumors (GIST). However, the role of minimally invasive surgery in the treatment of lesions with more than $2.5 \mathrm{~cm}$ in diameter is still debated for the higher risk of capsule rupture and peritoneal seeding. The aim of this study was to evaluate the feasibility and the outcome of laparoscopic approach for gastric GISTs.

Methods: We have retrospectively evaluated 8 patients with gastric GIST who underwent laparoscopic resection from January 2004 to April 2006. All lesions were located in the middle stomach. The mean size was $3.5 \mathrm{~cm}$ (range $2-5 \mathrm{~cm}$ ). Diagnosis was performed by gastroscopy and using an endoscopic ultrasound examination. Surgical approach was performed by use 3 or 4 trocars. The exicion was performed by use a linear stapler, the specimen was immediately placed and removed in a retrieval bag trough the incision of the port-site.

Results: All operations were completed with by laparoscopy. No major complications occurred. The pathological examination confirmed the diagnosis of GIST with negative surgical margins in all specimens. No recurrence was recorded at a mean follow-up of 21 months. 
Discussion: Laparoscopic resection for GIST seems to be a good alternative to laparotomy for the surgical treatment of gastric GISTs. We have successfully treated 6 lesions (75\%) with diameter $>2 \mathrm{~cm}$ and resection have been carried out safely without recurrence. Laparoscopic excision for GISTs is a feasible and safe technique if performed in selected patients with lesions size $<5 \mathrm{~cm}$.

\section{7}

\section{Surgical Treatment of Early Gastric Cancer \\ M. Sartelli, R. Scibé, G.C. Gesuelli, I. Patrizi \\ Chirurgia Generale, Ospedale Provinciale di Macerata, U.O. Chirurgia Generale, Macerata}

Introduction: Early gastric cancer is defined as a gastric carcinoma confined to the mucosa or submucosa regardless of lymph node status. The presence of lymph node metastasis in EGC is the most important prognostic factor. The actual therapeutic value of extensive lymphadenectomy in patients with early gastric carcinoma remains controversial. The most important risk factors associated with lymph node metastasis are large tumor size, undifferentiated histologic type, submucosal invasion and lymphatic-vascular involvement.

Methods: From January 1998 to April 2006 of 364 patients with primary gastric adenocarcinoma, 43 with EGC underwent potentially curative resection (R0) with lymphadenectomy.

Results: Of the 43 patients with early gastric cancer, 27 underwent sub-total gastrectomy and 16 total gastrectomy. 37 patients underwent D2 lymphadenectomy, 6 patients D1 lymphadenectomy. There was one post-operative death. Of the 43 cases, 36 were classified as $\mathrm{N} 0$ and 7 as $\mathrm{N}+$.

Discussion: In patients with EGC, the survival rate of patients with positive lymph nodes is significantly worse than that of patients with no lymph node metastasis. A standard D2 lymphadenectomy should be always performed in patients with risk of lymph node metastasis.

\section{8}

\section{CT Role on the Surgical Treatment of GIST:} A Pictorial Assay

\section{A. Sartori, F. Tauceri, V. Pistan, S. Scomersi, M. Belgrano, A. Rimondini, A. Spivach}

Objective: The aim of this study is to report CT aspects of the GIST to evaluate its efficacy in the identification of higher risk lesions of malignancy to plan the best surgical treatment.

Methods and Materials: Were evaluated retrospectively 26 patients that underwent to surgery with a diagnosis of GIST histological proven, localized to the stomach (20 cases), duodenum (1), cecum (1), small bowel (2), descending colon (1), and rectum (1). CT exams were performed with a single slice CT with a collimation of $5 \mathrm{~mm}$, before and after administering intravenous contrast medium.

Results: CT always allowed to define correctly site, size and structure of the lesions and in a few cases also signs of infiltration of neighbor structures. All the lesions presented solid density on the direct scan and a small enhancement after contrast medium, homogeneous pattern in 10 cases and inhomogeneous in 16; in a case histology revealed microcalcification not shown by $\mathrm{CT}$.

Conclusion: $\mathrm{CT}$, due to its panoramic view and high contrast resolution provide essential information for the therapeutic planning and the follow-up of the patients treated with surgery or chemotherapy and can modify the surgical approach to the lesion.

\section{9 \\ Role of Pelvic Floor Muscles Rehabilitation in Patients with Temporary lleostomy}

\section{A. Schiavone, A. Rinaldi, M.C. Gallo, A. Mauro, M. Gavioli, G. Natalini \\ Divisione di Chirurgia III, Azienda Policlinico, Universitaria di Modena}

Introduction: Recently, the pelviperineal rehabilitation has assumed a main role for the good treatment outcome in surgical proctology. In patients who underwent ileostomy, the rehabilitation executed in the period before and after stoma closing, was able to improve the sphincteric control.

Methods: The treatment is essentially based on the Kinesitherapy, Functional Electrostimulation and Biofeedback. The aim is that of reeducating patients to be aware of pelvic floor muscles, so that they do not lose the memory of ano-rectal functions (contraction-relax), during the period of sphincteric inactivity. Treatment starts at least 1 month before stoma closing using the three approaches in synergy. The Biofeedback allows estimating the effectiveness of the expressed muscular activity. However, an important active participation by the patients is required. The Functional Electrostimulation, in the preoperative phase, facilitates the consciousness of the muscles which have to be reactivated. Once stoma is removed, it is important to bring the patient back to normality with exercises of Kinesitherapy, which are useful to consolidate and keep the results obtained with ambulatory training.

Results: Our preliminary data show an improvement in treated patients of the physiological functions of the ano-rectal system with restored muscular force, endurance and motor coordination.

Discussion: We believe that a good rehabilitative treatment before and after ileostomy, is an important and indispensable tool in order to guarantee an improvement of quality life.

\section{0 \\ Retrorectal Dermoid Cyst: A Rare Entity. Case Report \\ G. Sciaudone, L. Brusciano, P. Limongelli, C. Di Stazio, 1. Guadagni, F. Selvaggi \\ I Division of General Surgery, Second University of Naples, Italy}

Introduction: Presacral cysts are included in the group of presacral tumours. An incidence of 1 per 40,000 hospital admissions has been estimated for this entity. The nature of these tumors is uncertain and treatment options are still debated. Such entity may simulate 
either anorectal sinus or fistula then causing problems in differential diagnosis.

Case: We report on a 39 years old female with a presacral mass incidentally found by a pelvic ultrasound performed after gynaecologic examination. The mass was evaluated by MRI and subsequently the patient was referred to our surgical unit. By performing a transperineal approach we carried out division of levator ani, medium gluteus and Waldayer fascia allowing the mass to be completely excised. Histological diagnosis was consistent with an epidermoid cyst. Patient was discharged 5 days after surgery and no complications or recurrence have been observed at 36 months follow-up.

Discussion: A complete excision of the mass seemed to be mandatory in order to clarify the nature of this kind of lesion and to prevent both its infection and malignant development. As what concerns the surgical approach, we chose a transperineal route, although alternative approach are described in literature, showing encouraging results that have to be confirmed in a large series of patients.

\section{1}

New Isoperistaltic Side-To-Side Strictureplasty for Long Stricture in Crohn's Disease: Preliminary Results

\section{G. Sciaudone, A. Giuliani, P. Limongelli, C. Di Stazio, I. Guadagni, F. Selvaggi \\ I Division of General Surgery, Second University of Naples, Italy}

Introduction: Strictureplasty is widely accepted as an alternative to resection in non-perforating jejunoileal Crohn's disease. The aim to preserve as much functional gut as possible, led surgeons to perform a strictureplasty instead of resection, particularly for patients with multiple or long stenosis.

Methods: We proposed a new type of isoperistaltic strictureplasty in 3 patients (1 male) with an obstructive disease and a long jejunoileal stenosis. Division of the ileum was performed distally to the diseased gut by calculating a distance from the stenosis similar to its length. This can allow the gut to be folded by anastomosing the stenosed tract to the healthy gut. By opening longitudinally both the diseased bowel and the healthy tract, a side-to-side entero-enteric anastomosis was performed. Then, an enterotomy was accomplished at the top of the loop and a side-to-end anastomosis was fashioned to replace bowel continuity in an isoperistaltic way.

Results: Patients presented with a bowel stenosis of 46, 57 and $61 \mathrm{~cm}$ in length respectively. There were no intraoperative complications. All patients had a normal postoperative course without any morbidity and mortality. Every patients underwent an upper gastrointestinal barium examination at 4 and 12 months after surgery, with no evidence of stenosis. At the last follow-up all patients were symptoms free.

Discussion: This technique seems to avoid the risk of inlet and outlet stenosis, likely because of the use of healthy tissue to perform the anastomosis. Furthermore, the proximal and distal edges of the anastomosis present a completely disease free tissue.

\section{2}

\section{Rectal Diverticula After Double-Stapled Transanal Rectotomy (STARR) for Obstructed Defecation}

\author{
G. Sciaudone, P. Limongelli, C. Di Stazio, I. Guadagni, \\ F. Selvaggi \\ I Division of General Surgery, Second University of Naples, \\ Italy
}

Introduction: The stapled transanal rectal resection (STARR) has been recently reported as a low-morbidity and effective operation for the treatment of obstructed defecation (OD). The following report involves a case of rectal diverticula performed after STARR procedure resulting in worsening of constipation.

Case: A 21-year-old female, nulliparous, presented a 9-years history of chronic constipation Physical examination revealed rectocele without genital or urological prolapse. Anorectal manometry showed increased threshold and maximum tolerated volumes and pudendal neuropathy was observed at electromiography. Defecography confirmed the diagnosis of rectocele and rectal mucosal prolapse without perineal descent. After failure of medical therapy (21/day of water, low-fiber diet and lactulose $10 \mathrm{~g}$ /day) a STARR procedure was performed. Two PPH-01 ${ }^{\mathrm{TM}}$ circular staplers were used. Patients was discharged on postoperative day two. Six months later she presented again with a severe constipation. Defecography pointed out a left postero-lateral rectal wall diverticular cavity $(24 \times 33 \mathrm{~mm})$ with an incomplete elimination of barium enema during evacuation. Proctoscopy confirmed this diagnosis. Transanal diverticulectomy and a direct rectal wall repair was performed without any complications. At 24 months follow-up patients showed a significant improvement of defecation.

Discussion: The management of OD should be mainly conservative and rarely surgical: according to the Mayo Clinic experience only $5 \%$ of constipated patients may benefit from surgical treatment. STARR procedure can produce new complications difficult to treat and early recurrence of symptoms requiring reoperation. This surgical technique should be reserved to expert colorectal surgeons with proved (truncated at 250 words).

\section{3}

\section{Cytoreductive Surgery and Intraperitoneal Chemohyperthermia for Peritoneal Carcinomatosis. Preliminary Results}

\author{
S. Virzi ', A. Grassi ${ }^{1}$, S. Bonomi', G. Navarra1', I. Imail', \\ S. Selva', A. Panetta ${ }^{2}$, G.L. Manna ${ }^{3}$ \\ UO Chirurgia Generale, Ospedale Bentivoglio Bologna
}

Introduction: Cytoreductive surgery and peritonectomy procedures (CRS) with perioperative intraperitoneal chemohyperthermia may allow prolonged survival for patients with peritoneal carcinomatosis.

Methods: From November 2003 to March 2006, we observed 79 patients with a peritoneal surface malignancy arising from colorectal cancer 32 , gastric cancer 10 , ovarian cancer 23 , appendiceal 
cancer 4, pseudomyxoma peritonei 4, uterus cancer 4, mesothelioma 1 , unknown origin 1 , who met the eligibility criteria for CRS and IPHP procedure. At laparotomy 7 patients were excluded from the combined treatment. In the remaining 72 patients, CRS was performed with peritonectomy procedures followed by IPHP using the closed abdominal technique. IPHP was performed using Cisplatin, Mitomycin-C or Doxorubicin for $60 / 90 \mathrm{~min}$ at mean intraperitoneal temperature of $42.5^{\circ} \mathrm{C}$.

Results: Seventy patients $97 \%$ were optimally cytoreduced CC$0 / 1$. Four patients were died in the post-operative period $5.5 \%$. Grade 3 morbidity was $19.4 \%$ : 3 intestinal perforation, 3 anastomotic fistula, 1 anastomotic dehiscence, 1 abdominal bleeding, 3 intraabdominal abscess and 3 ureteral fistula. Grade 3 toxicity was $15.2 \%$. After mean follow-up 14.5 months, 11 patients alive with disease, 43 patients alive without disease and 12 patients dead for disease.

Discussion: CRS combined with IPHP is confirmed to be a feasible (truncated at 250 words).

\section{4 \\ Prostatic Cancer Surgical Treatment vs. Non-Invasive Treatment}

S. Sorrenti, B. Vanni, M. De Stefano, S. Palermo, L. Di Battista, S. Guarino

\section{La Sapienza, Policlinico Umberto I, Roma}

Introduction: The carcinoma of the prostate is the fourth cause of death caused by tumour in the world. In our study we tried to identify the general principles of the treatment of this pathology whose management included the watchful waiting, invasive treatment and palliatives therapy

Methods: Our cases included 47 prostatic carcinoma, observed in our department. Our patients were divided in two groups: the first one (A) was made of 28 patients treated by radical prostatectomy and second one (B) made of 19 patients treated by conservative therapy: hormonotherapy, radiotherapy or chemotherapy.

Results: We performed a radical prostatectomy in group A; no perioperative mortality was observed. Few complications were experienced: 2 ureteral-bludder anastomotic-stenosys; 17 impotentia coeundi and a very high rate of incontinence in the first post-operative month, but in following ones the continence regained in most of the cases. Complications in group B: $52 \%$ of our patients died within 5 years: $90 \%$ of the deaths with a TNM between T3c and T4.

Discussion: Similar complications were observed either in surgical or in non-invasive approach. Radical prostatectomy was advise for patients with expectation of life over 10 years and with an organconfined illness, while hormone and radiotherapy was adviced for patients with advanced clinical stage and expectation life under 10 years.

\section{5}

\section{Surgical Treatment of Pancreatic Stump After Duodenocephalo-Pancreasectomy (DCP)}

\section{S. Sorrenti, S. Palermo, B. Vanni, M. De Stefano,} S. Guarino, L. Di Battista

Department of Surgical Sciences, Università 'La Sapienza', Rome

Introduction: DCP was performed in few patients with malignancy of the duodenopancreatic district because of the low rate of resecability of these tumours and because of the high mortality and morbidity rates connected to this surgery. We conducted this study to review the Literature about the treatment of the pancreatic stump after DCP. Analysing our casuistry we tried to establish the technique connected to the lowest rate of perioperative complications.

Methods: 44 patients underwent DCP in our Department. 28 affected by head pancreas cancer, 9 by choledoch adenocarcinoma, 4 by chronic pancreatitis, 2 by tumour of ampulla of Vater and 1 case by a pancreatic metastasis. In 23 cases we performed the classical Whipple technique and in the other 22 the Traverso-Longmire surgical variant.

Results: The global survival rate to 5 years was $25 \%$. Perioperative morbidity due to surgical complication was $9 \%$. Pancreatic leakage remained the major cause of morbidity. Pancreatic fistula was the most frequent complication but most of them were uncomplicated and healed with conservative treatment.

Discussion: The treatment of the pancreatic stump after DCP remains very controversial and the choice often derives from surgeon personal habits; our experience has brought us to identify the classic Whipple surgery with a double Roux-en-Y as the best technique to use.

116

\section{The Role of Preoperative Chemoradiation in Patients with Resectable Rectal Cancer: Preliminary Results}

\author{
S. Squillante, S. Di Palo, A. Vignali, A. Tamburini, \\ E. Orsenigo, C. Staudacher \\ Chirurgia Generale, IRCCS San Raffaele Milano
}

Introduction: To evaluate oncological and surgical outcome of patients submitted to neoadjuvant therapy for advanced rectal cancer.

Method: 138 patients ( 86 male, 52 female, mean age 61.4 years), with tumour of lower $(58 ; 42 \%)$, middle $(66 ; 48 \%)$, upper rectum $(14 ; 10 \%)$, showing a clinical stage II $(23 ; 17 \%)$ or III $(115 ; 83 \%)$ and with an average distance from anal verge of $6.5 \mathrm{~cm}$, submitted to fractionated 'long-course' RT with CT, locally staged by US and MR before and after neoadjuvant therapy and operated on after 4-6 weeks by its end.

Results: Surgical procedures (71 of which laparoscopic) were: 114 AR (83.8\%), 19 APR (14\%) and 3 TEM (2.2\%). Mean nodalsampling was 14.9. A complete o partial response was observed in $48.5 \%$ of the patients $(67 / 138)$. With a mean follow-up of 30 months, local recurrence rate was $5.7 \%$. Five-years overall survival and disease-free-survival were respectively 73 and $60 \%$. 
Discussion: We observed a significant clinical $(\mathrm{p}<0.004)$ and pathological $(\mathrm{p}<0.005)$ down-staging. Pre-treatment clinical stage was not significant. On the contrary, postoperative yTNM was significant for yT $(\mathrm{p}<0.001)$ and $y \mathrm{~N}(\mathrm{p}<0.0003)$. Non-responder patients had worse prognosis (5-years survival $30 \%)$. The variable with higher prognostic significance was $\mathrm{yN}(\mathrm{p}<0.0003)$, especially if we distinguish $\mathrm{N} 1$ by $\mathrm{N} 2(\mathrm{p}<0.0004)$. The response to neoadjuvant therapy represents a significant prognostic variable.

\section{7}

\section{Laparoscopic Total Mesorectal Excision}

P. Bisagni, S. Di Palo, E. Orsenigo, A. Tamburini, C. Staudacher

Osp San Raffaele, Milano

Background: We present the surgical technique of lap TME adopted in our Institution.

Method: We introduce Hasson and 4 ports. The inferior mesenteric vein is identified and Toldt's fascia divided. Then we continue to inferior mesenteric artery (IMA), sectioned once ureter is identified. The mobilization of splenic flexure is then completed. Using the IMA stump as a guide we divide mesorectal from presacral fascia. Dissection of the mesorectum is performed under direct vision using sharp technique. Particular attention is done to identify superior hypogastric plexus and right and left hypogastric nerves. The inferior hypogastric plexus is preserved by sectioning medially laterals ligaments of the rectum. Then we go on in front of Denonvillier's fascia. In all the phases we have great attention to preserve the integrity of visceral pelvic fascia. Once mesorectal dissection is completed, the rectum is divided at the pelvic floor. A $5 \mathrm{~cm}$ incision is made and the colon resected. After closing the incision, we laparoscopically perform a Knight-Griffen anastomosis.

Results: Conversion rate in 108 patients $12 \%$, morbidity $29.6 \%$ (no death), anastomotic leak $14.8 \%$, reoperation $6.5 \%$, hospital stay 10.1 days, lymphnodes sampling 14.3 , local recurrence rate $6.4 \%$ (median-follow-up 36 months).

Conclusion: Laparoscopic TME is a safe option and can be performed in the majority of patients.

\section{8}

\section{Sentinel-Node Mapping in Gastric Cancer: Preliminary Results in a Single Centre}

V. Tomajer, E. Orsenigo, M. Carlucci, S. Di Palo, A. Tamburini, L. Albarello', E. Masci², C. Doglioni ${ }^{1}$, P.A. Testoni²,

C. Staudacher

Dipartimento di Chirurgia, UO di Chirurgia

Gastroenterologica, 'Dipartimento di Anatomia Patologica, ${ }^{2}$ Dipartimento di Gastroenterologia, Università Vita-Salute San Raffaele Milano

Background: The first possible site of metastasis along route of lymphatic drainage from the primary lesion are known as sentinel-nodes
(SNs), detectable using injection of dyes or radioactive tracers. There are several matters of debate in performing the procedure, such as type of die, injection routes procedures (submucosal or subserosal), volume of tracer and observation timing. Our aim was to evaluate the feasibility of SN mapping in gastric cancer with submucosal blue die injection.

Methods: 29 cases of gastric adenocarcinoma without serosal invasion and distant metastasis were prospectively enrolled. Patency blue $2 \%$ was intraoperatively injected into the submucosa (four $0.5-2 \mathrm{ml}$ peritumoral injections). Positive blue lymph-nodes detected during first $15 \mathrm{~min}$ were considered as SNs. After then, a gastrectomy (total or subtotal) with a D2 lymph-node dissection was performed.

Results: SNs were identified in 17 of 29 patients; skip SNs were identified in 4 of $20(20 \%)$. Out of them, lymph-node metastases were retrieved in 7 patients. Four patients with negative hematoxylin and eosin staining had lymph-node metastasis in non SNs $(2 \mathrm{pN} 1,1 \mathrm{pN} 2$ and $1 \mathrm{pN} 3$ respectively). Positive and negative predictive value of SN staining was $100 \%$ and $71 \%$, respectively. Accuracy of SN mapping was $76 \%$, and sensitivity and specificity was $43 \%$ and $100 \%$, respectively.

Discussion: Intraoperative SN mapping in gastric cancer seems to be technically feasible. Complementary procedures like double tracers of dye and isotope, multiple sections of SNs and accurate pathological examination may be necessary to improve the results.

\section{9}

\section{Laparoscopic Splenopancreatectomy for Pancreatic Neuroendocrine Tumor}

\author{
V. Tomajer, E. Orsenigo, P. Bisagni, S. Di Palo, C. Staudacher \\ Dipartimento di Chirurgia, UO di Chirurgia \\ Gastroenterologica, Università Vita-Salute San Raffaele \\ Milano
}

Introduction: During the past, minimally invasive techniques were used for diagnostic laparoscopy in evaluating periampullary malignancy. Recently, laparoscopic distal pancreatectomy seems to be technically feasible and widely accepted. Indications for laparoscopic distal pancreatectomy include neuroendocrine neoplasms islet-cell tumors located in the pancreatic body or tail. We report the case of a 83-year-old woman in whom a computed tomography (CT) scan showed a solid, well-defined $3.5 \mathrm{~cm}$ tumor located in the pancreatic tail. We suspected a pancreatic nonfunctioning endocrine tumor. Fine needle examination demonstrated a neuroendocrine tumour. Patient has been scheduled for surgical laparoscopic treatment.

Methods: The procedure was undertaken under general endotracheal anaesthesia. She was placed in Lloyd-Davies position. Hasson Trocar was introduced in the epigastrium and three addictional $12 \mathrm{~mm}$ trocars were placed. Peritoneal cavity exploration revealed a nodular lesion located in the IV hepatic segment (frozen section did not reveal neoplasia). The gastrocolic ligament was divided using the ultrasonicactivated scissors (Sonosurg, Olympus). Inferior margin of pancreas was dissected with isolation of splenic vessels. The superior pancreatic border was dissected. Splenic vessels were divided by use EndoGIA. After the mobilization of pancreatic tail the splenopancreatectomy has been successfully completed. Proximal pancreatic stump has been reinforced by use fibrin glue and Tissufleece. 
Results: Postoperative course was uneventful. Patient has been discharged in the 6th postoperative day. Histopathological examination confirmed the diagnosis of neuroendocrine tumor.

Conclusions: Laparoscopic splenopancreatectomy is safe and technically feasible in the treatment of neuroendocrine pancreatic tumors.

\section{0 \\ Pneumatosis Cystoides Intestinalis of the Small Bowel Associated with Left Colonic Diverticular Disease: Case Report}

R. Bertelli, D. Cavaliere, M. Framarini, G. Mura, F Tauceri, G.M. Verdecchia

U.O. di Chirurgia e Terapie Oncologiche Avanzate, Ospedale G.B. Morgagni, L. Pierantoni di Forlì

Introduction: Pneumatosis cystoides intestinalis (PI) is a rare condition defined as the presence of gas within the bowel wall. The most common localization is the small intestine. It could be associated with a variety of disorders and procedures. It may present in a variety of ways, but complications are uncommon.

Methods and Results: We discuss the case of a 52-year-old man with a history of acute diverticulitis since 2001; subsequent colonoscopy and radiological studies confirmed the presence of left colonic diverticular disease. In July 2005 the patient presented an episode of severe haematochezia; the following colonoscopy described numerous and big diverticular localized in the descending colon with signs of recent bleeding. In January the patient underwent operation: intraoperative exploration revealed diverticular disease of the left colon and the occasional finding of massive pneumatosis cystoides of the small bowel; left colectomy was performed. The histological analysis confirmed the diagnosis of diverticular disease with mild peridiverticulitis. The postoperative course was uneventful and the patient is still well and alive.

Discussion: In case of (PI) surgery should be avoided unless there are signs of severe inflammation, metabolic acidosis or portal venous gas, which are indications of more serious disease; treatment of the primary disease is undertaken first.

\section{1}

Role and Value of the Predictive Factors of Common Biliary Duct Lithiasis in Preparation to the Laparoscopic Cholecystectomy. Retrospective Study

V. Neri, T.P. Valentino, A. Fersini, N. Tartaglia, F. Samele, A. Ambrosi, C. Santacroce

Department of Surgical Sciences, Division of General Surgery, Polyclinic of Foggia, University of Foggia, Foggia, Italy

Introduction: The aim of the study was to evaluate the predictive factors of common biliary duct stones (CBDS). These give the indication to perform the ERCP with endoscopic sphyncterotomy (ERCP/ES) before the laparoscopic cholecystectomy (LC).

Patients and Methods: In the period 1997-2005, were performed $102 \mathrm{ERCP} / \mathrm{ES} ; 76$ patients were examined in the period 1999-2005; were excluded the patients with acute biliary pancreatitis (48) because, in our opinion, the ERCP/ES has a therapeutic role. We present a retrospective study of $28 \mathrm{ERCP} / \mathrm{ES}$ before the LC with the suspicion of CBDS. The data were analyzed by univariate and multivariate statistical study.

Results: The univariate analysis identified alkaline phosphatase (ALP) $(\mathrm{p}<0.0001), \gamma$-gt $(\mathrm{p}<0.0001)$, direct bilirubin $(\mathrm{p}<0.0001)$ and CBD dilatation on abdominal ultrasonography (USG) $(\mathrm{p}<0.0001)$ as predictors of CBDS. A multivariate analysis identified ALP $(p<0.0001), \gamma$-gt $(p<0.0001)$ and direct bilirubin $(\mathrm{p}<0.0001)$ as independent predictive factors of CBDS; dilatation of the CBD ( $p=0.0759)$ did not have statistical significativity.

Conclusions: The concordance of cholestasis factors with the dilatation of the CBD has a statistical significativity for the diagnosis of CBDS and it represents the indication to execute ERCP/ES before $\mathrm{LC}$; instead, the ERCP/ES, as an invasive procedure, cannot be performed before the $\mathrm{LC}$, if it is present only the dilatation of the CBD and it is absent an increase of cholestasis factors.

122

Acute Biliary Pancreatitis: Prognostic Role of the Hemoconcentration (HC)

T.P. Valentino, C. Santacroce, A. Fersini, N. Tartaglia, F. Samele, A. Ambrosi, V. Neri

Department of Surgical Sciences, Division of General Surgery, Polyclinic of Foggia, University of Foggia, Foggia, Italy

Introduction: Studies that associate the hemoconcentration with the development of necrotizing acute pancreatitis (NAP), are present in literature. The aim of this study was to evaluate if the HC can be an early marker of necrotizing pancreatitis.

Patients and Methods: In the period January 1998-June 2005, 60 patients with acute biliary pancreatitis were admitted; 24 had a CT-scan within 36-72 h; Ranson's criteria were applied. Seven of the 24 patients had a NAP (Balthazar's score). The hematocrit (Hct) was retrospectively evaluated and associated with the CT-scan data.

Results: The regression analysis showed an association between NAP, by means of CT evaluation, and the HC. Hematocrit more than $43 \%$ in the males and more than $39 \%$ in the females within the first $24 \mathrm{~h}$, was a marker of severity and NAP. In 6 of the 7 patients with NAP there was critical value of Hct and only in 4 of the 17 patients with edematous pancreatitis there was a high value of Hct, showing the statistical significativity of the proposed criteria $(p<0.01)$. The negative predictive value of the $\mathrm{HC}$ was $94.7 \%$ for the evolution in pancreatic necrosis.

Conclusions: The prognostic value of the $\mathrm{HC}$ is comparable with the score of Ranson. So, it is an early and simple marker of the necrotizing evolution of the acute pancreatitis, because of its high negative predictive value: the patients with acute pancreatitis without HC will rarely develop a NAP. 


\section{3}

\section{Hemostasis in Thyroid Surgery: Role of Fibrinogen and Thrombin Pre-Applicated on a Collagen Sponge (FTPCS)}

\author{
A. Fersini, N. Tartaglia, F. Samele, L. Petito, P. Maglione, \\ F. Petruzzelli, F. Matrella, T.P. Valentino, A. Ambrosi, \\ C. Santacroce, V. Neri \\ Department of Surgical Sciences, Division of General \\ Surgery, Polyclinic of Foggia, University of Foggia, Foggia, \\ Italy
}

Introduction: The aim of this study was to evaluate, in thyroid surgery, the immediate advantages derived from the use of FTPCS.

Patients and Methods: In the period January-September 2005, 27 euthyroic patients were enrolled in the study (first group): 17 patients had a total thyroidectomy (TT) and 10 had an hemithyroidectomy (HT). In the same period 32 patients (second or control group) were studied: 21 had a TT and 11 a HT. All patients of the first group had a standard hemostasis and, so, had the application of the device object of the study. The patients of the control group were submitted to a standard hemostasis and, so, had the application of activated cellulose polymers. The evaluated parameters to control the device were: time necessary to have the hemostasis, maintenance and safeness of the same in the postoperative time $(24 \mathrm{~h})$, and simplicity in the use.

Results: In all 27 patients of the first group there was the hemostasis within about $5 \mathrm{~min}$. No patients of the first group were submitted to a re-operation because of the development of postoperative haematomas. The proposed device was simple and practical to use. In the control group there was a postoperative bleeding that required the re-intervention

Conclusions: The use of FTPCS, in thyroid surgery, demonstrated, in our experience, the following advantages: it was useful to speed up and become safer the operation and, overall, the postoperative course; it was no harmful for the recurrent nerve and it was a simple use device.

\section{4}

\section{Antegrade Dissection in Course of Videolaparocholecystectomy}

\section{Santacroce, T.P. Valentino, A. Fersini, N. Tartaglia, F. Samele, A. Ambrosi, V. Neri \\ Department of Surgical Sciences, Division of General Surgery, Polyclinic of Foggia, University of Foggia, Foggia, Italy}

Introduction: The aim of the study is to compare the results of a gallbladder partially antegrade dissection (GPAD) with the one of a group of previous laparoscopic cholecystectomy (LC) performed in a traditional retrograde way.

Patients and Methods: The operative procedure used since 2002 foresees the peritoneal incision from the edge of the infundibular-cystic region and continues along the edge of the gallbladder body until the fundus, clipping and cutting the cystic duct at the end of the dissection. With this methodic were performed 85 LC (first group):
50 simple cholelithiasis, 32 acute cholecystitis and 3 cholelithiasis in cirrhotic. We compared the results with 59 LC (second group) performed in the years 2000-2001 with a completely retrograde methodic: 48 simple cholelithiasis, 11 acute cholecystitis.

Results: In the first group there were no hemorrhagic complications, no lesions of the principal biliary duct, no cystic duct dehiscence, 5 conversions $(5.6 \%, 4$ acute cholecystitis and one cholelithiasis in cirrhotic), mean operative time 70 min, mean postoperative stay 3 days, residual choledocholithiasis in 1 patient $(1.1 \%)$; in the second group there were no hemorrhagic complications, no lesions of the principal biliary duct, cystic duct dehiscence in one case $(1.7 \%)$, no conversions, mean operative time $90 \mathrm{~min}$, mean postoperative stay 4 days, 1 residual choledocholithiasis (1.7\%).

Conclusions: GPAD was shown safe and efficacious, adding an ulterior increase of safety represented by the possibility of complete isolation of the cystic duct.

125
Hyper-Alpha-Amylasemia (HAA) Associated
with Multiple Myeloma (MM) in a Patient
with Asymptomatic Lithiasis of Gallbladder
and of the Principal Biliary Duct. Case
Report

T.P. Valentino, C. Santacroce, A. Fersini, A. Ambrosi, V. Neri

Department of Surgical Sciences, Division of General Surgery, Polyclinic of Foggia, University of Foggia, Foggia, Italy

Introduction: The HAA associated with hyper-lypasemia and hyper-pancreatic-amylasemia is present almost always in acute pancreatitis. The increase only of the $\alpha$-amylasemia can be associated to a pancreatic disease, or not. The literature shows the rare association between HAA and MM without pancreatitis. We present a patient with MM, with a great increase of the $\alpha$-amylasemia associated with an asymptomatic lithiasis of the gallbladder and of the principal biliary duct (PBD)

Case Report: 76 years old caucasian female with MM, in remission phase, IgG-lambda type, admitted for the casual comparison of a HAA $(1,694 \mathrm{U} / \mathrm{l})$ in course of a follow up for the haematological disease, and cholelithiasis. During the $72 \mathrm{~h}$ from the admission there was an increase of the $\alpha$-amylasemia (up to $3,436 \mathrm{U} / \mathrm{l}$ ), so, the patient was submitted to a trans-gastric echo-endoscopy, a MRCP and an ERCP that showed a stone in the PBD, so, a sphyncterotomy with the extraction of the stone was performed. At $48 \mathrm{~h}$ from the ERCP the patient was submitted to a videolaparocholecystectomy and after $72 \mathrm{~h}$ was discharged.

Conclusions: This clinical case, shows the possible association of the MM with the HAA without pancreatic disease; the concomitant presence of the asymptomatic lithiasis of the gallbladder and of the PBD, induced the execution of an articulated diagnostic-therapeutic route. 


\section{6}

\section{Haemorroidectomy in Local Anesthesia in a Day Care Unit}

V.M. Stolfi, P. Sileri, M. Venza, A. Mele, F. Susanna, D. Benavoli, A.L. Gaspari

Department of General Surgery, University of 'Tor Vergata', Rome

Introducion: The aim is to demonstrate that haemorroidectomy performed in a Day Care Unit in local anesthesia is a safe procedure with less postoperative complications than haemorroidectomy in general anesthesia.

Methods: 267 haemorroidectomies were performed in a Day Care Unit of Policlinico Tor Vergata of Rome. 166 cases were performed in local anesthesia with naropine $40 \mathrm{ml} 3.75$ (mean age $47.2 \pm 12.9,81 \mathrm{M}$ and $85 \mathrm{~F}, 63 \mathrm{III}$ stage haemorroids and $103 \mathrm{IV}$ stage). 101 cases were treated in local anesthesia with naropine $40 \mathrm{ml}$ $3.75+$ sedation with diprivan $20 \mathrm{ml}$ (mean age $45.5 \pm 13.3,58 \mathrm{M}$ and 27 F, 51 III stage, 50 IV stage). Stapled haemorroidectomy was performed in 74 cases, Ferguson in 69 cases, Milligan-Morgan in 36 cases and radiofrequency in 88 patients. Postoperative pain was evaluated using VAS scale. Statistical analysis was performed using t test.

Results: Postoperative pain was $4.0 \pm 2.9$ and $4.0 \pm 3.5$ $(\mathrm{P}=0.4)$ for the first day and $4.6 \pm 3.2$ and $4.8 \pm 2.9(\mathrm{P}=0.4)$ during first 3 days for haemorroidectomy performed in general and local anesthesia respectively. One patient operated with local anesthesia $(0.6 \%)$ and 4 patients operated with general anesthesia (3.9\%) developed urinary retention requiring new admission $\mathrm{P}=0.02$.

Conclusions: Haemorroidectomy in local anesthesia is a safe procedure feasible in Day Care. Patients treated in general anesthesia are at higher risk to develop urinary retention.

\section{7}

Treatment of Hemorrhoids in Day Care: Longo vs. Ferguson Technique

V.M. Stolfi, P. Sileri, M. Venza, A. Mele, F. Susanna, P. Gentileschi, A.L. Gaspari

Department of General Surgery, University of 'Tor Vergata', Rome

Introduction: The aim of the study is to compare Ferguson hemorrhoidectomy and Longo stapled prolassectomy for treatment of haemorrhoids in a Day Care.

Methods: 143 patients entered the study. 74 patients were treated with Longo technique (group A) 69 patients with Ferguson technique (group B).Visual analogue scale (VAS) was used for pain evaluation, $\mathrm{t}$ test for statistical evaluation.

Results: Postoperative pain with Ferguson and Longo technique was $5.2 \pm 4.8$ vs. $4.7 \pm 9.3(\mathrm{p}=0.06)$ respectively during first 3 days and $3.8 \pm 6.2$ vs. $3.0 \pm 7$ during next 5 days $(\mathrm{p}=0.001)$. Pain duration was $12.8 \pm 9.4$ in group A and $16.5 \pm 14.7$ in group $B(p=0.12)$. Discharge was $4.4 \pm 7.7$ in group A and $16.2 \pm 16.9$ in group B $(\mathrm{p}<0.001)$. Bleeding duration was $7.69 \pm 11.3$ and $14.1 \pm 16.1$ in group $A$ and $B$ respectively $(p=0.01)$. Return to work was
$17.3 \pm 10.8$ and $19.5 \pm 11.3(\mathrm{p}=0.31) .5$ patients in group A $(6.7 \%)$ developed anal fissure and none in group B $(\mathrm{p}=0.01) .3$ patients in group A (4\%) experienced recurrence and none in group B ( $p=0.04)$.

Conclusion: In both techniques postoperative pain did not differ during the first 3 days while it was significantly less in Longo technique during next 5 days. Bleeding and discharge lasted longer in patients treated with Ferguson technique. Patients underwent Longo technique experienced more recurrences and anal fissures compared with Ferguson technique.

\section{8}

\section{Treatment of Anal Fissure with Botulin Toxin in a Day Care Unit}

P. Sileri, V.M. Stolfi, A. Mele, M. Venza, F. Susanna, P. Gentileschi, G.S. Sica, A.L. Gaspari

Department of General Surgery, University of 'Tor Vergata', Rome

Introduction: Chronic anal fissure is associated with internal anal sphincter spasm. Botulinum toxin is effective in reducing internal anal sphincter pressure, relieving pain and promoting fissure healing. The aim is to evaluate efficacy and side effects of fissurectomy-botulinum toxin injection in the treatment of anal fissures resistant to medical therapy.

Methods: Retrospective nonrandomized study was performed on 12 patients who had previously undergone 8-week course of topical 0.2 percent glyceryltrinitrate and/or used anal dilators for 4 weeks. Both interventions had failed in promoting fissure healing. 20 units of botulinum toxin were injected in the internal sphincter after performing fissurectomy. Outcome measures was fissure healing, improvement of symptoms, need for surgical therapy and side effects.

Results: 12 patients underwent fissurectomy-botulinum toxin injection. Complete fissure healing was observed in 8 patients (66.6\%), 2 patients were improved symptomatically (16.6\%). In one case $(8.3 \%)$ therapy was not effective.

Conclusion: Fissurectomy-botulinum toxin injection is effective as a second-line therapy after an 8 -week course of $0.2 \%$ glyceryltrinitrate and/or anal dilators. It improved symptoms in $16.6 \%$ of cases and healed approximately $66.6 \%$ of fissures. Fissurectomybotulinum injection is a safe procedure and might be a valid alternative to sphyncterectomy.

\section{9}

'Video-Assisted' Surgery of the Colon is an Alternative to 'Totally Laparoscopic' Surgery: Rationale and Technique

N. Vettoretto, D. Fisogni, M. Giovanetti

az osp m.mellini Chiari (BS)

Introduction: Minimally-invasive surgery of the colon is about to enter the 'gold-standard' era, although still of difficult appraisal, expensive and longer in time. 
Methods: We purpose our initial experience in 'video-assisted' colorectal surgery, exploited by using minor laparotomic accesses for determined parts of each procedure. Laparoscopic time includes open access, mobilization and vascular section. Minilaparotomy is used for visceral section, eventual rectal isolation and anastomosis

Results: In the last 3 years we performed 32 colorectal operations, 9 right hemicolectomies, 3 resections of the splenic flexure, 6 left hemicolectomies, 5 sigmoidectomies, 9 low anterior resections of the rectum. We performed 17 (53.1\%) associated operations. Conversions to major laparotomy were $3(9.4 \%), 1$ for retrocolic abscess, 1 for adhesions and 1 for bleeding. Median operating time was $230 \mathrm{~min}$ (145-335), with 'open' time generally within $60 \mathrm{~min}$. We observed $2(6.2 \%)$ post-operative complications, one bleeding and one wound infection.

Discussion: Decreased pain and infections, faster ileus resolution and improved cosmesis characterize video-assisted as well as totally laparoscopic surgery; a reduction of costs and operating time, especially in initial series, might favor a major diffusion of the former, without significant differences in the outcome and patient's satisfaction.

\section{0}

\section{D3 Lymphadenectomy in the Treatment of Gastric Cancer}

\author{
A. Di Leo, S. Giacopuzzi, A. Minicozzi, G. de Manzoni \\ U.O. Chirurgia Generale, Ospedale Alto Garda e Ledro, \\ Arco
}

Background: The rationale for extended lymph nodes dissection is to achieve an R0 resection, nevertheless the benefit of D3 lymphadenectomy in gastric cancer surgery is steel debated. We studied the incidence of lymph node metastasis in relationship to the site of the tumour and $\mathrm{T}$ stage.

Methods: The frequency of node metastasis in N3 and M lymph node groups according with the IGCA was prospectively studied in 214 patients who underwent gastrectomy with D3 lymphadenectomy at the First Department of General Surgery of Verona in the period from September 1988 to October 2005. Eight patients with plastic linitis and three with carcinoma of the gastric stump were excluded from the analysis.

Results: Postoperative morbidity was $31 \%$ and mortality $2 \%$. The total number of dissected nodes was 10.176 with a mean of 47.6 per patient. Upper third: there was an involvement of N3 hepatoduodenal ligament nodes in $10 \%$, of $\mathrm{N} 3 / \mathrm{M}$ paraaortic nodes in $33 \%$ and of N2/N3 hepatic artery nodes in $15 \%$. Middle third: N2/N3 hepatic artery station was positive in $15 \%$, while N2/N3 hepatoduodenal ligament and N3/M paraaortic nodes in 7\% and 13\% respectively. Lower third: Metastasis in the N3 left gastroepiploic station was found in 3\%. $\mathrm{N} 3$ retropancreatic nodes were involved in 16\%. N2/N3 hepatoduodenal ligament and $\mathrm{N} 3 / \mathrm{M}$ paraaortic stations were both positive in $9 \%$.

Conclusion: These results suggest that D3 lymphadenectomy should be considered in curative surgery of advanced gastric cancer, especially if located in the upper third. Further studies with a larger number of patients required to confirm its prognostic value.
131

\section{Abdominal Wall Defect: Which Prosthetic Material?}

\author{
L. Leone, A.M. Bellotti, B. Battaglia, M. Catarci, G.B. Grassi \\ Department of General Surgery, San Filippo Neri Hospital, \\ Rome, Italy
}

The Incidence of wound hernia start from 3 to $11 \%$ on abdominal surgery. $23 \%$ patients have an origin from wound infection at the time of last surgery. The worst complications are obstruction (emergency surgery), relapse and infection. We describe the use of PTFE. PTFE is a gas under normal, or nominal conditions. Under some conditions the molecules of PTFE react combining each other, producing a substance called PTFE. Prevention of bowel adhesions. The intraperitoneal use of the ePTFE prosthesis is safe. It does not result in serious complications as reported with the use of Marlex mesh, such as bowel obstruction, bowel fistualization, and mesh migration'.

\section{Indications}

'Wall disaster'

Absence of peritoneum

Indications must to come from a specialist surgeon!!!

Small ventral hernia with peritoneum

polipropilene (lap option - PTFE)

Medium-large ventral hernia with peritoneum polipropilene (lap option)

Medium-large ventral hernia without peritoneum composix (dual mesh lap option)

Our experience in emergency surgery. In case of simple bowel resection a prosthesis can be implanted. In case of septical surgery (peritonitis with faecalis, biliary or urinary liquids) a direct wall suture or an adsorbable prosthesis can be done.

Conclusions: Today there are no general rules for ventral hernia treatment; there exist however several techniques and a number of materials. Our opinion is that the choice is to be made by the surgeon on the basis of his own professional persuasion, experience and knowledge.

\section{2}

\section{Sentinel Node Biopsy in Early Breast Cancer: Our Experience}

A. Mauro, M.C. Gallo, S. Guaitoli, A. Rinaldi, A. Schiavone, M. Gavioli, G. Natalini

Chirurgia3 Policlinico, Modena

Introduction: Axillary node dissection in patients suffering from early breast cancer provides staging information; lymph node metastasis is the most important prognostic factor for recurrence and survival. Unfortunately, morbidity is high after axillary lymph node dissection (ALND); therefore, sentinel lymph node biopsy (SLNB) is a valid alternative. The sentinel lymph node is the first node receiving lymphatic drainage from a tumor site. 
Materials and Methods: From January 1998 to December 2005,581 patients underwent SLNB. Patients with T1-T2 $<3 \mathrm{~cm}$ N0 were recruited. Localization of the sentinel node was carried out with radioactive albumine labelled with Tc99; pathological examination was carried out using standard methods of immunoistochemistry.

Discussion: Our initial experience was gained by performing SLNB with always ALND. In the first phase, 93 patients underwent SLNB and were subjected to axillary dissection, after. In the second phase, 488 patients underwent SLNB and axillary dissection was applied only if SLNB had resulted positive. Results I Phase: (93 patients): 59 patients had SLN negative and after ALND, 57 had all lymph nodes negative (97\%). Results II Phase: (488 patients): in 360 patients after SLNB were negative and they where not subjected to ALND; in 128 patients SLNB was positive and these ones underwent ALND. In these, 105 patients had only positive SLN (82\%), 23 patients had SLN and others lymph node positive (18\%).

Conclusion: In accord with literature data, our experience confirms that SLNB is a valid alternative to ALND, in the staging of patients with breast cancer (a less morbid and accurate staging).

\section{3}

\section{Resection of Metastatic Malignant Hemangiopericytoma of the Liver with Severe Hypoglycemia due to Production of Insuline-Iike Growth Factor IGF-II}

\section{S. Pachera, A. Ruzzenente, A. Valdegamberi, T. Campagnaro, M. Fontana, A. Guglielmi \\ Department of Surgery and Gastroenterology, University of Verona, Italy}

Introduction: A 47-year-old woman with meningeal hemangiopericytoma with liver, lung, bone and intracranial metastases developed severe recurrent hypoglycaemias after palliative treatment with chemio-embolization (TACE) with Lipyodol and Mitomicin C.

Methods: Specific examinations revealed low IGF-I ( $3.25 \mathrm{nmol} / \mathrm{l})$ and high value of IGF-II $(1,475 \mathrm{ng} / \mathrm{ml})$. Low serum glucose level was accompanied by undetectable insulin and $\mathrm{C}$ peptide serum level, and low levels of hGH, glucagone, catecholamines and cortisol. Non islet cell tumour induce hypoglycaemia (NICTH) was suspected.

Results: Patient was initially treated with continuous infusion dextrose and steroids, then a bridge treatment with steroids, glucagone and hGH was performed. Thereafter palliative resection of large $(18 \mathrm{~cm})$ liver metastases in IV, V, VI segments was carried out. Metabolic abnormalities were corrected after resection: biochemical controls showed normal glucose, insuline, serum $\mathrm{C}$ peptide, $\mathrm{GH}$ and IGF I serum levels and normalization of IGF II ( $813 \mathrm{ng} / \mathrm{ml})$. One year later no recurrences occurred in the liver.

Discussion: This is the first case of literature of severe hypoglycaemia after TACE for metastatic malignant hemangiopericytoma of the liver with production of IGF-II, whose metabolic abnormalities were corrected after surgical treatment.

\section{4}

\section{Surgical Treatment of GIST}

G. Calò, F. Catena, L. Ansaloni, G. Gagliardi, F. Gazzotti, L. D'Alessandro, A.D. Pinna, M. Taffurelli, G. Biasco,

D. Santini, V. Di Scioscio, P. Fusaroli ${ }^{1}$, G. Caletti ${ }^{1}$,

P. Castellucci, P. Tomassetti

St Orsola-Malpighi University Hospital, 1 Imola General Hospital, Bologna, Italy

Introduction: Gastrointestinal Stromal Tumors (GISTs) are a rare group of tumors of the gastrointestinal tract that have not yet been fully investigated. In this study the Authors present their experience on emergency and elective treatment of patients with such neoplasms and discuss the surgical approaches to these tumors.

Methods: A review of our 11-years experience was performed and 31 patients were found with the diagnosis of GIST.

Results: 10 patients had emergency presentation: 8 subjects with evidence of gastrointestinal blood loss and 2 patients with acute abdomen. 21 tumors were located in the stomach, 8 in the small bowel and 2 in the duodenum. Of the 31 patients 10 were treated with laparoscopic approach. All patients underwent curative resection and 2 had a recurrence and died.

Discussion: GIST treatment mainly involves surgical resection with the goal of complete removal which can be curative. In most cases laparoscopic approach is the ideal therapy. The histologic grade and tumor size are the most important prognostic factors.

135

\section{Quality Assessment of 65098 Inguinal Herniorrhaphies in Emilia-Romagna Region (Italy)}

S. Gagliardi, F. Catena, L. Ansaloni, R. Melotti ${ }^{1}$, R. Grilli, D. Fortuna ${ }^{1}$

St Orsola-Malpighi University Hospital Bologna,

${ }^{1}$ Emilia-Romagna Health Agency, Bologna, Italy

Introduction: Groin hernia repair is one of the most common surgical procedures, but there is no agreement about surgical or anaesthetic technique. Aim of our study was to investigate outcome results of groin hernia surgery in an Italian Region (Emilia-Romagna) of about 3,983,346 people.

Methods: We retrospectively analysed 65098 groin hernia repairs performed in Emilia-Romagna Region (Italy) from 2000 to 2004. Pearson $\chi$ test was used for statistical analysis.

Results: There were about $1 \%$ of laparoscopic operations. In 2004, $80 \%$ of patients was treated in day surgery admission. Prosthetic hernioplasty was carried out in the majority of patients (94\%). Recurrence rate was $1.93 \%$ in the non- prosthetic group whereas it was $0.86 \%$ in the prosthetic group $(\mathrm{p}<0.05)$. In laparoscopy procedures recurrence rate was $0.4 \%$ (vs. $0.93 \%$ in non- laparoscopic group; $p<0.05$ ). Out of 9,415 eligible patients for laparoscopic procedures (recurrent hernia, bilateral, athletes) only $1.98 \%$ was submitted to a laparoscopic operation.

Discussion: Mesh repairs have a lower recurrence rate than conventional open repairs. Laparoscopic operations have a limited 
use and only $2 \%$ of eligible patients are treated with this procedure. Systematic analysis of treatment and outcome variables in regional clinical database can improve the overall quality of surgical care.

\section{6}

\section{The LEONARDO Study}

\author{
L. Ansaloni, F. Catena, F. Agresta, S. Olmi \\ SPIGC LEONARDO Working Group
}

Introduction: In the literature a recent extensive systematic review were based on studies of mediocre quality, with very similar flaws, like unclear randomization method, lack of blindness, non intention-to-treat analysis and finally absence of the description of numbers of patients eligible and refusing trial participation. In order to compare the therapeutic effects of laparoscopic and conventional open appendectomy in the treatment of suspected acute appendicitis, we designed a randomised controlled trial, taking particular attention to answer to the following methodological issues: concealed randomization, double blinding, intention-to-treat analysis and finally the clear report of numbers of eligible, excluded and refusing patients.

Protocol Design: The LEONARDO (Laparoscopic Excision vs. OpeN Appendectomy multicentre Randomized, DOuble blind controlled trial) study is a multicentre prospective, randomised, doubleblinded comparison of laparoscopic versus open appendectomy. The outcome measured are the following: postoperative pain; wound infections; intraabdominal abscesses; duration of operation; length of hospital stay; return to normal activity; return of bowel function and cosmesis. The randomisation obtained through a computer-generated schedule is loaded in a web-site and assigned to the included patient via internet (concealment). Sample size, calculated to reach a confidence level of $95 \%$ with a power of $80 \%$ (considering the mean VAS for pain on day 1 in laparoscopic and open appendectomy 4 and $5 \mathrm{~cm}$ respectively) has to be of 193 patients for each group.

\section{7}

\section{The Surgery of GIST: Our Experience}

R. Torrini, G. Natalini

U.O.C. Primario Dott. Giovanni Natalizi, Ospedale 'S. Maria della Misericordia', Perugia

Introduction: We have treated 40 patients with GIST from 2000 until April 1, 2006. Mean age was 59 years old; $42.5 \%$ of which were male and $57.5 \%$ of which were female. As for the localization: 57.5 was gastric, $25 \%$ was in the small intestine, $7.5 \%$ was in the colonrectum, $10 \%$ in different organs such as retroperitoneum, pancreas, omentum e mesentery. 7 patients had more of one neoplasia and two of which had double neoplasia besides GIST. 50\% of which were epithelial neoplasm.

Methods: We performed the following operations: 3 total gastrectomies, 15 partial gastrectomies, 14 resections of multiple organs, 5 resections of the small intestine, 2 resections of retroperitoneal neoplasia, 2 resections of rectal polyp, one DCP, one resection of hepatic metastasis. Furthermore, 8 operations were performed with laparoscopic surgery. 4 patients had only biopsy because they were not suitable for the surgery therefore they had pharmacologic treatment. 7 patients had pharmacologic treatment and one patient died due to side effect of imatininb. 25 patients out of 30 at the follow up did not show signs of tumour recurrence.

Results: The knowledge of that pathology gives us the possibility to use a type of surgery which is precise and not too invasive.

Discussion: Currently the surgery is the first line treatment in GIST, however Imatinib is the best option in a few and specific cases.

\section{8}

\section{One-Stage Resection of Intravenous Leiomyomatosis Extending into the Right Ventricle: Report of a Case}

\author{
G.R. Fronda1, P. De Paolis', V. Maglione1, L. Mazza1, \\ E. Bertotti ${ }^{1}, M$. Mistrange/o ${ }^{2}$
}

${ }^{1}$ Chirurgia Generale 7 prof Fronda ASO S Giovanni Battista Molinette, ${ }^{2}$ Oncologia Medica dr Bertetto ASO S Giovanni Battista Molinette, Torino

Introduction: Intravenous leiomyomatosis (IVL) is a rare entity with only a handful of reports in literature. It has been described as an uterine tumor characterized by smooth cell proliferation within veins. We report a case of intracaval and intracardiac leiomyomatosis. Case report: A 54 year old female was admitted to our division for abdominal pain with abdominal mass. In 1997 she had undergone total hysterectomy with right adnexal conservation for IVL. Abdominal US revealed a pelvic mass. Thoraco-abdominal CT detected the pelvic tumor, an enlargement of IVC partially occupied by a thrombus originating from the right ovaric vein and multiple pulmonary nodules. Echocardiography demonstrated a $82 \times 35 \mathrm{~mm}$ mobile mass in the right cardiac cavity originating from the IVC with tricuspid regurgitation. A recurrence of IVL with intracaval and cardiac extension was suspected and the patient was submitted to surgery through a onestage operation under sternotomy and laparotomy with extracorporeal circulation. Histologic examination confirmed the leiomyomatosis. After an uneventful course, the patient was discharged on the 10th day. Adjuvant therapy consisting of gonadotropin releasing hormone agonist was administered because of pulmonary metastasis.

Discussion: Total surgical resection of the tumor is the treatment of choice to avoid complications and recurrences. The first complete resection was performed in 1982: a delayed laparotomy after resection of intracardiac thrombus. (1) Uchida reported only 30 cases of cardiotomy for intravenous leiomyomatosis extending to the heart in the past 30 years. (2) We consider the one-stage procedure mandatory in case like our, even if longer than two-stage surgery. 
139

Role of Surface-Coil 3T MRI with Rectal Lumen Distension in Local Staging of Rectal Cancer

\section{F. Sani, S. Martinelli, F. Bollino, M.V. Guerra, P. Torricelli, E. Gallo, M. Gavioli ${ }^{1}$}

Dipartimento integrato diServizi Diagnostici e per Immagine, Azienda Ospedaliero Universitaria Policlinico di Modena, 'Dipartimento integrato di Chirurgia Generale e Specialità Chirurgiche, Azienda Ospedaliero Universitaria Policlinico di Modena

Aim: To evaluate the diagnostic accuracy of surface-coil 3T MRI in the preoperative study of patients with rectal cancer.

Material and Methods: Thirty patients with hystologically proven rectal cancer underwent surface-coil 3T MRI, after positioning in the rectal lumen a thin probe with a hip baloon filled with water. In all patients TSE T2-w sagittal, para-coronal and para-axial sequences and TSE T1-w para-axial sequences before and after e.v. infusion of Gadolinium Dota were obtained. The slice thickness was $3 \mathrm{~mm}$ without gap, the FOV $24 \mathrm{~cm}$ and the matrix scan $\mathrm{x}$. The infiltration of rectal wall, perirectal fat and mesorectal fascia and the presence of perirectal limphonode were observed. All the patients underwent surgery. The MRI results were compared with anatomical evaluation.

Results: The mean diagnostic accuracy of 3T MRI in evaluating the local spread of the tumor was $85 \%$.

Conclusions: The high signal to noise ratio with a wide field of view enables surface-coil 3T MRI with rectal lumen distension to get high diagnostic accuracy in local staging of rectal cancer with a particular regard in assessing mesorectum and mesorectal fascia.

\section{Thoracic Surgery}

\section{0}

\section{Bronchial Suture Coverage with Intercostal Muscle Flap in Case of Bronchial Sleeve Resection or Right Pneumonectomy}

\section{Ampollini, A. Bobbio, P. Carbognani, L. Cattelani, A. Mazzeo, D. Stavroulias, M. Rusca \\ Unità Operativa di Chirurgia Toracica, Dipartimento di Scienze Chirurgiche, Azienda Ospedaliero-Universitaria di Parma, Parma, Italia}

Introduction: Broncho-pleural fistula is a faired complication after lung resection; we retrospectively evaluated the postoperative outcome of patients who had bronchial suture reinforcement with a pedicled intercostal muscle flap.

Methods: Between January 2000 and December 2005 all consecutive patients who underwent bronchial sleeve resection or right pneumonectomy had bronchial suture covered by an intercostal muscle flap. Preoperative clinical data, surgical reports, and postoperative outcome were reviewed; follow-up was completed in all patients.

Results: During the study period, twenty-eight bronchial sleeve resections and thirty-three right pneumonectomies were carried out. Among patients treated by bronchial sleeve resection there were five bilobectomies, fourteen upper right, seven upper left and two left inferior lobectomies. Median hospital stay was 9 days (range 5-30). Two deaths occurred: one for cardiac infarction and the other for acute respiratory failure. Post-operative complications were documented in 10 patients $(36 \%)$ : atrial fibrillation $(6 / 28,21 \%)$, pneumonia $(6 / 28,21 \%)$, persistent air-leaks $(5 / 28,18 \%)$. No bronchopleural fistula nor bronchial stricture were noted during early and late follow up. Among patients operated of right pneumonectomy, no death was registered. Median recovery time was 12 days (range 9-30). Postoperative morbidity accounted for $36 \%$ : atrial fibrillation $(9 / 33$, $27 \%)$, pneumonia $(2 / 33,6 \%)$ and one patient re-operated for superior vena cava thrombosis after partial caval resection. One case of bronchopleural fistula was noted on the 30th day postoperatively.

Discussion: Patients undergoing bronchial sleeve resection or right pneumonectomy are at high risk of post-operative complications. Bronchial suture coverage could be a valid method to avoid bronchopleural fistula.

\section{1}

Chemical Pleurodesis with Talc for Treatment of Recurrent Malignant Pleural Effusions

M. Anile, D. Diso, M. Di Stasio, V. Liparulo, C. Ricella, F. Venuta, G.F. Coloni

Dipartimento di Chirurgia Toracica del Policlinico Umberto I Università di Roma 'La Sapienza'

Objective: Recurrent pleural effusion represents a frequent complication of an advanced stage neoplasm. Sclerosant agents inserted during VATS or though thoracostomic tube may resolve pleural effusion improving the quality of life. We report our experience comparing these two procedures.

Methods: We observed 161 patients with recurrent malignant pleural effusion. Mean age was $61.4 \pm 13$ years (range 26-84 years). The localization of primitive cancer was lung in 58 patients $(36.1 \%)$, breast in $42(26.2 \%)$, bowel in $21(13.1 \%)$, pleura in $19(12 \%)$, skin in $7(4 \%)$ and other tissues in $14(8.6 \%)$. We performed videothoracoscopic talc poudrage (VTP) in 120 patients (mean age $60.2 \pm 13$ years), while 41 anaesthesiologic high-risk patients (mean age $70.6 \pm 10$ years) underwent to talc slurry (TS).

Results: One patient of VTP group died in the first postoperative day because of a reperfusion edema. There were no other major complications. Nine recurrence (7.5\%) occurred in VTP group and $4(9.7 \%)$ in TS group ( $\mathrm{p}=\mathrm{NS})$. Mean stay in hospital was $5.4 \pm 3$ days for VTP group and $11 \pm 8$ days for TS group $(\mathrm{p}=0.00001)$ and mean duration chest tube $4.5 \pm 1$ days and $8.2 \pm 4$ days $(p<0.00001)$, respectively. Continuous chest pain was present in 101 patients $(84.1 \%)$ of VTP group and in 21 patients $(57.1 \%)$ of TS group $(p=0.004)$. Overall median survival was 4.2 months (range 1-267 days). 
Conclusions: Pleurodesis with talc, both poudrage and slurry, is a palliative and sure treatment. In elderly and anaesthesiologic highrisk patients talc slurry can be an effective therapeutic option.

142

A Rare Case of Mixoid Lipo-Leiomyosarcoma of Pulmonary Artery Mimicking Pulmonary Thromboembolism Successfully Treated with Surgical Approach

\author{
D. Argnani, S. Sanna, M. Mengozzi, M. Monteverde, \\ M. Taurchini, A. Dell'Amore1, D. Dell'Amore \\ Thoracic Surgery Unit, G.B. Morgagni Hospital, Forlì, \\ ${ }^{1}$ Cardiac Surgery Unit, S. Orsola Hospital, Bologna, Italy
}

Introduction: Pulmonary artery liposarcomas are extremely rare, about 100 cases described worldwide. Rarely asymptomatic, it can cause dyspnea, chest pain or cough and is frequently misdiagnosed as pulmonary embolism. The diagnosis is often intraoperative and the prognosis is poor without intervention (1.5 months), 2-3 years after surgical and adjuvant therapy.

Methods: A 49-year-old man with a diagnosis of pulmonary embolism was admitted to our Institution for dyspnea and chest pain. The chest X-ray showed mediastinal widening, the angio-CT revealed an anterior mediastinal mass, occluding the right pulmonary artery (RPA) and compressing the lung and the superior vena cava (SVC). The diagnostic open biopsy through median sternotomy reveal a mesenchimal malignant neoplasm, infiltrating the origin of RPA at the common trunk, the lower part of SVC and the lung. A right intrapericardial pneumonectomy with arterial suture and superior vena cava reconstruction with pericardial patch was performed.

Results: Histologic finding was a mixoid low-grade liposarcoma-leiomyosarcoma of the RPA. The post-operative period was uneventful and the patient was discharged to the hospital after 8 days. The treatment was completed by adjuvant chemo-radiotherapy and actually the patient is disease free 15 months later.

Discussion: Surgical treatment should be considered, whenever technically feasible, as the most effective therapy for pulmonary artery liposarcomas because of their aggressivity and no curative effect of radiation-chemotherapy alone. Adjuvant therapy could be useful for incomplete resections and local recurrences.

\section{3}

\section{An Uncommon Cause of Pneumothorax}

T. Bartolotta, M. Cajozzo, A. Sunseri, F. Minervini', F. Cappello1, T.V. Bartolotta'2, G. Modica

Department of Oncology and Surgery, ${ }^{1}$ Section of Human Anatomy, ${ }^{2}$ Department of Radiology, University of

Palermo, Italy

Introduction: The most important secondary causes of pneumothorax include COPD, traumas and iatrogenic lesions.
Methods: An indirect trauma to the left shoulder occurred to a 28-year-old patient during a car accident. An immediate chest X-ray showed the presence of pnumothorax within the upper left lung resulting from a multi-fragmented longitudinal fracture of the lateral third of clavicle. A drainage catheter was inserted and connected to a Bulau water valve. A Desault bandage was applied in order to immobilize the shoulder and homolateral arm.

Results: Immediately after the thoracostomy there was an improvement in the general clinical condition of the patient; a subsequent chest X-ray showed that the pneumothorax had completely disappeared. A very accurate orthopaedic examination showed that the clavicular fracture had completely healed.

Discussion: About $30 \%$ of all lesions involving the shoulder are represented by clavicular fractures. Only in 1-2\% are cause of pneumothorax. Generally are caused by an indirect trauma and interest the inner third or the acromial portion of the clavicle, while fractures of the outer third of the clavicle are fairly rare. The dislocation and the angulation of the fragments of clavicular fracture cause a deformation of the area and may be associated with several complications due to the anatomical of the clavicle, such as lesions of the vascular structure (subclavian vein and brachial artery), to the nerves (brachial plexus) and secondary traumatic pneumothorax.

\section{4 \\ Virtual Bronchoscopy and Preoperative Assessment of Airways Stenosis \\ T. Bartolotta, M. Cajozzo, T. Balestrieri, T.V. Bartolotta', F. Cappello², G. Modica \\ Department of Oncology and Surgery, 'Department of Radiology, ${ }^{2}$ Section of Human Anatomy, University of Palermo, Italy}

Introduction: The virtual bronchoscopy (VB) allows to create 3D images visualizations from computed tomography image scans similar to those produced by current fiberoptic bronchoscopy (FB) procedures. VB holds significant promise to be complementary to the FB, supplant some real endoscopic examinations and become a standard screening practice for real endoscopy.

Methods: Fourteen patients ( 2 female and 12 male; age range 48-78 years) with endobronchial disease underwent VB. The VB findings, that included the presence or absence of obstructive lesions (defined as bronchial narrowing of $50 \%$ ), endoluminal masses or mucosal lesions (hemorrhage, erythema or tissue friability) were compared with those of FB.

Results: In 11 of 14 patients, VB was found to be accurate in the detection and grading airway stenosis to correlate closely with flexible bronchoscopy examination results. In two patients the deposition of mucus and in one coagulated blood has caused misinterpretation of VB findings on stenosis.

Discussion: Because VB is a non-invasive, reproducible and objective method that provides important information on anatomic and pathological details, should be used as complementary technique of examination airway stenosis (to measure the length and character) which is difficult to passable for FB permitting evaluation of airways stenosis, planning therapy and assessing treatment response. VB 
should be used in as elective technique in patients too ill to tolerate FB because many of the serious complications may be avoided.

\section{5 \\ Role of Video Assisted Thoracoscopy Procedure in the Diagnosis and Treatment of Malignant Pleural Mesothelioma}

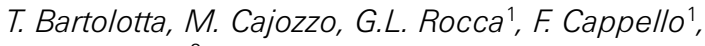 \\ T.V. Bartolotta'2, G. Modica \\ Department of Surgery and Oncological Sciences, \\ ${ }^{1}$ Section of Human Anatomy, ${ }^{2}$ Department of Radiology, \\ University of Palermo, Italy
}

Introduction: In the last years the video assisted-thoracoscopy (VATS) enter more widely in everyday practice of the thoracic surgeon. The aim of this study was to evaluate the results of VATS procedure for the assessment of malignant pleural mesothelioma (MPM) in patients with unusual clinical and radiologic presentation for the disease.

Methods: Eight consecutive patients in whom MPM was suspected not diagnosed by conventional methods was referred for histological diagnosis by VATS. In some of this patients, operative procedure drainage of neoplastic pleural effusion was performed where possible.

Results: All patients had a histological diagnosis of MPM from the material sent for biopsy. Two patients undergoing drainage of effusion subsequently were diagnosed of having mesothelioma. There have been neither morbidity nor mortality since VATS.

Discussion: Diagnostic resources of VATS are larger than these of the others diagnostical methods: is a safe and effective procedure for the assessment of MPM (detection of the tumor, depiction of contour and relation with other thoracic structures) and provides adequate tissue for histological diagnosis where other methods fail and avoid explorative thoracotomy. Therefore, at the same operative sitting it provides a therapeutic intervention: in this case results in significantly fewer hospital admission and appears to improve quality of life and length of survival.

\section{6}

\section{Treatment and Outcome of latrogenic Tracheobronchial Ruptures}

\section{S. Conforti, R. Paino, M. Ravini, M. Torre \\ Azienda Ospedaliera Niguarda Ca' Grande, Milano}

Introduction: Post intubation tracheobronchial ruptures are rare but severe complications with significant mortality unless treatment is used. We evaluated the presentation, the treatment and the outcome of these patients.

Methods: 20 cases observed from 1996 to 2006 were retrospectively analyzed. 19 were female, 1 male. Mean age was 66 years. In 13 patients the rupture was due to single-lumen intubation, in 4 to double-lumen and in 3 to tracheal cannula positioning. First symptom was mediastinal and subcutaneous emphysema. In all cases FBS revealed the injury.

Results: In 9 cases the lesion was in the distal trachea (length: 2-6 cm): 3 tracheal lesions, 2 of the carina and 4 of the main bronchi. In 11 cases the lesion was in proximal pars of the trachea. 9 of these patients were treated conservatively. 10 patients underwent surgical approach through right-side thoracotomy, only one through transcervical approach. The patients treated nonsurgically had a satisfactory outcome. No surgical mortality occurred. 2 patients died for preexistent associated diseases. No one had post-operatively stenosis.

Conclusions: Surgical treatment is mandatory in tracheobronchial ruptures under positive pression ventilation; conservative management may be recommended in selected patients with small and proximal ruptures of the trachea without mediastinitis; the use of endoscopical stent is not recommended.

\section{7}

\section{Inflammatory Myofibroblastic Tumor of the Lung}

M. Cicalese, G. De Luca, S. Galzerano, D. Amore, S. Spiezia, S. Griffo, C. Cecere

Università degli Studi di Napoli 'Federico II', Dipartimento Universitario di Scienze Chirurgiche, Anestesiologiche, Rianimatorie e dell'Emergenza, Area Funzionale di Chirurgia Toracica

Introduction: Inflammatory myofibroblastic tumor (IMT) of the lung is a rare benign tumor of uncertain aetiology.

Methods: A 38 years old male was admitted with chest pain, dry cough and haemoptysis for 2 months. He performed a chest Rx and $\mathrm{CT}$, bronchoscopy and FNAC under CT guidance. We suspected a lung cancer.

Results: Chest Rx revealed a small $(2 \mathrm{~cm})$ right lung opacity. CT scan showed a right superior lobe soft tissue density mass, FNAC was not diagnostic. The patient underwent a right anterolateral thoracotomy and wedge resection. Macroscopically the mass was wellcircumscribed, non encapsulated yellowish in colour on cut section. Microscopically the mass was composed of fibroblasts, collagen and inflammatory cells mainly of lymphocytes and plasma cells. There was no mitotic activity, so we concluded for IMT.

Discussion: IMT of the lung is a relatively uncommon neoplasm (0.04-2.7\%). Common symptoms include cough, dyspnoea, fever chest pain and haemoptysis, although many patients are asymptomatic. The exact pathogenesis remains uncertain, the IMT is generally considered to be a non neoplastic tumefaction composed of a variety inflammatory and other mesenchymal reactive cells. On radiographs, IMT typically appears as a solitary, peripheral, sharply circumscribed and lobulated mass. Therefore it, clinically and radiologically mimic a malignant process. The treatment of choice is surgery, and so the prognosis is excellent. 


\section{8}

\section{Posterior Mediastinum Neoplasms: A Case of Ancient Schwannoma}

\author{
G. De Luca ${ }^{1}$, F. Petteruti², A. Luciano², A. Lerro ${ }^{2}$, P. Pepino ${ }^{2}$ \\ ${ }^{1}$ Università degli Studi di Napoli 'Federico II', Dipartimento \\ Universitario di Scienze Chirurgiche, Anestesiologiche, \\ Rianimatorie e dell'Emergenza, Area Funzionale di \\ Chirurgia Toracica, ${ }^{2}$ Clinica Pineta Grande, Unità di \\ Chirurgia Cardiotoracica
}

Introduction: Schwannomas are benign tumors, deriving neural crest cells, can arise from a peripheral nerve.

Methods: A 56-year-old woman was admitted to our hospital with inspiratory dyspnea, dry cough, vague right chest and arm pain. She performed a chest X-ray, CT and magnetic resonance (MR), FNAC under CT guidance.

Results: CT revealed a huge, well-circumscribed heterogeneous, tumour with focal low density areas in the posterior mediastinum. MR imaging demonstrated a well defined lobulated mass, which demonstrated inhomogeneous enhancement after intravenous injection and excluded the possibility of intraspinal involvement. Cytological exam showed atypical features. She underwent total resection by means of posterolateral thoracotomy. Macroscopically the mass was encapsulated and lobulated. Microscopically, the tumor had a characteristic histological appearance composed of two distinct components: both richly hypercellular areas and loos mixoid areas with cystic degeneration and so we concluded for intercostal Ancient Schwannoma (AS).

Discussion: The intercostal AS can mimic pulmonary neoplasm in chest radiographs and CT. MR is the technique of choice in the assessment of peripheral nerve tumours. AS shows degenerative histological changes for which may lead to a mistaken diagnosis of malignant neoplasm. The definitive diagnosis of AS is possible only after histopathological and immunohistochemical examination.

\section{9}

\section{Is the Heimlich Valve Effective to Improve Outcome in General Thoracic Surgery?}

\author{
A. De Palma, D. Loizzi, N.P. Ardò, E. Spada, G. Garofalo, \\ M. Loizzi, F. Sollitto ${ }^{1}$
}

Cattedra di Chirurgia Toracica, Università degli Studi di Bari

Introduction: The Heimlich valves are commonly used in thoracic surgery for management of prolonged air leaks, to reduce hospital stay and costs. The objective of this study was to assess whether their extensive use immediately after lung resection significantly improves clinical outcome.

Methods: We enrolled in our study 56 patients who underwent lung resections for various diseases at our Institution. At the end of surgical procedure, 2 or 3 chest silicone tubes were positioned; patients were prospectively randomly assigned to one of two groups. In group $1(\mathrm{n}=28)$ drainages were placed on water seal. In group 2 $(\mathrm{n}=28)$ a Heimlich valve was applied to the anterior (and intermediate, if present) drainage and connected to a second hydraulic-valve system; the posterior drainage was placed on water seal. Chest tubes were removed when no air leak was evident and pleural effusion was less than $100 \mathrm{ml} / 24 \mathrm{~h}$.

Results: Mean chest tube duration was $8.3 \pm 3.9$ and $6.6 \pm 1.5$ days $(\mathrm{p}=0.047)$ in group 1 and 2 , respectively; mean post-operative hospital stay was $12.8 \pm 7.3$ and $10.8 \pm 3.7$ days $(\mathrm{p}=0.192)$ in group 1 and 2, respectively.

Discussion: The use of Heimlich valve compared to simple water seal system after lung resection surgery was associated with significantly shorter chest tube duration. This system could be routinely used in general thoracic surgery to improve clinical results.

\section{0 \\ Malignant Thymoma: Results of Surgical Resection}

\author{
D. Loizzi, A. De Palma, F. Tota, M. Genualdo, G. Capotorto, \\ R. Quercia, F. Sollitto, M. Loizzi
}

Cattedra di Chirurgia Toracica, Università degli Studi di Bari

Introduction: The authors report the analysis of their experience with patients submitted to surgical treatment for malignant thymoma, in order to evaluate survival related to histology, stage, completeness of surgical resection and myasthenic symptoms.

Methods: From 1985 to 2005 we operated on 158 patients ( 90 women, 68 men), mean age 46.8 years (range: 9-81 years), with thymic diseases. We performed 114 thymectomies (72\%) and 44 diagnostic biopsies (28\%).

Results: There was no operative mortality. Histological examination revealed malignant primary thymic tumor in 113 cases, whose mean follow-up was $64 \pm 49$ months (median 55 months, range: $0-172$ months). In the thymoma group ( $\mathrm{n}=68,39$ women, 29 men; mean age 54.4 years, range: $16-78$ years) 5 -year overall tumour specific survival rate was $90 \%$; 5 -year survival rate by histological subtype was $91 \%$ for mixed, $89 \%$ for lymphocitic, $77 \%$ for epithelial, $63 \%$ for spindle-cell one; by stage, $93 \%$ in stage I, $84 \%$ in stage II, $41 \%$ in stage III; by presence or absence of myasthenic symptoms, $90 \%$ and $80 \%$, respectively; in resected thymomas was $93 \%$ $(\% \mathrm{R} 0=84 \%)$, specifically $95 \%$ for R0, $85 \%$ for R $1-2$.

Discussion: Surgical resection is the gold standard for treatment of malignant thymomas; extended resections appear to be justified, as in our experience complete resection revealed to be the most important prognostic factor.

151

\section{Results of En-bloc Resection for T3 NSCLC: Our Recent Experience}

\section{Duranti, M. Lucchi, G. Fontanini, A. Mussi}

Department of Cardiac and Thoracic, Division of Thoracic Surgery, Pisa, Italy

Introduction: The surgery is the best treatment for non-small cell lung cancer invading the chest wall. We reviewed our surgical experience with the aim of debating about the indications and results. 
Materials and Methods: We investigated 93 patients who underwent en-bloc lung resection for NSCLC from January 2000 to December 2005. The mean age was 65.8 years. The pulmonary resection consisted of a pneumonectomy in 15 patients, lobectomy in 60 cases, segmentectomy in 3 cases and a wedge resection in 15 . We used a polyglactin-910 mesh for the prosthetic reconstruction of the chest wall just in 15 patients.

Results: The histology showed 50 squamous cell, 40 non-squamous cell and 3 small cell carcinomas. The post-operative staging was IIB (N0) in 66 and IIIA in 27 patients ( $8 \mathrm{~N} 1$ and 19 N2). 8 patients underwent a neoadjuvant treatment: 6 chemotherapy and 2 chemoradiotherapy. 12 out of the 93 patients were lost at the follow up. The overall 5-year survival was $41.7 \%$ (median $=29$ months). The stage disease (IIB vs. IIIA) and the N status (N0 vs. N1 vs. N2) affected significantly the survival $(\mathrm{p}=0.0003$ and $\mathrm{p}=0.0006)$.

Discussion: En-bloc chest wall resection is an effective treatment in case of NSCLC invading the chest wall. The prosthetic reconstruction is useful only for large anterior chest wall defects. In our experience the prognosis was affected only by the lymph nodal status and the stage of disease.

\section{2 \\ Multimodal Treatment of Malignant Pleural Mesothelioma (MPM): Our Experience}

\author{
L. Fino, M. Lucchi, P. Dini, A. Chella, A. Mussi \\ Department of Cardiothoracic, Division of Thoracic \\ Surgery, Pisa, Italy
}

Objective: MPM is a neoplasm arising from the pleura. We report the results of our experience on the multimodality treatment of MPM.

Methods: From 1999 to 2004, 73 patients with MPM (IMIG Stage $\leq 3$ ) underwent multimodal treatment consisting of pre-operative Intrapleural IL-2, pleurectomy/decortication (P/D) or extrapleural pleuropneumonectomy (EPP); intrapleural post-operative epidoxorubicin and IL-2, adjuvant hemithoracic radiotherapy (45-60 Gy), systemic chemotherapy (cisplatin-gemcitabine) and long-term subcutaneous IL-2.

Results: They were 56 male and 17 female (median age of 61.5 years; range 41-77). Histological diagnosis was made by a thoracoscopy: 58 (79.5\%) epitheliomorf; 5 (6.8\%) sarcomatous; 10 (13.7\%) bifasic. 64 patients underwent P/D (87.7\%) while 9 EPP $(12.3 \%)$. According to IMIG we had: stage IB in 4 patients $(5.5 \%)$, II in $19(26 \%)$; III in $50(68.5 \%)$. There were no mortality. We had 5 broncho-pleural fistula; 2 myocardial infarction, 2 pulmonary embolism. At December 2005, 25 patients were still alive while 48 died. 3 -year overall actuarial survival was $26.7 \%$ with a median survival of 24.4 months. Survival was significantly affected by the histotype, stage and performance status.

Conclusion: Multimodality treatment for stage I-III MPM was well tolerated with satisfying survival. New therapies are awaited to improve the outcomes.
153

\section{The Efficacy of Thoracoscopic Splanchnicectomy for Intractable} Pancreatic Pain

\section{A. Fiorello, G. Vicidomini, P. Laperuta, G. Busiello, V.G. Di Crescenzo, M. Santini}

Thoracic Surgery Unit, Second University of Naples, Naples, Italy

Introduction: Intractable pain is the most of distressing and debilitating symptom in patient affected by unresectable pancreatic carcinoma, chronic pancreatitis, celiac metastases. The purpose of this study was to evaluate prospectively the efficacy of thoracoscopic splanchnicectomy for refractory pancreatic pain due to pancreatic diseases.

Methods: Nine patients (mean age 62.3 years) underwent splanchnicectomy for refractory pancreatic pain: 5 patients with irresectable pancreatic cancer, 3 patients with pancreatic metastasis from gastric cancer and 1 patients with celiac metastasis. All patients were analgesic-dependent and unable to perform normal daily activities. In evaluating the effects on pain we used a visual analogue pain scale, reduction of analgesic use and change in lifestyle.

Results: All splanchnicectomies were performed by unilateral left videothoracoscopy. The mean operative time was $19 \pm 2.4 \mathrm{~min}$. No intra-operative complication occurred. The mean duration of follow up was 2 months. The average pain score reduced by $50 \%$ in all patients and drug addiction stopped. No patient required contralateral operation until death. The mean hospital stay was 5.7 days.

Discussion: Thoracoscopic splanchnicectomy represents the treatment of choice in intractable pancreatic pain. It is safe, offers pain relief, reduces consumption of analgesics and improves quality of life in these fragile patients with very short life expectancy. In our experience unilateral approach has been performed at first intention. In case of failure, an adjunctive procedure on the right side may be effective to obtain total pain relief.

\section{4 \\ Role of Diffusing Capacity in Predicting Complicat ions after Lung Resection for Cancer}

\author{
A. Fiorello, G. Vicidomini, G. Gallo, A. Perrone, \\ E. Polimeno, M. Santini
}

Thoracic Surgery Unit, Second University of Naples, Naples, Italy

Introduction: The assessment of operative risk for major lung resection is still a challenge. The use of spirometric evaluation as a predictor of postoperative morbidity and mortality is insufficient in patients with marginal predicted values of FEV1. The purpose of our study was to determine whether the assessment of diffusing capacity of the lung to carbon monoxide (DLCO), together with FEV1, could improve selection of patients with cancer for major lung resection.

Methods: A retrospective analysis of 76 patients who underwent lung resection for cancer was performed. We assessed the relationship 
of postoperative complications to both predicted postoperative FEV1 (ppoFEV1) and DLCO (ppoDLCO). Fifty-nine patients underwent lobectomy/bilobectomy and 17 had pneumonectomy. The postoperative events were classified as pulmonary or cardiac complications, overall morbidity and hospital mortality.

Results: Both ppoFEV1 and ppoDLCO were statistically significant predictors of morbidity $(52.6 \%)$ and mortality $(6.6 \%)$, but ppoDLCO showed to be a better predictor of pulmonary complications $(22.4 \%)$. In the group of patients with marginal ppoFEV1 $(<40 \%)$, this spirometric parameter failed to estimate the pulmonary complications risk; in contrast, ppoDLCO predicted pulmonary morbidity with considerably accuracy $(\mathrm{p}<0.005)$.

Discussion: Predicted postoperative diffusing capacity is a strong predictor of pulmonary complications after lung resection for cancer. Furthermore, our experience seem to suggest that in patients with marginal ppoFEV1 values, ppoDLCO can accurately estimate the postoperative pulmonary morbidity risk, allowing safe resection in patients with normal ppoDLCO values. However, prospective studies including larger series of patients are indicated to evaluate this hypothesis.

\section{5 \\ Local Relapses and Survival in NSCLC c-T1NO Diagnosed with Transpleural Techniques}

\author{
F. Granato, L. Voltolini, L. Luzzi, C. Rapicetta, S. Tenconi, \\ C. Ghiribelli, G. Gotti \\ Thoracic Surgery Unit, University Hospital of Siena, Siena, \\ Italy
}

Introduction: To assess if transpleural diagnostic techniques impact on local relaps and long-term survival.

Methods: 179 patients with NSCLC in clinical stage T1N0 undergone radical surgical treatment from 1994 to 2000 have been investigated. According to diagnostic procedure used, patients were divided into two groups: group 1 (62) in which diagnosis was obtained with bronchoscopy; group 2 (116) undergone to FNAB (59) or wedge resection (57) after negative bronchoscopy.

Results: The two groups did not differ significantly for age, sex, FEV1, comorbidity, histology, size of tumor and pathologic stage. After a follow-up of 48 months, local relapses were found in 6 patients $(9.5 \%)$ of group 1 and $15(12.5 \%)$ of group $2(\mathrm{p}=$ n.s.); metastasis developed in 18 patients $(28.6 \%)$ of group 1 and 16 patients $(13.8 \%)$ of group $2(\mathrm{p}=0.03)$. According to multivariate analysis, diagnostic procedure did not impact on incidence of local relapses. Survival of group 1 was significantly worse, with an high incidence of distant methastasis.

Discussion: FNAB and VATS wedge resection of tumor represent useful methods in diagnosing lung tumors not leading to iatrogenic tumor spread. Tumors diagnosed by bronchoscopy have a worse prognosis, probably due to a different biological behaviour and an earlier methastatic potential.

\section{6}

\section{Surgical Resection for Pulmonary Aspergillosis}

L. Libretti, P. Ciriaco, A. Bandiera, A. Puglisi, A. Carretta,

L. Ferla, P. Zannini

Ospedale San Raffaele, Milano

Objective: Pulmonary aspergillosis (PA) represents a devastating infection occurring in immunocompromised (ICP) and immunocompetent patients (ICT). Surgical resection (SR) might be advocated if medical treatment (MT) fails to eradicate the disease. We analyzed our surgical experience in PA in terms of perioperative complications and follow-up.

Methods: Within 11 years, 22 patients underwent surgery at our Institution for PA. ICP were 13 (12 hematologic disease, $1 \mathrm{HIV+)}$ and 9 ICT had hemopthysis. Preoperatory diagnosis was obtained in 18 patients by means of CT-scan, CT-guided biopsy and BAL. Neutropenia was present at the time of surgery in 5 ICP (white cell count $<500 \times 109 / 1)$ with a mean bone marrow aplasia of $21 \pm 10.3$ days SR included 15 lobectomies, 6 wedge resections and 1 explorative thoracotomy.

Results: Major postoperative complications occurred in 1 ICP (4\%) who died on postoperative day 25 of diffuse aspergillosis. Minor complications occurred in 7 patients, 6 of whom ICP (32\%). The immunological status was significantly correlated with onset of postoperative complications $(\mathrm{p}<0.05)$. Thirteen patients $(62 \%)$ are alive and disease-free (follow-up $63.4 \pm 50.8$ months). Mean survival was longer for ICT $(80 \pm 0.7$ vs. $39 \pm 53.6$ months $)$.

Conclusions: SR associated with MT can provide an effective treatment for PA although burdened with a high mortality and morbidity which are increased in ICP.

\section{7}

\section{Intraoperative 'Blood Patch' Prevention of Persistent Air Leak in Lung Resections}

F.L. Giudice, P. Fontana, A. Zaccaria, A. Ferronato, R. Salvi, S. Lovadina, V. Pagan

\section{Ospedale Umberto I, Venezia Mestre}

Introduction: Persistent air leak (PAL) is the most common complication after lung resection, requiring additional treatments and hospital stay. We consider 'blood patch' a successful procedure to avoid them.

Method: In a consecutive series of 570 lung resection, parenchymal suture air tightness was intraoperatively tested at endinspiratory pressure. Air escape beyond $30 \mathrm{~cm} \mathrm{H}_{2} \mathrm{O}\left(\mathrm{n}^{\circ} 437\right)$ was reputed at low risk for PAL. Air leak between 16 and $30 \mathrm{~cm} \mathrm{H}_{2} \mathrm{O}$ $\left(\mathrm{n}^{\circ} 93\right)$ were assumed as the main source of postoperative PAL and preventively treated by $50 \mathrm{cc}$ of blood left in the cavity for $3 \mathrm{~h}$.

Results: No early or late major complication were related to the procedure. The cumulative PAL incidence was $1.4 \%$, most of witch (6/8) belonging to the group leaking air in the pressure interval $16-30 \mathrm{~cm} \mathrm{H}_{2} \mathrm{O}$. Conversely, the PAL rate in the 437 patients reputed at low risk was $0.2 \%$. 
Discussion: According to our retrospective data, air leak occurring between 16 and $30 \mathrm{~cm} \mathrm{H}_{2} \mathrm{O}$ of pressure in the incubated lung was identified as the highest risk to become persistent postoperatively; intraoperative autoblood pneumostasis is a convenient and successful preventive method to minimize PAL occurrence in this group of patients.

\section{8}

\section{Surgical Treatment and Follow-up in Patients with Carcinomatous Pleuritis}

\author{
A. Ferronato, P. Fontana, A. Zaccaria, F. Lo Giudice, \\ R. Salvi, S. Lovadina \\ Ospedale Umberto I, Venezia Mestre
}

Introduction: In sporadic series, long term survival as been reported for patients operated on for lung cancer with secondary carcinomatous pleuritis (T4), either in the form of pleural dissemination or of malignant effusion or both, carries a very pour prognosis, with virtually no survival beyond 12 months. The usual treatment reserved to such IIIB stage tumors is palliative mainly aimed at controlling effusion by pleurodesis, while surgery is generally contraindicated point.

Method: In a retrospective study, of patients operated from 1999 to 2004 , we review the outcomes of 24 surgical patients (20 treated with standard lung resection \pm pleurectomy and 4 with extended pleuropneumonectomy) out of 48 individuals affected by pleural spread before or at thoracotomy.

Results: we observed a $16.6 \%$ major complication rate with no operative mortality; 5-year and median survival were $20 \%$ and 21 months, respectively. Time of diagnosis (pre- vs. intra/postoperative) or pattern (effusion vs. dissemination) of pleural disease, and type of resection (standard vs. extended) did not seem to influence the prognosis, while an adenocarcinoma histotype, completeness of excision and $\mathrm{N}_{0-1}$ were favourable prognostic indicators.

Discussion: Since most (90\%) of these IIIB stages are usually associated with $\mathrm{N}_{2-3}$ and/or unresectable tumor, it would seem reasonable to employ neo-adjuvant a treatment as the first approach, reserving surgical treatment to responders. Multicenter studies are necessary to better determine witch subgroup of patients with malignant pleuritis can most benefit from surgical therapy.

\section{9}

\section{Surgical Treatment of Primary Pulmonary Lymphoma}

\author{
A. Lomonaco, L. Di Carlo, A. Semeraro, N. Daddi, \\ M. Sciamannini, F. Puma, G. Daddi \\ Chirurgia Toracica, S. Andrea delle Fratte, Perugia
}

Introduction: Primary pulmonary lymphoma is an uncommon lung neoplasm.

Methods: From July 2000 to April 2006 we have observed three cases of primary lymphoma. 3 patients ( $2 \mathrm{~F}, 1 \mathrm{M}$; mean age 34.3 years, range 27-40) were examined with chest X-ray, CT scan and bronchoscopy. Most common symptoms were cough, fever, asthenia, weight loss and clubbing. Radiological findings were similar to primary pulmonary carcinoma. Bronchoscopy revealed bronchial stenosis (2) and no abnormal findings (1). Definitive diagnosis was possible by pathologic and immunohistochemical examination of the lung tissue obtained at surgery in 2 cases, in the remaining a preoperative diagnosis was attained by transbronchial biopsy. Tumor localization was in left upper lobe (2) and middle lobe (1). 2 patients underwent radical pneumonectomy and 1 left upper lobectomy. Surgical specimens were histologically proven to be Hodking's lymphoma in all cases. All patients received postoperative chemotherapy and 1 also preoperative.

Results: No perioperative mortality and morbidity with regular postoperative course. All patients are alive without evidence of disease at 27, 15 and 13 months.

Discussion: Primary pulmonary lymphoma is a rare type of malignant lung neoplasm without characteristic clinical features. Preoperative diagnosis is difficult because of the lack of specific characteristics and similarity to primary lung cancer. Treatment modalities include surgical treatment, radiotherapy and chemotherapy.

160

\section{Spontaneous Thoracic Esophageal Perforation Related to Chronic Alcohol Abuse Presenting as Pneumothorax: Report of a Case}

\author{
J.F. Sciuchetti ${ }^{1,2}$, F. Corti ${ }^{2}$, F. Formica ${ }^{2}$, D. Ballabio ${ }^{1}$ \\ ${ }^{1}$ Department of Cardio-Thoracic Surgery, Thoracic Surgery \\ Division, ${ }^{2}$ Department of Cardio-Thoracic Surgery, Cardiac \\ Surgery Division, San Gerardo Hospital, Milano-Bicocca \\ University, Italy
}

Case Report: We describe a case of a 36-year-old man with an history of alcohol abuse who referred to our thoracic division following the sudden onset of severe abdominal, chest pain and dyspnea after consuming one beer drink. Electrocardiogram showed no ischemic changes. An early chest radiograph revealed left pneumothorax, pneumomediastinum and left pleural effusion. Abdominal radiograph showed no abnormal findings. Chest computed tomography with oral contrast revealed large free leakage of contrast into the left pleural cavity with evidence of perforation of the lower part of the esophagus. Esophagoscopy showed a $1-\mathrm{cm}$ posterolateral open perforation located in the distal part on the left area of the esophageal wall. Surgical approach was performed $8 \mathrm{~h}$ from hospital recovery. At lateral left thoracotomy incision, remarkable mediastinitis and contamination of pleural cavity with gastric gists and food residues were found. Esophageal repair with separated $2-0$ stiches was performed through monolayer reconstruction. Mediastinum and pleural cavity were debrided and irrigated with betadine and saline solutions. Intraoperative esophagoscopy was executed to demonstrate no signs of fistula. Simultaneously, we operated a left minilaparotomy packaging a nutritional jejunostomy. Chest CT performed on postoperative day six described a small leakage of the esophagus. Under videoendoscopic and radioscopy guidance an endoprosthesis was inserted bridging the site of perforation. Next day a X-ray chest showed prosthesis dislocation to stomach that was successively removed. Chest irrigation with betadine and saline solution was then performed till sterilization of the pleural cavity and leakage 
closure documented at esophagoscopy control. Patient was discharged (truncated at 250 words).

\section{1 \\ Real Surgical Morbi-Mortality after Extrapleural Pneumonectomy \\ S. Lovadina, P. Fontana, A. Zaccaria, F. Lo Giudice, A. Ferronato, R. Salvi, V. Pagan \\ Chirurgia Toracica, Mestre}

Introduction: Extrapleural pneumonectomy (EPP) is increasingly employed in multimodal therapeutic protocol for malignant pleural mesothelioma (MPM) and others malignant or benign diseases. The potential benefits of EPP should be balanced against the spectrum of surgical complications. The conventional 30-day surgical morbid-mortality marker may underestimate the real risks associated with EPP. Some authors remarked that true (i.e. early and late) risks of pneumonectomy are significantly higher than that currently ascribed to the 30-days frame mortality; furthermore direct sequels of operation have been shown to adversely influence major lung surgery outcome far as long as 6 months.

Methods: A total of 78 patients undergoing EPP were retrospectively reviewed to better assess the early (30-days) and the late (6months) surgery related morbid-mortality rates, with a first subset of 35 consecutive patients between 1995 and 1999 vs. 43 in the period 2000-2005.

Results: Median age was 60 years (range 36-75 years). The majority of patients $(70.5 \%)$ undergone EPP for MPM. In the first group of patients $9(25.7 \%)$ had acute reesploration, early morbidity $68 \%$, late morbidity $28 \%$, early mortality $8.5 \%$, late mortality $12.5 \%$, overall morbidity $88 \%$ and overall mortality $20 \%$ vs. the second period with acute reesploration $3(6.9 \%)$, early morbidity $23 \%$, late morbidity $11.9 \%$, early mortality $2.3 \%$, late mortality $2.3 \%$, overall morbidity $35 \%$ and overall mortality $4.6 \%$.

Discussion: Morbidity and mortality after EPP occur not just under 30-days but as long as 6 months. Mortality and morbidity can be minimized with increasing experience.

\section{2}

\section{Thirty-days Hospital Readmission after Pulmonary Lobectomy: Prevalence and Characteristic}

P. Maniscalco, F. Quarantotto, L. Valieri, F. Acerbis, M. Santini, C. Brombin, C. Giorgio, G. Azzena

Department of General and Thoracic Surgery, Istituto di Clinica Chirurgica, S. Anna Universitary Hospital, Ferrara, Italy

Introduction: The aim of this study was to evaluate the rate, causes and variables related to 30 days hospital readmission.

Methods: From January 2003 to October 2004, 101 patients underwent pulmonary lobectomy and managed by clinical care pathway with target discharge on 5th postoperative day. Readmission was defined as the hospital recovery happened within 30 days from the last postoperative days. We have analyzed the prevalence, length and causes of recovery related to previous surgery recovery.

Results: Readmission rate was $8 \%$ and occurred after mean 19.7 days (ranged 3-28 days). The cause of readmission was: three pneumonia, two difficult pain control, one pleural effusion, a case of angina and one patients who died for tromboembolism. Three patients were readmitted in our Hospital (not in our surgical unit), and the other 5 patients were readmitted in another peripheric hospitals. The overall mean length of hospital stay was about $7 \pm 2.3$ days. No patients need surgical treatment. All the patients have had a previous recovery longer than 5 days and caratherizated by postoperative complication and delayed removal of tube thoracostomy.

Conclusion: Hospital readmission has been identified as an important outcome measure, especially after operation. In our experience readmission rate after lobectomy managed by clinical care pathways was $8 \%$ and it isn't related to short recovery.

\section{3}

\section{Standard Posterolateral or Auscultatory Triangle Muscle-Sparing Thoracotomy for Pulmonary Lobectomy}

\author{
P. Mendogni, A. Palleschi, L. Rosso, N. Bellaviti, \\ A. Schiraldi, G. Basilico, L. Santambrogio \\ U.O. Chirurgia Generale ad orientamento Toracico, \\ Ospedale Maggiore Policlinico, Mangiagalli e Regina \\ Elena, Milano
}

Introduction: Different thoracotomy techniques have been proposed to open the chest. When muscles are preserved, muscle strength, pulmonary function and pain could be reduced. However, no conclusive data exists.

Methods: From March 2005 to December 2005, 48 patients were enrolled in a randomised, prospective study. Posterolateral standard or auscultatory triangle muscle-sparing thoracotomy were performed in order to accomplish pulmonary lobectomy for stage I lung cancer. Patients with diabetes mellitus, previous thoracotomy and major psychiatric disease were excluded. The postoperative analgesia was standardized for both groups. Chest pain, thoracic muscles strength and pulmonary function were recorded before the operation and in day $1,7,30$, and 90 . Opening and closing time, total operative time, maximum opening of rib retractors, length of incision the occurring of rib fracture were also recorded.

Results: The preoperative and postoperative data regarding muscle strength, pulmonary function and pain were similar into the two groups. No difference was also recorded in length of incision, maximum opening of rib retractors, opening and closing time, total operative time and hospital stay. Seroma didn't occur in any patient. Multivariate analysis on the whole population revealed correlation only between length of incision and body mass index and between postoperative pain and operative time.

Discussion: Standard and muscle sparing thoracotomy are comparable in terms of postoperative pain and function impairment. Both provide excellent access to the pleural cavity to perform pulmonary lobectomy. 


\section{4}

Diagnostic Accuracy of PET in Detection of Lymph Node Involvement for Patients with Resectable Lung Cancer: A Preliminary Report

\author{
M. Monteverde, S. Sanna, M. Mengozzi, D. Argnani, \\ M. Taurchini, F. Matteucci' ${ }^{1}$, R. Galassi', D. Dell'Amore \\ U.O. Chirurgia Toracica, Ospedale G. B. Morgagni, 'Servizio \\ di Medicina Nucleare, Ospedale G. B. Morgagni, Forlì
}

Object: To assess the role of 18 FDG-Positron Emission Tomograhy imaging (PET) in detecting preoperatively thoracic lymph nodes involvement in patients with resectable bronchogenic carcinoma.

Materials and Methods: We present our preliminary report about 32 patients, 20 males and 12 females, mean age 69.5 years, affected by bronchogenic carcinoma. In 22 patients the diagnosis was preoperative, 10 patients were affected by undiagnosed solitary pulmonary nodule; 3 patients were considered resectable after neoadjuvant chemotherapy. All the patients underwent PET study to assess node involvement and were surgically treated with major resections and radical lymphadenectomy. The node histological feature was compared with the PET results to assess its sensibility, specificity and accuracy.

Results: In 5 cases a positive PET pathological node captation (3 hilar, 2 mediastinal) was observed, in 27 it was negative. 32 patients had 50 nodal stations ( 30 hilar/lobar, 20 mediastinal) surgically sampled. In comparison with histologic features we observed 4 PET true positive results ( 2 mediastinal, 2 hilar) and 2 false negative (1 hilar, 1 mediastinal, micrometastases). The true negative PET findings were 25 , one the false positive (hilar, reactive). The sensibility was $66 \%$, the specificity $96 \%$ and the accuracy $90 \%$.

Conclusions: In our preliminary study the FDG-PET imaging is accurate in detecting thoracic nodes involvement in patients with lung neoplasm. Negative PET studies would exclude the need for mediastinal nodal sampling prior to surgery, as far as PET positive results could preclude surgical intervention.

\section{5 \\ Surgical Management of Traumatic Diaphragmatic Rupture}

\author{
M. Paci, G. Ferrari, V. Annessi, S. de Franco, G. Guasti ${ }^{1}$, \\ G. Sgarbi \\ Division of Thoracic Surgery, ${ }^{1}$ Department of \\ Anesthesiology, Arcispedale Santa Maria Nuova, \\ Reggio Emilia
}

Introduction: Diaphragmatic injuries occurs in 1-7\% of major blunt trauma victims and in 10-15\% of patients with penetrating chest trauma. In conservatively managed patients the rate of initially missed diaphragmatic injuries range from 12 to $66 \%$. It must be remembered that a delayed diagnosis of diaphragmatic rupture is associated with a high mortality rate $(36 \%)$.

Methods: From 1994 to 2005, 13 patients with suspect diaphragmatic lesion (7 blunt and 6 penetrating) were evaluated.
Indications for surgical exploration were a penetrating chest injury (6 cases) or a blunt trauma with radiological suspicion of diaphragmatic injury ( 7 cases) as focal diaphragmatic discontinuity, diaphragmatic thickening, intrathoracic hernia, diaphragm elevation, hemothorax, or hemoperitoneum.

Results: A diaphragmatic injury was confirmed in 12 patients, 5 on the right side ( 3 blunt, 2 penetrating) and 7 on the left side (4 blunt, 3 penetrating). Six of them (4 penetrating, 2 blunt) were diagnosed and successfully repaired with thoracoscopic techniques, 3 (1 penetrating, 2 right acute blunt) were repaired after conversion to thoracotomy for large diaphragmatic lacerations, 2 left chronic hernia were repaired in thoracotomy and 1 patient (left acute blunt) was successfully treated by laparotomy. No complications occurred.

Discussion: There is no gold standard investigation and the best diagnostic modality is still direct inspection. Due to high associated morbidity and mortality of this lesion, in patients with severe blunt traumas or penetrating chest or upper abdomen injuries, we recommend an aggressive operative treatment.

\section{6 \\ Thoracic Surgery Division of Brescia Civic Hospital Experience in Surgical Treatment of Congenital Chest Deformations: Successful Treatment Using the Modified Ravitch Procedure and Minimally Invasive Repair of Pectus Excavatum}

\section{G.L. Pariscenti, S. Milano, D. Benetti, M. Benvenuti, G. Botticini, P. Bovolato \\ Ospedali Civili di Brescia, Chirurgia Toracica, Brescia}

Introduction: We describe our results in treatment of anterior chest wall malformations with 2 different operations: modified Ravitchminimally invasive correction of pectum excavatum 'Nuss': MIPEC.

Methods: From 1979 to April 2006 we treated 63 patients (52 M, $11 \mathrm{~F}$ age 6-41): 52 excavatum, 10 carinatum, 1 complex malformation in Poland's syndrome. 57 patients modified Ravitch technique: Sternocondroplasty (incision: median M-submammary transverse F) and 6 with MIPEC. Preoperative evaluation: chest $\mathrm{X}$-ray, thorax CT, pulmonary-cardiac evaluations.

Results: Sternocondroplasty morbidity: 1 wound infection of bilateral submammary access, 1 pericardial effusion recovered without problems; 1 hemothorax (reoperation). No operative deaths, bar dislocations, prolonged pain. 2 early bar removal for rupture. No MIPEC major complications; minor complications: self-resolving PNX, subcutaneous emphysema. Patients satisfaction and relief of medical symptoms was excellent in 59 patients, good in 3 patients, medium in 1 patients. (6/6 MIPEC excellent results in our low follow up).

Discussion: Both techniques provide excellent clinical results. MIPEC is blood-sparing, takes less operating time, but is associated with more severe prolonged pain. Surgical technique should be selected according to age and kind of malformation. Surgeon's experience is essential for optimal results of both techniques, especially MIPEC needs wide experience in thoracic surgery. 


\section{7}

\section{Surgical Treatment with IHC in Multimodality Approach of Malignant Pleural Mesothelioma}

\author{
G.L. Pariscenti, D. Benetti, M. Benvenuti, G. Botticini, \\ T. Ligabue, A.F. Bravo, P. Bovolato \\ Ospedali Civili di Brescia, Chirurgia Toracica, Brescia
}

Introduction: Accurate preoperative staging is important in predicting prognosis and appropriate treatment. Multimodality treatment gives today best results.

Methods: We treat MMP with multimodality approach: thoracoscopy/VATS, mediastinoscopy, laparoscopy-hydration protocolsurgery (EPP: extrapleural pnumonectomy pericardiectomy and diaphragmectomy, bovine pericardium reconstruction or PD: pleurectomy/decortication) argon beam cytoreduction-intraoperative hyperthermic chemotherapy (IHC), adiuvant chemo-radiant therapy recently IMRT.

Results: Until April 2006 we treated 101 patients: 67 EPP (29 IHC), 34 PD (5 IHC). Therapeutical results depend on: precocity of diagnosis-epithelioid histological variety. 2 years survival rate: stage I group (2 patients) 100\%; stage II (51 patients) 39.2\%; stage III (48 patients) $18.7 \%$. Low surgical mortality (EPP: 1.5\%-PD: $0 \%$, overall $1 \%$ ) and morbidity (4.9\%: 1 chylothorax, pulmonary embolism, pneumonia, heart dislocation, gastric hernia: all resolved).

Discussion: IHC neutralizes micro residual tumor sites, cell spreading, increases radio sensitivity and reaches very high tissue platin dosage in intrathoracic wall. IHC is a safe procedure, it doesn't worsen postoperative prognosis (no renal failure) due to our hydration protocol. This treatment seems to offer good results: IHC could improve survival rate, but comparative-randomized-prospective studies will be essential to support this protocol.

\section{8}

Lung Resection for NSCLC in Octogenarians: Analysis of 70 Consecutive Cases

\author{
C. Rapicetta, L. Luzzi, T. Ligabue, F. Granato, \\ S. Tenconi, G. Gotti \\ UOC Chirurgia Toracica, Siena
}

Introduction: To assess the presence of preoperative predictors of early and late mortality in octogenarians operated on for NSCLC.

Methods: From 1990 to 2004,70 patients older than 80 underwent lung resection for NSCLC. Age, presence of comorbidity, pathological stage, histology, extension of resection, Fev1, DLCO, $\mathrm{PaO}_{2}$, postoperative complications were analysed.

Results: The 70 patients underwent 49 lobectomies, 8 pneumonectomies, 7 segmentectomies, 4 wedge resections and 2 bilobectomies. Postoperative complications developed in 17 patients (13 major complications) and lead to perioperative death in 8 cases. At the multivariate analysis the presence of comorbidity $(p<0.001)$ and the pneumonectomy $(p<0.001)$ correlated with the development of major complications. The overall survival 5 year was $31.5 \%, 43 \%$ for stage I, $15 \%$ for stage II and $0 \%$ for stage III. At the multivariate analysis pathological stage $(\mathrm{p}=0.004)$ and segmental resection $(p=0.02)$ affected long-term survival. Survival in stage I was significantly better than in stage II and III ( $p=0.001)$.

Discussion: Only octogenarians in stage I have a long-term survival that justifies the risk of a lung resection. For this reason preoperative staging should be the most accurate as possible. Patients with comorbidity or scheduled for a pneumonectomy are prone to develop postoperative, sometimes fatal, complications.

\section{9 \\ Malignant Solitary Tumor of the Pleura: A Case Report}

\author{
R. Sguinzi, F. Raveglia, I. Abumalouh, A. Leporati, \\ M. Mezzetti
}

Università Degli Studi di Milano, Cattedra di Chirurgia Toracica, Unità Operativa Chirurgia II, Azienda Ospedaliera San Paolo, Milano

Introduction: A 55-year-old severe obese woman presented with a bulky right endothoracic mass diagnosed about twenty years ago. This tumor was characterized by slow and progressive growth and determined severe respiratory insufficiency (FEV1 0.79L, 31\% pred.). Thoracic CT-scan showed a disomogeneous mass with irregular enhancement occupying the whole medium and lower hemithorax and compressing, as evident at the flexible bronchoscopic examination, the right main stem.

Methods: The patient underwent a right posterolateral thoracotomy; an intrapleural large encapsulated tumor, arising from the lateral chest wall, dislocated the entire lower lobe which was involved. An extensive debulking procedure was carried out with a manual enucleation of the mass. A right lower lobectomy was performed, as the parenchyma was macroscopically infiltrated; we conserved the middle and lower lobe because completely re-expanded.

Results: The hystological exam of the multiple irregular fragments of the mass (diameter between 1.5 and $18 \mathrm{~cm}$, weight $4.5 \mathrm{~kg}$ ) showed a proliferation of atypical mesenchimal cells with high mitotic index $(>20$ mitosis/10 HPF $)$ infiltrating pulmonary parenchyma and suggesting for malignant solitary fibrous tumor of the pleura (SFT), III grade (FNCLCC), Ki67: 70\%.

Discussion: SFT is an infrequent neoplasm generally arising from the submesothelial mesenchymal cells and described in many organs and in the soft tissues with a favourable outcome when completely excised. The majority of SFT of the pleura are benign; however a substantial number of cases $(10-30 \%)$ recur or metastasize. 19th National Congress of the Italian Polyspecialist Society of Young Surgeons (IPSYS) 
170

\section{Askin's Tumor: Primitive Neuroectodermal Tumor of Chest Wall}

\author{
1. Abumalouh, F. Raveglia, R. Sguinzi, S. Meda, M. Mezzetti \\ Università Degli Studi di Milano, Cattedra di Chirurgia \\ Toracica, Unità Operativa Chirurgia II, Azienda Ospedaliera \\ San Paolo, Milano
}

Introduction: Primitive neuroectodermal tumours (PNETs) of chest wall are rare entities composed of small round cells with neural differentiation.

Clinical Features: We report the case of a 19-years old male diagnosed in our center.

Methods: The patient underwent a right posterolateral thoracotomy: triple wedge resection was performed.

Results: The histological exam reveals a small round blue-cell tumor suggesting a Ewing's Sarcoma/PNET of the chest wall.

Discussion: Treatment consists of extensive surgical resection, augmented by chemotherapy and radiotherapy. The overall survival is less than $40 \%$ at 2 years.

171

The Role of Thoracic Surgeon in Lymphangioleiomyomatosis: from Diagnosis to Therapy
G. Rizzardi', M. Loy'1, G. Marulli', A. Zuin'1, C. Giacometti², F. Calabrese ${ }^{2}$, F. Rea ${ }^{1}$
${ }^{1}$ Division of Thoracic Surgery, ${ }^{2}$ Department of Pathology, University of Padua, Padova, Italy

Introduction: Lymphangioleiomyomatosis (LAM) is a rare systemic disorder of unknown etiology that affect women during the childbearing years. It is characterized by abnormal smooth muscle proliferation in pulmonary interstitium and along the lymphatic vessels with cystic destruction of lung parenchyma. LAM can occur as a sporadic disease or as a manifestation of tuberous sclerosis. The commonest presenting symptoms are dyspnoea, pneumothorax and chylothorax.

Methods and Results: At our Institution over a 10-years period we made for LAM 8 diagnostic and/or therapeutic videoassisted thoracic operations (VATS). In one patient with recurrence of chylothorax we performed a thoracotomy for thoracic duct ligation, but 3 years later she presented again symptomatic chylous pleural effusion: so we performed parietal pleurectomy and instillation in pleural space of a iodine solution (Povidone). Moreover we performed for LAM 5 bilateral lung transplantations with good survival rate results ( $75 \%$ at 5 years) and we have not found, as described in literature, recurrence of LAM in transplanted lung.

Conclusions: The Thoracic Surgeon has an important role in LAM diagnosis and therapy. The VATS is an important diagnostic (lung biopsy) and therapeutic method for recurrent pneumothorax or chylothorax. Moreover in end-stage patients with severe respiratory failure lung transplantation should be consider a valid therapeutic option.
172

\section{Endo-Bronchial UltraSound Guided Trans- Bronchial Needle Aspiration (EBUS-TBNA) in the Evaluation of Mediastinal Lesions}

L. Rosso, A. Palleschi, P. Mendogni, A. Stanzi, R. Carrinola, M. Nosotti, A. Baisi, L. Santambrogio

Chirurgia Toracica Policlinico, Milano

Introduction: Lung cancer is the most common neoplasm in the western world and its treatment is based on a correct diagnosis and staging. The development of new minimally invasive techniques of tissue diagnosis from lymphnodes and solid lesions in the mediastinum can avoid the patient not required surgical procedures.

Methods: We perform EBUS-TBNA with the Olympus BFU160F ultrasonic video broncoscope in 30 patients $(22$ men and 8 women). Selection of the patients was based on suspicious lymphoadenopaty in lung cancer and suspicious mediastinal lesion on CT and PET scan. The procedures were perform in the operating room under sedation and local anesthesia.

Results: A total of 61 lesions were punctured using a 22-gauge needle after a power Doppler examination was performed. Diagnostic material was obtained in 28 patients. No major complications occurred. EBUS-TBNA demonstrated cancer in 15 patients and benign cytology in 9 patients. Non-small cell lung cancer was diagnosed in 14 patients and renal carcinoma in one patient.

Discussion: We consider EBUS-TBNA a successful, minimally invasive and safe diagnostic tool in patients with suspicious mediastinal and hilar lesions. This procedure may be able to replace more invasive methods in the staging of lung cancer.

\section{3}

\section{Role of VATS in Spontaneous Pneumothorax Secondary to Lung Giant Bullaes}

\author{
E. Rota, A. Bianca, L. Bottero, G. Faillace, A. Fantini, \\ P. Premoli, G. Turra, P. Stefanini, M. Longoni
}

Azienda Ospedaliera 'Ospedale Civile di Vimercate', U.O.C. di Chirurgia I, P.O.Sesto S.Giovanni

Introduction: Thoracoscopy in diagnosis and treatment of spontaneous pneumothorax in lung dystrophy.

Methods: A 57 years old man with an acute respiratory failure and a complete left spontaneous pneumothorax in bilateral large bullaes was admitted to our hospital. A thoracic tube was immediately positioned with a partial re-expansion of the lung. Six hours later he referred ingravescent dyspnea, rhynolalya and a small parts' emphysema was spread to face, neck, thorax and upper arms. A new X-ray chest revealed a complete collapse of the lung and a displacement of the drainage that was immediately replaced. The chest CT revealed an incomplete re-expansion of the superior left lobe, with a pneumomediastino and a bilateral bullous disease. After the stabilization of respiratory and cardiac functions, the patient was submitted to a VATS: firstly, a right thoracic drainage was positioned to prevent the rupture of the bullaes during monolateral ventilation; then, apical left bullectomy and talcage were performed. Two left thoracic tubes were 
positioned; on the 2nd p.o. day the right tube was removed and a chest $\mathrm{CT}$ confirmed the complete resolution of pneumomediastino and left pneumothorax. Inferior left tube was removed on the 8th p.o. day and on the 10th the superior.

Results: Patient discharged on the 11th p.o. day.

Discussion: VATS is efficacy, safe and reduces morbidity.

\section{4}

\section{Retrospective Clinical Analysis of Surgical Treatment's the Solitary Fibrous Pleural Tumor (SFPT)}

\author{
C. Ruggiero, C. Lavini, U. Moranti \\ Unità Operativa di Chirurgia Toracica, Policlinico \\ Universitario, Modena
}

Introduction: The solitary fibrous pleural tumor (SFPT) still represents a rare well known neoplasm in his histogenesis but with controversial appearances on his histological characterization and the grading of wickedness.

Methods: From 1985 to 2005 twenty-five cases have surgically been treated of which 13 males (52\%) and 12 females (48\%) with 59.9 -year-old middle age. The surgical approach has been in 23 cases (92\%) thoracotomy, in 2 cases videothoracoscopy. The performed operations have been: lobectomy (3), pneumonectomy (1), atypical resections (6), extrapulmonary resections (13). In two cases pericardial resections has been performed and in 2 cases partial diaphragmatic resections with plasty.

Results: The resection results complete (R0) in 22 cases, with borders microscopically infiltrated (R1) in 2 cases while in each case the borders of section are noticed macroscopically infiltrated by the neoplasm. The middle follow up has been of 111.6 months (range 13-210 months). Three cases of later recurrences have been recorded at 60 and 84 months from the surgical intervention. The global survival has been $92 \%$.

Discussion: The surgical treatment is with no doubt the fundamental treatment having a double value, therapeutic and diagnostic. The surgical, microscopic, histochemical and cytogenetical markers are important prognostic factors in the history of the neoplasm.

\section{5}

\section{Surgical Treatment of Giant Atherosclerotic Left Internal Mammary Artery Aneurysm: A Case Report}

\section{S. Sanna, M. Taurchini, D. Argnani, M. Monteverde, M. Mengozzi, A. Dell'Amore', D. Dell'Amore \\ Thoracic Surgery Unit, G.B. Morgagni Hospital, ${ }^{1}$ Cardiac Surgery Unit, S. Orsola Malpighi Hospital, Bologna, Forli}

Introduction: Giant atherosclerotic aneurysm of internal mammary artery (IMA) is extremely rare, usually related with a thoracic injury, as more frequent false aneurysm or artero-venous fistula of mammary trunk which could be confused with. The treatment of this pathology is not well established, an embolization or a stent position could be safe. Surgical repair is mandatory in giant aneurysms.

Case report: A 82 years old female presented on the chest radiogram a large left-sided mediastinal mass without any previous thoracic trauma or surgical intervention. The CT showed an aneurysm of $7 \times 5 \mathrm{~cm}$ of diameter without relationship with great vessels and supraaortic trunks. An angiography demonstrated the origin of the aneurysm from the left IMA. The patient underwent to aneurismectomy through median sternotomy, with isolation of the aneurysm and closure of upper and distal part of the normal left IMA. The postoperative course was uneventful and the histologic feature showed a fusiform aneurysm with atherosclerotic degeneration of arterial wall and intramural thrombosis.

Conclusions: We reported a very rare lesion of the IMA, but with clinical significance in terms of differential diagnosis with others mediastinal masses and because failure to identify and treat it may result in danger in case of sternotomy or thoracotomy or sudden rupture and death risk for the patient.

\section{6 \\ Favourable Tissue Effects of Quantum Molecular Resonance Bistoury (Vesalius ${ }^{\odot}$ ) Compared with Standard Electrical Bistoury}

\author{
A Novel Paradigm in Lung Surgery \\ M. Schiavon', F. Calabrese ${ }^{2}$, S. Nicotra1', G. Marulli', \\ G. Pozzato ${ }^{3}$, C. Giacometti ${ }^{2}$ M. Valente ${ }^{2}$, F. Rea ${ }^{1}$ \\ ${ }^{1}$ Department of Cardiac, Thoracic and Vascular Sciences, \\ Thoracic Surgery Branch, ${ }^{2}$ Department of Pathology, \\ University of Padova, Medical School, ${ }^{3}$ TELEA Electronic \\ Engineering, Quinto Vicentino VI, Italy
}

Introduction: Electrosurgical devices are largely employed in thoracic surgery but their use is burdened by extensive necrosis and tissue cooking. The Quantum Molecular Resonance (QMR) constitutes the theoretical basis of a new surgical bistoury called Vesalius. It uses a special spectrum of frequencies that produces energy adequate to break the molecular binding of the biological tissue, without increasing the temperature.

Methods: A rat model of thoracothomy was performed on fortysix adult male rats, divided into two groups using a standard electrobistoury (EB) and the new Vesalius ${ }^{\mathcal{C}}$ bistoury. In order to study acute and late tissue effects, skin, muscle and lung specimens were obtained immediately or 2 weeks after surgery. Necrosis, apoptotic cell death and inflammation were analyzed and quantified using a discrete semiquantitative scoring system in tissue samples for each group.

Results: We found that in the short term Vesalius produced less severe tissue damage than that of standard electrobistoury in skin $(\mathrm{p}=0.03)$, muscle $(\mathrm{p}=0.04)$ and lung specimens $(\mathrm{p}=0.01)$. Furthermore Vesalius did not increase apoptosis, thus leading to an absolute reduction in cell death. In the long-term in the Vesaliustreated rats inflammatory scores were significantly lower than in EBtreated rats, particularly in muscle tissue $(\mathrm{p}=0.005)$ and the pulmonary parenchyma $(\mathrm{p}=0.015)$.

Discussion: Our experimental study shows that the use of the QMR bistoury largely prevents heat damage after lung injury and may 
provide an interesting alternative to gold-standard electrosurgical devices in thoracic surgery.

\section{7}

\section{Oesophageal Duplication: A Case Report}

L. Di Carlo, A. Semeraro, N. Daddi, M. Sciamannini, A. Lomonaco, F. Puma, G. Daddi

Policlinico Monteluce, Perugia

Introduction: Oesophageal duplication (OD) is a rare congenital abnormality that arises during the third week of embryonic life. Between $25-30 \%$ of patients remain undiagnosed until adulthood. The most common site is in the middle oesophagus.

Methods: A 35-year-old woman was admitted complaining of dysphagia, epigastric pain and vomiting. Chest CT showed midoesophageal pseudodiverticulum with oesophageal double lumen from the thoracic inlet to the diverticulum. Oesophagoscophy revealed a true oesophageal lumen and pseudodiverticulum. Oesophagogram showed double lumen extending to the diaphragm. Bronchoscopy and abdominal ultrasound were normal.

Results: Surgical findings confirmed OD. Adhesions to mediastinal organs were present. The oesophagus was isolated, pseudodiverticulum with the mucosal duplication resected and the muscle coat reconstructed. The postoperative recovery was swift, discharge in 10 days. Follow-up at one year with a chest X-ray confirmed normal oesophageal lumen. The patient remains symptom-free.

Discussion: With OD the primary oesophagus and duplication are in the same muscle sheath and may communicate. Malignant degeneration has been reported. Two-thirds present with dysphagia and chest pain. Mediastinal mass and oesophageal diverticulum are the differential diagnoses. Chest radiograph reveals a mediastinal mass, oesophagogram shows abnormal oesophageal transit and sometimes double lumen if communicating with the oesophagus. CT scan is the gold standard. Oesophagoscopy occasionally might show the inlet and the possible outlet between duplication and oesophagus. Biopsy is contraindicated. Treatment, in symptomatic patients, is surgical and consists in the excision of the duplication with preservation of the muscular layer. Results are good with complete resolution of symptoms.

\section{8}

\section{Solitary Fibrous Tumors of The Pleura: Our Experience}

\author{
M. Sciamannini, L. Di Carlo, A. Semeraro, N. Daddi, \\ A. Lomonaco, P. Saccenti, F. Puma, G. Daddi \\ Policlinico Monteluce, Perugia
}

Introduction: Solitary fibrous tumors (SFT) of the pleura are rare and potentially malignant (5\% of pleural neoplasm).

Methods: From June 1988 to April 200618 patients underwent 21 surgical procedures. 6 males and 12 females; median age was 60 years (range 39-72). Predominant clinical symptoms were dyspnoea, chest pain and cough in 12 cases, hypoglycemia in 2, 6 patients were asymptomatic. Surgical approach was through posterolateral thoracotomy in 11 patients, lateral thoracotomy in 4 and VATS in 6.14 tumours arose from visceral pleura, 7 from mediastinal, diaphragmatic and parietal pleura. Removal of the tumor alone was possible in 1 case, in the remaining extended resection involving adjacent structures was performed ( 1 bilobectomy, 3 lobectomies, 14 wedge and 1 chest-wall resection, 4 diaphragmatic resections). In all cases radical surgical excision was achieved.

Results: There was no perioperative mortality or morbidity. All surgical specimens were histologically proven to be a localized benign tumor of the pleura. Immunohistochemical reactions were in 16 patients positive for CD34, negative for vimentin, S100, cytokeratin. Follow up was performed in 17 patients (range 17 years-10 months). Local recurrences occurred in 3 cases, in which sarcomatous degeneration was observed. 14 patients are still alive without evidence of disease, 3 are dead of disease.

Discussion: Even though the majority of SFT are benign, they should be considered as potentially malignant neoplasms. Radical resection as well as clinical and radiological follow up are highly recommended because of the potentially malignant biological behaviour and the lack of radical treatment options other (truncated at 250 words).

\section{9}

\section{A Therapeutical Protocol for Postoperative Pain Relief in Thoracic Surgery}

\section{A. Stefani, C. Casali, U. Morandi \\ Department of General Surgery and Surgical Specialities, Division of Thoracic Surgery, University of Modena and Reggio Emilia}

Introduction: Thoracic epidural analgesia is the most effective approach for pain control in thoracic patients but it is expensive, demanding to manage, local complications can occur and many patients reject this technique.

Methods: We start post-thoracotomy pain management with a paraspinal intercostal nerves block with ropivacaine, performed intraoperatively. Morphine (M) $0.1 \mathrm{mg} / \mathrm{kg}$ iv + ketoprofen lysine salt (KLS) $0.6 \mathrm{mg} / \mathrm{kg}$ iv are administrated before extubation. Analgesia is maintained with a continuous iv infusion of $\mathrm{M} 0.015 \mathrm{mg} / \mathrm{kg} / \mathrm{h}$ plus KLS $0.15 \mathrm{mg} / \mathrm{kg} / \mathrm{h}$. Supplemental doses of M are given intravenously, on patient's demand. After 3-4 days the dosage is reduced and the patient begins taking oral analgesics.

Results: This approach has provided good pain control in more than 900 patients, since 2002. Supplemental doses were needed in $30 \%$ but a careful titration improved pain control without oversedation. Side effects as nausea, urinary retention and constipation were frequent but easily managed and controlled. Significant respiratory depression was observed in only 3 cases.

Conclusions: We believe that continuous intravenous narcotic therapy can be a satisfying technique, provided that drugs are administered in the effective dosage, a flexible dosing regimen is prescribed (basal dosage plus on-demand administration) and that medical staff is awaken to the problem of pain and have familiarity with narcotics' management. 
180

Talc Poudrage vs. Talc Slurry in the Treatment of Malignant Pleural Effusion: A Prospective Trial

\author{
P. Natali, A. Stefani, C. Casali, U. Moranti \\ Università di Modena, Cattedra e U.O. di Chirurgia Toracica
}

Introduction: To investigate the effectiveness, safety and appropriate mode of administration of intrapleural talc for pleurodesis in the treatment of malignant pleural effusion (MPE).

Methods: Prospective not randomized trial to compare thoracoscopic talc poudrage (TP) to tube thoracostomy and talc slurry (TS). Six grams of talc were administered for each procedure. Only the patients with lung re-expansion after drainage entered the study. Patients at high risk for general anaesthesia, poor general conditions and short life expectancy received talc slurry through a chest tube. All other patients underwent videothoracoscopic talc poudrage, with a pneumatic atomizer, under general anaesthesia.

Results: 104 patients entered the study (69 TS, 35 TP). 61 patients in the TP group (88\%) and 25 in the TS group (71\%) had an immediate successful pleurodesis $(\mathrm{p}=0.03)$; 58 patients $(84 \%)$ and $21(60 \%)$ respectively had a life-long pleural symphisis $(\mathrm{p}=0.006)$. Adverse effects were generally mild: chest pain (37\% in TP patients, $53 \%$ in TS patients) and fever (42 and 38\% respectively).

Discussion: Intrapleural talc carries good results in the treatment of MPE. TP is more effective than TS. The main causes of failure were massive cancerous invasion of the pleura and delayed indication to pleurodesis. Both methods were safe but TS had a higher incidence of thoracic pain during the procedure.

\section{1 \\ Videothoracoscopic Resection of a Rare Case of Castleman's Disease

\author{
M. Taurchini, S. Sanna, M. Monteverde, M. Mengozzi, \\ D. Argnani, D. Dell'Amore \\ Thoracic Surgery Unit, Morgagni G.B. Hospital, Forlì
}

Introduction: Castleman's disease or angiofollicolar hyperplasia is a rare lymphoproliferative disorder described by Castleman in 1956, whose etiology is actually unknown. Young women are mostly affected. Two are the clinical groups: localized and disseminated disease and two the histologic variants: hyalinevascular and plasma cell. In localized disease, the most frequent, radical surgical treatment, with mininvasive approach if possible, is the main therapeutic indication.

Case Report: We report a case of a 48-year-old woman that was referred to our hospital because of an abnormal mass of the left thoracic wall, already diagnosed 7 years ago but in the last months progressively grown with concomitant thoracic pain. A thoracic MRI showed in the left posterior mediastinum a $5.8 \mathrm{~cm}$ well-defined round mass with no aortic involvement and disomogenous T2 weighted enhancement pattern, suspected for paravertebral neurinoma. The patient underwent videothoracoscopy with intraoperative evidence of a policicled highly vascularized lesion without communication with vertebral foramina. The mass was radically resected by videothoracoscopic approach. The postoperative course was uneventful. The pathological examination revealed a hyaline-vascular localized variant of Castleman's disease.

Discussion: Castleman's disease is a rare lymphoproliferative disorder, which can present as localized or disseminated disease. The gold standard treatment in localized lesions is radical surgical resection, with mininvasive technique, when possible, due to its good results, minimal invasiveness and patient's great compliance.

\section{2 \\ Sleeve Lobectomy (SL) after Induction Chemoradiotherapy (IT)}

\author{
A. Piraino, M.T. Congedo, A. Tessitore, G. Cusumano, \\ E. Meacci, P.M. Granone
}

Objective: SL is a good option in locally advanced NSCLC even when pneumonectomy is tolerated. We investigated the possible effects of IT, in increasing complications.

Methods: In 1995-2005 we performed 10 out of 33 standard SL after IT for NSCLC. Results of SL following IT, were statistically compared to SL without IT. IT consisted of a cysplatin-based regimen plus an average of 45 Gy radiation.

Results: Patients in both groups were comparable for demographic characteristics, with a prevalence of males. In the IT group clinical stage was significantly higher (prevalence of cIII). There was no clinical IV stage. Downstage to I-O was $70 \%$. Morbidity was $50 \%$ in the IT group vs. $17.3 \%$. There were two $(20 \%)$ anastomotic complications in the IT group. One patient (10\%) in the IT group had a post-operative death from broncho-vascular fistula. 1 year survival was respectively $100 \%$ in IT group vs. $85.7 \%$ in non-IT; 3 years survival $55.5 \%$ vs. $42.8 \%$; 5 years survival $44.4 \%$ vs. $28.6 \%$. There were no pIII or pIV stage in the IT group. Recurrence rate was $33.3 \%$ after IT vs. $35.7 \%$.

Conclusions: Although our experience is small, we observed an increase of morbidity and mortality after IT. There was not strong difference in survival and recurrence rate. We reported better results after IT, reflecting on the absence of advanced p-stages.

\section{3 \\ Spontaneous Pneumomediastinum (SPM): Personal Experience}

\author{
M.T. Congedo, A. Tessitore, A. Piraino, G. Cusumano, \\ M.L. Vita, E. Meacci, P.M. Granone
}

Objective: SPM is an uncommon clinical syndrome not correlated to trauma. Complications are rare, but potentially life-threatening. We report a single institution experience and a literature review.

Methods: We observed 6 cases ( 5 males and 1 female) of SPM from 1998 to 2006. Predominant symptoms were dyspnoea, chest/neck pain, dysphagia and cough. Diagnosis was usually made by chest X-ray and confirmed by CT scan. Barium swallow and bronchoscopy where done when complications were suspected. Antibiotics were always administered while anti-asthma therapy was given only when indicated. Pleural drain was inserted in two cases (33.3\%). 
Results: Subcutaneous emphysema was present in 3 cases (50\%); a trigger factor was found for three cases (asthma 2 cases and BPCO 1 case). Simple monitoring of the patient through chest X-ray and ECG, bed rest and antibiotics was sufficient for all the patients but two, in whom a chest drain was positioned. Clinical course was uneventful for everybody and neither complication or related-death were observed.

Conclusions: We confirmed a strong correlation between SPM and pre-existing respiratory disease as reported in literature. CT-scan as necessary to complete diagnosis and discover an underlying pathology. The evolution is usually indolent while antibiotics are useful to prevent infections.

\section{4}

\section{Latissimus Dorsi Reverse Flap to Substitute the Diaphragm after Extrapleural Pneumonectomy}

\author{
A.V. Bedini, D. Tosi, M. Guglielmetti, B. Conti, U. Pastorino \\ I.N.T., Milano
}

Background: The standard procedure for diaphragm reconstruction after extrapleural pneumonectomy for a malignancy consists of the use of prosthetic patches. Our original technique utilizing the reverse flap of the latissimus dorsi is evaluated.

Methods: Once the extrapleural pneumonectomy is performed, the distal portion of the latissimus dorsi, which has been divided with a standard posterolateral thoracotomy at the level of the fifth to sixth rib, is elevated into the chest through the passage obtained by resection of the tenth rib and sutured to the lower pericardium and to the chest wall. Thirty-one patients were evaluated.

Results: No operative death occurred. One case of flap-related complication was observed; no infection was postoperatively assessed. Twenty-four patients received adjuvant radiotherapy. No late complication was observed.

Conclusions: The distal latissimus dorsi can be used for total reconstruction of one hemidiaphragm, ensuring a watertight separation between the pleural and peritoneal cavities and avoiding paradoxical respiratory motion. In our opinion, the technique was easier, faster, and more reliable than the standard procedure employing prosthetic materials. We recommend that the procedure be integrated with the standard technique of extrapleural pneumonectomy.

\section{5}

\section{Reconstructive Tracheo-Bronchial Surgery: A Single Referral Center Experience}

\section{A. Viti, M.C. Ambrogi, A. Ribechini, A. Mussi}

Objectives: The surgical treatment of tracheo-bronchial lesions may result in a resolution of the disease, and in a complete reconstruction of the main airway. We reviewed our experience particularly referring to the surgical technique.
Methods: From 1995 to 2005, 42 patients underwent surgical treatment of tracheo-bronchial lesions. They were 21 male and 21 female, mean age of 58 years. Lesions were: 13 tracheo-bronchial traumas, 20 post-intubation stenosis, 2 tracheo-esophageal fistulas, 7 neoplastic lesions ( 3 carcinoids, 1 NSCLC, 1 adenoid-cystic tumor, 1 fibrolipoma, 1 papilloma). We performed 22 resection with end to end anastomosis, 5 wedge resections, 14 repairs of tears, one resection of the carina, throw 30 cervicotomies and 12 thoracotomies.

Results: We had no peri-operative mortality. One dehiscence of the anastomosis in 4th post-operative day was successfully repaired. Seven (10\%) patients required endoscopic procedures due to granulomas of the suture line. At a median follow up of 4.4 years, no patient with neoplastic lesion developed recurrence.

Conclusions: Surgical treatment of main airway lesions is effective and safe when employed in highly specialized institutions. The technique is now standardized, except for membranous wall lacerations. In this case, new recent surgical approaches, reducing invasiveness, have overthrew their management.

186

\section{Percutaneous Laser-Induced Thermoablation (LIT) of Non-Resectable Lung Metastases and Primari Lung Tumors: A Preliminary Evaluation of Technical Aspects and Local Efficiency}

E. Baracchini', E. Sattin², M. Cossaro², G. Masullo1, A. Morelli ${ }^{1}$, G. Aprile ${ }^{3}$, A. Vit', U. Livi

${ }^{1}$ Unit of Thoracic Surgery, Azienda Ospedaliera SMM, ${ }^{2}$ Unit of General Surgery, University of Udine, ${ }^{3}$ Department of Oncology, University of Udine, ${ }^{4}$ Department of

Radiology, Azienda Ospedaliera SMM, Udine

Introduction: Only a small part of patients in colorectal cancer or primitive lung tumors care can be radically resected. Thermal ablation is a mini-invasive local treatment that can be considered an alternative approach in non-resectable lesion or inoperable patients.

Methods: From March 2004 to April 2006, 22 patients treated with LIT. Nine patients had a small $(<5 \mathrm{~cm})$ non-resectable lung carcinoma, four patients a solitary lung metastases, mainly from colorectal cancer. Optical fibers were inserted into the tumor with CT guided percutaneous needle placement.

Results: All the patients tolerated LIT procedure well, with mild sedation and local anaesthesia. Easy manageable local side effects occurred. Post-treatment CT-scan demonstrated complete thermonecrosis in all the lesions smaller than $3 \mathrm{~cm}$. All the patients are still alive, with a local tumor control rate of $100 \%$ at radiological follow-up: no local progression has been observed in 19 patients with a follow-up of a least 6 months and in the 3 patients with a follow-up of at least 2 months.

Discussion: Percutaneous LIT of lung tumors permitted a complete ablation of lung metastasis and lung carcinomas with an optimal local tumor control rate at 6-month and a low complication rate. Complete necrosis was achieved only in lesions with maximum diameter smaller than $3 \mathrm{~cm}$. 


\section{7}

Percutaneous Laser-Induced Thermoablation (LIT) of Non-Resectable Lung Metastases and Primari Lung Tumors: A Preliminary Evaluation of Technical Aspects and Local Efficency

\author{
E. Baracchini ${ }^{1}$, E. Sattin ${ }^{2}$, M. Cossaro², G. Masullo ${ }^{1}$ \\ A. Morelli', G. Aprile ${ }^{3}$, A. Vit', U. Livi ${ }^{1}$ \\ ${ }^{1}$ Unit of Thoracic Surgery, Azienda Ospedaliera SMM, \\ 2Unit of General Surgery, ${ }^{3}$ Department of Oncology, \\ University of Udine, ${ }^{4}$ Department of Radiology, Azienda \\ Ospedaliera SMM, Udine
}

Introduction: Only a small part of patients in colorectal cancer or primitive lung tumors care can be radically resected. Thermal ablation is a mini-invasive local treatment that can be considered an alternative approach in non-resectable lesion or inoperable patients.

Methods: From March 2004 to April 200622 patients treated with LIT. Nine patients had a small $(<5 \mathrm{~cm})$ non-resectable lung carcinoma, four patients a solitary lung metastases, mainly from colorectal cancer. Optical fibers were inserted into the tumor with CT guided percutaneous needle placement.

Results: All the patients tolerated LIT procedure well, with mild sedation and local anaesthesia. Easy manageable local side effects occured. Post-treatment CT-scan demostrated complete thermonecrosis in all the lesions smaller than $3 \mathrm{~cm}$. All the patients are still alive, with a local tumor control rate of $100 \%$ at radiological follow-up: no local progression has been observed in 19 pts with a follow-up of a least 6 months and in the 3 pts with a follow-up of at least 2 months.

Discussion: Percutaneous LIT of lung tumors permitted a complete ablation of lung metastasis and lung carcinomas with an optimal local tumor control rate at 6-month and a low complication rate. Complete necrosis was achieved only in lesions with maximum diameter smaller than $3 \mathrm{~cm}$.

\section{Vascular Surgery}

\section{8 \\ Endovascular Aortic Repair (EVAR) in Abdominal Aortic Emergencies: A Single Center Initial Experience}

\author{
A. Angelini, E. Gatta, G. Pagliariccio, L. Carbonari, F.P. Aló \\ S.O.D. Chirurgia Vascolare, Azienda Ospedaliero- \\ Universitaria Ospedali Riuniti Umberto I - G.M. Lancisi - \\ G. Salesi, Ancona
}

Introduction: Mortality after open surgical repair (OR) in patients with ruptured abdominal aortic aneurysm (RAAA) remains high, and has not changed significantly in the last 30 years. Recently many series and few randomized prospective studies described the application of EVAR in emergency, even for RAAA. The open questions are: which is the proportion of patients eligible for emergency EVAR and what are the anatomical, clinical and technical conditions for emergency EVAR. Finally if outcome of emergency EVAR is better than OR.

Methods: 10 patients with an abdominal aortic urgency were treated with EVAR during a period of 18 months; 5 have haemodynamic instability while 3 presented with RAAA. In 6 patients an endoconversion on a previous aortic surgery or EVAR was performed. 4 bifurcated graft, 6 AUI and 2 iliac extension were placed.

Results: 30 days mortality was $0 \%$. Lower intensive care unit stay was observed; other parameters considered were: blood loss and transfusion, heart, renal and respiratory function. Considering patient presenting with RAAA, 25\% were suitable for EVAR and the inclusion criteria are other than haemodynamic instability.

Discussion: EVAR seems to be feasible in almost $40 \%$ of all presentations; haemodynamic instability does not appear to be a contraindication for EVAR in RAAA. This percentage might be improved by $\mathrm{CT}$ accuracy, team experience, and rapid access to treatment. EVAR gives better 30 days mortality results and a lower rate of cardiac, renal and respiratory complications.

\section{9}

\section{Aortic Prosthesis Infection: A New Strategy by Combined Homograft and Femoral Vein By-Pass}

\section{G. Regina ${ }^{1}$, G. Annunziata ${ }^{2}$, M. Ciavarella ${ }^{1}$ \\ ${ }^{1}$ Cattedra e Scuola di Specializzazione in Chirurgia Vascolare Universita' degli Studi di Bari, ${ }^{2}$ Cattedra e Scuola di Specializzazione in Urologia Universitaria I, Università degli Studi di Bari}

Graft infections are a dramatic complication of reconstructive vascular surgery. We in describe a new management strategy which utilizes a short aortic arch homograft supplied by a brachi-cephalic trunk, which is anastomosed to a long segment of autologous superficial femoral vein, itself anastomosed to the right femoral artery. Soon after a femoro-femoral cross over homograft by pass guarantees the left leg revascularization. This operation could be done through a retroperitoneal approach and offers the advantage of utilizing only a small part of aortic homograft which can short operative time two different teams, one preparing the femoral vein and the second opening the abdomen. Rate of aortic homograft fatal rupture is minimized. Eventual rupture of the femoro-femoral crossover by pass can be easily diagnosed and treated in short lapse of time.

Case Report: A 76 years old caucasian man was referred to our service with the diagnosis of aorta-bifemoral prosthetis graft infection. The previous Dacron graft in situ was completely excised and the infrarenal aorta was anastomosed with a criopreserved homograft using a $3 / 0$ polypropylene suture. The right femoral artery was then sutured with the SFV that was previously linked to the aortic arch homograft.

Discussion and Conclusion: The strategy of combining a homograft with a vein can overcame the shortage of the homografts and the variable diameters moreover avoids the possible the thoracic homograft is prone to dilatation and thrombosis if placed in abdominal position (truncated at 250 words). 


\section{0}

\section{Aortic Endografts with and Without Hooks:} Results at Mid-term Follow-up

M.E. Barzaghi, A. Griso, L. Nicolai, S.F. Ruffino,

F. Baratto, D. Kontothanassis, G. Camporese1,

G. Bellandi', A. Scuro

Vascular Surgery, University of Verona, ${ }^{1}$ Angiology, University of Padova, ${ }^{2}$ Vascular Surgery, Ospedale Civile, Arezzo

Introduction: To evaluate migrations and endoleaks in midterm follow-up of two different endografts with suprarenal fixation: Talent (Medtronic), hookless in its top stent and Zenith (Cook) with hooks.

Methods: We analyzed 119 patients underwent to EVAR with Talent or Zenith endografts, their ASA degree was 3. We treated 55 patients with Talent. Middle age was 72.48 years; 3 were females and 52 males. AAA middle diameter was $57.6 \mathrm{~mm}$ and the one of their proximal neck was $23.38 \mathrm{~mm}$ with a length of $21.3 \mathrm{~mm}$. The patients treated with Zenith graft was 64 . Females were 5 and 59 the males; with a middle age of 73.6. AAA middle diameter was $57.1 \mathrm{~mm}$ and the one of their proximal neck was $23.5 \mathrm{~mm}$ with a length of $26.8 \mathrm{~mm}$.

Results: During the follow-up we didn't find significant differences within Talent Endografts and Zenith one regarding number of endoleak, grow of proximal neck, sack and shrincage, number of reinterventions. We noted only significant difference about the number of proximal migrations between 5 and $15 \mathrm{~mm}$ : $16.3 \%$ for Talent vs. $0 \%$ for Zenith; no differences was found in the migration over $15 \mathrm{~mm}$. This result is independent of growth of proximal neck and these migrations don't cause an increase of major complications.

Discussion: The presence of hooks prevents the migration between 5 and $15 \mathrm{~mm}$. No differences was found in the clinical results.

\section{1}

\section{Evar: Correlations between Endograft's Radial Force, Proximal Neck Expansion and Migrations}

A. Griso, J. Ebner, L. Nicolai, M.E. Barzaghi, S.F. Ruffino, P. Benin, P. Candiani, A. Scuro

Vascular Surgery, University of Verona, Italy

Introduction: Correlations between endograft's radial force, proximal neck expansion and migrations.

Methods: With a sperimental model we calculated the radial force developed by different endografts at proximal aortic neck and correlated it with rate expansion and migrations; with a review of follow-up.

Results: With $20 \%$ oversize AneuRX develop a radial force of $160 \mathrm{~mm} \mathrm{Hg} / \mathrm{cm}^{2}$, Talent and Zenith $80 \mathrm{~mm} \mathrm{Hg}$, Excluder $70 \mathrm{~mm} \mathrm{Hg}$ and Endologix $40 \mathrm{~mm} \mathrm{Hg}$. We compared clinical results of AneuRX and Talent. Patients with minimum 12 months follow-up are 152; complete data are from 141 patients (60 Talent and 81 AneuRX). There aren't significant differences between the two groups about oversize applied (both $18.6 \%$ ) and middle diameter of preoperative proximal neck (24.4 Talent vs. 21.9 AneuRX). Proximal neck length is higher in patients with AneuRX (25.9 mm vs. $20 \mathrm{~mm}$ Talent). The radial force applied has been significantly higher with AneuRX than the one developed by Talent. There's a statistically significant correlation between migration $>10 \mathrm{~mm}(12.3 \%$ AneuRX vs. Talent 1.7$)$ and the radial force applied to aortic proximal neck.

Discussion: It's correct to correlate the evolution of proximal neck and the radial force applied (not with the oversize). The application of very high radial force on proximal neck could increase the incidence of complications during the follow-up.

192

Leiomyosarcoma of $\mathrm{VCl}$ and Venous Reconstruction by Palma's Crossover: A Case Report

A. Cotroneo, M.L. Spada, F. Mirenda, F. Benedetto, G. Roscitano, G. Passari, F. Spinelli

U.O.C. di Chirurgia Vascolare, Università degli Studi di Messina

Introduction: Leiomyosarcoma of the VCI is a rare retroperitoneum mesenchymal tumor. About 200 cases have been described in the literature without a long-term follow-up. Abdominal pain, no palpable abdominal mass and earlier diagnosis are associated with a better outcome. We performed 9 cases. Leiomyosarcoma is associated with venous occlusion. Venous reconstructions are rarely performed. Palma was the first to use a crossfemoral saphenous veno-venous by pass graft for unilateral iliac venous obstruction. Lalka reported an advantage for the use of ePTFE and an adjunctive AVF can improve patency.

Methods: We present a 69-year-old male with DVT of left leg assessed by ultrasonography. Abdomen-pelvis CT-scan showed a solid and irregural mass $(8 \times 7 \mathrm{~cm})$, at the left iliac confluence and in VCI. Cardiac hypertrophy, atrial fibrillation, mitralic insufficiency and NIDDM were concomitants pathologies. We performed a abdominal oblique laparotomy. A total resection of the tumor, the left iliac venous confluence and the left hypogastric artery was performed. The left ureter dislocated, was dissected and controlaterally anastomized. We treated the left leg edema after a week by a Palma's crossover with ePTFE and an AVF. Trombectomy of right femoral vein and placement of a cava filter has been necessary.

Results: No post-operative complications were founded. The histopathology confirmed a leiomyosarcoma. Adipous and linfatic tissue and ureter weren't infiltrated. The patient was discharged with chemiotherapy.

Discussion: Total resection is associated with a good result. Palma's crossover has been resolutive for the left leg edema. 


\section{3}

\section{Subintimal vs. Intraluminal Angioplasty in the Treatment of Critical Limb Ischemia (CLI)}

\author{
F. Benedetto, M.L. Spada, F. Mirenda, A. Cotroneo, \\ G. Roscitano, F. Spinelli \\ U.O.C. DI Chirurgia Vascolare, Università degli Studi di \\ Messina
}

Introduction: The authors compare the meaning time results between the different endovascular procedures (intraluminal and subintimal) in the treatment of CLI.

Material and Methods: From January 2004 to June 2005 we performed 81 limbs in 79 patients. The selected cases were 2 with severe claudication (Rutherford III), 34 with rest pain (Rutherford IV) and 43 with tissue loss (Rutherford V). Gangrene and long occlusion of tibials and peroneals arteries, showed by Doppler and B-mode ultrasounds, have been occluded for an endovascular treatment. The superficial femoral artery (17 cases) has been treated by intraluminal PTA in 10 cases and subintimal in 7 . In 35 occlusions, we performed 22 subintimal recanalizations and 13 intraluminal ( 6 stenting). In the popliteal-tibial pathology, we performed 38 intraluminal and 10 subintimal revascularization.

Results: Follow-up at 14.5 months showed 2 restenosis $(2.4 \%)$ of tibials arteries recanalized with subintimal technique and 7 occlusion $(8.6 \%)$ : 5 of SFA, treated with intraluminal recanalization and stenting and 2 peroneals occlusions after intraluminal recanalization.

Discussion: Subintimal angioplasty shows to be the best treatment of femoro-popliteal and tibials lesions (patency at 14.5 months $98 \%$ vs. $91 \%$ ) because reduces restenosis, acute occlusion and embolism.

\section{4}

\section{Thoracic Outlet Sindrome (TOS): A Case Report \\ G. Roscitano, A. Cotroneo, G. De Caridi, F. Benedetto, G. Passari, F. Spinelli \\ U.O.C. di Chirurgia Vascolare, Università degli Studi di Messina}

Introduction: The thoracic outlet syndrome (TOS) describes a spectrum of symptoms and signs which are all related to the passage of key anatomic structures through a narrow aperture on their way to the distal upper extremity. The form of TOS depends on the structure compressed: arterial, venous or nerve.

Material and Methods: We report a 9 years old female with pain, paresthesias and edema of right arm. After a positive Adson's test the MRN showed a venous compression of an hypertrophied pectoralis minor muscle. We choose to perform a transaxillary pectoralis minor muscle resection.

Results: The post-operative angiography confirmed the morphological result. We have noted a return to a normal state with absence of edema, pain and paresthesias.
Discussion: For estetic best results we performed the transaxillary access. Is very important to evaluate the correct cause of TOS to have a best clinical outcome.

195

\section{Two Cases of Hemispheric Reperfusion Syndrome after Carotid Artery Stenting Case Report and Review of the Literature}

\author{
G. Bianchi, D. Trevisan, S. Canale, R. Curci, A. Argenteri \\ Department of Vascular Surgery, Hospital of Lodi, Chair of \\ Vascular Surgery, University of Pavia, Italy
}

Introduction: Cerebral reperfusion syndrome after surgical or endovascular treatment of carotid stenosis is a rare, severe and unpredictable complication associated with a high mortality. Endocranial hypertension, due to cerebral oedema, is responsible of neurological symptoms such as headache, vomiting, and convulsions and can lead to cerebral haemorrhage ( $40 \%$ of the cases).

Material and Methods: We describe two cases of cerebral reperfusion syndrome after endovascular treatment for carotid re-stenosis. In both cases symptoms appeared at the end of the procedure as decreasing level of consciousness that lead patients to coma, complicated by seizure, hyperthermia and hypertension. In both cases a therapy based on anti-oedema and steroids drugs was able to reduce the cerebral oedema with an improvement of the neurological symptoms.

Discussion: Literature describes only 14 cases of cerebral reperfusion syndrome after carotid stenting, however the real incidence of this complication is uncertain because a confirmed definition of it does not exist yet and the few cases are usually published as 'Case Report'. The severe evolution of this acute disease urged the researchers to find predictive factors of this syndrome. Waiting for these results, rapid diagnosis and prompt treatment are the only two ways to reduce local and systemic complications of this disease.

\section{6 \\ Endovascular Treatment of Superior Mesenteric Artery Stenosis Case Report and Review of the Literature}

G. Bianchi, D. Trevisan, S. Canale, R. Curci, A. Argenteri

Department of Vascular Surgery, Hospital of Lodi, Chair of Vascular Surgery, University of Pavia, Italy

Introduction: Despite the new achievements in surgical and endovascular techniques, atherosclerotic disease of the superior mesenteric artery (SMA) remains a severe condition involving high mortality rate specially when associated to other lesions of the visceral vessels.

Material and Methods: From January 2000 to February 2006 we observed 15 patients with lesions to the SMA: 9 of them received surgical and 6 endovascular treatment. Surgical treatment has been reserved to those patients with acute occlusion or distal disease of the artery or that could not be submitted to endovascular treatment. 
Endovascular treatment has been performed in patients with proximal lesions of the SMA or couldn't face a surgical treatment because of their poor general condition. In five cases we performed SMA stenting and in one case a simple angioplasty. We observed two complications following endovascular procedures (both with stenting): in one case a dissection of the SMA distal to the treated segment and in the other case an intra-stent restenosis. Both of them have been subsequently treated surgically by means of aortic or iliac by-passes. In all patients we noticed an improvement of the symptoms and a rapid weight gain.

Discussion: Thanks to improvements in diagnosis and endovascular therapy, today we can offer patients with atherosclerotic lesions of the visceral vessels one more chance to face their disease with lower mortality and complications. Even if endovascular treatment fails after a few months, the improvement in general conditions allows the patient to undergo surgical intervention.

\section{7}

\section{Carotid Endarterectomy in Patients with Renal Insufficiency: Postoperative Complication Rate in Our Experience}

\author{
D. Trevisan, G. Bianchi, S. Canale, R. Curci, \\ A. Zolin, A. Argenteri \\ Department of Vascular Surgery, Hospital of Lodi, Chair of \\ Vascular Surgery, University of Pavia, Italy
}

Objective: Chronic Renal Insufficiency (CRI) is one of the major risk factors for cardiovascular disease, especially coronary and carotid atherosclerosis. The objective of this study is to show statistical correlation between postoperative complication rate after carotid surgery and CRI in 108 patients operated on at our Unit.

Methods: From March 2005 to February 2006, 108 consecutive cerebral revascularization procedures were performed on 102 patients (62 men, 60.1\%), 104 carotid endarterectomies (CEA) (96.3\%) and 4 stenting procedures $(3.7 \%)$. We divided the population into two groups: $\mathrm{A}=$ non-CRI; and $\mathrm{B}=\mathrm{CRI}$ (serum creatinine level $\geq 1.4 \mathrm{mg} / \mathrm{dl}$ ). Seven patients $(6.8 \%)$ had CRI; of these two were on haemodialysis. One patient $(14 \%)$ of group B was treated by carotid stenting. We observed the prevalence of postoperative complication rates in these two groups.

Results: A statistically significant difference was observed between the CRI group (B) and the control group (A) with respect to mortality: 0 deaths in group A vs. $1(14.2 \%)$ in group $B(p=0.001)$; ECG alteration: $23(24.2 \%)$ in group A vs. $4(p=0.04)$. No statistically significant difference was observed with respect to myocardial ischaemia $(p=0.056)$; major stroke $(p=0.71)$; TIAs $(p=0.20)$; wound haematoma $(\mathrm{p}=0.29)$; and appearance of headache on the first postoperative day $(\mathrm{p}=0.33)$.

Conclusions: Patients with CRI are at significantly greater risk of postoperative stroke and death. We advise CEA only for selected CRI patients with symptomatic carotid disease, acceptable surgical risk and good long-term life expectancy.

\section{8}

\section{Late Acute Gastrointestinal Bleeding after Infrarenal Aortic Grafting: A 16 Year Experience Review}

\section{P. Bianchi, I. Dalainas, S. Stegher, D. Dell'aglio, R. Casana, G. Nano, G. Malacrida, D.G. Tealdi}

University of Milan, Operative Unit of Vascular Surgery, Policlinico San Donato, San Donato Milanese, Milano, Italy

Introduction: This single-institution study reviews presentation and management of acute gastrointestinal bleeding related to secondary Aorto Enteric Fistula (AEF). This late complication of aortic grafting may present as communication between bowel and prosthesis or bowel's decubitus erosion on the graft (paraprosthetic AEF).

Methods: Between 1991 and 2006, 9 patients were treated in emergency for secondary AEF localized in duodenum $(78 \%)$ and ileum (11\%), with hematemesis/melena, and in sigmoid (11\%), with proctorrhage. Two were real AEF (22\%) while 7 were paraprosthetic AEF (78\%). Graft infection was present in 4 cases (45\%), while four (45\%) were proximal sterile pseudoaneurysms causing FAE. Surgical management included: graft removal with $(n=5)$ or without simultaneous extraanatomic bypass $(\mathrm{n}=1)$; 'in situ' reconstruction with Dacron graft interposition $(\mathrm{n}=3)$; ileo-duodenorrhaphy $(\mathrm{n}=8)$; sigmoidectomy with colostomy $(\mathrm{n}=1)$; secondary partial ileectomy $(n=1)$. Endografting $(n=2)$ was only a temporary measure to control bleeding.

Results: In-hospital mortality was $55 \%$. No deaths were observed intra-operatively, while 5 occurred during early postoperative period for major complications. Early postoperative major and minor morbidities were observed in all survived patients, causing prolonged ICU-stay and hospitalization.

Discussion: Secondary AEF is life-threatening, difficult to treat and associated with significant morbidity and mortality, particularly if associated with sepsis, hemodynamic instability and bowel resection.

\section{9}

\section{A Misdiagnosed and Badly Managed Acute Type B Aortic Dissection: Acute and Subacute Complications and Successful Endovascular Stent-Graft Repair}

\section{Bona, M. Fusari \\ Policlinico di Monza}

Introduction: Acute Stanford type B aortic dissection complicated with limb, visceral and renal ischemia is a clinical dilemma since surgical intervention carries high risk of mortality and morbidity. As catheterization techniques advanced over the past decade, endovascular treatment provides a less invasive alternative for management of such cases.

Methods: A misdiagnosed case of acute type B aortic dissection complicated with limb, visceral and renal ischemia was referred to our Hospital after $10 \mathrm{~h}$ of anuria. At arrival, the 40 -year-old man had severe hip pain radiating to the back while blood pressure was 240/120 mm Hg. Cardiothoracic surgeons deemed surgery a not 
survivable option because of organ damage; therefore, endovascular stent-graft repair was performed with stabilization of the dissection, right renal function rescue and perfusion of all major visceral branches and both common iliacs. Neither antihypertensive therapy nor left renal artery stenting were helpful to control hypertension.

Results: After emptying of a iatrogenic subdural hematoma complicating subacute course, the patient was discharged in XIX post-procedural day with good clinical conditions at three months follow-up.

Discussion: Complicated acute Stanford type B aortic dissection is a borderland pathology, which cardiologists, aneshetists, cardio-thoracic and vascular surgeons have to deal with Nevertheless the treatment of choice - surgical or endovascular - need a ready, comprehensive and correct clinical aspects interpretation: it can be safely managed only by a multidisciplinary, strongly integrated team.

\section{0 \\ Emergency Endovascular Treatment of Acute Type B Dissection and Aberrant Right Subclavian Artery with Kommerell's Diverticulum}

\author{
A. Bozzani, S. Pirrelli, V. Arici, P.N. Palmieri, A. Odero \\ Divisione di Chirurgia Vascolare, IRCCS Policlinico San \\ Matteo, Pavia
}

Introduction: The reported prevalence of aberrant subclavian artery is $0.4-2 \%$; association with Kommerell's diverticulum and secondary type B acute aortic dissection is extremely rare. For resistant chest pain and high mortality rate for surgical treatment of 'lusoria' artery aneurysms, endovascular treatment is mandatory in this patient.

Material and Methods: A 59-year-old men presented with chest pain and severe hypertension. Spiral CT demonstrated acute type B dissection associated an aberrant right subclavian artery with Kommerell's diverticlum $(3.5 \mathrm{~cm}$ of diameter). Previous 2 weeks hypothensive therapy, a Zenith Cook stent graft was placed, under general anesthesia, across the right subclavian orifice with successful exclusion of the aneurysm.

Results: Postoperatively the patient presented moderate persistent interscapular pain for a week and right arm easily fatigable with a weak radial pulse, but revascularization is not necessary. No paraplegia or central neurological defects was observed. Postoperative spiral CT demonstrated complete exclusion of aneurysm with flow in the distal subclavia via vertebral artery, and dissection false lumen thrombosis.

Conclusion: The descending thoracic aortic emergency open surgery is still subject to high mortality rate; endovascular technique represents, nowadays, a promising strategy for thoracic aorta acute syndrome treatment.

\section{1}

\section{Emergency Treatment of Descending Thoracic Aortic Aneurysms and Dissections}

\author{
A. Bozzani, S. Pirrelli, V. Arici, R. Corbetta, A. Odero \\ Divisione di Chirurgia Vascolare, IRCCS Policlinico San \\ Matteo, Pavia
}

Introduction: Many different diseases are grouped in the socalled Acute Aortic Syndrome. Recent works present an overall mortality in elective repair of $4.4 \%$. Those rates increase widely when urgent repairs are considered $(25 \%)$.

Material and Methods: Between 1991 and 2005, our case histories count 244 patients treated for thoracic aorta pathology. One third of the thoracic lesions involved the descending aorta ( 93 patients with DTAA). 73 (78\%) received elective repair, $20(22 \%)$, on the other hand, needed urgent repair. In 6 of the patients who underwent urgent repair $(30 \%)$, endovascular technique was used. In the same period, we observed 39 cases of acute type B aortic dissections. 32 of which were treated with medical therapy, and only 7 cases underwent urgent repair ( 3 with endovascular technique).

Results: Overall mortality and paraplegia rate during urgent repair of DTAA has been $40 \%$ and $14 \%$ respectively, superimposable to international case histories. Otherwise, endovascular technique had only one case of death out of 6 patients treated. Four out of 39 patients with acute type B dissection deceased during their stay in hospital. Paraplegia didn't show in our series of patients.

Conclusions: The descending thoracic aortic emergency open surgery is still subject to high mortality rate, while this rate, using endovascolar procedures, is meaningfully lower.

\section{2}

\section{Distal Bypass with Varicose Vein Covered by Prosthetic Mesh}

\author{
G. Carella, F. Stilo, A. Dattola, M.L. Spada, F. Spinelli
}

A 38 years old man was submitted, in another hospital, a popliteal tibioperoneal trunk bypass for treatment of popliteal arterial aneurysm. Risk factors: several trombophlebites and factor V Leiden mutation. A controlateral saphenous vein was used because the homolateral vein was varicose. In May 2005 the patient was referred us with critical limb ischemia. Angiography revealed a venous bypass ectasia and a pseudoaneurysm of distal anastomosis with occlusion of proximal tibial arteries and low flow at distal peroneal and tibial arteries due to previous embolization. We performed a bypass with homolateral varicose saphenous vein covered by prosthetical material (ProVena) from supragenicular popliteal artery to the supramalleolar anterior tibial artery. Post-operative outcome was useful. The follow-up performed at 1, 3 and 6 months with EcoColorDoppler didn't revealed hemodynamics alteration of bypass or medial and intimal thickening. After 3 months control aspect of same areas of neoangiogenesis had been observed through the mesh. In this young patient with thrombophilic risk factors, occlusion of tibial vessels and low-flow in pedal vessels, we used autologous saphenous vein even if it was varicose. The availability of a prosthetic external mesh gave us the possibility to 
avoid a synthetic graft. The occasional finding of neoangiogenesis through the mesh was a clue of fair tolerability.

\section{3}

\section{Angioplasty of Peroneal Lesions for Critical Limb Ischemia. Clinical Result a Prospective Single-Center Study}

\author{
T. Ceccacci, F. Maioli, G. Testi, A. Freyrie, M. Gargiulo, \\ A. Stella \\ Chirurgia Vascolare, Policlinico S. Orsola, Bologna
}

Introduction: In this prospective study we evaluated the role of angioplasty of peroneal TASC A-C lesions in patients with critical limb ischemia (CLI) and TASC D lesions of anterior (ATA) and posterior (PTA) tibial arteries.

Methods: We enrolled patients from October 2000 to March 2006, recording clinical limb's data (Wagner's and Texas' classification (TUC)), peroneal artery's runoff (I. Patency of anterior and posterior perforans; II. Patency of posterior perforan; III. Patency of anterior perforan; IV. Obstruction of both perforans), technical success, limb salvage and patients' survival at 1, 3, 6, 12, 18 months.

Results: 158 limbs underwent endovascular treatment; 58 arose peroneal TASC A-C lesions and ATA and PTA TASC D lesions. Among 49 limbs recruited, 48 there was a trophic lesion: 39 (79.6\%) Wagner 4-5.12 (24.5\%) TUC IIID. About peroneal runoff: type I was recorded in 13 cases (26.5\%), type II in 13 (26.5\%), type III in 14 (28.6\%), type IV in $9(18.4 \%)$. In all cases we reported a technical success. Limb salvage was $93.8 \%, 81.7 \%$ after 1 and $6,12,18$ months, respectively; patient survival was $98 \%$ (1 month), $98,88.2$ and $79.4 \%$ respectively. The variable significantly correlable to the limb amputation was trophic lesion TUC IIID $(p<0.05)$ in spite of the technical success.

Conclusion: Peroneal angioplasty is the first choice treatment in CLI, in patients with peroneal lesions TASC A-C and ATA and PTA lesions TASC D. In patients with trophic lesion TUC IIID this treatment does not guarantee limb salvage.

\section{4}

\section{Vascular Traumas}

\section{Cecere, P. Valitutti}

\section{Chirurgia Vascolare, Casa di Cura Calzoni, Agropoli}

Introduction: Actually, road accidents and job accidents represent the most frequent causes of the vascular traumas, not so frequent are the sporting accidents and the iatrogenic lesions.

Methods: Since July 1999 - January 2005, 60 patients traumatized with vascular lesions: 18 to the superior limbs (30\%), 28 to the inferior limbs (46.6\%), 7 to the abdomen (11.6\%) (1 abdominal aorta, 1 external iliac artery, 1 inferior cava vein, 2 hypogastric artery, 2 gluteal artery), 1 to the chest (1.6\%) (thoracic aorta), 6 to the neck (10\%) (2 int. carotid artery, 1 thyroid artery, 2 int. jugular vein. 1 subclavian artery). $40(66.6 \%)$ patients were of male sex and $20(33.3 \%)$ of female sex
(13-70 years). The typology of the vascular damage was: arterial thrombosis in 17 patients $(28.3 \%)$, complete arterial lesion in 21 patients $(35 \%)$, incomplete arterial lesion in 11 patients $(18.3 \%)$, venous lesion in 5 patients $(8.3 \%)$, decay of limb in 4 patients $(6.6 \%)$, FAV in 1 patients $(1.6 \%)$, pseudoaneurysm in 1 patients $(1.6 \%)$. Clinically the patients presented: 26 had a serious hemorrhage (43.3\%), 25 had a serious ischemia (41.6\%), 1 had an aching pseudoaneurysm $(1.6 \%), 11$ were affected by associated nervous lesions $(18.3 \%), 23$ were affected by associated arterial and venous lesions (38.3\%), 15 had serious muscular lesions (25\%), 17 had bony lesions (28.3\%).

Results: The surgical interventions have been: 10 arterial ligatures of which 4 for primary amputation, 2 venous ligatures, 5 direct arterial reparations, 9 resection-anastomosys T-T, 28 grafts or bypass in vein, 5 grafts or bypass in prosthesis, 1 embolization. In the follow-up (43 patients): the complete success in 34 patients $(79 \%)$, a partial success in 4 patients $(9.3 \%)$, serious sequences in 2 patients $(4.65 \%)$ and the amputation in 3 patients $(6.97 \%)$. Total mortality in 2 patients $(3.38 \%)$.

Discussion: The treatment of the vascular traumas depends either on the topography, or on the typology of (truncated at 250 words).

\section{5 \\ Treatment Strategy for Patients with Concomitant Abdominal Aortic Aneurysm and Diseases of Surgical Interest}

\author{
E. Cervi, S. Bonardelli, F. Viotti, M. Milesi, F. Nodari, \\ M. De Lucia, S.M. Giulini \\ Chirurgia Generale, Ospedali Civili di Brescia, Brescia
}

Introduction: The observation of patients affected by concomitant abdominal aortic aneurysm (AAA) and several abdominal pathologies of surgical interest is becoming common. The choice of correct therapeutic planes is still controversial for the risk of vascular prothesic graft infection after associated operations.

Method: Between January 1992 and December 2005, 1,545 patients (1,375 in election, 170 in emergency) underwent standard abdominal aortic repair for AAA. Concomitant abdominal pathologies of surgical interest were divided into three groups, according to their septic risk: aseptic (abdominal wall and renal diseases), potentially septic (cholelithiasis) and septic (peritonitis). 315 of 1,545 (20.4\%) patients presented concomitant abdominal pathologies. 152 of $315(48.3 \%)$ patients (136 in election, 16 in emergency) were treated with simultaneous surgical treatment.

Results: We treated 76 patients with no septic risk, 75 of potential septic risk and 1 with septic risk. There is no statistical difference in 30-day morbidity and mortality between patients belonging to onestage and two-stage procedures. During follow-up period of 6-140 months no graft infection were detected.

Conclusion: One-stage approach is indicated in pathologies with no septic risk and with potentially septic risk, but attention must be given to technical details and to rules of antisepsis. 


\section{6}

\section{Carotid Artery Stenting in Octogenarians: Our Experience}

\author{
E. Chisci, G. de Donato, P. Sirignano, M. Giubbolini, \\ C. Setacci
}

Chirurgia Vascolare ed Endovascolare, Universita' Di Siena

Introduction: The aim of this study was to evaluate whether, in our experience, Carotid Artery Stenting (CAS) performed in octogenarians really increases the procedure-related risk. Our secondary aim was to assess the incidence of complex anatomy of the aortic arch and supra-aortic vessels (cSVA) in patients $>80$ years old, which can increase the technical difficulty of CAS.

Methods: 812 CAS procedures were performed in our center. Patients were separated into 2 age categories: $<80(\mathrm{n}=718,88.4 \%)$ and $>80(n=94,11.6 \%)$. Death and stroke rate at discharge and at 30 days were analyzed.

Results: Three lesions in octogenarians could not be treated because of failure to access the vessel in one case and extremely tortuous arteries in two cases. The overall death and stroke rate at 30 days was $2.12 \%$ in the older group ( 1 fatal strokes, 1 minor stroke) and $1.11 \%$ in the younger group ( 2 deaths, 2 major strokes, 4 minor strokes; $p=40)$. A significantly high frequency of aortic arch type III and cSVA was observed in the older group $(p<0.001)$.

Discussion: In our experience CAS has proved to be safe and effective in elderly patients. Different age-related anatomical features can represent an adjunctive technical challenge, and these difficulties can be successfully managed without increased peri-operative risk if CAS is performed in high volume centers by highly skilled operators.

\section{7 \\ Is Color Duplex Us the Gold Standard for Peripheral Obstructive Arterial Disease?}

\author{
E. Chisci, G. de Donato, P. Sirignano, M. Giubbolini, \\ C. Setacci \\ Chirurgia Vascolare ed Endovascolare, Universita' Di Siena
}

Introduction: The aim of our study has been to estimate the reliability and the accuracy of color duplex US (dUS) for the diagnosis and the preoperative assessment of POD.

Methods: From 01.01.04 to 28.2.06, in our Unit of Vascular and Endovascular Surgery, were performed 1,674 dUS of the lower limb arteries. Were characterizes 476 cases of CLI. In all cases an intraprocedural angiography was performed, in order to compare it with the color duplex US report; primary endovascular revascularization was attempted in $88.7 \%$ and when it was not satisfactory we complete revascularization with open surgery.

Results: We organized our results in two groups: the femoropopliteal group and the infrapopliteal group. dUS revealed a sensibility of $97.2 \%$ and a specificity of $98 \%$ for the first group, and a sensibility of $73 \%$ and a specificity of $92 \%$ for the second one. Discussion in our experience, the dUS turns out the gold-standard for arteriosclerotic disease of the femoro-popliteal axis. The necessity to resort to a second level's examination has been dictated from the dif- ficulty to obtain a precise mapping of the lesions given the frequent presence of extended arterial wall calcifications, multiple stenoses, extended occlusions, oedema, extensive ulcers, or a very poor run-off. The angiography maintains its indication, exclusively like complementary examination, in the infrapopliteal axis disease.

208

Motor-Hoe popliteal Artery Trauma. Clinical Presentation and Surgical Treatment

\author{
G.L. Barbera, G.L. Marca, D. Di Lella, A. Martino \\ Department of Vascular Surgery, G. Di Cristina Civico \\ Hospital, Palermo
}

Introduction: The incidence of Inferior legs vascular trauma (ILVT) is $1-3 \%$ of all trauma, with an amputation rate ranging between $3.2 \%$ and $18 \%$. Popliteal Artery (PA) is affected in $10-25 \%$ of ILVT and is burdened with an amputation rate up to $50 \%$.

Methods: From January 1980 to December 2005, 17 male patients were treated because a PA trauma, in 17 limbs. The mean age was 40.3 (range: 28-69) (DS: 15.4). Thirteen patients (76\%) underwent popliteal graft, 2 patients were treated by end-to-end anastomosis, 1 patients $(6 \%)$ had a thrombectomy and in 1 patients $(6 \%)$ it was performed a fasciotomy.

Results: Postoperative patency rate was $82 \%$ burdened with $53 \%$ amputation rate. Matching results with clinical findings, we worked out that postoperative patency, in patients with $<10 \mathrm{~h}$ ischemia lag was 11 patients $(85 \%)$ vs. 2 patients $(50 \%)(\mathrm{p}<0.005)$, in patients younger than 40 years it was 8 patients $(89 \%)$ vs. 5 patients $(63 \%)(\mathrm{p}<0.005)$, and in patients without soft tissue infection it was 10 patients $(83 \%)$ vs. 3 patients $(60 \%)(p=n s)$. Limb salvage in patients with $<10 \mathrm{~h}$ ischemia lag was 10 patients $(77 \%)$ vs. 0 patient $(0 \%)(\mathrm{p}<0.005)$, in patients younger than 40 years we had 6 patients $(67 \%)$ vs. 4 patients $(50 \%)(\mathrm{p}<0.05)$, and patients without tissue infection it was 10 patients $(77 \%)$ vs. 1 patient $(25 \%)(p=n s)$.

Discussion: Our experience shows that $<10 \mathrm{~h}$ ischemic lag and $<40$ years age significantly increase postoperative patency and limb salvage rates. Because ILVT are usually (truncated at 250 words).

209

Lower Limb Revascularization with a New Bioactive Prosthetic Graft: Early and Mid-term Results

F. Di Carlo, G. Pratesi, W. Dorigo, M. Di Vito Francesco, A.A. Innocenti, R. Pulli, C. Pratesi

Department of Vascular Surgery, University of Florence, Florence, Italy

Aim: To retrospectively evaluate the results of femoro-popliteal bypasses performed with a new bioactive heparin-treated ePTFE graft in a single-centre experience.

Methods: From March 2002 to April 2006 we used in 49 patients undergoing lower limb revascularization a new bioactive 
prosthetic graft which consists of an ePTFE prosthetic graft with covalent end-point attachment of heparin to graft surface. Early and mid term results were assessed.

Results: Indications for surgical revascularization were critical limb ischemia in 35 patients and intermittent claudication in 14 patients. Interventions were performed for occlusion of native vessels in 35 cases and of a prosthetic graft in 10 patients; 4 patients were operated on for an occluded popliteal artery aneurysm. Intervention consisted of below-knee bypass in 32 patients; the remaining 17 had an above-knee revascularization. No perioperative deaths occurred. Cumulative 30-day graft patency was $90 \%$, with an amputation rate of $4 \%$ ( 2 cases); univariate analysis showed reintervention to be associated with 30-day graft failure. Mean duration of follow-up was 12 months. Cumulative estimated 14 months survival and primary patency rates were $97 \%$ and $85.9 \%$, respectively; corresponding limb salvage rate was $90 \%$. No influence on primary patency and limb salvage rates was found for reinterventions, level of revascularization and preoperative clinical status.

Conclusions: The use of a modified ePTFE graft with covalent end-point linkage of heparin molecules on graft surface provides good early and mid-term rates, with low rates of graft thrombosis and amputation.

\section{0 \\ Anaesthesiological and Surgical Strategy to Improve Intraoperative Care of Patients Undergoing Carotid Endarterectomy}

\section{A. Fargion, E. Barbanti, L. Azas, S. Matticari, W. Dorigo, N. Troisi, G. Pratesi, R. Pulli, C. Pratesi \\ Department of Vascular Surgery, University of Florence, Florence, Italy}

Objective: To evaluate the safety and effectiveness of a modified surgical and anaesthesiological protocol in improving the results of carotid endarterectomy.

Methods: From 1996 and 2005, 3,153 consecutive CEAs were performed. Surgical and anaesthesiological strategy changed during the years: until April 1999, all the interventions were performed under general anaesthesia, with SEPs monitoring and selective shunt insertion (group 1; 1,090 interventions). From May 1999 to December 2003, surgical approach was modified, with preliminary isolation and clamping of distal ICA, with a policy of wide use of patch (group 2; 1,474 interventions). Since January 2004, we have been using local anaesthesia with the modified surgical technique (group 3; 589 interventions). Early and long term results were compared.

Results: Neurological deficits at awakening were lower in group $3(0.1 \%)$ than in group 1 and $2(1.8$ and $0.4 \%$, respectively; $\mathrm{p}<0.001)$. Thirty-day stroke and death rate was lower in group 3 $(0.6 \%)$ than in group 1 and 2 (1.5 and $1.3 \%$, respectively). Mean duration follow-up was 23 months (SD 22.3). Estimated 24-months survival rates was significantly better in group $3(99.7 \%)$ and $2(98.3 \%)$ than in group 1 (95.65; $p=0.005, \log$ rank 15.2). There were no differences between the three groups in terms of estimated 24 months absence of any neurological event and of ipsilateral stroke.

Conclusions: The adoption of a multifactorial intraoperative prophylaxis of early postoperative events allowed, in our experience, to significantly improve early results of CEA, with a fall of complications rate largely below recommended standards.

\section{1 \\ Factors Affecting Outcomes of Lower Limb Revascularization in a Single Centre Experience}

N. Troisi, S. Bellandi, S. Massini, O. Mikhalyaeva, M. Di Mare, E. Chiti, G. Credi, C. Pratesi

Department of Vascular Surgery, University of Florence, Florence, Italy

Objectives: To analyze our results in surgical revascularization of lower limbs, with particular attention to the factors affecting early and late results.

Methods: From 2000 to 2004, 322 consecutive surgical lower limb revascularizations were performed. Data concerning these interventions were prospectively collected in a dedicated database. Early and late results were analyzed in terms of graft patency, limb salvage and survival. Univariate and multivariate analysis for the risk of early and late graft thrombosis were performed.

Results: Main indication for surgery was the presence of critical limb ischemia; nearly $45 \%$ of the patients had a below-knee femoropopliteal bypass. Thirty-day mortality and graft thrombosis rate were 1.5 and $17 \%$, respectively. Univariate analysis showed chronic renal failure, acute ischemia, urgent intervention, below-knee distal anastomosis, reintervention and the presence of large foot ulcers to significantly increase 30-day thrombosis; the last three features were confirmed also at multivariate analysis. Mean duration of follow-up was 20 months; 24-month graft patency and limb salvage rates were 68 and $84 \%$, respectively. Cox regression analysis showed the presence of large foot ulcers and the need for reintervention to significantly affect long-term graft patency.

Conclusions: Results of lower limb revascularization in our experience seem to be significantly affected by the clinical status of the limb and by the need for reintervention. These results should be carefully considered in planning surgical intervention, particularly when a redo surgery is needed.

212

\section{Endovascular Manage of Carotid Dissection during Carotid Stenting Using Cerebral Protection Filter Device}

A. Fontana, B. Marco, R. Moratto, G. Coppi

Department of Vascular and Endovascular Surgery, University of Modena and Reggio Emilia, Modena, Italy

Introduction: We report a case of complicated carotid stenting procedure with distal filter cerebral protection.

Methods: A patient underwent carotid artery stenting for a highgrade stenosis of the left internal carotid using a filter device never 
used before in own equipe. After crossing the thigh stenosis, internal carotid artery's dissection occurred with a complete flow blockage. There were no possibilities to carry on the guide without increasing the dissection. We rapidly changed the protection device with the occlusion Moma device, reversing flow and viewed of lumen in slowly angiographic control. So we proceeded to rapid stent deployment across the carotid lesion and ballon angioplasty of stenosis.

Results: We successfully concluded the endovascular procedure with a good result at intraprocedural angiographic control and without any clinic and instrumental neurologic sequelae. The ultrasound's control at 6 months demonstrated a good result without dissection signs.

Discussion: This case demonstrate that the use of protection filter can occur in complication like acute internal carotid dissection. This complication can be managed successfully with rapid application of carotid reversal flow device allowing to end endovascular procedure without emergent surgical intervention. The vascular surgeon could manage much more tools as possible to resolve with lowest damage the possible complication of endovascular procedures.

\section{3 \\ Endoscopic Perforating Vein Surgery in Chronic Venous Insufficiency: Our Experience \\ F. Stio, G. Guerriero, R.E. Stio, C. Chiesa, E. Masci, M. Marin, A. Pulcini \\ Dipartimento di Scienze Chirurgiche, Policlinico Umberto I, Universita' degli studi di Roma 'La Sapienza'}

Introduction: In the Western world, Chronic Venous Insufficiency (CVI) represents a serious economical and social problem both for the patients and for the health care system. The usual treatment includes phlebectomy and venous stripping. The patients in the advanced stages of the pathology (lipodermatosclerosis, ulcers) are very often affected by incompetence of the perforating veins. In these cases, the outcome of the open surgery for the ligature of the calf perforating veins (e.g. Linton and Felder Operation) is often worsened by serious complications of the wound. For this reason, the less invasive endoscopic ligature of the perforating veins has gained more and more ground.

Methods: From May 1996 to June 2005 our group performed a total of 500 Subfascial Endoscopic Perforating Vein Surgery (SEPS) procedures on 420 patients with CVI. The patients were classified according to CEAP classification: $41 \mathrm{C} 3$ (9.7\%), $187 \mathrm{C} 4$ (44.5\%), 90 C5 (21.5\%), 102 C6 (24.3\%). Before surgery, patients underwent the echo-color Doppler exam to localize the incontinent perforating veins.

Results: A total of 1,380 veins were located and dissected without serious complications (death or pulmonary embolism), nor was there significant morbidity. At the follow-up (6-120 months) all patients presented healing of the ulcers and resolution of the symptomatology.

Discussion: Our study has shown that SEPS can be effective in the treatment of patients affected by CVI and incontinence of the perforating veins. Therefore, these observations suggest that SEPS can be considered the preferred treatment for these patients.

\section{4}

\section{Radioguided Surgery of Chemodectoma at the Neck}

\section{A. Laurito, R. Massa1, F. Faccenna, R. Gabrielli, F. Benedetti-Valentini}

Chair of Vascular Surgery, 'Service of Nuclear Medicine, La Sapienza University, Rome, Italy

Aim of this perspective study was to assess whether ${ }^{111}$ In-pentetreotide scintigraphy (SRS) and subsequent intraoperative radiolocalization improve surgical radical excision of chemodectomas $(\mathrm{CHs})$ at the neck, reducing the risk of recurrence.

Eight patients with $12 \mathrm{CHs}$ and one with a neurinoma $(\mathrm{N})$ of the vagus nerve, all at the cervical location were investigated by SRS and SPECT. Planar images were obtained at 4 and $24 \mathrm{~h}$ post injection; SPECT was carried out $4 \mathrm{~h}$ post injection. Findings were compared with the results of duplex sonography, magnetic resonance imaging, CT-scan and angiography. Twelve CHs and $1 \mathrm{~N}$ were operated on and intraoperative $\gamma$ counting was carried out by a handheld probe before and after tumor excision.

Preoperative SRS showed high radiotracer uptake in all CHs but not in the $\mathrm{N}$ and correspondingly high $\gamma$ counting was recorded intraoperatively in all CHs but not in the N. All tumors were removed and confirmed by histology; two large lymphnodes with low counting were tumor free.

No false positive or false negative results was found. In $2 \mathrm{CHs} \gamma$ probe studies detected small leftovers, one at the carotid bifurcation was removed, the other one adhering to the vertebral artery at the base of the skull was left in place. No recurrence was recorded in a follow up from 5 months to 8 years.

Radioguided surgery improved surgical excision of $\mathrm{CHs}$ at the neck and contributed to differential diagnosis with tumors of different nature.

\section{5}

\section{Can 16-Slices CT Angiography be Used as a Basis for Planning Treatment of Critical Limb Ischemia in Diabetic Patients?}

\author{
F. Maioli, G. Testi, T. Ceccacci, A. Casadei, F. Giovanetti, \\ M. Gargiulo, G.L. Faggioli, A. Stella
}

Chirurgia Vascolare Policlinico S.Orsola Malpighi, Bologna

Introduction: Digital subtraction angiography (DSA) and sonography (US) are gold standards in diagnostic due to ankle and foot revascularization in diabetic patients (DP) with critical limb ischemia (CLI). Non invasive alternatives for accurate depiction of tibial and foot arteries are desirable.

Methods: We compare 16-slices CT angiography (CTA) with DSA in evaluating tibial and foot arteries disease in DP with CLI. A prospective study on 22 limbs (20 DP with CLI) that underwent CTA and DSA before revascularization. We divided limbs into 23 arterial segments. Arteries scanned by CTA and DSA were graded separately for stenosis. CTA sensitivity, specificity and accuracy in detection of stenosis $>70 \%$ (SL) were compared to DSA. 
Results: CTA and DSA scanning produced 1,012 arterial segments for evaluation. DSA showed 231 segments with SL. Sensitivity of CT for SL was $77.2 \%$ for the calf arteries and $73.5 \%$ for foot arteries; corresponding specificity and accuracy were $65,62.3$ and 71.8 , $68.2 \%$ respectively. The accuracy of CTA in SL detection of tibial and foot arteries was $72.7 \%$ anterior tibial, $77.3 \%$ tibio-peroneal trunk, $78.8 \%$ posterior tibial, $62.1 \%$ peroneal, $69.7 \%$ plantars, $54.5 \%$ dorsalis pedis and $77.3 \%$ plantar arch.

Discussion: CTA can replace DSA in most DP with CLI needing above knee revascularization. Patients predicted by CTA to peroneal artery or foot arteries treatment should undergo DSA or US to confirm.

\section{6}

\section{Contrast-Enhanced Ultrasonography with Second-Generation Agent in Detection of Type II Endoleaks after Abdominal Aortic Endografting}

G. Testi, R. Mauro, F. Maioli, T. Ceccacci, M. Gargiulo, A. Freyrie, A. Stella

Chirurgia Vascolare Policlinico S.Orsola Malpighi, Bologna, Italy

Introduction: Contrast-Enhanced Ultrasonography (CEUS) represent a new tool in endoleak detection after endovascular abdominal aortic aneurysm repair (EVAR). The diagnostic value of CEUS with a second-generation contrast agent (SonoVue?, Bracco, Italy) was prospectively compared with Color-Doppler Ultrasonography (CDU) and Computed Tomographic Angiography (CTA).

Methods: Between January 2005 and March 2006 were performed $68 \mathrm{CDU}$, CEUS and CTA examinations in 46 patients (43 men, 3 women), mean age 73 years, who underwent EVAR. The exams were done in the same day by three different operators that were blinded to the results of any other studies.

Results: No adverse reaction to contrast agent was observed. CTA, CDU and CEUS were in agreement for presence of a type II endoleak in 20 cases, and for absence in 39 cases. In 6 cases CEUS and CTA detected an endoleak despite CDU negativity. CEUS permitted to identify an endoleak not visualized with CTA in 3 cases. Sensibility of CEUS and CDU vs. CTA is respectively of 100 and $76 \%(\mathrm{p}<0.01)$. Moreover CEUS permitted in 16 patients to identify inflow and outflow vessels and in 9 patients to identify only inflow.

Discussion: In our experience CEUS showed a better sensibility in the diagnosis of type II endoleaks regards CDU. If this data will be confirmed by further studies, CEUS can support CTA in the follow-up of patients after EVAR.
217

\section{Role of Interactive Home Telehealth in the Planning of Carotid Endarterectomy in One Day Surgery}

N. Rousas, S. Mambrini, D. Mugnai, A. Robaldo, D. Palombo

Ospedale Mauriziano 'Umberto I' Torino

Introduction: To evaluate an experimental protocol aimed at early safety and protected discharge on the first day (One Day Surgery) after Carotid Endoarterectomy (CEA).

Methods: From October 2005 we selected 26 patients, with internal carotid stenosis $\geq 70 \%$, with a culturally adequate background. All patients underwent CEA; they were discharged on the first postop. day according to a system of 'Interactive Home Telehealth' (IHT). A bag containing an electronic artery pressure meter, an UMTS technology videophone and a questionnaire was given them. We monitored the surgical cut, arterial pressure, heart beat and the general conditions of the patients through a PC, linked to the Web, and a satellite video communication program. A collaboration has been reached with the emergency service so that an immediate action by the surgeon is possible.

Results: The image quality of the videophone is so good as to allow a correct judgement by the surgeon. A sense of insecurity emerged from the questionnaire because of the early discharge. This insecurity decreased after the first video connection and general satisfaction coming from the immediate return to the family. There were no surgical emergency; 3 patients had hypertension.

Discussion: CEA may be performed, in selected cases, as One Day Surgery. Our results suggested that the IHT protocol is a valuable and safety tool for decreasing the hospitalisation period.

\section{8}

\section{Role of EVAR in Acute Type B Aortic Dissection}

\section{A. Robaldo, S. Mambrini, D. Mugnai, N. Rousas, R. Mazzei, D. Palombo \\ Ospedale Mauriziano 'Umberto I' Torino}

Introduction: We report our experience of endovascular repair (ER) of acute type B aortic dissection (AtB-AD) and we evaluate feasibility, safety, and outcome of ER.

Methods: From 2005 to 20063 patients (mean age 72) with AtB-AD were treated with ER. Indications for treatment were: persistent severe pain in 1 patient and incipient aortic rupture in 2 . All cases were studied with CT-scan. Dissection goes from the left subclavian artery and extends to abdominal aorta in 1 case and to common iliac arteries in the others. Zenith endograft was placed in 1 patient. Valiant tube grafts were used in the remaining. True lumen access was obtained from a left brachial and femoral approach in all cases. Clinical status of each patient was monitored and CT-scan was performed postoperatively and at 3, 6, and 12 months follow-up (mean 6 months; range 3-12).

Results: ER with sealing of the primary tear was always successful with coverage of the left subclavian artery in 2 cases. Periprocedural complications didn't occur. All patients demonstrated 
complete resolution. No leakage, migration, neurological deficit or perfusion impairment was observed. Mean length of stay was 9 days after ER.

Discussion: Our experience suggests that ER of AtB-AD is a safe and feasible treatment option in urgency/emergency situations. However, prospective studies are needed to better define the longterm outcomes.

219

Preservation of Cholonic Circulation during EVAR for Aortoiliac Aneurysms in Patients with Previous Cholectomy

S. Mambrini, A. Robaldo, D. Mugnai, N. Rousas, R. Mazzei, D. Palombo

Ospedale Mauriziano 'Umberto I’ Torino

Introduction: Right emicholectomy (RE) or sigmoidectomy with rectal preservation (SwRP) affects colonic circulation by loss of pericolonic anastomosis and can induce intestinal ischemia during aortoiliac aneurysm (A) repair, if hypogastric artery (hya) are pathologic. We report our experience with EVAR.

Methods: We treated 3 patients with previous colonic resection for neoplasm. Patients 1 had a left common iliac (CI) A with a bilateral hyAs; patients 2 had an aortoiliac A involving the iliac bifurcations. Patients underwent previous RE. Patients 3 had a bilateral CIAs, involving the right hy with left hy stenosis. He underwent previous SwRP. All patients had multiple risk factors.

Results: Aortouniliac endoprosthesis was successful in all patients and required hy embolisation. Talent stent-grafts were used in 2 patients and a Zenith-Cook device in the other. Proximal landing zone was infrarenal in the first 2 patients and infra-inferior mesenteric artery in 1 . The first 2 patients underwent an external-internal iliac transposition via an extraperitoneal approach to preserve contralateral hya; CIAs were sutured. In patients 3 an occluder was placed in contralateral CIA and hy origin PTA was performed. Femoro-femoral bypass completed the interventions. Postoperative course and followup were uneventful.

Discussion: EVAR for aortoiliac As, in patients with previous cholectomy, is a feasible and safety procedure, but requires original solutions.

\section{0}

\section{Non Occlusive Carotid Thrombosis: Our Experience}

D. Mugnai, S. Mambrini, N. Rousas, A. Robaldo, D. Palombo

Ospedale Mauriziano 'Umberto I' Torino

Introduction: Non occlusive carotid thrombosis (NOCT) is an uncommon disease, due to thrombofilia and pre-existing lesions. Usually patients are symptomatic. Duplex-scan is enough for diagnosis.
Methods: From 19985 patients (mean age 57 years) were treated for NOCT. Medical history was relevant in 3: antiphospholipid syndrome, epilepsy, and piastrinosis respectively. First 2 patients had minor stroke. Patients 3 had TIA, patients 4 crescendo TIAs, and patients 5 amaurosis.

Results: Duplex-scan showed NOCT in all patients Carotid endarterectomy (CEA) was performed 7 days (patient 1), $6 \mathrm{~h}$ (patients 2 ), and $24 \mathrm{~h}$ (remaining patients) after the beginning of symptoms. Arteriotomy was performed after common and external carotid crossclamping, previous intraoperative echographic control of proximal point lesion. In this way, internal carotid backflow ejected thrombus. Thrombosis developed on: minimal lesions in the patients 1 and 3; severe stenosis in the others. In patients 3 a white thrombus was found. 3 patients underwent CEA standard and the others eversion technique. Post-operative course was uneventful. Symptoms improved or recovered. At follow-up none patients re-developed thrombosis.

Discussion: Urgent treatment is necessary in NOCT due to the risk of massive embolisation and occlusion, even if cerebral CT-scan demonstrates lesions. Surgery is preferable to anticoagulant therapy. Currently, limited data support carotid stenting.

\section{1}

\section{Laterocervical Paraganglioma: Our Experience in a Multidisciplinary Approach}

S. Mambrini, D. Mugnai, A. Robaldo, N. Rousas, R. Mazzei, D. Palombo

\section{Ospedale Mauriziano 'Umberto I' Torino}

Introduction: Laterocervical paranganglioma (LCP) is a rare, usually benign neoplams. LCP resection is associated with a high incidence of cranial nerve injury (CNI). To reduce CNI we performed a multidisciplinary approach.

Methods: From 1998 we treated 12 LCP in 10 patients ( 3 males; mean age 59 years). Presentation was an asymptomatic laterocervical mass, bilateral in 2 patients. Echo Duplex and CT-scan or MRI confirm the diagnosis. Carotid angiography with scleroembolisation (SE) was attempted in all patients. Surgery was performed $24-48 \mathrm{~h}$ after SE. In case of only partial resection we carried out a roentgentherapy (RT). CNI were treated intensively by prompt logopedic program.

Results: There were 6 type II and 6 type III LCP (Shamblin classification). The SE was successful in 9 cases. Presternocleidomastoidean incision and subadventitial tumor excision were performed in all patients. 1 patients with incomplete resection was successfully treated with RT. There was no perioperative stroke. CNI developed in 4 patients: 3 patients recovered in 1 week, 1 required a tracheostomy. 1 bilaterally treated patients developed a baroreflex failure syndrome. At mean follow-up of 67 months there were no signs of tumor recurrence.

Discussion: LCP excision remains a surgical challenge. A multidisciplinary approach with preoperative SE, RT if resection is partial, and a prompt logopedic program in case of CNI can improve the outcomes. 


\section{2}

\section{Urgent Carotid Stenting for Intimal Carotid Flap after Endoarterectomy: Value of Eco-Color-Doppler}

M. Manno, A. Mastromarino, R. D’adamo, E. Franceschini, M. Ventura

Chirurgia Vascolare, L'aquila

Introduction: The intimal flap post carotid endoarterectomy represent a rare but high risk condition that could be treated in urgent with stenting.

Methods: In our experience from 2001 to 2006 we have treated 504 carotid lesion. Of these in $468(93 \%)$ we performed traditional surgery while in 36 selected cases $(7 \%)$ we have performed a carotid stent. Five of the stents $(13.8 \%)$ were treated in urgent. These five cases were intimal flap diagnosed during the one month follow-up control of patients undergone to traditional surgery. All patients were asympomatic and were four males and one females (mean age 70 age). The eco-color-Doppler showed an intimal flap presence inside of the common carotid. The flaps were against the stream with consequently high risk of carotid thrombosis. Before starting the procedure we studied patient with a TC scan of the brain, EEG and a neurological evaluation. We have obtained an anatomic success in $100 \%$ of cases even at the angiography even at the eco-color-Doppler control.

Conclusion: Our experience shows as there will be and indication to carotid endovascular treatment in elective and urgent cases; also in particular cases such as intimal flaps the ultrasonographic evaluation is more accurate than angiography that at time of procedure did not show the lesions.

223

Temporary Laparotomy Closure in the Treatment of Ruptured Abdominal Aortic Aneurysms: Effectiveness of Simple Skin Approximation to Control Intra-Abdominal Pressure

C. Maraglino, C. Ricucci, L. Aiazzi

Dipartimento Cardiovascolare OORR Bergamo

Background: Multi-organ-failure (MOF) is cause of death in ruptured abdominal aortic aneurysms (rAAA) surgery. The abdominal compartment syndrome (ACS) due to elevated intra-abdominal-pressure (IAP) is the principal factor leading to such complication. The aim of this paper was to evaluate the efficacy of cutaneous abdominal closure to prevent ACS

Methods: From January 2004, 19 patients (17 \$\$e 2 \$ ) with rAAA underwent operative repair. In those surviving we performed skin edges approximation abdominal closure. IAP was quantified by bladder manometry at the end of operation and then every day. The patients undertook standard closure when IAP was below $20 \mathrm{~mm} \mathrm{Hg}$.

Results: We had 7 deaths (36\%), 4 (21\%) intraop. due to ongoing bleeding and $3(15 \%)$ postop. due to intestinal infarction ( 2 cases) and pulmonary failure (1 case). The mean IAP in the first postop. day was $18 \mathrm{~mm} \mathrm{Hg}( \pm 6 \mathrm{~mm} \mathrm{Hg})$. Only one of the 3 patients dead postoperatively presented a sudden rise of IAP over $30 \mathrm{~mm} \mathrm{Hg}$. Reexploration in this patient revealed a massive bowel ischemia. We managed to perform fascial closure in all cases before 5 th postop. day.

Discussion: A $20 \mathrm{~mm} \mathrm{Hg}$ IAP can cause more than $65 \%$ reduction in the portal vein flow with drop in tissue oxygen delivery. This bad perfusion is the trigger of MOF. An inadequate abdominal space evident postoperatively when the IAP begin to raise couldn't be at the end of operation. That's why we perform in all patients a delayed abdominal closure. Comparing these data with those of our overall experience with (truncated at 250 words).

\section{4}

\section{Endovenous Laser Treatment (EVLT ${ }^{\circledR}$ ) of Great Saphenous Vein Insufficiency in $\mathbf{2 2 3}$ Patients. Our Results on 60 Patients after Three Years}

\section{A. Mastromarino, L. Perilli, C. Petrassi, C. Spartera \\ Department of Vascular Surgery, University of L'Aquila}

Aim: The aim of this study has been to evaluate late $\left(\mathrm{EVLT}^{\circledR}\right)$ results of great saphenous vein.

Patients and Methods: From 1/09/2002 to 28/02/2006 we have treated 223 patients, 120 female (mean age 57aa) e 103 male (mean age 63aa). The more frequent symptoms were oedema, weariness and nocturnal cramps. We have performed a 3 years ecocolordoppler control in 60 patients, to evaluate the presence of saphena recanalization. The vein treated mean diameter of the patients was $12 \mathrm{~mm}$ (ranged 8 and $18 \mathrm{~mm}$ ). The mean joule/cm of saphenous vein given out has been 48 with pulsed emission of laser energy.

Results: We observed a complete vein obliteration in 56 patients $(93.3 \%)$ to ecocolordoppler. We checked the complete vein patency in 2 patients $(3.3 \%)$. The original vein diameter in those patients was 14 and $16 \mathrm{~mm}$. The great saphena was opened one month after procedure because of an insufficient laser energy emission. Only a patient was symptomatic. In other 2 asymptomatic patients $(3.3 \%)$ we observed a vein ricanalization in firstly $8 \mathrm{~cm}$ already seen one year follow-up. We didn't check deep vein thrombosis or phlebitis. 59 $(98.3 \%)$ patients would submit to laser treatment again.

Conclusions: In our experience EVLT treatment is a very efficacy technique with good late results. The vein recanalization is caused of a technical mistake.

\section{5}

A Case of Prosthetic Infection after Surgical Treatment of a Ruptured Abdominal Aortic Aneurysms

G. Mercandalli, G. Prouse, A.M. Settembrini, M Carmo

Department of Vascular Surgery, San Carlo Borromeo

Hospital, Chair of Vascular Surgery, University of Milan, Milan

A 63-year-old male came to the emergency room following sudden onset of pain in the left lateral abdominal region. An emergency 
contrast $\mathrm{CT}$ scan of the abdomen was performed. It showed the presence of a ruptured $9 \times 8.7 \mathrm{~cm}$ infrarenal aortic aneurysm. The patient underwent emergency aneurysmotomy followed by prosthetic grafting for aortic replacement with left distal anastomosis on the femoral artery and right distal anastomosis on the iliac artery. During the procedure the patient was pharmacologically treated twice for the onset of VF. The patient was transferred to the ICU where he underwent plasmatic ultrafiltration repeatedly after developing abdominal compartment syndrome with acute tubular necrosis. On the 23rd postoperative day he was transferred to our unit. A contrast CT scan was performed following the onset of fever, abdominal pain and diarrhea, with positive haemoculture. The CT scan showed the gathering of a small amount of fluid around the left branch of the graft, superiorly limited by the inguinal ligament. The patient was taken into surgery for the excision of the infected branch of the prosthetic graft and revascularization of the left inferior limb by creating a femorofemoral, right to left extra anatomical by pass graft, using the autologous, homolateral, inverted and valvectomized saphenous vein.

The result achieved through this procedure was excellent.

\section{6}

Vascular Lesion Management in Polytraumas

C. Micossi, N. Fermani, A. Pederzoli, M. Battistini

Department of Vascular Surgery, University of Tor Vergata, Rome, Italy

Introduction: The urgency to treats the polytraumatised patients is due to difficulty in creating controlled studies aimed to identifying guidelines and prognostic scores. The main problem is to reduce ischemic delays and the risks of primary and secondary amputation. The aim of this study is to identify the most reliable diagnostic methods and the most suitable therapeutic tactics for the polytraumatised patient.

Material and Method: Our experience comprises 101 patients selected from the ER as having undergone limb polytrauma with lesions not only of the vessels, but also of soft tissue and the surrounding bone structure.

Results: Revascularization in 91 patients (90\%); primary amputation in 3 cases $(2.9 \%)$ and secondary amputation in 6 cases $(5.9 \%)$, mortality was 1 cases $(0.9 \%)$.

Discussion and Conclusion: The ischemic delay is directly correlated with the percentage rate of surgical success. The use of intra-operative angiography finds support in all minor vascular traumas, and in all cases where an accurate study of the residual vascular anatomy is necessary in order to plan a vascular reconstruction operation. As a first-instance examination of the polytraumatised patient, we suggest the CT-scan, which offers the possibility to reduce diagnostic timing and pinpointing the most accurate therapeutic timing. First-instance amputation finds support if the limb has motor and sensitive deficit and loss of bone continuity greater than $6 \mathrm{~cm}$, or in patients with prolonged ischemy of over $6-8 \mathrm{~h}$ and showing an already unstable haemodynamic situation.
227

\section{Limb Vascular Injury: Report on 104 Consecutive Cases}

F. Napoli, M. Massucci, V. Genovese, G. lanni, G. Martinelli, A. Rached, A. Varroni, L. Capoccia, G. Bertoletti

Santa Maria Goretti Hospital, Vascular Unit, Latina, Italy

Introduction: We tried to analyze our results on limb vascular injuries admitted in the Vascular Unit of an urban hospital covering a large rural and tourist area.

Patients and Methods: Our retrospective analysis concerns 104 consecutive cases of extremities vascular trauma treated between August 1999 and March 2006.

Results: Demographic data show that Men/Women ratio is 4.5/1 and the median age (years) is 39.7. Injuries are equally distributed on upper $(55.7 \%)$ and lower (46\%) limbs. In fifty cases (48\%) arterial injury was an isolated lesion; in the 54 remaining patients $(51.9 \%)$ associated lesions were present: vein (33.3\%), vein-bone (31.4\%) and vein-bone-nerves $(22.2 \%)$. Popliteal artery has been the most injured vessel of lower limb (48.2\%) whereas radial and cubital arteries of the upper (63.1\%). Car accident was responsible of the $35.6 \%$ of injuries, industrial accident of $12.5 \%$, stab of $25.9 \%$, iatrogenic of $20.2 \%$. Firearm injury (low-velocity) in our series contributed only for the $5.8 \%$ of cases. All patients have been treated: six patients $(5.8 \%)$ by endovascular procedures, 98 patients by surgery, 2 patients received a hybrid procedure. The primary amputation rate was $2.8 \%$ ( 3 patients) and the delayed rate $3.8 \%$ ( 4 patients). Mortality rate was $0 \% .5 .7 \%$ of patients experienced permanent neurologic damages.

Discussion: Stab and firearms injuries are the least likely to lead to amputation in opposition to crushing trauma. Time before definitive treatment still remains the key prognostic factor. A dedicated team with two or three surgeons on call along with highly performing radiology Unit is (truncated at 250 words).

\section{8 \\ Emergency Thrombectomy of the Lower Caval Vein: Case Report}

\author{
F. Navarretta, G. lob, D. Piccolo, G. Vesce, \\ M. Bacchieri, L. Formato, H. Impérial, \\ M. Castagnola, F. Rutolo, N. Bellini, A. Antico
}

Introduction: The incidence of deep venous thrombosis is $0.16 \%$ and about $0.02 \%$ are complicated of pulmonary embolism (PE); with over 70,000 death a year in Italy, PE is the third leading cause of mortality.

Methods: A 24-year-old female patient was admitted to the emergency room with a lower left limb edema. Colour-Duplex-Scan revealed a left popliteo-femoral thrombosis extended to the external and common iliac veins, the caval and controlateral veins were patient. The Angio-Scan revealed the presence of thrombosis in the left ilio-femoral vein spreading to the lower cava where the thrombus appeared floating and reaching the emergence of the renal veins and even PE. A temporary caval filter was positioned in the lower 
suprarenal caval vein. An emergency caval trombectomy was then performed and a subrenal caval clip Adam-DE Weese inserted.

Results: The Angio-Scan obtained prior to discharge revealed a patent of lower caval vein. The patient was discharged on the ninth day.

Conclusions: Surgical thrombectomty of inferior caval vein may be indicated in selected cases. Current indications for surgical therapy include treatment of phlegmasia coerulea dolens, when fibrinolysis is not indicated; surgical therapy is also imposed by patient conditions and venous decompression is mandatory, or in supra or infrarenal caval thrombosis when a floating clot occurs and caval filter placing might expose to renal vein occlusion.

\section{9}

\section{Combined Carotid Endarterectomy and Coronary Bypass: A Feasible Procedure?}

\author{
A. Odero Jr, E. Franciosi, G. Poletto, A. Popovich, \\ P. Righini, P.L. Giorgetti \\ Chirurgia Vascolare, Università degli Studi di Pavia, Italy
}

Introduction: Carotid stenosis is important stroke risk factor in patients undergoing CABG: stroke risk is 3\% in monolateral asymptomatic carotid lesions, $5 \%$ in bilateral and $7-11 \%$ in occlusions. Predictive factors are prior stroke/TIA or carotid occlusion. Three are the different surgical management: staged, combined and reversed staged. The appropriate strategy is still controversial.

Methods: During 1997-2005 we have performed 150 CEA combined with CABG. Under general anesthesia and cerebral monitoring, the vascular surgeon performs CEA and thereafter cardiac surgeons performed CABG (ECC in 94\%).

Results: The 30-days mortality rate was $2 \%$, due to cardiac events; ischemic stroke were 1 minor and 2 major omolateral $(2 \%)$ and 2 controlateral (bilateral ICA stenosis $>90 \%$ ); minor cardiac complications were $29 \%$. 8 years FW revealed 23 death $(16 \%)$, any stroke, 1 TIA with afasia $(0.6 \%)$ and 3 emodinamic restenosis who underwent re-do.

Discussion: There is not significant differences between mortality rate $(2.0$ vs. $2.8 \%)$ and major neurologic morbility (1.36 vs. $0.6 \%$ ) between combined surgery and the data of each surgical equipe. Even if a $13 \%$ combined stroke/mortality rate has been reported by Hill 2005 in combined CEA/CABG, our data (3.4\%) suggest that combined approach is feasible due to single surgical stress, huge risks reduction than the staged and reversed do, low in-hospital stay and costs.

\section{0}

\section{Rupture of Aortic Aneurysm Secondary to Type III Endoleak}

\author{
A. Pilato, L. Sensi, F. Magnoni, L. Pedrini \\ Vascular Surgery, Ospedale Maggiore Bologna, Italy
}

Introduction: The study was aimed at describing a case of rupture of aortic endograft, 5 years after endovascular treatment.
Case Report: A 78 year old man, treated with endovascular repair (Talent) for aortic infrarenal aneurysm (diameter $70 \mathrm{~mm}$ ), 5 years ago; presented with acute abdominal pain and shock. Duplex scanning showed a large left pelvic haematoma and TC-multislide showed aortic aneurysm in association with direct bleeding in periaortic area. The diagnosis was rupture of aortic endograft. The patients was treated in emergency with laparotomy and sovradiaframmatic clamping. The endograft was slid and lacerated at the gate area, the same area where, during the first treatment, ballooning was performed for leak. The endograft was removed and was converted in aorto-bisiliac graft (Dacron). During the post-operatory period the patient was admitted to intensive unit and experienced temporary renal and lung failure.

Conclusion: Aortic rupture after type III endoleak, can be treated with endovascular approach only if patient's emodinamical conditions are stable. Surgical conversion is the treatment of choice in patients with shock.

\section{1}

\section{Carotid Stenting Procedures without Morphological Selection}

M. Ferri, G.L. Faggioli, M. Gargiulo, A. Freyrie,
F. Fratesi, L. Manzoli', C. Rossi', F. Losinno', A. Stella
Vascular Surgery, 'Section of Epidemiology and Public
Health, University G. D'Annunzio of Chieti, 'Unit of
Radiology, Policlinico S. Orsola, University of Bologna,
Italy

Introduction: Carotid stenting (CAS) is gaining popularity over standard carotid endarterectomy. However, no definitive conclusions on CAS efficacy have been drawn, especially on less severe patients. The study was aimed at evaluating CAS efficacy in patients with no morphological selection criteria, and to examine determinants of CAS inefficacy: neurological complications, technical failure and unsatisfactory result (UR) (the two groups combined).

Methods: From December 2004 to March 2006, patients with carotid stenosis $\geq 70 \%$ were selected for CAS on the basis of creatinine $<2.0 \mathrm{mg} / \mathrm{dl}$, tolerance to aspirin and clopidogrel, no bilateral iliac occlusion. Patients underwent Angio-MRI and Duplex scanning. Procedures were performed in local anaesthesia, by vascular surgeons with radiologist, cerebral protection and anaesthesiologist support. Logistic regression was used to examine the relationship between each outcome and age, gender, arch type, level of stenosis, type of plaque, contralateral stenosis or occlusion and symptoms. Follow-up was by Duplex scanning at 3,6 and 12 months.

Results: Of 167 patients undergoing CAS technical success was achieved in 147 cases $(88 \%) .13$ patients $(7.7 \%)$ experienced temporary neurological complications, 33 patients (19\%) had UR. At multivariate analysis, older age was significantly associated with increased risk of technical failure and UR. Arch III patients showed increased risk of neurological complications and UR. Plaque morphology were unrelated to CAS efficacy. The follow-up revealed one asymptomatic thrombosis at 6 months.

Discussion: CAS can be performed with acceptable complication in all type of carotid plaques. The increased risk of complication in type III arch, should be considered for CAS indication. 


\section{2}

\section{Hybrid Treatment of Aortic Arch, Thoracoabdominal and Multilevel Aorta Disease}

\section{G. Pratesi, L. Azas, R. Pulli, M. Di Vito Francesco, O. Mickalajeva, M. Di Mare, W. Dorigo, A.A. Innocenti, C. Pratesi \\ Department of Vascular Surgery, University of Florence, Florence, Italy}

Introduction: Aorta diseases can have a crucial localization, such as aortic arch (AA) and thoracoabdominal aorta (TAA). Moreover, the aorta can be affected by multilevel disease (MLD), involving abdominal and thoracic segments at the same time. The aim of our study was to analyze our experience in the hybrid treatment of these complex aorta lesions.

Methods: From January 2000 to January 2005, 966 patients underwent abdominal aortic aneurysm repair. During the same period, 72 patients were treated for different thoracic aorta diseases. A hybrid treatment was performed in 15 cases, 6 for AA, 4 for TAA, with revascularization of all visceral vessels, and for MLD in the remaining 6 cases.

Results: Technical and clinical success was obtained in all patients. One patient with TAA died in the third postoperative day for MOF. Among the 6 patients treated for MLD, one developed paraplegia and subsequently died in the 14th day for pulmonary complications; another patient required an adjunctive stent-graft placement for the treatment of a type III endoleak. The mean follow-up duration was $11.2 \pm 4.5$ months (range 1-36). No death, endoleak and reintervention occurred during the follow-up period.

Discussion: Our results suggest hybrid treatment of complex aorta lesions to be feasible and safe and associated with low peri and postoperative complications.

233

Acute Traumatic Aortic Rupture. A Comparison of Endoluminal Stent Grafts with Open Repair (Immediate or Dealayed) and Nonoperative Management: A Single Center Experience and Revision of Literature

\author{
C. Rivellini, G. Carignano, C. Novali \\ Divisione di Chirurgia Vascolare, Ospedale santa Croce, \\ Cuneo
}

Introduction: Blunt traumatic thoracic aortic rupture is a lifethreatening surgical emergency associated with high mortality and morbidity. The recent development of endovascular stent-graft prostheses offers a potentially less invasive alternative to open chest surgery, especially in patients with associated injuries. We sought to compare the results of conventional surgical repair and endovascular treatment of traumatic aortic rupture in literature and in experience of a single center.

Methods: Between 2003 and 2006, 9 patients were treated emergently for acute blunt traumatic aortic rupture. All patients had a lision limited to the isthmus, and associated injuries. Initial management included fluid resiscitation, treatment of other severe associated lesions and strict monitoring of blood pressure. Preoperative workup included chest computed tomography scan, transesophageal echography and angiography if possible.

Results: Stent graft placement successfully sealed the aorta in all patients with not conversion to open repair. In 2 patients the left subclavian artery was intenzionally covered with the device. Not patient in this group had paraplegia. No patient died and not procedure complications occurred in this group.

Discussion: Endovascular treatment of thoracic aortic rupture is a safe, effective and timely treatment option. Improvement of patient outcome can be achieved by delaying surgical repair until after management of major associated injuries if there are no signs of impending rupture.

\section{4}

\section{Subfascial Endoscopic Perforating Vein Surgery for Chronic Venous Insufficiency}

R. Cazzaniga, C. Ricchiuti, R. Romeo

ASL Vallecamonica Sebino, BS, U.O. Chirurgia Edolo

Background: Endoscopic ligation (Subfascial Endoscopic Perforating-vein Surgery - SEPS) is a minimally invasive technique for treatment of chronic venous insufficiency associated with incompetent perforating veins of the lower legs.

Patients: Over 4 years we performed SEPS in 26 patients with incompetent perforating veins preoperatively detected with Colorflow duplex US scanning. Mean age was $64.6(\mathrm{DS} \pm 9.62)$ and 16 $(61 \%)$ were female. 10 patients $(38 \%)$ had venous ulcers. The procedure was always performed with 2 trocars $(10$ and $5 \mathrm{~mm})$ and perforating veins were interrupted with bipolar coagulation. In 18 cases $(69 \%)$ was associated an internal saphenectomy. Mean operatory time was $75^{\prime}$ (54' in cases without superficial veins surgery).

Results: Mean follow-up was 27 months (range 4-54 months). We have in 2 cases trocar-site infection and in 1 patient oedema and persistent pain in the lower leg. Ulcerative lesions healed within 6 weeks in 7 cases $(70 \%)$ and show clinical improvements in 3 , with 1 ulcer recurrence within 6 months. In 14/16 patients without leg ulcers we observed reductions of symptoms. Even 4 patients, with persistence of some incompetent perforating veins postoperatively, showed anyway clinical benefit.

Conclusions: SEPS is a safe and effective procedure for treatment of chronic venous insufficiency related with incompetence of perforating veins. 19th National Congress of the Italian Polyspecialist Society of Young Surgeons (IPSYS) 


\section{5}

\section{The Treatment of Venous Malformations: Our Experience}

R. Serra, A. Luongo, D. Mastrangelo, G. Sabino, M.A. Pacilè, S. de Franciscis

Università Magna Graecia di Catanzaro, Cattedra di Chirurgia Generale, Scuola di Specializzazione in Chirurgia Vascolare

Introduction: Venous malformations are congenital lesions that can cause pain, decreased range of movement, compression on adjacent structures, bleeding, consumptive coagulopathy and cosmetic deformity. Sclerotherapy alone or combined with surgical excision is the accepted treatment in symptomatic malformations. Aim of this paper is to show our experience in the treatment of this kind of lesions.

Methods: Twenty-four patients with venous malformations were treated in the period 2000-2005. All patients performed ultrasound and MR evaluation. 12 patient underwent percutaneous sclerotherapy of peripheral venous malformations with ethanol $96 \%$ and 12 patients underwent surgical procedure to extirpate the malformation.

Results: All 12 Patients who underwent surgical procedure and 7 out of 12 patients who underwent percutaneous sclerotherapy showed complete improvement. The remaining 5 patients were considered as a treatment failure.

Discussion and Conclusions: MRI was essential to define the anatomic extent and involvement of various tissue layers, and to correlate treatable components of the malformation with patient symptoms. Treatment is decided by the need to alleviate clinical symptoms, and is dependent on the extent of the malformation as defined by MRI. In our experience surgical treatment showed better results than scleroterapy.

\section{6 \\ Vascular Lesions Secondary to Osteotomy for Corticotomy}

\section{F. Stilo, G. De Caridi, M.L. Spada, F. Mirenda, F. Spinelli \\ Cattedra e Unità Operativa Complessa, di Chirurgia \\ Vascolare, Policlinico Universitario di Messina}

Introduction: Management of vascular trauma are frequent delayed. A very rare situation is vascular injuries after elective operation for bone lengthening or correction of a deformity. We described 3 cases.

Methods: Case 1: M 22 aged, underwent corticotomy for bone lengthening, presented immediately acute limb ischemia for a partial lesion of popliteal artery, documented by US. After $7 \mathrm{~h}$ direct reconstruction of artery and fasciotomies were performed. Case 2: M 27 aged, underwent directional osteotomy for genu various correction. For 30 days constant increase of leg volume and decrease of function. US showed an important haematoma at popliteal level; arteriography documented partial lesion of under knee popliteal artery and voluminous false aneurysm. Direct correction of artery and fasciotomies were performed. Case 3: M 22 aged, underwent corticotomy for leg lengthening, immediately presented leg pain with distal decreased pulses. After $4 \mathrm{~h}$ increase of leg volume, arteriography showed a total lesion of under knee popliteal artery and AVF. Popliteo-tibial bypass with controlateral GSV and fasciotomies were performed. After 1 month e.v. closure of AVF.

Results: Recovery after 2 months with a minor leg insufficiency. Patency of bypass, absence of infection or delayed false aneurysms.

Discussion: Vascular injuries after elective orthopaedic procedures were very rare situations. Lesions were caused by osteotomy and corticotomy percutaneous performed. Variety of clinical presentation determined difficult diagnosis and delayed treatment. It's very important a quick diagnosis completed by arteriography.

\section{7}

Surgical Treatment of Pararenal Aortic Aneurysms: Management, Early Results and Risk Factors Analysis

\author{
C. Brioschi, E.M. Marone, S. Frigerio, Y. Tshomba, \\ G. Melissano, R. Chiesa \\ Chair of Vascular Surgery, 'Vita-Salute' University, \\ Scientific Institute H. San Raffaele, Milan, Italy
}

Background: To better define challenges, risks and results, we analysed our experience with the treatment of pararenal aortic aneurysms.

Methods: Between January 1993 and March 2005, 119 consecutive patients underwent surgery for pararenal aneurysms at our Institution. According to their localization, we treated 85 cases of juxtarenal aneurysms and 34 cases of suprarenal aneurysms. Four patients underwent emergency operation for a ruptured aneurysm.

Results: In the juxtarenal aneurysm group, the 30 days mortality rate was $4.7 \%(4 / 85)$ including 3 cases with ruptured aneurysm. In the suprarenal aneurysm group the 30 days mortality rate was $2.9 \%(1 / 34)$. Preoperative renal insufficiency was present in 16 cases. In 22 cases we registered a transient increase in creatinine levels with return to baseline levels within discharge. Four patients required long term dialysis. In 3 cases a short period of dialysis was required. Clamping time longer than $30 \mathrm{~min}$ is associated with a higher risk of transient postoperative deterioration of renal function $(p=0.0073)$. Preoperative renal insufficiency is associated with a higher risk of persistent postoperative deterioration of renal function $(\mathrm{p}<0.0001)$.

Conclusions: Morbidity and mortality of elective surgery for pararenal aneurysms is acceptable. One of the main risks of this surgery is renal morbidity. Preoperative renal insufficiency and long periods of renal ischemia are associated with a higher risk of postoperative deterioration of renal function that is often but not always reversible. 


\section{8}

\section{Laparoscopic Release of Celiac Artery Compression Syndrome by Median Arcuate Ligament: Report of $\mathbf{6}$ Consecutive Cases}

\author{
L. Dordoni, P. Baccari, E.M. Marone, Y. Tshomba, \\ L. Bertoglio, R. Chiesa \\ Chair of Vascular Surgery, 'Vita-Salute' University, \\ Scientific Institute H. San Raffaele, Milan, Italy
}

Introduction: Median arcuate ligament syndrome (MALS) is an unusual condition caused by compression of the celiac artery by abnormally low insertion of the arcuate ligament. Surgical treatment is performed through an upper abdominal midline incision. Successful laparoscopic approach has been recently reported.

Material and Methods: Between ' 88 and '06, at our Institution, 6 consecutive patients ( 4 men; mean age: 53 years) with postprandial abdominal pain and weight loss were treated for MALS. Preoperative MR or CT angiogram clearly demonstrated compression of the celiac axis by the arcuate ligament. All patients underwent laparoscopic release of celiac artery compression through four port sites under general anesthesia. In one case, percutaneous angioplasty and stenting of celiac artery was performed before laparoscopic treatment. The origin of the celiac artery was completely released with visible improvement of blood flow. CT scan was performed before discharge and annually thereafter. Duplex scan was performed every 6 months after the treatment.

Results: The treatment was successful in all cases (mean operative time $94.6 \mathrm{~min}$ ). We recorded no in-hospital deaths. Postoperative imaging studies demonstrated no residual arterial stenosis. Mean hospitalization time was 2.3 days. At mean follow-up of 34 months all patients reported complete resolution of their symptoms and weight gain and Duplex scan demonstrated markedly improved flow in the celiac artery.

Conclusion: Laparoscopy is a safe and effective method for treatment of MALS and may precede endovascular treatment of concomitant atherosclerotic stenosis.

\section{9}

\section{Thrombolysis and Stenting for Perioperative Occlusion of Aortofemoral Bypass: Late Perforation and Aortoenteric Fistula}

Y. Tshomba, A. Kahlberg, E.M. Marone, F. Setacci, D. Logaldo, R. Chiesa

Division of Vascular Surgery, 'Vita-Salute' University, Scientific Institute H. San Raffaele, Milan, Italy

Purpose: To present a case of late aortic perforation and aortoenteric fistula developed 6 years after thrombolysis and stenting of a perioperatively occluded aortofemoral bypass graft.

Case Report: We previously described the case of a 47-year-old man who experienced perioperative graft occlusion following infrarenal aortic endarterectomy and aorto-bi-femoral ePTFE bypass graft for occlusive disease. The patient was treated with local graft thrombolysis (rtPA) and stenting (Palmaz P4014) of an intimal flap at the level of the endarterectomized aorta next to the aortic anastomosis.
An urgent laparotomy performed 6 years later, showed aortic wall perforation by the stent with a large aortoenteric fistula. Surgical treatment included removal of the stent and of the aorto-bifemoral graft, bowel repair and axillo-bifemoral bypass grafting. The patient was discharged twelve days later with no early complications.

Conclusions: Although local thrombolysis and stenting may be a technically feasible technique instead of reoperation in early aortofemoral graft thrombosis, long-term results of this procedure may be affected by perigraft haematoma due to perioperative thrombolysis, increased risk of infection due to the prolonged intra-graft cannulation and risk of stent-related aortic injury, especially if an endarterectomy has been performed, definitely increasing the risk of late aortoenteric fistula. More experience with hybrid, open/endo procedures and more contemporary stent-based devices is needed to assess the efficacy and safety of this alternative treatment.

\section{0}

\section{Visceral Rotation during Thoracoabdominal Aortic Aneurysms Repair}

\author{
F. Calliari, E.M. Marone, F. Setacci, Y. Thsomba, \\ G. Melissano, R. Chiesa
}

\section{Cattedra e Scuola di Specializzazione in Chirurgia} Vascolare, Università 'Vita-Salute', IRCCS Ospedale San

Raffaele, Milano, Italy

Background: In spite to the new surgical adjuncts introduced in the last years in the treatment of thoracoabdominal aortic aneurysms (TAAA), early results of this major surgery are still complicated with significant mortality and morbidity rate. Surgical access to the thoracoabdomianal aorta plays a basic role in the development of such complications. Aim of the study is the analysis of our experience of TAAA surgery with trans-peritoneal and right visceral rotation to access the subdiaphragmatic aorta.

Methods: In our experience we used two surgical approach to thoracoabdominal aorta: thoraco-phreno-laparotomy in case of type I, II and III TAAA and bilateral subcostal laparotomy in case of type IV TAAA. In reason of new surgical adjuncts, in case of type I, II an III TAAA we prefer used as organ protection a left heart bypass (Biomedicusa pump) and cerebrospinal fluid drainage (CFD). From January 1993 to April 2006, to our institution, were treated 587 patients for thoracic (322 cases) and thoracoabdominal aortic aneurysms (265 cases).

Results: In 228 cases we used left heart bypass and in 233 cases we utilized CFD. The overall mortality was $13 \%$. Paraplegia in $8.5 \%$ of cases, Renal failure in $6.5 \%$ and respiratory failure in $18.4 \%$.

Conclusions: In spite to the evolution of technique, mortality and morbidity associated to TAAA surgery is sill high. The choice of ideal surgical approach may be improved the result in the management of this complex disease. 


\section{Obesity Surgery}

\section{1 \\ Pre-operative Esophageal Function Study in 75 Patients Affected by Morbid Obesity Candidate to Bariatric Surgery}

\author{
L. Antoniazzi, E. Masci, M. Pagani, E. Lattuada, \\ M.A. Zappa, G.C. Roviaro \\ Fondazione Policlinico, Mangiagalli e Regina Elena, IRCCS, \\ Milano, Italy
}

Introduction: Esophageal Function Tests remain fundamental in the diagnosis and management of esophageal benign diseases. Since 2005 we evaluated patients candidate to bariatric procedures to obtain a better pre-operative selection.

Methods: Between April 2005 and February 2006, we evaluated 75 patients affected by morbid obesity: $58 \mathrm{~F} / 17 \mathrm{M}$, mean age: $41.61 \pm 10.51$ years, mean BMI: $42.71 \pm 7.28$. We performed 75 esophageal motility and $35 \mathrm{pH}$-monitoring.

Results: $87.6 \%$ of patients were affected by hypotonic LES, intrathoracic in $91.7 \%$ of cases (r. pressure: $7 \mathrm{~mm} \mathrm{Hg}$ ). Incidence of motility abnormalities was 89\%: hypertonic waves (36.9\%), syncronous waves $(28.7 \%)$, esophageal pressurization $(20 \%)$, irrigative waves $(15 \%)$, increased intra-bolus pressure $(15 \%)$, hypotonic waves (13.6\%), brachiesophagus (5.4\%), diffuse esophageal spasm (4.1\%), a specific (30\%). We observed $38 \%$ of patients with increased pathologic reflux, even without statistically significative correlation with BMI.

Discussion: These findings resulted in pre-operative pharmacological therapy or endoscopic evaluation. Intra-operatively we proceeded to reduction of hiatus defect or hernia whenever present. Mean-term follow-up of patients submitted to gastric banding is ongoing with motility tests performed post-operatively. From preliminary data we observed a statistically significative increase of LES resting pressure with reduction of motility abnormalities detected pre-operatively.

\section{2 \\ Bioenterics Intragastric Balloon (BIB ${ }^{\circledR}$ ), Lap-Band System ${ }^{\circledR}$ (LB), Gastric Bypass (GBP) and Laparoscopic Biliopancreatic Diversion with Duodenal Switch (Lbpd/Ds): Experience in a Single Centre}

\section{Ciannella, V. Borrelli, M. Giuffrè, M. Lorenzo, U.A. Bassi, F. Persico, L. Angrisani \\ Endoscopic Surgery Unit and Gastroenterology Service, 'S. Giovanni Bosco' Hospital, Naples, Italy}

Four type of bariatric procedures are currently available in our centre. From January 2000 to December 2005, 891 procedures have been performed. BIB $^{\circledR}$ was implanted trans-orally under endoscopic control in 331 patients (134 M-197 F; mean age 39.1 years, range
17-64; mean BMI 57.9, range 35.7-81.3) as pre-operative treatment in view to surgical procedure. Six months after implantation, mean BMI was 51.2. LB was performed in 272 patients (63 M-209 F; mean age 33.4 years, range 17-64; mean BMI 45.3, range 35.1-58.3). Mortality was absent. 27 patients $(9.9 \%)$ underwent band removal for technical complications and/or unsatisfactory weight loss. Mean BMI at 3, 5, and 7 years follow-up was 32.7, 31.7 and 32.1 respectively. GBP has been performed in 224 patients, 184 (41 M-143 F; mean age 33.5 years, range $17-58$; mean BMI 45.7, range 38.1-57.1) by laparoscopic access and the other 40 patients (11 M-29 F; mean age 41.9 years, range 20-67; mean BMI 53.4, range 39-70.2) received this operation via laparotomic. $13(5.8 \%)$ patients were re-operated for surgical complications. Post-operative mean BMI at 3 and 5 years follow-up of laparoscopic and laparotomic GBP were 28.3, 25.2 and 33.1, 29.3, respectively. 22 patients ( $3 \mathrm{M}-19 \mathrm{~F}$; mean BMI 63.9, range 51-60) were scheduled for II stage LBPD/DS. Mean post-operative BMI after I and II stage was 54.9 and 46.8 respectively at 1 year follow-up. Criteria of choice for the different operations remains to be confirmed in the long term.

\section{3}

\section{Bionteric Intragastric Balloon (BIB ${ }^{\circledR}$ ) Reduce the Operative Risk in Patients Submitted to Biliopancreatic Diversion with Duodenal Switch (IBPD/DS) in Two Stages}

\section{P. P. Cutolo, V. Borrelli, M. Giuffrè, F. Persico, M. Ciannella, U.A. Bassi, M. Battaglini, V. Di Maio, L. Angrisani \\ Unit of Endoscopic Surgery, 'S. Giovanni Bosco' Hospital, 'Federico II' University of Naples, Naples, Italy}

Introduction: Considering BIB the least invasive method to obtain temporary weight loss, we have adopted the strategy of implanting this device as first line treatment in view of a three stage procedure: BIB (6 months) followed by laparoscopic sleeve gastrectomy (LSG) and finally laparoscopic biliopancreatic diversion with duodenal switch (LBPD/DS). This is a report of the clinical outcome obtained by this approach.

Methods: Twelve patients (3 M, 9F) mean age 36.4 (22-49 years) mean BMI 63.9 (47.4-79.5) mean weight $162.8 \mathrm{~kg}(130-191)$ mean EW\% 186.8 (115.4-261.4) entered this study. Twelve co-morbidities were diagnosed pre-operatively: hypertension (33\%), hypercholesterolemia $(25 \%)$, diabetes $(16.6 \%)$, sleep-apnea $(8.3 \%)$, osteoarthritis $(8.3 \%)$ and gastro-oesophageal reflux (8.3\%). BIB was implanted under conscious sedation and endoscopic assistance. LBPD/DS was planned 1 month after weight loss plateau.

Results: At time of LSG (1 month after BIB removal) mean BMI was 54.6 (43.7-62.2), mean weight $142 \mathrm{~kg}$ (120-176), EWL\% 21.1 and 9/12 co-morbidities were resolved. LBPD/DS was performed at mean BMI 46.9 (35.4-57.8), mean weight $124.2 \mathrm{~kg}$ (115-137), EWL\% 40.2 with 2 remaining co-morbidities. Both LSG and LBPD/DS were performed without laparotomic conversion and post-operative complications.

Discussion: BIB sharply reduce the weight and the comorbidities of candidates to the II stage LBPD/DS. 


\section{4}

\section{Brachioplasty After Bariatric Surgery and Massive Weight Loss}

\section{S. Gentileschi, R. Bracaglia, G. Mingrone1, R. Tacchino², G. Nanni², M. Foco ${ }^{2}$ \\ Department of Plastic Surgery, 'Department of Medicine, ${ }^{2}$ Department of Surgery, Policlinico A. Gemelli, Catholic University Sacred Heart, Rome, Italy}

Introduction: Arms are one of the most frequently area of the body, affected by massive weight loss. Each ex obese patient chooses a priority of corrective procedure and requires brachioplasty in about $20 \%$ of the cases, at the first consultation with the Plastic Surgeon. This operation restore arm contour and axillary ptosis, by an appropriate dermolipectomy.

Methods: The authors analyze their experience with the brachioplasty in 20 ex obese patients, undergone to bariatric surgery. 19 patients belonged to group 4, according to Teimourian et al., and 1 patient to group 3.

Results: The patient is marked in a standing position, to locate the scar in the medial brachial sulcus and in the axillary region, in a $\mathrm{T}$ shape. Only one case of a little dehiscence (about $2 \mathrm{~cm}$ ) occurred, that healed with 2 weeks of medications. The dehiscence was situated on the conjunction of the brachial with the axillary scar.

Discussion: It's very important to follow the contour of the arm with the skin markings and incisions, to avoid leaving skin excess, particularly near the elbow. It's mandatory to precisely locate the scars in the less visible area of the medial brachial sulcus, and to avoid excessive tension, which is not necessary for the correction of the defect. Correct surgical planning is very important for final location of the scar and correct evaluation of the amount if tissue to resect.

\section{5}

\section{Dermolipectomy of the Thighs after Bariatric} Surgery and Massive Weight Loss

\section{S. Gentileschi, R. Bracaglia, G. Mingrone ${ }^{1}$, R. Tacchino ${ }^{2}$, G. Nanni' ${ }^{2}$ M. Foco ${ }^{2}$ \\ Department of Plastic Surgery, 'Department of Medicine, ${ }^{2}$ Department of Surgery, Policlinico A. Gemelli, Catholic University Sacred Heart, Rome, Italy}

Introduction: Excess of dermoadipose tissue in the area of the thighs can cause difficulties to walk, to dress, intertrigo and psycologic troubles. Patients who have undergone bariatric surgery require thighs dermolipectomy in $24 \%$ of the cases at the first consultation with the plastic surgeon.

Methods: The authors performed 45 dermolipectomy of the thighs in ex obese patients, 25 of which had undergone bariatric surgery. Resection was planned, with the patient in standing position, performing skin markings to hide the scar in the groove between the thigh and the perineum, and in the medial aspect of the thigh, with a ' $L$ ' shape.

Results: All the patients achieved good correction of both aesthetic and functional problems. Patients who have undergone bariatric surgery had a higher incidence of wound dehiscence: $65 \%$ vs. $12 \%$.
Discussion: It's mandatory to avoid tension, perform minimal undermining and anchor the superficial fascia to the lateral border of the Colles fascia, for the high risk of wound dehiscence, in post bariatric patients. Critical steps of the surgical planning are the correct position of the scars and the evaluation of the amount of tissue to remove.

\section{6}

\section{The Eco-color-Doppler Guide CVC} in Obese Patients

\section{Docimo, L. Sparavigna, P. Papagno, A. Topatino, I. Verde, P. Capuano, V. Amoroso, G. Docimo}

Facoltà di Medicina e Chirurgia, Seconda Università degli Studi di Napoli, Cattedra di Clinica Chirurgica, Divisione di Chirurgia Generale e dell'Obesità

Introduction: The authors analyzed the results of 140 central venous catheterisms of internal jugular vein performed by eco-colordoppler.

Patients and Methods: Between January 2000 to December 2005 the authors examine obese patients candidated to bariatric surgery. It's a group of 140 patients, composed of 94 women and 46 men, whose age is between 24 and 60 years, and with a BMI between 32 and 90 . As the considerable adiposity and post-operation necessities, this procedure has been very useful.

Results: The eco-color-doppler guide CVC is a safe procedure with short performing time, low rate of failures and complications, and high rate success. The average performing time was $10 \mathrm{~min}$, $96.8 \%$ of success and $3.2 \%$ of impossibility (trombosis internal jugular vein bilateral).

Conclusions: It was very helpful in obese patients candidated to bariatric operation, allowing a comfortable and easy access venous, as well as the soministration of liquids otherwise with difficulty administering by peripheral way, correlated to corporal weight in qualitative and quantitative terms, such at improve the process of recovery postoperating.

\section{7}

\section{Lapaorscopic Roux en Y Gastric Bypass vs. Laparoscopic Gastric Banding for Morbid Obesity: A Single Institution Prospective Study}

\author{
A. Lazzaro, L. Franceschilli, M. Venza, F. Lirosi, D. Benavoli, \\ P. Gentileschi, A.L. Gaspari \\ Department of General Surgery, University of 'Tor Vergata', \\ Rome, Italy
}

Introduction: We prospectively evaluated two different bariatric procedures, laparoscopic Roux en Y gastric bypass (LRYGB) and laparoscopic gastric banding (LGB).

Methods: 80 patients entered the study. 50 patients received a LRYGB (62.5\% group A) and 30 a LGB (37.5\% group B). Mean age 
was 43.4 years in group A (23 to 66 years) and 41.2 years $(21-65$ years) in group B). Mean BMI was 46.8 (38-66) in group A and 44 (36-55) in group B. Mean operative time was $190 \mathrm{~min}$ (90-300 min) in group A and $120 \mathrm{~min}(60-240)$ in group B. Mean ospital stay was 8.2 days (6-12 days) in group A and 3.8 days (2-12) in group B. There were two major postoperative complications (4\%) in group A (one jejuno-jejunostomy leak and one subphrenic abscess) and 4 $(13.3 \%)$ in group B (three did not tolerate the prosthesis requiring removal and one acute slippage required re positioning). Long term surgical complications were none in group A and $5(16.6 \%)$ in group $\mathrm{B}$ (three band slippage requiring removal, one port infection, and one band erosion). At mean follow up (16 months in group A and 16.5 in group B) mean BMI was 30 in group A and 32 in group B.

Conclusions: LRYGB seems to have longer operative time and hospitalization, a lower incidence of long term complications and better weight loss results.

\section{Cardiac Surgery}

\section{8}

Adjustable-Size Sinotubular Junction for Aortic Valve Reimplantation Procedure: An In Vitro Study

\section{P. Bajona, D. Maselli, L. Bellieni, L. Arcieri, F. Chiaramonti, M. Croccia, F. Mangia, G. Minzioni}

Department of Cardiac Surgery, Azienda Ospedaliera

Pisana, Pisa, Italy

Introduction: Sinotubular junction (STJ) size in aortic valve reimplantation procedures is usually predetermined. We tested in vitro a method to realize an adjustable size sinotubular junction.

Methods: Eighteen $25 \mathrm{~mm}$ diameter scalloped porcine aortic roots were sutured inside $28 \mathrm{~mm}, 30 \mathrm{~mm}$ and $32 \mathrm{~mm}$ Valsalva grafts (Vascutek, Renfrewshire, Scotland) suspending commissures into the expandable region of the graft itself STJ size was then changed by wrapping the neo-commissural ridge with Dacron rings of decreasing size $\mathrm{Neo}$-aortic roots were statically pressurized up to $100 \mathrm{~mm} \mathrm{Hg}$ in a test circuit driven by a centrifugal pump Geometry of the aortic root shape of aortic valve leaflets and extent of their coaptation were analysed by direct endoscopic view and by ultrasound imaging techniques.

Results: Pressurizing unwrapped aortic root resulted in centrifugal displacement of commissures, aortic leaflets bending and central aortic regurgitation. Height and level of coaptation of aortic valve leaflets increased by wrapping the neo-STJ with Dacron rings of decreasing size with a maximum for a STJ size corresponding to $30 \mathrm{~mm}$ and decreased for further reduction of STJ size. Excess reduction of STJ size also resulted in prolapsed aortic leaflets and eccentric aortic regurgitation.

Discussion: An adjustable STJ size can be obtained by wrapping the expandable portion of a Valsalva graft after implanting commissures into its expandable region. Within certain limits reduction of the STJ results in increased coaptation height of aortic valve leaflets and optimization of aortic valve competence while maintaining normal range sinuses size.

\section{9}

\section{A Technique to Reposition Sinotubular Junction in Aortic Valve Reimplantation Procedures with the De Paulis Valsalva Graft}

\author{
L. Bellieni, D. Maselli, P. Bajona, F. Chiaramonti, \\ M. Croccia, F. Mangia, L. Arcieri, G. Minzioni \\ Department of Cardiac Surgery, Azienda Ospedaliera \\ Pisana, Pisa, Italy
}

Introduction: Sinotubular junction reconstruction in the reimplantation type of valve-sparing aortic procedure can present some problem when a Valsalva graft is used. Since in the Valsalva graft sinotubular junction height is predetermined, correct matching with native commissures height can be difficult. We propose a method by which it is possible to create a new sinotubular junction in Valsalva graft without altering its original configuration.

Methods: A 59-year-old man underwent David Operation with a Valsalva graft. Native sinuses were resected leaving coronaries on buttons. A Valsalva graft was selected and implanted. Commissures were fixed in the expandable skirt of the graft at the ideal height. The new sinotubular junction was realized by wrapping the expandable skirt of the graft with a ring obtained from the graft itself. By preventing expansion of the skirt the ring realizes a new sinotubular junction of proper height and size.

Results: The post-operative course was uneventful. Discharge echocardiography showed a nice neo-aortic root with a competent aortic valve and normal leaflets motion.

Discussion: Our technique is easy to realize, requires minimal modification of the graft limited to the reduction of the collar height. Our technique can be proposed for situation in which a correct matching between native sinuses and neo-sinuses is difficult to achieve.

250

The Ideal Theoretical Graft Over Sizing in Valve-Sparing Aortic Operations with a Standard Tubular or a Valsalva Graft

F. Chiaramonti, D. Maselli, L. Bellieni, P. Bajona,

M. Croccia, F. Mangia, L. Arcieri, G. Minzioni

Istituto Cardiotoracico, Pisa, Italy

Introduction: The aim of the present study was to determine, by a mathematical model, the ideal theoretical degree of ascending aortic graft over sizing needed to obtain normal sinuses dimension in the reimplantation type of valve-sparing aortic operations.

Methods: Echocardiographic studies were performed in 50 healthy subjects, to define aortic root dimension in a normal population. Size of sinuses of Valsalva was conventionally expressed as the area surrounding fully opened aortic cusps, the so-called beyond 
leaflets area (BLA). A mathematical relationship between aortic annulus diameter, aortic sinus diameter and resulting BLA was defined. By simulating intra-operative scenarios, the effect of different degrees of standard or Valsalva graft over sizing on BLA extension was tested.

Results: The same degree of graft over sizing resulted in a bigger beyond leaflets area for the Valsalva graft than for a standard graft. Over sizing degrees excending $+7 \mathrm{~mm}$ for a standard graft and $+3 \mathrm{~mm}$ for the Valsalva graft resulted in a beyond leaflets area over normal limits. To express the results, we have designed two different normograms, one for the standard graft and one for the Valsalva graft.

Discussion: A less pronounced graft over sizing is needed to achieve normal-range sinuses size when using a Valsalva graft, the ideal theoretical graft over sizing was $+7 \mathrm{~mm}$ for a standard graft and $+3 \mathrm{~mm}$ for the Valsalva graft. Our normograms can be helpful in selecting a proper graft size when performing a valve-sparing aortic procedure.

\section{1}

\section{Adjustable Length Polytetrafluoroethylene Chordae for Mitral Valve Repair: Preliminary Experience}

\author{
M. Croccia, D. Maselli, P. Bajona, L. Bellieni, \\ F. Chiaramonti, F. Mangia, G. Minzioni
}

Department of Cardiac Surgery, Azienda Ospedaliera

Pisana, Pisa, Italy

Introduction: We describe a new suture method by which length of artificial chordae tendineae can be repeatedly adjusted, after knot tying, without changing their papillary or leaflet anchoring.

Methods: Two $20 \mathrm{~mm}$ long loops are made at the middle of a double needle CV-5 ePTFE suture to realize the leaflet component. To realize the papillary component, a suture with five reference knots at a fixed distance of $2 \mathrm{~mm}$ each other is realized at the needle-less tip of two CV-4 semisutures, the two semisutures are fixed on papillary muscle tip. The two components are coupled by a loop that can be easily tightened and released and moved under a reference knot closer or farther from papillary muscle to respectively shorten or elongate the neo-chorda.

Results: 8 patients ( 4 male; age $67.1 \pm 12.6$ years) affected by degenerative mitral regurgitation (MR) received 23 artificial chordae ( 9 on anterior leaflet, 14 on posterior leaflet, mean $2.5 \pm 0.8$ ), P2 quadrangular resection was associated in 1 patient, ring annuloplasty in 7 patients. 10 post-implant length adjustment manoeuvres were performed. At discharge MR grade was none in five patients and trivial in three patients; at a mean follow up of $8.5 \pm 2.7$ months 1 patient experienced a worsening of MR, not related to neo-chordae implant, from trivial to $2+$ (truncated at 250 words).

Discussion: Our method allows easy and repeatable chordal length adjustment. Short-term results are encouraging.

\section{2}

\section{VAC System as Treatment of Deep Sternal Infections After Cardiac Surgery: Our Experience in 100 Cases}

\author{
A. Eusebio, A. Barbone, M. Bergonzini, E. Citterio, \\ M. Muretti, D. Ornaghi, F. Settepani
}

Istituto Clinico Humanitas, Rozzano, MI

Objective: Postoperative deep sternal wound infection occurs in $0.6-16 \%$ of patients who have undergone cardiac surgical procedures performed using median sternotomy. The variance in reported incidence levels may depend on differences in definitions of infection. To treat it we can use traditional methods as antibiotics irrigation and dressing changes daily, or a new method called VAC System.

Methods: From January 1997 to December 2005, 100 patients underwent to cardiac surgery operations in median sternotomy out of $5800(1.72 \%)$ presented deep sternal wound infections with a median age of 68,5 years (range 50-84 years); we compared the group A, composed by 55 patients treated with VAC System, to the group B, formed by 45 patients treated with traditional methods as twice a-day dressing changes.

Results: VAC System was removed after a mean of 14, 3 days, and the sternum was surgically closed there after 48 patients in VAC group had definitive surgical closure (87\%); In non-VAC group, the sternum was surgically closed after a mean of 17.5 days. 39 patients in non-VAC group had definitive surgical closure (86\%). None death was VAC-related. 30 days survival was $94.6 \%$ in group A and $95.6 \%$ in group B.

Conclusion: We consider the VAC System a convenient, fast and safe way to allow the development of a more suitable tissue granulation.

\section{3 \\ Edge to Edge Technique: A Simple Solution for Complex Lesions}

S. Gallieri, M. Pesso, F. Pirro, F. Massi, L. Fontanesi, I. Ghidoni

Department of Cardiothoracic Surgery, Hesperia Hospiatal Modena, Italy

Objective: The aim of this study is to report our experience with the Edge to Edge technique as treatment for the complex lesion and to demonstrate a better outcome with this simple and fast technique compared to a classic valve replacement or more demanding surgical procedures.

Material and Method: Between 1998 and 2005, 569 patients submitted to mitral valve repair, 71 (mean age $63.3 \pm 15.72$ ) underwent Edge to Egde correction. The most frequent etiology was degenerative (56 patients, $78.9 \%$ ). The most prevalent mechanism was prolapse of anterior leaflet in 27 patients, prolapse of both leaflets in 11 patients, prolapse of posterior leaflets with a very calcified posterior anulus in 17 patients and other complex mechanism in 16 patients. In 61 patients the Edge to Edge technique was the only surgical procedure on the mitral valve and in 10 patients the $\mathrm{E}$ to $\mathrm{E}$ technique was used to complete a classic repair of the posterior leaflets, as quadrangolar resection with sliding mono or bilateral, in order to 
correct a residual mild regurgitation on the original procedure. In 60 patients together with the E to E plasty we implanted a Carpentier ring which main diameter number was 32 . In 15 patients the mitral valve repair was associated to other cardiac procedure associated: 8 patients underwent to miocardial revascularization, 5 patients to aortic valve replacement, 1 patient to Morrow procedure and 1 patient to left ventricular aneurismectomy.

Results: The perioperative mortality was of two patients. Three patients died at the late follow-up, two (truncated at 250 words).

\section{4}

\section{Time-Course of Interleukin-6 and Neopterin After Left Ventricular Assist Device Implantation in Heart Failure End-Stage Patients}

\section{A. Garatti, R. Caruso, V. Sedda, F. Milazzo, A. Colombo, C. Russo, M. Lanfranconi, G. Bruschi, O. Parodi, E. Vitali}

A.O. Niguarda Ga' Granda; Dipartimento 'A.De Gasperis', Milano, Italy

Background: Left ventricular assist device (LVAD) has become a validated therapeutic option for bridging patients with end-stage heart failure (ESHF) to heart transplant. Aim of the study was to investigate the course of inflammatory status and the impact on clinical outcome.

Methods: Eleven patients (mean age $54 \pm 8,11 \mathrm{M}$ ) supported with LVAD for ESHF have been enrolled. Urine neopterin/creatinine $(\mathrm{Neo} / \mathrm{C}$ ), and plasma interleukin-6 (IL6) levels were analysed in samples collected before (T1) and $4 \mathrm{~h}$ (T2), $24 \mathrm{~h}$ (T3), $72 \mathrm{~h}$ (T4), 1 week (T5), 2 weeks (T6) and 4 weeks (T7) after LVAD implantation.

Results: Five out of 11 patients died (group A) between T5 and T6, of MOFS. The IL6 and Neo/C levels were not different between group A and survived patients (group B) at T1. Mean C-reactive protein and serum lactates were significantly higher in group $\mathrm{A}$ compared to group B at T1 $(\mathrm{CRP}=10.6$ vs. 3.2 ; Lactates $=3.4$ vs. 2.0 respectively; $p=0.05)$. By nonparametric analysis, IL6 levels were significantly changed in $\mathrm{A}(\mathrm{P}=0.021$ from $\mathrm{T} 1$ at $\mathrm{T} 5)$ with an increase at T2 after LVAD implantation compared to B. Only in A, a significant $(\mathrm{P}=0.027)$ increase of $\mathrm{Neo} / \mathrm{C}$ was observed.

Conclusion: These data showed that patients with unfavourable outcome showed a more pronounce inflammatory response and monocyte/macrophages activation after LVAD implantation, contributing to MOFS development.

\section{Small Intestine Capsule Endoscopy in Magnetic Suspended Axial Left Ventricular Assist Device Patient}

A. Garatti, G. Bruschi, C.M. Girelli, C. Russo, M. Lanfranconi, T. Colombo, E. Vitali

A.O. Niguarda Ga' Granda; Dipartimento 'A.De Gasperis', Milano, Italy

Background: Gastrointestinal bleeding is not uncommon in critically ill patients requiring intensive support and LVAD therapy, and seems to happen more frequently when continuous axial flow pumps are implanted. Capsule endoscopy (CE) is a new promising technology particularly useful in evaluating small bowel diseases. We report our experience with CE in a patient supported with Incor LVAD.

Case Report: A 45-year-old man with dilatative cardiomyopathy was implanted with Incor axial flow magnetically levitated bearings LVAD as bridge to transplant. The device was implanted via a median sternotomy and the immediate postoperative recovery was uneventful. However, during the subsequent course of LVAD support, multiple episodes of GI bleeding developed. Extensive evaluation with endoscopy showed no active bleeding lesions in the upper digestive tract nor in the colon. Beside, catheterization of superior and inferior mesenteric artery failed to reveal active bleeding lesions or arteriovenous malformations of small bowel. Capsule endoscopy of the entire digestive tract was then performed, failing to reveal any active bleeding lesions. No magnetic interference between devices occurred. Antiplatelet therapy was then stopped; no GI bleeding occurred during the remaining period of LVAD support. After 185 days of support, successful orthotopic cardiac transplantation was performed.

\section{6 \\ Ischemic Mitral Valve Repair with Future Band Ring: Early Clinical and Ecocardiographic Follow-up \\ F. Corti', F. Sangalli, F. Formica, O. Ferro, L. Colagrande, J.F. Sciuchetti, G. Paolini \\ Department of Cardio-Thoracic Surgery Science, Cardiac Surgery Clinic, San Gerardo Hospital, Milano-Bicocca University, Via Pergolesi, Italy}

Background: Severe coronary artery disease can be complicated by ischemic mitral regurgitation (IMR). Mitral valve repair is accepted as the procedure of choice to correct mitral regurgitation due to its numerous advantages over replacement and the choice of the appropriate prosthetic ring is the key factor in the efficacy of correction. The aim of the study is to verify the efficacy and safety of the Future Band (FB) (Medtronic Inc., Minneapolis, MN) ring, a partial semirigid band created to combine both remodelling and flexibility.

Methods: 30 patients with pure IMR underwent coronary revascularization and mitral valve annuloplasty with the FB ring. Clinical and echocardiographic data were scheduled for each patient before surgical repair, before discharge and during follow up. 
Results: All patient survived surgery and were discharged from hospital in the $4 \pm 1$ postoperative days. There were 3 late deaths but only one death was due to cardiac related events leading to a freedom from valve-related events of $96.6 \%$. Mean preoperative New York Heart Association (NYHA) class decreased from $2.7 \pm 0.4$ to $1.1 \pm 0.3(\mathrm{p}<0.005)$ at follow up. Echocardiographic data showed a statistically significant reduction in the grading of MR $(p<0.0001)$ and of left atrial dimension $(\mathrm{p}=0.027)$.

Conclusion: Our data suggests the clinical and echocardiographic safety and efficacy of the FB ring.

\section{7}

\section{Post Surgical Chronic Wound Healing with Allogeneic Platelet Gel-Enhanced: Initial Experience}

\author{
O. Ferro, M. Gianoli, F. Porta, F. Corti, F. Formica, \\ L. Colagrande, J.F. Sciuchetti, G. Paolini
}

Department of Cardio-Thoracic Surgery, Division of Cardiac Surgery, San Gerardo Hospital, Milano-Bicocca University, Via Pergolesi, Italy

Background: Many studies report that several platelet-derived growth factors (PDGF) have shown to induce fibroblast and mesenchimal cell proliferation in vitro. We investigated the release of PDGFs and transforming growth factor (TGF- $\beta 1$ ) in freshly prepared and in frozen/thawed samples to assess the improvement of chronic wounds healing rate.

Patients and Methods: Since October 2003 to February 2005, we investigated the use of allogeneic platelet gel (PG) to treat chronic surgical wounds. Ten patients ( 4 males) with $>30$ days non-healing wounds were enrolled. Each patient signed a consent form. Four patients had a dehiscent sternal wound and six suffered for postsaphenous vein graft harvesting chronic ulcers. Eight of them were diabetic and two had chronic peripheral vasculopathy. In four patients, infection was documented by microbiological tests. During the treatment period, we stopped systemic antibiotic therapy. PG was prepared using platelet concentrates (PC) and plasma from healthy blood donors, all fulfilling Italian Law requirement for blood donation. Twelve $35 \mathrm{~mm}$ Petri dishes containing pre-activated PG were obtained from each PC. Four to six dishes were used in each wound dressing according to wound surface, and the remaining were frozen at $-80^{\circ} \mathrm{C}$ for subsequent use after thawing. Every patient received one gel application weekly: first, the wound site was deterged with normal saline; second, the PG dressing was applied to cover the wound; third, a soaked sponge with ialuronic acid $(2 \mathrm{mg})$ was used to isolate the wound surface. Isomers PDGF AA, AB and $\mathrm{BB}$ and TGF- $\beta 1$ were determined in freshly (truncated at 250 words).

\section{8}

Pitfalls of Percutaneous Mitral Annuloplasty: An Anatomical Study of Human Coronary Sinus and Its Relation with Mitral Valve Annulus and Coronary Arteries

\author{
F. Mangia, D. Maselli, L. Bellieni, P. Bajona, F. Chiaramonti, \\ M. Croccia, L. Arcieri, G. Minzioni \\ Dipartimento cardiotoracico Osp.Cisanello, Pisa, Italy
}

Background: In order to allow performance of 'stand alone' mitral annuloplasty with a minimal invasiveness, percutaneous techniques consisting of delivery, into coronary sinus (CS), of devices intended to shrink the mitral valve annulus, have been recently tested in animal models. These techniques exploit the anatomic proximity of CS and mitral valve annulus. We sought to determine qualitative and quantitative anatomic relationship between $\mathrm{CS}$ and surrounding structures in human heart.

Methods: Distance of CS from mitral valve annulus and relationship between CS and surrounding structures were studied in 61 excised human heart.

Results: Maximal distance from CS to mitral valve annulus was found to be up to $19 \mathrm{~mm}$ (mean $9.7 \pm 3.2 \mathrm{~mm}$ ). A Diagonal or ramus branch, main circumflex artery or its branches were located between anterior interventricular vein/coronary sinus and mitral valve annulus respectively in 16.4 and $63.9 \%$ of cases.

Conclusions: Surgical anatomy suggests that, in human being, $\mathrm{CS}$ is located behind left atrial wall at a significant distance from mitral valve annulus. Percutaneous mitral annuloplasty devices probably shrink the mitral valve annulus only by an indirect traction mediated by the left atrial wall, a theoretical risk of compressing coronary artery branches exists. Chronic studies are needed to address this problem and determine long term efficacy of such methods.

\section{9}

\section{Intra-Aortic Balloon Pump: Prophylactic Treatment for High Risk Patients Undergoing Coronary Artery Bypass Grafting}

A. Miceli, B. Fiorani, G. Melina, U. Benedetto, R. Bianchini, A. Roscitano, F. Capuano, F. Barberi, E. Merico,

T.H. Danesi, E. Tonelli, R. Sinatra

Cardiochirurgia Ospedale S. Andrea, Roma, Italy

Introduction: Intra-aortic balloon pump (IABP) is a well-established treatment for postcardiotomy low cardiac output syndrome. The beneficial effects of IABP prophylactic insertion in high risk patients undergoing $\mathrm{CABG}$ is still controversial. Aim of this study was to evaluate whether preoperative IABP improves the early outcome of such patients.

Methods: From May 2004 to January 2006, a total of 39/346 patients (11.2\%) undergoing CABG had IABP support. Among these patients, IABP was inserted prophylactically in 17/39 (44\%), according to Christensons criteria.

Results: Mean age was $58.7 \pm 11$ years (range 40-84) and the mean preoperative ejection fraction $(\mathrm{EF})$ was $0.38 \pm 0.1$. The mean 
logistic EURO score for these patients was 9.3\%. The observed 30day mortality rate was $6 \%$ (1/17 patients). At discharge mean EF improved to $0.46 \pm 0.1$ ( $\mathrm{p}<0.001$ vs. preop.). There were no major complications related to IABP insertion.

Discussion: In our experience, prophylactic IABP treatment for high risk patients undergoing $\mathrm{CABG}$ shows a lower mortality rate when compared to the predicted risk, even if not statistically significant. A larger number of patients and possibly a randomized trial are needed to confirm these findings.

\section{0}

Traumatic Rupture of the Thoracic Aorta: Impact of Endovascular Stent-Graft Treatment in Emergent and Urgent Cases

\section{Botta', A. Dell' Amore', S. Turci', K. Buttazzi², E. Pilato', D. Pacini ${ }^{1}$, R. Di Bartolomeo ${ }^{1}$, R. Fattori ${ }^{2}$ \\ ${ }^{1}$ Departments of Cardiac Surgery, ${ }^{2}$ Radiology, University of Bologna, Bologna, Italy}

Introduction: Traumatic rupture of the thoracic aorta is a lifethreatening condition which can lead to immediate death in 75-90\% of cases. Conventional surgery for traumatic aortic transection is still associated to high morbidity and mortality rates. Endovascular stent graft treatment has been proposed as an alternative to open repair in traumatic ruptures.

Methods: From 1997 to 2006, 25 patients were referred to our institute for traumatic aortic rupture following road or occupational accidents. Endovascular procedures were performed within $24-48 \mathrm{~h}$ from symptoms appearance in 5 patients, within $48 \mathrm{~h}-14$ days in 8 patients and within 1 month in other 12 patients. CT-scan/MRI were diagnostic in all patients.

Results: Technical success was obtained in all patients. There were neither intraoperative nor perioperative deaths. No surgical conversions and paraplegia occurred. Cerebellar stroke occurred in 1 patient. The intraoperative angiography and CT-scan performed on discharge showed no endoleaks. At follow up ranging from 1 month to 8 years, there were neither stent related deaths nor complications.

Discussion: Traumatic rupture of thoracic aorta represents a continuing challenge for surgeons and interventistic radiologysts. Operative timing and management of this complex condition is nowadays matter of debate. Stent graft repair can be a less invasive, attractive and rational treatment option in descending aorta traumatic rupture. Our experience has shown a high percentage of technical success and low perioperative and midterm morbidity and mortality rates, even in high risk patients.
261

\section{Bipolar Radiofrequency Ablation of Atrial Fibrillation: Three Years of Experience}

B. Claysset, E. Mikus, L. Foteini, C. Savini, S. Martin-Suarez, E. Pilato, G. Marinelli, R. Di Bartolomeo

Department of Cardiac Surgery, University of Bologna, Bologna, Italy

Objectives: The Cox's maze procedure has been demonstrated to be the best procedure in curing Atrial Fibrillation (AF). Many sources of energy have been proposed to reproduce the same surgical lesions. Radiofrequency (RF) energy is nowadays the most widely utilized technique of ablation. The aim of this study is to present our experience by using bipolar RF ablation as surgical treatment of AF.

Material and Methods: Beginning May 2003, 120 consecutive patients underwent an ablation procedure with bipolar radiofrequency energy. Preoperative AF was permanent in $95.6 \%$ of patients. In all patients a concomitant operation was performed. In $32.4 \%$ of cases, concurrent operation was complex as pluri-valvular surgery or ascending aorta surgery. All patients underwent bilateral pulmonary vein isolation and excision of the left atrial appendage. Connecting lines between right and left pulmonary veins were always performed.

Results: In hospital mortality was of $2.5 \%$. Causes of death were not related to the ablation procedure. After the procedure $85.3 \%$ of patients were on sinus rhythm. Incidence of AF recurrence was of $16.2 \%$. At a mean follow-up time of $10.8 \pm 6.6$ months ( $\min 0.2-\max$ 21.6 ) the $88.1 \%$ of patients are on sinus rhythm.

Conclusions: Bipolar radiofrequency ablation can be used to replace the surgical incisions of the Cox maze procedure. Technically it is reproducible, easy and safe; sinus rhythm restoration rates are really satisfactory. We advise the use of this technology when atrial fibrillation is associated to a surgical cardiac pathology.

262

\section{Pulmonary Endoarterectomy Without Circulatory Arrest and Deep Hypothermia: Single Center Experience}
A. Dell'Amore, E. Pilato, S.M. Suarez, L. Botta, E. Mikus, P.M. Mikus, S. Pastore1, L. Di Marco,
G. Arpesella

Department of Cardiac Surgery, S.Orsola-Malpighi

Hospital, University of Bologna, Via Massarenti,

${ }^{1}$ Department of Cardiac Surgery, Anaesthesiology

S.Orsola-Malpighi Hospital, Bologna, Italy

Background: The current surgical strategy for pulmonary endoarterectomy (PEA) involves the use of extracorporeal circulation (ECC) and profound hypothermia $\left(18-20^{\circ} \mathrm{C}\right)$. The aim of the present study was to test the feasibility of a novel strategy of extracorporeal circulation, which could prevent bronchial back bleeding and allow a bloodless operating field, avoiding the risks associated with profound hypothermia and $\mathrm{CA}$ in patients undergoing pulmonary endoarterectomy. 
Methods: We present our clinical experience in eleven consecutive patients who underwent pulmonary endarterectomy with a different strategy of extracorporeal circulation, which permits a bloodless operating field, without the need for deep hypotermic circulatory arrest.

Results: We were able to perform pulmonary endoarterectomy avoiding circulatory arrest and deep hypothermia without scarify the effectiveness of the procedure.

Conclusion: The initial encouraging results have convinced us to apply systematically this technique in the cases operated in our center, even though further investigations are necessary to fully examine this technique.

\section{3}

\section{Endoscopic Vein Harvesting Reduces Recovery Time in Patients with BMI $>\mathbf{3 0}$ and Diabetes Mellitus}

\author{
A. Montalto, F. Ranocchi, G. Casali, F. Musumeci \\ S. Camillo, Roma, Italy
}

Introduction: The traditional technique of vein harvesting requires an extensive surgical incision associated with healing difficulties especially in patients affected by obesity $(\mathrm{BMI}>30)$ and diabetes with insulinic treatment.

Methods: We studied 70 patients randomized in two groups: group A (open technique); group B (endoscopic vein harvesting). Inclusion criteria were: indication to coronary revascularization; BMI $>30$ (men), >34 (women), more than 10 years history of insulin dependent diabetes mellitus. The two groups were similar in age, sex, number of by-pass effected, length of vein used.

Results: We obtained a significative difference between the two groups about recovery time, healing complications, mobilization time, with better results in group B.

Discussion: The healing difficulties are associated with longer recovery time and recovery costs. In our opinion the endoscopic vein harvesting can be considered a good choice in patients with high risk of complications.

\section{Laparoscopic Surgery}

\section{4}

\section{Incisional Hernia: Laparoscopyc Approch in Emergency}

M. Grande, C. Nigro, G. Galatá, F. Rulli, M. Villa, G. Attiná, A.M. Farinon

Department of General Surgery; Operative Unit of Laparoscopic Surgery, University of Tor Vergata, Hospital Tor Vergata, Rome, Italy

Introducion: Incisional hernia represents a frequent post-operative complication, $2-5 \%$ of all laparoscopys with peak of incidence that can achieve $50 \%$ in case of post-operative infections. The laparo- scopic repair at an incisional hernia has been demonstrated an effective and safe method with a low incidence of conversion and a substantial reduction of the hospital stay. The use of laparoscopic method has produced a substantial reduction of second repairs that passed from $25-52 \%$ to $3.4-9 \%$. Besides this procedure has shown to be particularly useful in case of the relapse incisional hernia.

Methods and Results: The laparoscopic approach has considered very useful also in patients with acute abdomen. We present the clinic case of a 45-year-old, obese and cardiopathic women, presented at emergency department complaining of approximately $12 \mathrm{~h}$ of acute progressive in epigatric quadrant pain. Phisical examination results were notable for not reducible incisional hernia. Laparoscopic repair was performed in emergency; the prosthesis (DualMesh of $19 \times 14 \mathrm{~cm}$-Tyco) was placed intraperitoneally and fixed with titanium spiral tacks (Protack). Operative time was $85 \mathrm{~min}$ and the hospital stay was 5 days. The patient to the follow up appeared in good state of health. Not recurrent in the short term.

Discussion: Laparoscopic incisional hernia repair is the optimal surgical treatment. A laparoscopic repair appears to shorter postoperative pain, hospital stay and decrease recurrence rates as compared to open incisional hernia repair.

\section{5 \\ Chronic Sigmoid Volvulus Associated with Dolichomegacolon: Report of a Case}

\section{Grande, C. Nigro, G. Galatá, G.M. Attiná, A.M. Farinon \\ Department of General Surgery, Operative Unit of Laparoscopic Surgery, University of Tor Vergata, Hospital \\ Tor Vergata, Rome, Italy}

Introduction: Sigmoid volvulus occurring with megacolon is an uncommon cause of bowel obstruction and various approaches to treatment have been proposed. Sigmoid volvulus is the third most common cause of colonic obstruction after cancer and diverticulitis. Etiologic factors include anatomic variation, chronic constipation, neurologic disease and megacolon. Accurate diagnosis is essential for optimal management. Most patients can be managed by nonoperative decompression, whenever there are no compromised intestines, followed by elective colic resection. When emergency laparotomy is necessary, resection is the best procedure.

Methods: We report herein a case of chronic sigmoid volvulus associated with dolicochomegacolon in which only the surgery finding allowed us to confirm.

Results: A 74-year-old women was admitted to the 'Tor Vergata' Hospital with a story of neuropsychiatric disorders, intermittent abdominal pains with obstinate stipsi. Phisical examination: abdomin pain, especially on hypogastrium, distension, severe costipation, peristalsis absent. In consideration of the clinical picture and the X-ray imagings (present of omega loop sign) an explorative laparotomy was performed. It revealed a distension of left colon and sigmoid volvulus in patient with dolichomegacolon. Operative detorsion of sigmoid volvulus and left emicolectomy was performed.

Discussion: The primary prevention is not possible. The surgical treatment becomes necessary in present of considerable dilatation with loss of the motility and the hope of preventing recurrent volvulus who present high mortality (20-30\%). 


\section{6}

\section{Laparoscopic Ventral Hernia Repair}

P. Bisagni, P. Baccari, C. Staudacher

Osp San Raffaele, Milano, Italy

Background: Postoperative ventral hernia is a common problem $(2-11 \%$ after abdominal surgery); recurrence after traditional repair is high even using a mesh (25\%). Laparoscopy has been recently suggested in ventral hernia repair as a safe and feasible method with shorter postoperative hospital stay, lower complication and recurrence risk.

Methods: From 2002, 114 patients with mean age of 60.6 (29-81; 60 Male, 54 Female) underwent laparoscopic ventral hernia repair. Laparoscopic repair was performed using 3-4 ports laterally to the fascial defect. Intraperitoneal onlay mesh technique was used with transfascial stapler fixation of the mesh.

Results: The mean ventral defect dimension were 112.7 (28.2-471) $\mathrm{cm}^{2}$. Mean BMI was 31.3 (22-41). In 19 cases (16.7\%) it was a recurrent ventral hernia. The majority of the defect were in the midline position $(88.6 \%)$. Conversion rate was $2.6 \%$ (3 patients), mean operative time was $78 \mathrm{~min}(40-210)$. The mean mesh dimension was 282.8 (78.5-647.6) $\mathrm{cm}^{2}$. Intraoperative bowel injury were 3 (2.6\%). Morbidity rate was $8.8 \%$. Mean postoperative hospital stay was 3 days. Recurrence rate is $1.7 \%$ ( 2 patients; mean follow-up 20 months).

Conclusions: Laparoscopic ventral hernia repair is feasible and safe with a shorter hospital stay.

\section{7}

\section{Laparoscopic Approach in Emergency Abdominal Surgery}

\section{A. Biancafarina, G. Ceccarelli, A. Bartoli, A. Spaziani, D. Conti, R. Bellochi, L. Casciola \\ Dipartimanto di Scienze Chirurgiche, Policlinico Umberto I, Roma, Italy}

Introduction: Several studies demonstrate that abdominal emergencies can be operated on through the laparoscopic approach. The approach can be diagnostic laparoscopy, surgery assisted by laparoscopy or laparotomy directed according to the findings of the laparoscopy. In the absent of any specific contra-indications for the specific laparoscopic procedures (haemodynamic instability or high operating risk, ASA IV), many abdominal diseases requiring emergency surgery can be performed with the laparoscopic approach: appendicitis, acute colecistitis, gastro-duodenal perforations, occlusions of the small intestine, abdominal traumas.

Methods: From January 1996 to January 2006, 495 patients underwent to laparoscopic approach in emergency: 327 patients for appendicitis, 63 for intestinal occlusion, 47 patients for gastroduodenal perforation, 12 patients for intestinal perforations, 28 patients for acute pelvic disease, 18 patients for abdominal trauma.

Results: The laparoscopic approach we allows to perform a correct intraoperative diagnosis in all cases. In this study were analyzed the rate of conversion, the median operative time, the median of post-operative hospital stay, the intraoperative and post-operative complications.

Discussion: With a correct selection of patients and the appropriated experience of the surgical team the results of laparoscopic treatment in emergency abdominal surgery are excellent. They are better than open surgery in relation to less infection of the wound, complications, hospital stay and postoperative pain.

\section{8}

\section{Laparoscopic Right Colonic Resection for Cancer}

\author{
P. Bisagni, S. Di Palo, E. Orsenigo, A. Tamburini, \\ C. Staudacher \\ Osp San Raffaele, Milano, Italy
}

Background: Role and technique of laparosopic right colectomy for cancer are still controversy. In the video we present the surgical technique adopted in our Institution.

Methods: Hasson trocar is introduced in left flank. We introduce 2 others ports. We identify the duodenum and prepare the right colonic vessels origin sectioning between clips or with EndoGIA. The mesocolon is sectioned and Toldt's fascia is mobilized (we look for gonadic vessels and urether which stay in retroperitoneal space, covered by Gerota's fascia). We prepare and section the ileocolic vessels and the last ileal loop. We go on sectioning the parietal colonic junction and mobilizing the right colonic angle. We divide the gastrocolic ligament and prepare transverse colon, sectioning the right branches of middle colonic vessels. We perform a $6 \mathrm{~cm}$ incision and section the transverse colon completing the resection. Ileo-colic anastomosis is performed with double layer running suture from the incision.

Results: $\mathrm{N}^{\circ}$ of right colonic resection (1998-2005): 182. Conversion rate: $4.5 \%$. Mean operative time: $141 \pm 41.6 \mathrm{~min}$. Mean nodes harvested: 18.7. Mean hospital stay: $8 \pm 2$ days, morbidity: $10.6 \%$, no mortality.

Conclusions: A good laparoscopic technique makes the laparoscopic right colonic resection safe and oncologically correct with a short postoperative stay.

269

Laparoscopic Ventral Hernia Repair: Advanced Surgery or Training Experience?

\section{Bencini, M. Paolina Mazzetti, R. Lulli \\ Chirurgia Generale, Ospedale Misericordia e Dolce, Prato}

Introduction: Since the nineties, some advocated the laparoscopic route in the correction of ventral hernias, due to reduction of morbidity and recurrences.

Methods: After a learning curve completed during the resident program, 20 patients (10 males and 10 females) with ventral hernias were operated in a large community hospital (2004-2006). Mean age of 58 years (30-74). Obesity (BMI > 28) was present in $8(40 \%) .12$ 
$(60 \%)$ had an incisional hernia, whereas $8(40 \%)$ a primary hernia. Defect size varied from 20 to $375 \mathrm{~cm}^{2}$ (mean 93 ). We used three trocars to reduce the hernia, and to displace the mesh (ePTFE). Four sutures gave adequate orientation, while fixation was obtained using a double circular line of helicoidal clips.

Results: Mean operation time was $112 \min (50-225)$. We had no major operative complications, but we collected 1 serious respiratory distress (HDU monitoring 24h). Analgesic requirement was small. Mean length of stay was 4 days (2-10). Seroma was detected in 1 (5\%) patient, but resolved without further treatment. No recurrences occurred after a mean follow-up of 12 months (1-27).

Discussion: Patients undergoing laparoscopic ventral repair are expected to have less complications, faster recovery and at least same recurrences, as compared to open traditional surgery, although very few evidence-based articles had been published. Moreover, some surgeons consider this as an advanced operation, due to technical difficulties and time consumption. Nevertheless, our good results suggest that a short specific training experience may be completed during the resident ship, and the experience developed in large community hospitals.

\section{0}

Laparoscopic Total Mesorectal Excision (FILM)

P. Bisagni, S. Di Palo, E. Orsenigo, A. Tamburini, C. Staudacher

Osp San Raffaele, Milano, Italy

Background: We present the surgical technique of lap TME adopted in our Institution.

Method: We introduce Hasson and 4 ports. The inferior mesenteric vein is identified and Toldt's fascia divided. Then we continue to inferior mesenteric artery (IMA), sectioned once ureter is identified. The mobilization of splenic flexure is then completed. Using the IMA stump as a guide we divide mesorectal from presacral fascia. Dissection of the mesorectum is performed under direct vision using sharp technique. Particular attention is done to identify superior hypogastric plexus and right and left hypogastric nerves. The inferior hypogastric plexus is preserved by sectioning medially laterals ligaments of the rectum. Then we go on in front of Denonvillier's fascia. In all the phases we have great attention to preserve the integrity of visceral pelvic fascia. Once mesorectal dissection is completed, the rectum is divided at the pelvic floor. A $5 \mathrm{~cm}$ incision is made and the colon resected. After closing the incision, we laparoscopically perform a Knight-Griffen anastomosis.

Results: Conversion rate in 108 patients $12 \%$, morbidity $29.6 \%$ (no death), anastomotic leak $14.8 \%$, reoperation $6.5 \%$, hospital stay 10.1 days, lymphnodes sampling 14.3 , local recurrence rate $6.4 \%$ (median-follow-up 36 months).

Conclusion: Laparoscopic TME is a safe option and can be performed in the majority of patients (truncated at 250 words).

\section{1}

Video-Thoracoscopy and Laparoscopy in the Treatment of Patients with ThoracoAbdominal Trauma

\author{
G. Ceccarelli, A. Biancafarina, A. Batoli \\ Divisione Chiruirgia Generale Vascolare e Mininvasiva, \\ Spoleto
}

Purpose: The purpose of this study is to assess the role of emergent laparoscopy and thoracoscopy as a diagnostic and potentially therapeutic modality in patients with thoraco-abdominal trauma. Diagnostic laparoscopy and thoracoscopy provides important information and is accompanied by improved diagnostic accuracy, reduction of non-therapeutic laparotomy and thoracotomy rates; reduction of post-operative morbidity and faster recovery.

Method: A 4-year period (April 2002-2006) retrospective review of a Level. Civil Hospital was made. The informations regarding patients who had operations for thoracic and abdominal trauma was abstracted, mechanism of injury, operative interventions, and patient outcomes were examined.

Result: There were 384 thoraco-abdominal trauma admissions, of which 47 had abdominal explorations for blunt (85\%) and penetrating $(15 \%)$ trauma; only 5 patients with thoracic trauma were operated ( 4 for penetrating and 1 blunt trauma), in 21 cases a simple drenage was enough. In the abdominal trauma, laparoscopy was performed in 18 cases and in 6 cases the conversion was necessary. Thoracoscopy in 2 cases. Laparotomy was avoided in $56 \%$ of these patients. There were no deaths in the laparotomy/thoracotomy and in the laparoscopic/thoracopscopic groups.

Conclusion: Laparoscopy in thoracoabdominal trauma is a safe method for the evaluation and treatment of selective blunt and penetrating abdominal injuries in hemodynamically stable patients. The conversion rate in high but when it is possible to perform the mininvasive treatment it reduces the morbidity of a negative laparotomy/ thoracotomy.

272

\section{The Fibrin Sealant Use for Mesh Fixation in Laparoscopic Transabdominal (TAPP) Hernia Repair}

G. Ceccarelli, A. Spaziani, A. Biancafarina

Divisione Chiruirgia Generale Vascolare e Mininvasiva, Spoleto

Background: Laparoscopic treatment of inguinal hernia with TAPP (trans abdominal pre peritoneal) technique is largely performed in our experience since about 14 years. The synthetic mesh used is polypropylene. Laparoscopic repair offers more rapid recovery than with the traditional open approach, and other advantages. However, injury to the nerves of the lumbar plexus with subsequent chronic pain has reported. These complications may be inherent to the use of staples for fixation of the mesh.

Methods: In our experience during the last 14 years, 2.870 consecutive laparoscopic hernia repairs were performed using 
laparoscopic approach (always transperitoneal TAPP technique). We give great importance to mesh fixation, to prevent recurrences; generally we use titanium clips, recently we have used in 53 cases, fibrin sealing (Tissucolâ), this new fixation method may be very interesting as it may reduce post-operative pain due to nerve irritation and potential vascular complication.

Results: The objective of this paper was to show our preliminary results using this new technique comparing two homogeneous groups of 53 patients. Post-operative pain, return to normal activity and complications were controlled.

Conclusions: Bilateral inguinal hernia, recurrences, associated abdominal disease are the main indications for laparoscopic transabdominal repair. The TAPP technique is considered more difficult with higher risk of serious complications in respect of visceral (especially bladder) and vascular injuries. However the data suggests less persisting pain, a faster return to usual activities following laparoscopic repair. In our opinion fibrin sealing may reduce the risks post-operative pain and other complication as incisional hernia or vascular.

\section{3}

\section{Laparoscopic Resection for Rectal Tumors}

U. Elmore, S. Bona, U.F. Romario, M. Zago, R. Rosati

Unità Operativa di Chirurgia Generale e Mininvasiva, Istituto Clinico Humanitas, Via Manzoni 56, Rozzano, MI

Introduction: We report our experience in laparoscopic surgery for rectal tumors with regard to perioperative course and medium term oncologic results.

Methods: Since 1997, 71 patients with rectal tumor (42 M, $29 \mathrm{~F})$ were candidate to laparoscopic resection. Tumor location was: low $(12$ patients $=16.9 \%)$, middle $(25$ patients $=35.2 \%)$ and upper rectum (34 patients $=47.8 \%)$.

Results: Conversion rate was $15.4 \%$ (11 patients), mostly for technical reasons or tumor size. Procedures completed laparoscopically were 57 anterior resections, 2 abdominoperineal resections, 1 Hartmann procedure. Median duration of laparoscopic procedure was $255 \mathrm{~min}$. Major complications (requiring reoperation) occurred in 12 patients (16.9\%): 8 had anastomotic leak, 3 had bowel perforation and 1 had ischemia of the transposed colon. Reoperation consisted of ileoor colostomy (10 patients), Hartmann procedure (1 patient), redo anastomosis (1 patient) and laparoscopic suture of small bowel perforation (1 patient). Median postoperative stay in non-complicated patients was 8 days (4-12 days). Among 60 patients who underwent laparoscopic procedures, 15 were stage Dukes A, 26 Dukes B, 16 Dukes $\mathrm{C}$ and 1 Dukes D. In 2 patients who underwent preoperative chemo-radiotherapy no evidence of carcinoma was found on specimen. Mean number of excised lymphnodes was 17 (4-40). Mean length of distal margin was $2.6 \mathrm{~cm}(0.5-10 \mathrm{~cm})$. Out of 54 patients with a follow-up of at least 12 months, 46 are alive and disease-free (mean f.u. 40.4 months); 3 patients with Dukes B1 and B2 tumor showed distant relapse (liver and lung) after 13.3 months.

\section{4}

\section{Surgical Tecnique of VI Subtotal Colectomy with Ceco-Rectal Anastomosis: Case Report}

M.G. Esposito, G. Conzo, U. Brancaccio, A. Palazzo,

F. Stanzione, S. Celsi, A. Livrea

Fourth Division of General Surgery and Endocrinosurgery, Second University of Naples, Italy

Introduction: A total colectomy with ileo rectal anastomosis may be indicated in the treatment of patients, non responding to medical treatment $(5 \%)$. A ceco-rectal anastomosis, after subtotal colectomy, might reduces the complications related to the total colectomy.

Case Report: A 20 years old young man affected by Chronic constipation from slow colonic transit, paradox diarrhoea, severe constipation, encopresis, and colonic dilatation. In 1995, the patient underwent internal sphincterotomy for erroneous diagnosis of Hirshprung's Disease. The instrumental diagnostic preoperative work up confirmed a chronic constipation from slow colonic transit; anorectal manometry showed normal values with significant rectal hypo sensibility. The patient underwent to a video laparoscopic subtotal colectomy and mechanical aniso-peristaltic latero-terminal ceco-rectal anastomosis. The operation was performed by 4 trocars $10 \mathrm{~mm} \varnothing$ in antiTrendelemburg position $\left(30^{\circ}\right)$. After mobilization of left colon, splenic and hepatic flexure, the colon went out from median mini laparotomy $<7 \mathrm{~cm}$ Ø. A subtotal colectomy with a preservation of $10 \mathrm{~cm}$ from ileocecal valve, appendectomy, and mechanical LT ceco- rectal anastomosis (EEAp34 Ethicon Endosurgery R) was performed. A trans rectal catheter Foley $24 \mathrm{Ch}$ was used for anastomotic decompression in the first p.o. days. In X p.o. the patient was submitted to a gastrographin microclisma X-ray, that showed anastomotic integrity.

Discussion: The preservation of ceco, ileum and ileocecal valve allows an inferior number of daily evacuations. In the case reported the postoperative course was satisfactory in term of intestinal function. After two months the patient reported 3 daily evacuations. VL subtotal colectomy with ceco-rectal anastomosis represents a good surgical option.

\section{5}

\section{Laparoscopic Use of Contour CCS}

F.S. Mari, L. Masoni, M. Gasparrini, A. Liverani, A. Milillo, A. Moscaroli

UOC Chirurgia B, Azienda Ospedaliera Sant'Andrea Universita' La Sapienza di Roma, II Facolta' di Medicina e Chirurgia

Introduction: We routinely perform laparoscopic resection of the rectum by a three to four trocar technique. Sectioning of distal rectum by Endo-GIA staplers, especially in the presence of large tumors, is often difficult and requires conversion by a Pfannestiel incision and manual completion of dissection and resection. The Contour CCS stapler, designed for open surgery, closes the bowel on two sides with a triple line of staples while cutting in the mid line at the same time. Differently from Endo-GIA staplers, this curved $5.5 \mathrm{~cm}$ long device closes the rectum quite always in a single shot. 
Methods: We have experimented an original technique based upon the insertion of the Contour CCS through a Lapdisc ${ }^{\mathbb{R}}$. A 6-7 cm suprapubic midline incision allows positioning of the Lapdisc. Gas leak along the shaft of the Contour can be limited by using a damp lint. Following complete preparation of the rectum up to the elevator ani, the stapler is positioned behind the bowel and by simple counterclockwise motion the rectum is included between the Contour jaws.

Result: The technique has been so far used in 8 cases of anterior resection of lower rectum, five patient had undergone previous neoadjuvant treatment by chemo-radiation and in all these cases a 'ghost ileostomy' was prepared. Should an anastomotic leak occur.

Discussion: This result is very promising and we hope that a modificated design with an circular shaft can simplificate this techniques.

\section{6}

\section{Laparoscopic Cholecystectomy in Day Surgery Preliminary: Experience of an University Hospital}

\author{
F.S. Mari, L. Masoni, M. Gasparrini, A. Liverani, \\ M. Cosenza, A. Milillo, M.L. Russo \\ UOC Chirurgia B, Azienda Ospedaliera Sant'Andrea \\ Universita' La Sapienza di Roma, II Facolta' di Medicina e \\ Chirurgia
}

Laparoscopic Cholecystectomy (LC) represents today the gold standard for the treatment of cholelithiasis. The advantages of LC over the open method are well know, the short post-operative course being between the most relevant. The reported average post-operative hospital stay is 2.6 days. In some Centers of excellence the proposal for LC as a Day Surgery or a One Day Surgery procedure dates back to 1996. Since November 2003, according to commonly accepted guide lines, we have instituted in our Hospital, 'Azienda Ospedaliera Sant' Andrea', a treatment protocol for cholelithiasis in Day Surgery. Our protocol now includes patients up to 65 years of age, without major contraindications for the general anesthesia (ASA I or II) and no acute complications related to gall bladder stones. Patients motivations and home support was also investigated. The day of surgery patients are admitted at 07:00 a.m. We use a 3-trocar technique, intraperitoneal splash of $10 \mathrm{cc}$ of $7.5 \mathrm{mg} / \mathrm{ml}$ Ropivacaine. A short term antibiotic prophylaxis is instituted. At the end of surgical procedure the patient is returned to the ward. By 02:00 p.m. a soup is served and he/she are allowed to walk. At 4:00 p.m. check of serum RBC, total and direct bilirubin, amylase and lipase is performed. At 6:00 p.m., the patient is discharged. Dietary instruction and drug prescription (oral analgesics and PPI) are given, together with the telephone numbers of the operative unit. We present our experience on the first 40 consecutive cases (16 males and 24 females, age ranging between (truncated at 250 words)
277

\section{The Use of Seamguard in Gastric Laparoscopic Surgery}

F.S. Mari, L. Masoni, D. Urbano, A. Liverani, M. Gasparrini, A. Milillo, F. Favi, S. Forte

UOC Chirurgia Generale B, Azienda Ospedaliera

Sant'Andrea, Università 'La Sapienza' di Roma, II Facoltà

di Medicina e Chirurgia

Introduction: Bioadsorbable Seamguard (BSG) is a randomfiber web of ePTFE that is completely absorbed within 6 months or less. The staple-line failure and his bleeding is the most frequent complication of the laparoscopic gastric surgery. This prosthetic device has been used with good results in bariatric and thoracic surgery as staple-line reinforcement allowing to reduce staple-line hemorrage and minimize the risk of leakage. So we believe that BSG may be ideal to use in gastric surgery.

Material and Methods: From June 2004 to April 2006 we used the BSG in 12 laparoscopic gastric resection performed on 7 males and 5 females, age ranging from 50 to 86 years. The procedures performed were 5 gastric wedge resections, 4 partial distal gastrectomies, 3 total gastrectomies.

Results: None clinically relevant anastomotic leakage or bleeding were observed during early postoperative period; at 6 months follow-up none stenosis has been developed. None patient needed of blood transfusion or even surgery. Mean hospital stay was 6.4 days (range 5-8).

Discussion: The use of BSG as a staple-line reinforcement seems safe and effective in preventing anastomotic leakage and bleeding without modification of suture line healing. Low anastomotic complication rate allows hospital stay reduction and leads to the tolerance of cost increasing due to this device.

\section{8 \\ Port-Site and Intraperitoneal Injection of Ropivacaine Does Not Improve Postoperative Pain after Minor Laparoscopic Procedures}

\author{
R. Cazzaniga, N. Botticchio ${ }^{1}$, G.L. Milesi ${ }^{1}$, C. Reguzzi ${ }^{1}$, \\ G. Spina1, C. Ricchiuti \\ ASL Vallecamonica Sebino, BS, U.O. Chirurgia Edolo, \\ ${ }^{1}$ IP BLOCCO OPERATORIO EDOLO, Italy
}

Background: Laparoscopic surgery is a minimally invasive technique associated with reduced postoperative pain. Nonetheless, pain after laparoscopy may be moderate or even severe for some patients. A controversy exists on the effectiveness of port-site and intraperitoneal administration of local anesthetics for postoperative analgesia. The effects of local anesthetics administration were therefore studied in a prospective, double-blind, randomised trial.

Methods: Seventy-two consecutive patients undergoing minor laparoscopic procedures (41 cholecystectomies and 29 appendectomies) under general anesthesia plus trocar-site and intraperitoneal local anesthetics or saline were studied. Patients present a mean age of 44.8 years (DS \pm 19.6 ) and $29 \%$ are male. Patients were randomly 
assigned to one of two groups of 36 patients each. Group 1 received $25 \mathrm{mg} /$ port and $100 \mathrm{mg}$ intraperitoneal of ropivacaine, group 2 (placebo) received $0.9 \%$ saline solution. Mean operation time was $67^{\prime}$ (Max 110' Min 30'). All patients received ketorolac $30 \mathrm{mg}$ i.v. at the end of procedure. Postoperative pain was assessed with VAS score during $48 \mathrm{~h}$ and further requests of analgesic drugs was registered. Two patients converted in open laparotomy ( 1 cholecystectomy and 1 appendectomy) and 1 patient that underwent early reoperation for postoperative bleeding, were excluded from the study.

Results: Pain was similar in both groups. No significant difference between ropivacaine and placebo were present for up to $48 \mathrm{~h}$. Analgesic consumption was the same. No side-effects occurred. Non significant differences were related to the age of the patients ( $\pm 50 \mathrm{aa}$.), to the procedure (cholecystectomy vs. appendectomy) or to the operatory time.

\section{9}

\section{Laparoscopic Resection of Rectosigmoid Endometriosis}

N. Osman, P. De Nardi, M. Carlucci, S. Ferrari ${ }^{1}$, C. Staudacher

Department of Surgery, 'Department of Obstetrics and Gynaecology, S. Raffaele Scientific Institute, University Vita-Salute, Milan, Italy

Aim: Pelvic endometriosis may involve the bowel wall causing severe bleeding and bowel obstruction. Aim of this study is to describe feasibility of laparoscopic resection of the rectosigmoid endometriosis.

Methods: From January 2004 to December 2005, 10 patients, mean age 32 (range 27-43) with pelvic endometriosis underwent laparoscopic treatment. Indications for surgery were symptoms of intestinal obstruction with cyclic rectal bleeding and pelvic pain. Diagnosis of endometriosis was performed by rectosigmoidoscopy, MRI and EUS.

Results: Laparoscopic rectal resection was performed in 10 cases, 4 were completed by laparotomic conversion; in 2 cases a left annexectomy and in one case a partial ureter resection were added; in 2 cases superficial serosal lesions were ablated with $\mathrm{CO}_{2}$ laser; a protective colostomy was performed in 2 cases. Complications occurred in 3 cases: 2 transient urinary disfunction and 1 rectovaginal fistulae, requiring a colostomy. At histology there was involvement of bowel muscularis propria in all cases. At a median follow-up of 12 months (range 4-24) all patients had resolution of both occlusion and bleeding, 6 patients also had improvement of pelvic pain.

Discussion: Treatment of symptomatic rectal endometriosis requires bowel resection. Laparoscopic approach can be effective on obstructive symptoms and bleeding. However pelvic colonisation and sclerosis lead to a lack of dissection plane. This make laparoscopic approach technically challenging requiring training and experience.

\section{0}

\section{Laparoscopic Right Emicolectomy (LRE), Our} Technique of Caudo-Cranial Dissection.

\author{
E. Ruggeri, L. Masoni, F.S. Mari, M. Gasparrini, F. Favi \\ Sant'Andrea, Roma, Italy
}

The LRE can be perform with latermedial technique. The difficulties of the direct approach to vessels are rap presented by the identification of the correct dissection plane. In fat patient the second portion of the duodenum isn't easy identifiable while in slim patient the mesentery of the right colon corner is thin and it's easy to fragment. We have developed an original technique of caudo-cranial dissection. The operation start with the ileo-colic vessels section at the origin. Than we open the mesentery and section of the last ileus handle. We continue, after the section of the Houston's ligament, with the identification on the retrocecal plan of Toldt wrap, with a cranial traction of the right colon. In this way we proceeded until the right colic corner following the plan in latero-medial sense towards the liver. When we complete the dissection in the mesenteric side appears the zone of traction of colic right vessels and the anastomotic Henle's venous branch. The section of the ileocolics vessels in lean patient can be executed after the preparation of the retroperitoneum until the third part of the duodenum. We have used this technique in 52 patients: 28 men and 24 women with a medium age of 68.3 years and a medium BMI of 25.6 (22.3-37.4). One patient have an anastomotic compliance 8 day after of operation the admit have a durate of $5.3 \mathrm{gg}$. We introduce a video that illustrates in detail our technique.

\section{Transplantation}

\section{1}

Intraportal Hepatocyte Transplantation in the Pig: A Hemodynamic and Histopathological Study

\author{
R. Ballarin' ${ }^{1}$ D. Neri², M. Masetti ${ }^{1}$, F. Di Benedetto', \\ N. De Ruvo', R. Montalti, A. Romano', G. Guerrini', \\ C. Longo ${ }^{2}$, G. Molteni ${ }^{1}$, M. Spaggiari' ${ }^{1}$ S. Di Sandro ${ }^{1}$, \\ G. Rompianesi ${ }^{1}$, G.E. Gerunda ${ }^{1}$ \\ University of Study Modena-Reggio Emilia, 'Liver and \\ Multivisceral Transplant Center, ${ }^{2}$ Clinica Chirurgica 3, \\ Padova, Italy
}

Background: Hepatocyte transplantation is an attractive treatment for various liver diseases. The intraportale route of transplantation is favored, but little information is available on the possible adverse effects in this technique. We investigated the influence of intraportale loads of hepatocytes on portal, pulmonary, and systemic hemodynamics in 13 pigs.

Methods: Under general anesthesia, pigs were provided with an arterial line, a Swan-Ganz catheter, and two intraportale catheters, one for cell infusion and one for heparin infusion and portal pressure 
measurement. Pig hepatocytes were infused at a rate of 25 million cells/min.

Results: The first six animals were used to develop the infusion technique. In the last seven animals, portal pressure increased linearly with cell load upon infusion of $400-2400 \times 10^{6}$ hepatocytes $\left(r^{2}=0.704 ; \mathrm{P}=0.05\right)$. Portal flow measured by Doppler sonography decreased by $23-66 \%$ below basal values. An inverse linear relationship was found between portal pressure and portal flow $\left(\mathrm{r}^{2}=0.679\right.$; $\mathrm{P}=0.05$ ), portal flow approaching zero for portal pressure $>40 \mathrm{~mm}$ $\mathrm{Hg}$. Pulmonary arterial pressure increased by $11-62 \%$. AST increased up to 10 -fold, and platelets decreased by $22-58 \%$. Hepatocytes-containing thrombi were present in segmental and in smaller portal branches. Hepatocytes were always identified in lung sinusoids $48 \mathrm{~h}$ after infusion, and a small basal pulmonary infraction was found in one animal.

Conclusion: These data suggest that up to $2.4 \%$ of total hepatocyte mass can be infused in this large animal model. However, the risk of significant thrombotic complications should be considered for clinical applications.

\section{2}

\section{Liver Transplantation in Three Sisters Affected by Familial Amyloidotic Polyneuropathy TTR Glu54Lys}

\section{A. Romano', M. Masetti', R. Montalti', F. Di Benedetto', N. De Ruvo ${ }^{1}$, A. Lauro², G. Guerrini' ${ }^{1}$ R. Ballarin'1, G. Molteni ${ }^{1}$, M. Spaggiari ${ }^{1}$, S. Di Sandro ${ }^{1}$, A.D. Pinna ${ }^{2}$, G.E. Gerunda ${ }^{1}$ \\ ${ }^{1}$ University of Study of Modena and Reggio-Emila, ${ }^{2}$ University of Study of Bologna (Liver and multivisceral transplant center)}

Introduction: Familial amyloidotic polyneuropathy (FAP) is an autosomal dominant hereditary disorder caused by mutation in transthyretin (TTR) gene. The most common of TTR-related-FAP type is caused by a substitution of methionine for valine at position 30 of the TTR gene. TTR is mainly synthesized by hepatocytes, so liver transplantation (LT) eliminates the source of the variant TTR molecule and is presently the only known curative treatment. In this paper we describe the first cases of liver transplantation performed for FAP TTRGlu54Lys, a very rare TTR variant.

Materials: Between May 2002 and July 2003 we performed three LT in three sisters affected by FAP TTRGlu54Lys variant. FAP diagnosis was based on compatible neurological symptoms and electromyography signs, family history, location of amyloid in abdominal fat and sural nerve. Symptoms of peripheral axonal neuropathy were graded according to Tashima's classification. Severity of diarrhoea, orthostatic hypotension, routine nerve conduction studies (NCS) electromyography (EMG) and echocardiogram were performed before and after LT.

Results: The three sisters are alive and well with normal liver function after more than 3 years from LT. Only one of them showed progression in neuropathy score. No patients showed improvements in gastrointestinal symptoms in the follow-up period. After LT, Case 1 and Case 3 showed improvement of hypotension, while Case 2 had worsening of symptoms. Eco-cardiography revealed increased thickness in the ventricular septal-wall in all of them.
Conclusion: In our study, FAP TTR Glu54Lys variant and its complications seems to be stopped by LT; longer follow-up its necessary to confirm.

\section{3 \\ Our Experience in 'de novo' Malignancies After Liver Transplantation}

\author{
D. Lorenzin1, G.L. Adani', U. Baccarani', M. Sainz-Barriga1, \\ G. Curro ${ }^{1}$, E. Benzoni ${ }^{1}$, C. Avellini' ${ }^{2}$, F. Bresadola ${ }^{1}$
}

${ }^{1}$ Department of Surgery and Transplantation, ${ }^{2}$ Institute of Pathology, University Hospital of Udine, Italy

Introduction: De novo malignancies after transplantation are a growing problem within solid organ transplant recipients, due to improved survival and longer follow-up and as a consequence of chronic immunosuppression.

Methods: From 1996 to May 2005, 14 de novo malignancies have been diagnosed in 14 recipients of 202 cadaveric orthotopic liver transplant patients surviving more than 12 months after the transplant for an incidence of 7\%. There were 12 male and 2 female. Indications for OLTx were end-stage cirrhosis due to alcohol in 6 cases, HCV in 4 cases, HBV in 2 cases and one case each due to primary biliary cirrhosis and primitive sclerosing cholangitis. Immunosuppression was based on Neoral $(23 \%)$ or Tacrolimus $(77 \%)$ associated with steroids $(100 \%)$ usually discontinued 3 months after transplant. The median elapsed time from transplant to the diagnosis of the de novo malignancies was 37 months (range 12-101 months) for liver transplants.

Results: Gastroenteric cancers were the most common within liver transplant recipients. Oropharyngeal and upper digestive tract tumors were always associated with a history of chronic alcohol consumption in liver recipients. Liver transplant recipients treated for acute rejection had a worst cancer prognosis than patients without rejection (1 and 2 year survival 83 and $63 \%$ vs. 36 and $17 \%, p=0.026$ ).

Conclusions: The estimated 1 and 2 years survival rate for all types of de novo malignancies was respectively 70 and $42 \%$, being 57 and $28 \%$ for solid organ tumors vs. 85 and $64 \%$ for skin cancers and PTLD $(\mathrm{p}=0.43)$ in liver transplants.

\section{4}

\section{Long Term Results of Liver Transplantation} for Hepatocellular Carcinoma in Our Center

\author{
D. Lorenzin1, U. Baccarani', G.L. Adani1, C. Avellini², \\ G. Currò ${ }^{1}$, E. Benzoni' ${ }^{1}$ A. Risaliti' , F. Bresadola ${ }^{1}$
}

${ }^{1}$ Department of Surgery and Transplantation, ${ }^{2}$ Institute of Pathology University Hospital Udine, Italy

Introduction: Liver transplantation (LT) is a treatment for HCC on cirrhosis within defined criteria. Aim of the study is to evaluate a single center experience in LT for HCC with respect to clinical and pathologic staging and long term results.

Methods: From 1996 to 200550 patients underwent LT for HCC. 43 male $(86 \%)$ and 7 female (14\%) mean age was 57 (range 37-67) years old. All patients fulfilled the Milan criteria. HCC diagno- 
sis was based on preoperative imaging and $\alpha$-fetoprotein, no tumor biopsy was performed. At histological examination we discovered 6 $(12 \%)$ incidentalomas and in $8(16 \%)$ cases no HCC was found. Finally we had 42 'true' HCC. Twenty-six patients (52\%) have been downstaged and $10(20 \%)$ upstaged at preoperative imaging; $15 \%$ were pT $1,45 \%$ were pT2, $27 \%$ pT3 and $13 \%$ pT $4 a$. $26 \%$ of cases exceeded the Milan criteria. One patient pT4a with microvascular invasion died of pulmonary metastasis 14 months after transplantation.

Results: No HCC recurrences within the liver were encountered at a median follow-up of 20 months (range 0-80 months). Overall the 1,3 and 5 years estimated survival rate were respectively $83 \%, 77 \%$ and $72 \%$. One, 3 and 5 years estimated survival rate were respectively $87 \%, 75 \%$ and $75 \%$ for $\mathrm{pT} 1$ and $\mathrm{pT} 2$ and $75 \%, 67 \%$ and $67 \%$ for $\mathrm{pT} 3$ and $\mathrm{pT} 4 \mathrm{a}(\mathrm{p}=0.99)$.

Conclusions: Based on our experience LT for HCC has long term results comparable to those without HCC also in the presence of significant number of cases exceeding the Milan criteria at pathological staging.

\section{5}

\section{Urological Complications in Kidney Transplantation: Uretheral Stenosis}

R. Bertelli, B. Nardo, R. Montalti, F. Grammatico, P. Beltempo, V. Pacilè, L. Puviani, F. Neri, D. Prezzi,

M. Tsivian, G. Varotti, G. Fuga, A. Faenza

Dip. di Discipline Chirurgiche, Rianimatorie e dei Trapianti, SSD Chirurgia Trapianti di Rene, Ospedale S. Orsola,

Università di Bologna

Introduction: To evaluate incidence, risk factors, therapy and outcome of uretheral stenosis after kidney transplantation.

Methods: From 1991 to 2004, 895 patients underwent kidney transplantation, of whom 879 single kidney transplantation (836 from cadaveric donor, 43 from living donor) and 16 double kidney transplantation; $848(94.7 \%)$ were first transplants, 47 (5.2\%) were retransplantations. $535(59.8 \%)$ urinary anastomosis were uretherocystostomy and 359 (40.1\%) uretherouretherostomy. Endouretheral stent was positioned in 115 cases.

Results: $26(2.9 \%)$ early uretheral stenosis (EUS, within 3 months from transplant) were observed of which 22 in first kidney transplant and 4 in retransplant $(p=0.06)$. One case of stenosis was observed in living donor transplant. 16 EUS underwent anastomosis recontruction and 10 were treated with cystoscopic endouretheral stent positioning. All the treatments were successful. $6(0.67 \%)$ late uretheral stenosis (LUS) were reported (5 on uretherocystostomy, 1 on uretherouretherostomy), all resolved by treatment ( 3 reoperations, 3 endouretheral stent positioning).

Discussion: The recipient's and donor's parameters, as well as perioperative variables do not seem to be related to uretheral stenosis incidence. We believe that EUS determined by technical factors should be treated surgically; whereas stent positioning is more suitable in LUS.

\section{6}

\section{Urological Complications in Kidney Transplantation: Urinary Fistulas}

R. Bertelli, B. Nardo, R. Montalti, F. Grammatico, V. Pacilè,

L. Puviani, F. Neri, D. Prezzi, M. Tsivian, G. Varotti, G. Fuga,

A. Faenza

Dip. di Discipline Chirurgiche, Rianimatorie e dei Trapianti, SSD Chirurgia Trapianti di Rene, Ospedale S. Orsola,

Università di Bologna

Introduction: To evaluate incidence, risk factors, therapy and outcome of urinary fistulas after kidney transplantation.

Methods: From 1991 to 2004, 895 patients underwent kidney transplantation: 879 single kidney transplantation and 16 double kidney transplantation; $848(94.7 \%)$ first transplant, 47 (5.2\%) retransplantations. $535(59.8 \%)$ urinary anastomosis were uretherocystostomy and 359 (40.1\%) uretherouretherostomy. Endouretheral stent was positioned in 115 cases.

Results: 48 (5.4\%) early urinary fistulas (EUF, within 3 months from transplant) were observed ( 45 on first transplant, 3 in retransplant) all in single kidney transplants. 45 cases were resolved with surgical treatment, 2 with conservative treatment, 1 needed transplantectomy. No correlation was found with perioperative and recipient's parameters. Donor's male sex, trauma or post-anoxic encephalopathy as cause of death increased EUF incidence. Out of $4(0.44 \%)$ late urinary fistulas (LUF) reported: 1 needed transplantectomy, 1 anastomosis reconstruction and 2 were conservatively treated.

Discussion: In our experience EUF are related to male donors or deceased of trauma or post-anoxic encephalopathy, whereas stent presence isn't a protective factor. The incidence of urological complications is similar in both techniques of urinary anastomosis. In our opinion surgical reanastomosis is the treatment of choice in case of EUF.

\section{7}

\section{Surgical Aspects on Double Kidney} Transplantation in Our Experience

\author{
R. Bertelli', V. Pacilè ${ }^{1}$, P. Beltempo ${ }^{1}$, R. Montalti ${ }^{1}$, \\ L. Puviani ${ }^{1}$, G. Cavallari ${ }^{1}$, M. Tsivian ${ }^{1}$, D. Prezzi ${ }^{1}$, F. Neri ${ }^{1}$, \\ G. Fuga' ${ }^{1}$ S. Stefoni ${ }^{2}$, B. Nardo' ${ }^{1}$, A. Faenza ${ }^{1}$
}

${ }^{1}$ Department of Surgery, ICU and Transplantations, ${ }^{2}$ Nephrology, Dialysis and Transplantation Unit, University of Bologna, Italy

Introduction: Double-kidney transplantation (DKT) uses marginal organs not suitable for single transplant. We aimed to verify DKT results in terms of graft and patient survival and complications.

Methods: Between 2001 and 2006 we performed 25 DKT. Recipients' mean age was 62 . The indications were: polycystic disease (3), chronic glomerulonephritis (17), hypertensive nephroangiosclerosis (4), reflux nephropathy (1). Mean HLA A, B, DR mismatches was: 3.6. Donors' mean age was 65,10 with at least one renal function risk factor (hypertension, smoke, diabetes), mean creatinine clearance $78 \pm 30 \mathrm{ml} / \mathrm{min}$. The kidneys were perfused with 
Celsior (mean cold ischemia time: $16 \pm 2 \mathrm{~h}$ ). Biopsy was performed (mean score: 4.2). Immunosuppression included basiliximab, corticosteroids, tacrolimus and mophetil micophenolate.

Results: 16 patients had a good postoperative renal function. 9 had acute tubular necrosis (ATN), 2 of which had no renal function recovery. 1 acute rejection occurred. Urinary complications incidence was $31 \%$; there were 2 surgical revisions for intestinal perforation. Graft and patient survival was 78 and $100 \%, 78$ and $94 \%$ at 3 and 36 months.

Discussion: DKT is a safe way to face organ shortage. The score used in this study is useful to determine whether a kidney should be refused or accepted. Our results are encouraging, but this series is too small to consent a conclusive statement.

\section{8 \\ Percutaneous Transluminal Coronary Angioplasty in Heart Transplant Recipients}

P. Colombo, G. Bruschi, I. Bossi, S. Campisi, P. Silva, A. Garatti, F. Trazza, G. Pedrazzini, M. Frigerio, E. Vitali, S. Klugmann

Cardiology and Cardiac Surgery 'A. De Gasperis', Niguarda Ca' Granda Hospital, Milan, Italy

Background: Cardiac Allograft Vasculopathy (CAV) remains a major factor limiting long-term survival after cardiac transplantation (Htx). Aim of this study was to examine the outcomes of percutaneous coronary intervention (PCI) for localized and diffused CAV.

Methods: At our Institution from November 1985, 753 patients have been heart transplanted, to date $46 \mathrm{Htx}$ patients underwent 52 PCI, resulting in 76 treated lesions. Patients characteristics are reported on Table.

Results: The indication for PCI was progressive asymptomatic coronary vasculopathy in 28 cases $(61 \%)$. Mean time from Htx to PCI was $96.3 \pm 58.3$ months (range 6-239). Multivessel procedures were $18(34.6 \%)$. We used angioplasty only in $17 \%$ lesions and $43 \%$ lesions were treated with DES. Primary success was obtained in $94.7 \%$ lesions with only one periprocedural myocardial infarction. Angiographic follow up was obtained in 48 lesions at $18 \pm 21$ months (range 2-130). We had recoil in 14\% of angioplasty only, restenosis in $45 \%$ of BMS and in $11 \%$ of DES with a successful retreatment in $77 \%$ pf cases. All the patients obtained a clinical follow up in a mean time of $35.3 \pm 38.3$ month (range 1-162). Death occurred in $33 \%$ of patients, hospitalization for AMI in $2 \%$ and for heart failure in $15 \%$.

Conclusions: In Htx recipients, PCI can be performed safely with high rates of primary success. Restenosis rates are still high. DES seems to favorably impact restenosis, even if further studies should be designed.

\section{9}

\section{Combined Heart-Kidney Transplantation: Report on Nine Cases}

\author{
G. Bruschi ${ }^{1}$, S. Campisi ${ }^{1}$, S. Trunfio ${ }^{1}$, T. Colombo', \\ G. Pedrazzini', F. Turazza1 , A. Garatti ${ }^{1}$, G. Di Stefano ${ }^{1}$, \\ A. O. Slim², C.V. Sansalone ${ }^{2}$, M. Frigerio ${ }^{1}$, E. Vitali \\ ${ }^{1}$ Cardiology and Cardiac Surgery 'A. De Gasperis', \\ 2Department of Abdominal Organ Transplantation, \\ Niguarda Ca' Granda Hospital, Milan, Italy
}

Background: Combined heart-kidney transplantation has become a therapeutic solution for patients with coexisting, irreversible heart and kidney failure.

Patients and Methods: Between April 1989 to January 2006 at our Centre, nine patients with cardiomyopathy and severe renal insufficiency, underwent combined heart-kidney transplantation from the same donor. Seven patients were male, mean age at transplant was $45.2 \pm 10.12$ years (range $24-54$ years). Seven patient $(77.7 \%$ ) were undergoing hemodialysis. Seven donors were male, ages ranged from 18 to 69 (mean $29.3 \pm 16.2$ years). Heart and kidney harvestering, preservation and grafting were performed according to the routine technique.

Results: Mean ischemic time was $132.2 \pm 57 \mathrm{~min}$ (range 29-220 $\mathrm{min}$ ) for the cardiac allograft and ranged from 5 to $8 \mathrm{~h}$ for the kidney. Crossmatch was negative in all cases. Hospital mortality was $11.1 \%$, one patient. No documentated early rejection occurred. Prompt recovery of good cardiac function with low inotropic support and immediate diuresis from the transplant kidney occurred in 8 patients $(88.8 \%)$. Renal function normalized in 7 patients between the 7 th and 11th post-operative day. At a mean follow-up of $9.2 \pm 5.2$ years two patients died (at 3 and 6 years after transplant) for neoplasm and acute cerebral event.

Conclusions: Combined cardiac and renal transplantation with allografts from the same donor has acceptable short- and long-term outcomes for patients with coexisting end stage cardiac and renal failure.

290

Intestinal Transplantation in Children A Single Center Study
A. Bussotti ${ }^{1}$, T. Kato², G. Selvaggi², A. Davide², J.J. Gaynor ${ }^{2}$, A. Tzakis ${ }^{2}$
'Department of General Surgery 'Paride Stefanini', University of 'La Sapienza', Rome, Italy; 'Department of Surgery, University of Miami Miller School of Medicine, Miami, Florida, USA

Objective: To describe a single center experience of pediatric intestinal transplantation (Itx) and to provide an overview of the children who underwent this procedure along with their outcomes.

Summary Background Data: Pediatric Itx presents multiple challengers because of the very young ages at which patients require transplantation and their higher susceptibility of infectious complications. 
Methods: We have performed 141 Itx in 123 children with a median age of 1.37 years. Primary grafth included isolated intestine $(n=28)$, liver and intestine $(n=27)$, multivisceral $(n=61)$, and multivisceral without liver $(\mathrm{n}=7)$. Two protocol modifications were introduced in 1998, daclizumab induction and frequent rejection surveillance. In 2001 indications for multivisceral transplantation were expanded, and induction with campath $1 \mathrm{H}$ was introduced.

Results: Actuarial patient survival at 1 and 3 years for Group 1 (January 1994-December 1997, $\mathrm{n}=25$ ), Group 2 (January 1998-March 2001, $\mathrm{n}=29$ ), Group 3a (April 2001-present, daclizumab, $\mathrm{n}=51$ ) and Group 3b (April 2001-present, Campath $1 \mathrm{H}, \mathrm{n}=18$ ), was $44 / 32 \%, 52 / 38 \%, 83 / 60 \%$, and $44 / 44 \%$, respectively $(\mathrm{p}=0.0003$ in favor of Group 3$)$. Severe rejection implied a dismal prognosis (65\% mortality at 6 months). Observed incidence of severe rejection in Groups $1-4$ was $32 \%, 24 \%, 11 \%$ and $11 \%$ respectively. In multivariable analysis, use of multivisceral (with or without liver) transplant $(\mathrm{p}=0.002)$, induction with daclizumab $(\mathrm{p}=0.005)$, patient at home prior to transplant $(\mathrm{p}=0.007)$, and age at transplant $>1$ year $(\mathrm{p}=0.02)$ favorably influenced patient survival. Multivisceral transplant was protective with respect to the mortality rate due to rejection, while an older at transplant was associated with both a lower incidence rate of developing (truncated at 250 words)

291

\section{Endoscopy Findings to Nodulate Immunotherapy in Small Bowel Transplantation}

\section{A. Dazzi, M. Di Simone, A. Lauro, C. Zanfi, A. Amaduzzi, F. Pezzoli, L. Golfieri, G. Ercolani, L. Pironi, A.D. Pinna \\ Chirurgia Generale, Università di Bologna}

Purpose: Graft monitoring after intestinal transplantation is one of the key factors to obtain good results in terms of graft and patients survival. Histology evaluation is actually the gold standard feature to modulate immunotherapy in these patients. Endoscopy findings can assume a relevant role in graft monitoring, especially after the introduction of zoom endoscopy, allowing to see directly villi of the mucosa.

Patients and Methods: We evaluated zoom endoscopy findings compared to histologic patterns.

Results: From our results endoscopy images can match histology responses as well, with good specificity and sensibility. Villi appearance was modified in $100 \%$ of acute rejection episodes, while crypt widening was altered in about $80 \%$ of mild acute rejection episodes.

Conclusion: Zoom endoscopy can be a validal feature to graft monitoring after intestinal transplantation. Graft monitoring requires endoscopy, histologic and clinical findings as well.
292

\section{Liver Transplantation in HIV Patients: Preliminary Results After a 30-months Follow-Up}

\author{
S. Di Sandro, F. Di Benedetto, N. De Ruvo, M. Masetti, \\ R. Montalti, A. Romano, G. Guerrini, R. Ballarin, \\ M. Spaggiari, G. Molteni, G.E. Gerunda \\ Centro Trapianti di Fegato e Multiviscerale, Azienda \\ Ospedaliero-Universitaria Policlinico di Modena
}

Introduction: Between June 2003 and March 20068 HIV+ patients received liver transplantation: $5 / 8$ for HCV-related cirrhosis, $2 \mathrm{HBV}$-related and $1 \mathrm{HBV}-\mathrm{HCV}$-related. 5/8 patients were also affected by hepatocellular carcinoma (HCC).

Methods: All patients were treated with HAART before transplantation, whose interruption at transplant day was restarted an average 8 days thereafter. The HIV viral load before transplant was $<50$ copies $/ \mathrm{ml}$ in all cases, as requested by protocol; CD4 cell count ranged before transplant between $144-530 \mathrm{c} / \mathrm{microl}$. All patients received a cadaver whole liver but 1 (split II-III segment LTx). 3/8 patients died after transplant: one for cardiac tamponade, one MOF, one for Kaposi's visceral sarcoma (overall patients and grafts survival $62.5 \%$ at 30 months). Immunosuppression was based on CsA and steroid weaning for $6 / 8$ patients, $2 / 8$ patients Tacrolimus and steroid weaning.

Results: Infectious complications were due to CMV reactivation in $5 / 8$ cases, and $3 / 8 \mathrm{EBV}$ reactivations; $1 / 8$ had a coinfection with HHV6 and HHV1. All these complications were treated pre-emptively. One patients suffered from a colestatic HCV recurrent hepatitis, treated with pegINF and Riba antiviral therapy.

Discussion: The outcome of liver transplant in HIV patient in our group has been influenced by infections (HCV, CMV, EBV), and Kaposi's sarcoma. The risk of cardiovascular complications (maybe related to lypo-distrophy) must be taken in due account.

293

\section{Radiologic Imaging of the Transplanted Bowel}

A. Pecchi', M. De Santis' ${ }^{1}$ F. Di Benedetto ${ }^{2}$, A. Pinna ${ }^{3}$,
P. Torricelli1
'Department of Radiology, ${ }^{2}$ Liver and Multivisceral
Transplant Center, University of Modena and Reggio
Emilia, ${ }^{3}$ Department of Surgery, Liver and Multiorgan
Transplant Division, University of Bologna, Italy

Purpose: Radiologic evaluation of the transplanted bowel is still largely unknown and rather complex, as it involves several techniques depending upon indications and times that have not yet been fully defined.

Materials and Methods: From December 2000 to November 2002, 11 patients with transplanted bowel were studied with different methods: traditional radiologic evaluation with contrast agent, evaluation of transit time with radiopaque markers, US of the intestinal wall and Doppler of the vascular axes, CT and MR. 
Results and Conclusion: The study of transit is useful in patients with chronic intestinal pseudo-obstruction for to identify the normalization of motility. Traditional examination of the gastroenteric tract permits to evaluate the graft anatomy and the recovery of motility both of the residual native bowel and of the transplanted loops. Moreover, it plays a crucial role in the detection of the postoperative complications. CT examination is crucial for the detection and drainage of collections and abscesses, and in the follow-up of patients with Gardner's syndrome as it permits to investigate all the sites in which desmoids might arise and their relationship with the graft. Considering the fertile age of patients transplanted, MR may represent an effective method and can therefore substitute traditional radiologic evaluation. US represents a quick examination technique, which plays a role in the follow-up of the transplanted patient and in the identification of the major postoperative complications.

294

\section{Radiological Imaging in Patients Requiring Intestinal Transplants}

\author{
A. Pecchi ${ }^{1}$, M. De Santis ${ }^{1}$, F. Di Benedetto ${ }^{2}$, A. Pinna ${ }^{3}$, \\ P. Torricelli ${ }^{1}$ \\ ${ }^{1}$ Department of Radiology, ${ }^{2}$ Liver and Multivisceral \\ Transplant Center, University of Modena and Reggio \\ Emilia, ${ }^{3}$ Department of Surgery, Liver and Multiorgan \\ Transplant Division, University of Bologna, Italy
}

Purpose: The intestinal failure-general indication for transplant - is an inability to feed oneself and/or maintain a hydro-electrolytic balance without parenteral support and may be due to the lack of an intestinal surface, or the lack of an absorbent function. The pre-operative evaluation of there patients involves radiology, through the different methodology.

Materials and Methods: From December 2000 to November 2002, 16 patients with intestine transplants underwent traditional radiological study of the gastro-enteric tract with and without mdc per os, study of transit time through the use of radio-opaque markers, US, TC.

Results and Conclusion: The evaluation of intestine via traditional methodology shows the length of the residual intestine and the presence of organic or functional changes on the remaining loops. The radio-opaque markers, help in diagnosis of pseudo-obstruction. The US with Color Doppler, helps in choosing which type of transplant to perform and in defining the level of hepatic sufferance. The TC permits a panoramic evaluation of the thoracic-abdominal vascular axis, detecting the presence/absence of patency, the presence of possible anatomical changes and provides information supplementary to that of the traditional (position of loops within the abdomen, condition of the mesentery, presence of extrinsically-developed lesions attached to the wall). Angiography was used, in the cases that certain doubts were encountered after using TC. RM was occasionally used in order to obtain supplementary information.
295

\section{Rejection Episodes and 3-years Graft Survival Under Sirolimus and Tacrolimus Treatment after Adult Intestinal Transplantation}

A. Lauro, A. Dazzi, G. Ercolani, C. Zanfi, L. Golfieri A. Amaduzzi, F. Pezzoli, T. Lipparini, T. Lucci, S. Nucaro, A. D'Errico ${ }^{1}$, M. Di Simone, S. Faenza, L. Pironi², A.D. Pinna

U.O. Chirurgia dei Trapianti di Fegato e Multiorgano,

${ }^{1}$ Anatomia e Istologia Patologica, Istituto F. Addarii,

${ }^{2}$ Centro di Riferimento Insufficienza Intestinale, University of Bologna, Policlinico S.Orsola-Malpighi, Italy

Purpose: mTOR inhibitors have been recently introduced in clinical practice after intestinal transplantation. We focused on Sirolimus (Rapamycin ${ }^{\circledR}$ ) prolonged effect on rejections and graft survival following intestinal transplantation.

Patients and Methods: Twenty isolated intestinal recipients and 5 multivisceral (2 with liver) were considered in our series and divided in two groups: 15 recipients were started on Sirolimus because of nephrotoxicity or biopsy-proven rejection. Control group was represented by 10 recipients who never received rapamycin or received rapamycin for a period shorter than 3 months.

Results: During prolonged treatment combined with primary immunosuppression based on Tacrolimus (Prograf ${ }^{\mathbb{R}}$ ), the Sirolimus group showed decreased number of ACRs $(\mathrm{p}<0.01)$. Cumulative 3year graft survival was $86 \%$ in Sirolimus group and $54 \%$ in control group $(\mathrm{p}<0.05)$. Four recipients out of $15(26 \%)$ discontinued Sirolimus because of side effects.

Discussion and Conclusion: In our experience the use of mTOR-inhibitors in combination with calcineurin-inhibitors seems to be more effective than monotherapy in reducing the number of rejections and improving long-term graft survival after intestinal transplantation. Side effects can limit its use as maintenance therapy.

\section{6}

Intestinal Failure-Associated Liver Disease in Adult Patients: The Role of Small Bowel Transplantation

\author{
C. Zanfi, A. Lauro, A. Dazzi, A. Amaduzzi, L. Golfieri, \\ F. Pezzoli, L. Pironi ${ }^{1}$, A.D. Pinna
}

UO Chirurgia Trapianti Fegato e Multiorgano, ${ }^{1}$ Centro Insufficienza Intestinale, Policlinico S. Orsola-Malpighi, Bologna, Italy

Purpose: To evaluate liver function recovery after isolated intestinal transplantation in adults with irreversible intestinal failure.

Patients and Methods: Over a 5 years period we transplanted 34 adult patients: 25 received an isolated intestinal graft, 4 a multivisceral graft without liver, and 5 a multivisceral graft with liver. Among the group of patients transplanted with the isolated graft without liver we selected 14 recipients with clinical pre-transplant liver dysfunction which was considered when bilirubin was superior than $2 \mathrm{mg} / \mathrm{dl}$ 
and/or transaminases more than $100 \mathrm{IU} / \mathrm{ml}$ (N.V.: 37/40); other inclusion criteria were: TPN period $>3$ months, no diagnosis of portal hypertension or cirrhosis. Two patients had a biopsy-proven liver fibrosis.

Results: At discharge all patients recovered liver function normal values: mean bilirubin blood level was $0.9 \mathrm{mg} / \mathrm{dl}$ and mean transaminases was $32 / 35 \mathrm{IU} / \mathrm{ml}$. After a mean follow-up of 3 years only 1 patient has elevated ALT without clinical signs of liver disease: mean bilirubin level is $0.8 \mathrm{mg} / \mathrm{dl}$ (range $0.3-1.2 \mathrm{mg} / \mathrm{dl}$ ), mean transaminases level $22 / 24 \mathrm{IU} / \mathrm{ml}$ and PT value is $72 \%$. Patients receiving a successful isolated intestinal transplant or a multiviscearl one without liver didn't experience further liver deterioration. Pre-transplant liver disease didn't impact on survival rates. No one is on TPN.

Conclusion: In selected cases isolated intestinal or multivisceral graft without liver can represent a 'liver salvage therapy' for early failing liver in adult patients with irreversible intestinal failure. Pre-transplant liver disease isn't a negative prognostic factor.

\section{7 \\ Antiaggregant Prophylaxis Reduces the Incidence of Late Hepatic Artery Thromobosis After Liver Transplantation in High Risk Patients

\author{
G.L. Barba, M. Vivarelli, A. Cucchetti, A. Lauro, \\ M. Del Gaudio, M. Ravaioli, G.L. Grazi, A.D. Pinna \\ U.O. Chirurgia Trapianti fegato e multiorgano, Policlinico \\ S. Orsola Malpigli, Bologna, Italy
}

To minimize the incidence of late hepatic artery thrombosis (LHAT) after liver transplantation (LT) antiaggregant therapy was reviewed in 824 adult LT performed from April 1986 to August 2005. LT with donor who died of vascular accident and/or required arterial conduit for artery reconstruction were considered at High Risk for LHAT ( $=499)$; LT without these characteristics were considered at Low Risk $(n=587)$. From January 2003 antiaggregant therapy started after LT when INR was above 1.2 and/or platelet count was above $60.000 \times \mathrm{mm}^{3}$, with a median time from LT of 8 days (0-29). LHAT occurred in 14 cases $(1.69 \%)$ with a median time from LT of 500 days, ranging from 50 to 2405 days. 13 patients out of 587 (2.21\%) who did not received prophylaxis experienced LHAT and only one patient out of $237(0.42 \%)$ who received prophylaxis experienced the event $(\mathrm{p}=0.07)$. In the High-risk group, of the 161 patients who received antiaggregant therapy LHAT occurred in 1 case $(0.6 \%)$ significantly lower in comparison to the remaining 338 patients who did not received antiaggregant therapy in which LHAT occurred in 12 cases $(3.6 \% ; p=0.04)$. In conclusion, antiaggregant therapy after LT seems to be protective against LHAT development after LT in patients receiving grafts from donor who died from vascular accident and/or requiring arterial conduit for artery reconstruction.

\section{8}

\section{Quality of Life in Adult Intestinal Transplantation: A Subjective Analysis of Recipients}

L. Golfieri, A. Lauro, E. Tossani' ${ }^{1}$ A. Dazzi, C. Zanfi, A. Amaduzzi, F. Pezzoli, G. Ercolani, M. Vivarelli, M. Del

Gaudio, G.L. Grazi, M. Ravaioli, M. Cescon, G. Varotti, S. Grandi ${ }^{1}$, A.D. Pinna

OU Liver and Multiorgan Transplant Surgey, University of Bologna, ${ }^{1}$ Psychological Department, University of Bologna, Italy

Introduction: Intestinal transplantation has become an accepted therapy for individuals permanently dependent on parenteral nutrition (TPN) with life-threatening complications. Quality of life and psychological well-being can be seen as an important outcome measure of transplantation surgery.

Methods: We evaluated 22 intestinal transplant recipients and 21 healthy subjects (a control group). All subjects were administered the Italian Version of a Psychological Well-Being Scales by C. Ryff (PWB) and the World Health Organization Quality of Life-Brief (WHOQOL). Quality of life and psychological well-being were assessed in transplant recipients in relation to rejection number, admission number and immunosuppressive protocol.

Results: Transplant recipients reported significantly higher scores in the 'Autonomy' $(\mathrm{p}=0.02)$ and lower scores in 'Positive relation with others' $(\mathrm{p}=0.025)$ dimension of PWB, compared to control. Transplant patients showed only lower scores in psychological domain $(p=0.007)$ of quality of life. Transplant recipients with less rejections showed higher scores in psychological domains of quality of life $(\mathrm{p}=0.014)$.

Conclusion: Improvement of psychological well-being in transplant population may be related to the achievement of the goal of transplantation: recovery of bowel function. But the data confirm that the transplant experience require a long and difficult adaptation trial to the new condition of 'transplant recipient'.

299

\section{Longitudinal Analysis of Kidney Function in Orthotopic Liver Transplantation Patients: Risk Factors and Kidney Function Assessment}
L. Mandalà, C. Caccamo, M. Artero, P. Salis, V. Cosentini, D. Cintorino, S. Gruttadauria, M. Spada, R. Verzaro,
M. Spampinato, E. Luna, A. Arcadipane, C.S. Foglieni, B. Gridelli

Istituto Mediteranneo per i Trapianti e le Terapie ad alta Specializzazione (IsMeTT), UPMC, Italy

Introduction: To determine post OLTx Chronic Renal Failure (CRF) incidence and identify a correlation between cystatin $\mathrm{C}$, serum creatinine and creatinine clearance.

Methods: 152 liver recipients (108 male/ 48 female; mean age: 50.9 years) were included in a database obtained collecting demographic, 
pre- and post-transplant variables able to affect kidney function. Immunosuppression was based on tacrolimus and steroids. Kidney function was assessed measuring serum creatinine 1, 6 months and 1 year post liver transplant. In patients with serum creatinine $\geq 1.5 \mathrm{mg} / \mathrm{dl}$, kidney function was reassessed measuring serum creatinine, creatinine clearance and cystatin C. Survival was determined using the Kaplan-Meier method. The Cox regression was used for significance analysis. Correlation between cystatin $\mathrm{C}$, serum creatinine and creatinine clearance was assessed using a multiple-regression model.

Results: 1 year post-transplant survival on CRF group was $70 \%$ (estimated incidence of $34 \%$ ). Creatinine and cystatin $\mathrm{C}$ correlation was extremely significant $(\mathrm{r}=0.67 ; \mathrm{p}<0.0003)$. A significant yet weaker correlation was noted between creatinine clearance and cystatin $\mathrm{C}-0.48(\mathrm{p}<0.005)$. No significant relation was found between $24 \mathrm{~h}$ proteinuria and cystatin $\mathrm{C}$.

Discussion: Cystatin $\mathrm{C}$ was the most sensitive parameter to identify post liver transplant CRF mainly caused by nephrotoxic drugs.

\section{0}

'Adult Intestinal and Multivisceral Transplantation from Trauma Donors'

\author{
F. Pezzoli, A. Lauro, A. Amaduzzi, A. Dazi, L. Golfieri, \\ C. Zanfi, A.D. Pinna
}

Chirurgia dei trapianti di fegato e multiviscerale, Policlinico Sant'Orsola-Malpighi-Bologna, Italy

Introduction: Further expansion of organ transplantation may be limited by the availability of donors organ and maximizing yield from the more conventional donor pool could be an important factor. The aims of this study are to estimate the availability of donor organs resulting from trauma victims and to investigate the specific reasons of organ procurement failure.

Methods: From 2000 to 2005 we evaluated intra-operatively 49 donors for intestinal grafting.

Results: 14 donors $(28.6 \%)$ were not suitable for bowel transplantation: 8 of them $(57.1 \%)$ were trauma victims and their grafts were not suitable because of bowel edema or ischemia ( 3 cases), vascular abnormalities ( 2 cases), donor hypotension ( 2 cases) and death of recipient ( 1 case); 6 of them $(42.9 \%)$ were death for haemorrhages and their grafts were not suitable because of bowel edema or ischemia ( 2 cases), vascular abnormalities ( 1 case), recipient's factors ( 2 cases), suspect of carcinoma ( 1 case). 16 of 35 donors suitable for transplantation $(45.7 \%)$ were trauma victims.

Discussion: Our data indicate that the availability of organs from donors death for trauma or for haemorrhages is similar. The factor that seems to limit organ availability from trauma victim is the physiologic support of potential donor. Our results suggest the potential role of a specific training for the medical staff employed in this field.
301

\section{Laparoscopic Live Donor Left Nephrectomy in the Presence of Renal Vascular Anomalies}

\author{
R. Sampietro, E. Orsenigo, C. Socci, V. Zuber, \\ C. Staudacher \\ Department of Surgery, San Raffaele Scientific Institute, \\ University Vita-Salute, Milano, Italy
}

Background: Although laparoscopic technique for left living donor nephrectomy has been standardized and widely applied in highvolume transplant centres, some technical items remain debated. Moreover, the feasibility and safety of pure laparoscopic approach to vascular aberrancies of the hilum such as retroaortic left renal vein is not well defined.

Materials and Methods: In the video a case of left laparoscopic live donor nephrectomy in presence of a retroaortic renal vein (the commonest renal venous anomaly) has been explained. A fourport transperitoneal approach was used and kidney has been withdrawn through a Pfannenstiel incision by use an endocatch bag.

Results: The procedure was successfully performed without vascular complications by use a totally laparoscopic technique. Operative time was $230 \mathrm{~min}$ and warm ischemia time $2.5 \mathrm{~min}$. Recipient kidney transplant was successfully performed in a standard fashion.

Conclusions: The presence of a major renal vascular aberrancy such as retroaortic renal vein does not contraindicate the minimallyinvasive approach for left living donor nephrectomy. Nevertheless, a preoperative identification of all anomalies is mandatory.

\section{2 \\ Right Laparoscopic Living Donor Nephrectomy \\ R. Sampietro, E. Orsenigo, C. Socci, F. Gavazzi, C. Staudacher \\ Department of Surgery, San Raffaele Scientific Institute, University Vita-Salute, Milano, Italy}

Background: Due to anatomical reasons, left kidney procurement is preferred for live donation. In open live donor nephrectomy the right kidney is selected if arterial or venous anomalies are present in the left kidney. In laparoscopic living donor nephrectomy, further reluctance to procure the right kidney is related to the more difficult exposure and the possible extreme shortening of the right renal vein due to stapled transection.

Materials and Methods: The video shows a case of right laparoscopic living donor nephrectomy. A four-port transperitoneal approach has been used. The kidney has been withdrawned through a Pfannenstiel incision.

Results: The procedure was successfully performed. Operative time was $200 \mathrm{~min}$. Warm ischemia time was $2 \mathrm{~min}$. The recipient kidney transplant was performed under optimal vascular conditions with immediate function.

Conclusions: Right laparoscopic living donor nephrectomy is a technically feasible, safe and reliable option for minimally invasive 
organ donation and should be considered when the left kidney is not suitable for transplantation.

\section{3}

\section{Timing and Problems in Pancreas Retransplant}

\section{S. Soldano, A. De Roberto, P. Aseni, O. Rossetti, C.V. Sansalone}

Kidney and Pancreas Transplantation Unit, Niguarda Hospital, Milan, Italy

Introduction: Pancreas transplant (PT) is the treatment of choice in selected patients with IDDM. Since that some patients can develop early or late graft loss due to surgical or medical complications, the opportunity for a second pancreas graft should be considered. We present our early experience on Re-PT in a diabetic population who received a PT in our transplant unit.

Methods: From October 1994 to March 2006, 75 diabetic patients (mean age 32.7 years, M/F 48/27) were submitted to PT. Seventy of them with ESRD received a combined pancreas-kidney transplant (PKT) while 5 patients with good renal function received a pancreas transplant alone (PTA). Sixteen patients had bladder diversion while 59 patients had enteric diversion. All patients received quadruple cyclosporine-based immunosuppression. In the PKT group 8 patients who lost their pancreatic graft either for post-operative complications ( 5 patients) or chronic rejection ( 3 patients) received a second graft.

Results: One patient died for recurrent venous thrombosis and massive pulmonary embolism, 1 patient lost his graft for enteric fistula, three patients developed chronic rejection and resumed insulin therapy 8, 11 and 23 months after Re-PT and three patients have good graft function 11, 16 and 28 months after the second surgical procedure.

Discussion: Re-PT is a correct indication in diabetic patients who lost their first pancreatic graft. Patient and graft survival is better when Re-PT is performed later.

\section{4}

\section{Cardiovascular Disease and Renal Transplant}

\section{S. Soldano, A. De Roberto, P. Aseni, O. Rossetti, C.V. Sansalone}

Kidney and Pancreas Transplantation Unit, Niguarda Hospital, Milan, Italy

Introduction: Cardiovascular disease (CVD) is the first cause of death after kidney transplant (KT). CVD is present in more than $10 \%$ of uremic population on waiting list for KT. We present our experience on patients with CVD who received a KT.

Methods: From January 2000 to December 2003, 18 patients (mean age 54.9 years; M/F 14/4) with CVD (LVH 4 patients, IHD 10 patients, CHF 3 patients, HT 1 patient) underwent KT. Mean time from cardiac event to KT was 8, 7, 4, 1.5 years for LVH, HT, IHD and $\mathrm{CHF}$ respectively. All patients received quadruple cyclosporine-based immunosuppression.
Results: Patient with HT died on operating room 30 min after graft reperfusion because of ventricular fibrillation. All transplanted patients had ICU observation period for a mean time of $72 \mathrm{~h}$ and 13 of them had immediate active diuresis. In 4 patients the need of tight blood pressure control was the main adverse event. With a mean follow up of 28 months one patient died because of IHD, and two patients lost their grafts for chronic rejection and lymphoma respectively. Overall patient and graft survival was 88.8 and $77.7 \%$ respectively.

Discussion: Uremic patients with CVD who receive a KT, have a higher risk of morbidity and mortality compared to the general uremic population.

\section{5 \\ Simultaneous Iliac Artery Prosthetic Replacement and Kidney Transplantation: A Case Report}
A. Vecchi, N. Cautero, S. De Luca, P. Garelli, G. Frascà', G. Gaffi ${ }^{1}$, D. Taruscia ${ }^{1}$, A. Giovagnoni ${ }^{2}$, A. Risaliti
Clinica di Chirurgia Epatobiliare e Trapianti d'Organo, ${ }^{1}$ U.O. di Nefrologia e Dialisi, ${ }^{2}$ Radiologia Clinica, Azienda Ospedali Riuniti Umberto I-G.Lancisi-G.M.Salesi-Ancona

Introduction: Aortoiliac atherosclerosis (AA) frequently affects kidney transplant (KTx) candidates. Reconstructive surgery represent a formidable surgical challenge and KTx may be contraindicated. Staged or simultaneous vascular reconstruction, commonly with an artificial prosthesis, is feasible at reasonable risk.

Methods and Results: A 49-year-old male was listed for KTx after standard clinical evaluation. Peripheral vascular assessment with abdominal X-ray and Doppler ultrasound showed a normal ultrasound patency without iliac calcifications so angiography was not performed. A left kidney was transplanted into the right iliac fossa. An unexpected severe arteriosclerosis with almost complete stenosis of common, external and internal right iliac artery was observed. A right iliac unsuccessful thromboendoarterectomy was attempt so a PTFE prosthesis replacement of iliac trunk was accomplished. The KTx was completed in usual fashion performing the arterial anastomosis on prosthesis. The postoperative clinical course was uneventful and the patient was discharged after 16 days with a good graft function and a normal ultrasound pattern. A MRI after 4 months demonstrate the regular patency of anastomotic tracts.

Discussion: KTx candidates may have severe AA without symptoms or Doppler alterations so some groups consider angiography as a mandatory step in the pre-transplantation work-up. In KTx candidates with severe AA disease the controversy remains whether aorto-iliac repair and KTx should be done simultaneously or in two stages. Our experience and those reported in the literature prove that patients can safely undergo simultaneous iliac prosthetic reconstruction and KTx. We should therefore be familiar with the prosthetic materials in order to achieve optimal results. 


\section{6}

Combined Radiological and Surgical Approach for Treatment of Hepatic Portal Venous Gas in a Patient with Complicated Sigmoid Diverticulitis

\author{
P. Garelli, N. Cautero, S. De Luca, A. Vecchi, \\ G. Svegliati-Baroni ${ }^{1}$, E. Antico ${ }^{2}$, A. Giovagnoni ${ }^{2}$, U. Negro ${ }^{2}$, \\ G. Valeri ${ }^{2}$, M. Verdecchia ${ }^{2}$, A. Risaliti \\ Clinica di Chirurgia Epatobiliare e Trapianti d'Organo, \\ ${ }^{1}$ Clinica di Gastroenterologia, ${ }^{2}$ Dipartimento di Radiologia, \\ Azienda Ospedali Riuniti Umberto I-G.Lancisi-G.M. \\ Salesi-Ancona
}

Introduction: Hepatic portal venous gas (HPVG) is a rare condition associated with a variety of abdominal diseases. Overall mortality rate of HPVG is $75 \%$ and a correct diagnosis is mandatory for a proper management. We describe a case of sigmoid diverticulitis complicated by HPVG.

Methods and Results: A 37-year-old man was observed due to persistent acute abdominal pain, fever, jaundice, and chest pain. Abdominal US shoved hepatomegaly, cholecistitis and intraperitoneal free fluid; an abdominal X-ray examination and a CT scan evidenced abnormal gaseous distension of the portal and inferior mesenteric veins. A colonscopy evidenced sigmoid diverticula. Splenoportography demonstrated a communication between a peripheral branch of the left colic vein and a sigmoid diverticulum, tight stenosis of portal trunk with a mesenteric cavernoma. The patient underwent embolization of the left colic vein followed by a superior mesenteric vein balloon angioplasty plus stenting. Two days after, control studies demonstrated a fair diameter of treaded veins and progressive disappearance of HPVG. Liver function remained stable throughout. With a stable patient the enterovascular fistula was treated with sigmoidectomy. Patient was discharged after a normal postoperative clinical course.

Discussion: HPVG is a rare condition often detected accidentally in patients with acute abdominal pain. HPVG usually predicts a survival rate $<25 \%$. In detection of HPVG ultrasound and computed tomography are very sensitive and help in therapeutic decision. In our case the selective embolization and stenting of mesenteric-portal tree reduced the splancnic congestion and the risk of hemorrage of the planned sigmoidectomy.

\section{7}

\section{Biliary Cystadenocarcinoma: A Case Report}

\author{
A. Vecchi, N. Cautero, S. De Luca, P. Garelli, I. Bearzi', \\ A. Risaliti \\ Clinica di Chirurgia Epatobiliare e Trapianti d'Organo, \\ ${ }^{1}$ Anatomia Patologica, Azienda Ospedali Riuniti Umberto \\ I-G.Lancisi-G.M.Salesi-Ancona
}

Introduction: Biliary cystadenocarcinoma (BCAC) is a rare hepatic neoplasm that may be difficult to distinguish from simple hepatic cysts. Prognosis is better than other malignant hepatic tumours. Cystadenomas are often incorrectly diagnosed as simple cysts which results in inadequate therapy; the possible malignant transformation and recurrence are also consequences of primary incorrect diagnosis than a tardive or incomplete surgical excision.

Methods and Results: A 42-year-old woman with a previous diagnosis of benign angioma was admitted to our Unit because of abdominal discomfort. A previous sonographic and computed tomographic evaluation was suggestive of cavernous haemangioma about $6 \mathrm{~cm}$ of diameter. A magnetic resonance imaging (MRI) features demonstrated a liver mass about $10 \mathrm{~cm}$ diameter with both cystic and solid components with a central scar. The symptomatic patient was listed for surgical exploration and an extended left hepatic resection was planned. An intraoperative accident occurred; the portal vein was sectioned, during preliminary dissection, because the hepatic ilum was displaced about the mass. After prompt portal vein reconstruction the hepatic resection was completed. The microscopic examination confirmed a diagnosis of BCAC without metastasis. The postoperative clinical course was uneventful and the patient was discharged after 7 days. A MRI after 4 months demonstrate the regular liver regeneration.

Discussion: BCAC is difficult to diagnose because its clinical manifestations are similar to other cystic lesions. Imaging examination is helpful and demonstrates the presence of internal septa that highly suggests the diagnosis of cystadenoma. Cystadenomas require complete excision to prevent recurrence or neoplastic evolution.

\section{8 \\ Surgical Complications in Pancreas Transplantation}

\section{F. Vistoli, U. Boggi, M. Del Chiaro, S. Signori, C. Moretto, I. Mosca, L. Morelli, A. Sgambelluri, G. Amorese, \\ P. Marchetti, G. Rizzo, F. Mosca \\ Regional Referral Center for the Treatment of Pancreatic Diseases, Pisa, Italy}

Introduction: Surgical complications (SC) have shaped the history of pancreas transplantation (PT) and frequently result in graft loss and increased mortality.

Methods: Between May 1996 and February 2006, 220 PT were performed: 114 simultaneous pancreas kidney transplants (SPKTx), and 106 solitary PT (SPTx). Venous effluent was created in the portal vein in most instances (52 SPKTx and 76 SPTx). Drainage of exocrine secretions was enteric (Roux en-Y), in 80 SPKTx and 101 SPTx, or urinary, in 34 SPKTx and 5 SPTx. SC were defined as the need for relaparotomy (R) during the first 3 months post-PT or during the initial hospital stay, if longer than 3 months.

Results: SC occurred in $13.2 \%$ recipients (SPKTx $=12.3 \%$ vs. SPTx $=14.2 \%)$ after a mean period of 10.3 days (SPKTx $=11.2$ days vs. SPTx $=9.7$ days). Thrombosis (SPKTx $=5.2 \%$ vs. SPTx $=3.8 \%$ ) and hemorrhage (SPKTx $=4.4 \%$ vs. SPTx $=3.8 \%$ ) accounted for most R. Duodenal segment leaks occurred in $1.7 \%$ of SPKTx and in $0.9 \%$ of SPTx. R had no impact on recipient mortality but was associated with decreased kidney graft survival in SPKTx $(p=0.008)$ and poorer insulin independence in SPTx $(\mathrm{p}=0.005)$. One- and 5-year patient survival with or without SC were $90.0 \%$ vs. $95.1 \%$, and $90.0 \%$ vs. $90.8 \%$, respectively. Equivalent figures for kidney grafts (SPKTx) were $61.5 \%$ vs. $94.9 \%(p=0.008)$, and $61.5 \%$ vs. $87.1 \%(p=0.008)$, respectively. Pancreas graft survival was $50.0 \%$ vs. $92.7 \%$ $(p=0.0001)$, and $37.3 \%$ vs. $81.4 \%(p=0.0001)$, respectively. 
Conclusions: A SC rate below $15 \%$ has been consistently achieved thanks to standardized operative techniques and strict protocols of post-transplant surveillance.

\section{9}

\section{Surgery Complications Following Kidney's Transplant: Our Experience}

\author{
R. Torrini, M. Piervittori, G. Natalini \\ UOC generale Osp, 'R. Silvestrini', Perugia
}

Introduction: Our centre performed n.297 kidney's transplants from dead body with the mean age of 42 years old. The transplants were performed from August 1988 until December 2005. Our waiting list is between 8 up to 24 months. The aim of our audit was to evaluate post-surgery complications.

Methods: 40 patients of $297,13.4 \%$ of the total patients, had surgery's complication: 24 that required an conservative treatment and 16 required an surgery treatment. $50 \%$ of surgery complications (20 cases, $6.7 \%$ of all transplants) were prolonged lymphorragie and lymphoceles. At the beginning, all cases were, treated with conservative treatment with a good outcome in 18 patients $(98 \%)$. Two patients had complication and they undergone to surgery again: laparoscopic opening lymphocele and confection of peritonel pocket. $20 \%(8$ cases) of the surgery complications were vascular complications. $15 \%$ of the surgery complications were urinary complications. One patient had hernia of surgical scar.

Results and Discussion: An impairment of the kidney's functionality, was presented in 3 patients that received a second surgery.

Conclusion: Our audit shows that our over the years, our centre has improved for both quality of the post surgery and increment of the number of transplants. The improvement is due to an improvement of selection of the patients who need the kidney's transplant as well as to an improvement of capacity detect quickly all possible post surgery complications. Furthermore, the development of immunosuppressive treatment is an important step which give us the possibility to reduce the use of steroids.

\section{Urology}

\section{0 \\ Laparoscopic Hand Assisted Adrenalectomy: When and Becouse}

\author{
G. Regina ${ }^{1}$, G. Annunziata², M. Ciavarella ${ }^{1}$ \\ ${ }^{1}$ Cattedra e Scuola di Specializzazione in Chirurgia \\ Vascolare Universita' degli Studi di Bari, ${ }^{2}$ Cattedra e \\ Scuola di Specializzazione in Urologia Universitaria I, \\ Università degli Studi di Bari
}

Introduction: The laparoscopic access represent today the goldstandard to the surgical treatment of the adrenal gland tumor. The only limit is the size of the mass $(<8 \mathrm{~cm})$ and the possible close relation with near organs.

Case Report: The patient is a male 48 years old, with diagnosis of hepatitis $\mathrm{C}$ and diabetes. Incidentally haematic analis shows abnormal transaminasi and so he has been exposed to abdminals ecography, that revealed the presence of ipo-iperecogena area in the zone of right adrenal gland with maximum diameter of $12 \mathrm{~cm}$. The TAC and the RMN of the abdomen confirmed the presence of the mass and the liver (right lobe) appeared partially compressed by this mass and the below kidney, in bad position, was lower transfer. This formation to RMN was ipointensa and iperintensa in all study phases after the administration of MDC and was possible to see area of recent bleeding into the neoplasia. The not clear origin of the illness, the big dimension of this, the close relation with other organs and the suspect of the second illness at the limphonodes made as to choice for the surgical way to consider our experience of great account in the laparoscopia hand assisted we had use this technique to avoid classic lumbotomic access and all collateral effects with that operation. To the introduction of the Device (lap disk) we had adopted an abdomen tranperitoneal right incision of the dimension of $7 \mathrm{~cm}$. and after we introduced 3 trocars of $10-12 \mathrm{~cm}$. In the (truncated at 250 words).

311

\section{Expressed E-Cadherin mRNA Levels Analysis in Evaluating Urothelial Bladder Cell Carcinoma Natural History}

\author{
T. Cai ${ }^{1}$, G. Nesi ${ }^{2}$, M. Piazzini ${ }^{3}$, I. Sardi ${ }^{4}$, R. Bartoletti ${ }^{1}$ \\ ${ }^{1}$ Department of Urology, ${ }^{2}$ Department of Pathology and \\ Oncology, University of Florence, ${ }^{3}$ Medical Genetics Unit, \\ Department of Clinical Physiopathology, ${ }^{4}$ Department of \\ Pediatrics, University of Florence, Italy
}

Introduction: Many authors indicate that the E-Cadherin (ECAD) loss expression is associated with poor prognosis in bladder urothelial cell carcinoma (UCC). The aim of this study is to define the role of E-CAD mRNA expression on recurrence, progression and survival in UCC of the urinary bladder over a long follow-up period. 
Methods: From 30 patients with bladder UCC, enrolled in our previous study, 27 were selected for this study. All patients were reanalyzed in terms of clinical and tumor characteristics, tumor pathological analysis and tumor E-CAD mRNA expression. All data were correlated to 12 years follow-up results.

Results: Significant correlations between stage $(p=0.002)$, grade $(\mathrm{p}=0.008)$ and E-CAD mRNA levels were reported. E-CAD did not show any correlation in predicting recurrence or progression in bladder UCC. The survival analysis demonstrated a significant relationship $(p=0.019)$ between patients with expressed E-CAD mRNA levels and cancer-specific survival. The univariate analysis demonstrated that expressed E-CAD mRNA levels is an independent prognostic factor in terms of cancer-specific survival in UCC of the urinary bladder $(\mathrm{p}=0.002)$.

Discussion: Our study demonstrated that mRNA extraction and Northen Blot analysis is to be considered a good and reliable method to evaluate E-CAD mRNA levels for predicting survival rate in patients affected by urothelial bladder cancers.

\section{2 \\ Transdermic Hydroelectrophoresis in the Treatment of Peyronie's Disease}

\section{T. Cai, A. Gavazzi, N. Mondaini, R. Bartoletti}

Department of Urology, University of Florence, S. Maria Annunziata Hospital, Florence, Italy

Introduction: The aim of the study was to test toxicity and efficacy of a new device useful for transdermic hydroelectrophoresis on patients affected by Peyronie's disease.

Methods: 16 patients were enrolled in the study. 10 patients had a plaque $(<1 \mathrm{~cm}), 2$ of them had a plaque $(>1 \mathrm{~cm})$ while 4 had more than one plaque. 14 patients reported pain. All patients were studied by clinical evaluation, sonography and self-photography. A hydroelectroforesis machine (Winform ${ }^{\circledR}$ ) was used to treat penile plaques twice a week, by using a gel containing various compounds, such as EDTA, lidocain $2 \%$, verapamil and dezametason. The intensity of the electrical impulses was $15-20 \mathrm{~mA}$ for $20 \mathrm{~min}$ per treatment. Pain during erection was fully resolved at most after 2 treatments.

Results: No side effects were found. The comparative sonographic evaluation of the plaques prior to and after treatment demonstrated a complete plaque resolution on 4 patients $(25 \%)$, a plaque size reduction in 6 of them $(37.5 \%)$ while the clinical situation of 6 other patients remained unchanged. The penile recurvatum was unchanged in 7 cases, while reduced in 8 , and completely resolved in 1 patient.

Discussion: The local administration of various compounds by transdermic hydroelectrophoresis in the treatment of Peyronie's disease, has many advantages in terms of efficacy and tolerability, compared with classic infiltrative treatments.
313

\section{Restaging Transurethral Resection (reTUR) in T1HG Bladder Cancer}

\author{
L. Carbone, P. Cozzupoli, M. lerace, O. Sicuro, E. Sgro, \\ D. Veneziano
}

UO Urologia, Reggio Calabria

Objective: We report our experience with repeat transurethral resection (reTUR) in T1HG bladder tumors, evaluate residual tumors and prognostic significance.

Material and Methods: 44 patients underwent to second endoscopic resection 4-12 weeks later first TURV. The reTUR consisted of resection of the tumour scar bed and separated resection of margin.

Results: $13 \mathrm{pz}$ underwent to reTUR $(29.5 \%)$ presented residual disease $(\mathrm{R}+)$ and $31(70.5 \%)$ resulted negative (R0). The median follow-up of R+ pz was 24 months; 4/13 (30.8\%) infiltrated the muscle. First-time recurrence was 14.5 months; $5 / 13 \mathrm{R}+(38.5 \%)$ recurrence. ReTUR modified therapy in $5 \mathrm{R}+(38.4 \%) .7 / 13 \mathrm{R}+(54 \%)$ achieved progression disease and metastasis in follow-up. $2 / 13 \mathrm{R}+$ achieved a state of complete response. 2 patients died to a colon/liver cancer. One patient was lost $(7.6 \%)$. One patient presented recidive of low grade/stage. The mean follow-up of $31 \mathrm{R} 0$ patients was 22 months. 2/31 R0 (6.4\%) had recurrence of some stage ( $\mathrm{T}_{1 \mathrm{HG}}$ tumor). First-time recurrence was of 19.3 months. 25/31 R0 (57\%) achieved complete response. $4 \mathrm{R} 0(13 \%)$ presented recurrence of $\mathrm{T}_{\mathrm{aLG}}$ disease.

Conclusion: Residual tumor is present in $30 \%$ of pz. The procedure provide important prognostic information, reduces tumor recurrence and appears to delay early tumor progression at follow-up period. R0 patients can be considered a good prognostic factor. $\mathrm{R}+$ patients are at high risks of recurrence and progression in short and median follow-up period.

\section{4 \\ Z-plasty Technique in Surgical Treatment of Phimosis}

\author{
A.V. Pagliari, F. Klinger, C.A. Amodeo, B. Banzatti, \\ M. Klinger
}

Istituto di Chirurgia, Plastica e Ricostruttiva dell'Università degli Studi di Milano, I.C. Humanitas, Italy

Introduction: Phimosis is a common pathologic condition characterized by a unretractable foreskin or a circular band of tight prepuce preventing full retraction. Medical and conservative therapy require steroid oinment and stretching exercises. Surgical therapy is reserved to severe resistant cases. Different techniques of preputioplasty have been described in last years.

Methods: In our series the constricted ring of the phimosis is interrupted with a Z-plasty. In the procedure no skin is removed and it is sufficient to expose the glans penis painlessly. Patients were operated under general anesthesia with an average time of $15 \mathrm{~min}$.

Results: No post-operative complications were observed and in any patients verified a rest enosis. At follow-up the cosmetic and functional results were satisfactory. 
Discussion: In severe phimosis Z-plasty technique is a safe and rapid technique alternative to the more radical circumcision and allows to obtain excellent cosmetic and functional results.

\section{5}

\section{A Rare Case of Leiomyoma in the Funicolo \\ G. Benedetto, M. Titta, F. Nigro, G. Abatangelo, A. Tasca \\ Department of Urology, Hospitals, Bortolo, Vicenza}

Introduction: The leiomyoma of funicolus of the testis is a rare cancer. Among benign tumors of the epididimis and the testis, leiomyoma is the common one.

Materials and Methods: A 71 years old man came to our attention in July 2005, cause of a left inguino-scrotal mass, it was as a walnut, not dolorable. Ultrasound documented solid lymphatic lesion of the diameter of $5 \times 2.7 \times 4.5 \mathrm{~cm}$ in left inguino-scrotal center, no linphadenopathy nor distant mathastases were seen. $\alpha$ fetus protein, $\beta$ HCG and LDH turned out in the norm. Surgical examination demonstrated a solid mass that was excided. At frozen section we documented a leiomyoma, therefore we decided to preserve the didimis, cause of the benignity of the lesion. A leyomioma was confirmed to the definitive histological examination. A thoracal and abdominal TC was negative for repetitive lesions. The follow-up to 8 months is negative, the patient is alive and disease free.

Results and Conclusion: The leiomyoma of funicolus of the testis is a rare cancer. Explorative inguinotomy represents the only modality of diagnosis and treatment. The prognosis is favourable.

\section{6}

\section{The Antegrade Sclerotherapy Sec. Tauber for Varicocele Therapy. Our Experience}

\section{G. Benedetto, M. Titta, G. Abatangelo, F. Nigro, A. Tasca}

Department of Urology, S. Bortolo Hospital, Vicenza

Aim of the Study: The antegrade scleroteherapy of spermatic veins proposed by Tauber and Johnsen is a fast, sure and reliable procedure. We reported our experience with this mini-invasive surgical technique.

Materials and Methods: From March 2003 to December 2004, 145 patients with primitive varicocele (medium age 25.9 years), underwent antegrade sclerotherapy sec. Tauber: 134 cases $(92.4 \%)$ on the left side, 1 on the right side $(0.6 \%)$ and $10(6.8 \%)$ bilaterally. All patients were clinically examined, before surgery and again 6 months post-surgery, by objective examination, scrotal color Doppler ultrasound, and semen analysis.

Results: Medium operative time was 25 min (range 15-35); all patients have been discharged in the same day of surgery. The proceedings have been executed in local anesthesia. No allergic reaction to the sclerosante liquid was described. Post-operative complications were scrotale ematomia $(2 \%)$ and testis pain $(2.7 \%)$. No orchiepididimitis, hydrocele or infection were described. Spermiogramma was improved in 98 cases $(67.5 \%)$ and among 23 patients, 12 obtained pregnancie $(48 \%)$.
Conclusions: Antegrade sclerotherapy sec. Tauber is a miniinvasive surgical technique well accepted by the patients, with little complication and rare cases of recidive with a good percentage of improvement of the seminal parameters.

\section{7 \\ Mucinous Tubular and Spindle Cell Carcinoma: A Case Report}

\section{Pola, S. Benzone, G. Artuso, P. Seren, E. Cossaro, E. Andretta, F. Laganà, F. Sercia, E. Nisi ${ }^{1}$, R. Manconi ${ }^{1}$, P. Bastianello \\ Department of Urology, 'Department of Pathology, Dolo Hospital, Italy}

Aim of the Study: Renal mucinous tubular and spindle cell carcinoma is a rare epithelial neoplasm with a favourable prognosis. We describe a case of type of tumor occurred in our Institute.

Materials and Methods: A 74 years old man came to our attention cause of a left renal mass, incidentally found by an ultrasound study. Abdominal tomography confirmed the presence of a neoplasm apparently confined to the left kidney, no distant methastases nor linphadenopaty were described. The patient was treated by left radical nephfrectomy, with no post-operative complications. At 6 months from nephrectomy the patient is alive and disease free. Histologically the tumor was a mucinous and spindle cell carcinoma confined to the kidney (pT2N0).

Results: Renal mucinous tubular and spindle cell carcinoma is a renal epithelial low grade neoplasm histologically composed of tightly packed, small, tubules separated by pale mucinous stroma, the parallel tubular arrays often have a spindle cell configuration. Mitoses are often infrequently. These tumors have a complex immunophenotype and stain for many cytokeratins, is commonly presented the epithelial membrane antigen. Cause of the presence of these tubular arrays and spindle cells these tumors were before classified as collecting duct carcinomas or sarcomatoid tumors, but they are a low grade tumor and in the prognosis, in the few cases reported in the literature, was favourable.

Conclusions: Renal mucinous tubular and spindle cell carcinoma is a rare neoplasm. Treatment is represented by radical nephrectomy. Right histological characterization is fundamental to formulate a correct prognosis.

\section{8 \\ Bladder Stones and Clean Intermittent Catheterism in Neurogenic Bladder \\ L. Pola, S. Benzone, G. Artuso, P. Seren, E. Cossaro, E. Andretta, F. Laganà, F. Sercia, P. Bastianello \\ Department of Urology, Dolo Hospital, Italy}

Aim of the Study: We reviewed the cases of bladder stones (BS) occurred to our patients in clean intermittent catheterism (CIC) cause of neurogenic bladder to evaluate the reasons of this complication. 
Materials and Methods: From $01 / 1990$ to $12 / 2005$ we surveyed 6 man with BS, among 139 patients in CIC (8.3\%) in follow-up in our Department. In 3 patients, 2 with spinal cord injury and 1 with myelomeningocele, mean diureses was $500-800 \mathrm{cc}$, a patient made an autonomous reduction of CIC per day, from 5 to 3 , he was affected by hyperuricemia too. In one case iperreflexia and high detrusorial pressure caused recurrent urinary infection and calculi of struvute in a patient who refused urological follow-up. In another case a kidney stone was the primitive nucleus for a BS.

Results and Conclusion: BS is an uncommon complication in subjects who performed CIC. Usually BS formation depends on situations easily identifiable as introduction of hair in bladder, poor hydratation, bad CIC management, recurrent urinary infection or impossibility to eject upper urinary tract stones. We found BS in $8.3 \%$ of our patients in CIC for neurogenic bladder, most of all caused by reduced compliance of patients to urologist prescription.

\section{9}

\section{Transobturator Tension-Free Vaginal Tape (TVT-O) Procedure in the Treatment of Female Stress Urinary Incontinence: A Single Centre Experience}

\section{M.A. Cerruto, P. Curti, R. Bertoloni, S.Z. Antoniolli, W. Artibani ${ }^{1}$, F. Zattoni \\ Cattedra e Divisione Clinicizzata di Urologia, Università di Verona, ${ }^{1}$ Clinica Urologica, Università di Padova, Italy}

Aim of the Study: The aim of the work was to report our experience using transobturator Tension-Free Vaginal Tape (TVT-O) procedure with and without associated pelvic organ prolapse (POP) surgery in the treatment of female stress urinary incontinence (SUI).

Material and Methods: From October 2004 to December 2005, 51 female patients (mean age 61 years, range 41-85), complaining of SUI with or without POP, underwent TVT-O procedure. Preoperatively in all cases a complete uro-gynaecological work-up, urodynamics and a perineal ultrasound were obtained. Physical examination showed a stage II POP in 10 cases. Seven patients had urgency due to idiopathic detrusor overactivity and 4 presented with recurrent SUI after anti-incontinence surgery. Followup evaluation included history, physical examination, urinalysis, post micturition residual, uroflow and perineal ultrasound.

Results: TVT-O procedure as single operation was performed in $41 / 51$ patients $(80.39 \%)$. Neither perioperative nor immediate postoperative complications were recorded. In the patient group with TVT-O alone the mean hospitalization was 2.5 days and the catheter was removed within $24 \mathrm{~h}$. At a 13-month mean follow up, got out of 32/51 patients $(62.74 \%)$, a de novo urgency was recorded in $9.37 \%$ of cases and abnormal uroflow parameters were documented in 7 patients. Overall $81.25 \%$ of patients got a complete and/or satisfactory urinary continence.

Conclusion: Concerning peri and postoperative complications our experience is in line with the literature data. Our lower cure rate might be due to the preoperative clinical complexity of the unsuccessful cases.

\section{0}

\section{Posterior Reconstruction of the Rhabdosphincter Allows a Rapid Recovery of Continence After Transperitoneal Video Laparo Radical Prostatectomy: A Two Arm Prospective Randomised Trial - Preliminary Report}

\author{
B. Rocco, A. Gregori, S. Stener, A. Bozzola, S. Galli, \\ R. Knetz, F. Scieri, A. Scaburri, F. Gaboardi \\ Istituto Europeo di Oncologia, Milano, Italy
}

Introduction: Urinary incontinence is one of the major drawbacks of radical retropubic prostatectomy (RRP). One reason of this urinary incontinence is a postop. deficiency of the rhabdosphincter (RS). It has been demonstrated that reconstruction of the posterior aspects of the RS allows a rapid recovery of continence. The aim of this study was to evaluate the application of this technique in laparoscopic prostatectomy (VLRP), assessing the early continence rate.

Materials and Methods: In order to avoid caudal retraction of the RS, before the urethro-vesical anastomosis, the posterior part of the RS is joined to the residuum of the Denonvilliers fascia and then fixed to the posterior bladder wall, $1-2 \mathrm{~cm}$ cranially and dorsally to the bladder neck. A 2 arm prospective randomised trial was carried out with 31 patients recruited for each arm. The A group underwent VLRP and the B group underwent VLRP with RS reconstruction (VLRP-R). Continence was defined as 0 pads or 1 diaper/day and was assessed 3, 30, and 90 days after the procedure.

Results: $74.2 \%$ vs. $25 \%$ of patients were continent at catheter removal with the VLRP-R technique vs. VLRP. A statistically significant difference was present at 30 days. At 90 days the difference, although still present, was not statistically significant.

Conclusion: The posterior reconstruction of the RS allows a shortening of continence recovery time after VLRP.

\section{1}

\section{Periprostatic Nerve Blockade in Transrectal Ultrasound Guided Biopsy of the Prostate: Is this Procedure Useful and Safe?}

B. Santoni, R. Bertoloni, A. Mulonia, R. Spinadin, R. Zennaro, F. Pittarello, V. Gasparella

Department of Urology, Piove di Sacco Hospital, Italy

Introduction: Transrectal ultrasound (TRUS)-guided prostate biopsy $(\mathrm{PB})$ is the standard procedure to diagnose prostate cancer. It can be associated with significant discomfort and pain; up to $90 \%$ of patients report pain during $\mathrm{PB}$, up to $20 \%$ of patients reject a second PB without any anesthesia. In this study we assessed the utility, benefit, morbidity of periprostatic nerve blockade (PNB) for pain management during PB.

Methods: From September 2004 to March 2006, 178 men underwent PB in our institution by the same operator. Patients were assigned into 2 groups: group 1 (91 patients) received no local anes- 
thesia; group 2 ( 87 patients) received a periprostatic injection of $12 \mathrm{ml}$ $1 \%$ lidocain $+0.50 \%$ bupivacain $(6 \mathrm{ml}$ bilaterally) prior PB as Nash's technique. In the last group were included 28 patients who underwent rebiopsy ( 7 asked general anesthesia to repeat biopsy). All patients were asked to answer to a post-procedural questionnaire: the pain was evaluated by visual analogue pain scale (score: $0-10$ ).

Results: There was a significant difference in the mean pain score (MPS) into 2 groups, the MPS was significantly lower in group 2 vs. group $1(2.7$ vs. $4.9 \mathrm{p}<0.05)$. All patients who underwent rebiopsy answered to repeat $\mathrm{PB}$ again without any problems if necessary with this technique. No complications were observed in all group during procedure.

Conclusions: PNB during transrectal ultrasound PB results in less discomfort and pain. It is easy to perform, safe and should be considered in all patients, especially in younger men and for high number of biopsies.

\section{2}

\section{Healthcare Professionals Training in the Area of Urology in Gash Barka Region - Eritrea}

\author{
M. Savino, F. Salvestrini \\ Department of Urology, Siena University School of \\ Medicine, Italy
}

Introduction: In 2005, the Eritrean Ministry of Health and the Italian co-operation Eritrea entered into a partnership for a project concerning human resources development for hospitals in the Gash Barka region. 'Project Gash Barka' focuses on improving surgical services in the region through specialised classroom and on-the-job training. During the course of the project it has become evident that there is a specific need to develop the knowledge of Eritrean healthcare professionals in the area of urology. The Department of Urology, Siena University School of Medicine expressed the willingness to participate in the above mentioned initiative.

Methods: Training plan is divided into three parts: first phase, including the three main hospitals (Barentù, Tesseney and Akurdet) evaluation and the characterization of specific needs in area of urology; second phase, starting after the sign of the agreement, dealing with prevention and basic urological instruments; third phase, including training for diagnosis (ultrasonography, cistoscopy) and treatment of principal urological pathologies. First phase has developing in January 2006.

Results: After first phase, both parties have agreed to work in co-operation in the following areas:

- Give the necessary training to the required health professionals for the prevention, diagnosis and treatment of urological health implications;

- Provide on-the-job training to health professionals working at the three major hospitals in the Gash Barka region;

- Collaborate with the Barentu Associated Nurses School concerning the training of student nurses.

Discussion: The Eritrean Ministry of Health agreed our proposal in April 2006. We're looking for resources and we (truncated at 250 words).

\section{3}

\section{Medical-Expulsive Treatment of Distal Ureterolithiasis: Nifedipine and Tamsulosin}

B. Santoni, R. Bertoloni, A. Mulonia, L. Pola, R. Spinadin, R. Zennaro, F. Pittarello1, V. Gasparella

Division of Urology, 'Division of Radiology, Piove di Sacco Hospital, Italy

Introduction: Expulsive medical theraphy (EMT) of ureterolithiasis is accepted. Many recent studies demonstrate that different drug combinations increase the rate of spontaneous passage of distal ureteral stones.

Objectives: The aim of our study was to assess the efficacy of nifedipine and tamsulosin in addition to a corticosteroid drug during watchful waiting in patients with lithiasis of lower ureter.

Methods: From December 2002 to December 2005 a total of 107 symptomatic patients with distal ureteral calculi smaller than $1 \mathrm{~cm}$ were randomly divided into 3 groups. Group 1 included 35 patients and was acted as the control group, group 2 included 36 patients who received tamsulosin $0.4 \mathrm{mg}$ daily, group 3 included 36 patients who received nifedipine $30 \mathrm{mg}$ slow-release daily. Each group was given an antibiotic prophylaxis and the groups 2 and 3 also a corticoid drug (deflazacort $30 \mathrm{mg}$ daily per 10 days max). The end points were: stone passage rate, expulsion time and analgesic use.

Results: No significant differences were found between the groups for age, sex and mean stone size. The stone espulsion rate was $48.5 \%$ ( 17 of 35 patients) for the group 1, 83\% ( 30 of 36 patients) for the group 2 and $78 \%$ (28 of 36 patients) for the group 3 . The difference in groups 2 and 3 was significant respect group 1. Average expulsion time for groups 1-3 was $14,7.5$, and 8.1 days respectively, significantly lower in the groups 2 and 3 . Finally mean ketorolac dosage for the (truncated at 250 words).

\section{4}

Medical-Expulsive Treatment (MET) of Distal Ureterolithiasis: Nifedipine vs. Tamsulosin

\section{B. Santoni, R. Bertoloni, A. Mulonia, L. Pola, R. Spinadin,} R. Zennaro, F. Pittarello ${ }^{1}$, V. Gasparella

Division of Urology, 'Division of Radiology, Piove di Sacco Hospital, Italy

Introduction: Many studies demonstrate that different drug combinations increase the rate of spontaneous passage of distal ureteral stones. We assessed the efficacy of nifedipine and tamsulosin in addition to a corticosteroid drug in patients with lithiasis of lower ureter.

Methods: From December 2002 to December 2005, 107 symptomatic patients with distal ureteral calculi ( $\mathrm{size}<1 \mathrm{~cm}$ ) were randomly divided into 3 groups. Group 1 included 35 patients and was acted as the control group, group 2 included 36 patients who received tamsulosin $0.4 \mathrm{mg}$ daily, group 3 included 36 patients who received nifedipine $30 \mathrm{mg}$ slow-release daily. For groups 2 and 3 was added deflazacort $30 \mathrm{mg}$ (10 days). The end points were: stone passage rate, expulsion time and analgesic use. 
Results: No significant differences were found between the groups for age, sex and mean stone size. The stone espulsion rate was $48.5 \%$ ( 17 of 35 patients) for the group 1, 83\% (30 of 36 patients) for the group 2 and $78 \%$ ( 28 of 36 patients) for the group $3(\mathrm{P}<0.05)$. Average expulsion time for groups 1-3 was 14, 7.5, and 8.1 days respectively $(\mathrm{P}<0.05)$. Finally mean ketorolac dosage for the control of pain was significantly lower in the groups 2 and 3 especially for the tamsulosin.

Discussion: MET for the ureterolithiasis without complications should be considered, before invasive treatment, always. Both, nifedipine and tamsulosin increased stone expulsion rate, decreased expulsion time and reduced the use of analgesics especially for tamsulosin.

\section{5}

\section{Healthcare Professionals Training in the Area of Urology in Gash Barka Region - Eritrea}

\section{Savino, F. Salvestrini \\ Department of Urology, Siena University School of Medicine}

Introduction: In 2005, the Eritrean Ministry of Health and the Italian co-operation Eritrea entered into a partnership for a project concerning human resources development for hospitals in the Gash Barka region. 'Project Gash Barka' focuses on improving surgical services in the region through specialised classroom and on-the-job training. During the course of the project it has become evident that there is a specific need to develop the knowledge of Eritrean healthcare professionals in the area of urology. The Department of Urology, Siena University School of Medicine expressed the willingness to participate in the above mentioned initiative.

Methods: Training plan is divided into three parts: first phase, including the three main hospitals (Barentù, Tesseney e Akurdet) evaluation and the characterization of specific needs in area of urology; second phase, starting after the sign of the agreement, dealing with prevention and basic urological instruments; third phase, including training for diagnosis (ultrasonography, cistoscopy) and treatment of principal urological pathologies. First phase has developing in January 2006.

Results: After first phase, both parties have agreed to work in co-operation in the following areas: give the necessary training to the required health professionals for the prevention, diagnosis and treatment of urological health implications; provide on-the-job training to health professionals working at the three major hospitals in the Gash Barka region; collaborate with the Barentu Associated Nurses School concerning the training of student nurses;

Discussion: The Eritrean Ministry of Health agreed our proposal in April 2006. We're looking for resources and we hope to start until the end of this year. We believe that it will be represent a special opportunity for human and professional growth.

\section{Experimental Surgery}

326

The Effect of Meraxin a New IL8 Inhibitor in
Protection from Ischemia-Reperfusion
Damage in Kidney Transplantation

L.Puviani ${ }^{1}$, F. Neri ${ }^{1}$, V. Pacilè ${ }^{1}$, R. Bertelli', P. Beltempo', G. Cavallari', M. Tsivian ${ }^{1}$, D. Prezzi ${ }^{1}$, M. Pariali ${ }^{2}$, R. Bertini ${ }^{3}$, A. Faenza ${ }^{1}$, B. Nardo

${ }^{1}$ Department of Surgical Disciplines, ICU and

Transplantations, ${ }^{2}$ Biomedical Research Center, University of Bologna, ${ }^{3}$ Dompè Itd, L'Aquila, Italy

Introduction: Assessing the efficacy of meraxin, a new IL8 inhibitor, in kidney trasplantation (KT) in rats, in protection from ischemia reperfusion damage.

Methods: Syngenic Lewis (CT1) rats of $\sim 250 \mathrm{~g}$ were used. Left kidney was harvested in the donors $(n=24)$ and preserved by simple hypothermy in Celsior for $4 \mathrm{~h}$. The recipients underwent nephrectomy and were divided in 4 groups $(\mathrm{n}=6)$ : $\mathrm{G1}$ (control): only KT, G2: KT and $3 \mathrm{mg} / \mathrm{kg}$ of meraxin, G3: KT and $10 \mathrm{mg} / \mathrm{kg}$ of meraxin, G4: $\mathrm{KT}$ and $30 \mathrm{mg} / \mathrm{kg}$ of meraxin. The drug was administered e.v. $2 \mathrm{~h}$ prior to reperfusion of the trasplanted organ. At $24 \mathrm{~h}$ after the reperfusion we assessed renal function with determination of serum creatinine levels and the inhibition of the polimorphonucleate leucocytes infiltrate formation in transplantated kidney.

Results: Renal function improved in rats treated with meraxin vs. G1 rats. Among the treated animals the lowest creatinine levels were observed in G4 $(1.59 \pm 0.4 \mathrm{mg} / \mathrm{dl})$, with a significant difference from G1-G3, whose creatinine levels were higher $(\mathrm{G} 1=2.54 \pm 0.5 \mathrm{mg} / \mathrm{dl}$, $\mathrm{G} 2=2.21 \pm 0.7 \mathrm{mg} / \mathrm{dl}, \mathrm{G} 3=2.20 \pm 0.5 \mathrm{mg} / \mathrm{dl}$ ). Tissutal kidney damage in G4 was lower than in the other groups, with a reduction of the inflammatory response.

Discussion: Meraxin treatment in syngenic rats with KT has shown itself efficacious in protecting transplanted organs from ischemia-reperfusion damage. The optimal dose was $30 \mathrm{mg} / \mathrm{kg}$.

\section{7}

A Pilot Experimental Study on Controlled Portal Vein Arterialization with an

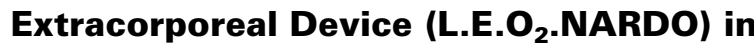
Swine Model of Subtotal Hepatectomy

\author{
V. Pacilè, M. Tsivian, D. Prezzi, F. Neri, L. Puviani, \\ R. Bertelli, G. Cavallari, R. Montalti, M. Pariali, P. Beltempo, \\ D. Cianciavicchia1, A. Pinna, B. Nardo \\ Department of Surgery and Transplantations, S. Orsola \\ Hospital, University of Bologna, 'Bellco Srl, Mirandola, \\ $\mathrm{MO}$, Italy
}

Introduction: To determine whether physiologically oxygenated blood reversed in the portal system by portal vein arterializa- 
tion (PVA) through an extracorporeal device namely L.E.O 2 .NARDO (Liver Extracorporeal Oxygen. NARDO) is effective in treating swine with subtotal hepatectomy leading to acute liver failure (ALF).

Methods: 10 pigs with ALF induced by $85-90 \%$ liver resection and $5 \mathrm{~min}$ of ischemia-reperfusion injury were randomly divided in two groups: 5 animals received PVA extracorporeal treatment and 5 were untreated (control group). An extracorporeal device was interposed between iliac artery and portal system, treatments lasted $6 \mathrm{~h}$. Serum and liver samples were collected. Survival was assessed at 1 week.

Results: The PVA treatment yielded beneficial effects: lower serum ammonia, transaminases and total bilirubin as compared to control group. INR recovered rapidly and the 7-day survival was significantly higher in the treated group $(\mathrm{p}<0.05) .4$ swine in the treated group survived at 1 week vs. 0 of the control group; mean survival time was: $144 \mathrm{~h} \pm 13 \mathrm{~h}$ and $24.4 \mathrm{~h} \pm 5 \mathrm{~h}$ in the PVA-extracorporeal and control groups, respectively.

Discussion: Arterial blood supply in the portal system through the extracorporeal device is easily applicable, efficacious, safe and cost effective and may represent a novel approach for swine ALF induced by subtotal liver resection and ischemia-reperfusion.

\section{8}

\section{Protective Effect of Portal Vein}

Arterialization in Acute Liver Failure Induced by Hepatectomy in Normal and Fatty Liver Rats

\author{
L. Puviani ${ }^{1}$, D. Prezzi ${ }^{1}$, F. Neri ${ }^{1}$, M. Tsivian ${ }^{1}$, R. Bertelli ${ }^{1}$, \\ G. Cavallari', P. Beltempo' ${ }^{1}$ P. Caraceni ${ }^{2}$, M. Pariali ${ }^{3}$, \\ A.M. Pertosa ${ }^{3}$, M. Bernardi ${ }^{2}$, A. Pinna ${ }^{1}$, B. Nardo ${ }^{1}$ \\ ${ }^{1}$ Department of Surgery and Transplantations, \\ ${ }^{2}$ Department of Internal Medicine, Cardioangiology and \\ Hepatology, ${ }^{3}$ Biomedical Research Center, University of \\ Bologna, Italy
}

Introduction: To determine whether oxygenation of portal blood by portal vein arterialization (PVA) is protective in normal and fatty liver (FL) rat with hepatectomy-induced acute liver failure (ALF).

Methods: ALF was induced in rats with normal and FL (cholinedeficient diet) by $70 \%$-hepatectomy. Rats were divided in PVA-group, (left renal artery connected to splenic vein with a stent following nephrectomy and splenectomy, $\mathrm{n}=16$ ), and control group (nephrectomy and splenectomy, $\mathrm{n}=16$ ). Liver regeneration was assessed by bromodeoxyuridine (BrdU) incorporation and liver/body weights ratio. Liver injury was evaluated by ALT levels. The animals were sacrificed at $24 \mathrm{~h}, 48 \mathrm{~h}$ and 7 days collecting blood and parenchymal samples for biochemical, histological-immunohistochemical assay.

Results: PVA significantly improved the 7 day survival compared to controls in both ALF models (hepatectomy of normal liver: 90 vs. $30 \%$; hepatectomy of FL: 75 vs. $25 \%$ ). ALT levels were slightly lower in PVA groups vs. non-PVA groups $(\mathrm{p}=\mathrm{ns})$. The BrdU staining was significantly greater in PVA rats in both models. Liver/body weight ratio after hepatectomy recovered significantly faster in arterialized rats.

Discussion: An arterial oxygenated blood supply by PVA promotes a rapid regeneration leading to a faster restoration of liver mass after hepatectomy in normal and FL and resolution of ALF.
329

\section{The 3R's in Education and Training Project: New Tools to Deal with the Early Microsurgical Training}

\author{
F. Carú1, B. Cozzi', C. Ballarin², A. Peruffo², M. Isola ${ }^{3}$, \\ D. Sozzi ${ }^{4}$, S. Gatti ${ }^{5}$ \\ ${ }^{1}$ Institute of Experimental, Transplantations and \\ Cardiovascular Surgery, University of Milan, ${ }^{2}$ Department \\ of Experimental Veterinary Sciences, ${ }^{3}$ Department of \\ Veterinary Clinical Sciences, University of Padua, \\ ${ }^{4}$ Department of Maxillofacial Surgery, University of \\ Milan, Bicocca, ${ }^{5}$ Fondazione IRCCS Ospedale Maggiore \\ Policlinico Mangiagalli Regina Elena, Milan, Italy
}

Introduction: The aim of the 3R's in Education and Training Project is to develop new alternatives to the animal's use for research and education, mainly for the early microsurgical training, in accordance with the 3R's principles (Refinement, Reduction, Replacement) on animal's use for scientific purposes.

Methods: The project is composed by: (1) the handbook Anatomy of laboratory animals (Rodents and Lagomorphs), (2) the CDrom Microsurgery Interactive Course, (3) the device Hydraulic trainer for experimental microsurgery, (4) the handbook Textbook on the anatomy of swine in preclinical research.

Parts 2-3 are specifically developed for preclinical microsurgery: (2) The Microsurgery Interactive Course is a multimedia support giving an easy-handling tool to bear comparison with principal needs of surgical knowledge and expertise, (3) The Hydraulic trainer for experimental microsurgery is an hydraulic simulator featuring the same conditions of in-vivo models (rodents).

Results: (1) The handbook has been published in Italian (CEA Edizioni, Milan, 2006), an English version is under preparation, (2) The CD ROM is in the last revision phase, (3) The device's setting up is ongoing, a patent application will be requested, (4) The text book is in the very early phase of preparation.

Discussion: At time this project had been partially implemented at Biotechnologies and Experimental Surgery courses, as target young surgeons, students and personnel working with laboratory animals. Hopefully it will contribute to improve the education and learning processes, giving significative benefits (saving of animals, avoidance of unjustified animal sacrifice) still assuring the highest quality of experimental and, consequently, clinical microsurgery.

\section{0 \\ No Evidence for Substantial Islet Graft Loss During Early Post Transplantation Phase}

\author{
W. Moritz, R. Zuellig, G. Cavallari, M. Weber \\ Clinic for Visceral and Transplantation Surgery and \\ Division of Endocrinology and Diabetology, University \\ Hospital Zurich, Switzerland
}

Introduction: Since the introduction and the subsequent success of the Edmonton protocol with an improved isolation procedure and immunosuppressive strategy, islet transplantation (tx) has been 
newly implemented into the program for the treatment of IDDM in numerous clinical centers. However, a substantial part of grafted islets is thought to succumb to the adverse effects of prolonged ischemia subsequent to the embolization.

Methods: We investigated the early events of islet engraftment related to hypoxia in a model of syngeneic intrahepatic islet tx in Streptozotocin-induced diabetic rats by histological and metabolic assessment.

Results: Based on these investigations, we can not confirm a dramatic graft loss within the first 14 days after tx, even though a subtherapeutic islet mass was chosen. However, insulin immunostaining and glucose tolerance test indicated a reduced insulin content and secretory capacity of islet grafts in diabetic recipients when compared to islets grafts in normoglycemic or diabetic, insulin treated rats.

Discussion: Our results suggest that loss of graft performance in the immediate early phase after tx is more likely a consequence of exhaustion rather than of an acute and substantial islet cell death. Our observations underline the need of a tight blood glucose control posttx, especially when only a marginal graft tissue is available for tx.

\section{1}

Effects of TIMP-2 on Vascular Remodelling after ePTFE Grafting. An Experimental Study

\author{
G. Cavallaro, G. Mosiello, A. Polistena, A. Cucina, \\ B. Randone, P. Coluccia, G. De Toma, A. Cavallaro
}

Department of Surgery, 'P. Valdoni', Policlinico Umberto I, University of Rome, 'La Sapienza'

Introduction: In reconstructive vascular surgery, myointimal hyperplasia contribute to the adverse outcome of synthetic grafts. This phenomenon is due to unregulated extracellular matrix degradation and remodelling, and excessive smooth muscle cell proliferation. Matrix metallopreoteinase 2 (MMP-2) is known as an important contributor to these events. The aims of our study were to investigate the effects of selective MMP-2 inhibitor (TIMP-2) in endothelialization rate, SMC proliferation and myointimal hyperplasia in experimental ePTFE arterial grafts.

Methods: In 20 male Lewis rats, a $1 \mathrm{~cm}$ long ePTFE graft has been inserted at the level of the abdominal aorta. Animals were randomised in 2 groups (10 animals each): group A received 6 subcutaneous inoculations of TIMP-2 $(2.5 \mu \mathrm{g})$ after surgery, group B received only the vehicle of TIMP-2.

Results: Myointimal hyperplasia and SMC density were augmented in group B, whereas EC density was augmented in group A, and these findings were statistically significant. In group A SMC were better organized, just like SMC of thoracic aorta. In group B SMC were no organized. Furthermore, anti-TIMP-2 and anti-MMP-2 coloration revealed higher levels of TIMP-2 and lower levels of MMP-2 in group A vs. group B.

Conclusions: Use of TIMP-2 affects the neointimal formation of experimental ePTFE arterial grafts, leading to a better organized neointima, with improved endothelialization.
332

\section{Tolerance-Inducing Strategy in Small Bowel Transplantation in Swine}

J. Viganò, S. Zonta, M. Alessiani, M. Bardone, M. Doni,

F. Lovisetto, T. Dominioni, M. De Martino, M. Mazzilli,

N. Mevio, C. Filisetti, A. Bottazzi ${ }^{1}$, P. Morbini², P. Dionigi

Epatopancreatic Surgery Unit, IRCCS Policlinico San Matteo, Pavia, Department of Surgical Sciences,

University of Pavia, ${ }^{1}$ Anaesthesiology and Reanimation

Unit, IRCCS Policlinico San Matteo, ${ }^{2}$ Pathology Institute,

Pharmacology Institute, IRCCS Policlinico San Matteo,

Pavia, Italy

Introduction: One of the strategies under development to enhance survival of allografts and to reduce immunosuppressive treatment, is the Donor Bone Marrow infusion (DBMi). In this large-animal study, we compared a immunosoppression (IS) short course based on tacrolimus (TAC) and MMF with the same IS regime combined with DBMi.

Methods: We performed 17 intestinal transplantation (ITx) in piglets: Group $1(\mathrm{n}=5)$ : ITx with no IS; Group $2(\mathrm{n}=7)$ : ITx and 60 days of IS with TAC +MMF; Group $3(n=5)$ ITx + DBMi, and 60 days of IS with TAC+MMF. Follow-up time after IS withdrawal was 4 months.

Results: Gr1 pigs died of graft acute rejection (ACR) after a median of 11 days. In Gr2, 2 pigs died of infection and other 2 of ACR within 90 days; 3 animals (43\%) were sacrificed at day 180 and their grafts showed no signs of ACR. In Gr3, 2 pigs died of infection and 1 of Graft vs. Host Disease (GVHD) within 80 days; the 2 surviving animals at day 180 showed sign of chronic rejection in the allograft.

Discussion: Total IS withdrawal is followed by sudden and lethal ACR/ACR-related infection in more than $50 \%$ of the recipients. When a tolerance-inducing strategy (DBMi) is applied to the same model, GVHD may also occur. The important observation in this study is the similar successful postoperative course of animals with/without DBMi. However, when DBMi is used, we observed a tendency to develop chronic rejection in allograft, probably associated with a decline with time of donor-leukocytes chimerism, as recently demonstrated in rats.

333

\section{Carbohydrate Absorption Is Not Altered by Malononitrilamide FK778 in a Pre-Clinical Model of Total Small Bowel Transplantation}

M. Doni, S. Zonta, M. Alessiani, J. Viganò, M. Bardone,

F. Lovisetto, T. Dominioni, M. De Martino, M. Scaglioni,

A. Biroli, A. Bottazzi ${ }^{1}$, M.D. Molinaro ${ }^{2}$, P. Poggi ${ }^{3}$,

P. Dionigi

Epatopancreatic Surgery Unit, IRCCS Policlinico San Matteo, Pavia, Department of Surgical Sciences, University of Pavia, ${ }^{1}$ Anaesthesiology and Reanimation Unit, ${ }^{2}$ Pharmacology Institute, ${ }^{3}$ Analyitical Laboratory, IRCCS Policlinico San Matteo, Pavia, Italy

Introduction: Malononitrilamide 715 (FK778) is a new class of low-molecular weight immunosuppressant that inhibits both T-cell 
and B-cell function acting on the pathway for the de novo pyrimidine biosynthesis. Pyrimidines are important for intestinal trophism and their inhibition may predispose to metabolic and functional impairment, such as diarrhea and malabsorption. In this study we assessed the absorptive capacity of intestinal allograft in a large-animal model of small bowel transplantation (SBTx) in pigs chronically treated with FK778.

Methods: Ten outbred pigs underwent total orthotopic SBTx. Immunosuppression consisted of oral tacrolimus (trough levels between 5 and $15 \mathrm{ng} / \mathrm{ml})$ and oral FK778 $(4 \mathrm{mg} / \mathrm{kg} /$ day) administered for 60 days. The D-xylose absorption test was performed at day 60 to evaluate carbohydrate absorption.

Results: Results were compared to normal controls. Eight of the ten animals were alive and in good condition at day 60. All their allograft were free from rejection. The animals had a mean maximal weight loss of $6.4 \%$ during the study period; the final weight was comparable with the initial weight $(\mathrm{p}>0.05)$. Diarrhea was present in all animals (mean $16 \%$ of postoperative days). The $\mathrm{D}$-xylose curves showed that absorption in the transplanted animals at day 60 is similar to that in the untreated controls $(\mathrm{p}>0.05)$. The absence of differences was confirmed by the statistical analysis.

Discussion: In conclusion, our pre-clinical study in pigs shows that chronic treatment with FK778 in combination with tacrolimus does not impair carbohydrate absorption of the allograft after SBTx.

\section{4 \\ FK778 Is Superior to Mycophenolate Mofetil or Rapamycin in Tacrolimus-Based Immunosuppression After Small Bowel Transplantation in a Large Animal Model (PIG)}

S. Zonta, M. Alessiani, J. Viganò, M. Doni, M. Bardone,

F. Lovisetto, T. Dominioni, M. De Martino, M. Podetta, M. Scaglioni, E. Vicini, A. Bottazzi' , M.D. Molinaro², P. Morbini', P. Dionigi

Epatopancreatic Surgery Unit, IRCCS Policlinico San Matteo, Pavia, Department of Surgical Sciences, University of Pavia, ${ }^{1}$ Anaesthesiology and Reanimation Unit, ${ }^{2}$ Pharmacology Institute, ${ }^{3}$ Pathology Institute, IRCCS Policlinico San Matteo, Pavia, Italy

Introduction: Tacrolimus (TAC) and Mycophenolate Mofetil (MMF) or Rapamycin (RAP) are used in combined therapy after clinical small bowel transplantation. Malononitrilamide (FK778), recently showed its efficacy when administrated in combination with TAC in heart, kidney and liver transplantation. The aim of this study is to assess, in a swine small bowel transplantation model (SBTx), the effect of a combined therapy of TAC + FK 778 vs. a combined therapy of TAC + MMF or TAC + RAP.

Methods: We performed 26 SBTx: Gr.1 $(n=10)$, TAC+oral FK778 (100 mg/daily); Gr.2 ( $=10)$ TAC + oral MMF $(10 \mathrm{mg} / \mathrm{kg}$ twice daily); Gr.3 $(\mathrm{n}=6)$ : TAC + oral RAP (trough level ranging 5-11 ng/ml);

Results: Follow-up time was 60 days. Survival rates were $90 \%$, $90 \%$, and $70 \%$ at 30 days and $90 \%, 80 \%$, and $60 \%$ at 60 days in groups 1, 2, and 3, respectively. Statistical differences were significant between group 1 vs. $2(p=0.05)$; group 1 vs. $3(p=0.02)$. None of the immunosuppressed animals developed evidence of graftversus-host disease. At the end of the study, 2 animals in Gr.2 and 2 in Gr.3 showed histological signs of moderate-to-severe acute cellular rejection (ACR). The incidence of infection was higher in Gr.3 (2.1 episodes/pig) compared with Gr.1 (0.3 episode/pig) and Gr.2 (1.25 episodes/pig).

Discussion: In our large animal model, the immunosuppressive regimen with TAC and FK778 showed superior results in terms of survival and ACR control compared to the combined TAC and MMF or TAC and RAP therapy. Thus, this study support the use of FK778 in clinical intestinal transplantation.

\section{5}

\section{Antibiotic Prophylaxis in Severe Acute Pancreatitis: Experimental Evaluation of Ertapenem Efficacy}

\author{
F. Gazzotti, F. Catena, L. Ansaloni, S. Gagliardi, \\ S. Di Saverio, L. D'Alessandro, A.D. Pinna
}

Transplant, General and Emergency Surgery DPT, St Orsola-Malpighi University Hospital, Bologna, Italy

Introduction: Secondary infection of the inflamed pancreas is the principal cause of death after severe acute pancreatitis (AP). Prophylactic antibiotics that were used in AP were always initiated after hours from induction of pancreatitis. The effectiveness of antibiotics initiated earlier is unknown. The aim of this study was to evaluate the effectiveness of ertapenem initiated when AP is induced in rats.

Methods: 20 Sprague-Dawley rats were studied. AP was induced in rats by intraductal injection of 3\% taurocholate. Rats were divided randomly into two groups: group I rats received normal saline as a placebo, group received ertapenem $15 \mathrm{mg} / \mathrm{kg}$ after AP induction. At $24 \mathrm{~h}$, all rats were killed for quantitative bacteriologic study. A point-scoring system of histological features was used to evaluate the severity of pancreatitis.

Results: All rats showed pathologic signs of acute pancreatitis. Ertapenem administrated after induction of AP significantly reduced the prevalence of pancreatic infection and mortality as compared to controls.

Discussion: Early antibiotic prophylaxis with ertapenem reduces pancreatic infection rate after $\mathrm{AP}$ and it has a beneficial effect on survival. 
Day Surgery

\section{6}

Local Anesthesia and Day Surgery for Stapled Prolapsectomy: Our Experience and Results After 340 Procedures

\author{
P. Mariani, G. Arrigoni, R. Cadoni, A. Guadalaxara, \\ L. Rampinelli, C. Facchinetti, G. Perrone \\ Chirurgia Generale, Ospedale Bolognini, Seriate, BG
}

Introduction: Stapled prolapsectomy for the treatment of hemorrhoidal disease is characterized by minimal post-operative discomfort and faster resumption of activities. Some limitations to applicability of the Longo's procedure could be attributed to the anesthetic technique; after a prospective trial on the feasibility of this procedure under local anesthesia and on outpatient basis, we report our experience and results after 300 procedures with focus on the efficacy of the same day discharge.

Material and Methods: From April 2002 to March 2006, we performed 340 consecutive stapled prolapsectomy with local anesthesia (ropivacaina $7.5 \mathrm{mg} / \mathrm{dl}$ ), using a modified variant of Nivatvong's technique of infiltration of the anorectal region. Our series included 190 females and 150 males with a mean age of 45.7 (range 23-68) years; $60 \%$ had prolapsed third-degree hemorrhoids, while 40 percent had fouth-degree hemorrhoids.

Results: In all patients it was possible to perform the procedure under local anesthesia, obtaining complete relaxation and absence of pain in the anal canal during the introduction of the circular anal dilatator and minimizing the discomfort in the hypomesogastric region during the stapler firing. Three-hundred and fifteen patients $(93 \%)$ were discharged $6 \mathrm{~h}$ after the operation, whereas twenty-five required an overnight stay for early complications (pain, urinary retention and early bleeding).

Conclusion: The stapled prolapsectomy procedure is feasible and can be performed safely under local anesthesia and as day surgery, with good pain control and minimal complications rate.

\section{7}

\section{Day Surgery Operative Unit: Organizational Model}

M.G. Muzi, C. Ridolfi, G. Galatà, D. Amabile, F. Andreoli, M.B. Silvi, M. Villa, L. Bonanno, C. Nigro

Department of Surgery, Tor Vergata University Hospital, Rome

Introduction: Day surgery is an assistance model that permits procedures to be performed during a daytime hospitalization. Economical healthcare difficulties and the need to reduce expenses favoured the diffusion of this model obtaining the approbation of Hospital administrations and of patients. Although the operations performed are characterized by short-medium duration and low incidence of complications, strong operative severity and accurate selection are necessary to limit post-operative complications and patient's discomfort.

Material and Methods: First approach for patients is the examination to assess if they are fit for day surgery including anaesthesiological evaluation following SIAARTI recommendations. If operation is performable in day surgery unit patient is included in operating list; then is phone-called and all the informations are given to him. Type of anaestesia is chosen by the anaesthetist in accordance with the surgeon and is characterized by a rapid recovery of physiologic functions, social relations, and a reduction of incidence of postoperative collateral effects. After dismission a letter that includes all information about operation, following check-up, therapy, contacts to call in case of necessity is given to the patient.

Results and Conclusions: The assistance model of day surgery satisfies the need to reach a quality-cost balance such as to give quality treatment but minimize costs through reduction of the period of hospitalization without increasing the risk of complications.

\section{8 \\ Nasal Reconstruction in Cutaneous Cancer Therapy}

F. Klinger, A.V. Pagliari, F. Caviggioli, D. Ventura, F. Villani, S. Giannasi, B. Banzatti, C.A. Amodeo, J. Hassibi, M. Klinger

Istituto di Chirurgia Plastica e Ricostruttiva dell'Università degli Studi di Milano, I.C. Humanitas

Introduction: Basal cell carcinoma is the most frequent cutaneous cancer of the nose and is characterized by its local spreading and exceptionally rare tendency to metastasize. Since a significant advantage has been seen in surgery compared to other treatments surgical excision ensuring the highest chance of cure is frequently employed. Ecision defects of the nose may be covered with either local flap or a full-thickness skin graft.

Methods: In our series nasal reconstruction was performed according to the topographic aesthetic subunits of the nose. In reconstructive planning were also considered skin texture, colour and contour.

Results: There were no cases of flap loss and all patients were recontructed in a single stage procedure with an excellent final cosmetic appearance. No recurrence case was observed.

Discussion: Nasal reconstruction are often dicatted by size and location of the defect. Patients treated with regional flap had good healing and good simmetry and required minimal revision. Single stage reconstruction with local flap requires both proper flap design and extensive undermining of the entire nasal soft tissue cover. Nasal simmetry is maintained despite the defect and donor flap being on the same side of the nose. 


\section{9}

\section{Treatment of Pilonidal Sinus with Primary Closure: A Twenty Years Experiences}

\section{Persico Stella, S. Toccaceli, R. Dandolo, M. Diana, \\ A. Budak, C. Tasciotti, M. Tasciotti, G.E. Casciaro, \\ E. Spaziani, F. Stagnitti \\ U.O.C. Chirurgia Universitaria, Ospedale Fiorini, Terracina, Università degli Studi 'La Sapienza', Polo Pontino}

Introduction: Pilonidal sinus is a common disease among young adults after the puberty while it is quite rare in subjects older than 40 years. The incidence in the third and fourth decade and the prevalence of the disease in males, with a rate of 3:1 are probably related to androgen secretion. In case of abscess most of the authors suggest a conservative approach with incision and drainage under local anesthesia, delaying the definitive treatment by complete excision of the sinus. Nevertheless, surgical treatment of the sinus has an high recurrence rate and as shown by the several techniques proposed, choice primarily depends on the experience of the surgeon and on the consensus of the patient.

Methods: From 1985 to 2004, 229 patients (171 M, 58 F) aged between 16 and 35 years, affected by pilonidal sinus were treated by complete excision of the sinus and primary closure.

Results: Primary healing was achieved in 208 patients (91\%) in an average time of 11.9 days. Secondary healing was achieved in 21 patients (9\%) in 16-19 days. Mean postoperative hospital-stay was 1.9 days and average time off work was 16 days. Recurrent pilonidal sinus was observed in 10 patients with a mean follow-up of 30 months (range 12-60).

Discussion: On the basis of their experience, the authors conclude that complete excision of pilonidal sinus with primary closure provides good results in terms of healing, morbidity, early return to work and recurrence rate and can be considered the treatment of choice of pilonidal sinus.

\section{0}

Management of Pilonidal Sinus with Excision and Open Granulation Procedure. A Prospective Study

\author{
M. Rastrelli, A. Simmini, B. Ferraro, P. Frasson \\ Division of General Surgery, S. Antonio Hospital Padua, \\ Chirurgia Generale Ospedale S. Antonio, Padova, Italy
}

Introduction: Pilonidal Sinus (PS) is a common surgical disease in young people. The most probably etiophatogenesis consider PS an inflammatory disease due to reversed hair development in the derma of intergluteous space. In this way the inflammatory reaction creates a cystic cavity that can be infected. The optimal treatment is still matter of discussion.

Methods: Between 1/1/1998 and 31/12/2004, 400 patiens (357 male and 43 female; average age $25.02 \pm 3.80$, range $15-51$ years) were operated on for primary or recurrent PS with open procedure. We have built a database for valutation of effectiveness and acceptability of this procedure by the patiens. We have done also a follow-up after 2 years for evaluation of recurrent number.
Results: Duration of operation was $20 \mathrm{~min}$ (range 15-50), 12 patiens bleeded after surgery (3\%), length of hospital stay was $5 \mathrm{~h}$ (range 3-7), postoperative pain lasted for 3 days (range 1-6), postoperative disability and social disruption (evaluated by indirect questions about ability to drive the car and the position assumed while at home) lasted for 4 days (range 2-7), time to resumption of work was 18 days (range 7-24), time for complete wound healing was 50 days. There was $3.4 \%$ of PS recurrence after a mean follow up of 28 months.

Conclusions: This method is a gold standard for the treatment of PS because is simple and well accepted by the patiens. The number of recurrent PS, with open procedure, is the lowest. Our number of recurrent $\mathrm{PS}$ is lower than in international licterature.

\section{1 \\ Inguinal Hernia Repair with PAD in Day Surgery}

C. Talarico, N. Papandrea, M. Deonofrio, G. Gullà, A. Lioi

Surgical Unit, 'San Biagio' Hospital, Chiaravalle C.le, CZ

Introduction: In this video the authors present their personal experience with the PAD (Valenti's dynamic self-regulating prothesis) in the treatment of inguinal hernia.

Methods: From January 2000, 547 male patients were operated for inguinal hernia. The operation was performed under local anesthesia on Day Surgery bases. In this report the authors analyze patients characteristic, length of operations, postoperative analgesia, hospital stay length and postoperative complications.

Results: The median age was $\mathbf{5 8 . 3}$ years, the type of hernia was direct in 185 cases, indirect in 346 and double combined hernia in 16. In 35 cases the hernia was recurrent. Duration of operation varied from 20 to $100 \mathrm{~min}$. The postoperative analgesia was unnecessary in 335 patients, while in 155 single dose of Ketorolac was sufficient to control the pain (in 57 double somministration). The discharged was conducted in all cases within the $24 \mathrm{~h}$ from admission and the median time of dismission was $6.3 \mathrm{~h}$. The postoperative complication were: 3 local haematomas, 3 wound infections, 3 acute urinary ritenction. The recurrency of hernia was found, under follow-up in 6 cases.

Discussion: The treatment of inguinal hernia with PAD patch in Day Surgery is safe and well tolerated in a large series of patients. This technique reduce the duration of operation and postoperative doses of analgesic drugs and permit to discharge the patients earlier. 


\section{Surgical Oncology}

\section{2 \\ Synchronous Adenomatous Polyps and Colorectal Carcinoma}

\section{T. Bartolotta, M. Cajozzo, A. Sunseri, G. Tomasello, C. Randazzo, T.V. Bartolotta1, F. Cappello², G. Modica \\ Department of Surgical and Oncological Sciences, ${ }^{1}$ Department of Radiology, ${ }^{2}$ Section of Human Anatomy, University of Palermo}

Introduction: Most colorectal cancers (CRC) arise from adenomatous polyps (AP). The aim of this study is to underscore the incidence of synchronous AP in patients with CCR and to emphasise the importance of their perioperative detection and management.

Methods: 1904 consecutive patients who underwent a complete colonoscopy between May 2002 and February 2006 for CCR screening. The principal indications for this procedure are rectal bleeding, iron deficient anemia, positive fecal occult blood test and family history of CCR. If there were technical complications to perform a complete colonoscopy as lack of cleaning of the colon the patients were excluded from the study.

Results: A total of 152 patients were diagnosed with CCR. AP detected concomitantly with a CCR were noted in $14.8 \%$ of cases. The distance between synchronous lesions was less than $30 \mathrm{~cm}$ in $56.1 \%$ of all the patients in the study. The CCR associated with AP have a higher prevalence in the worst prognostic stages.

Discussion: Synchronous polyps in patients with CCR are a frequent event and in some of this patients, naturally, the planned surgical procedure may alter as a consequence of the colonoscopic findings. Detection and removal of AP with colonoscopy is known to be the best preventive method for the CCR. With removal of synchronous colon polyps, the risk of metachronous cancer seems to be decreased effectively.

\section{3}

\section{Gastrointestinal Carcinoids: Diagnosis and Treatment}

P. Cianci, C. Verrusio, G. Guarino, B. Puppio, M. Ciuffi,

S. Mazzuoli, G.L. Torre

U.O. di Chirurgia Oncologica-Centro di Riferimento

Oncologico di Basilicata-Rionero in Vulture, PZ

Background: Carcinoids are rare neoplasms. The aim of this study is to consider such pathology in its nosological, diagnostical and therapeutical aspects.

Methods: From September 2002 to December 2005, at our Institute, 7 patients with carcinoid of the gastrointestinal tract were examined, (4 F, $3 \mathrm{M}$ ) with mean age of 56 years. In 5 patients, location was gastrical, single in 3 cases and multiple in 2.2 carcinoids of duodenal onset were found, one of which was multifocal. Through a care- ful anamnestic investigation, dosage of the main disease markers (Serotoninemy, gastrinemy, 5-HIAA urinary) and recourse in instrumental endoscopic and eco-endoscopic examinations, a correct preoperative diagnosis was reached. All of the lesions surgically treated presented a diameter $>1 \mathrm{~cm}$ and an invasion of the submucosa. 3 gastroresections for single gastric carcinoids and 2 total gastrectomies for multifocal ones were carried out. The 2 cases of duodenal onset underwent gastroduodenal resection according to Billroth I.

Results: In the immediate post-operating period we observed an abrupt reduction of the disease markers. Early and tardive post-operative complications were not found, with patients discharge on average on the 8th day. All the patients had six-monthly follow-ups for the first 2 years. Until today no patient has presented relapse and secondarisms.

Conclusions: Progress in diagnostic sectors have permitted the arrival at an increasingly precocious diagnosis, and thus, therapeutical success. The surgical treatment remains until today the only real resolutive therapeutical approach.

\section{4}

\section{Hypoparathyroidism Post-Thyroidectomy}

B. Puppio, C. Verrusio, G. Guarino, P. Cianci, P. Di Leo, A. Traficante, G.L. Torre

U.O. di Chirurgia Oncologica-Centro di Riferimento Oncologico di Basilicata-Rionero in Vulture, PZ

Background: Hypocalcemy due to temporary and definite hypoparathyroidism is the main, sometimes serious, post-operating complication after total and sub-total thyroidectomy. This study wants to evaluate the incidence and the severity of total post-thyroidectomy hypocalcemy.

Methods: From September 2002 to December 2005, we analyzed 140 patients, all of which had undergone to a total thyroidectomy, at our Institute. In the period before and after the operation, the plasmatic concentration of calcium was evaluated in all patients. Four of them received a parathyroid gland transplant in the sterno-cleidmastoid muscle (SCMm).

Results: Transitory hypocalcemy was found in 65 patients (46\%), $56(80 \%)$ of which had been operated for a benign pathology and 14 (20\%) of which for a malignant one. A 2-week post-operation revealed check-up during which time, patients had taken orally calcium carbonate, that serum levels had returned within the normal range. Only 4 patients $(2.8 \%)$ presented permanent hypoparathyroidism.

Conclusions: Post-thyroidectomy hypocalcemy can be due to the removal of all the parathyroids, vascular apparatus ligature or damage, direct or secondary hematoma lesion of the parathyroid parenchyma. The stimulus produced by transitory hypocalcemy is often enough to determine compensation by the residual glands. Respect of the surgical technique rules and the use of some precautions, such as the transplant of devascularized glands in the (SCMm) muscle or an incision of the capsula with the purpose of decompression, are essential measurements in order to reduce permanent hypoparathyroidism. 


\section{5}

\section{Endoscopic Ultrasonography in Pre-Operative} TNM Staging for Gastric Cancer

\author{
G. Guarino, P. Cianci, B. Puppio, C. Verrusio, O. Ignomirelli, \\ A. Capobianco, G.L. Torre \\ U.O. di Chirurgia Oncologica-Centro di Riferimento \\ Oncologico di Basilicata-Rionero in Vulture, PZ
}

Background: This study wants to evaluate the utility of endoscopic ultrasonography (EUS) in the pre-operative TNM staging of gastric cancer.

Methods: The series included 24 patients $(18 \mathrm{M}, 6 \mathrm{~F})$ with gastric cancer, proved by EGDS and biopsies pre-operatively, and evaluated with EUS, according to TNM classification, from September 2002 to December 2005 in our Institute. The final analysis included 21 patients ranging in age from 47 to 81 years (mean 68.6 years). Diagnostic accuracy of EUS for TNM staging of gastric cancer was determined by comparing pre-operative EUS with subsequent postoperative histopathologic findings.

Results: The diagnostic accuracy of EUS in preoperative determination of cancer invasion depth was $100 \%(3 / 3)$ for T1, $0 \%(0 / 3)$ for T2, $83.3 \%(18 / 15)$ for T3. The overall accuracy was $85.7 \%$. The diagnostic accuracy of EUS for detection of nodal involvement was $100 \%(12 / 12)$ for N0 and 33\% (3/9) for N+ and 72.4\% (15/21) overall. EUS tended to overstage T criteria in $14.2 \%(3 / 21)$ and understage $\mathrm{N}$ criteria in $66 \%(6 / 9)$. Radical gastrectomy and subtotal gastrectomy with D2 dissection was proposed for 9 and 12 patients respectively on the basis of the staging. Preoperative staging accurately predicted the operative strategy for $87.5 \%$ of the patients.

Conclusions: EUS is a useful diagnostic method for pre-operative staging of gastric cancer for $\mathrm{T}$ and $\mathrm{N}$ parameters and in accorde to the stage can suggest the most appropriate surgical treatment. However, EUS evaluation of malignant lymph nodes is still unsatisfactory.

\section{6 \\ Experimental Studies on the Modification of Urinary Alanine Aminopeptidase (AAP) and N-Acetyl- $\beta$-D-Glucosaminidase (NAG) in Patients Submitted to Intraperitoneal Hyperthermic Chemoperfusion (HIIP) \\ T. Cioppa, D. Marrelli, C. Pedrazzani, A. Neri, S. Caruso, G. De Marco, E. Pinto, F. Roviello \\ Department of General Surgery and Surgical Oncology, University of Siena, Italy}

Introduction: NAG and AAP have been utilized as indicators of renal disease or tubular damage. This study was aimed at analysing the variation of enzymes value in patients submitted to HIIP.

Methods: The 24-hours urinary escretion of NAG and AAP was measured on p.o. day 3 in 46 patients who underwent HIIP for peritoneal carcinomatosis (TG) and in 25 patients submitted to major surgical procedures $(\mathrm{CG})$.

Results: In the CG, a significant difference between mean preoperative and p.o. values of NAG (5.8 vs. $10 ; p=0.04)$ were observed. A difference between mean p.o. values of NAG (25.4 vs. $10 ; p=0.01)$ and AAP (29 vs. $16.8 ; p=0.07)$ was observed in TG with respect to CG. In TG, complications were observed in $41.3 \%$. No differences in mean value of NAG and AAP were observed according to overall complications $(\mathrm{p}=0.20$ and $\mathrm{p}=0.28)$, also considering surgical $(p=0.11$ and $p=0.45)$ or medical separately $(p=0.53$ and $p=0.80)$. An extremely high value of urinary enzymes was observed in patients with intestinal fistula and fluid collection (NAG: 62.7 and 67; AAP: 27.2 and 81.9). In TG, a significantly higher value of enzymes was observed for patients with grade 2-3 renal toxicity (NAG: 59.6; $\mathrm{p}=0.001$; AAP: $61.9 ; \mathrm{p}=0.006$ ). No difference was observed according to other variables.

Discussion: p.o. value of NAG and AAP was significantly higher in TG in relation to tubular damage induced to treatment. p.o. assessment of enzymes should play a role in prediction of septic surgical related complications as well as severe renal toxicity.

\section{7 \\ Anatomical vs. Wedge Hepatic Resection. Our Experience about 31 Consecutive Patients with Hepatic Metastases from Colorectal Cancer}

G. De Marco, D. Marrelli, G. Cerullo, M.E. Perrotta,

T. Cioppa, S. Caruso, Y. Lambert, C. Pedrazzani, F. Mariani,

A. Neri, F. Roviello

U.O. Chirurgia Oncologica, Università degli Studi di Siena

Introduction: Surgical resection may be the only treatment modality for the cure of colorectal liver metastases (CLM). This study was aimed at analysing our experience on hepatic liver nonanatomical or anatomical resection for colorectal metastases.

Methods: 31 patients who underwent anatomical or wedge hepatic resection for CLM between 1996 and 2006 were analysed.

Results: The metastases were synchronous in 17 cases $(54.8 \%)$ and metachronous in 14 (45.2\%); they were located in a single lobe in 22 cases $(71 \%)$, while both hepatic lobes resulted to be involved in 9 cases. The treatment choice was conditioned by the number of the metastases and their localization: 21 wedge and 10 anatomical resections were performed. No p.o. deaths were observed and the overall morbidity rate was $12.9 \%$, without significant differences between two groups. The overall 3-year survival rate was $34.1 \%$. Patients with synchronous metastases, bilobar localization or more than 3 metastases had a worse prognosis. Anatomical and wedge resection had a 3 -year survival rate of 45 and $24.6 \%$, respectively $(p=0.59)$.

Discussion: Hepatic resection for metastatic colorectal cancer provides long-term survival in selected patients both with wedge or anatomic resections. The choice of the technical drift from the number and the site of metastases. 


\section{8}

Correlation between Jaundice and CA 19-9 Serum Levels in Benign and Malignant Conditions

S. Caruso, D. Marrelli, G. De Marco, G. Cerullo,

M.E. Perrotta, T. Cioppa, Y. Lambert, C. Pedrazzani,

F. Mariani, A. Neri, F. Roviello

U.O. Chirurgia Oncologica, Università degli Studi di Siena

Introduction: The study was aimed at evaluating the relation between CA 19-9 level and obstructive jaundice.

Methods: Data from 88 patients referred for obstructive jaundice were analyzed.

Results: A primary malignancy was diagnosed in 62 patients (MG) while a benign disease in 26 (BG). Although, elevated CA 19-9 levels were found in a part of BG $(61.5 \%)$ the median value was significantly lower $(173.2$ vs. $2,709.9 \mathrm{U} / \mathrm{ml})$. In the MG the normalization of CA 19-9 was mainly related to radical resection of the tumor, while a partial decrease was observed also after endoscopic stenting. In fact, 6 of the 15 malignant cases submitted to endoscopic stenting demonstrated a significant decrease in CA 19-9 levels (average $50.3 \%$ ). Otherwise, in $\mathrm{BG}$ resolution of the acute condition lead to normalization of serum CA 19-9 that resulted to be strictly related to the corresponding variation of bilirubin concentration.

Discussion: In presence of obstructive jaundice, distinguish between benign and malignant disease processes on the basis of elevated CA 19-9 is not reliable. However, the increase in CA 19-9 in BG is usually lower and relief of jaundice was constantly associated with a normalization of serum CA 19-9. A rising level in the absence of further jaundice is suggestive of an underlying malignancy, although a falling value does not exclude the diagnosis.

\section{9}

\section{Clinical Utility of Serum Tumor Markers Assay in Patients Admitted for Intestinal Occlusion}

S. Caruso, D. Marrelli, G. De Marco, G. Cerullo, M.E. Perrotta, T. Cioppa, Y. Lambert, C. Pedrazzani, F. Mariani, A. Neri, F. Roviello

U.O. Chirurgia Oncologica, Università degli Studi di Siena

Introduction: CEA, CA 19-9 and CA 72-4 are the tumor markers commonly used in colorectal cancer patients. The study analyzed the clinical utility of commonly used tumor markers in patients with intestinal occlusion.

Methods: In 47 patients afferent to hospital for intestinal occlusion quantitative determinations CEA, CA 19-9, CA 72-4 were performed.

Results: $19.1 \%$ were diagnosed having colorectal cancer while $80.9 \%$ patients presented a benign condition. The rate of abnormal CEA, CA 19-9 and CA 72-4 level were 17\% (8 patients: 6 benign and 2 malignant), $23.4 \%$ (11 patients, 8 benign and 3 malignant) and $0.2 \%$ (1 patient, benign). The sensitivity of CEA and CA 19-9 for colorectal cancer was $22.2 \%$ and $33.3 \%$. The specificity was $84.2 \%$ and $78.9 \%$.
The combination of CEA with CA 19-9 increase sensitivity to $44.4 \%$. The positive predictive value of CEA and CA 19-9 for colorectal cancer were $25 \%$ and $27.3 \%$. The negative predictive value were $84.6 \%$ and $82.8 \%$.

Conclusion: Elevated serum levels of CEA and CA 19-9 were found in both benign and malignant occlusion conditions. The finding of elevated serum markers may induce in the diagnosis of cancer by prompting a more extensive search for intrabdominal cancer. The low specificity (markers elevation in benign conditions) of CEA and CA 19-9 should be taken into account.

350

Vascular Resection during Pancreatectomies

U. Boggi, M. Del Chiaro, C. Croce, S. Signori, F. Vistoli, I. Mosca, F. Sgambelluri, V. Perrone, N. De Lio, F. Mosca

Università di Pisa, Divisione di Chirurgia Generale e Trapianti

Background: Traditionally pancreatic resections are not indicated for pancreatic cancer involving main peripancreatic vessels.

Aim: To analyze the outcome of pancreatectomies associated to vascular resection (PaVR).

Methods: From November 1987 to December 2004, we performed 138 PaVR (including 102 vein, 16 arterial and 20 venousarterial resections), whose results were compared with conventional pancreatectomies (CP) and palliative procedures (PP) for locally advanced pancreatic cancer.

Results: PaVR, CP and PP morbidity and mortality rates were 37.6, 36.2 and $25.8 \%,(p=N S)$ and 5.8, 5.7 and $3.2 \%(p=N S)$, respectively. One, 3 and 5-year actuarial survival rates of patients diagnosed with pancreatic cancer were 55.3, 17.6 and 7.9\%, respectively: they were comparable to CP ( $p=N S$ ) and significantly better than PP group $(p<0.0001)$. In PaVR group patients diagnosed with ductal adenocarcinoma showed a statistically relevant difference in 1 , 3 and 5-year survival rates in case of absent or present involvement of tunica intima $(63.8,21.6$ and 10.7 vs. 36.5, 4 and 4; $p<0.001)$. At a multivariate analysis better survival rates were associated to antiblastic therapies, tumor grade $\mathrm{G}_{1-2}$ and no infiltration of tunica intima.

Conclusions: Deep of tumor vascular infiltration, histological grading and adjuvant or neoadjuvant treatments seem to be the most important prognostic factors in the outcome of PaVR.

\section{1}

\section{Neoadjuvant Therapy in Colorectal} Metastases: A New Chance for the Patient?

\author{
E. Malvezzi, E. Ciferri, M. Castagnola, O. Municinò, \\ G.M. De Silva, G.S. Bondanza \\ U.O. Chirurgia, Genoa-North Hospital, Italy
}

Introduction: Mean survival time of patients with colorectal mts is $4-12$ months. Less than $30 \%$ of patients is operable. Surgery allows a 5 years' survival of $30 \%$. Neoadjuvant therapy, using the 
newly drugs L-OHP and CPT11 allows to extend surgical indications to $15 \%$ of the responders and to obtain a longer survival in the others.

Methods: From 1997 to 2005, we operated on 237 patients with colorectal cancer; of these 58 had metastatic disease: 32 sincronous and 26 metacronous. Metastatic patients can be further divided in: Group A, 11 patients did not receive any therapy; Group B, 10 patients underwent primary surgical resection; Group C, 37 patients had a neoadjuvant therapy.

Results: Mean survival was 6 months (range 1-13) in Group A and 24 months (range 6-72) in Group B. Among Group C 13 patients obtained a downstage: in particular 10 underwent surgery with a 27 months survival (range 7-95), while 3 patients are still on therapy with a mean survival of 20 months (range 17-22). 24 patients after a period stable disease had a progression and the final median survival was 18 months (range 5-33). 4/58 patients are still alive, desease free and without therapies since 1 year.

Discussion: Therapeutic algorithm used actually allows to $27 \%$ of patients with colorectal mts without surgical indication to obtain a mean survival and a quality of life similar to patients firstly operated on.

\section{2 \\ Anal Malignancies Associated to Condyloma Acuminata}

M. Mistrangelo, P. Cassoni ${ }^{1}$, I. Castellano ${ }^{1}$, V. Ghisetti ${ }^{2}$, S. Delmonte ${ }^{3}$, S. Rondoletti ${ }^{3}$, V. Costamagna, A. Mussa

Sezione di Chirurgia Oncologica dell'Università degli Studi di Torino, Ospedale Molinette, Torino

Introduction: HPV infection is an important factor in the oncogenesis of various squamous cancers. HPV DNA was found in $88 \%$ of anal cancer in one study and in more than $90 \%$ of cervical squamous cell carcinoma.

Patients and Methods: At the Oncological Surgical Department, 643 patients were diagnosed as affected by anal condylomata in a period from October 1999 to August 2005. 9.7\% of patients were $\mathrm{HIV}+, 6.7 \% \mathrm{HBV}+$ and $3.1 \% \mathrm{HCV}+.93 .9 \%$ presented a perianal localization, $62.5 \%$ an endoanal (3.4\% only endoanal), and $35.8 \%$ a genital localization. 510 patients were submitted to surgery.

Results: Histological exam revealed a degeneration in 21 patients $(4.1 \%) .11$ of these patients were HIV $+(52.4 \%$ of degenerations); and 2 further patients were immunosuppressed $(9.5 \%)$. Histology revealed 5 Buschke Lowenstein, 4 Bowen disease; 4 Bowenoid papillomatosis; 3 carcinomas in situ; 1 high displasia; 1 basocellular and 3 squamous carcinomas. HPV tipization was performed in 135 patients: 105 patients low risk; 18 high risk and 12 low/high risk. Patients with diagnosis of Buschke Lowenstein tumour was sumbitted to radical surgery; patients with anal carcinoma to RT + CT or to surgical excision; and other patients to surgical excision. Patients are in follow up.

Conclusions: Histopatological examination should be performed to confirm the diagnosis and to detect precancerous or cancerus changes.

\section{3}

\section{PET-CT in Patients Affected by Anal Cancer}

M. Mistrangelo, S. Cornaglia, E. Pelosi ${ }^{1}$, G. Bisi ${ }^{1}$, A. Mussa

Sezione di Chirurgia Oncologica dell'Università degli Studi di Torino, Ospedale Molinette, Torino

Introduction: In anal cancer 2 studies tried to assess the impact of PET with CT simulation in radiation therapy planning and the presence of recurrence after primary therapy. Preliminary results seems good.

Patients and Methods: At the our Department, 9 patients were studied with PET-CT. In the first 2 cases we performed PET-CT after RT and CT for a squamous anal cancer. In the other 7 patients ( 5 basaloid and 2 squamous) we applied a protocol consisting in a staging PET-CT and 2 following exams 1 and 3 months after Nigro's therapy.

Results: In one case PET-CT revealed a signal at the level of a lower limb, diagnosed as a desmoid after a biopsy. In a second patient, treated with RT and CT, PET-CT resulted positive for recurrence with a previous biopsy negative. A new biopsy confirmed the presence of recurrence. In the other 7 patients PET-CT and biopsy were negative for recurrence after therapy. In 2 patients, treated surgically but with surgical margins positive for neoplasm, PET-CT was negative for residual disease (false negative).

Conclusions: PET-CT is a promising radiological exam to study recurrence in anal cancer. Biopsy, CT and MNR are inaccurate to reveal recurrence after RT and CT. In our initial experience we obtained good results with PET-CT in the study of recurrences in anal cancer. Worse results are observed in the detection of residual disease after surgery.

\section{4 \\ Sentinel Node Biopsy in Patients Affected by Anal Carcinoma}

M. Mistrangelo, A. Mobiglia, M. Bellò' ${ }^{1}$ P. Cassoni², G. Beltramo 3 , E. Milanesi4 , B. Mussa, A. Mussa

Sezione di Chirurgia Oncologica dell'Università degli Studi di Torino, Ospedale Molinette, Torino

Introduction: Anal carcinoma is rare. Inguinal lymph node status is an important prognostic factor and it is an indipendent prognostic factor for local failure and overall mortality.

Patients and Methods: At our department 24 patients affected by an anal neoplasm were studied between October 2001 and December 2005. Histology revealed 14 squamous; 8 basaloid carcinomas; 1 carcinoma in situ and 1 spinocellular epithelioma. Patients were injected in the four cardinal points around the neoplasm with $37 \mathrm{MBq}$ of nanocoll-Tc-99 m. Lymphoscintigraphy revealed 10 cases of monolateral and 14 cases of bilateral migration. Patients were submitted to inguinal sentinel node biopsy.

Results: Detection rate was $100 \%$. Postoperative complications: lymphorrea in 12 patients $(50 \%)$; in 1 case a lymphedema of lower limb, and in 1 case a lymphocele treated surgically. Nodal metastases were found in 6 patients $(25 \%)$, bilateral in 2 cases. These patients were treated also with inguinal radiotherapy. During follow up 2 patients 
died and 4 recurred. We have not observed any inguinal metastases in patients negative at the first exam.

Conclusions: SN in anal cancer is a feasible procedure. Elective irradiation of bilateral inguinal areas may not be recommended, patients negative for inguinal metastases could be spared the prophylactic treatment. Further studies are required to confirm these results.

\section{5}

Asymptomatic Colorectal Cancer with Un-Resectable Liver Metastases: Immediate Colorectal Resection or Upfront Systemic Chemotherapy?

\author{
A. Muratore, D. Zorzi, H. Bouzari, E. Sperti, F. Leone, \\ M. Aglietta, L. Capussotti \\ Istituto Per La Ricerca e La Cura Del Cancro Candiolo, TO
}

Objective: Surgical treatment of relatively asymptomatic colorectal cancers with unresectable liver metastases is still a matter of discussion. Aim of the study is to evaluate the rate of complications related to the un-resected colorectal tumour and requiring surgical treatment.

Method: From January 2000 to December 2004 we have prospectively collected data on 35 consecutive cases treated straight away by chemotherapy without primary tumour resection. All the patients underwent FOLFOX6 as first-line chemotherapy.

Result: The median follow up was 18.5 months. Fifteen of the 35 patients $(42.9 \%)$ were down-staged to surgery; the mean interval between chemotherapy start and colon-rectum cancer resection was 6.5 months. Twenty patients did not underwent any curative surgery; the mean interval between chemotherapy start and the end of the follow-up was 17.7 months. Only one patient $(2.8 \%)$ developed clinical signs of intestinal occlusion 5.6 months from the start of chemotherapy and required a colostomy.

Conclusion: The rate of complications related to the non-resected colorectal tumour is very low using oxaliplatin as first line chemotherapy. Non-operative management of relatively asymptomatic colorectal cancers with un-resectable liver metastases is a safe approach.

\section{6 \\ GIST: The Multidisciplinary Approach as the Best Management in Diagnosis and Therapy}

B. Oreggia, C. Rocha, F. Botti, A. Carrara, E.Contessini Avesani

Introduction: GIST can arise anywhere in the gastrointestinal tract and can present a great variety of clinical and radiology features, depending mostly on size and location. These tumours are difficult to be discovered preoperatively, there are problems in the interpretation of radiological imaging and, when incidental intraoperative finding occurs, histological examination is fundamental for the definitive diagnosis and surgical treatment respecting oncologic eradication standards.
Methods: We present 3 cases of patients with GIST treated in our institution: we describe a rare case of a 31-year-old man affected by Crohn's disease with an intraoperative diagnosis of synchronous colon GIST, a 71-year-old woman with a gastric GIST with only an abdominal mass completely asymptomatic and an 81-year-old man with a small bowel GIST conditioning anemia diagnosed preoperatively by video-capsule.

Results: In the first case we observe a case of incidental finding in a patient with clinical and radiological presentation confused by symptoms and images of an IBD where the explorative laparotomy was resolutive. In the second case the histological characteristics were fundamental to confirm diagnosis and perform a radical surgery. In the third one a new diagnostic tool as video-capsule had the main role in discovering the jejunal mass and guiding the surgery.

Discussion: It is necessary to coordinate the efforts of radiology, surgery and pathology in a multidisciplinary approach to perform the best management in the suspect of a GIST.

\section{7 \\ Reconstruction of the Nipple-Areola Complex: An Update}

F. Klinger, A.V. Pagliari, B. Banzatti, C.A. Amodeo, D. Ventura, F. Caviggioli, S. Giannasi, J. Hassibi, F. Villani, M. Klinger

Istituto di Chirurgia Plastica e Ricostruttiva dell'Università degli Studi di Milano, U.O. Chirurgia Plastica II, I.C. Humanitas

Introduction: Along with continuing progress in reconstructive surgery of the breast after mastectomy numerous techniques of nipple-areola complex recontruction have been devoloped. Each has its own advantages and disvantages. Special attention was taken to the long-term deterioration of the projection of the neo-nipples.

Methods: We review and discuss the concepts and techniques of nipple-areola reconstruction that have evolved over the past decades. Furthermore those principles and techniques are pointed out that fulfil best the criteria of an ideal nipple-areola complex with emphasis on different approach of breast reconstruction and individual anatomical conditions of the patients.

Results: The principle criterion for a pleasing nipple-areola complex is simmetry regarding several parameters: colour, texture, size and projection. A simmetic size and execellent cosmetic aspect with controlateral nipple-areola complex was obtained in quite all unilateral cases combining local and free graft and tattoo.

Discussion: A carefully selection of the surgical technique in nipple-areola complex reconstruction, attention to each individual anatomical situation determinate a satisfactory result with a great benefit for the patients both aesthetically and psicologically. 


\section{8}

\section{Monitoring of Chest Chemo-Perfusion After Surgery and Our Treatment Protocol in Malignant Pleural Mesothelioma}

\author{
G.L. Pariscenti, M. Benvenuti, D. Benetti, G. Botticini, \\ T. Ligabue, A.F. Bravo, P. Bovolato \\ Ospedali Civili di Brescia, Chirurgia Toracica, Brescia
}

Introduction: We describe our MMP treatment protocol, feasibility, results with intraoperative hyperthermic high dosage chemotherapy, platin dosages.

Methods: Our protocol: Thoracoscopy/vats, mediastinoscopy, laparoscopy for staging-Hydration Protocol before/after surgeryextended EPP (bovine pericardium reconstruction) or PD-Argon beam cytoreduction-after definitely chest closure IHC $\left(42.5^{\circ} \mathrm{C} 60^{\prime}\right.$ Cisplatin $150-200 \mathrm{mg} / \mathrm{m}^{2}$ ). Thoracoscopy + intrathoracic wall tissue biopsy. Adiuvant chemo-radiant therapy recently IMRT.

Results: Until April 06 we treated 101 patients : 67 EPP (29IHC), 34 PD (5IHC). Results depend on: precocity of diagnosis-epithelioid histological variety. Low surgical mortality (EPP: 1.5\%-PD: 0\%, overall $1 \%$ ); morbidity $4.9 \%$ not IHC correlated. We describe platin dosages of washing-chemo solution before/after IHC, intrathoracic tissue-blood-plasma-urine, 2 years survival.

Discussion: IHC neutralizes micro residual tumor sites, cell spreading, increases radio sensitivity; it's a safe procedure, no collateral effects:it doesn't worsen postoperative prognosis, no renal failure even if blood-urine platin dosages are high, due to our hydration. Superficial intrathoracic wall tissue platin dosage is about 100 times superior to systemic chemotherapy). This protocol seems to offer good results; IHC could improve survival rate, but comparativerandomized-prospective studies are essential to demonstrate it.

\section{9}

\section{Analysis of the Response to Neoadjuvant Chemotherapy in Gastric Cancer}

\section{R. Persiani, A. Biondi, M. Zoccali, D. D’Ugo \\ Department of Surgery, Catholic University of Rome}

Introduction: Aim of this study is the evaluation of the effects induced by neoadjuvant chemotherapy (NACT) and its impact on survival on a series of locally advanced gastric carcinomas (LAGC).

Methods: Downstaging was assessed comparing pre-treatment clinical and laparoscopic staging with pathologic staging on 30 consecutive patients who completed a 3-year follow-up. T-downstaging (qualitative analysis) and the grade of pathologic response (quantitative analysis) were included in a statistical correlation between tumor regression and 3-year survival.

Results: In this series tumor downstaging was obtained in $13 / 30$ patients $(43.3 \%)$. After 3-year follow-up, overall survival was $>37.5$ months with an overall survival rate of $56.7 \%$; this figure reached up to $70.8 \%$ in those cases $(24 / 30)$ who benefited from a R0-resection. A significant association with survival was demonstrated for T-downstaging $(p=0.04)$, but not for the grade of pathologic response. At $\chi^{2}$ test T-downstaging showed a significant association with R0-resection $(\mathrm{p}=0.02)$. The $\mathrm{R}$ factor was the only independent prognostic factor in this series.

Discussion: In this study, the 3-year survival for LAGC treated by NACT prior to D2-gastrectomy compares favourably with historical series treated by surgery alone. Patients who obtained T-downstaging and subsequently benefited from a R0-resection had a definitely better chance of cure.

\section{0 \\ Pattern of Recurrence following Curative Resection of Gastric Cancer}

\author{
R. Persiani, S. Rausei, F.C.M. Cananzi, D. D’Ugo \\ Department of Surgery, Catholic University of Rome
}

Introduction: This study investigated the timing and potential predictors of recurrence of gastric cancer (GC) after curative resection.

Methods: 223 patients underwent curative resections for GC; the median follow-up was 45 months. Patterns of failure were classified according to first site of relapse. Recurrence-free survival was estimated by the methods of Kaplan-Meier and log-rank test was used to determine univariate significance. Cox regression was used for multivariate analysis.

Results: Disease-free survival rates were 73.5, 64.1 and 59.6\% at 12,24 and 36 months, respectively. Recurrence was detected in $37.7 \%$ of patients (84/223): 16 patients showed anastomotic relapse (AR), 21 lymphatic relapse (LR), 14 peritoneal relapse (PR) and 33 distant metastases (DM). pT-stage and tumor site resulted independent predictors of recurrence. Proximal location was significantly associated with AR and PR and advanced T-stage was associated with DM and LR. The mean time to recurrence was 19.1 months and 19.9 months for AR and PR, respectively; the mean time to LR and DM was 24.2 months and 25.9 months, respectively.

Discussion: Clinicopathological features of the primary tumor are sensible predictors of risk and timing for different patterns of recurrence of GC. Grading the level of risk for recurrences could be helpful in guiding individualized adjuvant treatment.

\section{1 \\ Locally Advanced Gastric Cancer: Long-Term Survival After Neoadjuvant EEP Chemotherapy and D2-Gastrectomy}

\section{R. Persiani, V. Vigorita, S. Rausei, A. Biondi, D. D’Ugo \\ Department of Surgery, Catholic University of Rome}

Introduction: We report the results after a 5-year follow-up of a study aimed at evaluating a neoadjuvant chemotherapy (NACT) in resectable locally advanced gastric cancer (LAGC).

Methods: Between February 1996 and May 2000, 24 patients with LAGC, staged by laparoscopy, underwent D2-gastrectomy after 3 preoperative cycles of chemotherapy (Epidoxorubicin, Etoposide, Cisplatinum). Three further cycles of chemotherapy were planned after surgery. The survival rate was calculated after a 5-year follow-up 
and differences were assessed by the log-rank test. Multivariate analysis was performed using the Cox regression.

Results: The main toxicity was grade 3-4 neutropenia, which occurred more frequently during the postoperative chemotherapy. R0 resection was achieved in $83.3 \%$ of patients. Complete pathological response was never documented, but a tumor downstaging (pTNM vs. lap-TNM) was obtained in $41.7 \%$ of patients (10/24). Median survival was 38 months and 5-year survival rate was $45.8 \%$. At univariate and multivariate analysis, $\mathrm{R} 0$ resection was the most important prognostic factor.

Discussion: NACT seems to be a promising modality for increasing the rate of R0 resection for LAGC. New treatment schedules must be investigated, in order to obtain higher rates of pathological response. Long-term results in our series suggest the presence of a survival benefit for LAGC patients treated by NACT and D2-gastrectomy.

\section{2}

\section{Extension of Lymph Node Metastases in Melanoma with Positive Sentinel Lymph Node}

\author{
T. Piardi, B. Compagnoni, M. Valota, G.L. Baiocchi, \\ E. Ngassa, G.A.M. Tiberio, S.M. Giulini \\ Clinica Chirurgica, Brescia
}

Introduction: The treatment of nodal metastasis of skin melanoma is still lacking. The value of the extended lymphectomy in patients with $\mathrm{SL}+$ is discussed. We report our experience, with reference to the extension of lymph node metastases in patients submitted to radical lymphectomy after SL+.

Methods: 74 patients were operated on for lymph node metastases from 1998 to 2005. 49 (66\%) submitted to radical lymphectomy on the basis of the SL+ were the object of this study. The characteristics of the primary lesion, of the sentinel lymph node, of the lymphatic status were registered.

Results: The location of primitive tumour was: trunk $37.8 \%$, arms $13.6 \%$, legs $48.6 \%$, with Breslow $0.75-1.50 \mathrm{~mm}$ in $21.6 \%$, $1.51-4$ in $46 \%$ and $>4 \mathrm{~mm}$ in $32.4 \%$. Axillary lymphectomy was done in 22 and inguinal-iliac-obturatory lymphectomy in 27 . The total number of removed nodes was 842 (range 6-37), 39 of which (4.6\%), from 13 patients $(26 \%)$, were positive for metastases. There were 5 micro metastases, 1 incomplete metastasis and 33 massive metastases, 2 of which showed extracapsular extension. The mean number of positive nodes was 3 for patient (1-11).

Discussion: Nearly $1 / 4$ of the patients with $\mathrm{SN}+$ harbour massive nodal metastases in the region of the harvested sentinel node. Simple chemotherapy without lymphectomy could represent a suboptimal treatment in these cases, where locoregional extended lymphectomy is indicated.
363

\section{Small Bowel Tumors: Personal Experience}

E. Piazzese, V. Pacilé, Bonsignore, F. Fiumara, M. Livio, L.G. Angiò

Università degli Studi di Messina, Scuola di Specializzazione in Chirurgia Generale I

Introduction: The small bowel tumors (SBT) are rare and represent less than the $6 \%$ of the gastrointestinal neoplasias (GIN) and less than the $2 \%$ of only malignant forms. The non-specific symptomatology and the limits in the imaging technique determine a delay in the diagnosis, that is usually formulated in the advanced stadium, as find unexpected during a laparotomy performed for the onset of complication.

Patients and Methods: From 1997 to 2005 we have observed 12 SBT, equal to him $1.1 \%$ of all the GIN treated in the same period, of different nature: 4 GIST, 2 lymphomas, 4 adenocarcinomas, 1 carcinoide and 1 adenoma, located in $75 \%$ of the cases in the ileum. In 9 patients $(75 \%)$ intestinal occlusion was observed, in $2(16.6 \%)$ hemorrhage and in $1(8.3 \%)$ perforation; all have been submitted in urgency to exploratory laparotomy and intestinal resection.

Results: The perioperative mortality has been void; the morbidity has been of $16.7 \%$ and the postoperative middle hospitalization of 6 days. Thin to today (middle follow-up of 48 months) the evolution of illness is observed in 4 cases, with a $66.7 \%$ global survival.

Conclusion: Our experience, even though numerically limited, confirmation the frequent delay in the diagnosis of the SBT, that can jeopardize the radicality of surgical action (however valid to resolve the complication) above all for the most greater risk of happened neoplastic diffusion.

\section{4 \\ Radiofrequency Thermoablation in Liver Neoplasms: The Laparoscopic Approach}

R. Cazzaniga, C. Ricchiuti

ASL Vallecamonica-Sebino, U.O.Chirurgia Edolo, BS

Radiofrequency (RF) thermoablation represents a consolidated technique for treatment of primary and metastatic liver neoplasms. The laparoscopic approach, less common than percutaneous treatments, presents three main advantages. The echolaparoscopic exploration (LUS) allows a better sensibility than Helical-CT scan documenting small lesions, not detected before and allows to perform a US-guided biopsy, modifying in some cases the therapeutic schedule. In case of peripheral lesions laparoscopic approach allows direct control of near organs, particularly stomach colon and gallbladder, and their protection from thermo damages during the treatment: this permits such treatments not allowed with percutaneous approach. The visual control of the procedure allows to directly verify haemostasis when the needle must be taken out. This taking out is usually bloodless thanks to the coagulation obtained in the needle's way. The needle can reach a temperature of 75-80 degrees stopping the saline infusion into the lumen of the RF electrode. In case of small bleeding the entrance point is tightly compressed by the US probe and it is possible to perform an haemostasis with mono or bipolar coagulation. 
Technical Notes: The patients is under general anaesthesia. A $10-\mathrm{mm}$ blunt trocar is inserted through an umbilical incision with open technique. After the laparoscopic exploration a second 10-mm trocar is inserted at right hypocondrium or flank. In some cases a third 10 or $5-\mathrm{mm}$ trocar can be inserted. LUS examination of the liver is performed, stressing in almost $30 \%$ of our cases focal lesions not underlined by $\mathrm{CT}$ or percutaneous preoperatory US.

If necessary a (truncated at 250 words).

\section{5}

\section{Short Course Radiotherapy (SC-RT) and Delayed Surgery for Low Rectal Cancer: Preliminary Results}

\author{
D. Scala, V. Albino, F. Cremona, F. Ruffolo, R. Palaia, \\ D.N. Idà, C. Guida, B. Pecori, M. Elmo, B. Morrica, \\ V. Parisi, P. Delrio. \\ Surgical Oncology 'C', ${ }^{1}$ Radiotherapy, Istituto Nazionale \\ dei Tumori di Napoli
}

Introduction: We present the preliminary results of (SC-RT) and delayed surgery in patients with moderate risk low rectal cancer.

Methods: Since January 2003 to December 200512 patients ( 8 F, 4 M; median age 57.8 years) with stage II-III rectal cancer were treated. Patients underwent $5 \times 5$ Gy RT. Proctectomy with TME was planned 30 days after RT. Toxicity, pathological response and surgical results have been recorded.

Results: Pre-treatment stage was: 2 T2 N0, 10 T3 N0. Median tumor distance from the anal verge was $5.5 \mathrm{~cm}$ (range 1.5-8.9). 8 anterior resections with temporary stoma, 2 Miles' and 2 Hartmann's procedures were performed at a median interval of 30 days. Pathological staging was as follows: 5 pT1 N0, 5 pT2 N0, 1 pT3 N1, 1 pT3 N2. Median number of retrieved nodes was 36.7. Circumferential margin was free of tumor in all cases. Tumor regression grade (TRG) was as follows: 3 TR G2, 2 TR G3, 5 TR G4, 2 TR G5. Downstaging occurred in 9 cases $(75 \%)$. Two cases of heritema, 1 grade 1 diarrea, 1 anal pain were recorded. Among early postoperative complications 2 anastomotic fistulas were recorded. Median postoperative stay was 11.5 days. No local or distant relapses has been recorded.

Discussion: A significative downstaging of rectal tumors with low impact on TRG may be obtained delaying the interval between a SC-RT and surgery, with an acceptable rate of postoperative complications. The impact on disease free and overall survival has to be determined with a longer follow up.

\section{6 \\ Laparoscopic Removal of a Bladder Leiomyoma: Case Report}

D. Scala, F. Ruffolo, S. Perdonà', D.N. Idà, V. Parisi, P. Delrio

Surgical Oncology, ${ }^{1}$ Urology, Istituto Nazionale Tumori, Napoli, Italy

Introduction: Bladder leiomyomas are unusual findings among pelvic benign tumors. Clinical presentation may include acute and chronic pelvic pain. Reported treatment is cystoscopic or laparotomic resection. We report on a case of bladder leiomyoma successfully treated by a laparoscopic approach.

Case Report: A 59 years old female with acute pelvic pain was admitted to our Division. Nine years before she underwent a total vaginal hysterectomy. Abdominal US showed a $3 \mathrm{~cm}$ mass in the right lateral wall of the bladder, previously biopsied with an unconclusive histology by a CT guided FNA. Cystoscopy showed the bladder mass covered by normal mucosa. The patient underwent a laparoscopic excision of the tumor. Incision of the pelvic peritoneum along the right lateral border of the bladder exposed the peri-bladder space. The mass was then visualized and carefully excised from the bladder wall. Postoperative course was uneventful and the patient was discharged at day 3 after urinary catheter removal. Histology showed a leiomyoma $($ actin + desmin + ) with a low $\mathrm{Ki}-67$. US control at three months showed no sign of relapse.

Discussion: Bladder leiomyoma may be excised by trans-urethral resection. Postoperative course is characterised by prolonged transurethral catheterization, bladder calcareous plaques and eventual removal of remnants with a second operation. Laparoscopic approach obtains diagnosis and a complete en-bloc excision of the tumor in one step. A 3 trocars mini-invasive procedure can be performed and carries no late complications or distorsion of bladder anatomy with functional sequels. Laparoscopic removal of these benign tumors is safe and effective.

\section{7 \\ Benign Schwannoma of the Pancreas \\ M. Spaggiari, F. Di Benedetto, N. De Ruvo, M. Masetti, R. Montalti, A. Romano, G. Guerrini, R. Ballarin, G. Molteni, S. Di Sandro, G.E. Gerunda \\ Centro Trapianti di Fegato e Multiviscerale, AZ, \\ Ospedaliero, Universitaria Policlinico di Modena}

Introduction: This article regards the study of a benign Schwannoma, developed in a 47-year-old man, in the body-tail of the Pancreas. The Schwannoma of the pancreas is a rare disease actually reported in only 24 cases in the English and European literature (30 patients), and one review of 10 cases in the Japanese literature. This tumor originates from the Schwann cells that line the nerve sheath and is sometimes referred to as neurilemmoma or neurinoma. Pancreatic schwannomas arise from either autonomic sympathetic or parasympathetic fibers. Schwann cells can give rise to two types of tumors: Schwannomas and Neurofibromas. Neurofibromas are associated with von Recklingh-ausen's disease and can undergo malignant degeneration more frequently than Schwannomas.

Methods: We have analysed the clinical manifestations, radiological findings, surgical evidences, treatment indication and histological analysis of an isolated mass of $2.5 \mathrm{~cm}$ in the body-tail of the pancreas, involving, without infiltrating, the splenic vein, which underwent a distal spleno-pancreatic resection.

Results and Discussion: The most common location is in the head of pancreas (40\%). Preoperative diagnosis is very difficult, not as to the detection of the pancreatic mass, but relatively to radiological differential diagnosis from other aggressive pancreatic tumors such as those of epithelial or neuroendocrine origin. Schwannomas can be 
treated with simple enucleation depending on their size and location, even if, in literature, only 4 patients $(10 \%)$ underwent this procedure.

\section{8 \\ Chemo Embolization as Rescue Treatment of Resected Hepatoblastoma in Adult}

\author{
G. Molteni, F. Di Benedetto, N. De Ruvo, M. Masetti, \\ R. Montalti, A. Romano, G. Guerrini, R. Ballarin, \\ M. Spaggiari, S. Di Sandro, G.E. Gerunda \\ Centro Trapianti di Fegato e Multiviscerale, Az. \\ Ospedaliero-Universitaria Policlinico di Modena
}

Introduction: Hepatoblastoma is the most common malignant liver tumor in children, but is extremely rare in adult: only 25 cases have been collected in English literature and only 2 cases among 4,765 histologically proven cases of primary liver cancer in Japan. Survival after resection is very poor, therefore the problem is the management of this rare tumor.

Methods: We report a case of a 33 years old man who had been suffering from nausea, vomiting, loss of weight and abdominal pain in right upper quadrant for 3 months. A MRI of the liver showed a tumor of the left hemiliver, $18 \times 12 \times 10 \mathrm{~cm}$ of size and infiltrating the stomach. A left hepatectomy with sub-total gastrectomy and regional linfoadenectomy was performed. The histological diagnosis was hepatoblastoma. Recurrence in the right lobe occurred after 1 month, and the patient was referred to chemotherapy with cisplatinum and adriamicin. As the tumor was not responsive to therapy, we performed 3 cycles of chemoembolization of the right hepatic artery.

Results: The chemoembolizations were well tolerated by the patient and their efficacy was confirmed by the downfall of $\alpha$-fetoprotein level and by serial computed tomography. The patient survived 11 months with recurrence of disease and died 1 year after resection with lung metastasis.

Discussion: Hepatoblastoma in adult is a very rare and aggressive tumor. Chemotherapy is not effective. We obtained very good results with intra-arterial chemoembolization, performed when the $\alpha$-fetoprotein level was going up, even if the computed tomography showed no vital tissue in the nodules.

\section{9}

\section{Surgical Treatment of Gastric Gist. Our Experience}

F. Torelli, G. Izzo, A. Cosenza, A. Renzi, L. Vicenzo,

L. Monaco, A. Basciotti, A. Brillantino, M. Schettino,

F. La Mura, N. Di Martino

VIII Chirurgia Generale e Fisiopatologia Gastroenterologica, Seconda Università degli Studi di Napoli

Introduction and Purposes: Gastrointestinal stromal tumors are an extremely wide group of tumors and represent only the $1 \%$ of all gastrointestinal tumors. Surgical treatment keeps being the golden step in the therapeutical approach.
Materials and Methods: The purpose of the actual trial is to show our experience towards 4 patients that we observed in 2005 with gastric GIST: a 59-year-old male (Patients n.1), and 3 females (Patients n. 2, 3, 4) $(50,53$ and 60 years old respectively). In the first patient the tumor, with a $2 \mathrm{~cm}$ diameter, took part in the over pyloric region towards the lesser curvature; the number 2 patients was affected by a gastric fundus formation, easily bleeding; in the number 3 patients the tumor was in the under cardiac fundic region, while in the last patient (number 4) we found a $3 \mathrm{~cm}$ formation occupying the gastric body towards the lesser curvature.

Results: The 2 patients with tumor located towards the lesser curvature underwent a video laparoscopy resection (Wedge resection) with wide edge of unaffected tissue. The other 2 patients (n. 2-3) were treated by the 'open' way and they both underwent a total gastrectomy.

Conclusions: Gastric lesions of the anterior wall and of the greater curvature tend to be removed more easily via laparoscopy, while the laparotomic procedure is to be preferred for iuxtacardiac or prepyloric lesions for the risk of stenosis. To end up, laparoscopic surgery can surely be considered in the treatment of gastric GIST.

\section{0 \\ Treatment of Peritoneal Carcinomatosis from Colonic Cancer and Pseudomixoma Peritonei by Cytoreduction and Hyperthermic Antiblastic Peritoneal Perfusion, HAPP}

C. Bergamini, M. Vaira, M. De Simone

Department of Oncological Surgery, Osp. S. Giuseppe, Empoli, FI

Introduction: Peritoneal carcinomatosis can be treated, with encouraging results, by surgical cytoreduction, peritonectomy and hyperthermic antiblastic peritoneal perfusion (HAPP).

Materials and Methods: After complete cytoreduction, in semiclosed HAPP, the abdominal wall is partially closed and hanged to an autostatic retractor. We performed 185 HAPP, carcinomatosis arose from colorectal cancer in 36 cases; from PMP in 47 cases. About colonic cancer, in 23 patient we used Cisplatinum and C-Mitomycin; in 11 cases Oxaliplatinum and in 2 cases C-Mitomycin alone. About the 47 PMP, 3 patients have been treated in 2 steps, and one patient in 3 steps.

Results: Colonic cancer: morbidity rate was $28.8 \%$ (5 re-intervention). Perioperative mortality was $2.8 \%$. For 23 patients treated with CDDP and C-mytomicin, the median survival time was 14.5 months, lower than we expected. In the last 11 patients, selected with PCI $<15$ and treated with oxaliplatinum, results are encouraging. PMP: 20 patients $(42 \%)$ presented perioperative complications $(5$ reoperation). No perioperative mortality. The $81 \%$ of the patient are NED at a FU with a range of 1-120 months.

Conclusions: Colonic cancer: interesting results if correct selection and complete cytoreduction. New drugs may improve results. PMP: aggressive cytoreduction combined to HAPP consent good results, with long DFI in more than $80 \%$ of the patients treated. 
371

\section{Hyperthermic Isolated Limb Perfusion (ILP) for the Treatment of In Transit Metastases from Limb Melanoma}

\author{
M. Vaira, C. Bergamini, M. De Simone \\ U.O.C. Chirurgia Generale, Ospedale S.Giuseppe, Empoli
}

Introduction: Isolated limb perfusion (ILP) for in transit metastasis from melanoma offers high drug concentration in the tumor area and low systemic toxicity.

Materials and Methods: After lymphnodes dissection and isolation of the limb from systemic circulation, ILP is performed with L-Pam (at $41.5^{\circ} \mathrm{C}$ ) for $60 \mathrm{~min}$ with extracorporeal circulation technique. From 1995 to April 2006, 80 patients with in transit metastases from melanoma have been treated with 88 ILP ( 6 patients treated twice and 1 three times). 77 patients were at stage III (29 stage IIIa; 48 stage IIIAB), 11 patients at stage IV (for limb-salvage), 84 lower limbs; 4 upper limbs.

Results: No perioperative mortality. Morbidity: edema of limb, 8 transitory leucopenia, 1 retroperitoneal hemorrage, 1 chronic ischemia damage. Complete response on 41 patients (46.6\%); partial response (necrosis $>50 \%$ ) on 42 patients $(47.7 \%)$. Overall median survival time: 692 days; Stage IIIA: 1,099 days; stage IIIAB: 707 days; stage IV: 256 days.

Discussion: ILP is treatment of choice for in transit melanoma of the limb, is safe, with low morbity rate and no mortality rate in our series. Involvement of regional limphnodes and systemic disease are related to lower survival rate (Stage IIIAB and IV). Also in partial response allows control of bulky disease and good quality of patient's life.

\section{2}

\section{Liver Haemostasis by Tachosil after Glisson's Capsule Removal in Cytoreduction Plus Hyperthermic Antiblastic Peritoneal Perfusion (HAPP) for Pseudomixoma Peritonei}

\author{
M. Vaira, C. Bergamini, M. De Simone \\ U.O.C. Chirurgia Generale, O.S. Giuseppe, Empoli
}

Introduction: In pseudomixoma peritonei (PMP), cytoreduction often requires the removal of Glisson's capsule affected from peritoneal carcinomatosis. Sometimes may be necessary an extraperitoneal approach, consisting in remove diaphragmatic peritoneum and Glisson's capsule en bloc. After this procedure, bleeding from liver should create some troubles during and after perfusion. This time we tried Tachosil to stop liver bleeding.

Materials and Methods: In this case, as often happens, it has been necessary an extraperitoneal approach to right diaphfragm, with removal of diaphragmatic peritoneum and Glisson's capsule en bloc. After this procedure, a bleeding area of more or less $5 \mathrm{~cm}^{2}$ on liver was difficult to treat, for its position, with common surgical procedures. So we placed a Tachosil sheet on this area, then completed the cytoreduction. After this procedures we performed a semi-closed HAPP with Cisplatinum and C-Mitomycin for $60 \mathrm{~min}$ at a median intraperitoneal temperature of $41.7^{\circ} \mathrm{C}$.
Results: After the HAPP, we checked the peritoneal cavity; Tachosil has never been removed from HAPP and bleeding had stopped.

Conclusions: Tachosil ensures bleeding control and remains placed-on also if submitted to high temperature and continue washing of peritoneal cavity for $60 \mathrm{~min}$, so it should be used safely also if surgery is followed from $\mathrm{H}$.

\section{3}

\section{Epithelioid Angiomyolipoma of the Kidney with Renal Vein Calcification in a Young Girl}

F. Vasta, A. Ricciardello, G. Banno, M. D'Agostino, R. Blandino, A. Pistritto, S. Ristagno, L. Puzzo, V. Panebianco

Surgical Oncology Group, S. Vincent Hospital, Taormina, ME, Italy

Introduction: Epithelioid angiomyolipoma is a recently recognized variant of angiomyolipoma, characterized by the presence of polygonal cells with densely eosinophilic cytoplasm and varying degrees of nuclear atypia. The epithelioid cells had pleomorphic and hyperchromatic nuclei with densely eosinophilic cytoplasm.

Methods: We report a case of epithelioid angiomyolipoma of the kidney with renal vein calcification that occurred in a 31-year-old woman. Left nephrectomy was performed. Grossly, a well-demarcated, spheroid, largely hemorrhagic tumor measuring $10 \mathrm{~cm}$ in diameter occupied the superior pole of the left kidney. Left renal vein thrombosis with $3.5 \mathrm{~cm}$ necrotic tissue partially calcified.

Results: Patient recovered uneventfully and was discharged 5 days after surgery. HMB-45 was detected in the epithelioid cells. There was no expression of cytokeratin, epithelial membrane antigen or desmin. The patient showed no evidence of recurrence or metastatic disease 3 months after nephrectomy.

Discussion: Previous report has suggested that epithelioid angiomyolipoma have the potential to be malignant, and thus regular postoperative surveillance is mandatory. Preoperative immaging can lead to an erroneous diagnosis of renal cell carcinoma or sarcoma.

\section{4}

Huge HYDATIC CYST: A 'Familiar' Case

F. Vasta, A. Ricciardello, G. Banno, M. D'Agostino, R. Blandino, A. Pistritto, S. Ristagno, L. Puzzo, V.

Panebianco

Surgical Oncology Group, S. Vincent Hospital, Taormina, $\mathrm{ME}$, Italy

Introduction: Hydatid disease caused by Echinococcus often manifests as slowly growing cystic mass and mainly affects the liver or lung and rarely other parts of the body such as brain, heart, bone and muscle.

Methods: Two Mediterranean brothers present with symptoms as such: Subject 1: 46 years old male admitted in our surgical department with complaints of diffuse abdominal pain. A Chest and Abdominal CT 
scan was performed which revealed a right liver lobe cyst $(18 \mathrm{~cm})$. Subject 2: 39 years old male admitted in our surgical department with complaints of dyspnea. A chest and Abdominal CT scan was performed which revealed a cyst of $18315 \mathrm{~cm}$ on the inferior lobe of the left lung. Pre operatively all ecchinococcus tests were negative. Both patients underwent surgical procedure for the removal of the cyst.

Results: Patients had an uneventful post operative period and were sent home on POD no 6. The histological results were consistent with the hypothesis of an HYDATIC CYST with presence of scoliosis and laminated membranes.

Discussion: Not all the hydatid cysts present with the characteristic radiological finding. Huge cysts should be considered in the differential diagnosis of space-occupying lesions of the lung and of the liver, especially in endemic areas.

\section{Breast Surgery}

\section{5}

\section{Controversies and New Issues in the Spreading use of Sentinel Lymph Node Biopsy (SInb): Detection of Micrometastases in Patients with Final Diagnosis of Intraductal Carcinoma of the Breast, DCIS}

S. di Saverio, F. Catena, D. Santini', L. Ansaloni, A. Leone, T. Fogacci, F. Gazzotti, A. De Cataldis, S. Gagliardi,

T. Komatsu, M. Taffurelli,

Chirurgia d'Urgenza, 'Istituto di Anatomia Patologica, Policlinico S.Orsola-Malpighi, Università di Bologna, Italy

Background: By definition DCIS does not metastatize. The low rate $(1-2 \%)$ of axillary metastases is attributed to undetected invasive foci. Axillary lymph nodes dissection (ALND) and SLNB are not usually necessary. In selected cases of DCIS 'High-Risk' (H-R) SLNB can be performed.

Materials and Methods: Among 408 patients with DCIS, 107 underwent ALND and 32 SLNB. $46 \%$ of patients treated with ALND had preoperative diagnostic procedures (FNAC or Directional Vacuum-Assisted Biopsy) while out of 32 patients with SLNB 25 had a previous DVAB and 2 FNAC.

Results: The rate of node positivity was $3.6 \%$. Among 107 patients who had ALND, 1 patient revealed metastases. Of the 32 SLNB patients, 4 were found to have micrometastases $(12.5 \%)$ of which 3 showed a DCIS 'H-R', while the 4th has a DCIS G1 and low VNPI. Surprisingly all of these patients underwent a previous DVAB and the nodal positivity was only detected by IHC with a similar morphologic pattern.

Conclusions: The clinical significance of SLN micrometastases IHC-detected remains uncertain. We hypothesize the anatomic disruption after preoperative bioptic procedures increases the likelihood of epithelial cell displacement and the frequency of IHC-positive SLN and both of which are directly proportionate to the degree of manipulation. These micrometastases can not be considered true biologic metastases but rather the 'benign transport' of breast epithelium by preoperative manipulation
376

\section{Patients with DCIS of the Breast Applying USC/VAN Nuys Prognostic Index:} A Retrospective Review
S. Di Saverio, F. Catena, D. Santini', L. Ansaloni,
A. De Cataldis, G. Calò, T. Fogacci, F. Gazzotti, S. Gagliardi,
T. Komatsu, M. Taffurelli,

Chirurgia d'Urgenza, Chirurgia d'Urgenza 'Istituto di Anatomia Patologica, Policlinico S.Orsola-Malpighi, Università di Bologna, Italy

Introduction: The VNPI is a useful score for predicting the risk of local recurrence (LR) in patients with DCIS conservatively treated. This score combines 3 independent predictors of LR. VNPI has recently been updated with the addition of age as a 4 th parameter into the scoring system.

Materials and Methods: Applying the USC/VNPI we reviewed retrospectively 259 patients with DCIS who were treated with breast conserving surgery with or without radiotherapy (RT); of which $63.5 \%$ had a low VNPI, 32\% intermediate and $4.5 \%$ a high score. In the first group, the major part of patients underwent conservative surgery $(\mathrm{CS})$ without RT; in the intermediate group, almost half of the patients received RT; whereas $78 \%$ of the patients with high VNPI were treated with CS + RT.

Results: Twenty-one LR were observed (8\%), 16 were invasive, with a mean follow up of 130 months. Statistically, there were no significant differences in all groups between patients treated with CS or CS + RT. Disease-free survival at 10 years was $94 \%$ with low VNPI, and $83 \%$ in intermediate and high score range $(\mathrm{p}<0.05)$. On the contrary no significant differences were observed in the subgroups of VNPI. The overall survival breast cancer specific was $99 \%$ and no differences were observed between groups $(p=n s)$.

Conclusions: Although in our series there isn't a significant difference in LR rates by parameter of age, VNPI is a useful and simple scoring system for therapeutic management of DCIS.

\section{7 \\ Partial Intraoperative Sentinel Node
Evaluation in Breast Cancer Surgery}

A. Moscaroli, A. Lombardi, S. Maggi, M.L. Russo, M. Pezzatini, C. Amanti

U.O.C. Chirurgia Generale B, U.O.S. Chirurgia Senologica, AZ, Ospedaliera Policlinico Sant'Andrea Roma

Introduction: Sentinel node biopsy (SLB) has been developed as a standard operative procedure for staging of breast cancer in patients with axillar clinically negative nodes. It combines the potential advantage of decreased morbidity with a more careful examination of the first node and consequently with high accuracy in the prediction of axillary lymph node involvement. However, this procedure requires a second operation to complete axillary dissection in case of sentinel node metastasis. In alternative it is possible to perform a total and definitive intraoperative examination of sentinel node, effective procedure, but very expensive in terms of time and resources. The aim of our study was to 
demonstrate the role of partial intraoperative sentinel node evaluation to reduce the number of reoperations for sentinel node positivity, performing when necessary an axillary clearance in only one procedure.

Material and Methods: Sentinel node biopsy was performed in 141 breast cancer patients. A partial intraoperative examination of sentinel node was detected in 136 cases, followed by a definitive pathologic evaluation; in 5 patients $(3.5 \%)$ it wasn't possible to find $\mathrm{SN}$ and in these cases a total axillary dissection was made at once.

Results: Thirty-seven patients $(27 \%)$ resulted positive at permanent pathologic SN examination in according with data of literature; 21 of whom had been found positive at partial intraoperative examination $(56.7 \%)$. False-negative SN resulted in 16 cases $(11.7 \%$ of total), 11 of these, positive only for micrometastasis. Partial intraoperative examination allowed to reduce the percentage of a second surgical procedure for sentinel node.

\section{Emergency Surgery}

\section{8}

\section{Spontaneous Gallbladder Rupture Causing Haemoperitoneum}

W. Bugiantella, E. Facchiano, A. Pisciaroli, M. Paganelli, A. Patriti, E. Cavazzoni, A. Donini

Department of Surgery, Section of General and Emergency Surgery, University of Perugia, Santa Maria della Misericordia Hospital, Perugia, Italy

Introduction: Haemoperitoneum due to non traumatic gallbladder rupture is a very rare condition. Despite perforation of the gallbladder accompanies about $3-10 \%$ of lithiasic cholecystits, bleeding from the gallbladder wall is uncommon because of flogistic thrombosis of the blood vessels caused by acute or chronic inflammation.

Methods: A 77 years old woman was admitted at our department for acute upper abdominal pain and rapid fall of hematocrit. She had no history of trauma or GI disorders with the exception of gallbladder stones and she was under Aspirin for recurrent cerebrovascular disorders. CT scan of the abdomen suspected a rupture of the anterior wall of the gallbladder and showed free abdominal fluid and perihepatic fluid collection. Laparotomy was performed and a rupture of the infundibulum of the gallbladder was found, associated with a conspicuous haemocholeperitoneum. A $3.5 \mathrm{~cm}$ stone was also found free in the peritoneal cavity.

Results: Few cases of spontaneous gallbladder rupture causing massive hemoperitoneum are reported in literature; the great majority of the patients presented both gallbladder stones and a condition favoring spontaneous bleeding such anticoagulant or steroid therapy, complicated pregnancy, lupus, vascular disorders, cystic artery aneurysm, gallbladder tumors.

Discussion: Although traumatic gallbladder rupture associated with a major peritoneal bleeding is uncommon, this disorder should be considered in elderly patients under anticoagulant therapy and with an history of gallbladder stones presenting with acute abdomen of unknown origin and suspicious haemoperitoneum.

\section{9}

\section{Acute Bowel Obstruction due to Intestinal Malrotation with Volvulus: Case Report of Two Adults}

\author{
F. Coccolini, P. Cambrini, S. Gagliardi, F. Gazzotti, F. Catena, \\ S. Di Saverio, L. D'Alessandro, M. Taffurelli, L. Ansaloni, \\ A.D. Pinna \\ Transplant, Emergency and General Surgery DPT, \\ Policlinico S. Orsola-Malpighi, Bologna
}

Introduction: Malrotation with volvulus is a quite frequent condition presenting in the neonate and the young child, but rare in adults. Very rarely presents with episode of acute abdominal pain in an adult requiring urgent surgery. We report 2 patients with intestinal malrotation in urgent setting.

Case Report: Case 1. A 26-year-old woman presented with a sudden onset of abdominal pain of 6-hours duration. At laparotomy the entire small bowel, caecum, ascending colon and half of transverse colon were necrotic due to a volvulus. Right hemicolectomy and partial small bowel resection with ileostomy was performed. $48 \mathrm{~h}$ later a second look was performed to check for the viability of the residual small intestine. 1 month later a ileo-colic anastomosis was performed. Case 2. A 63-year-old woman presented with a $12 \mathrm{~h}$ duration abdominal pain. At laparotomy the entire small bowel, caecum, ascending colon and half of transverse colon were cyanosed. A volvolus affecting the cecum and the ascending colon was evident due to a mobile cecum and ascending colon. Art. hemicolectomy with ileo-transverse anastomosis was performed. The postoperative period was uneventful and the patient was discharged in the 8th postoperative day.

Discussion: Malrotation with volvulus should be considered in adults who present with unexplained acute onset abdominal pain.

\section{0}

\section{A Very Early Closure of an Open Abdomen:} Retrospective Study

\author{
M. Montesi, E. Bartolucci, V. Corso, T. Fogacci, L. Veneroni, \\ G. Francioni \\ Ospedale Infermi Rimini, Chirurgia Generale, Rimini
}

Introduction: In the modern care of trauma and no trauma patients, the open abdomen is a valid technique employed more frequently in specific circumstances.

Material and Methods: We undertook a retrospective review of 46 patients who underwent early closure of an open abdomen from 2001 to 2006 on the Division of General Surgery of 'Infermi Hospital' of Rimini. All open abdomen patients were treated by using nonadherent plastic drapes (Steri-drape) that's inexpensive, biologically inert, minimize fluid loss and it's easy to remove for daily inspection of the underlying viscera. Progressive fascial advancement was used during the re-operations using simple interrupted sutures.

Results: We treated 30 patients from 18 to 85 years. The majority of patients was an open abdomen for peritonitis, only one for compartmental syndrome. The average length of time the abdomens were 
open before fascial closure was $62 \mathrm{~h}$. We had a mortality of $30 \%$. No major complications that need re-intervention after we closed the abdomen were seen. We had 4 intra-abdominal abscess, 9 wound infection, 4 hernia formation.

Conclusion: We think that all open abdomens may be closed very early during the initial treatment without major complications. Our technique with closure of the fascia within $96 \mathrm{~h}$ lead to treat abdominal hypertension and to prevent ACS and to reduce complications.

\section{1}

\section{The Open Abdomen and the Young Surgeon}

V. Corso, M. Montesi, T. Fogacci, E. Bartolucci, M. Prandi, M. Todaro, L. Veneroni, G. Francioni

Ospedale Infermi Rimini, Chirurgia Generale, Rimini

Introduction: The idea to treat a patient with a laparostomy and leaving abdominal wall open, goes back to the last century. But what can be at present the young surgeon's role in preventing, in the diagnosis and in the treatment of patients with abdominal hypertension and in patient with severe peritonitis?

Material and Methods: We've evaluated all the patients treated with an open abdomen from 2001 to 2006 on the Division of General Surgery of 'Infermi Hospital' of Rimini. In every patients we have analyzed the SAPS II score, the APACHE II and the SOFA score at the admission, the PEV on the closure of laparostomy, on the second, third and fourth post-operative day. One young surgeon was present in every surgical procedure and he taked care to treat the patients until the demission and for the follow up.

Results: What have we young surgeons learned on the treatment an on the prevention of abdominal hypertension? Why we leave an open abdomen and what a young surgeon do? The paths to the open abdomen occurs for three reason: we are unable to close at the end of the case because it's just too tight and the peak of inspiratory pressure are high; we are unwise to close because of the devasting intraabdominal infections; we need to reopen the abdomen that has been closet without recognition of ACS.

Discussion: We think that all young surgeons must be able to recognize and to treat AI, particularly they must know the mechanisms involved in the formation of the AI and the surgical choices for its treatment. Further studies are needed in this relatively new field of emergency surgery and all we young surgeons can create a multicentric study for the treatment of ACS in Italy.

\section{2}

Blunt Liver Trauma in Children: Conservative Operative Treatment. Case Report

D. Loffredo, G.G. Manco, F. Zabatta, F. Pastena, F. Lojodice, D. Del Ciampo, A. Evangelista, N. Causa, G. Cioffi, P. Falco, A. Marvaso

U.O.C. Chirurgia, P.O. Rizzoli, Lacco Ameno, Ischia, NA

Introduction: Blunt trauma of the liver and consequent bile duct injuries after abdominal contusion are rare in children.
Improvement in diagnostic techniques and intensive care management have reduced mortality and morbility even in operative and nonoperative treatment.

Material and Methods: This report describes a case of blunt liver injury in a 6 years old child. The little patient had abdominal pain by visiting in Emergency Room, Blumberg sign positive blood pressure was $90 / 40 \mathrm{~mm} \mathrm{Hg}$, F.C. $110 \mathrm{~b} / \mathrm{m}$, HB $11 \mathrm{~g} / \mathrm{l}$. Emergency ultrasound and TC was performed. The surgeon decided for operative management because of rupture of the VI and VII hepatic segment and haemodinamic instability of the patient. Surgery was performed by suture of a portal branch and position of Floseal Tm, fibrin glue (Tissucol Tm) and Tabotamp and direct suture of the hepatic parenchyma.

Results: Postoperative management was without complications. Drains were removed on third postoperative day. Hospital demission was made on seventh post-operative day. Two months after surgery ultrasound non showed bilioma or hepatic abscesses.

Conclusions: Operative conservative management of blunt liver trauma can be safely performed in children with haemodinamic instability if other treatments are not possible.

\section{3}

\section{Topic Hemostats and Sealants in Emergency Surgery: Pros and Cons}

\section{S. Panicucci, F. Filidei, F. Scassa, M. Barlettai, C. Bagnato}

Introduction: Currently, indications for use and choice of topic hemostats and sealants (THS) are heterogeneous, mostly based on individual surgeon's preferences. Even surgeons who use THS frequently, appear to have a poor knowledge about these products. The most recent literature and our personal experience show that correct use of THS can significantly improve surgery, but suggest that their improper use can worsen things too.

Methods: The study was based on literature's review, a multidisciplinary surgical questionnaire sent in 30 surgical units and on a direct experience with various types of THS.

Results: Questionnaires were completed by 21 surgical units and their results confirm a surprisingly scattered use of THS. On 14 commercially available products only 4 were known by more than $30 \%$ of the units. Most frequently used agents were fibrin glues (47\%), oxidized cellulose (62\%) and collagen sponge (19\%). Our experience shows positive results in cost/effectiveness using reusable fibrin glues (Quixil) and oxidized cellulose (Tabotamp Nu-Knit) along with fibrin based products.

Discussion: As for other procedures in surgery, there is no reason to assume that a learning curve in use of THS should be unnecessary. Our study shows that the employment of THS in our area is done with a poor methodical approach. The high cost of THS imposes the definition of guidelines to optimize cost/effectiveness. 
384

\section{All Comers Policy for Laparoscopic Approach in Right Acute Abdomen}

R. Cantisani, V. Gentile, C. Galatioto, F. Filidei, S. Panicucci, S. Sergiampietri

Introduction: Right Acute Abdomen (RAA) with initial diagnosis of acute appendicitis (a.a.) is one of the most common surgical admissions. However, Literature reports up to $30 \%$ of 'normal appendices' found at surgery. An 'all comers policy' for laparoscopy in RAA is therefore justified because of its reliability, both as a diagnostic and therapeutic procedure.

Methods: From January 2001 to December 2005, 623 patients (321 M/302 F; 3-89 years) were admitted to our Unit, with a diagnosis of RAA. All patients were submitted to a laparoscopy and the following data were recorded: diagnostic effectiveness, operative time (OT), surgical procedures and outcome.

Results: In 489 patients $(78.5 \%)$ a diagnosis of a.a. was confirmed. In the remaining 134, laparoscopy showed the following: 96 (15.4\%) PIDs, 18 (2.9\%) bowel inflammatory disease, 9 (1.4\%) sigmoiditis, $4(0.6 \%)$ perforated duodenal ulcers; $3(0.5 \%)$ pyelonephritis; $3(0.5 \%)$ omental necrosis; $1(0.2 \%)$ caecal perforation. Conversion to open surgery was needed in 44 patients $(7 \%)$. The conversion rate for Laparoscopic Appendectomy (LA) was 5.7\% (28 patients). Mean OT was $59.5 \mathrm{~min}(15-150)$ in LA and $134 \mathrm{~min}(60-240)$ when a conversion was carried out; p.o. complications $(2.6 \%)$ were observed in a.a. only.

Discussion: Laparoscopy is recommended as a primary invasive tool in RAA. Our study demonstrated its safety and efficacy, allowing further treatment.

\section{5}

\section{Liver Trauma: The III-IV Segment Tears}

G. Galatà, F. Rulli, C. Micossi, C. Ridolfi, M. Grande, M. Villa, A.M. Farinon

Department of General Surgery, University of 'Tor Vergata', Rome, Italy

Introduction: The liver is the most commonly injured intraabdominal organ. The liver's mass is the key factor in determining the extent of the inertial force and consequently of damage in the case of sudden deceleration. Speed accidents, usually, produce characteristic lesions where the III-IV segment tear onto the hepatic ligament causing grade I-III liver injuries (LI). 5 cases of such injuries are described.

Methods: All trauma patients admitted at our hospital who sustained a blunt abdominal injury from January 1 to December 312004 were identified. Clinical records were reviewed for demographics, severity of LI, associated concomitant injuries, management scheme. Grade of LI was determined from initial CT or from intraoperative findings.

Results: A total of 159 patients were identified. In 14 (8.8\%) one or more liver lesion/s were associated: low-grade injuries of which 3 were grade I, 8 were grade II. $40 \%$ were high-grade injuries comprised of 6 grade III and 1 grade IV. In this series, the more fre- quent occurrence was a tear between the hepatic segments III and IV, by the acute impact of the liver onto the hepatic ligament.

Conclusions: An hepatic injury caused by the round ligament has been intraoperatively diagnosed in 1 of 5 liver trauma patients $(35.7 \%)$ in our one-year abdominal blunt trauma series. Our clinical contribution underlines the high frequency of such lesions that seems to be related and characteristic of high speed trauma. In these cases immediate deceleration due to the impact may be relevant in the pathophysiology of the lesion.

\section{6 \\ Emergency Surgical Treatment of Respiratory Failure due to Thyroid Metastasis from Renal Carcinoma}

\author{
A. Gurrado, G. Lissidini, W. Lavermicocca, D. Lardo, \\ L. Nacchiero, M. Testini \\ Department of Application in Surgery of Innovative \\ Technologies, University of Bari, Italy
}

Introduction: The clinical presentation of secondary tumours to the thyroid is uncommon; the autopsy incidence of metastases to the gland is more frequent, ranging from $1.2 \%$ in unselected patient series to $24 \%$ in patients with widespread tumours. The acute respiratory insufficiency is relatively common due to a massive haemorrhage into a thyroid nodule, a mediastinal goiters and a rapidly enlarging of anaplastic neoplasm or thyroid lymphoma. A rare case of acute airway failure due to laryngeal compression and infiltration by metastasis of renal clear cell carcinoma is reported.

Case Report: A 73-year-old man with a previous history of multinodular goitre presented with sudden dysphonia, stridor, dyspnea and dysphagia. Eight years before, the patient underwent a right radical nephrectomy for a renal clear cell carcinoma. An urgent CT scan diagnoses a complete obstruction of the upper airway due to a massive infiltration of a thyroid mass. An emergency operation with tracheostomy and total thyroidectomy was immediately performed. Histological findings showed a metastatic renal cell carcinoma, with invasion into adipose tissue and removed limph nodes. The recovery was uneventful and the patient was discharged ten days after the operation.

Conclusion: The acute respiratory failure by obstruction is a severe clinical manifestation from compression or infiltration of upper airways and it requires an urgent tracheostomy. Renal metastases to the thyroid are not as rare as believed, but no previous cases of acute airway obstruction presenting as first clinical manifestation of a metastatic renal carcinoma in the gland and determining emergency operation have been reported. 


\section{7}

\section{Massive Mesenteric Ischemia Resulting from} Giant Umbilical Hernia

\author{
D. Merlicco, G. Lissidini, A. Gurrado, D. Lardo, \\ L. Nacchiero, M. Testini \\ Department of Application in Surgery of Innovative \\ Technologies, University of Bari, Italy
}

Introduction: The $90 \%$ of mortality after emergency hernia repair occurs in patients with cardiopulmonary diseases.

Case Report: A 73-year-old female was transferred in emergency at our observation with diagnosis of intestinal obstruction due to a giant strangulated umbilical hernia. Anamnesis revealed that patient didn't undergo previous surgical hernia repair for the high operative risk due to cardiovascular and pulmonary severe diseases with frequent recurrence of pulmonary oedema. The clinical examination showed an enormous strangulated umbilical hernia, measuring $40 \mathrm{~cm}$ in its long axis, with acute abdomen. Emergency laparotomy showed a huge peritoneal umbilical sac containing massive mesenteric ischemia starting from $40 \mathrm{~cm}$ after the Treitz ligament and extended to the right colonic flexure. A near-total resection of the small bowel and a right hemicolectomy with a terminal jejunostomy were performed. The recovery was complicated by atrial fibrillation and pulmonary sub-oedema. Twenty days after the operation the patient was discharged and begun domiciliary total parenteral nutrition. 24 months after surgical treatment the patient is still alive with no recurrence of the hernia.

Conclusion: The reported case presented a high preoperative risk (ASA IV), due to severe chronic cardiopulmonary disease. Emergency hernia repair was performed as she previously refused elective surgery. We recommend elective hernia repair in elderly patients in the presence of a high pre-operative risk too, because emergency surgery is frequent and it is burdened by a major risk.

\section{8 \\ Management and Surgical Treatment of Extreme Complications Resulting from Abdominal Wall Hernias}

\author{
W. Lavermicocca, G. Lissidini, A. Gurrado, D. Lardo, \\ L. Nacchiero, M. Testini \\ Department of Application in Surgery of Innovative \\ Technologies, University of Bari, Italy
}

Introduction: Hernia repair is the commonest surgical procedure performed in favourite areas. Elective surgery for abdominal wall hernias is a safe and easy-to learn procedure associated with few complications and no mortality. Morbidity and mortality significantly increase in emergency treatment with a range from 19.5 to $58 \%$ and 3.5 to $22 \%$ respectively. How can these mortality figures after operation for a benign condition be explained?

Case Reports: Case 1: A 70-year-old female in gasping syndrome was operated for biliary peritonitis due to multiple intestinal perforations resulting from a strangulated ventral recurrent hernias. The original surgical choice is reported. Case 2: A 73-year-old female operated for a massive mesenteric ischemia resulting from agiant strangulated umbilical hernia is reported. Case 3: A 30-year-old male operated for a huge strangulated inguinal hernia with a pachyvaginalitis. Case 4: A 71-year-old man operated for a malignant peritoneal mesothelioma mimicking a strangulated groin hernia. CASE 5: A 63year-old female operated for an incisional strangulated hernia containing a carcinoma of the caecum. All presented cases are still alive with a follow-up ranging from 18 to 36 months after the emergency operation.

Discussion: ASA grade, emergency surgery, type of hernia, severity of complications, and delay in the treatment of a complicated hernia are the factors determining the high mortality rate. Surgical outcome is affected by age, concomitant diseases, and rapidity in diagnosis, hospitalization, and emergency surgical treatment.

\section{9 \\ 'Abdominal Compartment Syndrome (ACS) and Acute Severe Pancreatitis: Do Exists an Ideal Surgical Treatment?'}

\author{
G. Martino, E. Tarantino, G. Martino, M. Marengo, \\ G. Fanello, M. Benedetti, G. Spera, V. Frantellizzi, G. Fusco, \\ M. Cardi, R. Caronna, P. Chirletti \\ Università degli Studi di Roma 'La Sapienza' Azienda \\ Policlinico Umberto I, U.O.C. Chirurgia d'Urgenza Pronto \\ Soccorso e Trauma B, Scuola di Specializzazione in \\ Chirurgia Generale V ad indirizzo d'Urgenza
}

Introduction: Abdominal Compartment Syndrome (ACS) is a clinical picture due to the acute increase of the abdominal pressure, with consequent multiorgan failure (MOF). ACS is frequent in trauma patients but also during Severe Acute Pancreatitis (SAP), and represent a frequent indication for operative treatment. The aim of the study was to discuss the incidence and treatment of ACS in a series of over 60 consecutive patients admitted and treated for AP in our institution.

Methods: From 2000 to 2004, 63 patients with AP (severe in 26) were admitted in our surgical department. Standard medical treatment and laboratory and imaging diagnosis were made in all patients for the diagnosis of AP. ACS was diagnosed when measurement of the bladder pressure according to the Cheatam and Safcsak technique was above $25 \mathrm{~mm} \mathrm{Hg}$.

Results: In 4 cases $(15.4 \%$ ) with SAP (Ranson $>6$, Apache II score $>20$, Balthazar III-IV) an ACS has been diagnosed (IAP $>25 \mathrm{~mm} \mathrm{Hg}$ and organ failure). All these patients underwent surgery with necrosectomy, cholecystectomy and/or CBD exploration and removal of stones, placement of peritoneal drains for postoperative continue peritoneal lavage, and temporary abdominal wall closure (Gore-Tex mesh). All patients were sent to ICU for assisted ventilation and CVVDH. Mortality was $11.5 \%$.

Discussion: Incidence of ACS in SAP varies in literature from 10 to $80 \%$, with mortality up to $45 \%$ of the cases. From the results of our study, IAP $>25 \mathrm{~mm} \mathrm{Hg}$ has to be considered, with other variables (Ranson, Apache II score, Balthazar) a valid indication for early operative treatment. 


\section{0}

\section{Occult Bleeding from lleal Angiodysplasia. Diagnostic and Therapeutic Options}

F.M. Keinan, G. Fanello, M. Cardi, B.G. Romeo,

S. Mangioni, G. Martino, A. Vespa, M. Marengo,

M. Benedetti, G. Spera, G. Fusco, V. Frantellizzi,

V. Crispino ${ }^{1}, R$. Caronna, P. Chirletti

Università degli Studi di Roma 'La Sapienza', Azienda

Policlinico Umberto I, UOC Chirurgia d'Urgenza Pronto

Soccorso e Trauma B, Scuola di Specializzazione in

Chirurgia Generale V ad indirizzo d'Urgenza

Introduction: GI bleeding of ileal origin is $3-5 \%$ of all digestive haemorrhages. In 3 to $20 \%$ is caused by angiodysplasia and almost always present with acute haemorrhage. Occult bleeding is the most unfavourable condition for diagnosis that can be done with angiography, scintigraphy with marked erythrocytes or endoscopeincluded study with capsule. The choice of treatment modality essentially depends from interested line and bleeding activity.

Case Report: 52 years old woman, with serious sideropenic anaemia from 5 years, hospitalized for serious GI bleeding $(\mathrm{Hb}$ $6.5 \mathrm{~g} / \mathrm{dl}$ ). EGDS and RSCS were negative. Digestive series, CT and MRI led to the suspicion of ileal haemorrhage. Red blood cell scintigraphy shows bleeding from distal ileum. Capsular endoscopy was difficult for the presence of haematin but revealed bleeding in the last ileal loop. Exploratory laparotomy was performed with intraoperative enteroscopy that confirmed the presence of angiodysplasia. Ileo-caecal resection was performed. Histology confirmed the presence of artero-venous malformations.

Discussion: In patients over 50 years with chronic anemia, an ileal pathology has to be suspected, when other locations are endoscopically excluded. Digestive haemorrhages from angiodysplasia are rare but pose serious problems of location often of difficult resolution. Currently, the diagnostic tool with greater diagnostic accuracy seems to be capsular endoscopy. It locates precisely the lesions but it can be hindered from the presence of abundant haematin. In these cases ileal resection driven by intraoperating enteroscopy is suitable.

\section{1}

\section{Complex Abdominal Trauma}

S. Mangioni, G. Fanello, G. Martino, M. Marengo,

G. Spera, M. Benedetti, G. Fusco, V. Frantellizzi, M. Cardi,

R. Caronna, P. Chirletti

Università degli Studi di Roma 'La Sapienza', Azienda Policlinico Umberto I, UOC, Chirurgia d'Urgenza Pronto

Soccorso e Trauma B, Scuola di Specializzazione in

Chirurgia Generale V ad indirizzo d'Urgenza

Introduction: Complex abdominal trauma is a trauma that simultaneously interest different organs for which an immediate surgical treatment is required with frequent involvement of different surgical specialities. The authors present their experience in the clinical and surgical management of these patients.
Methods: From January 2003 to December 2005, 55 patients with multiple trauma were admitted in our institution. Nine met the criteria for a diagnosis of complex abdominal trauma (ISS $>29$ and APACHE II score $>20$ ). Mean age was 38.4 years. Operation was decided upon hemodynamic instability, ISS $>35$ and positive FAST. Indications to damage control surgery was made preoperatively upon the presence of hypothermia, coagulative disorders and metabolic acidosis, and intraoperatively for ongoing hemorrhage and lesions for which time-consuming procedures would have been necessary.

Results: Solid viscus were interested in 8 patients $(88.8 \%)$, hollow viscus in $3(33.3 \%)$, diaphragm in $1(11.1 \%)$, vascular in 2 $(22.2 \%)$. A damage control surgery was decided in 3 patients. Mean ICU stay was 23.6 days. Morbidity was $88.8 \%$ (sepsis in 7 , ARDS in 7, ACS in 3 and MOF in 1). Mortality was $33.3 \%$.

Discussion: Incidence of complex abdominal trauma is increasing due to higher speed in MVA and better trauma care at the site of the accident, during transportation and the in-hospital treatment. ISS is frequently $>30$ and the APACHE II score $>20$. Time and clinical judgement are of paramount importance for survival. Early laparotomy and DCS are the main clues to reduce preventable deaths.

392

\section{Diagnosis and Treatment of Face Lesions in Polytraumatized Patients}

\author{
F. Klinger, A.V. Pagliari, F. Caviggioli, S. Giannasi \\ D. Ventura, J. Hassibi, F. Villani, C.A. Amodeo, \\ B. Banzatti, M. Klinger
}

Istituto di Chirurgia Plastica dell'Università degli Studi di Milano, U.O.C. Plastica II, I.C. Humanitas

Introduction: Polytrauma belongs to the most serious injuries due to their extent because in most of them the life of patients is directly threatened. When vital functions are stabilised diagnostic procedures and treatment of injuries that are not directly life-threatened are maked. Face injuries may become life severely disabling if not diagnosed and treated promptly.

Methods: In diagnostic and therapeutic strategies of face lesions, involving a multidisciplinary team, a pivotal role is played by plastic surgeon. In polytraumatized patients a repeated clinical examinations of the face and early computed tomography are essential for a correct patients evaluation.

Results: A adequate treatment of face soft tissue damage and maxillofacial bone fracture allows a impaired quality of life after major trauma with a acceptable social and working life.

Discussion: Face injuries in polytraumatized patients are not critical for survival but for the later quality of life. Maxillofacial injuries are frequently misinterpreted in association with polytrauma and could lead to severe functional impairment. 


\section{3}

\section{The Acute Diverticulitis in Emergency}

L. Persico Stella, S. Toccaceli, R. Dandolo, M. Diana,

A. Budak, M. Tasciotti, C. Tasciotti, G.E. Casciaro,

E. Spaziani, F. Stagnitti

Introduction: Treatment of acute diverticulitis still remains complex and not univocal today, surgical mortality being between 5 and $45 \%$.

Methods: We have observe 76 patients with diagnosis of acute diverticulitis. In 61 cases the inflammatory and sub-occlusive symptomatology has been faced with medical therapy, with resolution of inflammatory symptomatology in 47 cases. In 14 patients the resolution of inflammatory symptomatology has been had but only partially of the sub-occlusive picture and therefore, it has been provided to surgical operation in election. 15 patients ( 9 male, 6 female) in an average time of 69.3 years, introduced a clinical picture of acute abdomen and therefore they have been undergone to surgical operation in emergency.

Results: All the patients had been operated within the $24 \mathrm{~h}$ from the entry in the first aid; in 13 patients we have found a diverticular tumour, with diastasical perforation in 2 of us, while in 2 cases was present a diverticulum perforation. In all the patients we have executed a primary resection with manual anastomosis in double layer. We haven't registered intra operating mortality only in 1 case we have had a anastomotical dehiscence, that have not needed of a re-operating. In 3 cases we have registered a wound infection treated with antibiotic therapy.

Discussion: In our experience the adoption of an operating in an only time, when is not deferrable, seems to be the most suitable and the less expensive procedure. In the greater part of the cases is preferable to treat the patients, when possible.

\section{4}

\section{Diaphragm Lesion from Steel Weapon}

L. Persico Stella, S. Toccaceli, C. Tasciotti, R. Dandolo,

M. Diana, A. Budak, M. Tasciotti, G.E. Casciaro,

E. Spaziani, F. Stagnitti

U.O.C. Chirurgia Universitaria, Ospedale Fiorini, Terracina, Università degli Studi 'La Sapienza', Polo Pontino

The authors report here a case of perforation of diaphragm from an injury from a steel weapon. The patient, a man of 21 age, of Indian nationality, reached in the first-aid reporting to have been assaulted approximately $12 \mathrm{~h}$ before and to have been hit in the abdomen and the thorax with a blade. The patient presented with dysponea, thoracic and abdominal pain and a state of shock. On examination there was double penetrating wound in the left dorsal region, an untreatable abdomen and a left hemithorax with clinical signs of pleural effusion. According to hemogas analysis was present an unamissing ipossya. The x-ray examinations showed us a left pleural effusion with the presence of intestinal loops in emithorax. The ultrasonographic examination evidenced an abundant deposit in the abdomen and therefore it was decided to operate surgically through a xifo-umbilical median incision. At the opening of the peritoneum there was an abundance emoperitoneum with presence of lesion from cuts to the diaphragmatic dome near to esophageal hiatus with laceration of the small omentum but with any lesion of the other intra abdominal organs. We provided to mend of the diaphragmatic lesion and a water-drainage was placed in the Douglas, one under the liver and one left thorax drainage according to Boulau. During the operation there was recovery with reinfusion intra operating of approximately $800 \mathrm{cc}$ blood. The post operative period was regular and so the patient was dischanged on the 18th day.

\section{5 \\ Inguinal Hernioplasty with PROLOOP SYSTEM}

S. Peruzzi, F. Catena, L. Ansaloni, S. Gagliardi, F. Gazzotti,

S. Di Saverio, L. D'Alessandro, A.D. Pinna

Transplant, General and Emergency Surgery DPT St

Orsola-Malpighi University Hospital Bologna, Italy

Introduction: In order to answer to polypropylene disadvantages (postoperative pain, long-term discomfort, infections, intestinal obstruction and fistulisation) this material has been improved like in PROLOOP SYSTEM. Parallel Flex ${ }^{\mathrm{TM}}$ monofilament construction was the base of the standard for low profile hernia mesh repair. Atrium's knitted polypropylene has superior biocompatibility and strength character. The handling qualities and the thinner profile of the original Prolite mesh explain the outcomes of enhanced patient comfort, less pain, and fewer recurrences or complications. The ProLoop ${ }^{\mathrm{TM}}$ Plug is a non-absorbable, lightweight, pre-formed, three-dimensional plug constructed of knitted rows of monofilament polypropylene with multiple protruding monofilament loops ('hairy' plug). The 'hairy' plug has an innovative shape that simply conforms to the defect, stays in place, sutures easily, and will not collapse or 'pop out' during the procedure.

Methods: A case-control comparison of mesh-plug repair of inguinal hernia with PROLOOP SYSTEM and a control group with common polypropylene mesh-plug repair was carried out at the Department of Emergency Surgery of St Orsola-Malpighi University Hospital with the participation of 4 surgeons who accept to standardise the technique.

Results: From January 2004 up to now 41 patients submitted to mesh-plug repair of inguinal hernia with PROLOOP SYSTEM with a 6 month minimum follow-up were enrolled. There were not intraoperative or postoperatively complications. No recurrences and wound infections were observed. The post-hernioplasty acute and chronic pain/discomfort (tested with visual analogue scale and simple verbal scale) and parenteral/oral analgesic consumption were statistically significant lower in PROLOOP SYSTEM compared to control group.

\section{6}

\section{Liver Trauma: Personal Experience}

E. Piazzese, V. Pacilè, F. Fiumara, A. Bonsignore, S. Incardona, L.G. Angiò

Università degli Studi di Messina, Scuola di Specializzazione Chirurgia Generale I

Introduction: Element that conditions the prognosis and treatment of liver trauma (LT) is the hemodinamic status of patient and presence of associated abdominal lesions (AAL), susceptible of surgical treatment. 
Patients and Methods: We have hospitalized 18 patients with LT (2000-2005), in 17 cases from closed traumas, in 1 from fire weapon; the lesions have been classified according to AAST's criteria: 3 of II degree, 6 of II degree, 5 of III degree, 3 of IV degree, 1 of $\mathrm{V}$ degree. In 9 cases were present AAL, in 6 were extraabdominal lesions. 9 patients, with hemodinamic stability and without AAL (group A), have been submitted to conservative treatment. The remainders 9 patients, with hemodinamic instability and with AAL (group B), has been submitted to laparotomy, surgical liver haemostasis (in 3 cases with resection) and to specific treatment of AAL.

Results: In group A has not been recorded mortality and morbility correlated to liver traumas. In group B is deceased a patient (liver lesion of $\mathrm{V}$ degree associated to speenic rupture and diaphragm'lesion) for haemorrhagic shock; the morbility has been of $11 \%$.

Conclusion: Therapeutic choice in LT has to be driven, above all, from the hemodinamic status and from the presence of AAL, and only relatively from the degree of liver lesion. In the case of conservative treatment is necessary however a narrow clinical and diagnostic monitoring, possibly performed in an Intensive Care Unit.

\section{7}

\section{Fournier's Syndrome: Cases Report}

E. Piazzese, V. Pacilè, D. Oliva, A. Bonsignore, F. Fiumara L.G. Angiò

Università degli Studi di Messina, Scuola di Specializzazione Chirurgia Generale I

Introduction: Fournier's syndrome (FS) is a rare form of acute gangrene of masculine genital ones characterized by plurymicrobial eziology, sudden onset, rapid evolution and high mortality.

Patients and Methods: From 1998 to 2005 we have observed 6 patients (age 23-73 years), with FS and with the following illnesses of general order: diabetes ( 5 cases), obesity (4 cases), cardiovascular pathologies ( 3 cases), neurological illnesses ( 1 case). All the patients have been treated in initial phases through antibiotic therapy to ample spectrum of action and through surgical intervention of necrosectomy. Subsequently the patients have been submitted to antibiotic specific therapy, to daily local cured and essays through oxygen therapy in iperbaric room.

Results: We have recorded 1 death for septic shock (mortality $16.6 \%$ ) in a cardiopathic and diabetic patient not compensated; the postoperative course of remainders 5 patient has been regular getting the recovery for second intention and through plastic surgery. The middle hospitalization has been of 18 days.

Conclusion: Our experience confirms that precocious diagnosis of FS associated to the specific physician-surgical treatment, fundamentally contemplated to the complete elimination of the infection, reduce the risk of deadly complication, especially in the patients already compromises or affections from other pathologies of general order.

\section{8}

\section{Hemoperitoneous from Rupture of Hepatocarcinomas in Healthy Liver and Spleen Lesion Secondary to Closed Abdominal Trauma: Case Report}

\author{
V. Pacilè, E. Piazzese, M. Lauretta, A. Bonsignore, \\ F. Fiumara, L.G. Angiò \\ Università degli Studi di Messina, Scuola di \\ Specializzazione in Chirurgia Generale I
}

Introduction: While the spontaneous rupture of the hepatocarcinomas (HCC) is frequent event $(2-26 \%)$, that secondary results exceptional and among the causes it needs to enumerate the iatrogenes and the abdominal traumas. In this last case, the liver lesions, represent a factor of risk for the onset of an hemoperitoneus if these are subglissoniane, in relief, on the anterior face and of big dimensions; gravity can increase in case of cirrhosis, coagulative disease, portal hypertension and other organs lesions, raising the rate of mortality and morbility.

Case Report: GC, 77-year-old man, diabetic in antiaggregant therapy, are hospitalized for abdominal trauma. Documentated anemizzation and hemoperitoneous whose origin is attributed by the TC to an laceration of the liver inferior face. The explorative laparotomy confirmed the hemoperitoneous and documented the presence of a lacerated liver tumor and a lesion of the spleen; therefore proceeded to liver wedge-resection and to splenectomy. The histological examination deposes for HCC on healthy liver.

Conclusion: After consultation of the medical literature, we can affirm the oneness of the case observed for the characteristics of the HCC (small dimensions, located on the inferior liver face, in absence of cirrhosis), for the association with the spleen lesion and for the antiagregant treatment followed by the patient (responsible of the gravity of the hemorrhage).

399

\section{Complicated Small Bowel Diverticulosis: Personal Experience}

V. Pacilè, E. Piazzese, A. Ardizzone, F. Fiumara, A. Bonsignore, L.G. Angiò

Università degli Studi di Messina, Scuola di Specializzazione Chirurgia Generale I

Introduction: Small bowel diverticulosis (SBD) is an infrequent pathology $(0.01-5 \%)$ that results, nevertheless, under esteemed because of the objective difficulties of diagnosis and for the absence or poverty of specific symptoms, often manifesting for onset of an acute complication (perforation, occlusion, hemorrhage).

Patients and Methods: From 1995 to 2005 we have hospitalized 5 patients for secondary acute abdomen to SBD, complicated from perforation in 3 cases and from occlusion in 2 patient. In all cases, for impossibility to express a preoperative diagnostic judgment on the cause of complication, we have proceeded to a explorative laparotomy and, in relationship to intraoperative find, to the resection of intestinal segment center of diverticular illness. 
Results: Mortality has been void and morbility of $20 \%$. The postoperative middle hospitalization has been of 6 days. Any short bowel syndrome has been recorded.

Conclusion: Our experience, even though limited, already brings a contribution to descriptions of SBD present in literature and confirmation the difficulty in the formulation of correct eziologic diagnosis in preoperative phase. The surgical attitude must be regulated in relationship to intraoperative find, avoiding the too wide intestinal resections, that can cause a short bowel syndrome.

\section{0 \\ Spontaneous Spleen Rupture in Infectious Mononucleosis: Case Report}

A. Bonsignore, E. Piazzese, V. Pacilè, F. Fiumara, M. Pettinato, G. Galarco, L.G. Angiò

Università degli Studi di Messina, Scuola di

Specializzazione Chirurgia Generale I

Introduction: Spontaneous spleen rupture (SSR) recognizes manifold causes distinguished in infectious, hematological, oncologiche and of the miscellany. We bring the case of SSR in progress of infectious mononucleosis (IM).

Case Report: PM (woman, 16 years), hospitalized for left abdominalgia, insurgent 10 days after a clinical picture of fever, sore throat and laterocervical lymphoadenopaty. For progressive worsening of general conditions and of abdominal objectivity (Kehr sign), for comparison of acute anemia and abdominal ematic payment (to ultrasoound and TC), the patient has been submitted to exploratory laparotomy, that documented hemoperitoneum anf spleen rupture; preceded therefore to splenectomy and hystopatologic find of spleen is compatible with location of IM, confirmed by the sierologic investigation. The postoperative course has been regular and the patient has been submitted to vaccination specifies and to periodic controls.

Conclusion: SSR, also being a rare complication $(0.1-0.5 \%)$, the most common cause of death remains for IM $(30 \%)$. Such eventuality, that usually happens during the third week from the debut of IM, must be suspects in the patient with tachycardia, hypotension, left abdominalgia, splenomegalia and positiveness of Kehr sign. The management of SSR in progress of IM is traditionally represented by the splenectomy that only therapeutic strategy remains in the patient unstable.

\section{1 \\ Laparoscopy in Abdominal Trauma: Indications and Limits \\ S. Sergiampietri, C. Bagnato, F. Scassa, R. Cantisani, V. Gentile}

Introduction: At present, the role of Video Laparoscopy (VL) in abdominal trauma (AT) is still debated because of various factors: operative length, necessity of hemodinamic stability, surgeon's experience etc. The purpose of the study is to evaluate the safety and efficacy of VL as a diagnostic and therapeutic procedure in selected ATs.
Methods: From March 2001, 170 trauma patients who required surgery were examined. 152 patients $(89 \%)$ suffered from blunt ATs and 18 patients (11\%) from penetrating ATs. VL was performed in 26 hemodynamically stable patients $(15 \%)$ and was followed by open surgery in 5. Main indications for VL were a pre-existing diagnosis of free intraperitoneal fluid and evaluation of injury depth in penetrating thoraco-abdominal traumas.

Results: VL demonstrated to be a valid diagnostic and therapeutic procedure in 21 patients $(73 \%)$. In 11 patients with penetrating injuries, VL allowed the exclusion of an involvement of visceral structures. In 8 patients with blunt ATs, VL identified a self limited intraperitoneal bleeding. In the remaining 2 patients, a hemostasis of hepatic bleeding was also performed. In 5 patients $(19 \%)$ a visceral perforation and a failure to control bleeding required an open procedure.

Conclusions: In spite of many limiting factors, a VL exploration in stable patients can be effective both as a diagnostic and therapeutic procedure, avoiding surgical exploration in selected cases

\section{2}

\section{Gallstone Obstruction of the Left Colon Associated to Colonic Diverticular Stricture. Report of a Case and Review of Management}

\author{
A. Vagliasindi, R. Bertelli, G. Mura, M. Framarini, \\ D. Cavaliere, F. Tauceri, G. Solfrini, G.M. Verdecchia \\ U.O. di Chirurgia e Terapie Oncologiche Avanzate. \\ Ospedale G.B. Morgagni, L. Pierantoni di Forlì
}

Introduction: Gallstone ileus is a rare disease; colonic involment is unusual. Concomitant cardiorespiratory or metabolic illness are responsible for the high mortality rate related to this disease and is moreover associated with delayed diagnosis

Methods and Results: We discuss the case of an 76-year-old woman presented to the emergency department with a 3 days history of abdominal pain in the left upper abdominal quadrant, related to colonic gallstone obstruction, in a patient with cholecystocolic fistula and left colonic diverticular stenosis. The patient was referred for urgent surgery. Cholecystectomy was therefore performed to remove the cholecystocolic fistula, with total colectomy being necessary due to a multiple involvement of the whole colonic frame. The surgical operation was completed by rectal stump closure and by terminal ileostomy. After surgery, the patient's clinical situation was significantly impaired, with hemodynamic and hydroelectrolytic imbalance secondary to both obstruction and sepsis. Her clinical conditions progressively improved. 25 days after surgery, the patient was discharged. Discussion. Only an early and punctual diagnosis of gallstone ileus will improve the mortality and morbidity rates associated with this disease, by a reduction of the incidence of post-surgery complications and the design of the best therapeutic approach. 19th National Congress of the Italian Polyspecialist Society of Young Surgeons (IPSYS) 


\section{3}

\section{Acute Peritonitis due to Ropture of Tuboovarian Actinomycotic Abscess in Presence of Intrauterine Contraceptive Device: A Case Report}

\section{R. Bertelli, M. Framarini, D. Cavaliere, G. Mura, F. Tauceri, G.M. Verdecchia \\ U.O. di Chirurgia e Terapie Oncologiche Avanzate, Ospedale G.B. Morgagni, L. Pierantoni di Forlì}

Introduction: Actinomycosis of the female genital tract is a rare cause of tubo-ovarian abscess (TOA). Intrauterine contraceptive devices (IUD) are often implicated. TOAs from actinomycosis are often misdiagnosed as ovarian malignancies. Clinical presentation is variegated.

Methods and Results: We discuss the case of a 33 years old woman with an IUD since 5 years and a recent negative PAP test, who presented to the emergency department with a history of one day of sudden and increasing abdominal pain in the lower abdominal quadrants and fever $39^{\circ} \mathrm{C}$. The abdominal examination revealed Blumberg sign present and a right pelvic mass. The US examination described right hydro uretero nephrosis and confirmed the presence of a dishomogeneous pelvic mass involving right adnexa. After removal of the IUD, the patient underwent emergency surgery. The intraoperative exploration revealed acute peritonitis and broken right ovaric abscess. Right salpingoophorectomy and toilette of the peritoneal cavity was performed. The postoperative course was uneventful and the patient discharged after 6 days. Histopathologic examination revealed tuboovaric actinomycosis. The patient was treated with antibiotic therapy.

Discussion: Preoperative diagnosis of tuboovarian actinomycosis is difficult even a PAP test is performed. Clinical presentation is aspecific. Correct antibiotic therapy is mandatory.

\section{4}

\section{Transcatheter Arterial Embolization in Blunt} Abdominal Trauma

\section{Morandi, M. Gavioli ${ }^{1}$ \\ Struttura Complessa di Radiologia 1, ${ }^{1}$ Struttura Complessa di Chirurgia 3, Azienda Ospedaliera Universitaria \\ Policlinico di Modena}

Introduction: The first description of transcatheter embolization of the internal iliac artery to control hemorrhage was published in 1972. Since that time, the role of interventional radiologists in trauma has evolved from the initial diagnosis of vascular and solid organ injuries to temporizing or definitive treatment. Many technical innovations in imaging and angiographic equipment, as well as new developments in transcatheter therapy, have paved the way for this trend in nonoperative management. These include: the following state-of-theart digital subtraction angiography, helical CT, microcatheters, steerable and hydrophilic guidewires, and coaxial guiding catheters and sheaths. Indications for emergency catheter angiography in the trauma patient include clinical signs or symptoms of hemorrhage or CT evidence of ongoing hemorrhage or vascular injury.

Method and Materials: During a 15-month period, 6 patients who were hemodynamically stable underwent spiral CT followed by 2 splenic arteriography, 1 liver arteriography and 3 renal.Vessel were embolized with permanent occluding agents or with temporary occluding agent.

Results: Upon embolization the bleeding stopped in all patients.

Conclusion: Transcatheter embolization of active hemorrhage or vascular injury often is considered preferable to surgical treatment alternatives, such as in the following instances: when rapid occlusion is desired, when surgical access is difficult, when the patient is a poor operative risk, when selective transcatheter embolization may limit the amount of normal tissue or parenchyma necrotized.

\section{Gynaecology}

405

Farnsworth Technique for Prosthetic Vaginal Repair: Cure of Severe Prolapse

D. De Vita, F. Araco' 1 E. Piccione ${ }^{1}$

Department of Obstetric and Gynaecology, S. Francesco D'Asissi Hospital, Oliveto Citra, Salerno, 'Department of Obstetric and Gynaecology, Tor Vergata University, Rome, Italy

Introdution and Aim: The objective of this original technique is the efficacy on prolapse cure, to improve functional symptoms and to reduce vaginal erosion. A retrospective case review is performed of 23 patients who underwent a total vaginal reconstruction using a polypropylene mesh for III-IV degree prolapse involving in all vaginal compartments. Patients were assessed prospectively for both objective and functional outcomes. Surgical complications were also documented.

Material and Methods: New techniques, enveloped to Centre for Pelvic Reconstructive Surgery at Sydney Adventist Hospital, have been used to Department of Obstetric and Gynaecology 'S. Francesco D'Assisi', Oliveto Citra, on 23 patients, subdivided in three different groups, with III and IV stage genital prolapses.

Results: Patients were followed up for an average of 12 months. The average age of the patients was 50 years. Objective surgical success was recorded in all patients. Urgency/frequency symptoms were cured or improved in $74 \%$. Three patients performed to total vaginal reconstruction have been post-operative obstructed defecation symptoms. Only three cases of minor erosion were observed.

Discussion and Conclusion: The aim of this innovative technique is to create a side walls need to be secured to prevent further prolapse, by precisely positioned tapes. 


\section{6}

\section{Tensile Strength and Host Response Towards Different Implant Materials Used for} Augmentation of Fascial Repair in a Rat Model

\section{F. Spelzini, M. Konstantinovic, J. Deprest}

Università degli Studi di Milano

Introduction: There is a wide range of implant materials available for urogynecological surgery. There is still a lack of information regarding the host behaviour towards implanted materials and the mechanical consequences of integration processes.

Materials and Methods: Was analysed the behaviour of four different implant materials available on the market: two biologic materials $\left(\right.$ Pelvicol $^{\odot}$, Pelvisoft ${ }^{\odot}$ ), one synthetic material $\left(\right.$ VyproII $^{\odot}$ ) and a composite synthetic-biologic material (Pelvitex ${ }^{\odot}$ ). Implants were studied in an animal model for the fascial defect repair. Explants were analysed macroscopically and microscopically. 96 animals were operated and sacrificed at 7, 14, 30, 90 days.

Results: Biologic materials caused the mildest acute and chronic inflammatory reaction, the composite material showed a milder inflammation than the synthetic one and was related to the lowest grade of intraperitoneal adhesion. The tensile strength, was high for all the explants at 90 days. The materials with a synthetic component showed a higher grade of neovascularisation at the interface.

Conclusions: Synthetic materials are related to a more pronounced inflammatory reaction and a stronger fibrotic processes. Biologic materials induce milder inflammation and lower structural integration. The collagen coating, seems to improve the biocompatibility without compromise a strong mechanical integration.

\section{7}

\section{The Ultrasonographic Diagnosis of Pelvic Floor's Dysfunctions}

\section{A. Suprani, P. Busacchi, S. Presepi, D. De Aloysio \\ Department of Obstetrics and Gynaecology, University of Bologna, Italy}

Introduction: Aim of this study is to evaluate the effectiveness and the utility of introital ultrasound as an imaging diagnostic tool for women with cystocele.

Methods: 57 patients affected by cystocele and a control group of 15 women without prolapse underwent an urogynaecological examination and an introital ultrasound. U.S. parameters were: (1) H parameter (distance between the line crossing the lower margin of the pubic symphysis and the bladder's base), at rest (H1) and after Valsalva maneuver (H2); (2) C parameter (distance between the pubic symphysis and the bladder's neck) at rest (C1) and under strain (C2); (3) shift vectors IH1H2I and IC1-C2I (absolute descensus values of the bladder's base and neck); (4) cystocele's fascial defect (central, lateral or mixed).

Results: The results showed a correlation between the clinical prolapse degree and the detected U.S. measures. A U.S. diagnostic value has been assigned to the $\mathrm{H} 2$ parameter for the descensus of bladder's base and to the shift vector for the mobility of bladder's neck. Besides, the hypotheses of a correlation between a high degree of the cystocele and mixed fascial defects and a relationship between the hypermobility of bladder's neck and lateral and mixed fascial defects were confirmed.

Discussion: Introital ultrasound is a valid alternative to standard methods in the diagnosis of pelvic organs prolapse as it allows a reliable evaluation of pelvic floor's anatomical and functional integrity.

\section{Orthopaedic}

408

\section{Treatment Options in Spine Metastases of} Renal Cell Carcinoma

M. Cappuccio, S. Bandiera, A. Gasbarrini, L. Mirabile, G.B. Brodano, L. Boriani, S. Boriani

U.O. Ortopedia e Traumatologia, Chirurgia del Rachide, Ospedale Maggiore, Bologna

Introduction: Seventy-five patients with symptomatic spine metastases secondary to Renal Cell Carcinoma (RCC) were reviewed between 1974 and 2006.

Methods: The mean age was 58.6 years (min: 28; max: 79). The metastases was located in 44 cases in the lumbar spine, in 26 cases in the thoracic spine and in 5 cases in the cervical spine. Thirteen patients were submitted only to radiation therapy. Different procedure was done in the remainder cases: in 16 cases a palliative procedure, in 34 cases a curettage and in 12 cases a en bloc resection. Seventeen cases were operated on for neurologic dysfuction, the remainder only for the pain.

Results: All the cases were followed for at least 6 months (6-69 months, average: 18). The local recurrence percentage was 9 of 34 for curettage and 0 of 12 for en bloc resection. The mean survival was 7 months of the 13 patients, who were treated only with RTR, 16 months of patients submitted to palliative surgery, 22 months of patients submitted to curettage and 26 months of patients submitted to en bloc resection.

Discussion: The treatment of spine metastases of RCC is multidisciplinary: the eligibility of patients to en bloc resection both on the basis of general conditions and local tumor expansion is critical for the local control of the disease. If en bloc resection is not feasible, combination of SAE, complete intralesional excision, RTR, immunotherapy seem to be the most appropriate treatment.

\section{9}

\section{Treatment of Lower Limb Epiphyseal} Fractures

\section{L.M. Reggiani, M. Magnani, O. Donzelli \\ 8 Division of Pediatric Orthopedics and Traumatology, Istituti Ortopedici Rizzoli, Bologna, Italy}

Introduction: The epiphyseal fracture represent almost $30 \%$ of fractures; they are more frequently caused by indirect trauma and they 
are more common in males. Lesions involving phisys and epiphysis can be responsible for growth problems.

Methods: The most widely used classification is the one made by Salter and Harris who divide epiphyseal fractures into 5 kinds of lesions according to $\mathrm{X}$-ray images. The 5th kind is a compression lesion of the phisys, accompanied by a compromission of its vascularization, can be dignosed only retrospectively and can produce a growth problems. MRI, carried out within 10 days after lesion, can help to classify the fracture but it offers only a qualitative analysis. Technetium scintigraphy carried out after at least 6 months from the trauma can be useful to evaluate the qualitative and quantitative activity of the epiphyseal cartilage.

Results: The treatment of the epiphyseal fractures aims to anatomically reduce the fracture and to stabilyze it; in some cases it can be necessary to treat the possible deformities caused by the fractures.

Discussion: Because of the difficulty of evaluate the degree of the phisys lesion and the compromission of the vascularization, it is often difficult to predict, in an ealy phase, the evolution of this kind of fractures, using only X-ray and MRI images.

\section{0 \\ Flow-Chart for Treatment of Spinal Metastases}

\author{
L. Mirabile, A. Gasbarrini, S. Bandiera, M. Cappuccio, \\ G.B. Barbanti, L. Boriani, S. Boriani \\ Department Orthopedics and Traumatology, Maggiore \\ Hospital, Bologna, Italy
}

From 1996 to 2002 we treated 284 localizations of spinal metastases in 168 patients (108 males and 60 female). We studied preoperatively all the patients with standard X-rays and scintigraphy followed by CT scan or MRI. The average age at diagnosis was 56.6 years (range 14-89). Surgical indications are: instability of the spine, pain, paresis, life expectancy and local control of the disease. We found 22 different istotypes of metastases; kidneys, lung and breast were the most common primary sites $(61.2 \%)$. Treatment was: in 80 cases three-dimensional decompression of nervous structures, in 57 cases an intralesional aggressive curettage (debulking of the lesion), and in 19 cases it was possible to perform an en-bloc resection of the tumoral lesion. All cases were then treated with instrumental stabilization. 37 cases $(24 \%)$ was treated in emergency. The poor general condition of the patient, the aggressiveness of the tumoral growth, the sensitivity of the metastases to adjuvant therapy or the absent risk of pathological fractures prompted us to proceed with a non-surgical approach of the metastases in 12 cases. Survival depended on anatomic site of primary carcinoma, preoperative neurological deficit, extent of disease, number of vertebral bodies involved, tumoral location and age. Of all the patients treated 104 died at an average of 12 months (range 1-50) after surgery, and 41 patients are still alive with an average follow up of 21 months (ranges 6-66).

Conclusions: Spinal metastases are only apparently similar lesions but they are really different considering the large varieties of istotypes.

\section{1}

\section{Optimal Timing and Procedure of Osteosynthesis in Polytrauma Patients}

\author{
G. Restuccia, A. Faldini \\ I Clinica Ortopedica, Pisa, Italy
}

Introduction: High energy accidents in multi-traumatized patients can results in combined fractures of lower and upper extremities. Primary stabilization of long bone fractures is depending on concomitant injuries.

Methods: In this study we evaluate the criteria of treatment indications in polytrauma with poly fractures, including patients with organic lesions. We report the experience of the I Orthopaedic Clinic of Pisa in the treatment of polytrauma patients.

Results: We had very good results after external fixation in polytrauma injuries, but intramedullary nailing, its timepoint modulated by trauma severity, seems a safe and reliable procedure, particularly in shaft femur fractures.

Discussion: Operative treatment of combined fractures in multi-traumatized patients depends on the amount of tissue damage, combination of fracture site and concomitant injuries. These situations influence also the choise of osteosynthesis. The experience gained at the I Orthopaedic Clinic of the University of Pisa is based on using early bone stabilization in all polytrauma patients. Frequently we use external fixation for primary stabilization. It's a minimally invasive method, and it allows closed reduction and biological healing of fracture. Sometimes, in specific situations, the external fixation is converted in intramedullary nailing.

\section{2 \\ High Tibial Osteotomy and Arthroscopic Implantation of Autologous Chondrocytes for the Treatment of the Chondral Defects in the Varus Knee

\author{
F. Franceschi, G. Rizzello, U.G. Longo, L. Ruzzini, \\ R. Papalia, G. Vadalà, A. Marinozzi, E. Denaro \\ Department of Orthopaedics, University Campus \\ Bio-Medico, Roma, Italy
}

Introduction: The aim of this study was to determine the outcome of patients with varus malalignment who underwent an arthroscopic implantation of autologous chondrocytes and a high tibial osteotomy.

Methods: We performed 9 arthroscopic implantations of autologous chondrocytes in conjunction with an opening wedge osteotomy on the medial side of the proximal tibia in patients with chondral defects of the medial tibial plateau in varus knee. Each patient was evaluated for clinical history, clinical examination, conventional radiographs, MRI and arthroscopy, pre and postoperative IKCD, pre and postoperative Lysholm, pre and postoperative Tegner score and pre and postoperative VAS.

Results: The Lysholm Score, IKCD, Tegner score used for evaluation showed a statistically significant improvement from preoperative average rating to postoperative average score. 
Discussion: Treatment of chondral lesions involving the articular surface of the knee remains a formidable therapeutic challenge because articulate cartilage has limited capacity for regeneration. HTO alone and conventional treatments that abrade or penetrate the subchondral bone known to produce fibrocartilaginous repair. Only the autologous chondrocyte implantation and the transplantation of osteochondral grafts provides adequate hyaline articular cartilage.

413

\section{Histologic Changes in Human Macroscopic Intact Supraspinatus Tendon as Pathogenic Precursors of Rotator Cuff Tears}

\author{
F. Franceschi, G. Rizzello, U.G. Longo, L. Ruzzini, \\ R. Papalia, A. Marinozzi, E. Denaro \\ Department of Orthopaedics, University Campus \\ Bio-Medico, Roma, Italy
}

Introduction: The aim of this study was to analyze the morphological features of the human surgical specimens of normal supraspinatus tendon from patients with rotator cuff tears.

Methods: 41 subjects were recruited for the study. 20 subjects (group 1) sustained a rotator cuff tear and proceeded arthroscopic repair of the lesion. 21 subjects (group 2) underwent surgery due to glenohumeral instability. During surgery, under arthroscopic control, a full thickness supraspinatus tendon biopsy was harvested in the middle portion of the tendon. All slices were processed for histological analysis.

Results: On surgical specimens of supraspinatus tendon from patients with rotator cuff tears, but not from patients with instability, we found increased preponderance of hyaline degeneration, fibrocartilaginous or chondroid metaplasia, calcification, lipoid degeneration, mucoid or myxoid. Degenerative changes were more evident on the articular side of the rotator cuff.

Discussion: The present study provides a description of the histological architecture of human surgical specimens of normal supraspinatus tendon from patients with rotator cuff tears. Preexisting degenerative change in the supraspinatus tendon seems to be the main cause of rotator cuff tears.

\section{4}

\section{Should the Long Head of the Biceps Tendon be Detached After Tenodesis? Outcome Analysis of Two Different Techniques}

\author{
F. Franceschi, G. Rizzello, U.G. Longo, L. Ruzzini, \\ R. Papalia, M. Ippolito, A. Marinozzi, E. Denaro \\ Department of Orthopaedics, University Campus \\ Bio-Medico, Roma, Italy
}

Introduction: The aim of this study was to determine whether or not detaching the biceps tendon from the glenoid after the tenodesis results in improved outcome.
Methods: From 1999 and 2001, 22 patients had an arthroscopic rotator cuff repair and associated biceps tendon lesions that were repaired with two new arthroscopic techniques of tenodesis incorporating the biceps tendon in the rotator cuff suture. Patients were randomized into one of two groups: tenodesis without tenotomy (group 1) and tenodesis with tenotomy (group 2). Preoperative and postoperative function were assessed by means of modified UCLA rating scale and shoulder ROM. The mean follow up period was 47.2 months (range 36-59).

Results: The UCLA rating system used for evaluation showed a statistically significant improvement from preoperative to postoperative average score in the two groups. No statistically significant difference in UCLA scores was found when comparing repairs performed with or without tenotomy.

Discussion: This study suggests that there is no difference between detaching and not detaching from the glenoid the long head of the biceps after including it in the rotator cuff suture. These new arthroscopic techniques of tenodesis may be an acceptable intervention for the treatment of long head biceps pathology.

415

\section{Single-Row vs. Double-Row: Outcome Evaluation by MR-Arthrography After 2 Years of Follow-Up}

F. Franceschi, G. Rizzello, U.G. Longo, L. Ruzzini, R. Papalia, A. Marinozzi, E. Denaro

Department of Orthopaedics, University Campus Bio-Medico, Roma, Italy

Introduction: The purpose of the present study was to evaluate the outcome of arthroscopic repair in two focused groups of patients with large and massive rotator cuff tears treated with single and double row arthroscopic technique and to correlate these results with the integrity of the cuff as determined by clinic and MR-Arthrography evaluation.

Methods: 45 arthroscopic rotator cuff repairs were randomized into one of two groups: single row and double row suture anchor repair technique. All shoulders had pre and postoperative evaluation by a modified UCLA and a postoperative assessment by the use of MR-Arthrography scans at 1 year from surgery.

Results: We did not find a statistically significant difference in total UCLA score when comparing single row and double row. MR-Arthrography examination at 2 years of follow up showed that double row suture anchor technique provides a better structural outcome (6 partial-thickness defects and 1 full-thickness defect) if compared with single row suture anchor repair ( 9 partial-thickness defects and 2 full-thickness defect) $(\mathrm{p}<0.05)$.

Discussion: Restoring the anatomical footprint is appealing, but in our work we did not found a better clinical outcome in patients underwent double row suture anchor technique vs. single row suture anchor repair. 


\section{6}

Effect of a Mucolytic Agent on Collagen Fibres: An Optical and Polarized Light Histological Study

\author{
A. Di Martino, V. Costa, V. Denaro \\ Department of Orthopaedics, University Campus \\ Bio-Medico, Roma, Italy
}

Introduction: In this study the authors consider the effect of a mucolytic agent on collagen fibres using an experimental model of fibrosis.

Materials and Methods: We injected a mucolytic agent in the context of the Achilles' tendon of 7 sheep. Animals were grouped on the base of the time of sacrifice: (1) Group I: time 0; (2) Group II: killed at 3 weeks time; (3) Group III: killed after 6 weeks; (4) Sham control group. All the specimens were retrieved and subjected to standard and polarized light analysis histological analysis.

Results: In Group I it is possible to observe absence of histological alterations of tendineal fibres of the animals, while it developed after 3 weeks, time in which an active process of mesenchimal proliferation infiltrates collagen fibres substituting them. This observation was supported by the polarized light analysis. These structural changes interestingly resolved within 6 weeks from the inoculation, leaving a normal tendineal structure.

Discussion: We studied the effect of a mucolytic agent on collagen fibres and observed its fibrinolytic effect which modifies tendineal structure, resulting in a cellular infiltration. Mucolytic agents induce the breakdown of disulphuric bonds bridging the mucopolisaccaride's molecules. We postulate it could exert a similar effect on collagen fibres with breakdown of disulphuric bonds between polypeptidic chains.

\section{7}

\section{Effect of Electromagnetic Fields Generated by Titanium Alloys Used in Spinal Fusion on Human Osteoblast Cultures}

\author{
V. Denaro, N. Papapietro, G. Rizzello, S.A. Barnaba, \\ G. Vadalà, L. Ruzzini \\ Department of Orthopaedics, University Campus \\ Bio-Medico, Roma, Italy
}

Introduction: The aim of this study was to verify if galvanic current and micro-electromagnetic fields (ELF) generated by Titanium implants could be involved in osteolysis around spinal instrumentation.

Methods: We made a galvanic cell using Titanium screws and bars prelevated from one of the patients which presented periprosthetic osteolysis and we calculated the current values that generate in vivo. After that we calculated the entity of ELF. We prepared human osteoblasts cultures initiated from femoral heads. Micro-ELF were applied to the cultures and we considered cell growth and alkaline phosphatase (ALP) expression.

Results: We found that human osteoblast cultures exposed to micro-ELF, had lower rate of growth and a decreased ALP activity if compared with control cultures.
Discussion: Since many years Titanium bars, plates, and screws are used in spine fusion to achieve stability of an unstable vertebral segment. Failure causes are not yet completely known. Our work shows that the electromagnetic fields generated by the coupling of Titanium alloys have significant effect on the osteoblast growth and differentiation reducing cell number and ALP expression. This modification can increase peri-prosthetic osteolysis and can cause failure of spinal instrumentation.

\section{8}

Shoulder Ganglion Cyst with Suprascapular
Nerve Compression: Arthroscopic Treatment

\author{
G. Rizzello, F. Franceschi, F. Leonardi, C. Amato, \\ U.G. Longo, V. Denaro \\ Department of Orthopaedics, University Campus \\ Bio-Medico, Roma, Italy
}

Introduction: Para-labral glenoideal ganglion cysts can be responsible for suprascapular nerve compression. We describe our experience with arthroscopic treatment of shoulder ganglion cysts with suprascapular nerve compression.

Methods: Three female patients were referred to our institution for counselling affected by shoulder pain and weakness. Electromyography showed partial denervation of the supraspinatus muscle with decreased motor conduction rate. MRI showed the presence of a ganglion cyst below the suprascapularis muscle at the level of the incisurae of the scapula. Through a probe into the labral defect, cyst decompression was performed directly from the joint, then labrum was repaired.

Results: At 4-6 months follow-up, patients recovered from symptoms. Electromyography showed absolute recovery from preoperative nerve conduction defects, and MRI showed absence (and no recurrence) of the ganglion cyst.

Discussion: Suprascapular nerve compression by a ganglion cyst is a rare cause of a painful shoulder. Electromyography allows for nerve damage localization and entity. MRI is quintessential to show the cyst and recognize labral lesions. Shoulder arthroscopy can be useful in cyst decompression and labral lesions suturing, and represents the gold standard for contemporary treatment of this disease.

\section{9 \\ Treatment of Hallux Valgus Associated with Luxation of the Second Metatarsal- Phalangeal \\ F. Cancilleri, G. Rizzello, A. Marinozzi, M. Ippolito, \\ U.G. Longo, E. Denaro \\ Department of Orthopaedics, University Campus \\ Bio-Medico, Roma, Italy}

Introduction: We suggest an algorithm, based on our experience, for treatment of hallux valgus associated with luxation of the second metatarsal-phalangeal (MF). 
Methods: In Haddad first and second stages a reducible griffe of second toe proximal interphalangeal is frequentely observed, in these cases we use the technique of long flexor tenotomy and dorsal transposition. In third and fourth stages, when subluxation is greater than $50 \%$ a careful planning is suggested. When luxation is easily reducible and there is not painful plantar overload, long flexor tenotomy and dorsal transposition associated to the correction of hammer deformity is recommend. A stabilization period of 18-20 days, with $\mathrm{K}$. wire, is required. When luxation isn't reducible and a painful plantar overload is associated, we advice the use of hedge-shaped dorsal osteotomy, according to Gauthier, combined with long flexor tenotomy and hammer deformity correction. Weil osteotomy is reserved both to severe dorsal luxation of MF and luxations associated to rheumatoid arthritis.

Results: We have observed a clear improvement in AOFAS score, from 32.9 on a preoperative overage to 81.8 on a 6 months postoperative follow-up.

Discussion: We have used this algorithm for the last two years, obtaining successful results.

\section{0 \\ Are Intervertebral Discs of Different Level the Same? Morphological Study in Cynomolgus Monkey (Macaca Fascicularis)}

\author{
U.G. Longo, F. Franceschi, G. Rizzello, \\ A. Marinozzi, R. Papalia, F. Forriol, E. Denaro \\ Department of Orthopaedics, University Campus \\ Bio-Medico, Roma, Italy
}

Introduction: The aim was to analyze the morphological differences of the intervertebral disc and endplates at different levels.

Methods: Forty-five vertebral motion segments were obtained from the spine of nine 3-4-year-old cynomolgus monkeys (Macaca fascicularis). From every spine, five discs were sectioned (C5-C6, T3-T4, T9-T10, L2-L3, L4-L5). In all the groups, tissue samples were collected and sections were stained with Masson's trichrome, Safranine-O and van Gieson's connective tissue stain to analyze the intervertebral discs. Immunohistochemistry was performed, to detect collagens I and II.

Results: There were no differences existing in annular fibers anchoring on the endplate between discs of different levels. There were no differences between discs of different levels in the collagen I and II localization. The height of the discs varied along the spine. The smallest value was measured in T3-T4, with a larger increase caudally than cranially. The highest value was measured in L2-L3. A cervical disc was $55 \%$ the height of a lumbar one. The endplate height increased along the length of the spine. The inferior EP was always higher than the superior.

Discussion: The study provides a detailed structural characterization of the intervertebral disc and may be useful for further investigations on the disc degeneration process.

\section{1}

\section{Bone Substitution: Osteoconduction of a New Biomimetic Hydroxyapatite Doped with Magnesium (Mg-HA)}

\author{
G. Logroscino, E. Pola, M. Venosa, F. Visci, L. Proietti, \\ G. Maccauro, R. Bedini ${ }^{1}$, A. Tampieri \\ Department of Orthopaedics, University Campus \\ Bio-Medico, 'Department of Orthopaedic and \\ Traumatology, Catholic University of Rome, Rome, Italy
}

Introduction: Substitutes are highly requested in orthopaedic surgery, neurosurgery and otorinolaringoiatric surgery. The problems related to autologous, homologous and heterologous graft increase the research of new sinthetical materials reproducible on large scale. Hydroxyapatite showed to be one of the most effective biomaterials for bone substitution. When doped in specific sites with different ions HA may be more osteoconductive.

Methods: A new biomimetic HA obtained by doping HA with magnesium ions was made to realize a material more similar to natural bone. Granular samples of Mg-HA were tested in living bone in vivo (NZW rabbits) and compared with traditional granular HA. Macroscopic, radiographic, light microscopic, histomorphometric and SEM analysis were periodically performed up to 12 weeks.

Results: Both the biomaterials demonstrate optimal bone apposition, biocompatibility and osteoconductivity. Mg-HA had more osteoconduction and biodegradation than traditional HA.

Conclusion: This study demonstrates the effectiveness of $\mathrm{Mg}-$ $\mathrm{HA}$ as bone substitute in orthopaedic surgery. A long term study (40 weeks) is on course to assess the total degree of resorption and bone substitution of the material.

\section{2}

\section{Clinic and Ecographic Study of Rotator Cuff in Antegrade Intramedullary Nail}

\author{
S. Cerciello, F. Visci, M. Venosa, G. Logroscino, \\ G. Maccauro, T. Nizegorodcew
}

Department of Orthopaedics, University Campus Bio-Medico, Roma, Italy

Introduction: Antegrade intramedullary nail is one of the possible treatments of humeral shaft fracture.

Results: Whether in case of post-operative functional recovery or in case of biological healing of fracture, are actually analyzed and valued in Literature.

Aim: The aim of this study is to estimate the possible damage of rotator cuff after antegrade intramedullary nail.

Methods: From May 2002 to December 2004, 29 patient with humeral shaft fracture have been operated with UHN; 10 (7 men and 3 women) have been followed with an average follow-up of 27.9 months; all fractures were traumatic except 1 case that was a pathologic fracture (bone metastasis). Clinical evaluation was performed with Constant Score; the radiographic assessment was executed by 3 projection (neutral, internal and external rotation); the tendon of $\mathrm{m}$. sovraspinatous was evaluated by echografic examination. 
Discussion: Healing of fractures was obtained after 2 months from surgery in all cases; Constant Score's average was 78.5. In only 1 case we have had impingment sindrom originated by excessive length of the nail that was resolved after the nail removal. In 7 cases the tendon of $\mathrm{m}$. sovraspinatous ecographic exam was normal. In 2 cases a signicative iperecogen area, that probably was a scare, was found. We have never observed a significant damage of the sovraspinatous tendon in all its thickness.

Conclusion: The results of our study show that antegrade intramedullary nail for humeral shaft fractures is a reliable method that allow good results based on clinical and instrumental point of view. Damage of (truncated at 250 words).

423

\section{Computer-Assisted Surgery in Knee Prosthesis}

\section{A. Spinarelli, B. Moretti, V. Pesce, S. Patella, L. Moretti, V. Patella \\ 2nd Department of Orthopedics and Trauma, Bari University, Italy}

Introduction: Computer-assisted surgery is becoming more and more largely used in orthopaedic surgery.

Methods and Results: The choice of a prosthetic system is usually justified by biological and mechanical reasons even though in daily practice this choice is often due both to experience and to the 'philosophical' choice of the surgeon one without taking into consideration the bibliographic evidence. These claims become more significant in the case that they refer to controversial as well as still debatable issues. The surgeon who approaches computer-assisted surgery has to know the traditional technique and the pitfalls of CAOS so that verify step by step what is going to do. There's the necessity to know the traditional technique and its limits; absolute necessity to know standard technique and its limits. In the beginning is predictable to use navigation as parameter of validation of standard technique (learning curve) and not to perform surgery.

Discussion: The aim of this study is to consider CAS correlated problems, so that to underline the importance of a correct approach to the surgery with the purpose to avoid, as far as possible, worse consequences often of those described in the standard technique.

\section{Maxillofacial Surgery}

\section{4 \\ 'Orbito-Maxillary Advancement in Craniofacial Dysostoses'}

\author{
A. Cassoni, T.M. Marianetti, V. Terenzi \\ Chirurgia Maxillo-Facciale, Policlinico Umberto I, Roma, Italy
}

Introduction: Since first advocated by Tessier for treating the craniofacial dysostoses, such as Crouzon and Apert syndrome, the Le Fort III operation has been an integral component of the process of normalizing facial appearance in affected patients. Subsequently, distraction techniques have been applied to the upper midface. Potential advantages of LF III distraction are: the possibility to obtain more stable advancement and decreased relapse; no need for bone grafts and intermaxillary fixation; improved airway results; less morbidity and decreased cost. On the other side, disadvantages are secondary procedures needed to remove the devices and cheek scars.

Materials and Methods: From 1997 at today, at the Department of Maxillo-Facial Surgery, Policlinico Umberto I, 10 cases have been treated in which internal distraction system were used for midface advancement following Le Fort III osteotomies for the treatment of syndromic craniosynostosis. Bilateral bured subcutaneous distracters were anchorated anteriorly to the lateral orbit rim and body of the zygoma and posteriorly to the temporal bone. Distraction was carried out at the rate of $1 \mathrm{~mm}$ for day, beginning on the seventh post-operative day after intra-operative expansion.

Results: Complications, such as infections or intracranial migration of pins, were experienced in no cases. Aestethic and functional results were satisfactory in all cases.

Discussion: Distraction technique has demonstrated to be a valid surgical method to obtain orbito-maxillary advancement also when an advancement of more then $10 \mathrm{~mm}$ is required.

\section{5}

\section{Craniofacial Advancement: Comparison between External and Internal Distraction Devices}

\section{G. Gasparini, G. Longobardi, R. Boniello, A. Di Petrillo \\ Universita' Cattolica del Sacro Cuore (sede di Roma) Unità Operativa di Chirurgia Maxillo-Facciale}

Objective: To compare external and internal craniofacial distraction devices used for craniofacial advancement.

Patients and Methods: We analyzed 4 patients affected with craniosynostosis syndromes. Surgical complications are reported together with the possible effects of osteogenic distraction on pain, sleeping difficulty, speech, eating problems, disturbance of recreational activities and sensory alterations.

Results: The external distraction device had a greater impact on the psychological well-being of patients and daily activities. In one 
case, the pins of the external device were accidentally placed on a cranial suture, causing fracture during surgery. However the external device is advantageous in terms of control of the vector of distraction, which is modifiable as needed by the operator. Internal distraction devices are better tolerated by patients from a psychological point of view and have a more favorable impact on daily activities. However they have a greater complication rate since the vector of distraction is difficult to control. In fact the only way to correct vector distraction is through additional surgical intervention. In one case decubitus of the internal distraction device occurred, with subsequent loss of liquor, necessitating removal of the device.

Conclusions: Comparable efficacy of the two devices was seen regarding the distraction osteogenesis and histogenesis. However, external devices offer better results in craniofacial advancement.

\section{6}

\section{Treatment of Severe Maxillary Atrophy by Means of Prefabricated Free Vascularized Fibula Flap}

\author{
R. Boniello, G. Longobardi, G. Gasparini, M. Clementini
}

Universita' Cattolica del Sacro Cuore (sede di Roma) Unità

Operativa di Chirurgia Maxillo-Facciale

Objective: Treatment of severe maxillary atrophy despite complex major surgery often ends up with an unsatisfactory result. This paper presents the augmentation of the maxilla with a prefabricated free vascularized fibula.

Methods: The key points of this treatment are (I) preoperative planning and fabrication of the maxillary template by means of TC scans (II) prefabrication of the fibula and performing of a 'vestibuloplasty' using a skin graft; (III) reconstruction of the maxilla using the prefabricated fibula as free vascularized flap.

Results: The reconstructions with the fibula flaps were fast and successful. This method granted an excellent remodelling of the free microvascular flap. In this way an ideal adjustment on the receiving site and a very satisfactory facial aesthetic immediately after the surgical treatment have been obtained.

Conclusions: We highly recommend performing a surgical simulation using a craniofacial model of the patient's anatomy created using CT image data. The procedure provides almost ideal maxillary and mandibular contours.

\section{7}

\section{Osteonecrosis of the Jaws in Oncologic Patients Due to Adverse Effect of Biphosphonates: Our Experience}

\author{
G. Longobardi, G. Gasparini, R. Boniello
}

Universita' Cattolica del Sacro Cuore (sede di Roma) Unità Operativa di Chirurgia Maxillo-Facciale

Objectives: Biphosphonates make up a class of pharmaceuticals which have been used in treating bone pathologies for over 15 years.
They have mainly been used in cases of osteoporosis due to their ability to inhibit bone reabsorption. In time however, they have steadily been more frequently used for oncologic pathologies like Paget's Disease, multiple myeloma, malignant hypercalcemia and they are now part of the therapeutic protocol for oncologic patients with bone metastasis. At the beginning of this century, literature began to report some cases of maxillary osteonecrosis lesions in oncologic patients undergoing therapy with the above mentioned drugs.

Patients and Methods: In the present work the authors report and discuss the clinical aspects and therapy over 21 clinical cases.

Results: Despite it is suggested to not make surgery on osteonecrosis lesion by biphosphonates to avoid clinical unsucces, a lot of patient treated with surgery by the authors showed resolution of osteonecrosis.

Conclusion: The surgical treatment showed in the most of the cases a resolution of osteonecrosis. The suspension of biphosphonates therapy and the general clinical conditions seem to be essential for the success of the treatments.

\section{8}

\section{The External Cantoplastic in the Reconstruction of Palpebral Inner and Achieved Malformations.}

\section{S. Fontana1, P. Amaddeo, A. Barbaglio, F. Faldi}

U.S.C. Chirurgia Maxillo-Facciale Ospedali Riuniti di Bergamo, ${ }^{1}$ Scuola di specializzazione in Chirurgia MaxilloFacciale Università degli Studi Milano, Bicocca

Introduction: The upper edge of the lower eyelid, usually, is tangential to the scleral-corneal edge; the exposion of a share of the sclera, between the scleral-corneal edge and the eyelid edge, is known as scleral-show. The scleral-show has to be differentiated both from ectropion, because in this case the upper edge of eyelid is upset and sticks to the lower eyelid, or ocular-eyelid diastase, in this case there is a loss of touch between the free edge of the eyelid and the globe without ectropion, but mostly, without epiphora, because there is no loss of contact between the eyelid and conjunctive. The scleral-show has different causes: congenital, hereditary, hyatrogena, senile and traumatic.

Methods: Four cases of scleral-show are reported by the authors, each of them related to a specific cause. They all have been treated throughout external cantoplasty. The first case is a correction of exposition after basocellular carcinoma recurrence excision. In this particular situation the repositioning of the eyelid, secondary to a relapse removal, is associated with a frontal flap. Second and third cases figure out a blefaroplasty complication knows as 'oeil round'. The last case is a coloboma congenital defect.

Results: In all the case we have obtained a correct palpebral lock, avoiding corneal exposition and ulcer. The cantoplastic with lateral cantolysis, allows to achieve the correction of a scleral-show no matter the cause and origin.

Discussion: It's a relatively simple and quick technique, you can also perform on local anaesthesia, that warrants a good suspension of the lower eyelid, avoiding palpebral fissure anomalies. 


\section{9}

\section{Cranio-Facial Fractures: Diagnostic Procedures, Therapeutic Protocols, Short and Long-Term Results}

\author{
P. Formillo, S. Marelli, R. Monteverdi, A.B. Gianni \\ Istituto Ortopedico Galeazzi, Milano, Italy
}

Introduction: Cranio-facial traumas involve the region between the cranium and the face, which includes the anterior cranial base, the naso-ethmoid-orbital region and the frontal sinuses. Their empty structure accounts for the comminution of the midface and the anterior cranial base which result from direct high-speed traumas and difficultly lead to restitutio ad integrum.

Methods: 8 patients with cranio-facial traumas were observed at Galeazzi Ortopedic Institute of Milan, Italy, Department of Maxillofacial Surgery, from January 2002 to December 2004. All patients were males and the mean age was 32.9 years (range 14-65 years). In 7 cases was involved the frontal sinus, in 4 cases the ethmoidal bone, in 7 cases the nasal bones, and in all cases the orbit.

Results: Good functional end esthetical results were obtained in all patients. None of the endocranic structures underwent infection, nor did any mucocele form at distance from the surgical procedure. We observed 3 post-operative complications: a bifrontal access bone flap infection, a case of epiphora due to secondary obstruction of the naso-lacrimal ducts after laceration and one case of pneumocephalus.

Discussion: Bone comminution doesn't account for the use of residual bone fragments by means of rigid fixation, requiring autologous bone grafts, harvested from calvarial bone, or alloplastic materials such as porous polyethylene and hydroxyapatite. The study of an adequate operative strategy, other than correct surgical procedures is essential. Surgical timing is therefore mandatory, in order to achieve a complete morpho-functional restoration of the anatomical structures.

\section{0}

\section{Three-Dimensional Mandibular Movements at Maximum Mouth Opening in Patients Rehabilitated After a Mandibular Condyle Fracture. Preliminary Report}

\author{
P. Formillo, R. Monteverdi, A.B. Gianni, C. Sforza², \\ G.M. Tartaglia'2, N. Lovecchio ${ }^{2}$, A. Ugolini2 ${ }^{2}$ V.F. Ferrario ${ }^{2}$ \\ Istituto Ortopedico Galeazzi, Milano, Italy
}

Introduction: The aim of this study was to quantitatively assess the percentage contribution of rotation and translation movements of the mandible at maximum mouth opening in patients rehabilitated after a mandibular condyle fracture.

Methods: Free, habitual movements of mouth opening were recorded in 9 patients aged $18-58$ years using an optoelectronic 3-D motion analyzer. Data were compared to those collected in 27 healthy subjects. For each subject, the mandibular movements at the interincisor point (occlusal plane) were reconstructed, and, using suitable mathematical algorithms, divided into their rotation and gliding components. The relative contribution of the two components to the total movement was calculated for each frame of motion. In particular, the situation at maximum opening was assessed.

Results: At maximum mouth opening, on average, the patients had a similar total displacement of the mandibular interincisor point ( $86 \%$ of the reference value, $p>0.05$, Student's $t$ ), with a somewhat reduced vertical displacement $(84 \%$ of the reference value, $\mathrm{p}=0.012$ ). The percentage of mandibular movement explained by rotation at maximum mouth opening was significantly larger in the patients $(82 \%)$ than in the reference subjects $(77 \%, \mathrm{p}=0.005)$.

Discussion: Overall, the patients had a good recovery as far as the total mandibular movement was concerned, but a modification in the rotation and translation components of mouth opening.

431

\section{Custon Made Implants to Cranio-Facial Defect Repair}

\section{Gervasoni ${ }^{1,2}$, S. Valsecchi ${ }^{2}$, M. Meroni ${ }^{2}$, E. Balzaretti ${ }^{2}$, P. Nannei ${ }^{2}$, L. Colombo ${ }^{2}$, A. Di Francesco ${ }^{2}$ \\ ${ }^{1}$ University school of Maxillofacial surgery, University of Milano Bicocca, Maxillofacial Unit Sant'Gerardo Hospital Monza, ${ }^{2}$ Maxillofacial Unit Sant'Anna Hospital Como}

Introduction: Autologous bone represent the gold standard in reconstruction of bony defects in the cranio-maxillofacial region. Donor site morbidity, shaping difficulties with longer surgical times and instability of the final result determined the development of alloplastic materials. In our experience the most commonly used alloplastic materials are porous hydroxyapatite or polyethylene due to their bone bonding behaviour and to the ease of their shaping. Technically, it is easy to work with; they can be carved, contoured, adapted, and fixated to obtain a precise three-dimensional construct. In addition, these biomaterials are strong substance that does not resorb or degenerate. It demonstrates long-term stability, high tensile strength, resistance to stress and fatigue, and a virtual lack of surrounding soft-tissue reaction.

Materials and Methods: Between January 2002 to April 2006, 11 patient with post-traumatic or postoperative cranial defect were treated using custom made implants ( 8 polyethylene and 3 porous hydroxyapatite).

Results: In our experience, the use of custom-made device provides to obtain an implant exactly matching the bone defect in shape and thickness, obtaining good aesthetic results, reduction of surgical times with no evidence of resorption, migration or construct failure during follow up.

Discussion: Polyethylene and porous hydroxyapatite custom made device are a suitable choice to traditional autologous bone graft because the lack of donor site morbidity and reduction of surgical time. In addition the porous structure seem to allow substantial vascular and soft-tissue ingrowth into the cranial defect with an excellent stability of the reconstruction. 


\section{2}

\section{Orbital-Maxillary Reconstruction Using Microvascular Free-Flaps}

\author{
S. Mazzoni, A. Bianchi, C. Marchetti \\ S. Orsola, Bologna, Italy
}

Introduction: The orbito-maxillary complex is an intricate three-dimensional entity with important functional and aesthetic purpose. Reconstruction of such defects should (1) support the orbital contents or fill the orbital cavity in cases of exenteration, (2) obliterate any communication between the orbit, nasopharynx, oral cavity, (3) achieve facial symmetry and good aesthetic result. We report our experience using microvascular free-flaps for the restoration of orbito-maxillectomy defects.

Methods: From January 2003 to April 2006, 17 patients underwent reconstruction using microvascular free-flaps for partial/total maxillectomy defect and/or orbital defect. In 11 cases ALT free-flap was performed; in 4 patients we harvested a fibula osteocutaneous free-flap, in 1 case a rectus abdominis free-flap and in 1 patient a forearm free-flap.

Results: One total flap failure was reported owing to vascular deficiency. Three partial necrosis were noticed: loss of the iliac graft used for the orbital floor reconstruction related to infection in one case; loss of skin paddle with an oral-nasal transitory fistula in 2 patients. One patient died for local recurrence and one for systemic disease during the follow-up (from 2 to 28 months).

Discussion: Patients with midface defects involving the maxilla and the orbit can undergo free tissue transfer and have satisfactory restoration of the complex three-dimensional anatomy with stable functional and aesthetic results. Free flap reconstruction creates a permanent separation of the oral cavity from the sinonasal/orbital cavity in a single stage procedure with the restoration of masticatory and phonatory function. Free flap reconstruction also provides an appreciable cosmetic results, improving patients' overall (truncated at 250 words).

\section{3}

Reconstruction of Craniofacial Defects with Alloplastic Materials: Our Experience

\author{
M. Morabito ${ }^{1}$, S. Paulli ${ }^{1}$, D. Frattini ${ }^{2}$, A. Griner ${ }^{3}$ \\ ${ }^{1}$ Maxillo-Facial Surgery, U.O.C. Legnano, ${ }^{2} \mathrm{Head}$ of Maxillo- \\ Facial Surgery, U.O.C. Legnano, ${ }^{3}$ Neurosurgery Legnano, \\ Italy
}

Introduction: The modern surgical techniques allows, in the treatment of the poli-traumatized patient, an useful access to the most important anatomical structures and when necessary an immediate reconstruction of the skeletal defects. The primary aim is to restore the normal anatomy and the function of the cranio-facial district. Often the aesthetic defect, of the primary reconstruction, may result insufficient and inadeguate for the patient 's public relationship life.

Methods: The authors describe the use of three different alloplastic implants to repair craniofacial defects. Various materials has been proposed for cranial reconstruction. The ideal material should be strong, to protect the underlying structures and maintain its desired form, biocompatible, easily shaped at the operating table and inexpensive. The bone defects were the result of craniectomy for infections, brain edema, trauma, neurosurgery such as subdural hemorrage, cerebrovascular aneurysms or brain tumor surgery.

Results: The authors used in the closure of skull defects different materials such as porous polyethylene, CMW bone cements and hydroxyapatite to cover little, medium and large deficits with good aesthetic results and no implant related complications.

Discussion: A variety of materials have been proposed and used for aesthetic and reconstructive surgery, but no one material is universally accepted as successful. The aim of this clinical study was to emphasize the effectiveness of computer-generated models and the safety of implantable biomaterials, which has become integral part in modern cranio-maxillo-facial surgery.

\section{4}

\section{Demolition and Restoration in Cranio- Maxillo-Facial Oncology: The Patient and Surgeon Need}

\author{
S. Paulli', M. Morabito ${ }^{1}$, D. Frattini ${ }^{1}$, \\ S.S. di Spilimbergo ${ }^{2}$ \\ ${ }^{1}$ Maxillo-Facial Surgery Unit, Hospital Legnano, ${ }^{2}$ Maxillo- \\ Facial Surgery Unit, Hospital San Bortolo Vicenza, Italy
}

Introduction: The authors present few cases of maxillo-facial carcinoma in which the restoration was made with traditional flaps or freeflaps according to the patient health status and her/his expectations.

Methods: The authors treated benign and malignant neoplasm of the cervico-facial district such as oral spinocellular carcinomas, odontogenic cysts of the jaws and orbital neoplasm. The reconstruction was made use with axial flaps, submentalis and 'naso-genieno', and revascolarized free flaps (antero-lateral thigh flap; fibula; iliac crest; chienese; lateral arm flap; rectus abdominis myo-cutaneous flap).

Results and Discussion: The modern methodics of micovascular restoration of the cranio-facial district provide different rehabilitative solutions; in the common aim of the morpho-functional re-establishment, our choice was made with the need to satisfy so much the standards of a radical oncology as at same time to offer an efficacious restoration. During our experience, we try to achieve the most appropriate reconstructive technique: we took in consideration the extention of the resection and the patient health status and even more the patient own will and expectations.

\section{5 \\ Oral Rehabilitation Using Bony Free Flap with Endosseous Implants}

\author{
G. Nicolai, B. Lorè \\ Chirurgia Maxillo-Facciale, Policlinico di Tor Vergata, Roma, \\ Italy
}

Introduction: Prosthetic implant rehabilitation after maxillary and mandibular reconstruction using bone free flaps represents the less 
invasive method for the replacement of one or more dental elements; such method is however influenced by the skeletal bases conditions.

Methods: In our department 115 reconstructions have been performed with bony free flap because of maxillo-mandibular deficit. Of such reconstructions 76 were made with fibula free flap; 31 with iliac crest, 7 with scapula and big dorsal muscle and 1 with radial + radio. The Authors prefer to insert the implants after the bony reconstructions with free flap and not simultaneously.

Results: The $30 \%$ of the patients have been rehabilitated with prosthetic implant. The rehabilitations have been successful; however they have been influenced by bone quality, characteristics of periimplant tissue and CT-RT treatments. To solve the peri-implant tissue problem, the Authors prefer to use palatal mucosa grafts around the implants.

Discussions: The authors want to point out that the use of bone free flap in maxillo-mandibular reconstruction has revolutionized the treatment of the cases with complex substance losses. The success of the rehabilitation is influenced by the characteristics of the bone, as well as by the timing of plant insertion, by the peri-implant tissue and the possible treatment with CT-RT in oncological patients.

\section{6}

Traumas of the Middle and Superior Third of the Face: Reconstructive Dilemmas

\author{
A. Saltarel, E. Ventucci, A. Battisti, G. Nicolai \\ Chirurgia Maxillo-Facciale, Policlinico di Tor Vergata, \\ Roma, Italy
}

Introduction: Injuries to the facial bones, orbits and adjacent soft tissue structures are common. Orbital injuries can occur alone but are most often associated with other injuries, such as malar, frontal, naso-ethmoidal, Le Fort II-III fractures and skull fractures. Frequent causes of these injuries are attributed to automobile accidents, physical altercations, gunshot wounds, home accidents, athletic injuries, work injuries and other injuries. The management of orbital injuries is one of the most interesting and difficult areas in facial trauma. Correct diagnosis and treatment of these fractures is extremely important to avoid functional and aesthetical outcomes.

Methods: In this work authors present their experience in management of complex facial trauma. A 5-year retrospective clinical and epidemiologic study evaluated the patients treated for maxillo-facial fractures from 2000 to 2005 at the departments of maxillo-facial surgery of the University of Rome 'La Sapienza' and 'Tor Vergata'.

Results: The goal of treatment of these fractures is to restore normal anatomy and therefore normal function of the craniofacial complex. The authors emphasize how the early treatment of craniofacial injuries is important to achieve the better functional and cosmetic results.

Discussion: It is now generally accepted that the best results are obtained with early treatment of these fractures to avoid functional and aesthetic outcomes. Delays inevitably occur as life-threatening conditions clearly need to take precedence, in these cases late treatment needs an accurate planning to reduce the higher risk of complication.
437

\section{Reconstruction of the Superior and Middle} Third of the Face: State of the Art

\author{
V. Valentini, G. Nicolai \\ Chirurgia Maxillo-Facciale, Policlinico di Tor Vergata, Roma, \\ Italy
}

Introduction: Reconstruction of wide defects of the superior and middle third of the face underwent progressive evolution during the last years, depending upon evolution of surgical techniques. Although limited defects may be treated by local flaps, wide reconstructive procedures may range from prosthesis to pedicled and free flaps.

Methods: In this work, the Authors report their experience on reconstructive procedures of the superior and middle third of the face in the Department of Maxillo-Facial surgery of the University of Rome 'La Sapienza' and 'Tor Vergata'.

Results: The evolution of surgical techniques for reconstructive procedures of the superior and middle third of the face resulted in optimal functional and aesthtic outcome.

Discussion: The orbito-maxillary region plays a critical role as the anatomical and functional key, of the face, as the orbit, the nasal cavity, the oral cavity. Defects of the upper maxilla and the orbitomaxillary region result functional (breathing, feeding, speech) and psychological impairment. Therefore, the objectives of surgical separative treatment employ closure of the defect, reconstruction of the surgical defect, complete functional, and best aesthetic rehabilitation.

\section{8}

\section{Bony Haemangioma of the Orbito-Zygomatic Region: Reconstructive Aspects}

\author{
B. Lorè, I.V. Aboh, G. Nicolai, A. Torroni, A. Battisti, \\ V. Valentini \\ Chirurgia Maxillo-Facciale, Policlinico di Tor Vergata, Roma, \\ Italy
}

Introduction: Osseous haemangioma is a rare lesion, its incidence being $0.5-1 \%$ of all osseous neoplasm. They are considered vascular neoformation of bony district. The bones of the maxilo-facial region mainly affected by this tumor are: mandible, zygoma, maxillary sinus, nasal bone and frontal bone. Different reconstructive options have been illustrated to guarantee a good functional and aesthetic outcome. In this work, the authors present the case of a 57-yearold man affected by bony haemangioma of the left zygoma. They also focus their attention on the reconstructive aspects.

Methods: A 57 years old man came to our attention complaining a painful swelling of the left zygomatic region, which was first noticed four years before. The lesion was initially small and asymptomatic, but it enlarged gradually causing an aesthetic deformity. TC scan showed a $1.5 \mathrm{~cm}$ osteolytic lesion. Histology report was for osseous haemangioma. The patient underwent surgery. Removal of the neoformation and reconstruction with rib graft was performed.

Results: Three weeks after surgery the patient had a complete improvement of both functional and aesthetic aspect. No scar, 
swelling or depression were seen on the left orbito-zygomatic region. No diplopia was referred. There was a satisfactory symmetry of the face.

Discussions: Cranio-maxillo-facial bony heamangiomas represent a high challenge for maxillo-facial surgery. Reconstruction of the orbito-zygomatic structure is very important because of face symmetry and eye function. Defects of orbito-zygomatic bone can lead to aesthetic deformity and huge eye motility problems. For major defects free flaps are (truncated at 250 words).

\section{9}

\section{Face Burn Sequelae Treatment}

F. Klinger, A.V. Pagliari, F. Caviggioli, D. Ventura, S. Jannasi, J. Hassibi, C.A. Amodeo, B. Banzatti, M. Klinger

Istituto di Chirurgia Plastica, Ricostruttiva dell'Università degli Studi di Milano, U.O. Chirurgia Plastica II, I.C. Humanitas

Introduction: With improved acute care a higher percentage of more severely injured facial burn patients are surviving their burns. Patients who have survived thermal injuries to the face suffer severe disfigurement from devastanting deformities of full-thickness facial burns.

Material and Method: When burned face needs correction and reconstruction different plastic techniques are employed in multidisciplinary approach to the patients.

Result: Early grafting of eylied burns with full-thickness grafts prevent the development of recurrent cicatrical ectropion. A rhinoplasty was performed to create a better appearance and correct any internal or external nasal deviations. Lip vermilion is restored with oral mucosal flaps. Pressure therapy as well as the positional splinting must be maintained for many months until the deleterious wound forces abate. Good results were obtained with filiform douche of thermal water.

Conclusion: The face is a focal point of interpersonal communication and reconstruction of extensive deep burns of the face is a challenging problem and needs special emphasis.

\section{0 \\ Lip Reconstruction following Trauma: 2 Case Reports}

\section{S. Spotti, A. Baj, A.B. Gianni}

Department of Maxillofacial Surgery of the Graduate School of Medicine of Milan, University of Milan, Italy

Introduction: The lips are complex, laminated structures consisting of skin, subcutaneous tissue, muscle, submucosa and mucosa. Clearly when the lip is lost through injury or disease, it presents a complex reconstructive challenge: the facial artery muscular mucosal flap (FAMM), harvested from lateral cheek, is a composite flap with many features which make it an excellent option for lip and vermilion reconstruction. Lip amputation are rare but possible, for example after a dog bite, in this case microsurgical replantation must be systematically tried to restore form and function in one step.
Methods: The authors present 2 cases. A 2 years old girl with medial lower lip vermilion avulsion reconstructed with FAMM flap tailored from the inside of the left cheek A 30 years old girl with upper lip amputation after a dog bite: revascularization of the amputated segment was obtained by arterial anastomosis with the corresponding labial coronary artery Venous drainage was obtained by inducing bleeding and by postoperative application of leeches.

Results: Aesthetic and functional results were evaluated as being good, with reestablishment of labial continence and recovery of protective sensitivity.

Discussion: Various reconstruction techniques are used in the correction of defects effecting over $1 / 3$ of the inferior lip and in particular the vermilion. The FAMM flap is the most favourable technique because the flap is rotated from the cheek and therefore does not interfere with mouth opening. Microsurgical replantation of severed lip parts can be accomplished with a high degree of reliability despite the paucity of vessels and small vessel size.

\section{1 \\ Orbital Floor Fractures: Proposal of a Flow Chart}

\section{S. Spotti, D. Rocchetta, G. Bellocchio, A.B. Gianni \\ Department of Maxillofacial Surgery of the Graduate School of Medicine of Milan, University of Milan, Italy}

Introduction: The increasing emphasis on open reduction in the management of orbital fractures has led to an extensive debate as to which approach provides adequate exposure for these fractures. This retrospective study compares the exposure provided and the rate of complications between transconjunctival, subciliary and lower lid incisions for orbital rim and floor fractures.

Methods: The charts of 130 orbital and orbito-zygomatic fractures, treated with open reduction and internal fixation, were reviewed. There were 32 orbital blow-out fractures and 108 orbitozygomatic fractures. A total of 72 transconjunctival (55\%), 33 subciliary (25\%), 25 lower lid (20\%) incisions had been performed, and the adequacy of exposure as well as intraoperative and postoperative complication rates were compared.

Results: We used always transconjunctival preseptal approach in pure orbital blow-out fractures. Scar appearance was similar in all three groups, except when an isolated conjunctival incision was used, which produced an invisible scar. We found no transient ectropion and a $15 \%$ rate of permanent scleral show with the subciliary approach compared with no transient ectropion and a $3 \%$ rate of permanent scleral show with transconjunctival approach.

Discussion: We found a higher rate of complications with the subciliary and lower lid approach. We believe that the transconjunctival approach provides excellent exposure with less risk of postoperative eyelid retraction and ectropion and, therefore, advocate transconjunctival incision for the management of orbital blow-out fractures and in all case without comminuted orbital rim fractures. According to international literature indications we propose rational flow-chart to chose the best access to orbital floor. 


\section{2}

Maxillo-Mandibular Advancement (MMA) in the Treatment of Obstructive Sleep Apnea Syndrome

\author{
S. Spotti, G. Beltramini, F. Laganà, A.B. Gianni \\ Department of Maxillofacial Surgery of the Graduate \\ School of Medicine of Milan, University of Milan, Italy
}

Introduction: Obstructive sleep apnea syndrome (OSAS) is a potentially serious clinical condition, associated with cerebrovascular and cardiovascular diseases, metabolic alterations, car accidents and mood disturbances. Recent epidemiology data show a prevelance of OSAS in 1-4\% of the population aged 20-60 years and a mortality of $20 \%, 4$ years after diagnosis. Maxillo-mandibular advancement represents a valid therapeutical option, with a percentage of success ranging from 90 to $100 \%$.

Methods: From January 2003 to April 2006, 11 patients (mean age 46 years) suffering from mild-severe OSAS (mean Respiratory Disturbance Index, RDI, 66) were treated with maxillo-mandibular advancement. All patients were studied by means of polysomnography, fibroscopy of the upper airways and cephalometric analysis Orthodontic treatment preceded the surgical procedure. Surgery was programmed on models of the dental arches.

Results: Six months after the surgical procedure, all patients were asymptomatic and the polysomnographic findings were normal $(\mathrm{RDI}<10)$. Pre-operative orthodontia guaranteed a valid occlusion. Patients were satisfied of the esthetical results No major complications arose.

Discussion: Maxillo-mandibular advancement restores anatomical airway perviousness with the three-dimensional repositioning of the splancnocranium and no interference between the function of the organs. It is an efficacious technique and results in low morbidity Orthodontic therapy and accurate pre-surgical programming in eumorphic patients as well allow for therapeutical success and satisfying esthetical and occlusion results.

\section{3 \\ Parothydectomy in a General Surgery Unit. Personal Experience}

C. Talarico, N. Papandrea, M. Deonofrio, G. Gullà, A. Lioi

Surgical Unit, 'San Biagio' Hospital, Chiaravalle C.le, CZ

Introduction: The authors present their personal experience about the surgical treatment of parothyd neoplasms. The surgical operations were performed by general surgeon, technique and results obtained were investigated.

Methods: From January 1996, 22 patients were operated for parothyd neoplasm, 12 male and 10 female with age ranged for 22-76 years. In 12 cases the neoplasm was pleomorphic adenoma, in 5 Warthin neoplasms, in 2 oncocitomas, in 1 linphoma, in 2 poor differentiated carcinomas. The operations were performed under general anaesthesia, skin incision always a ' $\mathrm{S}$ ' type and the parothydectomy was total in all cases. Magnification, with loops $(2.4 \mathrm{x})$ and bipolar coaugulation is a standard technique to expose the facial nerve.
Results: The median duration of operation was: $190 \mathrm{~min}$. The postoperative complication were: 1 permanent facial nerve palsy (in case of aggressive carcinoma infiltrating the trunk of the nerve), in 4 temporary nerve palsy with complete regression within 3 months, 1 wound infection. The follow-up (range 2 months-10 years) of the totality of patients shows that no one of this have local recurrence.

Discussion: Total parothydectomy is the treatment of choice in case of neoplasm. This surgical procedure if performed with respect of facial nerve anatomy is safe in hands of non dedicated surgeons too. Magnification with optical loops and bipolar coagulation permit to reduce the rate of facial nerve palsy.

\section{4 \\ La Ricostruzione Del Mascellare Con Lembi Locali}

\section{A. Torroni}

Introduction: The reconstruction of the upper jaw has evolved to the point that the use of free flaps is today almost routine. Even though microvascular flaps are the best reconstructive choice for aesthetic and functional problems caused by deficits in the upper maxilla, such procedures have a significant biological and healthcare cost and, in addition, are not suited for all patients. Therefore, it remains as important as ever for all reconstructive surgeons of the head-and-neck district to be competent in traditional reconstruction methods using local or locoregional flaps.

Materials and Methods: In the period between 1990 and 2002, 114 patients underwent reconstructive surgery of the upper jaw. In 69 patients the reconstruction followed a surgical excision of benign or malign tumors; in 15 patients the reconstruction followed traumas, and in 30 cases (not including cleft palate) the reason for reconstruction was malformation. The reconstruction of the upper maxilla was done with local flaps in 8 cases; a temporal muscle flap by itself in 25 cases, with bone grafts in 43 cases, or with alloplastic material grafts in 20 cases; free flaps in 13 patients; and obturator prostheses in 5 cases.

Results: Several traditional techniques for the reconstruction of the maxillary bone defects are presented and discussed.

Conclusion: The traditional methods of reconstruction of the upper jaw remain, still day in a period of microsurgery, valid therapeutic options in certain circumstances. These include a generally compromised condition of the patient, the impracticality and/or failure of other reconstructive methods. 


\section{5}

\section{Ricostruzione Mediante Limbo Libero Miocutaneo di M. Retto Dell'addome Dopo Glossectomia Totale: Presentazione di Un Caso Clinico}

\author{
C. Carnevale, A. Torroni, P. Gennaro, G. Nicolai, V. Valentini
}

Introduction: When a total glossectomy is performed the functional recovery of swallowing and articulation is extremely important in maintaining the patient's quality of life. Reconstruction with rectus abdominis free tissue transfer is a viable method for rehabilitation, improving quality of life after total or subtotal glossectomy with laryngeal preservation.

Material and Method: The mobile tongue and the suprahyoid muscles were reconstructed in a 60 years old female presented to us with squamous cell carcimona of the left side of the tongue. She had undergone four cicles of neoadjuvant chemotherapy and after 6 months she underwent to resection of the mobile tongue with the oral floor and bilateral functional neck dissection. A rectus abdominis musculocutaneous flap with the intercostal nerve anastomosed to the remaining hypoglossal nerve was used for the reconstruction. The rectus abdominis muscle was inserted between the mandible and the hyoid bone, taking account of the anatomical positions of the tongue and suprahyoid muscles. The skin island, of the rectus abdominis myocutaneous flap is created about $20 \%$ larger than the defect in both width and length and it is sutured to the defect such that the excess skin.

Results: After the operation, the reconstructed tongue showed no sign of atrophy and the hyoid bone could be raised. In the early post-operative stage after rehabilitation program the patient had good functional outcomes measured by independent feeding, speech and swallowing.

Conclusion: Reconstruction of the tongue with free flaps is a feasible method to restore the functional outcomes in deglutition and speech (truncated at 250 words).

\section{6 \\ Microvascular Reconstruction of the Mandible in Irradiated Patients}

\section{I.V. Aboh, B. Lorè, G. Longo, A. Torroni, P. Gennaro, V. Valentini}

Introduction: In this work, the authors focus their attention on the use of revascularized free flaps for the reconstruction of the major defects of the mandible secondary to removal of high stage tumour, in irradiated patients. They also illustrate all problems involve these particular cases and the best reconstructive options according to international literature and to their experience.

Methods: Three patients ( 2 females and 1 male) previously treated with a mandibulectomy and radiotherapy for malignant neoplasms underwent secondary reconstruction of the mandible and soft tissue with microvascular free flaps. An osteomiocutaneous DCIA free flap was used in two cases, whereas a double flap (fibula free flap + rectus abdominis free flap) was used in the other case.

Results: The indications to mandibular reconstruction using revascularized free flap depend on: extension and localization of the bone defect, necessity for an immediate or later reconstruction, condition of the donor site and the general conditions of the patients. All patients treated showed a satisfactory outcome.

Conclusions: Nowadays it is possible to guarantee a satisfactory renewal of function and morphological appearance in the majority of patients who present with mandibular defects. There is also the possibility to correctly apply osteo-integrated implants for a good occlusal restructuring process.

\section{7 \\ Different Surgical Solutions in Lip Reconstruction}

F. Klinger, A.V. Pagliari, F. Caviggioli, S. Giannasi, D. Ventura, J. Hassibi, F. Villani, B. Banzatti,

C.A. Amodeo, M. Klinger

Istituto di Chirurgia Plastica e Ricostruttiva dell'Università degli Studi di Milano, U.O. Chirurgia Plastica II, I.C.

Humanitas

Introduction: Lip represent a distinct anatomic unit with great functional and aesthetic importance. Lips retain saliva and food during mastication and play a pivotal role in speech artuculation, facial expression and emotion. Lips reconstructions represent a complex field and none single technique satisfying all situations.

Methods: From single clinical cases we showed the recontruction techniques employed to repair lip defects according their site, thickness and width. Local flap closure is the technique of choice when simple closure would yield unsatisfactory results.

Results: In our series lip defects arise from tumor excision, trauma and burn. In all patients was maintained oral competency and aperture with excellent cosmetic results.

Discussion: Lips reconstruction is for plastic surgeon a challenge for their unique character. A careful pre-operative planning and ability to use the tissue adjacent to the defect with identical texture facilitates adequate reconstruction with acceptable deormity.

\section{8 \\ Different Surgical Solutions in Lip Reconstruction}

F. Klinger, A.V. Pagliari, F. Caviggioli, S. Giannasi,

D. Ventura, J. Hassibi, F. Villani, B. Banzatti,

C.A. Amodeo, M. Klinger

Istituto di Chirurgia Plastica e Ricostruttiva dell'Università degli Studi di Milano, U.O. Chirurgia Plastica II, I.C. Humanitas

Introduction: Lip represent a distinct anatomic unit with great functional and aesthetic importance. Lips retain saliva and food during mastication and play a pivotal role in speech artuculation, facial expression and emotion. Lips reconstructions represent a complex field and none single technique satisfying all situations. Methods From single clinical cases we showed the reconstruction techniques 
employed to repair lip defects according their site, thickness and width. Local flap closure is the technique of choice when simple closure would yield unsatisfactory results.

Results: In our series lip defects arise from tumor excision, trauma and burn. In all patients was maintained oral competency and aperture with excellent cosmetic results.

Discussion: Lips reconstruction is for plastic surgeon a challenge for their unique character. A careful pre-operative planning and ability to use the tissue adjacent to the defect with identical texture facilitates adequate reconstruction with acceptable deormity.

\section{Phlebological Surgery}

\section{9}

\section{Aneurysms of the Saphenous Veins}

M. Bucalossi, S. Mancini, L. Gazzabin, A. Comandi, F. Berna, G. Botta

Chirurgia Generale 3, Siena, Italy

Introduction: We report experience about the primitive aneurysms of the superficial venous system of the lower limb in patients with chronic venous insufficiency. Such dilatation doesn't meet spontaneous broken, by only for accidental trauma, so the most dangerous complication remains the venous thromboembolism.

Methods: From January 1995 to April 2006, 72 patients underwent to surgical treatment for primitive aneurysmatic dilatation of superficial venous system. The dilatation was occasionally found during a duplex scan. The diameter was inclusive in a range between 22.4 and $38.6 \mathrm{~mm}$. None has injured the deep venous system. The aneurysmatic dilatation was localized in LSV (55), SSV (11) and in ASV (6). In all patients it was localized at S-F or S-P junctions. All the patients underwent to surgical treatment by crossectomy and stripping of long, short or anterior saphena, with exeresis of the dilatation.

Results: The histologic studies underlined a venous wall weakness, elastic elements fragmentation and smooth muscolar cells depletion. No patients undergone to surgical therapy met mortality, morbidity and thromboembolic episodes.

Discussion: Due to the clinical experience on 72 patients, we can observe as surgical exeresis of aneurysm, with treatment of saphenic insufficiency, is an efficient therapy, giving indubitable results on the point of view prognostic.
450

\section{Prevention of Recurrent Varicose Veins after} Surgery

M. Bucalossi, S. Mancini, L. Gazzabin,

A. Comandi, F. Berna, G. Botta

Chirurgia Generale 3, Siena, Italy

Introduction: We report experience about the primitive aneurysms of the superficial venous system of the lower limb in patients with chronic venous insufficiency. Such dilatation doesn't meet spontaneous broken, by only for accidental trauma, so the most dangerous complication remains the venous thromboembolism.

Methods: From January 1995 to April 2006, 72 patients underwent to surgical treatment for primitive aneurysmatic dilatation of superficial venous system. The dilatation was occasionally found during a duplex scan. The diameter was inclusive in a range between 22.4 and $38.6 \mathrm{~mm}$. None has injured the deep venous system. The aneurysmatic dilatation was localized in LSV (55), SSV (11) and in ASV (6). In all patients it was localized at S-F or S-P junctions. All the patients underwent to surgical treatment by crossectomy and stripping of long, short or anterior saphena, with exeresis of the dilatation.

Results: The histologic studies underlined a venous wall weakness, elastic elements fragmentation and smooth muscolar cells depletion. No patients undergone to surgical therapy met mortality, morbidity and thromboembolic episodes.

Discussion: Due to the clinical experience on 72 patients, we can observe as surgical exeresis of aneurysm, with treatment of saphenic insufficiency, is an efficient therapy, giving indubitable results on the point of view prognostic.

451

\section{Varicose Veins Redo in One Day Surgery}

P. Muselli, A. Giardino, G. Barugola, S. Partelli, G. Armatura, G. Lipari, E. Baggio

Surgical Sciences Department, University of Verona

Introduction: Redo surgery for varicose veins is a frequent problem in this kind of treatment. The recurrence is normally due to technical errors (mainly for the short saphenous vein surgery), neoangiogenesis and to natural evolutivity of the varicose disease. Purpose of our work is to verify the day surgery redo surgery feasibility.

Methods: Between January 1991 and January 2005 we treated 17 patients with short saphenous vein and 108 patients with long saphenous vein recurrences. Our preoperatory planning concern preoperative assessment with clinical examination and Duplex-colour scanning; exceptionally we performed a contrast varicography. Regarding the surgical technique we use a modified $\mathrm{Li}$ approach in $91.7 \%$, and a frontal approach in the remaining $8.3 \%$ for the long saphenous vein. In $98.6 \%$ we utilize local anaesthesia joined with e.v. sedation (propofol and others). Only in 1 case (1.4\%) we utilize general anaesthesia (refuse of the patient for the local anaesthesia). In all operations for short saphenous vein relapse we utilize only local anaesthesia without other kind of anaesthesia, approaching directly to the point of leak 
Results: We have no conversion from local to general anaesthesia. All the patients were discharged the same day (true One Day Surgery); we hospitalised only one patient (the same that refused the local anaesthesia).

Discussion: The redo surgery for varicose veins of the lower limbs, despite a greater technical difficulty, is perfectly compatible with Day Surgery, without technical changes.

\section{Plastic Surgery}

\section{2 \\ 'Microsurgical Vascular Training: A Model to Face it During the Residency'}

L. Annacontini, G. De Salvia, A. Campanale, A. Maiorella, G. Gozzo

Plastic and Reconstructive Surgery Department, University of Foggia, Italy

Introduction: Microsurgery prove how fine and accurate could be patient reconstruction after oncological demolition, trauma or congenital malformation. Thanks to appropriate instruments, magnification and suturing material the microsurgeon can carefully manipulate vessels and nerves. The authors present their training model on vessels starting on laboratory rats, ending in the operation theatre.

Methods: First exercises always start on latex and biological models. T-T and T-L anastomoses, by-pass and vascular grafts must be learnt by the resident in training. We refer on our training path with $\mathrm{T}-\mathrm{T}$ anastomose on femoral artery and femoral vein graft vs. femoral artery. It lasts 3 weeks, under a tutor supervision. Basic training foresee 15 Wistar rats for each technique. A mixture of ketamine and xylazine is used to induce anesthesia. All the anastomoses are performed with the Carrel technique.

Results: Success rate both for T-T suture and vein graft pass from $0-20 \%$ in the first week to $80-100 \%$ in the last week. In 3 weeks suturing confidence raise from $120-40 \mathrm{~min}$ for T-T suture and from 180 to $50 \mathrm{~min}$ in vein grafting. After this period resident can be involved as assistant in microsurgical procedures.

Discussion: Laboratory training should never stop. A fine technique is the result of years of practice both in the Lab and operation theatre. To obtain the best result, pre-operative planning, a dedicated operation theatre, surgical instruments and an Equipe in harmony are mandatory since microsurgery remains a long, difficult, stressful surgery but fascinating.
453

'Monstrous Wart' Eradication with an Integrated Surgical Approach: Case Report

\section{Annacontini, M. Grieco, G. Gozzo, A. Campanale,} A. Maiorella

Plastic and Reconstructive Surgery Department, University of Foggia, Italy

Introduction: Wart Vulgaris is a benign tumour due to Human Papilloma Viruses. Surgical protocol foresee diatermocoagulation and excision. Authors present the case of a big plantar wart on the left foot healed in 3 months both with surgical and ambulatory treatment.

Methods: Female, 71 years old, with hypertension, arthrosis and L.E.S. The wart, $10 \times 5 \times 3 \mathrm{~cm}$, dated 15 years and obliged patient to wheelchair. 1990-2001: all dermatological attempts failed. 2001-2002: two skin grafts also failed. Authors visited the patient on June 2005. A biopsy confirmed the previous diagnoses; deep excision, to the plantar fascia, was carried out and the defect repaired with INTE$\mathrm{GRA}^{\circledR}$; after 16 days it was removed due to infection and the presence of Musca Domestica grubs. 2 V.A.C. therapy cycles followed come before by a surgical toilette. Good wound bed was obtained in 3 weeks and a skin graft applied; taking rate: $80 \%$ at 7 days, $100 \%$ at 18 days.

Results: Elastic band, orthopedic shoes and a drain plantar were suggested. 6 months follow up showed functional restoration and wound recovery.

Discussion: Authors underline how in this singular case excision to the superficial fascia layer, V.A.C. therapy and skin graft allowed the only possible radical tumour excision. Other procedures failed certainly due to the incomplete 'monstrous wart' excision plus an inadequate wound bed preparation.

\section{4 \\ Rectus Abdominis Flap: Use on Abdominal Wall Reconstruction}

\section{G. Basso, G. De Salvia, D. Massariello, L. Annacontini,} A. Maiorella

Chirurgia plastica e ricostruttiva, Università degli Studi di Foggia

Introduction: Primary tumors or metastasis from abdominal organs can involve abdominal wall (skin, fascia, muscle). The treatment consists in huge demolition of the tumor (including muscle). The reconstruction can be performed through muscular flap or synthetic mesh. The authors present their experience with pedicle muscular flap of rectus abdominis.

Methods: From 2002 till 2006, 8 patients have been treated, $5 \mathrm{M}$, $3 \mathrm{~F}$, age 57-72: 7 affected by primitive tumor of the abdominal wall, 1 affected by colon's carcinoma metastasis. Dimension between $10 \times 9$ and $20 \times 18 \mathrm{~cm}$. All the patients were treated with huge demolition and secondary reconstruction with pedicled muscle flap of controlateral rectus abdominis. A dermal layer reconstruction utilizing a dermal-adipose flap was putted on muscular reconstruction.

Results: Postoperative period was uneventful in all patients. All patients returned to daily activities after 4 weeks. Medium follow-up 
is 4 years. RMN or TAC controls showed no complications (hernias or laparoceles).

Discussion: Reconstruction of the abdominal wall can be performed with muscular flaps or synthetic mesh. Reconstruction by muscular flaps needs longer operative time, plastic surgery equipe, but, in our hands, we can observe no risk of reject, easier postoperative period and faster recovery, no synthetic prosthesis needed, a global minor cost per patient, a satisfactory anatomic reconstruction.

\section{5}

\section{The Silicone Replica Technique in Plastic Surgery}

\section{S. Burrai, F. Farace, A. Puddu, C. Rubino}

University of Sassari, Departement of Plastic and Reconstructive Surgery, School of Specialization in Plastic Reconstructive and Aesthetic Surgery

Introduction: The silicone replica technique is a quantitative, non-invasive method to evaluate superficial skin texture. This procedure derives from a orthodontic polyvinylsiloxane procedure. We use the silicone replica to analyze full-tickness skin graft, anti-ageing techniques, atypical nevi and melanomas. We describe the technique and outlook its different future use.

Methods: To obtain the replicas polyvinylsiloxane and a catalyst are mixed together and applied onto the skin. Silicone solidifies and the cast is removed. Then a three-dimensional positive contra-replica is obtained and analyzed by scanning electron microscopy. Skin topographic parameters studied are: roughness, anisotropy, microsulcus number and width. Our application fields are: differential diagnosis between melanoma and nevi (30 applications); topic glycolic acid application vs. multivitamin and moisturizing cream product efficacy (30 patients); postoperative full-thickness skin graft orientation (20 patients).

Results: Our results show that the melanoma has a very irregular surface, with marked reduction or disappearance of cutaneus sulci, formation of new lines with varying direction. Topic glycolic acid is more effective than multivitamin and moisturizing products. Fulltickness skin graft modifies its dermis and change overlying superficial skin pattern.

Conclusion: Silicone replica is a versatile method and may help in diagnosis of early stage M.M or in differential diagnosis. This procedure is able to detect antiageing product efficacy and may be employed even to test laser-therapy or bo-tox.

\section{6 \\ Microsurgical Anastomosis with the Airborne Suture Technique}

\section{E. Cigna, N. Scuderi}

Department of Plastic Surgery, University of Rome 'La Sapienza', Italy

Introduction: Refinements in microvascular techniques have been introduced with the aim to improve the vessel patency rate, decrease the time spent during the anastomosis making the technique easier and faster and lessen the frustration associated with the process. In certain situations reduce the ischemia time is a prerequisite.

Methods: The airborne suture tying technique requires maintaining the suture ends in the air with instruments and allows tying the knot without leaving the suture end. Two methods are possible; each one places the final knot in a different direction. This technique has been consecutively applied on 20 microsurgical anastomoses by the same surgeon.

Results: Compared to conventional techniques, this method allowed the same surgeon to save approximately $15-20 \%$ of time. Moreover reducing the handling of the suture is reduced the risk of damaging the intima of the vessel. The technique reduces the cumbersome process of picking the suture off the surrounding structures and allows for a quicker completion of the anastomosis.

Discussion: Minimizing ischemia time is critical for replantations or complex reconstructions. This suture-tying technique, helps the surgeon to avoid the occasional frustration encountered when attempting to pick up a suture end that is embedded in the surrounding adventitia. It also helps the surgeon to save time in performing the anastomosis. This airborne technique can be used routinely or only when there is a tendency for the suture to adhere to the adjacent tissues. The end result is a quicker, more elegant anastomosis with less frustration for the surgeon.

\section{7 \\ Analisys of 372 Cases of Nose Skin Carcinoma, Operated in 19 Years}

\section{S. Gentileschi, A. Bruno, M. Schirosi, E. Farallo, A. Seccia \\ Department of Plastic and Reconstructive Surgery, Policlinico A. Gemelli, Roma, Italy}

Introduction: The incidence of epithelial tumors of the nose is high, accounting for about $27 \%$ of all the skin carcinomas of the body and for $35 \%$ of the only head and neck skin carcinomas.

Methods: The Authors analyzed their experience about 372 patients, affected by skin carcinoma of the nose, operated between 1985 and 2004.

Results: Patients aged from 26 to 93 , with a higher incidence between 60 and 80 (65\% of the patents), $57 \%$ were male and $43 \%$ female. 346 tumours were basal cells carcinoma (93\%) and 26 squamous cells carcinoma (7\%). The most frequent clinical form was nodular (50\%). Dimensions were between $0.5 \mathrm{~cm}$ and $4 \mathrm{~cm}$ (average $1.5 \mathrm{~cm})$. More frequently involved areas were the dorsum $(30 \%)$ and the ala (26\%). Reconstructive procedures were local flaps (57\%), primary closure (25\%) and skin graft (18\%). Mostly employed flaps were, in order of frequency: nasolabial, frontal, dorsum of the nose. In 8 cases reconstruction was delayed because of oncologic surveillance. In all the other cases reconstruction was performed in one time stage.

Conclusion: Nose skin cancer demonstrated, in our experience, great trend to infiltrate deep structures (cartilages). They must be treated very radically, necessitating often complex aesthetic reconstruction, following aesthetic subunit. 


\section{8}

\section{Analisys of 334 Cases of Eyelids Skin Carcinoma, Operated in 21 Years}

\author{
S. Gentileschi, A. Bruno, M. Schirosi, E. Farallo, A. Seccia \\ Department of Plastic and Reconstructive Surgery, \\ Policlinico A. Gemelli, Roma, Italy
}

Introduction: Non melanoma skin cancer is one the most frequent cancer in the world, in the white race, accounting for 2,750,000 new cases every year. Face presents higher risk, because of sun exposure. Lid involvement is more frequent on lower eyelid and implies complex reconstructive problems.

Methods: The Authors analyzed their experience about 334 patients, affected by skin carcinoma of the eyelids, operated between 1983 and 2004.

Results: Patients aged from 38 to 85 , with a higher incidence between 60 and $80,53 \%$ were male and $47 \%$ female. 314 tumours were basal cells carcinoma and 20 squamous cells carcinoma. The most frequent clinical form was nodular. Dimensions were between $0.5 \mathrm{~cm}$ and $4 \mathrm{~cm}$ (average $2 \mathrm{~cm}$ ). More frequently involved areas were lower eyelid (45\%) and inner canthus (35\%). Reconstructive procedures were local flaps $(60 \%)$, primary closure $(33 \%)$ and skin graft $(7 \%)$. Mostly employed flaps were, in order of frequency: glabellar, nasolabial, frontal, Mustardè, Tripier. In 11 cases exenteratio orbitae was necessary, reconstructed by frontal, temporal or microvascular flap.

Conclusion: Lid skin cancer demonstrated, in our experience, great trend to infiltrate adjacent structures (muscle, conjunctiva). They must be treated very radically for high trend to recurrence.

\section{9 \\ Correction of Breast Ptosis and/or Hypoplasia After Bariatric Surgery and Massive Weight Loss}

\section{S. Gentileschi, R. Bracaglia, G. Mingrone1, R. Tacchino², G. Nanni², M. Foco ${ }^{2}$ \\ Department of Plastic Surgery, 'Department of Medicine, ${ }^{2}$ Department of Surgery, Policlinico A. Gemelli, Catholic University Sacred Heart, Rome, Italy}

Introduction: Correction of breasts ptosis and hypoplasia accounts for about $24 \%$ of the claims of the patients who have undergone bariatric surgery or massive weight loss, at their first consultation with the plastic surgeon.

Methods: Authors analyze techniques employed with $35 \mathrm{ex}$ obese patients undergone to breast lift and/or augmentation. 15 of them had undergone bariatric surgery.

Results: Breast implants were judged indicated in 29 patients ( $83 \%)$, united to the correction of the ptosis. In the patients were we executed only breast lift, Planas technique was employed with dermoparenchimal inferior pedicled flap, anchored to pectoralis major muscle, used as an autoprosthesis. We only had 1 case of haematoma (8 months after surgery) for traumatic laceration of periprosthetic capsule.

Discussion: Planas technique allows good reshaping of the breast, achieving even some fullness of the upper pole. Often involu- tion of both adipose and glandular components of the breasts, in ex obese patients, imposed the use of implants. Soft tissues are extremely thin and loose in post bariatric patients. So to prevent implants evidence in its upper pole and its 'weigh' on soft tissues, we made the pocket under the serratus, in the lower pole, and under the pectoralis in its upper pole. To better fill the inferior pole and avoid double profile, if tissues laxity recurr, anatomic shape with high projection was preferred.

460

\section{Sentinel Node Biopsy in Cutaneous Melanoma: Our Experience}

S. Gentileschi, R. Bracaglia, A. Seccia, M. Sturla, M. Salgarello, E. Bartoccioni, R. Capizzi', A. Paradisi', I. Proietti ${ }^{1}$, E. Farallo, P. Amerio ${ }^{1}$, E. Capoluogo ${ }^{2}$, G. D'Errico ${ }^{3}$, L.M. LaRocca ${ }^{4}$, A. Mulè ${ }^{4}$

Department of Plastic and Reconstructive Surgery, ${ }^{1}$ Institute of Dermatology, ${ }^{2}$ Institute of Biochemistry and Clinical Biochemistry, ${ }^{3}$ Institute of Nuclear Medicine, ${ }^{4}$ Institute of Pathologic Anatomy and Histology, Policlinico A. Gemelli, Roma, Italy

Introduction: Sentinel node biopsy (SNB), in cutaneous melanoma, has both a prognostic and therapeutic importance. Eventual presence of metastasis has an undiscussed negative prognostic value, at the point to be part of the staging system of the AJCC. More studies are necessary to demonstrate the superiority of the elective lymphoadenectomy over the elective or therapeutic one.

Methods: The authors analyzed their experience about 75 patients, affected by cutaneous melanoma, all belonging to the stage I or II, at the moment of diagnosis, who underwent to SNB. 26 patients showed a Breslow (BR) thickness $<1 \mathrm{~mm}, 41$ a BR included between 1 and $4 \mathrm{~mm}, 5$ patients a BR $>4 \mathrm{~mm}$, and 3 a not specified BR, because of wide regression.

Results: 17 patients of the 75 resulted positive to SNB. 3 showed thin melanoma, 10 BR between 1 and $4 \mathrm{~mm}, 3$ with BR $>4 \mathrm{~mm}, 1$ with not specified BR. These patients underwent complete lymphoadenectomy of the involved region, and 4 of them showed further metastatic nodes. Follow-up was of 18 months (between 1 and 70 months) and a strong association between disease free time, survival and SN involvement was evident.

Conclusion: Our experience confirmed the importance of the $\mathrm{SN}$, as a prognostic factor, in cutaneous melanoma. We think that regression, ulceration and Clark level IV or V should be considered as indications to the execution of SNB even in presence of thin melanomas. 
461

\section{Plastic Surgery After Massive Weight Loss and Bariatric Surgery}

\section{R. Bracaglia, S. Gentileschi, G. Mingrone1, R. Tacchino²,} G. Nanni ${ }^{2}$, M. Foco ${ }^{2}$

Department of Plastic Surgery, ${ }^{1}$ Department of Medicine, ${ }^{2}$ Department of Surgery, Policlinico A. Gemelli, Catholic University Sacred Heart, Rome, Itlay

Introduction: Massive weight loss, due either to diet or bariatric surgery, causes excess of cutaneous and subcutaneous tissues, implicating intertrigo, chronic infections and sometime difficulties of mobility and dressing. The Plastic Surgeon is called to correct these dermoadipose excesses, when weight loss is complete.

Methods: The authors discuss the techniques employed in the treatment of the areas mostly affected by weight loss, and the more frequently requested procedures.

Results: The areas mostly affected are the abdomen, the thighs, the arms, the back and the breasts. Most executed procedures are abdominoplasty, thigh and arms dermolipectomy, breast lift and reduction or augmentation, and, in the male patient, thoracic dermolipectomy. We never associate more procedures in the same operation, in patients who have undergone bariatric surgery, for their delicate general postoperative management and their higher risk of wound dehiscence $(17.6 \%)$, seroma (13\%), infection $(7 \%)$, in our experience.

Discussion: Critical steps in the surgical planning are the positioning of the final scars and the amount of tissue to be removed, to avoid excessive tension. Patients, usually show good motivation but must be well informed about final scars and about the real possibilities of improvement. In these conditions this surgery can provide satisfying final results and a better life quality to these patients.

\section{2}

Planas Technique for Abdominal Contouring after Bariatric Surgery and Massive Weight Loss

S. Gentileschi, R. Bracaglia, G. Mingrone', R. Tacchino², G. Nanni' ${ }^{2}$ M. Foco ${ }^{2}$

Department of Plastic Surgery, 'Department of Medicine, ${ }^{2}$ Department of Surgery, Policlinico A. Gemelli, Catholic University Sacred Heart, Rome, Italy

Introduction: Ex obese patients often require Plastic Surgery procedures, for the correction of cutaneous redundance, in different areas of the body. Abdomen is the most frequently requested procedure, accounting for $37 \%$ of the claims, at the first consultation with the plastic surgeon. Abdominal region presents peculiar features, according to the type of obesity, to the physique and to the eventual presence of scars, due to bariatric surgery.

Methods: The authors analyze their experience with the Planas technique, called 'Vest over Pants' technique, employed for 37 ex obese patients abdominoplasty.

Results: Planas technique showed to be faster if compared to traditional technique, less heavy for both surgeon and assistants to per- form, and only minor complications occurred, such as wound dehiscence $(12 \%)$, seroma ( $7 \%$ ) and superficial infection of the wound $(4 \%)$.

Discussion: Planas technique allows faster execution, minor undermining and higher respect of vascularization. This is important particularly in the patients who have undergone bariatric surgery, who often present decreased vascularization of the dermis and scars, which can affected healing of the wound. In these patients, we never associate this procedure to other dermolipectomies, in the same operation. If even orizontal skin laxity is present modification of the incisions must be considered, to widen skin resection, and achieve the proper correction.

\section{3 \\ Employment of Compressive Polyethylene Device for Treatment of Hypertrophic Scars}

\section{S. Gentileschi, R. Bracaglia, R. Fortunato, M. Laineri, G. Lorini, F. Del Monte \\ Department of Plastic and Reconstructive Surgery, \\ Policlinico A. Gemelli, Roma, Italy}

Introduction: Hypertrophic scars are a complex problem to treat and compression, applied during the maturation period, is one of the most believed effective therapy. A good compressive device should be enough soft to be comfortable, but with a sufficiently hard tract, over the scar area, to best perform compression.

Methods: The authors analyze their experience with 32 young patients, presenting a hypertrophic scar of the face, treated by a new kind of brace, made of an elastic structure and a hard part of polyethylene $2 \mathrm{~mm}$ thick. Mean age of the patients was 20 . The treatment was beginned between the first and sixth months, from the healing of the cutaneous wound, and carried on until the scar had become flat and light. The mean period of treatment was 1 , continued during the day.

Results: The scars improved, in all the cases, very significantly, according both to surgeon and patient opinion. The interruption of treatment was decided, in agree with patient, when improvement achieved was judged satisfying.

Discussion: This new device, made of transparent polyethylene and elastic fibres presented the advantage to be light, soft and pliable, if compared with the other kind of braces, particularly with the carbon fibres devices. As the polyethylene is rigid it is possible to create an open elastic structure, leaving covered only a little part of the face, with less discomfort for the patient.

\section{4}

\section{Application of a Albumin - Glutaraldehyde Based Glue in Plastic Surgery}

\section{S. Gentileschi, R. Bracaglia, R. Fortunato}

Department of Plastic and Reconstructive Surgery, Policlinico A. Gemelli, Roma, Italy

Introduction: Bioglue ${ }^{\circledR}$ (Cryolife inc) is a biologic glue, mostly employed for repair of acute aortic dissection. It acts as tissue sealant and prevent bleeding through needles holes. 
Methods: The authors analyze its employ in 4 patients. In 1 case it was used to fix the deep surface of a flap employed to cover the repair of a myelomeningocele to the underlying dura, in a few hours newborn; in 1 case to fix the deep surface of a mucoperiosteal flap to the palatal bone in the correction of a many times operated cleft palate fistula; in 2 cases to fix a dorsal bone graft to the nasal bones, during a secondary rhinoplasty.

Results: In all the 4 cases the glue well succeeded in fixing the tissues very quickly and hardly. No seroma was observed under the flaps, which had no fixation stitches on the deep surface and no drain.

Discussion: Bioglue showed to be very easy to apply, and fast to act. It was useful where stitches were not appliable, such as on meninx and bone. Particularly in the case of the newborn, no stitch or drain could be applied under the flap, in contact with the dura, and Bioglue completely prevented a dead space and seroma formation. Even the palatal flap and nasal bone graft were well stabilized by Bioglue. We believe that it can be particularly useful in those cases where the deep surface of a flap must be fixed to the recipient bed, but stitches are not appliable (truncated at 250 words).

\section{5 \\ Skin Reducing Mastectomy (SRM) and Immediate Reconstruction}

\section{L.M. Lapalorcia, G. Bistoni, S. Chiummariello \\ Department of Plastic and Reconstructive Surgery, University of Perugia, Italy}

Introduction: In 1991 Tooth proposed SRM, a safe treatment for breast cancer. A Wise pattern, inferior pedicle based, breast reduction incision provides good access and opportunity to reshape and improve the breast, avoiding undesired skin retractions and pathologic scarring post-radiotherapy. Immediate placement of Becker 35 implant guarantees adequate volume and projection to the reconstructed breast.

Methods: From 10/2005 to 4/2006 11 patients underwent SRM at Our Institution; mean age was 57, median 58.6. 5 patients had (T1) DCIS (46\%), 4 had infiltrating G1 DC (T2, N1) (36\%), 1 had fibrocystic mastopathy already biopsied twice ( $9 \%$ ), 1 had already undergone radical mastectomy and was treated with reconstruction and symmetrization (9\%). Image studies were performed for the staging. Becker 35 implant was placed in the surgical pocket and symmetrization was performed when required. SLNB was performed.

Results: Aesthetic result was judged satisfactory by Surgeons and patients in $73 \%$ of the cases. Of the remaining (27\%), 1 developed seroma, 1 partial necrosis of the NAC, 1 infection treated with removal of the implant and placement of a new one 7 months later.

Conclusions: Indications are the same as for SSM. Dermis must be free of cancer. Providing a secure procedure with good aesthetic results encouraged us to keep on performing it on selected patients. The experience at our Breast Unit had been encouraging and demonstrated how valuable the cooperation between the Oncologic and the Plastic Surgeon is. Longer follow up is required for monitoring of long term results and recurrence.
466

\section{Role of the Pedicled Myocutaneous Latissimus Dorsi Flap in Reconstruction of the Complex Wounds of the Trunk}

\author{
N. Panizzo', M. Candiani', L. Moretti ${ }^{2}$, G.P. Faini', \\ E.R. Cordaro ${ }^{1}$, P.C. Parodi ${ }^{1}$, C. Riberti ${ }^{2}$ \\ ${ }^{1}$ Department of Plastic Surgery, University of Udine, \\ ${ }^{2}$ Department of Plastic Surgery, University of Ferrara, Italy
}

Introduction: Reconstructive options for managing and coverage of complex wounds are manifold, but in our experience the use of miocutaneous latissimus dorsi flap fulfills the necessity of large superficial loss of substance coverage as well as coverage of the deep ones.

Methods: During the 1995-2005 decade we have treated 32 patients with complex wounds of the trunk of different etiologies (oncologic, traumatic, iatrogenic), all resolved with the use of pedicled latissimus dorsi flap. 20 patients were affected by wounds of the thoracic wall, 8 patients by wounds at the level of the dorsal region and 7 patients presented wounds at the level of scapular girdle. In the $85 \%$ of the cases we prepared the latissimus dorsi flap pedicled on the dominant thoraco-dorsal artery stalk, while in the remaining percentage the vascularization has been granted by the minor pedicles of the flap. In the $19 \%$ of the cases we utilized the flap in its muscular variant.

Results: The minor complications rate $5.7 \%$ with an average healing time of 23 days. 3 patients presented major complications. The thickness of the flap and the textural dorsal skin qualities, matching with the receiving site, allowed us to obtain very satisfying aesthetic results.

Discussion: The Latissimus Dorsi flap represents our first reconstructive choice when considering the management of the complex wounds of the trunk, because allows us to obtain a long lasting coverage, necrotic tissue debridement and a good control of infective processes, thanks to its rich vascular network and good versatility.

\section{7 \\ Tracheostomy Management and Microsurgical Reconstruction of the Oral Cavity. Our Experience with Ring Stitches}

\section{P. Mulas, F. Farace, V.E.E. Fois ${ }^{1}$, C. Rubino \\ Department of Plastic Reconstructive Surgery, University of Sassari, School of Specialization in Plastic \\ Reconstructive and Aesthetic Surgery, 'Department of Otorhinolaryngology}

Introduction: Patients operated for oral cavity cancer and immediate reconstruction with free or pedicled flaps usually undergo elective tracheotomies to avoid respiratory deficiency. Tracheostomy tube keeps open tracheotomy inflating a cuff in its inferior third and avoids secretion diffusion in the inferior airway. Tube management includes periodical post-operative cleaning. External fixation is necessary to prevent cannula expulsion. If the tube comes out, tracheotomy flaps may join together leading a sudden respiratory deficiency. Neckband is often employed to affix tracheostomy tube, but band compression and tissue edema may seriously damage flap pedicle. Stitches avoid 
direct compression, but postoperative management is really painful for patients. We report a modified stitch technique to prevent problems related to neckband and classic stitches: the ring stitches.

Methods: This technique has been employed in 10 pectoralis major, 7 RFFF, 2 fibula and 1 VRAM reconstructions. 4 stitch-loops performed on the skin, 2 for each side of the cannula, under general anaesthesia. Everyone forms a 'buttonhole' in which fixing tracheostomy tube through other ring stitches between buttonholes and tube.

Results: None of the flaps underwent necrosis and no accident connected with tracheostomy happened.

Conclusions: Cannula management is realized removing only the latter stitches, while loops on the skin remain on site. Therefore, we may clean or change the cannula without pain. In fact this technique avoids postoperative pinpricks on the skin and does not press on the pedicle.

\section{8 \\ Wise Pattern Mastectomy Breast Reconstruction: Benefits and Pitfalls}

\section{G. Paolini, G. Cotroneo, M. Angelini, G. Volpes, C. Amanti, F. Santanelli \\ Sant'Andrea Hospital-II Faculty-University La Sapienza of Rome, Rome, Italy}

Introduction: Wise pattern mastectomy is the gold standard technique in macromastia patients requiring skin sparing mastectomy for breast cancer, because of its optimal reconstructive potential. We review our series in order to highlight risks and benefits.

Materials and Methods: In 2 years activity 17 among 150 treated patients, underwent 22 wise pattern mastectomies with immediate reconstruction and controlateral reduction, for 14 infiltrating ductal and 8 lobular carcinomas. Average age was 47.2 years. Nineteen implants, two expanders and 1 Diep flap were used for reconstruction. Average operative time was $3.1 \mathrm{~h}$. The NAC was preserved on a thin dermal pedicle 16 times, sacrificed 5 times and transplanted as a free graft one time. Follow-up is 14 months.

Results: Overall success rate was 64\%. Complications (36\%) were 3 total and 2 partial NAC necrosis, and 3 partial skin necrosis. 4 cases underwent skin revision ( 2 bilateral implants substituted by expanders). 1 patient was reoperated for cancer occurrence in contralateral reduced breast. Patient and surgeon satisfaction were respectively 8 and 7.5 according to VAS scale.

Discussion: The wise pattern mastectomy reconstruction is a satisfactory technique, because of optimal lower pole fullness, skin envelope adaptation and easy symmetry match. Is anyway hampered by a high chance of mastectomy skin flaps necrosis with risk of implant exposure. To increase success good cooperation with senologist, careful handling of mastectomy and dermal flaps and intraoperative evaluation of NAC viability are mandatory.
469

\section{Infections in Plastic and Reconstructive Surgery: The S'Andrea Hospital Experience}

\section{G. Paolini, S. Giordano, B. Longo, L. Renzi, F. Santanelli}

Sant'Andrea Hospital-II Faculty, University La Sapienza of Rome, Rome, Italy

Introduction: Currently no guidelines on antibiotic treatment in plastic surgery exist and it is abused. To verify our practice a prospective study on infections incidence was performed.

Materials and Methods: Clinical profile (ASA status), risk factors (Obesity, diabetes, etc), etiology (41\% bariatric, 30.4\% oncologic, $12 \%$ traumatic, $16.6 \%$ miscellanea), procedure $(19.4 \%$ breast reductions, $17.3 \%$ abdominoplasties, $9.2 \%$ breast reconstructions, $7.1 \%$ free flaps, $3.9 \%$ rhinoplasties, $43.1 \%$ miscellanea), operative time ( $2 \mathrm{~h}$ ), drainage usage ( $42 \%$ ) and complications of 283 patients (72.4\% females, $27.6 \%$ males, mean age 46 years) undergoing surgery were recorded. Antibiotics were given intravenously from before surgery until discharge (on 1st-2nd day) and usually passed orally for five days. Contaminated wound discharge diagnosed infection. F-up was 8 months. T-Student test analysis was performed.

Results: Overall infection rate was $2.47 \%$. Male-female ratio was $3 / 4$, risk factors present in $86 \%$. Aetiology ( 3 bariatric, 3 oncologic, 1 trauma), procedures ( 4 free flaps, 3 addominoplasties), operative time (6h), discharge (24 days), and isolated germs (4 S. Epidermidis and MSSA and 3 MRSA) of infected cases were the abovementioned. Longer operative time was the only factor positively correlated $(\mathrm{p}<0.05)$ to higher infection incidence.

Discussion: The low infection incidence may be due to prevalence of elective surgery and residential saprophite germs. The positive correlation to operative time persuaded us to adjust our antibiotic protocol according to the type of the procedure more than to patient profile.

\section{0}

\section{An Unusual Flap for the Reconstruction of Nasal Tip Defects}

S. Ricci, G. Franco Guarneri, P.C. Parodi, F. De Biasio, F. Consiglio

Unit of Plastic and Reconstructive Surgery, University of Udine

Introduction: Aesthetic reconstruction of the nasal tip defects is still a challenge for the surgeon. The tip is the aesthetic focal subunit of the nose and the commonly used flaps for repair, frequently esitate in evident scars, irregularities in colour and texture and distortion of the nasal contour that can compromise the aesthetic outcome.

Methods: The authors suggest a modified nasalis flap that is elevated including the nasalis muscle and preserving the angular artery. It can be mono or bipedicled and the donnor site is closed with a V-Y flap or with a interpositional flap from the lateral alae.

Results: All patients treated with the modified nasalis flap had good results with any complications. In some patients treated with the other techniques the authors have noted partial flap necrosis, distortion 
of the nasal tip and visible scars in the donor site with poor aesthetic results.

Discussion: This flap offers a valid alternative for reconstruction of central and lateral nasal tip and supratip defects of small to moderate size up to $2 \mathrm{~cm}$ in diameter. It is easy and fast to execute and gives good aesthetic results with scars well concealed, nasal contour minimally altered and excellent colour and texture matches.

\section{1}

\section{A Case of Reconstruction of the Axillary Region Using the Toracodorsal Perforator Flap in a Patient Affected by Metastatic Melanoma}

\section{Gelati, L. Fabiocchi, G. Giannini, G. Zannetti, R. Cipriani \\ Chirurgia Plastica, Policlinico S Orsola, Bologna, Italy}

Introduction: Nowadays the use of perforator flaps to repair big defects after tumor resection is spread out all over the world. We would like to discuss the advantages and disadvantages of the thoracodorsal perforator-based flap in a 50 years patient, affected by metastatic melanoma. This patient presented an axillary metastasis of $18 \times 15 \mathrm{~cm}$. We repaired the loss of substance by using the toracodorsal perforator flap.

Materials: After tumor resection, a cutaneous flap was prepared on the muscolocutaneous perforators arising from the lateral branch of thoracodorsal artery. The flap was harvested in range of size up to as large as $20 \times 15 \mathrm{~cm}$.

Results: The flap survived completely without even marginal necrosis. Cosmetic results were satisfactory from the patient's point of view. The improvement in range of motion was even satisfactory if compared to other surgical techniques of repair such as skin graft.

Discussion: The thoracodorsal perforator flap can be safely raised to meet any size required even in the most severe defects. The donor site scar may be considered as acceptable considering the functional advantage gains.

\section{Endoscopic Surgery}

\section{2 \\ New Endoscopic Technique for Intragastric Balloon Removal}

G. Diamantis, L. Magno, L. Sivero, P. lovino, G. Galloro

Dip. di Chirurgia Generale, Geriatrica, Oncologica and

Tecnologie avanzate, Area di endoscopia digestiva

operativa, AUP Federico II, Napoli

Introduction: Placement and removal of intragastric balloon for obesity are performed endocopically often in general anaesthesia. We propose a new safer and faster technique for endoscopic removal of intragastric balloon during standard sedation.

Methods: In 87 obese patients we performed three removal techniques: by standard gastroscope and foreign body forcep, by standard gastroscope and retrival snare, by double channel gastroscope and foreign body forcep plus symmetrical polipectomy snare model shark. Balloon retriving time, number of times the grasping devices loosed the balloon, amount of antispastic drug, symptoms cumulative score and VAS score for discomfort were evaluated.

Results: The technique by double channel gastroscope and foreign body forcep plus symmetrical polipectomy snare model shark shows a significantly lower balloon retriving time, number of loosed balloons, total number of ampoules used, symptoms cumulative score and VAS score compared to the other two (Dunn's $p<0.05$ ). The number of loosed balloons was positively associated with number of antispastic ampoules used, balloon retriving time and VAS score.

Discussion: The technique by double channel gastroscope and foreign body forcep plus symmetrical polipectomy snare model shark, allows balloon removal safely, quickly and easily avoiding to loose the balloon with a good endurance from patients.

\section{3 \\ Schistosomal Colonic Lipoma-Like Lesion Treated with Endoscopic Mucosal Resection \\ G. Diamantis, L. Magno, L. Sivero, P. Iovino, G. Galloro \\ Dip. di Chirurgia Generale, Geriatrica, Oncologica e \\ Tecnologie avanzate, Area di endoscopia digestiva operativa, AUP Federico II, Napoli}

Introduction: The polyp-like lesions of the colon are not usual in the infestation from Schistosoma mansoni. Clinical manifestations are relatively rare in relationship of the duration and the intensity of the infestation.

Methods: A 52-year-old woman, complaining of intermittent abdominal pain on the right lower quadrant, abdominal bloating and alternating bowel habit, was referred from her family practitioner to our unit of surgical digestive endoscopy to perform a colonoscopy. The endoscopic examination revealed an intramural lesion with $1.5 \mathrm{~cm}$ diameter in the ascending colon. This lesion seem to be 
covered by an apparent normal mucosa, moderately yellowish, without bleeding stigmata, presenting a positive 'pillow sign' at the touch of the closed biopsy forceps, tipical of the lipoma-like lesions. Endoscopic mucosal resection 'en block' of the lipoma-like lesion, was successfully performed by polipectomy flat snare.

Results: Histopathologic evaluation revealed granulomatous inflammation around a single schistosomal ova. Further questioning determined that the patient had lived for 1 year in Brasil a few months before the arising of the clinical symptoms. She was treated with praziquantel $(20 \mathrm{mg} / \mathrm{kg})$ in an effort to destroy any viable eggs.

Discussion: We report an unusual case of schistosomal colonic lipoma-like lesion treated with endoscopic mucosal resection.

\section{4 \\ Right Adrenalectomy with Retroperitoneoscopic Approach for HCC Metastasis in a Living Related Liver Transplant Patient}

\author{
L. Mandalà', P. Marchesa' ${ }^{1}$ D. Cintorino' ${ }^{1}$, S. Gruttadauria1, \\ M. Spada', R. Verzaro'1, M. Spampinato', E. Luna1, \\ P. Mezzatesta2 , G. Barranco², G. Vizzini', A. Arcadipane1, \\ M. Ida Minervini ${ }^{1}$, C.S. Foglieni ${ }^{1}$, B. Gridelli ${ }^{1}$ \\ ${ }^{1}$ Istituto Mediteranneo per i Trapianti e le Terapie ad alta \\ Specializzazione (IsMeTT), UPMC, ${ }^{2}$ Ospedale Civico, Italy
}

Introduction: HCC metastasis is a well known long-term complication after living donor liver transplantation. We present a right adrenalectomy with retroperitoneoscopic approach in a living related liver transplant recipient for an HCC metastasis.

Methods: A 58-year-old gentleman with a liver $\mathrm{HCC}$ on $\mathrm{HCV}$ related cirrhosis, underwent a living related liver transplant with no post-surgical complications in May 2003. A pathology exam showed two HCC nodules in the native liver. Two years later an abdominal CT scan showed a $10 \mathrm{~mm}$ solid lesion in the right adrenal gland. In 6 months the lesion volume increased to $22 \mathrm{~mm}$. A retroperitoneoscopic right adrenalectomy was performed in September 2005. The patient was placed in prone Jacknife position and three trocars were used in the right lumbar space.

Results: Operative time: $3 \mathrm{~h}$; hospitalization time: 3 days. No post-operative complication was occurred. The pathology of the specimen showed metastatic hepatocellular carcinoma with the following pattern of special stains: Hep Par strongly positive; CAM 5.2 focal rare tumor cells positive; $\mathrm{p}$ CEA positive with canalicular pattern. Six months later, a total body CT scan did not show trocar site metastasis or retroperitoneal recurrence.

Discussion: We recommended the retroperitoneoscopic approach in selected cases and for patient with previous major abdominal surgery.

\section{5}

\section{Self-Expandable Metallic Stents in Acute Colon-Rectal Occlusions: Personal Experience}

\section{Pacilè, E. Piazzese, A. Bonsignore, F. Fiumara, M. Licursi, L.G. Angiò}

Università degli Studi di Messina, Scuola di

Specializzazione Chirurgia Generale I

Introduction: Employment of self-expandable metallic stents (SEMS) in the acute colon-rectal occlusions (ACO) it represents a valid therapeutic alternative that allows the general riequilibrium of patient and rapids decompression of bowel; it also favors the appeal to a surgical approach in election improving the results in terms of mortality and morbidity.

Patients and Methods: From 1997 to 2004, 21 patients with ACO ( 20 for $\mathrm{K}$ colon left, 1 for results of ischemic colitis post-resection of sigma), have been treated through positioning of SEMS to purpose palliative in 5 cases $(23.8 \%)$ and as bridge to surgery in $16(76.2 \%)$.

Results: 16 patients, after 4-6 days positioning of SEMS, colic resection have been submitted to in once with a post-operating hospitalization of 5-9 days, a morbidity of $6.5 \%$ and $0 \%$ mortality. During the procedure as complication is recorded 1 colic perforation, 1 occlusion (fecal impact), 1 hemorrhage and 2 SEMS migration.

Conclusion: Employment of SEMS represents a valid therapeutic option for the treatment of ACO operable (bridge to surgery) and not operable (palliative), with a technical-clinical success varying from 64 to $93 \%$ of cases. This procedure is characterized by a varying risk of complication $(0.5-50 \%)$, correlated to the technique, to the experience of operator, to the devices type and the center of obstruction: they owe to be used therefore only in presence of correct indication.

\section{6 \\ Biliary Stents: Technical Considerations and Indications \\ C. Luigiano, V. Pacilè, E. Piazzese, L.G. Angiò, C. Famulari \\ Università degli Studi di Messina, Scuola di \\ Specializzazione in Chirurgia Generale I}

The use of biliary stents has increased significantly during the last 2 decades and has increased the indications for stent therapy in biliary diseases. Plastic stents were introduced in 1979 and are widely applied in the management of malignant and benign biliary disorders. Stents are available in various material (Polyethylene, Teflon), size (5-12 Fr), shape (straight, curved, single or double-pigtail) and length $(4-15 \mathrm{~cm})$. In general, plastic stents are placed using a pusher tube over a guidewire with or without a guiding catheter. Delivery systems are now available for plastic stents that combine the guiding and pusher catheters or eliminate the need for a guiding catheter. Self-expandable metal stents (SEMS) were introduced in clinical practice in 1989. Different types of SEMS are available, all these stents are assembled over a 7.5-12 French delivery catheter; SEMS are composed of either stainless steel or nickel shape-retaining titanium (Nitinol), are covered 
and un-covered and are available in various size $(6-10 \mathrm{~mm})$ and length (4-8 cm). Malignant biliary obstruction (pancreatic and biliary neoplasms), benign biliary strictures (chronic pancreatitis or postoperative injuries), primary sclerosing cholangitis (treatment of strictures with clinical and biochemical improvement), postoperative bile leaks (reducing the transpapillary pressure gradient), biliary complications after OLT (bile leaks after T-tube removal and biliary strictures) and biliary stones (patients who cannot undergo sphincterotomy and/or complete stone extraction and in selected high-risk patients with unextractable stones). Biliary stenting is an established method for the management biliary disorders with reduction of mortality, morbidity and hospitalization if compared to surgery.

\section{Endocrine Surgery}

\section{7}

\section{Metastases to the Thyroid Gland: Report of 12 Cases}

F. Calzolari ${ }^{1}$, P. Sartori ${ }^{2}$, C. Talarico ${ }^{3}$, D. Parmeggiani, G. Bovo ${ }^{4}$, P. Sperlongano ${ }^{5}$, M. Monacelli ${ }^{1}$, C. Misso ${ }^{1}$, M. d'Ajello $^{1}$, F. Uggeri' ${ }^{2}$ N. Avenia ${ }^{1}$

${ }^{1}$ Area Funzionale Omogenea Interaziendale di Endocrinochirurgia del Collo e Tessuti Molli, Regione Umbria, ${ }^{2}$ Dipartimento Chirurgico, Ospedale S. Gerardo di Monza Università degli Studi Milano Bicocca, ${ }^{3}$ Divisione di Chirurgia Generale, Ospedale San Biagio, Chiaravalle C.le, Catanzaro, ${ }^{4}$ Servizio di Anatomia Patologica, Ospedale S. Gerardo di Monza Università degli Studi Milano Bicocca, ${ }^{5}$ Dipartimento di Scienze Anestesiologiche, Chirurgiche e dell'Emergenza, V Divisione di Chirurgia Generale e Tecniche Chirurgiche Speciali, Seconda Università degli Studi di Napoli, Italy

Introduction: Management of intrathyroid metastases (MTST) is unclear. We retrospectively reviewed clinical data of patients with metastatic disease to the thyroid gland.

Methods: Between January 1995 and December 2005, 7,957 patients were operated for thyroid pathologies. The 12 patients with MTST $(0.15 \%)$ had a mean age of 68 (range 24-72). The primary tumours were: renal $(n=5)$, colon $(n=3)$, lung $(n=2)$, melanoma $(\mathrm{n}=1)$. In one patient no primary site was detected. The surgical procedures were: total thyroidectomy $(\mathrm{n}=8)$, thyroid lobectomy $(\mathrm{n}=2), \operatorname{MRND}(1)$, lymphadenectomy $(\mathrm{n}=3)$, tracheotomy $(\mathrm{n}=1)$, tracheal stent $(\mathrm{n}=1)$.

Results: The procedures were curative in 9 and palliative in 3 patients. Complete macroscopic resection $(\mathrm{R}=0)$ was achieved in 6 patients, positive margins resection (R1) in 2 patient and incomplete macroscopic resection (R2) was registered in 2 patients. Multifocality of MTST occurred in $57 \%$ of patients. 8 patients died of their disease after 5 years, 1 patient is alive without disease, 1 patient is alive with disease and we lost the follow-up in 2 patients. The mean follow-up was 2.8 years.
Discussion: MTST should be considered in all patients who had cancer history (especially renal cancer) and FNAB should be always considered. When MTST appear isolated, total thyroidectomy should be considered because incidence of the multifocality $(57 \%$ in our series).

\section{8 \\ Non-Invasive Adrenal Imaging in Primary Aldosteronism \\ C. De Crea, C.P. Lombardi, M. Raffaelli, V. Rufini, E. Traini, L. Sessa, G. Treglia, R. Bellantone \\ Division of Endocrine Surgery and Institute of Nuclear Medicine, Università Cattolica del S. Cuore, Rome, Italy}

Introduction: Bilateral adrenalectomy rarely cures bilateral idiopathic hyperaldosteronism (IHA), while adrenalectomy usually results in biochemical cure of patients with aldosterone-producing adenomas (APA) and unilateral primary adrenal hyperplasia (PAH). Adrenal venous sampling (AVS) has been advocated as an essential tool in differentiating unilateral from bilateral aldosterone hypersecretion, although invasive procedure with variable success rate. We reviewed the reliability of noninvasive preoperative imaging studies in our experience.

Methods: Demographic, clinical characteristics and follow-up of 50 consecutive patients with PA were evaluated. Outcome after adrenalectomy was determined biochemically and clinically.

Results: All the patients underwent CT scan. 35 patients underwent adrenalectomy for APA, basing on the CT scan results, and were biochemically cured. Blood pressure improvement rate was $97 \%$. Fifteen patients underwent dexamethasone suppression adrenal cortical scintiscan (ACS). Eleven of these prosecuted medical treatment because of a bilateral uptake. Adrenalectomy was performed in the remaining 4 patients with unilateral uptake, with histology of APA in 3 and PAH in 1 . All these patients were cured with blood pressure improvement. The overall accuracy of combined noninvasive imaging procedures was $100 \%$.

Discussion: Noninvasive adrenal imaging studies accurately differentiates unilateral and bilateral aldosterone hypersecretion. AVS should be indicated when preoperative non-invasive imaging studies do not localize aldosterone hypersecretion.

\section{9}

Parathyroid Hormone Level $4 \mathrm{H}$ after Surgery and Post-Thyroidectomy Hypocalcemia: A Critical Appraisal

\author{
A.M. D'Amore ${ }^{1}$, C.P. Lombardi ${ }^{1}$, M. Raffaelli ${ }^{1}$, P. Princi ${ }^{1}$, \\ C. Dobrinja1, C. Carrozza ${ }^{2}$, C. Zuppi ${ }^{2}$, R. Bellantone ${ }^{1}$ \\ ${ }^{1}$ Division of Endocrine Surgery, ${ }^{2}$ Institute of Biochemistry, \\ Università Cattolica del S. Cuore, Rome, Italy
}

Introduction: In a small series of patients, iPTH levels $4 \mathrm{~h}$ after surgery $(4 \mathrm{~h}-\mathrm{iPTH})$ seemed the most accurate and earliest predictor. 
Aim of this prospective study was evaluating its reliability as predictor of hypocalcemia in a larger series of patients.

Methods: 523 consecutive patients who underwent total thyroidectomy (TT). Serum iPTH was measured $4 \mathrm{~h}$ after the end of surgery and serum calcium in the first and the second postoperative days. iPTH levels were correlated to hypocalcemia and symptoms. Hypocalcemia was defined as serum calcium $<8.0 \mathrm{mg} / \mathrm{dl}$, even if in one single measurement.

Results: 191 patients developed hypocalcemia. Five patients were still under vitamin D/oral calcium 6 months after surgery. 73 patients experienced slight symptoms. $4 \mathrm{~h}$-iPTH levels were significantly reduced in hypocalcemic patients $(28.8 \pm 15.3$ vs. $11.2 \pm 11.6$ $\mathrm{pg} / \mathrm{ml})(\mathrm{P}<0.001) .4 \mathrm{~h}$-iPTH was within the normal range $(10-65$ $\mathrm{pg} / \mathrm{ml}$ ) in 332 patients (299 normocalcemic) and subnormal in the remaining 191 (121 hypocalcemic, of whom 62 were symptomatic). Overall accuracy of $4 \mathrm{~h}$-iPTH levels below the normal range $(<10$ $\mathrm{pg} / \mathrm{ml}$ ) in predicting post-TT hypocalcemia and symptoms was $80.3 \%$ and $73.2 \%$, respectively. False negative results were observed in 33 hypocalcemic patients $(6.3 \%), 11$ of whom symptomatic $(2.1 \%)$. All the symptomatic patients had a $4 \mathrm{~h}-\mathrm{iPTH}$ level $<20 \mathrm{pg} / \mathrm{ml}$.

Discussion: $4 \mathrm{~h}$-iPTH below the normal range alone is not accurate in predicting clinically relevant postoperative hypocalcemia. The definition of new cut off levels and the integration with pre- and postoperative serum calcium levels should be investigated.

\section{0 \\ Intraoperative PTH Monitoring: Is it Possible to Improve the Accuracy?}

\author{
E. Traini ${ }^{1}$, C.P. Lombardi ${ }^{1}$, M. Raffaelli ${ }^{1}$, E. Di Stasio ${ }^{2}$, \\ C. Carrozza ${ }^{2}$, C. Zuppi ${ }^{2}$, L. Oragano ${ }^{1}$, R. Bellantone ${ }^{1}$ \\ ${ }^{1}$ Division of Endocrine Surgery, ${ }^{2}$ Institute of Biochemistry, \\ Università Cattolica del S. Cuore, Rome, Italy
}

Introduction: The quick parathyroid hormone assay (QPTHa) is a useful tool to verify the adequacy of resection during parathyroidectomy for primary hyperparathyroidism (pHPT). The accuracy in predicting cure is related to blood sample timing and the criteria for interpretation of the results. The Miami criterion (MC) (intact PTH drop $\geq 50 \%$ from the highest of either pre-incision or pre-excision level at $10 \mathrm{~min}$ after gland excision) has been demonstrated to be the most accurate, even if characterized by some false positive (FP) results. In this prospective study we evaluated the possibility to improve the specificity and the overall accuracy of the QPTHa.

Methods: Seventy one consecutive patients with pHPT selected for minimally invasive parathyroidectomy were prospectively evaluated. PTH was measured before the incision and the excision, 5 (T5), 10 (T10), 20 (T20) and 30 (T30) minutes after the gland excision. A kinetic analysis and a mathematical model for PTH decay interpretation were constructed. The established criteria were compared with the $\mathrm{MC}$ in predicting operative outcome.

Results: The MC correctly predicted outcome in 65/71 patients $(91.5 \%)$. Kinetic analysis showed that a $\geq 50 \%$ drop from the highest pre-excision value at $10 \mathrm{~min}$ after parathyroidectomy combined with the difference $(\Delta)$ between the T10 and T20 levels allows for the most accurate prediction of cure. Overall accuracy of this model was $98.6 \%$.
Discussion: Extending the sampling period and evaluating the $\Delta$ between the post-excision samples (T10 and T20) result in a lower FP rate and a higher overall accuracy when compared with the MC.

481

Video-Assisted Thyroidectomy for Papillary Thyroid Carcinoma: Seven Years Experience

\author{
P. Princi, C.P. Lombardi, M. Raffaelli, C. De Crea, \\ A.M. D'Amore, D. Maccora, R. Bellantone \\ Division of Endocrine Surgery, Università Cattolica del \\ S. Cuore, Rome, Italy
}

Introduction: In spite of some concerns, video-assisted thyroidectomy (VAT) has been proposed also in case of small papillary thyroid carcinoma (PTC). Aim of this study was to evaluate the completeness of the surgical resection in patients underwent VAT for PTC.

Methods: Among 583 patients who underwent VAT between June 1998 and November 2005, 198 showed at final histology a PTC and were included in the study.

Results: There were 171 women and 26 men with a mean age of $43.4 \pm 12.9$ years. 212 video-assisted procedures were successfully accomplished. In 16 cases a video-assisted central neck node clearance was accomplished. Final histology showed central neck lymph node metastases in 16 patients. Postoperative complications were 4 transient recurrent nerve palsies, 45 transient hypocalcemias, 3 permanent hypoparathyroidisms, 1 post-operative haematoma. The cosmetic result was excellent. Follow up has been completed in 124 patients with a mean length of $22.2 \pm 16.4$ months (range 4-65). Mean postoperative serum thyroglobulin was $0.1 \pm 0.4 \mathrm{ng} / \mathrm{ml}$ on LT4 suppressive treatment. Postoperative ultrasound showed no residual thyroid tissue in all patients. Mean RAIU at postoperative scintiscan was $2.4 \pm 4.7 \%$. One patient underwent lateral neck node dissection because of jugular lymph node recurrence 2 years after VAT.

Discussion: VAT is feasible and safe in case of PTC. The completeness of the surgical resection seems comparable to that reported for conventional surgery. VAT should be considered a valid option for the treatment of small PTC and could be proposed to low risk patients, at least in selected referral Centres.

\section{2 \\ Video-Assisted Central Neck Lymph Node Dissection for Papillary Thyroid Carcinoma}

\author{
M. Raffaelli', C.P. Lombardi ${ }^{2}$, P. Princi ${ }^{2}$, A.G.S. Ibarrola², \\ M. Salvatori ${ }^{1}$, P. Castaldi ${ }^{1}$, C. Contini ${ }^{2}$, R. Bellantone ${ }^{2}$ \\ ${ }^{1}$ Institute of Nuclear Medicine, ${ }^{2}$ Division of Endocrine \\ Surgery, Universitá Cattolica del Sacro Cuore, Rome, Italy
}

Introduction: Video-assisted thyroidectomy (VAT) has been proposed for small Papillary Thyroid Carcinoma (PTC). We attempted to remove central neck lymph nodes unexpectedly found enlarged during VAT for PTC. Herein we report our experience of 
video-assisted lymph nodes dissection $\quad($ VALD $=$ video-assisted lymph node dissection).

Methods: 250 patients underwent VAT for PTC. Among them, 78 patients underwent concomitant VALD. Only macroscopically enlarged lymph nodes were removed in 78 patients. In 16 patients a complete video-assisted central compartment neck dissection (VACCD) was performed

Results: There were 69 women and 9 men with a mean age of $42.2 \pm 13.8$ years (range: $19-71$ ). Mean number of removed lymph nodes in case of VA-CCD was $8 \pm 3.5$ (range: 6-18). The following complications were registered: 6 transient and 3 definitive postoperative hypocalcemias, one transient recurrent nerve palsy. Final histology showed lymph node metastases in 16 patients. Mean follow up was $17.9 \pm 10.6$ months (range: $2-40$ ). Mean postoperative serum thyroglobulin on LT4 was $<1 \mathrm{ng} / \mathrm{ml}$ and postoperative ultrasound didn't show residual thyroid tissue in all the patients. The mean preablation radio-iodine up-take (RAIU) was $1.4 \%$ (range: $0.1-6.8$ ). All the patients considered the cosmetic result excellent. One patient underwent lateral neck node dissection because of jugular lymph node recurrence two years after VAT.

Discussion: In our experience VA-CCD is feasible and safe, with results comparable to those of conventional surgery. Nevertheless, larger series and longer follow-up are necessary to definitively validate this procedure.

\section{3 \\ Total Parathyroidectomy with Subcutaneous Autotrasplantation for Seconday Hyperparathyroidism}

M.G. Esposito, F. Stanzione, A. Palazzo, U. Brancaccio,

S. Celsi, G. Conzo, A. Livrea

Fourth Division of General Surgery and Endocrinos, Second University of Naples, Italy

Objective: The Authors have evaluated medium long term results of surgical treatment of 60 patient operated on of total parathyroidectomy (PTXt) with and without subcutaneous Autotransplantation for Secondary Hyperparathyroidism (HPTs).

Materials and Methods: From January 1999 to March 2006, 60 patient were submitted to PTXt (29 females and 31 males meanage 67 years, mean age haemodialysis 11 years), 37/60 patient with transplantation of autologus parathyroid tissue into the forearm subcutaneous tissue. Surgical indications according to Kidney Disease Outcomes Quality Initiative (K/DOQI) guidelines included: persistent serum level of iPTH $>800 \mathrm{pg} / \mathrm{ml}$; associated with hypercalcaemia $>10 \mathrm{mg} / \mathrm{dl}$; and/or hyperphosphatemia $>6.5 \mathrm{mg} / \mathrm{dl}$. Pre-surgical work-up included: serum iPTH, calcium, phosphorus, and alkaline phosphatases; FT3, Ft4, TSH, calcitonin, tireoglobulin, thyroid and parathyroid Ultrasound with color power doppler, Scintigraphy 99 Tc sestamibi; laryngoscope examination. Preoperative iPTH levels ranged $650-3,000 \mathrm{pg} / \mathrm{ml}(10-65) \Sigma 1500.00$, blood calcium ranged $10.04-12.05 \mathrm{mg} / \mathrm{dl}(8.1-10.4) \quad \Sigma 10.20$; phosphorus ranged $4.8-6.7 \mathrm{mg} / \mathrm{dl}$ (2-4); $\Sigma 6.5$; ALP ranged 210-1516 U/1 $(91-258) \Sigma 750.00$. Persistent itching was present in 60/60; joint pains in $56 / 60$; spontaneous bone fracture in $6 / 60$; fatigue in $58 / 60$; cardiovascular diseases: 48/60; gastric diseases 24/60. Evaluating age, symptoms, and mean age of dyalisis 37/60 patients underwent PTXt and subcutaneous forearm transplantation of parathyroid tissue; 21/60 PTXt and 2/60 subtotal PTX.

Results: 4 parathyroid glands were found in 47/60 patient; 9 glands in 1/60; 3 glands in 3/60. Postoperative normal PTH range was observed in 51/60 patient; hypoparathyroidism in 4/60, while in $5 / 60$ a persistent hyperparathyroidism was present. At follow-up (range-time 6-36 months) blood levels of bilateral iPTH showed higher gradient $(5-10 \mathrm{pg} / \mathrm{ml})$.

Conclusions: PTXt with transplantation of autologus parathyroid tissue represents usefulness surgical treatment of HPTs. Subcutaneous tissue represents a good side of implantation for the increase of the parathyroid tissue and eventually for removing in local anaesthesia.

\section{4 \\ Comparison between Thyroidectomy and Hemithyroidectomy in Treatment of Thyroid Nodules Identified as Follicular Lesions by Fine-Needle Aspiration}

\author{
I.J. Fernandez, M.C. Raimondi, O. Cavicchi, O. Piccin, \\ A.R. Ceroni \\ ENT Department, Sant'Orsola Hospital, Bologna \\ University, Italy
}

Introduction: 'Follicular lesions' of the thyroid represent the grey area of FNAB diagnosis and require an histological examination. The initial surgical approach for solitary nodules identified as follicular lesions could be either total thyroidectomy (TT) or hemithyroidectomy (HT), two procedures that have a different morbidity rate.

Methods: Patients with cytological diagnosis of 'follicular lesion' who underwent thyroid surgery in the ENT Department of Sant'Orsola Hospital, over a 5 years period (2001-2006), were selected retrospectively. FNAB diagnosis were compared with histological diagnosis, in order to identify TT that were an over-treatment, and HT that require a further operation, that represent an under-treatment.

Results: 39 of 80 patients $(48.75 \%)$ had a malignant lesion. HT were performed in 44 patients $(55.0 \%)$ and a TT in $36(45.0 \%)$. Postoperative morbidity was present in 10 patients $(27.7 \%)$ who underwent TT ( 1 recurrent nerve palsy, 7 transitory ipoparathyroidism, and 2 post-operative bleeding), in 1 patient $(2.2 \%)$ who underwent HT ( 1 recurrent nerve palsy) and in 2 patients $(15.7 \%)$ that need further operation (1 transitory ipoparathyroidism, and 1 transitory recurrent nerve palsy).

Discussion: Morbidity related to HT was lower than morbidity of TT. Additionally we observe that HT was an under-treatment in $29.5 \%$, while TT an over-treatment in $44.4 \%$. However $\chi^{2}$ test analysis does not demonstrate a statistical relevant difference between these procedures. Because of the low morbidity, the high rate in which HT is a sufficient treatment, and the low rate of re-operation morbidity, we advocate HT as the initial surgical approach for 'follicular lesions'. 


\section{5}

\section{Preoperative Embolization of Thyroid Arteries in a Patient with Large Non-Hodgkin Thyroid Lymphoma}

\section{G. Galatà ' , F. Rulli ${ }^{1}$, M. Villa ${ }^{1}$, M. Grande ${ }^{1}$, A.M. Farinon ${ }^{1}$, G. Simonetti ${ }^{2}$ \\ ${ }^{1}$ Department of Surgery, ${ }^{2}$ Department of Radiology, Tor Vergata University Hospital, Rome, Italy}

Introduction: Primary thyroid lymphoma is a rare disease. It can be defined as a lymphoma that arise from the thyroid gland and usually is of the non-Hodgkin type that can be further divided into indolent cell and aggressive types. Treatment of thyroid non-Hodgkin lymphoma (TNHL) is based on a combined-modality of chemotherapy and radiotherapy. Surgery is utilized in cases of operable disease so the minimal disease is present before the combined-modality therapy.

Methods: We herein report a case of a 61 years old man affected by an aggressive type of TNHL presenting with a large goiter with extension to the mediastinum and compression of the trachea causing severe dyspnea, dysphagia and stridor. Because of the extent of the goiter and furthermore the potential of significant blood loss in an attempt to reduce the goiter size and minimize surgical risks, preoperative embolization was performed six days before surgery under conventional angiography.

Results: This procedure allowed a significant reduction in blood perfusion to the gland which resulted in a decrease on the size of the goiter facilitating surgical removal of the gland.

Conclusion: We consider preoperative embolization of thyroid arteries a successful and feasible procedure and could be considered as a primary step before surgical and chemo-radiotherapy treatment in the case of aggressive TNHL.

\section{6 \\ Parathyroid Cancer and Atypical Parathyroid Adenoma: Intra-Operative Diagnosis and Treatment}

\author{
G. Ippolito', F.F. Palazzo', F. Sebag', C. De Micco², \\ J.F. Henry
}

${ }^{1}$ Department of Endocrine Surgery, La Timone University Hospital, ${ }^{2}$ Department of Pathology, Nord Hospital, Marseille, France

Introduction: The differentiation between parathyroid cancer (PC) and atypical parathyroid adenoma (APA) is difficult at the time of surgery. The aim of this study was to determine whether parathyroid tumours classified as PC or APA have a different clinical patterns and long term outcomes.

Methods: A retrospective review of suspicious or malignant parathyroid tumours treated between 1974 and 2005 was performed. We defined PC as a lesion with vascular or adjacent tissue invasion. APA was defined as neoplasm showing the presence of fibrous bands, mitotic figures, trabecular growth and nuclear atypia.

Results: Twenty-seven cases of suspicious or malignant parathyroid tumours were identified. After histological review, diagnosis of
PC was confirmed in 11 patients and 16 tumours were classified as APA. In most cases the clinical presentation and operative findings of PC and APA were indistinguishable. In PC group, at initial surgery 7 patients underwent an en-bloc resection (BR) with recurrent nerve (RLN) sacrifice in one case and 4 patients had parathyroid tumour resection (TR) only. Four of the 7 patients that underwent an BR had recurrences and died of hypercalcemia. No recurrences were observed in the other 7 patients with a median follow-up of 65 months. In APA Group, 8 patients had an BR with RLN sacrifice in 3 cases and 8 patients had TR alone. There was no recurrence with a median follow-up of 91 months.

Discussion: Operative findings of presumed malignancy are not always reliable in differentiating APA from PC. Without evidence of macroscopic local invasion and the value of ER during initial surgery is debateable.

487

\section{A Single Institution 25 Year Review of True Parathyroid Cysts}

G. Ippolito', F.F. Palazzo1, F. Sebag', M. Sierra1, C. De Micco' ${ }^{2}$, J.F. Henry ${ }^{1}$

${ }^{1}$ Department of Endocrine Surgery, La Timone University Hospital, ${ }^{2}$ Department of Pathology, Nord Hospital, Marseille, France

Introduction: Parathyroid cysts are rare and their origin is a subject of debate. They have been described as either functional causing hyperparathyroidism or non functional in eucalcaemic patients.

Methods: We have performed a 25 year departmental review of parathyroid cysts. Features studied included the clinical presentation, intra-operative findings and an histological review was performed. Cases of cystic degeneration of parathyroid adenomas and pseudocystic change were excluded.

Results: Over 25 years 22,009 thyroidectomies and 2,505 parathyroidectomies were performed in our department. Amongst these 38 non functional parathyroid cysts were documented in 37 patients. The mode of presentation included incidental findings on routine chest X-ray, compressive symptoms or an asymptomatic palpable neck mass. Aspiration was the initial treatment in 14 patients and was curative in 10 of these. Four out of 14 patients underwent surgical procedures for recurrence of the cyst that occurred 6-48 months after aspiration. In 27 patients surgery was performed and all identified parathyroid cysts were localized in the inferior parathyroid glands. Histologically the cyst wall consisted in associations of lymphoid, muscular, thymic, salivary, adipose and mesenchymal tissues.

Conclusions: Parathyroid cysts are rare but should be included within the differential diagnosis of a neck lump. True parathyroid cysts are non-functional. Pathological and immunohistochemical findings are suggestive of a branchial origin. Fine needle aspiration may be curative and is diagnostic due to the characteristic appearance of the fluid and high PTH levels on assay. 


\section{8}

\section{Parathyroid Mediastinic Adenoma: A Case Report}

G. Ferrocci, R. Stano, P. Maniscalco, F. Zanzi, C. Gregorio, G. Resta, G. Cavallesco, G. Azzena

Department of General and Thoracic Surgery, Istituto di Clinica Chirurgica, S. Anna Universitary Hospital, Ferrara, Italy

Background: Parathyroid mediastinal cysts are really rare diseases with different expressions, from clinically silent forms to clinical relevant conditions characterized by hyperparathyroidism or compression syndromes.

Methods: A female patient, aged 77, was admitted to our surgical unit for persistent cough and found affected with mediastinal parathyroid adenoma; a thoracoscopic treatment was performed.

Results: At the admission the patient reported a 5 months history of persistent cough; lab data showed increased serum calcium and decreased serum phosphorus levels. A CT scan showed an enlargement of the postero-lateral mediastinum due to a large $(7 \mathrm{~cm})$ mass which dislocated and compressed the trachea and the oesophagus. A right thoracoscopic exploration of the mediastinum was performed and the mass was excised. Final histopathology revealed a cystic parathyroid adenoma. Serum calcium and phosphorum levels normalized in the early postoperative period and the patient was discharged 6 days after surgery without complications.

Discussion: Primary hyperparathyroidism is rarely related to mediastinal parathyroid adenomas; differential diagnosis between these neoplasms and other benign or malignant masses is required in order to decide the best therapeutic strategy. Surgical thoracoscopic resection represents the gold standard technique to treat these neoplasms.

\section{9}

\section{Total Thyroidectomy: Choise Treatment for Multinodular Toxic Goiter}

\author{
A. Marziani, F. Condorelli, A. Cavallaro, S. Aversa, \\ M. Costanzo, M.A. Cannizzaro \\ Università di Catania, Dip Sc chir Trapianti d'Organo e \\ Tecnol, Avanzate, Dottorato Ricerca Metodol Sperimentali \\ e Applicaz Teconologiche in chirurgia, U.O. \\ Endocrinochirurgia P.O.U. S. Luigi, S. Currò
}

Introduction: The aim of this study was to focus the surgical indications for MNTG (multinodular toxic goiter) and analyse surgical results to settle on the surgical choise treatment.

Methods: We analysed 452 patients operated on for thyroid pathology from January 2003 to December 2005 . 85 patients $(18.8 \%)$ were hyperthyroid: 55 (64.7\%) were MNTG with autonomous areas (1 with follicular carcinoma), 10 (11.7\%) were adenomas in MNG, 3 were Plummer's adenomas, 7 Grave's disease, 9 MNG in limphatic thyroiditis ( 2 with papillary carcinoma), 2 limphatic thyroiditis.

Results: The indications for surgery were: 39 compressive goiters, 22 cervico-mediastinal goiters, 3 antidrug intolerance, 2 follicular nodules, 2 recurrent disease and 3 persistent hyperthyroidism. The mean post-operative hospital stay was 2.8 days (2-7). Transient hypocalcemia occurred in 11 patients and transient unilateral laryngeal nerve injury in 4 patients. One patient had permanent hypocalcemia and another one had permanent RLN injury. In almost all cases total thyroidectomy was performed. Only 2 patients underwent loboisthmusectomy because of exclusive unilateral localization of nodules. All patients became hypothyroid after operation and relieved their symptoms.

Discussion: Total thyroidectomy results in a rapid, reliable resolution of hyprthyroidism and removal of $\mathrm{MNG}$, requires no re-treatment, removes any coexisting malignancy and post-surgical hypothyroidism is simple to treat.

\section{0 \\ Minimally Invasive Videoassisted Thyroidectomy (MIVAT): Results after 8 Years Experience}

\author{
G. Materazzi, P. Berti, D. Galleri, M. Massi, J. D’Agostino, \\ G. Donatini, C.E. Ambrosini, G. Frustaci, A. Fosso, \\ P. Miccoli
}

Dipartimento di Chirurgia, PISA, Italy

Introduction: Minimally invasive video assisted thyroidectomy (MIVAT) was introduced in 1998. Its results after an acceptable relapse can now be evaluated.

Patients and Methods: The procedure is based on a unique incision in the central neck, $2 \mathrm{~cm}$ above the sternal notch, using small conventional retractors and needlescopic $(2 \mathrm{~mm})$ reusable instruments. Haemostasis is achieved by a harmonic scalpel. 958 patients underwent MIVAT since June 1998. There were 826 females and 132 males. Lobectomy was carried out in 349 patients, total thyroidectomy in 593, total thyroidectomy and central compartment lymph nodes clearance in 16 cases.

Results: Mean operative time of lobectomy was $34.8 \mathrm{~min}$; for total thyroidectomy was $47.7 \mathrm{~min}$. Indications for MIVAT included papillary carcinoma in 285 cases, follicular nodule in 263 , multinodular goiter in 244 , Graves' in 37 , gene RET mutation carriers in 16 cases. Conversion was required in 21 cases; complications were represented by transient monolateral nerve palsy in 21 cases $(2.1 \%)$, definitive monolateral nerve palsy in 12 cases (1.2\%). Twenty-four patients exhibited a hypoparathyroidism (3.9\%), but only 2 showed a permanent hypoparathyroidism $(0.3 \%)$.

Conclusion: MIVAT can be considered safe, offering significant cosmetic advantages with possible new promising indications such as prophylactic thyroidectomy in RET gene mutation carriers (truncated at 250 words) 


\section{1}

\section{Radioguided Parathyroidectomy for a Recurrent Adenoma in Forearm Graft}

\section{G. Ardito, L. Revelli, P. Princi, E. Giustozzi, A. D'Amore,} M. Boscherini, M. Raffaelli, C.P. Lombardi

Division of Endocrine Surgery, Catholic University of Sacred Heart, Rome, Italy

Introduction: Surgery for secondary hyperparathyroidism included total parathyroidectomy with forearm autograft implantation. Recurrence can occurs and is most likely caused by hyperplasia of the small fragments of parathyroid tissue in the brachioradialis muscle.

Methods: Radioguided surgery for hyperparathyroidism combines parathyroid scanning, using $99 \mathrm{~m}$ Tc-sestamibi, with a handheld intra-operative detector that guides the surgeon to the uptake area. This method has been described for surgery for recurrent or aberrant adenomas of parathyroids (MIRP: minimally invasive radioguided parathyroidectomy). We report a peculiar case of a recurrent hyperparathyroidism in a patient with chronic renal failure and positive sestamibi scanning for an adenoma in forearm graft implanted seven years before, after total parathyroidectomy. The patient, a $37-$ year-old man in dialysis, have a diagnosis of HPT based on elevated intact parathyroid hormone levels and a hyperconcentrating area on $99 \mathrm{~m} \mathrm{Tc}$-sestamibi scintiscan and ultrasounds. Because no sutures or clips were placed to identify the location of the parathyroid tissue in the forearm we recurred to radioguided surgery to facilitate the location of the parathyroid fragments. Tc-sestamibi radioisotope $(10 \mathrm{mCi})$ was administrated 60 min preoperatively. Using an incision of $3 \mathrm{~cm}$ around the scar on the forearm the surgical procedure was guided by probe. In this case radioguidance was particularly helpful on detecting all microareas of residual hypercapting tissue.

Results: Postoperative calcium and PTH levels were normal (follow up 12 months).

Conclusions: Radioguided surgery is sensitive for the intraoperative localization of parathyroid recurrence in the forearm muscle. MIRP is indicated on a clearly positive parathyroid (truncated at 250 words).

\section{2}

\section{Functional Neck Dissection in Papillary Thyroid Carcinoma: A Less Invasive Surgical Treatment}

G. Ardito, L. Revelli, E. Giustozzi, P. Princi, A. D'Amore, M. Boscherini, M. Raffaelli, C.P. Lombardi

Department of Surgery, Catholic University of Sacred Heart, Roma, Italy

Introduction: Total thyroidectomy with functional neck dissection is the treatment of choice of papillary thyroid carcinoma with lymph node involvement. The lymph node dissection of the lateral compartment is usually performed through a Kocher incision prolonged along anterior border of the sterno-cleido-mastoideus muscle. In selected cases we perform a lymph node dissection of the lateral compartment through a less invasive approach acceding to the lymphnodal system via retro sterno- cleido-mastoideus muscle space.
Methods: Twenty-nine consecutive patients with papillary thyroid carcinoma and cervical lymph nodes metastases were included. Functional neck dissection (FND), unilateral or bilateral, was performed through a traditional Kocher incision, running along the superficial fascia of the neck, posteriorly to the sterno-cleido-mastoideus muscle (SCM).

Results: Mean age was $28.0 \pm 20.7$ years. There were 24 women and 5 men. Mean tumor size was $2.5 \pm 1 \mathrm{~cm}$, while the greatest metastatic lymph node was $4.5 \mathrm{~cm}$. Minimally invasive selective functional neck dissection was performed in all patients associated to total thyroidectomy and central compartment lymph nodes clearance. Metastatic lymph nodes were found in 192 out of 459 of the lymph nodes dissected.

Discussion: Minimally invasive neck dissection seems to have carry a lower risk in terms of specific morbidity and allows a quicker recovery and better aesthetic result. This access has to be considered as a less invasive procedure comparing to the other surgical accesses for the radical or modified lateral neck dissection. With this access a systematic selective compartment-oriented lymphadenectomy is feasible removing cervico-lateral lymph nodes from level (truncated at 250 words).

\section{Neurosurgery}

\section{3 \\ Intracranial Pressure Monitoring in Severe Traumatic Brain Injury \\ C. Capuano, L. Arpino, C. De Rosa, A. Franco \\ Division of Neurosurgery, 'San Giovanni Bosco' Hospital Naples, Italy}

Introduction: Intracranial hypertension is a common occurrence in patients with severe traumatic brain injury (TBI)-induced coma. It may be caused by diffuse cerebral edema, swelling of focal contusions, postoperative hematomas. We propose our experience with the ICP monitoring system with intraparenchimal or ventricular sensor.

Methods: 12 patients ( $8 \mathrm{M}-7 \mathrm{~F})$ with severe TBI were ICP monitored using the intraparenchimal system and 3 using an EVD (January 2005-November 2005). The diagnosis of admission was TBI-induced coma in 12 cases and post-hemorrhagic hydrocephalus in 3 . The procedures associated with PIC monitoring were EVD in 3 cases and decompressive craniectomy in 3 .

Results: In our hands, PIC monitoring was an important procedure in order to evaluate the prognosis of the patients and a powerful tool in order to determine the appropriate therapy. The values recorded were considered critical when superior to $20 \mathrm{~mm} \mathrm{Hg}$; in those cases, antiedema therapy was administered, with consistent results in terms of postoperative disability.

Discussion: Consensus has emerged regarding indications for ICP monitoring in patients with TBI, based on the identifications of groups at risk of developing intracranial Hypertension, where at highest risk are patients with a GCS score of $<8$ and abnormal CT scan. 
ICP monitoring thus seems to be a safe and effective tool in the management of severe traumatic brain injury.

\section{4}

\section{Floseal as a Hemostatic Agent for Spinal Surgery}

\author{
L. Arpino, C. Capuano, P. Nina, A. Franco \\ U.O.C di Neurochirurgia, P.O. San Giovanni Bosco, Napoli
}

Introduction: Control of operative bleeding is important in spinal surgery and rapid and effective hemostasis allows the surgeon to visualize the surgical site, minimizing the potential injury to nerve roots. Most of the hemostatic agents have shown difficulty in application, especially in locations difficult to access. We tested Floseal (a combination of gelatin-based matrix and thrombin solution) in our spinal procedures.

Methods: 46 patients undergoing spinal surgery were treated intraoperatively with Floseal from December 2004 to October 2005 (25 M-21 F). The procedures were: 15 lumbar discectomy, 6 lumbar laminectomy, 12 anterior cervical discectomy with fusion, 7 lumbar posterior fusion and 5 cervical laminectomy.

Results: Hemostasis success was defined as cessation of bleeding within 10 min of topic hemostatic agent application; in our series the control of bleeding was satisfactory in $90 \%$ of cases at first bleeding site and in $93 \%$ at all bleeding sites. Another important issue is the procedure time, always shorter in the patients treated with Floseal.

Discussion: Intraoperative control of bleeding during spinal surgery is an issue of great importance and a wide variety of products are available in order to reach this goal. In our hands, the use of Floseal showed to be effective in $90 \%$ of the cases and allowed a shortening in procedures time.

\section{5}

Effectiveness of Fibrin Sealant in Prevention of Cerebrospinal Fluid Leaks or Tension Pneumocranium for Patients Undergoing Brain Surgery

L. Arpino, C. Capuano, P. Nina, A. Franco

U.O.C. di Neurochirurgia, P.O. San Giovanni Bosco, Napoli

Introduction: Cerebrospinal fluid (CSF) leaks are one of the most common complications after brain surgery. At times, tension pneumocranium may occur for the same procedures. The ordinary use of fibrin sealant can prevent these problems.

Methods: From October 2004 to October 2005 we performed 126 intracranial procedures requiring duraplasty (48 tumors and 78 injuries). We usually perform duraplasty with a simple interrupted or a running locked suture, regularly using fibrin sealant to achieve a watertight closure. We on average performed a CT control after surgery and at 1-month follow-up.

Results: We never found features of tension pneumocranium in CT control after surgery for brain tumors. We sometimes (6/78) found this complication on $\mathrm{CT}$ control after surgery for brain injuries but it naturally cleared up on CT control at 1-month in 4 patients. We never found clinical evidence of CSF leaks in all operated cases.

Discussion: CSF leaks and tension pneumocranium may occur after brain surgery. Sometimes a new procedure is required to work out this complication. The ordinary use of fibrin glue to get a watertight duraplasty may prevent these problems. We reconsidered our cases in last year: according to the literature, this retrospective review indicates that the fibrin sealant reduce the incidence of postoperative CSF leaks and tension pneumocranium while reducing overall management costs.

\section{6 \\ ACDF with Bioabsorbable Implant: Report of 16 Operated Cases}

\author{
A. Franco, P. Nina, L. Arpino, C. Capuano \\ U.O.C. di Neurochirurgia, P.O. San Giovanni Bosco, Napoli
}

Introduction: We report our experience about 16 anterior cervical discectomy and fusion (ACDF) with bioabsorbable implant to treat symptomatic cervical spondylosis.

Methods: From Jun 2003 to Sep 2004 we performed 16 ACDF using dense cancellous interbody allograft with bioabsorbable anterior plate. Surgery was indicated for patients with radiculopathy unresponsive to conservative management or with progressive cervical myelopathy. For the follow-up we used JOA score for myelopathy and VAS for pain. Follow-up ranged from 12 to 25 months. A retrospective analysis was performed.

Results: At neuroradiological examination, $90 \%$ of the patients showed signs of fusion. At the most recent follow-up, 6 patients $(5$ with radiculopathy and 1 with myeloradiculopathy) reported a complete remission of neurological signs while the remaining patients with myelopathy the JOA score varied on average from 9.63 prior surgery to 15.63 after it.

Discussion: Symptomatic cervical spondylosis is a pathology quite frequent after the third decade of life. Anterior cervical plate fixation significantly improves arthrodesis after ACDF. However, the metallic materials employed may be responsible for some complications relative to their properties. The use of bioresorbable implants seems to not be liable of these adverse reactions and recently their use in spinal surgery has increased.

\section{7 \\ Brain Metastases from Carcinoid Tumors}

M. Esposito, C. Peca, G. laconetta, R. Donzelli, P. Cappabianca, M.L. Del Basso De Caro'1, F. Maiuri

Department of Neurosurgical Sciences, Neurosurgical Clinic, and Department of Biomorphological Sciences, 'Section of Pathology, 'Federico II' University, Naples, Italy

Carcinoid tumors very rarely metastasize to the brain, as confirmed by lack of intracranial localizations in almost all series of 
carcinoids and the only 70 well documented cases with brain metastases. This study includes 5 cases arising from carcinoids in our series of 380 brain metastases operated upon between 1980 and 2004 $(1.3 \%)$. The primary tumor was in the lung in 2 cases, in the pancreas in 2 and in the ileum in one; its size ranged from 4 to $6 \mathrm{~cm}$. The site of the single brain metastasis was supratentorial in 2 cases and infratentorial in 3 . All five patients underwent surgical resection, followed by irradiation (one case), chemotherapy (one case) or both (3 cases). Immunohistochemical staining showed positively for synaptophysin, neuron-specific-enolase, chromogranine and cytocheratine and negativity for S-100 and HMB-45. The median survival ranged 10 months. Carcinoid tumors with brain metastases are more often located in the lung $(70 \%)$ and are of large size $(>3-4)$; the localization to the brain occurs late in the course of the neoplastic disease, corresponds to a tumor progression and indicates a poorer prognosis. Factors associated to a worse outcome include age $>65$ years, $<1$ year interval from the diagnosis of the primary tumor, and presence of symptoms of carcinoid syndrome. Surgery and adjuvant treatments result in short survival $(<1$ year $)$ in most cases.

\section{8 \\ Spheno-Orbital Meningiomas: Tumor Location and Surgical Approaches}

\author{
G. Buonavolontà', G. Mariniello², M. Esposito², A. Colella², \\ F. Maiuri2 \\ ${ }^{1}$ Department of Ophtalmology, Section of Orbital Surgery, \\ 'Federico II' University, ${ }^{2}$ Department of Neurological \\ Sciences, Neurosurgical Clinic, Naples, Italy
}

Meningiomas of the sphenoid wing often extent into the orbital cavity through the bone, optic canal and superior orbital fissure. The present series includes 60 patients with sphenorbital meningiomas operated on in the Neurosurgical and Ophthalmological Clinics of the 'Federico II' University of Naples; these account for $6.3 \%$ of all intracranial meningiomas and $3.4 \%$ of all orbital neoplasms. Prevalent symptoms and signs include proptosis, blurred vision, disturbances of the eye movements and optic disc pallor, whereas signs of brain involvement were more infrequent. According to their intraorbital locations, the tumors were classified as: (1) lateral and supero-lateral; (2) medial and infero-medial; (3) orbital apex; (4) diffuse. Three different surgical approaches were used: lateral orbitotomy in 15 cases; supraorbital-pterional approach in 42; fronto-temporal-orbito-zygomatic approach in 3 . The postoperative results, considered in 52 patients with sufficient follow-up, were excellent in 26 , good in 16 , moderate in 6 and poor in 4 . The long-term recurrence rate was $42 \%$. In conclusion, most spheno-orbital meningiomas may be safety approached by lateral orbitotomy or supraorbital-pterional approach, whereas more extensive approaches are usually unnecessary. However, a really radical removal may be rarely accomplished and the recurrence rate is high, because of the tumor extent and its anatomical relationship.

\section{9}

\section{Indications and Role of the Surgical Treatment of Multiple Brain Metastases}

\section{A. Colella, A. Giamundo, M. Gangemi, M. Esposito, F. Magro, P. Vergara, F. Maiuri \\ Department of Neurosurgical Sciences, Neurosurgical Clinic, 'Federico II' University, Naples, Italy}

In the last years surgery is indicated with increasing frequency also in very selected cases of multiple brain metastases (up to 3), when the primary tumor is absent or stable, there are no systemic metastatic localizations, and in patients of $<70$ years and with good ( $>70) \mathrm{KPS}$. We have operated on 16 patients with multiple (2-3) brain metastases ( $4 \%$ of the overall material of 400 intracranial metastases operates in our Neurosurgical Departement). Among the 16 cases, 5 had two contiguous lesions, which were removed in one-stage operation through the same craniotomy. The other 11 patients with distant brain metastases were operated on because of the large tumor size with clinical progression ( 7 cases), the presence of a cerebellar metastasis with hydrocephalus ( 2 cases) or for intratumoral hemorrhage ( 2 cases); in this group only 3 patients underwent removal of both lesions, whereas in the other 8 removal of one (larger) lesion followed by stereotactic radiosurgery of the other was performed. The results of our study, compared with those from other series of the literature, confirm that in selected patients with multiple (up to 3 ) brain metastases (both contiguous and distant), if located in cortical regions or deep with matter, mainly in non-critical areas, the surgical resection may improve the outcome and allows a survival similar to that of single brain metastases.

\section{0 \\ Neuroendoscopic Management of Hydrocephalus: Indications, Technique and Potential of the Third Ventriculostomy}

\section{F. Magro, V. Seneca, A. Colella, P. Vergara, M. Esposito, M. Gangemi \\ Department of Neurosurgical Sciences, Neurosurgical Clinic 'Federico II' University, Naples, Italy}

For many years, ventriculo-peritoneal shunt implant has been the treatment of choice in patients affected by obstructive hydrocephalus of different etiology. With the development of modern neuroendoscopes, endoscopic third ventriculostomy (ETV) has gained a pre eminent role in the surgical management of these pathologies, permitting to avoid the complications of shunt implantation, such as malfunction or infection. Today, endoscopic third ventriculostomy has become the highest standard in the resolution of obstructive hydrocephalus, and its indications are expanding to different forms of 'communicating' hydrocephalus. In this work, the authors list the indications of ETV through a review of international literature and on the basis of physiopathological considerations, explain and the operatory technique by analyzing surgical videos of diverse cases, and draw conclusions on the state-of-the-art of this procedure today. 


\section{1}

\section{'One Side Shutter' Approach for Surgery of Foramen Magnum Tumours}

\author{
D. Catapano, V. Monte, M.P. Luigi, V. Carotenuto, \\ L. Innocenti, V. D'Angelo
}

Neurosurgical Operative Unit, 'Casa Sollievo della Sofferenza' Hospital, I.R.C.C.S., San Giovanni Rotondo, FG, Italy

Introduction: The 'far lateral' approach to the foramen magnum is widely utilized for surgical treatment of the anterior or antero-lateral tumours. The main problem in surgery is the prevention of injury to the neurovascular structures. This goal is generally achieved by partial or total condilectomy. Sometimes even partial condilectomy can be enough for safe tumour removal and can produce postoperative instability. In order to modulate lateral extension of bone removal we introduce the concept of 'one side shutter window'. For extra-axial tumours the surgical window is determined on CT-scan by two lines diverging from an extreme distal implant of the lesion. One line is fixed and is conditioned by the dislocation of the neuraxis; the other one is variable according to the bone removal. Neuronavigation is particularly helpful to this goal.

Material: From January 1992 to December 2005, 33 patients with foramen magnum neoplasms have been surgically treated at our department. In 21 patients the implant of tumor was anterior or antero-lateral. Age range 9-78 years (mean: 52 years); sex ratio was M/F 1:1.5.

Results: Preoperative planning of surgical approach was performed on $\mathrm{CT}$ and MR of the foramen magnum region. In all patients a lateral approach was performed. The patients with clivus chordoma for complete removal needed also a transoral approach. Total condilectomy was never performed and a partial (1/3 medial) condilectomy was necessary only in 8 patients. In the remaining 13 cases the removal of anterior/antero-lateral tumours was achieved without any condylar mass removal. In all patients the (truncated at 250 words).

\section{2}

\section{Comparison Between the Microscope and the Endoscope in the 'Direct' Endonasal Extended Transsphenoidal Approach: An Anatomical Study}

\author{
D. Catapano, C.A. Sloffer, G. Frank', E. Pasquini², \\ V.A. D'Angelo ${ }^{3}$, G. Lanzino ${ }^{1}$ \\ Microsurgical Laboratory, Department of Neurosurgery, \\ Illinois Neurological Institute, University of Illinois College \\ of Medicine at Peoria, USA; ' ${ }^{1}$ Department of Neurosurgery, \\ 'Bellaria' Hospital, 'Department of Otolaryngology, \\ 'Sant'Orsola-Malpighi' University Hospital, Bologna, \\ ${ }^{3}$ Neurosugical Department, 'Casa Sollievo della \\ Sofferenza' Hospital, San Giovanni Rotondo, FG, Italy
}

Object: We compare the exposure afforded by the surgical microscope and the endoscope in the 'direct' endonasal extended transsphenoidal approach to the sella, suprasellar and parasellar region.

Methods: Five formalin-fixed silicon-injected adult cadaveric heads were studied. A 'direct' endonasal transsphenoidal approach was performed via the right nostril pushing over the nasal septum, was performed via one nostril.

Results: The 'direct endonasal' approach provides a slightly off midline view. With the combined use of the microscope and the endoscope a good view of midline structures as well as the immediate contralateral paramedian structures is obtained.

Conclusions: Using the microscope and the endoscope it is possible to achieve an adequate exposure of midline suprasellar and infrasellar/upper clival region through a simple 'direct' endonasal exposure. The microscope offers the unique advantage of tridimensional vision particularly useful when dealing with delicate and fine structures such as small perforators. The endoscope affords a more panoramic view which allows extension of the area covered by the surgical microscope. From a direct endonasal route, there is a preferential visualization of the contralateral (the approach) structures, although addition of the endoscope allows for ipsilateral extension of the exposure.

\section{Orl Surgery}

\section{3 \\ Adenotonsillectomy for Treatment of Obstructive Sleep Apnea in Children}

\author{
A. Ranieri ${ }^{1}$, M. W. Rossetti ${ }^{1}$, E. Vetrano ${ }^{2}$ \\ ${ }^{1}$ Functional Unity of Otorhinolaryngology, S. Rita Clinic, \\ Atripalda, AV, ${ }^{2}$ E.N.T. Clinic, Second University of Naples, \\ Italy
}

Introduction: Obstructive sleep apnea (OSA) is an increasingly common indication for adenotonsillectomy in children. OSA has been implicated in poor school performance, failure to thrive, and cor pulmonale in children, and hypertension and automobile accidents in adults. Current treatment options for OSA include pharmacologic agents, nasal continuous positive airway pressure, and surgery. Uvulopalatopharyngoplasty improves OSA in fewer than $60 \%$ of adult patients, while nasal continuous positive airway pressure in effective in up to $20 \%$ of patients. In adults with OSA, surgery in often considered only after nasal continuous positive airway pressure has failed. In children with OSA, surgery (usually adenotonsillectomy) is often the initial treatment. The rationale for adenotonsillectomy comes from observations of the increased frequency of enlarged adenoids and tonsils in children with OSA; moreover several studies have shown improvement in nocturnal oxyhemoglobin saturation of children who have undergone tonsillectomy for recurrent tonsillitis.

Materials and Methods: Sixty-nine children, 41 boys and 28 girls, aged between 2-14 years (mean age 6.9), referred to our observation from November 2003 to January 2005, because of suspected OSA, were eligible for the study. Patients with obvious neurologic or craniofacial obnormalities were excluded. History taking, a physical examination, and a preoperative nocturnal PSG were performed on all patients. The following data were recorded: age, race, weight, snoring, apneas witnessed by the parents, mouth breathing, excessive daytime sleepness, nasal patency, and tonsillar size. The decision to perform adenotonsillectomy was made after considering the patient's history and physical findings as well as the PSG. 


\section{4}

\section{Postoperative Complications in Patients Undergoing Thyroidectomy}

C. Bruzzi, S. Zucchini, M. Sarmati, N. Furxhi, O. Piccin,

O. Cavicchi, A.R. Ceroni

ENT Department, S. Orsola Hospital, University of Bologna, Italy

Introduction: In this study we estimated the postoperative complications in a group of patients treated for thyroid disease.

Methods: Retrospective review of 479 patients that underwent thyroid surgery in the ENT Department of University of Bologna, from January 2002 to February 2006. The incidence of iatrogenic complications was estimated in relation to the thyroid pathology confirmed by the histological examination and to the type of surgical technique adopted. Postoperative hemorrage requiring surgical review, transitory hypocalcemia and inferior laryngeal nerve paralysis were considerated.

Results: 397 patients underwent total thyroidectomy and 82 underwent lobo-isthmusectomy. Postoperative hemorrage requiring surgical review occurred in $8(2 \%)$ patients. There were 124 case of transitory hypocalcemia (24\%). Inferior laryngeal nerves at risk were 876; palsy occurred in 21 patients treated with total thyroidectomy $(2.4 \%)$ and 5 of them recovered successfully within 6 months. Meanwhile 2 patients $(0.2 \%)$ presented bilateral paralysis requiring tracheostomy. Later on, in both cases, one of the vocal folds begun to function normally and tracheostomy was closed.

Conclusions: In this study the postoperative complications resulted uniformly distributed in the different types of thyroid pathology. The incidence of transitory hypocalcemia resulted statistically significant in patients affected from Basedow Disease and Papillary Carcinoma because of the more enquiring surgical approach due to higher risk of hemorrage for Basedow and malignancy.

\section{5}

\section{Multilevel Surgery for the Treatment of Obstructive Sleep Apnea}

\section{S. Zucchini, C. Bruzzi, C. Di Lieto, O. Piccin, G. Scaramuzzino, G. Sorrenti \\ ENT Department, S. Orsola Hospital, University of Bologna, Italy}

Introduction: The purpose of this study was to evaluate the effectiveness of multilevel surgical approach for the treatment of patients with obstructive sleep apnea (OSA).

Methods: 27 male patients who underwent uvulopalatopharyngoplasty (UPPP) in conjunction with genioglossal advancement (GA) for sleep disorder, were retrospectively evaluated. All patients had preoperatively and postoperatively polysomnography to evaluate the efficacy of surgical treatment.

Results: The mean age of the patients was 49.8 years (range 35-61), with a mean BMI of 27.8 (range 24.3-31.1). The group had moderate-severe OSA with a mean RDI of 44.68 (range 19-94). No patient referred symptoms as speech or swallowing difficulties. The mean postoperative RDI was 25.74 , that is a reduction of $42.4 \%$. The surgical cure rate was $48.1 \%$ (13 patients).

Conclusions: Although in our experience the success rate was unsatisfactory, multilevel surgery is a correct approach in patients with oro-hypopharyngeal obstruction. Furthers studies with larger population and statistical analysis to evaluate predictive factors for surgical outcome are necessary.

\section{6 \\ Vocal Fold Augmentation in Patients with Unilateral Vocal Fold Paralisys by VOX IMPLANT ${ }^{\mathrm{TM}}$. Our Experience}

S. Zucchini, C. Bruzzi, C. Bordonaro, O. Piccin, O. Cavicchi, A.R. Ceroni

ENT Department, S. Orsola Hospital, University of Bologna, Italy

Introduction: The purpose of this study was to evaluate the effectiveness of a new minimally invasive procedure to move the vocal cords to a medial position by injection of polydimethylsiloxane (PDMS) particles in patient's with unilateral vocal cord paralysis.

Methods: Eight patients with unilateral vocal paralysis underwent endoscopic vocal cord injection of a soft tissue bulking agent (polydimethylsiloxane elastomers-VOX IMPLANT ${ }^{\mathrm{TM}}$ ) to treat their voice symptoms. Laryngeal function was assessed by videostroboscopy and further characterized by the objective parameters of voice profile obtained by MDVP and maximum phonation time, Jitter, Shimmer and NHR parameters.

Results: All the patients affected by vocal cord paralysis improved their quality voice as assessed by MVDP and by Jitter, Shimmer and NHR parameters. All the patients improved also their maximum phonation and referred a reduction of voice stress.

Conclusions: Our preliminary experience allows to asses that VOX IMPLANT ${ }^{\mathrm{TM}}$ offers good results in patients with vocal cord paralysis and demonstrates its effectiveness in term of voice's quality.

\section{7 \\ New Ideas and Innovations in Plastic Surgery: Nasal Reconstruction Local Perforator Flaps}

\section{Fallahdar, G. Cavenaghi, M. Guzzo, T. Ibba, G. Scaramellini \\ National Cancer Institute, Milan, Italy}

Introduction: The reconstruction of the nose presents a particular challenge point. We propose an innovative technique of two different island flaps, based on two perforators: (1) the angular artery and (2) the lateral nasal artery.

Methods: Two patients underwent radical excision of nasal BCCs, with immediate reconstruction. The first case involved skin resection of the nasal tip and dorsum. An island flap was harvested in 
the nasiolabial groove, based on the angular artery perforator, superiorly. A subcutaneous tunnel from the donor to recipient site, was created. The entire defect was reconstructed with no discrepancy between the flap thickness and the skin surrounding the recipient site. In the second case, resection of the ala was undertaken. After nasal lining, and following identification of the lateral nasal artery perforator, a sidewall nasal island flap was designed and elevated. The flap was based solely on the perforator, excluding subutaneous tissue, surrounding the vessel, a V-Y advancement flap was then performed.

Results: The flaps survived completely and secondary revisions were not required. Both functional and aesthetic results were excellent.

Discussion: The perforator vessels offer versatile tailor-made flaps.

Advantages: One stage procedure, a large arc of rotation, reliable vascularity, excellent texture, colour, and thickness match, and an inconspicuous donor site scar.

\section{8}

\section{The Postsurgical Role of Botulinum Toxin a Injection in Patients Affected by Head and Neck Cancer}

\author{
M.R. Marchese, A. Giorgio, F. Bussu \\ Istituto di Clinica ORL, Policlinico 'A. Gemelli' Università \\ Cattolica del Sacro Cuore, Roma, Italy
}

Botulinum toxin (BTX) has evolved from a poison to a versatile clinical tool with an expanding list of uses particularly in otolaryngology. The wide applicability of BTX results from its particular properties. The effects can be transient, non-destructive, localized to the target area and their degree controlled by dose with minimal systemic side effects. Its injection is able to depress the secretory capacity of the glands. Application of BTX seems to be of great clinical relevance in post-surgery tumour diseases. The study investigates the effect of local injections of BTX type A (Dysport) into the parotid glands in various states of relative hypersalivation with the aim to present new indications for its application. A total of 100 units of BTX were injected into each parotid glands of 6 patients with hypersalivation resulting from head and neck carcinoma: 4 cases affected by pharyngocutaneous fistula following total laryngectomy and 2 cases underwent oncological excisional and reconstructive surgery of the oral cavity and maxillary sinus respectively. The patients were clinically examined to assess the severity of symptoms and the saliva secretion was assessed used visual analogical scale (VAS). Quantitative saliva measurements showed an objective reduction in all patients. Fistula closed without revision surgery within 1 week. All 6 patients reported distinct improvement in their symptoms within 4 days after injection and VAS scores showed an improvement in the mean rate. In the current study intraglandular application of BTX is a reliable, easy and side-effect-free therapy for the oncological head and neck surgery complications.

\section{9 \\ Extensive Solitary Fibrous Tumor of the Retropharyngeal Space}

M. Marchetti, V. Seccia, A. Santoro, L. Muscatello, A.P. Casani

ORL 1-Pisa

Introduction: Solitary fibrous tumor (SFT) is a rare neoplasm whose histologic diagnosis poses significant problems in differential diagnosis. Although most of these neoplasms arise at the level of the pleura, there have also been reports of extrapleural origins. The most frequent localization in the head and neck region is in the nasal cavity.

Methods: We describe the case of an 81-year-old patient with an SFT that arose in the retropharyngeal space. Symptoms were solid food dysphagia associated with modest dyspnea.

Results: Physical examination revealed the presence on the posterior pharyngeal wall of an apparently circumscribed, multilobed mass, which was compact in consistency and partially obstructed the upper aerodigestive tract. After radiologic assessment to carefully evaluate its size and relationship to surrounding structures, the tumor was surgically removed.

Discussion: The importance of immunohistochemical findings in the histologic differential diagnosis are discussed. Follow-up plays a crucial role in evaluating the possible recurrence of such tumors, because parameters for determining their aggressiveness are still controversial.

\section{0}

\section{Cochlear Implant Soft Surgery and Residual Hearing}

P. Melillo, I. Cantore, F. Cianfrone, W. Di Nardo, G. Paludetti

Institute of Otorhinolaryngology, Catholic University of the Sacred Heart, Rome, Italy

Objective: Preservation of residual hearing should be a desirable outcome of implant surgery. The aim of this study is to evaluate residual hearing after minimally-invasive cochlear implantation surgery performed with two different kinds of electrode arrays, with and without rigid introductor (stylet).

Methods: We report the results on 39 patients with measurable pre-operative hearing thresholds. 21 of the implants had the rigid introductor (stylet). Surgery was performed by using soft surgery criterias: 4-5 cm retroauricolar incision, $1.2 \mathrm{~mm}$ cochleostomy, careful electrode array insertion. In all patients we obtained a complete insertion of the electrode array. Responses to pure-tone stimuli were measured after surgery.

Results: After implantation 5 patients (13\%) showed no variation of hearing threshold, 25 (64\%) preserved an appreciable hearing threshold level in the implanted ear, 9 (23\%) had a total loss of residual hearing. On comparing the 2 groups not statistically significant differences were found.

Discussion: Data seem to suggest that cochlear function is less sensitive than expected to implant surgery mechanical trauma and 
that some theories concerning cochlear physiology should be revisited. Besides, electrode array stiffness seems not to influence preservation of cochlear residual functional integrity.

\section{1 \\ Management of Head and Neck Necrotizing Fasciitis}

O. Piccin, C. Bruzzi, O. Cavicchi, E. Pasquini, D. Saggese

ENT Department, University of Bologna, Italy

Introduction: Necrotizing fasciitis (NF) is a potentially lethal soft tissue infection characterized by widespread necrosis of subcutaneous tissue and fascia, vascular thrombosis and extreme systemic toxicity. It is a rare occurence in the head and neck region and occur most frequently in immunodeficient patients or diabetics. The pathogenesis of this entity is polymicrobial with a combination of aerobic and anaerobic organisms.

Methods: Retrospective review of 9 cases of head and neck NF treated at the ENT Department of Bologna University.

Results: All patients underwent CT scan that detected the presence of soft tissue gas in the deep space of the neck. In two patients CT identified mediastinic involvement. All patients had parenteral broad spectrum antibiotics administration followed by surgical debridement within $24 \mathrm{~h}$. The two patients with mediastinitis underwent sternotomy. Adjuvant treatment with hyperbaric oxygen was necessary in six patients. All patients had a successful outcome.

Discussion: Head and neck NF is still a rare but potentially fatal disease. Only early diagnosis and surgical debridement may reduce mortality. CT is the most useful imaging modality for the diagnosis of this disease and chest scan is necessary in all cases because of the high risk of mediastinal involvement. Broad spectrum antibiotic coverage is necessary and more than one antibiotic is required.

\section{2}

\section{Palliative Surgical Treatment of Locally Invasive Head and Neck Neoplasm}

\section{A. Santoro, M. Marchetti, V. Seccia, A.P. Casani, S.S. Franceschini, L. Moscatello \\ Otorinolaringoiatria, Università di Pisa, Italy}

Introduction: Surgical treatment of locally invasive neoplasm without guarantee of radical excision represents an ethical and therapeutic dilemma. Although the decision to treat is driven by the hope to alleviate symptoms and increase life expectancy, the quality of life may be severely affected and patients may require long term hospital care.

Methods: We report a case series of eight patients surgically treated in the last year. None of the patients was eligible for radiotherapy (already performed or not clinically suitable). Clinical decision was: palliative treatment or surgical intervention. With regard to the diagnosis: three patients out of eight had cervical limphonodal metastases $\mathrm{N} 3$, one patient had a maxillary carcinoma with invasion of the check skin, one had a recurrence of an oral carcinoma with extension to the oropharynx and larynx, one had an extensive recurrent differentiated thyroid carcinoma, one had a poorly differentiated and highly progressive parotid sarcoma, one had a cutaneous spread of oropharyngeal carcinoma from iatrogenic cause.

Results: Five patients out of eight achieved a satisfactory control of pain and an improvement in terms of quality of life. Three of the remaining patients suffered significant postoperative complications with serious disability, one died following postoperative complications.

Discussion: The decision to treat with extreme surgery is generated by weighting risks and benefits of either observation or surgical management but also by clinician experience. Although general conditions of these patients are often severely impaired and may deteriorate following surgery, in a good number of cases oncologic surgery leads to satisfactory results.

\section{3}

\section{Treatment of Pediatric Emergencies with Mininvasive Techniques}

\section{F. De Bernardi, G. Delù, C. Cambria, F. Simoncello, P. Bossolesi, P. Palma, P. Castelnuovo \\ Deptartment of Otorhinolaryngology, University of Insubria, Varese, Italy}

Introduction: Transnasal endoscopic techniques have increasingly been used in recent decades also in pediatric patients. We describe the application of endoscopic surgery in the treatment of complications and emergengies in pediatric population.

Materials and Methods: From January 1996 to May 2006 we surgically treated 7 newborns for bilateral choanal atresia, 10 patients with acute complicated rhinosinusitis and 8 patients with fulminant mycotic sinusitis in pediatric age at the Department of Otolaryngology of Pavia and Varese.

Results: The age of our patients ranges from 10 days to 16 years (mean age 7.5 years). All patients were treated with an endonasal endoscopic approach either a single modality or in combination with an external approach (4 cases with complicated rhinosinusitis). Nine children underwent medication in general anestesia. Three patients treated for complicated sinusitis needed a revision surgery. All the patients are followed with endonasal endoscopic controls and the follow up period ranges from 1 month to 10 years (mean 2 years). Three patients affected by fulminant mycosis are dead, among whom one for disease.

Conclusion: We belive that endoscopic sinus surgery should be the gold standard for pediatric patients. It is a mininvasive approach and it does not require skin incision so offering a reduction of cosmetic and functional disabilities. Endonasal approach does not alterate the midface growth of the child and it affords a lower surgical trauma. The intraoperative bleeding is reduced, the recovery is quicker and the hospital stay is shorter. External approaches remain still necessary but its application has (truncated at 250 words). 


\section{4}

\section{Complications in Oncologic Endonasal Endoscopic Sinonasal Surgery}

\section{P. Battaglia ${ }^{1}$, M. Bignami ${ }^{1}$, F. Sberze ${ }^{1}$, A. Pistochini ${ }^{2}$, G. Padoan', P. Palma', P. Castelnuovo ${ }^{1}$ \\ ${ }^{1}$ Department of Otorhinolaryngology, University of Insubria, Ospedale di Circolo e Fondazione Macchi, Varese, ${ }^{2}$ Department of Otorhinolaryngology, University of Sassari, Cliniche Universitarie, Sassari, Italy}

Introduction: The gold standard in the treatment of malignant lesions of the sinonasal and anterior skull base tract is craniofacial approach followed by radiotherapy. We present our initial experience in the treatment of this lesions with the endonasal 'multi-layer centripetal technique' or 'cranioendoscopic technique' followed by radiotherapy and the eventual complications associated.

Materials and Methods: From June 1997 to March 2006 we treated 88 patients with malignat tumors, 68 with pure endoscopic approach and 20 with cranioendoscopic approach. M:F = 54:34. Mean follow up was 29.8 months.

Results: In pure endoscopic surgery we had no intraopertive death, 1 patient had post-operative stroke, 1 had meningitis 65 days later, 1 had inferior tubinate radionecrosis, 3 had postoperative rhinoliqorrhea after duraplasty and 1 of these underwent revision surgery, and 2 lumbar drainage positioning. Now all these patients have resolution of complication. 1 patient had frontal stent encasement that was removed in local anesthesia. In cranioendoscopic technique 2 patient died for surgery, 1 for cerebral edema and the other for cerebral hematoma. 1 patient had extracerebral access 5 months later and recovered with medical technique. 1 had osteomyelitis post radiotherapy, 1 had duraplasty defect 5 months later and both undergone surgical revision.

Conclusions: 'Multi-layer centripetal technique' and 'cranioendoscopic technique' are innovative approach for sinonasal malignancies feasible in carefully selected patients. The operation should also be performed in centers where the cooperation of a neurosurgeon is readily available. Complication are not rare and surgeon must have longstanding experience both in endonasal and external procedures.

\section{5}

Pre- and Intraoperative Evaluation of Glottic Lesions in the Planning of Excisional Biopsy; Personal Experience in 60 Patients

\section{Giudice, F. Sberze, G. Delù, P. Palma, P. Castelnuovo}

Department of Otorhinolaryngology, University of Insubria, Ospedale di Circolo e Fondazione Macchi, Varese, Italy

Introduction: Macroscopic aspects of glottic epithelial abnormalities during endoscopic examination are not specific and even preoperative clinical assessment is not able to define the final pathology. The same clinical appearance can correspond to different types of lesions ranging from keratosis with or without atypia to invasive carcinoma.
Methods: Between January 2003 and April 2006 we applied to a cohort of 60 patients with mid-vocal cord leukoplakias a preoperative examination with laryngostroboscopy (LS) and intraoperative workup employing rigid endoscopes and hydrodissection (HD) into Reinke's space. These tests were done in order to appraise the potential invasion of the layered structure of the lamina propria and consequently to predict and to determine the deep extent of the excisional biopsy to be performed.

Results: After pre- and intraoperative work-up results, we performed carbon dioxide laser cordectomies, according to the European Laryngological Society (ELS) Classification, as follows: Type I cordectomy in 27 Patients, Type II in 16, Type III in 17 respectively. Histopathological diagnoses demonstrated keratosis without atypia in 22 patients, keratosis with mild atypia in 8, moderate atypia in 4, severe dysplasia in 5, microinvasive carcinoma and invasive carcinoma in 20 , fibromiosarcoma in 1 . Finally we estimated the number of under- and overtreatments analysing the histopathological reports related to the type of resection.

Discussion: Unilateral glottic lesions of the mid-vocal cord without involvement of the anterior commissure and impairment of vocal cord mobility may be treated with excisional biopsy alone. Lesions ranging from keratosis without atypia to invasive carcinoma without infiltration of the (truncated at 250 words).

\section{6 \\ Primary Systemic Amyloidosis with Cervical Lymph Nodes Involvement: A Case Report and Review of the Literature}

\section{Seccia, M. Marchetti, A. Santoro, L. Muscatello, S.S. Francheschini}

Introduction: Amyloidosis (A.) is the deposition of amyloid, an extracellular fibrillar protein with unique ultrastructural, x-ray diffraction and biochemical properties, in one or more sites of the body. We report a rare case of primary systemic A. with massive neck involvement.

Method: A 53-year-old woman was referred to our centre already diagnosed with primary systemic A. (hepatic involvement and multiple localizations to axillaries, inguinal and bilateral neck lymph nodes). The patient underwent bilateral modified radical neck dissection.

Results: Local symptoms (neck pain, dysphagia) were reduced. No significant complications occurred. Currently the patient is locally disease-free.

Discussion: Head and neck A. is a rare disease and it may represent a local amyloidoma (more frequent) or a manifestation of a systemic disease (rare); cervical lymph nodes involvement can be considered exceptional in both cases, especially in the latter. A review of the literature shows only 5 other similar cases but with the neck lymph nodes only partially involved and only towards the end of the medical course. Systemic A. is a progressive and fatal disease that is normally treated medically, but in our case the aim of the procedure was to reduce local symptoms and to improve medical management and radiotherapy efficacy. 


\section{7}

\section{Endoscopic Removal of Ectopic Esthesioneuroblastoma Arising in the Pterygopalatine Fossa - A Case Report}

V. Seccia, M. Marchetti, A. Santoro, L. Muscatello, S.S. Francheschini

Introduction: Esthesioneuroblastoma (ENB) is a malignant tumour derived from the olfactory neuroepithelium, adjacent to the cribriform plate. We report a rare case of primary ectopic ENB of the pterygopalatine fossa, treated with endoscopic surgery (ES) and radiotherapy.

Method: A 69-year-old woman complained of V2-V3 dysaesthesias and was referred to us after a brain MRI revealed a mass in her left pterygopalatine fossa, extended intracranially without dural involvement. The biopsy showed ectopic ENB. The patient underwent ES of the mass.

Results: The mass was completely removed and p.o. radiotherapy was performed. Currently the patient is disease-free.

Discussion: ENB is a rare neuroectodermal tumour usually located in the nasal vault; it is rarely ectopic (maxillary/ethmoid sinus, pituitary gland). Literature showed no reported previous cases of ENB of the pterygopalatine fossa. ENB is characterized by its local invasiveness. Our case showed local endocranial diffusion with no metastases. Symptoms are related to the site of origin of the tumour: obviously our patient complained of trigeminal dysaesthesias. The existing classification systems do not anticipate an ectopic pterygopalatine tumour. The standard therapy consists of surgery and radiotherapy: advantages of the ES are the complete removal of the mass with a less invasive procedure, no facial scars and minimum hospital stay.

\section{8}

\section{Different Managements of Periorbital Cellulitis}

G. Tenti, C. Di Lieto, M.A. Fernandez, E. Pasquini, G. Macrí, V. Sciarretta, P. Farneti

ORL Ospedale S. Orsola Bologna, Italy

Introduction: Acute infection of the paranasal sinus is a rather frequent pathology, on the contrary the local and particularly orbital complications are rare. Endonasal and orbital features don't permit a correct classification and don't identify the presence of orbital abscess, consequently it is mandatory to perform a CT scan. The aim of this presentation is to describe our therapeutic management in 7 patients with periorbital cellulitis.

Materials and Methods: Between 2000 and 2006, 7 patients, 6 younger than 9 and one 22 years old came to our department with marked edema of eyelids of sudden onset. In 4 patients the medical treatment permitted a complete resolution 2 patients presenting abscessual cavity underwent urgent surgical procedure. 1 patient received programmed surgical treatment after a second episod of rhinosinusitis complicated with proptosis. All patients received CT at the diagnosis of periorbital cellulitis. A wide spectrum antibiotic iv therapy associated with systemic and local corticotherapy was begun. When an abscess was found at CT scan surgical endoscopic endonasal procedure were performed. Only in the case of eyelids abscess an external incision was necessary.
Results: Since first days the chemosis and proptosis appeared reduced. The endoscopy confirmed the endonasal improvement where not sign of infection was evident.

Discussion: The cases reported underwent different treatments depends on their different clinical stages. We recurred to surgery when despite $24 \mathrm{~h}$ medical therapy the clinical signs weren't reverted, when at CT an abscess was evident, and when the clinical features were recurrent after an initial regression.

\section{9}

\section{Foreign Body in the Ethmoid Region}

G. Tenti, V. Sciarretta, G. Macrí, E. Pasquini

ORL Ospedale S. Orsola Bologna, Italy

Introduction: Foreign body of paranasal sinuses is a rare occurrence. Recent developments in surgical techniques using telescopes have permitted a less invasive mode for removal. We report a case of a metallic nail located within the left posterior ethmoid and breaching through the ipsilateral lamina papiracea.

Material and Methods: A 29-year-old-man was struck by a nail inferiorly to the left eyebrow while mowing the grass by the lawnmower. Suddenly this patient complained of pain at level of left orbit and a wound of the eyelid was detected at the ER admission. Subsequent neurologic and ophthalmologic evaluations were unremarkable. After the repair of the wound a facial x-ray was performed and it demonstrated the presence of a loop shaped nail close to the left orbit. This patient underwent through a CT scan, that pointed out a foreign body breaching through the left lamina papiracea. Thus an endoscopic transnasal approach was accomplished to remove the foreign body.

Results: The endoscopic approach was characterized by an antero-posterior ethmoidectomy and it was successful to remove the foreign body.

Discussion: Foreign body within paranasal sinuses can be safely removed by mininvasive endoscopic approaches with lower morbidity if compared to traditional external approaches. The preoperative radiologic assessment through CT scan is of utmost importance to predict risks and drawbacks.

\section{0}

\section{New Ideas and Innovations in Plastic Surgery: The Proximal Reversed Brachioradialis Perforator (PRBP) Free Flap and the Proximal Reversed Radial Artery (PRRA) Free Flap in Head and Neck Reconstruction}

D. Fallahdar, M. Guzzo, T. Ibba, G. Cavenaghi, F. Mattavelli, R. Bianchi, G. Scaramellini

National Cancer Institute, Milan, Italy

Introduction: The Radial Forearm Flap as one of the most commonly used in reconstructive microsurgery. However, the incidence of donor site morbidity is significant. To reduce the donor site aestheticfunctional complications, we have developed a new techique, The 
PRBP and PRRA free flaps with advantage in donor site primary closure; and it's based on the proximal brachioradialis perforator or on the proximal septocutaneous vessels, situated along the proximal $1 / 3$ of the radial artery.

Methods: The flap designed on the proximal $1 / 3$ of the volar face of the forearm, $4-5.5 \mathrm{~cm}$ width. Primary incision is made on the medial side. Upon identification of perforator vessels, dimensions \& position of the flap can be determined, as well as the length of the vascular pedicles required. The radial artery is subseqently clamped to its origin to verify the retrograde blood supply to the flap. The PRBP and PRRA free flaps used in head\&neck reconstruction in 2 consecutive patients in October 2005.

Results: Both patients had excellent functional-aesthetic results, uneventful recovery of their donor sites, and no complications/flap loss experienced.

Discussion: Primary closure of the donor site results in a recovery time of less than 1 week, which is both benficial to the patient, and is cost-effective. This new technique is recommended, which is applicable in most microsurgical reconstructive cases.

\section{Pediatric Surgery}

\section{1 \\ Diagnosis and Management of a Migrated Esophageal Foreign Body: Case Report}

\author{
O. Adorisio, R. Mattei, N. Centonze, L. Landi, \\ B. Noccioli \\ Department of Pediatric Surgery, Neonatal Surgery Unit, \\ Children's Hospital 'Anna Meyer', Florence, Italy
}

Introduction: Foreign body ingestions in children often present with minimal symptoms. Typical approaches to acute ingestion depending on the type, shape and position of the foreign body include simple observation awaiting spontaneous passage, or endoscopic removal. In rare and often dramatic occasion, a sharp ingested body which remains in the esophagus can perforate it migrating extraluminally to cause complications such as mediastinal abscess, mediastinitis, tracheoesophageal or esophago-aortic fistula, and death.

Methods: An 11-year-old girl presented in the Emergency Department with dysphagia and a right laterocervical neck mass. An X-ray study showed a very thin wire, two centimetres in length, migrated in the right side of the neck outside the oesophagus; esophagogram did not show any leakage. A previous ingestion of a foreign body was suspected and the patient underwent surgical excision under fluoroscopic guide.

Discussion: Foreign body ingestion or inhalation associated to acute symptoms such as cough, cyanosis, dysphagia or dyspnoea must lead to prompt investigation and treatment because of the potential for severe complications. The most common site of perforation are esophageal physiologic strictures, ileo-cecal junction and recto sigmoid region. In the esophageal region if the event is no suspected or when the patient is asymptomatic, diagnosis can be delayed with potential severe complications. The migration of foreign body may remain silent with infection or abscess formation.

Conclusions: Foreign body ingested and retained into the esophagus should lead to prompt endoscopic removal. No suspected ingestion usually brings to delayed diagnosis, so leading to potential severe complications.

\section{2 \\ Peritoneal Dialysis (PD) as Rescue Therapy in Two Anuric Extremely Low Birth Weight (ELBW) Newborn}

\author{
M. G. Di Napoli, V. Rossi, F. Macchini, D. Fontana \\ Chirurgia Pediatrica, Fondazione Policlinico IRCCS, Milano, \\ Italy
}

Introduction: Severe acute renal failure (ARF) is common and accounts for high mortality in newborn intensive care units. Frequently it is acquired because of hypoxic ischemic injury and toxic insults. Renal replacement therapy is necessary for giving these neonates a chance of life and may be provided with PD, intermittent hemodialysis or hemofiltration.

Methods: We describe the case of two anuric ELBW newborn. Both received, without success, two cycles of indomethacin for a patent ductus arteriosis, underwent to the surgical closure of ductus followed by progressive renal failure with: abnormal biochemistry, severe decrease of urine output and anasarca. After a laparotomic insertion of a single-cuff Tenckhoff catheters both received continuous flow peritoneal dialysis (CFPD). We used low volume CFPD $(<20 \mathrm{ml} / \mathrm{kg} /$ exchange) with this prescription: dialysis solution with $2.5 \%$ dextrose, dwell time 30-45 min, drain time 15-20 min. CFPD was discontinued when adequate weight loss had occurred and patient's daily urine output exceeded their fluid intake.

Results: There were no complications associated with the use of dialysis catheter insertion or CFPD therapy. To start immediately the $\mathrm{PD}$ and prevent leakage we applied some fibrin glue around the cutaneous exit point of catheter. Both patients survived requiring only minor ventilatory parameters' changes.

Discussion: In newborn with severe ARF when vascular access may be difficult the use of PD is the only chance of life.

\section{3 \\ Effectiveness of Fluticasone Propionate in the Treatment of Esophageal Stenosis Due to Eosinophilic Esophagitis in Children}

\author{
F. Macchini, V. Rossi, M.G. Di Napoli, A. Valadè \\ Pediatric Surgery Department, Fondazione IRCCS \\ Policlinico Mangiagalli Regina Elena, Milano, Italy
}

Introduction: Eosinophilic esophagitis (EE) is an isolated eosinophilic inflammation of the esophagus, leading to esophageal stenosis. Suggested treatments include strict elementary diet or systemic immunosuppression, with problems of compliance and side 
effects. Two children with esophageal stenosis caused by EE were treated with topical application of steroids as alternative treatment.

Methods: Two children (1 male), 15th and 8th years old, with an history of epigastric pain and vomiting, were studied for occurrence of progressive dysphagia. An X-ray contrast study of the esophagus showed in both a stenosis in the middle portion, confirmed by an upper endoscopy. Biopsies specimens showed a mucosal eosinophilia, defined as large number of eosinophils ( $>20$ /high power field) in the squamous epithelium of proximal and distal esophagus. Both children were treated with Fluticasone Propionate (FP) 2 puff by mouth twice daily (110 mcg/puff) without inhaling.

Results: A quick relief of symptoms was observed after 15 days treatment. Resolution of the stenosis and a reduction of the eosinophilic infiltrate were demonstrated by endoscopy after a month. At the follow-up both children were asymptomatic after 6 months.

Conclusion: EE is considered an emerging diagnostic problem in the pediatric population. It should always be considered in the differential diagnosis of GERD. FP seems effective for a quick improvement of mucosal inflammation and stenosis. FP is characterized by a good compliance in pediatric age, without side-effects.

\section{4}

\section{Prolapse of Urethral Mucosa in Female Children}

\author{
V. Rossi, M.G. Di Napoli, F. Macchini, S. Fedeli, A. Sartori, \\ M. Torricelli \\ Chirurgia Pediatrica, Fondazione Policlinico di Milano, \\ IRCCS, Italy
}

Introduction: Urethral prolapse denotes the evertion (circular or segmental) of the urethral mucosa through the external meatus. Two different entities exist: premenarcheal and menopausal urethral prolapse. Premenarcheal prolapse is predominantly asymptomatic and is usually brought to medical attention by vaginal bleeding.

Method: Five patients (age from 2-10 years) with urethral segmental prolapse were treated at Pediatric Surgery division of University Policlinico Hospital of Milan during a 6-year period. Patients were identified by perineal bleeding (two cases) and diagnosed by physical examination.

Results: In three cases medical approach consisted of antibiotic and antiinflammatory treatment, combined with local antiseptic for 2-3 weeks. In two cases surgical ligation was performed. In all the patients prolapse was resolved.

Discussion: The results, with follow-up for 4-12 months without recurrence, suggest that urethral segmental prolapse in children can be managed without surgical intervention (truncated at 250 words).

\section{5}

\section{Initial Experience with Two-Stage Hypospadias Operation (Bracka)}

\section{Rossi, A. Sartori, M.G. Di Napoli, F. Macchini, S. Fedeli,} M. Torricelli

Chirurgia Pediatrica, Fondazione Policlinico di Milano, IRCCS, Italy

Introduction: Despite the introduction of more refined surgical techniques, the optimal treatment of the most severe form of hypospadias remains to be determined.

Materials and Methods: We report the use of a 2 stage repair with preputial graft interposition and subsequent tubularization of the urethral plate applied it to all severe cases of hypospadias with significant chordee or small glans.

Results: Both stages of the procedures were completed in 20 patients. Complications were observed in 3 patients $(15 \%)$ : included 1 glans disruption and 2 fistulas. Cosmetic and functional out-come after a 1 year follow-up was optimal in all cases with a normally located 'slit' meatus and straight penile shaft.

Discussion: Although the controversy between use of grafts and flaps will probably continue forever, we believe that our 2-stage approach should be considered as a valid alternative for the most severe forms of hypospadias.

\section{6}

\section{Testicular Microlithiasis in Pediatric Age}

V. Rossi, M.G. Di Napoli, F. Macchini, S. Fedeli, A. Sartori, M. Torricelli

Chirurgia pediatrica, Fondazione Ospedale Maggiore

Policlinico di Milano IRCCS, Italy

Introduction: Testicular microlithias (TM) is a rare disease, small number of cases has been reported in pediatric age. The wide usage of high resolution US in the evaluation of scrotal diseases has resulted in a coincidental increase in the number of TM cases. Testicular microlithiasys is a pathology of which actual cause is unknown and that is belived to resulted from the degeneration of the seminiferous epithelium wiped into the tubular lumen.

Methods: Five patients (age 4-12 years) underwent US examination for scrotal trauma (2 cases), uniteral criptorchidism (2 cases) and varicocele ( 1 case). Follow-up US examination were performed.

Results: 2 of the 5 patients underwent 3 US examinations, the remaining 3 patients underwent 2 US examinations. Microliths were bilateral in 3 patients and unilateral in 2 patients. In 3 cases microliths were distributed throughout the testis. In the remaining 2 cases they were present in limited areas of parenchyma. As to the importance of microliths, it was defined as mild in 3 cases and moderate-severe in 2 cases.

Discussion: The use of high-frequency US transducers (10-13 $\mathrm{MHz}$ ) has recently allowed an easier demonstration of this disease in children. In pediatric age, if testicular microlithiasis represents an isolated sign, patients need non-invasive US follow-up until adult age. 
Only if TM is in association with focal lesions of testis parenchyma is it mandatory to perform biopsy or surgical treatment.

\section{7}

\section{Transcrotal vs. Inguinal Orchidopexis}

A.C. Agliardi, V. Rossi, M.G. Di Napoli, S. Fedeli, F. Macchini, A. Sartori, M. Torricelli

Chirurgia Pediatrica, Fondazione Policlinico IRCCS, Milano, Italy

Introduction: Transcrotal approach has been described, when indicated, as an alternative to the inguinal route in the management of cryptorchidism in 1989.

Methods: We reviewed the notes of the cryptorchid children operated on in our Department since 1988 to 2004. 1597 operations (on 1376 patients), mean age 4.9 years: 1,325 inguinal approach (group A) $83 \%$ and 134 scrotal approach (group B) 8\%, others $9 \%$. Follow-up 5 months- 6 years.

Results: The complications observed in the group of children operated on inguinal route (group A) was: 9 testicular hypotrophies $(1 \%)$ and 7 recurrences $(1 \%)$. In the group B of children operated on scrotal route we observed none testicular hypotrophies and $6(4 \%)$ recurrences.

Discussion: Children with primary palpable undescended, gliding or trapped testes can be managed successfully through the transcrotal route in majority of cases. In our experience the scrotal approach, when correctly indicated and performed presents positive results. This technique allows a proper repositioning of the testis in the scrotum and good aestetic results.

\section{8}

\section{Follow-Up Examination of Patients with Undescended Testicles}

\section{A.C. Agliardi, V. Rossi, A. Sartori, M.G. Di Napoli, F. Macchini, M. Torricelli \\ Chirurgia Pediatrica, Fondazione Policlinico di Milano, IRCCS, Italy}

Introduction: The literature concerning cryptorchidism suggests that an abnormal testicular descent reduces human fertility. The aim of this paper is to present the result of a retrospective study of 60 adult patients, who underwent orchidopexy at an age between 2-12 years, in order to evaluate the results of hormonal and surgical treatment on spermatogenesis after 10-19 years.

Methods: 3 patients (5\%) were father; 57 patients underwent orchidopexy (47 patients for monolateral undescended testis $(82 \%)$, 10 patients for bilateral undescended testis (18\%), mean age 5 years. Bilateral cryptorchidism were treated by orchidopexy combined with human corionic gonadotrophin therapy. All young men underwent to clinical examination, blood sampling for Testosterone, FSH and LH serum basal level, testicular ultrasound and semen analysis. Patients were divided into 2 groups according to their age at the time of orchi- dopexy: group A (37 patients over 4 years $65 \%$ ) and group B (20 patients $2-3$ years $35 \%$ ).

Results: The data suggest that a better sperm density and motility is observed in early orchidopexy. A significant difference of testicular size was also noticed when patients with unilateral cryptorchidism were managed with early surgical and hormonal therapy. FSH level increase inversely with spermatogenesis; elevated Lhhormone levels suggest Leydig cell dysfunction and gonadal atrophy.

Conclusions: Early operation at 2 years of age at the latest is the only possibility to prevent infertility or sub-infertility. The intervention prevents secondary damage to retained and the controlateral testis.

\section{9 \\ Timing of Surgery for Congenital Diaphragmatic Hernia (CDH): Report of Our Experience in 27 Cases}

\author{
V. Rossi, M.G. Di Napoli, F. Macchini, G. Bordin, \\ A. Fagnani, A. Sartori, M. Torricelli \\ Division of Pediatric Surgery, Fondazione Policlinico- \\ Mangiagalli, IRCCS, Milano, Italy
}

Introduction: $\mathrm{CDH}$ is a relatively common birth defect; its incidence range widely around 1/4000 live births and it usually presents with severe respiratory failure. Reported survival averages $50 \%$ but may be significantly lower. Differed surgery, with the emphasis on preoperative ventilatory management up to including high frequency oscillatory ventilation (HFOV) and inhalated nitric oxide (INO) for the management of pulmonary hypoplasia and vascular anomalies, has now become the widely accepted approach to the management of $\mathrm{CDH}$.

Method: 37 infants with $\mathrm{CDH}$ were managed at our Neonatal Intensive Care Unit between November 1996 and June 2002. Surgical repair of the diaphragm defect occurred in 27 infants (mean BW $3244,1660-4500 \mathrm{~g})$ mean GA 38 weeks (30-41 weeks), 17 infants had stomac dislocated in thorax.

Results: $3 / 27$ infants underwent urgent surgical intervention within the $24 \mathrm{~h}$ of life; none of these survived. 24/27 underwent a nonurgent, differed intervention after stabilization (mean 7 days 3-13 days): all underwent HFOV, 18 received surfactant therapy and 10 underwent inhaled no therapy. 19/24 infants survived and were discharged from NICU. 7 infants had wide defect in diaphragm: 3/7 correction needed Goretex patch (survival rate 33\%), while 4/7 underwent abdominal muscolar flap (survival rate $75 \%$ ).

Conclusion: The timing of surgical repair depends on the degree of stability or liability of the pulmonary vascular bed. We consider differed intervention the best time management to critical $\mathrm{CDH}$. 


\section{0}

\section{Retrospective Review of Pediatric Patients with Acute Scrotum}

\author{
V. Rossi, M.G. Di Napoli, E. Itri, A. Sartori, M. Torricelli \\ Chirurgia Pediatrica, Fondazione Policlinico IRCCS, Milano, \\ Italy
}

Introduction: The objective of the study is to compare historical features, physical examination findings, and testicular color Doppler ultrasound in pediatric patients with epididymitis, testicular torsion and torsion of appendix testis.

Methods: 90 patients were included in the study (64 with epididymitis, 13 with testicular torsion and 13 with torsion of appendix testis). Historical features did not differ among groups except for duration of symptoms. Of 13 with testicular torsion all had a tender testicle and an absent cremasteric reflex. 62 (97\%) patients with epididymitis had a tender epididymis and $43(67 \%)$ had a scrotal erytema/edema. Doppler ultrasound showed decreased or absent blood flow in 8 patients, 7 of whom were diagnosed with testicolar torsion. 10 out of 13 patients with testicular torsion had a salvageable testicle at the time of surgery.

Results: The physical examination is helpful in distinguishing among epididymis, testicular torsion and torsion of appendix testis. Patients presenting with a tender testicle and an absent cremasteric reflex were more likely to have a testicular torsion rather than epididymitis or torsion of appendix testis.

Conclusions: An absent cremasteric reflex was the most sensitive physical finding for diagnosing testicular torsion. Color Doppler ultrasound is a useful adjunct in the evaluation of the acute scrotum when physical findings are equivocal. Most boys presenting with acute scrotum don't have testicular torsion. The history and physical examination can reliably identify those boys who can be managed conservatively. Routine surgical exploration is no longer justified in all boys with the acute scrotum.

\section{1}

\section{Topical Steroid Therapy for Phimosis}

V. Rossi, M.G. Di Napoli, R. Arnoldi, A. Sartori, M. Torricelli

Chirurgia Pediatrica, Fondazione Policlinico IRCCS, Milano, Italy

Introduction: Topical steroids are an effective alternative to circumcision for the treatment of phimosis.

Material and Methods: We evaluated 27 boys with phimosis for possible circumcision because of genitourinary anomalies (4), a history of urinary tract infection (11) or both (7), or balanoposthitits (5). Patient age ranged from 12 to 31 months. In no case could the prepuce be retracted to visualize the meatus. Patients were treated with $0.05 \%$ betamethasone cream applied to the distal aspect of the prepuce twice daily for 40 days.

Results: Of the boys 20 (74\%) had fully retractable foreskin after topical steroid therapy and $5(18 \%)$ had a partial response at 1 month, with the prepuce becoming fully retractable during month 2 . No adverse side effects were noted. Of the 2 patients in whom treatment failed 1 subsequent underwent circumcision while the parents of the other refused further therapy.

Conclusions: Topical steroids application for the treatment of unretractable prepuce complaints seems to be effective and safe, and is therefore recommended before surgical intervention is considered.

532

The Impact of Post-Operative Chylothorax in Infants with Congenital Diaphragmatic Hernia

F di Loreto, O. Louisma, G. Casaccia, I. Capolupo, B. lacobelli, A. Nahom, F. Morini, P. Bagolan

Chirurgia Pediatrica, Ospedale Bambino Gesu', Roma

Background: Though pleural effusion (PE) is frequently encountered in newborns with congenital diaphragmatic Hernia $(\mathrm{CDH})$, its consequences over the postoperative course have never been studied in details.

Methods: The carts of $138 \mathrm{CDH}$ patients consecutively treated at our institution between 1997 and 2005 were reviewed. Patients were diveded into two groups according to whether their course was complicated (Group 1) or not (Group 2) by $>$ PE. The groups were compared with regard to demographics (sex, weight, > gestational age, birthweight, long of stay (LOS) and clinical determinants $>$ including mecanical ventilation ( $>$ or $<96$ hours, hernia side, need of patch repair, associated malformations, and mortality rate. Statistical analysis was performed by t-test or Chi square test as appropriate.

Results: Thirty one patients were in Group 1 and 107 in Group 2. The two groups had comparable gestationalage, birthweight and prevalence of associated malformations. No statistical association with PE was found with regard to hernia side, type of hernia and patched or direct closure. Mortality rate was also not statistically different (20\% in Group 1 and $22 \%$ in Group 2). A statistically significant association was found with LOS (92 days in Group 1 vs 54 days in Group 2, p < 0.05). patient who were mecanically ventilated less than 96 hours, PE was found in 2 cases while it occurred in 29 cases when longer than 96 hours. in particular, all patient ventilated for longer than $400 \mathrm{~h}$ developed PE.

Conclusion: FOllowing repair of $\mathrm{CDH}$; $\mathrm{PE}$ is common. In infants with $\mathrm{CDH}$, the developement of post operative Pe significantly affets the pronosis, being with a prolonged LOS and the need of a longer mecanical ventilation. 


\section{Oculistic Surgery}

\section{3}

\section{Orbital Rhabdomyosarcoma: Clinical Aspects and Therapy}

\author{
S.C. Di Simplicio ${ }^{1}$, A.G. Resti ${ }^{1}$, C. Meazza ${ }^{2}$, A. Ferrari², \\ F. Spreafico ${ }^{2}$ \\ ${ }^{1}$ Servizio di Chirurgia Oftalmoplastica e dell'orbita, U.O. di \\ Oftalmologia, Università Vita e Salute San Raffaele, ${ }^{2}$ U.O. \\ di Oncologia Pediatrica, Istituto Nazionale dei Tumori, \\ Milano, Italy
}

Introduction: Although rhabdomyosarcoma is a rare tumor among the entire group of mesenchymal malignancies, it is a relatively common lesion and significant challenge for the ocular oncologist in terms of its diagnosis and management. Our Center collaborates with Pediatric Oncology Center at the National Tumor Institute of Milan for the management of the patients. In our study we present the results of the Study Group in 25 years of experience.

Methods: 20 patients, 14 males and 6 females, with mean age of 7 years. Of these patients 11 underwent only to biopsy, 8 had a partial exeresis and 1 underwent to exenteratio orbitae. 19/20 patients underwent to chemotherapy, and 18/20 underwent to radiotherapy.

Results: With the right management, DFS at 5 years was $78.9 \%$. All patients with recurrence or progression of the malignancy deceased.

Discussion: Rhabdomyosarcoma is not a 'killer', but is tumor which can be cured. The role of the ophthalmologist if essential to recognize the pathology and to address the patient to the oncologist who must execute the therapy. Surgery is only the last choice.

\section{4}

\section{Dietary Lutein, Vitamins and Fish Intake and Age-Related Macular Degeneration} Prevalence

\section{Scorolli, A. Meduri, M. Morara, S.Z. Scalinci, \\ D. Molinaro, R.A. Meduri \\ Pathophysiological, University, Bologna, Italy}

Introduction: To measure prevalence of age-related macular degeneration (ARMD) and examine the intake of lutein, vitamins $\mathrm{A}-\mathrm{C}-\mathrm{E}$ as well as fruits and vegetables, and the intake of $\omega-3$ polyunsatured fatty acids present in fish, and then evaluate their function in altering the prevalence of ARMD.

Methods: People ( $\mathrm{N}=3000 ; 1857$ women, 1143 men) aged from 43 to 87 years old. Patients with ARMD were identified by an ocular fundus exam in direct ophthalmoscopy and then classified in 4 different clinical categories in order to gravity. They were also placed in order to age and sex in 4 groups to quantify as well as possible the prevalence in each one. A 100-items self-administered, semiquantitative food frequency questionnaire was fairly completed by all participants and was used to assess intakes of lutein, vitamins A-C-E and $\omega-3$ polyunsatured fatty acids. All people were also subjected to the Amsler's grid test to evaluate visual impediments.

Results: Our epidemiologic data indicate a relationship between lutein, vitamins and $\omega-3$ polyunsatured fatty acids and Age-Related Macular Degeneration: prevalence observed in our population-based study resulted inferior than the AREDS's one, suggesting those compounds can play a protective role in the eye.

Discussion: An intake of dark green leafy vegetables, fruits and regular consumption of fish, like in Mediterranean diet, appears to be beneficial in protecting retinal tissues, but this is not proven. Future investigations on the role of nutrition may better explain the association with risk of ARMD.

\section{5 \\ Role of Cysteine in Corneal Reepithelization After Photorefractive Keratectomy}

L. Scorolli, A. Meduri, M. Morara, S.Z. Scalinci,

S.D. Esposti, D. Molinaro, R.A. Meduri

Pathophysiological, University, Bologna, Italy

Introduction: To evaluate the effect of cysteine oral supplements in the process of corneal reepithelization after PRK.

Methods: 100 patients (200 eyes), divided into two groups (Groups 1 and 2) with the same age and comparable refractive errors, each made of 100 patients, have been monitored. It was administered to patients in Group 1 oral L-cysteine, with a daily dose of $200 \mathrm{mg}$ for 15 days starting from 1 week before surgery, using the other group (Group 2) as a control. After surgery, patients have been daily monitored starting from $24 \mathrm{~h}$ following surgery, through biomicroscopy, to measure the epithelial diameter defect; patients were then monitored on 1 th, 2 th, 3 th, 4 th week after surgery through confocal microscopy, to value epithelial corneal thickness.

Results: Eyes in Group 1 showed a time of reepithelization inferior to Group $2(102 \pm 15$ vs. $159 \pm 9 \mathrm{~h})$. Mean value of corneal epithelium thickness before surgery was $51 \pm 5 \mu \mathrm{m}(51 \pm 4 \mu \mathrm{m}$ in Group 1 and $52 \pm 4 \mu \mathrm{m}$ in Group 2). At week 1 the value was: $29 \pm 4$ $\mu \mathrm{m}$ in Group 1 and $22 \pm 3 \mu \mathrm{m}$ in Group 2. At week 2: $37 \pm 5 \mu \mathrm{m}$ in Group 1 and $29 \pm 2 \mu \mathrm{m}$ in Group 2. At week 3: $45 \pm 4 \mu \mathrm{m}$ in Group 1 and $35 \pm 4 \mu \mathrm{m}$ in Group 2. At week 4: $50 \pm \mu \mathrm{m}$ in Group 1 and $43 \pm 3 \mu \mathrm{m}$ in Group 2 .

Discussion: Cisteyne, administered orally in a daily dose of $200 \mathrm{mg}$, is shown to be effective in reducing time of corneal reepithelization after PRK and also aids the return of the epithelial thickness to the original levels. 19th National Congress of the Italian Polyspecialist Society of Young Surgeons (IPSYS) 


\section{6}

Treatment of Cystoid Macular Edema (CME) in Retinitis Pigmentosa (RP) with Intravitreal Triamcinolone Acetonide

\section{Scorolli, M. Morara, A. Meduri, R. Provenzano, S.Z. Scalinci, R.A. Meduri \\ Ottica Fisiopatologica Policlinico Universitario S. Orsola Bologna, Italy}

Purpose: To evaluate the anatomic and visual outcomes of treatment with intravitreal triamcinolone acetonide injection in patients with cystoid macular edema (CME) secondary to retinitis pigmentosa (RP).

Methods: Study including 20 eyes of 20 patient affected by CME secondary to RP, mean age was $40.2 \pm 13$ years (group A). A control group was selected (group B) of 20 eyes of 20 patients, mean age $=39.5 \pm 11.3$ years affected by CME secondary to RP composed by patients who refused any kind of treatment. All the treated eyes received an intravitreal injection of $0.1 \mathrm{ml}$ of triamcinolone acetonide Kenacort-A $40 \mathrm{mg} / \mathrm{ml}$. Evaluation parameters were best corrected visual acuity (BCVA), central macular thickness measured by OCT. Total follow-up was 12 months.

Results: BCVA showed a not significant improvement after 3 and 6 months in group A compared with group B. Central macular thickness was similar before treatment. At 3 months the decrease was higher, to a mean value of $272.78 \pm 64.6 \mu \mathrm{m}$ in group A and $462.37 \pm 76.17 \mu \mathrm{m}$ in group B. At 12 months values resulted similar to baseline thickness in group A: $442 \pm 95.5 \mu \mathrm{m}$ In group B was $476.58 \pm 74.72 \mu \mathrm{m}$.

Conclusions: Intravitreal triamcinolone acetonide may be useful for selected cases of CME in patients affected by RP but the efficacy seems to be limited in time and to obtain a good anatomic result and an improvement of BCVA is necessary a retreatment after 6 month.

\section{7}

Evaluation of Basics Fibroblast Growth Factor (bFGF) Level in Pig Eyes that Underwent Surgical Adypocytes Implant Under the Scleral Plane

\section{Scorolli, A. Meduri, M. Morara, S.Z. Scalinci, R. Provenzano, R.A. Meduri \\ Pathophysiological, University, Bologna, Italy}

Objective: To evaluate the level of bFGF release in pig eyes after surgical adipocytes implant under the scleral plane compared to bFGF level in normal eyes.

Methods: 18 pigs, 6 pigs underwent adipocytes implant in both eyes (group A), 6 pigs underwent implant in only one eye, chosen in a randomized way (group B), and 6 pigs did not undergo any surgical operation (group C). After 4, 8, 12 months from the implant 6 pigs (2 from group A, 2 from B and 2 from C) were sacrified. Explanted eyes were divided into two groups: group 1 (operated eyes) and group 2 (non-operated eyes). We performed on all explanted eyes bFGF dosage by means of immunoistochimical technique.

Results: We evaluated levels of endobulbar bFGF in vitreal fluid $(50 \mu \mathrm{l})$. After 4 months FGF levels were $7.76 \pm 0.64 \mathrm{pg} / \mathrm{ml}$ in group 1 , and $0.82 \pm 0.16 \mathrm{pg} / \mathrm{ml}$ in group 2 . After 8 months bFGF levels were $6.39 \pm 0.72 \mathrm{pg} / \mathrm{ml}$ in group 1 , and $0.75 \pm 0.26 \mathrm{pg} / \mathrm{ml}$ in group 2. After 12 months bFGF levels were $7.21 \pm 0.52 \mathrm{pg} / \mathrm{ml}$ in group 1 , and $0.71 \pm 0.22 \mathrm{pg} / \mathrm{ml}$ in group 2 . The difference between bFGF levels in the two group showed a good tropism and good vascularization of peduncle of orbital flat implanted.

Conclusions: After surgical adipocytes implant, an increase of intraocular bFGF level can be observed. These data may justify clinical efficacy of this procedure in the rational treatment of Retinitis Pigmentosa, in which levels of bFGF are characteristically decreased.

\section{Anaesthesiologist}

\section{8 \\ Intravenous Anesthesia with Propofol and Remifentanyl Provides Better Surgical Conditions than Sevorane-Remifentanyl During Endoscopic Sinus Surgery}

\author{
V. Altimari, R. D'Andrea, R.M. Melotti, A. Potalivo, \\ A. Rosada, M. Valbonetti
}

Introduction: The purpose of this study was to compare surgical conditions for functional endoscopic sinus surgery (FESS) under general anesthesia during controlled induced hypothension using either propofol-remifentanil or sevorane-remifentanil.

Methods: After consent ethical committee twenty patients undergoing elective FESS under general anesthesia were randomly allocated into two groups. After induction, the group 1 received propofol (Target Controlled Infusion $4-6 \mathrm{mcg} / \mathrm{ml}$ )-remifentanil $(0.1-1 \mathrm{y} / \mathrm{kg} / \mathrm{min})$ and group 2 sevorane (1.5-2.5)-remifentanil $(0.1-1 \mathrm{y} / \mathrm{kg} / \mathrm{min})$. Heart rate (HR), systolic blood pressure (SBP), diastolic blood pressure (DBP) e mean arterial pressure (MAP) were monitored continuously in the radial artery. The surgeon, blinded to the hypotensive agent used and the haemodinamic variables, performed all the operations. The surgeon used a category scale $(0-5)$ to assess surgical conditions: being the numeric value $4-5$ the ideal ones on the scale established.

Results: Blood pressure was not different between the two groups, but heart rate was lower in the group $1(61 \mathrm{bpp} / \mathrm{min}, 95 \%$ confidence interval, $51-73)$ than the group 2 (75 bpp/min, $95 \%$ confidence interval, 65-81). Surgical conditions were rated to be significantly better $(\mathrm{P}<0.05)$ during anesthesia with propofol-remifentanil (median rating: 4.7 compared with group 2 median rating 3 ).

Conclusions: Anesthesia with propofol-remifentanil provides better surgical conditions compared with a traditional balanced anesthesia technique using sevorane-remifentanil. 


\section{9}

Mid Latency Auditory Evoked Potential (AEP) Allow to Value Depth Anaesthesia and Modulate Blood's Concentration of Propofol by Target Controlled Infusion During Breast Cancer Surgery

\author{
L. Piancastelli, A. Potalivo, G.F. Di Nino, R.M. Melotti, \\ M. Agulli, S. Blandino, S. Cassetti \\ Anaesthesiology DPT St Orsola-Malpighi University \\ Hospital, Bologna, Italy
}

Introduction: The processed auditory evocked potential (AEP) with the AAI-index (A-Line Auditory Evoked Potential Monitor, version 1.4, Danmeter A/S; Odense, Denmark) is a technology used to assess the functional depression of the central nervous system during general anesthesia. The purpose of this study was to value the reliability of AEP's monitoring to predict Propofol concentration during breast surgery under general anesthesia using propofol-remifentanyl.

Methods: Fifty patients ASA I-II undergoing elective breast cancer surgery under general anesthesia received propofol for the induction and propofol and remifentanyl for the maintenance by target-controlled infusion (propofol $4-6 \mathrm{mcg} / \mathrm{ml}$-remifentanyl $0.1-1$ $\mathrm{y} / \mathrm{kg} / \mathrm{min}$ ). Deepness of anaesthesia was assessed using the observer's assessment of anaesthesia and sedation (OAAS) scale and by AEP monitor. Heart rate (HR), systolic blood pressure (SBP), diastolic blood pressure (DBP), mean arterial pressure (MAP), blood's oxygen saturation and $\mathrm{ETCO} 2$ were monitored during all surgery.

Results: The transition between consciousness and unconsciousness (OAAS levels 3 and 2) takes place at a value of AAI index between 30-40 while the surgical anaesthesia under a value of 25 . The blood's propofol and remifentanyl concentration may be changed in relation to AAI-index to maintain a OAAS level under 2.

Conclusions: Our results suggest that mid-latency auditory evoked potential may be used to monitor anaesthesia level, to modulate blood's propofol concentration and decrease main anesthetic consumption during total intravenous anaesthesia.

\section{0}

\section{Toracotomy in Thoracic Trauma \\ E. Gamberini, S. Bonarelli, A. Cecchi, G. Malewsky, M. Benni}

Thoracotomy is an uncommon option in early treatment of thoracic trauma according to ATLS guidelines. Massive haemothorax is the major cause of supine thoracotomy which must be performed to prevent injury to often uninvestigated spinal cord and to reduce time from anaesthesia induction to surgery beginning. Special attention should be paid to controlateral PNX, even if simple, which had to be drained before positive pressure ventilation is attempted. Anaesthesia must be carried on avoiding Propofol, Thipentone and volatile agents because of their vasodilatative side effect, dangerous if haemorragic hypovolemia is present. Rapid Intubation Sequence with Ketamine, Suxamethonium and Sellick manoeuvre should be the first choice in traumatized, full stomach patient. Double Lumen Tube (DLT) is the first choice for pulmonary exclusion technique. Pulmonary Occlusion Catheter is an option in difficult intubation with DTL. Third choice is a standard endotracheal tube positioned in a main bronchus guided by a flexible bronchoscope to exclude controlateral lung. Pressure Controlled Ventilation with Plateau Pressure lower 35 mbar, PEEP 4-8 mbar, high $\mathrm{FiO}_{2}$ if needed, should be instituted to prevent VILI to the already injured lung, expecially during one lung ventilation. Fluid infusion should be titrated to maintain permissive hypotension $(\mathrm{PH})$ until haemostasis is obtained to limit bleeding and overload postoperative complications. PH must be avoided if brain injury is suspected. Completion of severe trauma route, ventilatory weaning, and invasive haemodynamic monitoring must be attempted postoperatively in ICU.

\section{1}

\section{Dopamine: A Drug to Forget?}

\section{A. Potalivo, R.M. Melotti, R. D'Andrea, M. Villa, V. Moscaritolo, M. Agulli, V. Altimari, A. Guglielmetti, P. Mascitti}

Anaesthesiology DPT St Orsola-Malpighi University Hospital, Bologna, Italy

Introduction: Dopamine is a natural inotropic agent with specific receptors in the kidney and mesenteric blood vessels and was considered an essential drug, expecially in the intensive care unit, to support cardiac output and blood pressure in patients with cardiac failure and distributive shock and for its supposedly protective effects on renal function and splanchnic mucosal perfusion.

Methods: We made a literature review of randomized control trials published on Pubmed in the last five years about the modern utilization of dopamine in ICU.

Results: There is no evidence that low 'renal' dose DA has any beneficial effect on renal function, on hepatosplanchnic circulation, and recent data suggest that DA may even have detrimental effects on splanchnic oxygen uptake or on the outcome of patients with ARF.

Discussion: The current evidence is that low-dose dopamine, besides not achieving the preset goal of organ protection, may also be deleterious because it can induce renal failure in normo and hypovolemic patients. Furthermore, dopamine may cause harm by impairing mucosal blood flow and by aggravating reduced gastric motility and also suppresses the secretion and function of anterior pituitary hormones aggravating catabolism and cellular immune dysfunction and inducing central hypothyroidism. Dopamine blunts the ventilatory drive, increasing the risk of respiratory failure in patients who are being weaned from mechanical ventilation. There is now ample scientific evidence demonstrating that the use of low-dose dopamine ineffective and its use as a vasopressor may also be questioned. 


\section{2}

\section{Monitoring Severe Sepsis in a Patient After Total Colectomia with Terminal lleostoma for Crohn's Disease}

\author{
S. Campi, A. Potalivo, A. Pigna, H.V. Oven, I. Aprile, \\ E. Zangheri, M. Celotti, G. Simoncini, R.M. Melotti, G.F. Di Nino
}

Anaesthesiology DPT St Orsola-Malpighi University Hospital, Bologna, Italy

Introduction: Sepsis is one of the most frequent causes of morbidity and mortality in patients undergoing major surgery. Studies regarding physiopathology have underlined a straight connection between loss of infection-coagulation response control and clinical progress in severe sepsis, septic shock and MOF. Administration of activated Protein C (PC) showed its effectiveness reducing mortality, but in elevated bleeding risk its use is not recommended. We used PC concentrate $(\mathrm{PCc})$ in a patient suffering from severe sepsis after total colectomia.

Case Report: Patient $q$ of 31 years old affected by Crohn's disease after total colectomia in emergency, was recovered with diagnosis of acute respiratory failure due to pulmonar edema. SAPS: 28, SOFA: 9. On recovery non invasive ventilation was applied, with cardiocirculatory support and large spectrum antimicrobial therapy. The worsening of her clinical conditions required tracheal intubation, mechanical ventilation, liquid therapy, infusion of amines and FFP. SOFA 9, MAP-CVP 52.3, $\mathrm{SvO} 243.5$. Considering the clinical conditions, haemodynamical monitoring data and results of coagulation blood samples, AT together with a bolus of PCc (60 UI $/ \mathrm{kg})$ was administered, followed by continuous infusion $(10 \mathrm{UI} / \mathrm{kg} / \mathrm{h})$ for $48 \mathrm{~h}$. The follow-up of clinical conditions, together with monitoring of haemodynamical status, pharmacological support and nutrition, permitted the stabilisation of clinics and biochemical blood values within $72 \mathrm{~h}$.

Conclusions: The acquired deficit due to PCc waste is straightly connected to multiorgan failure and high mortality. Infusion of PCc in a post-surgical context did not cause any complications and has contributed to full recovery of the clinical conditions.

\section{3}

Organization, Function and Implementation of an Acute Pain Service

\author{
F. Carosi', B.G. Samolsky-Dekel, A. Potalivo, \\ R.M. Melotti, G.F. Di Nino
}

Department of Surgical and Anesthesiological Sciences, Post Graduate School of Anesthesiology and Intensive Care, University of Bologna, Italy

Introduction: The implementation of Acute Pain Service (APS) is mandatory to improve postoperative pain management. ${ }^{1,2}$ In 2004 we established an APS in the S. Orsola-Malpighi Hospital, Bologna. The service is conducted by anesthesiology residents, coordinated by an anesthesiologist and provides pain evaluation and control every 6h. In 2005 the APS followed $n=1997$ patients. The aim of this study was to assess our APS efficacy during 2005. Measured outcomes were: mean pain intensity, proportions of patients whose pain treatment proceeded over $48 \mathrm{~h}$ and patients level of satisfaction with postoperative pain management.

Methods: Overall 1213 patients, who underwent General, Vascular, Thoracic, Otolaryngology, Maxillo-Facial or Plastic surgery, were included. Based on international multimodal therapy guidelines these patients were assigned to different pain management protocols. During the first $48 \mathrm{~h}$ following surgery vital sign, pain intensity and the occurrence of side effects were recorded. Patient satisfaction (insufficient, sufficient, good, optimal) was registered on the $48 \mathrm{~h}$.

Results: Mean pain intensities were $1.23( \pm 1.67)$ at rest and $2.35( \pm 2.01)$ at movement on a $10-\mathrm{cm}$ visual analogue scale (VAS). Pain intensity was $>3$ in 92 patients $(7.6 \%)$ at rest and in $347(28.6 \%)$ at movement. Pain treatment was needed and hence proceeded after the first $48 \mathrm{~h}$ in $50 \%$ of the patients. Patient satisfaction turned to be good $(41.6 \%)$ or optimal $(58.1 \%)$.

Discussion: Evidence suggests that the introduction of an APS may reduce surgery patients' pain intensity through pain assessment, multi-modal pain treatments and continuous quality process evaluation. ${ }^{1,2}$ Our data further confirm this hypothesis. 


\section{European Surgical Research}

Abatangelo, G. 315, 316

Aboh, I.V. 438, 446

Abumalouh, I. 169, 170

Acerbis, F. 162

Adani, G. 7

Adani, G.L. 5, 6, 8, 9, 283, 284

Adorisio, O. 521

Agliardi, A.C. 527, 528

Aglietta, M. 355

Agresta, F. 136

Agresti, M. 50

Agulli, M. 52, 539, 541

Aiazzi, L. 223

Albarello, L. 118

Albino, V. 365

Alessiani, M. 332, 333, 334

Altimari, V. 52, 538, 541

Amabile, D. 337

Amaddeo, P. 428

Amaduzzi, A. 291, 295, 296, 298, 300

Amanti, C. 377,468

Amato, C. 418

Ambrogi, M.C. 185

Ambrosi, A. 39, 40, 121, 122, $123,124,125$

Ambrosini, C.E. 490

Amerio, P. 460

Amodeo, C.A. 314, 338, 392, 439

Amodeo, C.A. $357,447,448$

Amore, D. 147

Amorese, G. 308

Amoroso, V. 246

Ampollini, L. 140

Andreoli, F. 337

Andretta, E. 317, 318

Angelici, A.M. 83

Angelini, M. 468

Angiò, L.G. 93, 94, 95, 96, 97 , 98, 99, 363, 396, 397, 398, $399,400,475,476$

Angrisani, L. 242, 243

Anile, M. 141

Annacontini, L. 452, 453, 454

Annecchiarico, M. 1, 2

Annessi, V. 165

Annunziata, G. 189, 310

Ansaloni, L. 52, 134, 135, 136, $335,375,376,379,395$

Antico, A. 228
Antico, E. 306

Antoniazzi, L. 241

Antoniolli, S.Z. 319

Apicella, A. 88, 89, 91

Apicella, F. 72, 73

Aprà, F. 50

Aprile, G. 186, 187

Aprile, I. 542

Araco, F. 405

Arcadipane, A. 299, 474

Arcieri, L. 248, 249, 250, 258

Ardito, G. 491, 492

Ardizzone, A. 399

Ardò, N.P. 149

Argenteri, A. 195, 196, 197

Argnani, D. 142, 164, 175, 181

Arici, V. 200, 201

Aricò, F. 95

Armatura, G. 451

Arnoldi, R. 531

Arpesella, G. 262

Arpino, L. 493, 494, 495, 496

Arrigoni, G. 71, 336

Artero, M. 299

Artibani, W. 319

Artuso, G. 317, 318

Aseni, P. 303, 304

Attene, F. 41

Attiná, G. 264

Attinà, G.M. 42, 265

Avanzolini, A. 31

Avellini, C. 283, 284

Avenia, N. 88, 89, 91, 477

Aversa, S. 489

Azas, L. 210, 232

Azzena, G. 15, 16, 17, 18, 162, 488

Baccarani, U. 5, 6, 7, 8, 9, 283, 284

Baccari, P. 238, 266

Bacchieri, M. 228

Baggio, E. 451

Bagnato, C. 383, 401

Bagolan, P. 532

Baiano, L. 16

Baiocchi, G.L. 3, 35, 362

Baisi, A. 172

Baj, A. 440

Bajona, P. 248, 249, 250, 251, 258

Balestrieri, T. 144
Ballabio, D. 160

Ballarin, C. 329

Ballarin, R. 74, 281, 282, 292, 367, 368

Balzaretti, E. 431

Bandiera, A. 156

Bandiera, S. 408, 410

Banno, G. 373, 374

Banzatti, B. 314, 338, 357, 392 , 439, 447, 448

Baracchini, E. 186, 187

Baratto, F. 190

Barba, G.L. 297

Barbaglio, A. 428

Barbanti, E. 210

Barbanti, G.B. 410

Barbarisi, A. 88,89

Barbarisi, A. 91

Barbato, G. 86, 87

Barbera, G.L. 208

Barberi, F. 259

Barbone, A. 252

Bardone, M. 332, 333, 334

Barlettai, M. 383

Barnaba, S.A. 417

Barranco, G. 474

Bartoccioni, E. 460

Bartoletti, R. 311, 312

Bartoli, A. 267

Bartolotta, T. 143, 144, 145, 342

Bartolotta, T.V. 143, 144, 145, 342

Bartolucci, E. 380, 381

Barugola, G. 451

Barzaghi, M.E. 190, 191

Basciotti, A. 36, 369

Basilico, G. 163

Bassi, U.A. 242, 243

Basso, G. 454

Bastianello, P. 317, 318

Batoli, A. 271

Battaglia, B. 66, 131

Battaglia, L. 4

Battaglia, P. 514

Battaglini, M. 243

Battisti, A. 436, 438

Battistini, M. 226

Bazzocchi, F. 31

Bearzi, I. 307

Bechi, P. 61

Bedini, A.V. 184
Bedini, R. 421

Belardi, A. 66

Belgrano, M. 108

Bellandi, G. 190

Bellandi, S. 211

Bellantone, R. 478, 479, 480, 481, 482

Bellaviti, N. 163

Bellieni, L. 248, 249, 250, 251, 258

Bellini, N. 228

Bellò, M. 354

Bellocchio, G. 441

Bellochi, R. 267

Bellotti, A. 66

Bellotti, A.M. 131

Beltempo, P. 285, 287, 326, 327, 328

Beltramini, G. 442

Beltramo, G. 354

Benavoli, D. 126, 247

Bencini, L. 269

Benedetti, M. 389, 390, 391

Benedetti-Valentini, F. 214

Benedetto, F. 192, 193, 194

Benedetto, G. 315, 316

Benedetto, U. 259

Benetti, D. 35, 166, 167, 358

Benin, P. 191

Benni, M. 540

Benvenuti, M. 35, 166, 167, 358

Benzone, S. 317, 318

Benzoni, E. 5, 6, 7, 8, 9, 283 , 284

Bergamini, C. 370, 371, 372

Bergonzini, M. 252

Berna, F. 13, 14, 20, 69, 70, 449,450

Bernardi, M. 328

Bertelli, R. 37, 38, 120, 285, 286, 287, 326, 327, 328, 402, 403

Berti, P. 33, 490

Bertini, R. 326

Bertoglio, L. 238

Bertoletti, G. 227

Bertoloni, R. 319, 321, 323, 324

Bertotti, E. 138

Bettini, D. 31

Bezzi, M. 81, 82

Bianca, A. 173

\section{KARGER}

(C) 2006 S. Karger AG, Basel

Fax +41 613061234

E-Mail karger@karger.ch

www.karger.com

Accessible online at:

www.karger.com/esr 
Biancafarina, A. 267, 271, 272

Bianchi, A. 432

Bianchi, G. 195, 196, 197

Bianchi, P. 198

Bianchi, R. 520

Bianchini, R. 259

Biasco, G. 134

Bignami, M. 514

Biolchini, F. 64

Biondi, A. 359, 361

Biondi, P. 91

Biroli, A. 333

Bisagni, P. 10, 43, 117, 119, 266, 268, 270

Bisi, G. 353

Bissolotti, G. 43, 44

Bistoni, G. 465

Blandino, R. 373, 374

Blandino, S. 52, 539

Bobbio, A. 140

Bodeci, A. 79, 80

Boggi, U. 308, 350

Bollino, F. 139

Bona, S. 273

Bona, V. 199

Bonanno, L. 337

Bonardelli, S. 205

Bonarelli, S. 540

Bondanza, G.S. 351

Bongini, G. 24, 27

Boniello, R. 425, 426, 427

Bonomi, S. 113

Bonsignore 363

Bonsignore, A. 93, 94, 95, 96, 97, 98, 99, 396, 397, 398, $399,400,475$

Bonuso, C. 2

Bordin, G. 529

Bordonaro, C. 506

Boriani, L. 408, 410

Boriani, S. 408, 410

Borioni, G. 76, 77

Borrelli, V. 242, 243

Borsellino, G. 21

Borzellino, G. 46

Boscherini, M. 491, 492

Bossi, I. 288

Bossolesi, P. 513

Botta, G. 13, 14, 20, 449, 450

Botta, L. 260, 262

Bottazzi, A. 332, 333, 334

Bottero, L. 173

Botti, F. 356

Botticchio, N. 278

Botticini, G. 166, 167, 358

Bottura, R. 4

Bouzari, H. 355

Bovo, G. 477

Bovolato, P. 166, 167, 358
Bozzani, A. 200, 201

Bozzola, A. 320

Bracaglia, R. 244, 245, 459, 460, 461, 462, 463, 464

Brancaccio, U. 58, 274, 483

Bravo, A.F. 35, 167, 358

Bresadola, F. 5, 6, 7, 8, 9, 283, 284

Brillantino, A. 36, 369

Brioschi, C. 237

Brodano, G.B. 408

Brombin, C. 162

Bruno, A. 457, 458

Bruschi, G. 254, 255, 288, 289

Brusciano, L. 110

Bruzzi, C. 504, 505, 506, 511

Bucalossi, M. 13, 14, 20, 69, 70, 449, 450

Budak, A. 339, 393, 394

Bugiantella, W. 45, 378

Buonavolontà, G. 498

Burrai, S. 455

Busacchi, P. 407

Busiello, G. 153

Bussotti, A. 290

Bussu, F. 508

Buttazzi, K. 260

Caccamo, C. 299

Cadoni, R. 336

Cadoni, R. 71

Cai, T. 311, 312

Cajozzo, M. 143, 144, 145, 342

Calabrese, F. 171, 176

Calandra, G.F. 3

Caletti, G. 134

Calliari, F. 240

Calò, G. 134, 376

Calopresti, A. 96, 98

Calvieri, S. 11

Calzolari, F. 477

Cambria, C. 513

Cambrini, P. 379

Cammisa, F. 90

Campa, R. 83

Campa, R.D. 81,82

Campagnaro, T. 103, 104, 133

Campanale, A. 452, 453

Campi, S. 542

Campisi, S. 288, 289

Camporese, G. 190

Canale, S. 195, 196, 197

Cananzi, F.C.M. 360

Cancilleri, F. 419

Candiani, M. 466

Candiani, P. 191

Cannistrà, M. 29

Cannizzaro, M.A. 489

Cantisani, R. 384, 401
Cantore, I. 510

Cantu, M. 35

Capizzi, R. 460

Capobianco, A. 345

Capoccia, L. 227

Capoluogo, E. 460

Capolupo, I. 532

Capone, D. 51

Capotorto, G. 150

Cappabianca, P. 497

Cappellini, P. 72, 73

Cappello, F. 143, 144, 145, 342

Cappuccio, M. 408, 410

Capuano, C. 493, 494, 495, 496

Capuano, F. 259

Capuano, P. 246

Capussotti, L. 355

Caraceni, P. 328

Carbognani, P. 140

Carbone, E. 45

Carbone, L. 313

Cardi, M. 389, 390, 391

Cardona, R. 30, 68

Carella, G. 202

Carignano, G. 233

Carlucci, M. 10, 118, 279

Carmo, M 225

Carnevale, C. 445

Caronna, R. 389, 390, 391

Carosi, F. 543

Carotenuto, V. 501

Carpino, A. 29

Carrara, A. 356

Carretta, A. 156

Carrinola, R. 172

Carrozza, C. 479, 480

Carú, F. 329

Caruso, R. 254

Caruso, S. 346, 347, 348, 349

Casaccia, G. 532

Casadei, A. 215

Casali, C. 179,180

Casali, G. 263

Casana, R. 198

Casani, A.P. 509, 512

Casciaro, G.E. 339, 393, 394

Casciola, L. 267

Casolino, C. 1, 2

Cassetti, S. 52, 539

Cassoni, A. 424

Cassoni, P. 352, 354

Castagnola, M. 228, 351

Castaldi, P. 482

Castellano, I. 352

Castelli, A. 19, 21, 22, 46

Castellini, G. 31

Castellucci, P. 134

Castelnuovo, P. 513, 514, 515

Catapano, D. 501, 502
Catarci, M. 66, 131

Catena, F. 134, 135, 136, 335, $375,376,379,395$

Cattelani, L. 140

Causa, N. 59, 60, 382

Cautero, N. 305, 306, 307

Cavaliere, D. 37, 38, 120, 402, 403

Cavallari, G. 287, 326, 327, 328,330

Cavallaro, A. 12, 331, 489

Cavallaro, G. 11, 12, 331

Cavallesco, G. 17, 488

Cavallesco, G.N. 15

Cavazzoni, E. 45, 378

Cavenaghi, G. 507, 520

Cavicchi, O. 484, 504, 506, 511

Caviggioli, F. 338, 357, 392, 439, 447, 448

Cazzaniga, R. 234, 278, 364

Ceccacci, T. 203, 215, 216

Ceccarelli, G. 267, 271, 272

Cecchi, A. 540

Cecere, C. 147

Cecere, D. 204

Celiku, E. 79, 80

Celotti, M. 542

Celotti, S. 47

Celsi, S. 58, 274, 483

Centonze, N. 521

Cerbone, D. 86, 87, 90

Cerciello, S. 422

Cerea, K. 3

Ceroni, A.R. 504, 506

Ceroni, A.R. 484

Cerqua, R. 85

Cerruto, M.A. 319

Cerullo, G. 347, 348, 349

Cervi, E. 205

Cescon, M. 298

Chattat, R. 52

Chella, A. 152

Chessa, A. 25, 26, 48, 49, 63

Chiacchio, A. 85

Chiaramonti, F. 248, 249, 250, 251, 258

Chiesa, C. 213

Chiesa, R. 237, 238, 239, 240

Chirletti, P. 389, 390, 391

Chisci, E. 206, 207

Chiti, E. 211

Chiummariello, S. 465

Cianchi, F. 61

Cianci, P. 343, 344, 345

Cianciavicchia, D. 327

Cianfrone, F. 510

Ciannella, M. 242, 243

Ciavarella, M. 189, 310

Cicalese, M. 147 
Ciferri, E. 351

Cigna, E. 456

Cimmino, G. 85, 86, 87, 90

Cintorino, D. 299, 474

Cioffi, G. 60, 382

Cioppa, T. 346, 347, 348, 349

Cipriani, R. 471

Ciriaco, P. 156

Citterio, E. 252

Ciuffi, M. 343

Civitelli, S. 24, 25, 26, 27, 48, 49, 63

Claysset, B. 261

Clementini, M. 426

Clerico, G. 23, 32

Cobani, E. 79, 80

Coccolini, F. 379

Cojutti, A. 5, 6, 7, 8, 9

Colagrande, L. 256, 257

Colella, A. 498, 499, 500

Colella, G. 91

Colognesi, A. 18

Colombo, A. 254

Colombo, L. 431

Colombo, P. 288

Colombo, T. 255, 289

Coloni, G.F. 141

Coluccia, P. 331

Comandi, A. 13, 14, 20, 69, 70, 449,450

Compagnoni, B. 362

Condorelli, F. 489

Conforti, S. 146

Congedo, M.T. 182, 183

Consiglio, F. 470

Consiglio, F.M. 14

Contessini Avesani, E. 356

Conti, B. 184

Conti, D. 267

Contine, A. 45

Contini, C. 482

Conzo, G. 58, 274, 483

Coppi, G. 212

Corbetta, R. 201

Cordaro, E.R. 466

Cornaglia, S. 34, 353

Corsi, C. 53

Corso, V. 380, 381

Cortesini, C. 61

Corti, F. 160, 256, 257

Cosentini, V. 299

Cosenza, A. 36, 369

Cosenza, M. 276

Cossaro, E. 317, 318

Cossaro, M. 186, 187

Costa, V. 416

Costamagna, V. 78, 352

Costantino, A. 99

Costanzo, M. 489
Cotroneo, A. 192, 193, 194

Cotroneo, G. 468

Cozzi, B. 329

Cozzupoli, P. 313

Credi, G. 211

Cremona, F. 365

Crispino, V. 390

Croccia, M. 248, 249, 250, 251, 258

Croce, C. 350

Crocoli, A. 42

Cuccaro, P. 51

Cucchetti, A. 297

Cuccomarino, S. 50

Cucina, A. 331

Curci, R. 195, 196, 197

Curro, G. 283

Currò, G. 284

Curti, P. 319

Cusumano, G. 182, 183

Cutolo, P. P. 243

D'adamo, R. 222

Daddi, G. 159, 177, 178

Daddi, N. 159, 177, 178

D’Agostino, J. 490

D'Agostino, M. 373, 374

d'Ajello, M. 477

Dalainas, I. 198

D’Alessandro, L. 134, 335, 379, 395

D'Ambra, M. 51

D'Amore, A. 491, 492

D'Amore, A.M. 479, 481

Dandolo, R. 339, 393, 394

D'Andrea, R. 538, 541

Danesi, T.H. 259

D'Angelo, V.A. 501, 502

D'Antonio, D. 59

Dattola, A. 202

Davide, A. 290

D'Avino, R. 1

Dazi, A. 300

Dazzi, A. 291, 295, 296, 298

De Aloysio, D. 407

De Bernardi, F. 513

De Biasio, F. 470

De Caridi, G. 194, 236

De Cataldis, A. 52, 375, 376

De Crea, C. 478, 481

de Donato, G. 206, 207

De Fina, S. 92

de Franciscis, S. 235

de Franco, S. 165

De Lio, N. 350

De Luca, G. 147, 148

De Luca, M. 19, 21, 22

De Luca, R. 56, 57

De Luca, S. 305, 306, 307
De Lucia, M. 205

de Manzoni, G. 130

De Marco, G. 346, 347, 348, 349

De Martino, M. 332, 333, 334

De Micco, C. 486, 487

De Nardi, P. 279

De Nicola, E. 71

De Palma, A. 149, 150

De Paolis, P. 138

De Roberto, A. 303, 304

De Rosa, C. 493

De Ruvo, N. 74, 281, 282, 292 , 367, 368

De Salvia, G. 452, 454

De Santis, M. 293, 294

De Silva, G.M. 351

De Simone, M. 370, 371, 372

De Stefano, M. 114, 115

De Toma, G. 11, 12, 331

de Tullio, D. 15, 16, 17, 18

De Vita, D. 405

Degrate, L. 92

Del Chiaro, M. 308, 350

Del Ciampo, D. 59, 60, 382

Del Basso De Caro, M.L. 497

Del Gaudio, M. 297, 298

Del Monte, F. 463

Del Viscovo, L. 88

Delconte, C. 53

Dell' Amore, A. 260

Dell'aglio, D. 198

Dell'Amore, A. 142, 175, 262

Dell'Amore, D. 142, 164, 175, 181

Della Vecchia, A. 51

Della Volpe, N. 1, 2

Delmonte, S. 78, 352

Delrio., P. 365

Delrio, P. 366

Delù, G. 513, 515

Denaro, E. 412, 413, 414, 415, 419,420

Denaro, V. 416, 417, 418

Deonofrio, M. 341, 443

Deprest, J. 406

D'Errico, A. 295

D'Errico, G. 460

Di Bartolomeo, R. 260, 261

Di Battista, L. 114, 115

Di Benedetto, F. 74, 281, 282, 292, 293, 294, 367, 368

Di Betta, E. 54

Di Carlo, F. 209

Di Carlo, L. 159, 177, 178

Di Crescenzo, V.G. 153

Di Fabio, F. 54

Di Francesco, A. 431

Di Vito Francesco, M. 209, 232
Di Lella, D. 208

Di Leo, A. 130

Di Leo, P. 344

Di Lieto, C. 505, 518

Di Lillo, S. 51

Di Maio, V. 243

Di Marco, L. 262

Di Mare, M. 211, 232

Di Mari, N. 48

Di Martino, A. 416

Di Martino, N. 36, 65, 369

Di Muria, A. 57

Di Napoli, M.G. 522, 523, 524, $525,526,527,528,529,530$, 531

Di Nardo, W. 510

Di Nino, G.F. 539, 542, 543

Di Palo, S. 10, 43, 44, 105, 106,

$116,117,118,119,268,270$

Di Petrillo, A. 425

Di Sandro, S. 281, 282, 292, 367, 368

Di Saverio, S. 335

di Saverio, S. 375

Di Saverio, S. 376, 379, 395

Di Scioscio, V. 134

Di Simone, M. 291, 295

Di Simplicio, S.C. 533

di Spilimbergo, S.S. 434

Di Stasio, E. 480

Di Stasio, M. 141

Di Stazio, C. 110, 111, 112

Di Stefano, G. 289

Diamantis, G. 472, 473

Diana, M. 339, 393, 394

Dibra, A. 79, 80

Dini, P. 152

Dionigi, G.L. 33

Dionigi, P. 332, 333, 334

Diso, D. 141

Dobrinja, C. 55, 479

Docimo, G. 56, 57, 246

Docimo, L. 57, 246

Doglioni, C. 118

Dominioni, T. 332, 333, 334

Donatini, G. 490

Doni, M. 332, 333, 334

Donini, A. 378

D’Onofrio, M. 103

Donzelli, O. 409

Donzelli, R. 497

Dordoni, L. 238

Dorigo, W. 209, 210, 232

D'Ugo, D. 359, 360, 361

Duranti, L. 151

Ebner, J. 191

Elmo, M. 365

Elmore, U. 273 
Ercolani, G. 291, 295, 298

Esposito, M. 497, 498, 499, 500

Esposito, M.G. 58, 274, 483

Esposti, S.D. 535

Eusebio, A. 252

Evangelista, A. 60, 382

Fabiocchi, L. 471

Faccenna, F. 214

Facchiano, E. 378

Facchinetti, C. 336

Faenza, A. 285, 286, 287, 326

Faenza , S. 295

Faggioli, G.L. 215, 231

Fagnani, A. 529

Faillace, G. 173

Faini, G.P. 466

Falco, P. 59, 60, 382

Faldi, F. 428

Faldini, A. 411

Fallahdar, D. 507, 520

Famulari, C. 476

Fanello, G. 389, 390, 391

Fantini, A. 173

Farace, F. 455, 467

Farallo, E. 457, 458, 460

Fargion, A. 210

Farinon, A.M. 42, 264, 265, 385,485

Farneti, P. 518

Faro, G. 93

Fattori, R. 260

Favero, A. 5, 6, 7, 8, 9

Favi, F. 277, 280

Fedeli, S. 524, 525, 526, 527

Ferla, L. 156

Fermani, N. 226

Fernandez, I.J. 484

Fernandez, M.A. 518

Feroci, F. 61

Ferrari, A. 533

Ferrari, F. 29

Ferrari, G. 165

Ferrari, S. 279

Ferrario, V.F. 430

Ferraris, C. 50

Ferraro, B. 340

Ferri, M. 231

Ferro, O. 256, 257

Ferrocci, G. 15, 488

Ferronato, A. 157, 158, 161

Fersini, A. 39, 40, 121, 122 , $123,124,125$

Ferulano, G.P. 51

Filidei, F. 383, 384

Filisetti, C. 332

Finelli, F. 50

Fino, L. 152

Fiorani, B. 259
Fiore, F. 53

Fiorello, A. 153, 154

Fisogni, D. 129

Fiumara, F. 93, 94, 95, 96, 97, 98, 99, 363, 396, 397, 398, $399,400,475$

Foco, M. 244, 245, 459, 461, 462

Fogacci, T. 375, 376, 380, 381

Foglieni, C.S. 299,474

Fois, V.E.E. 467

Fontana, A. 212

Fontana, D. 522

Fontana, M. 103, 104, 133

Fontana, P. 157, 158, 161

Fontana, S. 428

Fontanesi, L. 253

Fontani, A. 49

Fontanini, G. 151

Formato, L. 228

Formica, F. 160, 256, 257

Formillo, P. 429, 430

Fornari, F. 11

Forriol, F. 420

Forte, S. 277

Fortuna, D. 135

Fortunato, R. 463, 464

Fosso, A. 490

Foteini, L. 261

Fracastoro, G. 46

Framarini, M. 37, 38, 120, 402, 403

Franceschi, F. 412, 413, 414, $415,418,420$

Franceschilli, L. 247

Franceschini, E. 222

Franceschini, S.S. 512

Francheschini, S.S. 516, 517

Francioni, G. 380, 381

Franciosi, C. 92

Franciosi, E. 229

Franco, A. 493, 494, 495, 496

Frank, G. 502

Frantellizzi, V. 389, 390, 391

Franzini, P. 4

Frascà, G. 305

Frasson, M. 106

Frasson, P. 340

Fratesi, F. 231

Frattini, D. 433, 434

Freyrie, A. 203, 216, 231

Frigerio, M. 288, 289

Frigerio, S. 237

Fronda, G.R. 138

Frustaci, G. 33, 490

Fuga, G. 285, 286, 287

Furxhi, N. 504

Fusari, M. 199
Fusaroli, P. 134

Fusco, G. 389, 390, 391

Gaboardi, F. 320

Gabriele, M. 490

Gabrielli, R. 214

Gaffi, G. 305

Gagliardi, G. 134

Gagliardi, S. 135, 335, 375, 376, 379, 395

Galarco, G. 400

Galassi, R. 37, 164

Galatá, G. 264

Galatà, G. 265, 337, 385, 485

Galatioto, C. 384

Galleri, D. 490

Galli, S. 320

Gallieri, S. 253

Gallo, E. 139

Gallo, G. 154

Gallo, M.C. 62, 101, 102, 109, 132

Galloro, G. 472, 473

Galzerano, S. 147

Gamberini, E. 540

Gangemi, M. 499, 500

Ganio, E. 23, 32

Garatti, A. 254, 255, 288, 289

Garcea, D. 31

Gardani, M. 3

Gardini, A. 31

Garelli, P. 305, 306, 307

Gargiulo, M. 203, 215, 216, 231

Garofalo, G. 149

Gasbarrini, A. 408, 410

Gasparella, V. 321, 323, 324

Gaspari, A.L. 126, 127, 128, 247

Gasparini, G. 425, 426, 427

Gasparrini, M. 275, 276, 277, 280

Gatti, S. 329

Gavazzi, A. 312

Gavazzi, F. 302

Gavioli, M. 62, 101, 102, 109, $132,139,404$

Gaynor, J.J. 290

Gazzabin, A. 20

Gazzabin, L. 13, 14, 20, 69, 70, 449,450

Gazzotti, F. 134, 335, 375, 376, 379,395

Gelati, C. 471

Genco, B. 21, 22

Gennaro, P. 445, 446

Genovese, V. 227

Gentile, V. 384, 401

Gentileschi, P. 127, 128, 247
Gentileschi, S. 244, 245, 457, 458, 459, 460, 461, 462, 463, 464

Genualdo, M. 150

Gerunda, G.E. 74, 281, 282 , 292, 367, 368

Gervasoni, C. 431

Gesuelli, G.C. 107

Gheza, F. 3

Ghidoni, I. 253

Ghinassi, S. 66

Ghiribelli, C. 155

Ghisetti, V. 78, 352

Giacometti, C. 171,176

Giacopuzzi, S. 130

Giamundo, A. 499

Giani, I. 23, 32

Giannasi, S. 338, 357, 392, 447, 448

Giannì, A.B. 429, 430, 440, 441,442

Giannini, G. 471

Giannini, P. 4

Gianoli, M. 257

Giardino, A. 451

Giordano, S. 469

Giorgetti, P.L. 229

Giorgio, A. 508

Giorgio, C. 162

Giori, A. 53

Giovagnoni, A. 305, 306

Giovanetti, F. 215

Giovanetti, M. 129

Girelli, C.M. 255

Giubbolini, M. 206, 207

Giudice, F. Lo 158, 161

Giudice, F.L. 157

Giudice, M. 515

Giuffrè, M. 242, 243

Giuliani, A. 111

Giulini, S.M. 3, 35, 205, 362

Giustozzi, E. 491, 492

Golfieri, L. 291, 295, 296, 298, 300

Gotti, G. 155,168

Gozzo, G. 452, 453

Grammatico, F. 285, 286

Granato, F. 155, 168

Grande, M. 42, 264, 265, 385, 485

Grandi, S. 298

Granone, P.M. 182, 183

Grassi, A. 113

Grassi, G.B. 66, 131

Grassi, I. 24, 25, 26, 27, 48, 49, 63

Grazi, G.L. 297, 298

Gregori, A. 320

Gregorio, C. 488 
Gridelli, B. 299, 474

Grieco, M. 453

Griffo, S. 147

Grilli, R. 135

Griner, A. 433

Griso, A. 190, 191

Gruttadauria, S. 299, 474

Guadagni, I. 110, 111, 112

Guadalaxara, A. 336

Guaitoli, S. 102, 132

Guarino, G. 343, 344, 345

Guarino, S. 114, 115

Guarneri, G. Franco 470

Guasti, G. 165

Guerra, M.V. 139

Guerriero, G. 213

Guerrini, G. 74, 281, 282, 292, 367,368

Guglielmetti, A. 541

Guglielmetti, M. 184

Guglielmi, A. 103, 104, 133

Guglielminetti, A. 52

Guida, C. 365

Gullà, G. 341, 443

Gullà, N. 45

Gurrado, A. 386, 387, 388

Guzzo, M. 507, 520

Hassibi, J. 338, 357, 392, 439, 447, 448

Henry, J.F. 486, 487

Iacchetta, F. 62

Iacobelli, B. 532

Iaconetta, G. 497

Ianni, G. 227

Ibarrola, A.G.S. 482

Ibba, T. 507, 520

Idà, D.N. 365, 366

Ierace, M. 313

Ignomirelli, O. 345

Imail, I. 113

Impérial, H. 228

Incardona, S. 396

Innaro, N. 28

Innocenti, A.A. 209, 232

Innocenti, L. 501

Intini, S. 7, 9

Iob, G. 228

Iovino, F. 56

Iovino, P. 472, 473

Ippolito, G. 486, 487

Ippolito, M. 414, 419

Irlandese, E. 56

Isola, M. 329

Itri, E. 530

Iusco, D. 64

Izzo, D. 65

Izzo, G. 36, 65, 369
Jannaci, A. 50

Jannasi, S. 439

Jovine, E. 64

Kaci, M. 79, 80

Kahlberg, A. 239

Kato, T. 290

Keinan, F.M. 390

Klinger, F. 314, 338, 357, 392, 439, 447, 448

Klinger, M. 314, 338, 357, 392 , 439, 447, 448

Klugmann, S. 288

Knetz, R. 320

Komatsu, T. 375, 376

Konstantinovic, M. 406

Kontothanassis, D. 190

Laganà, F. 317, 318, 442

Lagoussis, P. 47

Laineri, M. 463

Lambert, Y. 347, 348, 349

Lancisi, G.M. 188

Landi, L. 521

Landolfo, G. 64

Lanfranconi, M. 254, 255

Lanzino, G. 502

Lapalorcia, L.M. 465

Laperuta, P. 153

Lardo, D. 386, 387, 388

LaRocca, L.M. 460

Lattuada, E. 241

Lattuneddu, A. 31

Lauretta, M. 398

Laurito, A. 214

Lauro, A. 282, 291, 295, 296, 297, 298, 300

Lavermicocca, W. 386, 388

Lavini, C. 174

Lazzari, A. 64

Lazzaro, A. 247

Leonardi, F. 418

Leone, A. 375

Leone, F. 355

Leone, L. 66, 131

Leopardi, F. 19, 22

Leporati, A. 169

Lerro, A. 148

Lerro, F. 64

Libretti, L. 156

Licursi, M. 475

Ligabue, T. 167, 168, 358

Liguori, G. 55

Limongelli, P. 110, 111, 112

Linguerri, R. 75, 76, 77

Lioi, A. 341, 443

Lipari, G. 451

Liparulo, V. 141
Lipparini, T. 295

Lirosi, F. 247

Lissidini, G. 67, 386, 387, 388

Liverani, A. 275, 276, 277

Livi, U. 186,187

Livio, M. 363

Livrea, A. 58, 274, 483

Llukaci, A. 79, 80

Loffredo, D. 59, 60, 382

Logaldo, D. 239

Logroscino, G. 421, 422

Loizzi, D. 149, 150

Loizzi, M. 149, 150

Lojodice, F. 59, 60, 382

Lombardi, A. 377

Lombardi, C.P. 478, 479, 480, 481, 482, 491, 492

Lomonaco, A. 159, 177, 178

Longo, B. 469

Longo, C. 281

Longo, G. 446

Longo, U.G. 412, 413, 414, $415,418,419,420$

Longobardi, G. 425, 426, 427

Longoni, M. 173

Lorè, B. 435, 438, 446

Lorenzin, D. 5, 6, 7, 8, 9, 283, 284

Lorenzo, M. 242

Loreto, F di 532

Lorini, G. 463

Losi, L. 62

Losinno, F. 231

Louisma, O. 532

Lovadina, S. 157, 161

Lovadina, S. 158

Lovecchio, N. 430

Lovisetto, F. 332, 333, 334

Loy, M. 171

Luc, A. Realis 23

Luc, A.R. 32

Lucchi, A. 31

Lucchi, M. 151, 152

Lucci, T. 295

Luciano, A. 148

Lucisano, A.M. 28, 68

Luigi, M.P. 501

Luigiano, C. 476

Lulli, R. 269

Luna, E. 299, 474

Luongo, A. 235

Luppi, G. 62

Luzzi, L. 155, 168

Maccauro, G. 421, 422

Macchini, F. 522, 523, 524, $525,526,527,528,529$

Maccora, D. 481

Macrí, G. 518, 519
Maggi, L. 72

Maggi, S. 377

Maglione, P. 123

Maglione, V. 138

Magnani, M. 409

Magno, L. 472, 473

Magnoni, F. 230

Magro, F. 499, 500

Maioli, F. 203, 215, 216

Maione, F. 2

Maiorella, A. 452, 453, 454

Maiuri, F. 497, 498, 499

Malacrida, G. 198

Malewsky, G. 540

Malvezzi, E. 351

Mambrini, S. 217, 218, 219, 220, 221

Mancini, S. 13, 14, 20, 66, 69, $70,449,450$

Manco, G.G. 59, 60, 382

Manconi, R. 317

Mandalà, L. 299, 474

Manfredelli, S. 81, 82, 83

Mangia, F. 248, 249, 250, 251, 258

Mangioni, S. 390, 391

Maniscalco, P. 17, 162, 488

Manna, G.L. 113

Manno, M. 222

Manzoli, L. 231

Maraglino, C. 223

Marca, G.L. 208

Marchesa, P. 474

Marchese, M.R. 508

Marchetti, C. 432

Marchetti, M. 509, 512, 516, 517

Marchetti, P. 308

Marco, B. 212

Marelli, S. 429

Marengo, M. 389, 390, 391

Marfisi, C. 31

Margarita, V. 50

Mari, F.S. 280

Mari, F.S. 275, 276, 277

Marianetti, T.M. 424

Mariani, F. 347, 348, 349

Mariani, P. 71, 336

Marin, M. 213

Marinelli, G. 261

Mariniello, G. 498

Marino, F. 23, 32

Marinocosentino, L. 66

Marinozzi, A. 412, 413, 414, 415, 419, 420

Marone, E.M. 237, 238, 239, 240

Marrelli, D. 346, 347, 348, 349

Martellucci, J. 49 
Martinelli, G. 227

Martinelli, S. 139

Martini, F. 72, 73

Martino, A. 208

Martino, G. 389, 390, 391

Martin-Suarez, S. 261

Martuzzi, F. 64

Marulli, G. 171, 176

Marvas, A. 59

Marvaso, A. 60, 382

Marziani, A. 489

Masci, E. 118, 213, 241

Mascitti, P. 52, 541

Maselli, D. 248, 249, 250, 251, 258

Masetti, M. 74, 281, 282, 292, 367, 368

Masoni, L. 275, 276, 277, 280

Massa, R. 214

Massariello, D. 454

Massi, F. 253

Massi, M. 490

Massini, S. 211

Massucci, M. 227

Mastrangelo, D. 235

Mastrangelo, L. 64

Mastromarino, A. 222, 224

Masullo, G. 186, 187

Materazzi, G. 33, 490

Matrella, F. 123

Mattavelli, F. 520

Mattei, R. 521

Matteucci, F. 37, 164

Matticari, S. 210

Mauri, M. 47

Mauro, A. 62, 101, 102, 109, 132

Mauro, R. 216

Mazza, L. 138

Mazzei, R. 218, 219, 221

Mazzeo, A. 140

Mazzetti, M.P. 269

Mazzilli, M. 332

Mazzoni, L. 52

Mazzoni, S. 432

Mazzuoli, S. 343

Meacci, E. 183,182

Meazza, C. 533

Meda, S. 170

Meduri, A. 534, 535, 536, 537

Meduri, R.A. 534, 535, 536, 537

Mele, A. $126,127,128$

Melillo, P. 510

Melina, G. 259

Melissano, G. 237, 240

Melotti, M.R. 52

Melotti, R. 135
Melotti, R.M. 538, 539, 541, 542, 543

Mendogni, P. 163, 172

Mengozzi, M. 142, 164, 175, 181

Mercandalli, G. 225

Merico, E. 259

Merlicco, D. 387

Meroni, M. 431

Messina, F. 15, 16, 17, 18

Mevio, N. 332

Mezzatesta, P. 474

Mezzetti, M. 169, 170

Miccoli, P. 33, 490

Miceli, A. 259

Mickalajeva, O. 232

Micossi, C. 226, 385

Mikhalyaeva, O. 211

Mikus, E. 261, 262

Mikus, P. M. 262

Milandri, C. 37

Milanesi, E. 354

Milano, S. 166

Milazzo, F. 254

Milesi, G.L. 278

Milesi, M. 205

Milillo, A. 275, 276, 277

Milone, P. 50

Minervini, F. 143

Minervini, M. Ida 474

Mingolla, G.P. 75, 76, 77

Mingrone, G. 244, 245, 459, 461,462

Minicozzi, A. 130

Minzioni, G. 248, 249, 250, 251, 258

Mirabile, L. 408, 410

Mirenda, F. 192, 193, 236

Misso, C. 477

Mistrangelo, M. 34, 78, 138, $352,353,354$

Mobiglia, A. 354

Modica, G. 143, 144, 145, 342

Molinaro, D. 534, 535

Molinaro, M.D. 333, 334

Molteni, G. 281, 282, 292, 367, 368

Monacelli, M. 477

Monaco, L. 36, 369

Mondaini, N. 312

Montalti, R. 74, 281, 282, 285 , 286, 287, 292, 327, 367, 368

Montalto, A. 263

Monte, V. 501

Montesi, M. 380, 381

Monteverde, M. 142, 164, 175, 181

Monteverdi, R. 429, 430

Monticoni, C. 50
Morabito, M. 433, 434

Morandi, C. 404

Morandi, U. 179

Moranti, U. 174, 180

Morara, M. 534, 535, 536, 537

Moratto, R. 212

Morbini, P. 332, 334

Morelli, A. 186, 187

Morelli, L. 308

Moretti, B. 423

Moretti, L. 423, 466

Moretto, C. 308

Morgagni, D. 31

Morgagni, P. 31

Morini, F. 532

Moritz, W. 330

Morrica, B. 365

Mosca, F. 308, 350

Mosca, I. 308, 350

Moscaritolo, V. 52, 541

Moscaroli, A. 275, 377

Moscatello, L. 512

Mosiello, G. 331

Mugnai, D. 217, 218, 219, 220, 221

Mulas, P. 467

Mulè, A. 460

Mulonia, A. 321, 323, 324

Municinò, O. 351

Mura, F. La 36, 369

Mura, G. 37, 38, 120, 402, 403

Muratore, A. 355

Muretti, M. 252

Muscatello, L. 509, 516, 517

Muselli, P. 451

Mussa, A. 34, 78, 352, 353, 354

Mussa, B. 354

Mussi, A. 151, 152, 185

Musumeci, F. 263

Muzi, M.G. 337

Nacchiero, L. 67, 386, 387, 388

Nahom, A. 532

Nannei, P. 431

Nanni, G. 244, 245, 459, 461, 462

Nano, G. 198

Napoli, F. 227

Nardo, B. 285, 286, 287, 326 , 327,328

Nascimbeni, R. 54

Nasti, A.G. 81, 82, 83

Natali, P. 180

Natalini, G. 62, 101, 102, 109, 132, 137, 309

Navarra, G. 113

Navarretta, F. 228
Negro, U. 306

Nella, G. 4

Neri, A. 346, 347, 348, 349

Neri, D. 281

Neri, F. 285, 286, 287, 326, 327,328

Neri, V. 39, 40, 121, 122, 123, 124, 125

Nesi, G. 311

Ngassa, E. 362

Ngassa, I. 54

Nicolai, G. 435, 436, 437, 438, 445

Nicolai, L. 190, 191

Nicotra, S. 176

Nigro, C. 42, 264, 265, 337

Nigro, F. 315, 316

Nina, P. 494, 495, 496

Nisi, E. 317

Nizegorodcew, T. 422

Nobili, C. 92

Noccioli, B. 521

Nodari, F. 205

Nosotti, M. 172

Novali, C. 233

Novelli, E. 32

Nucaro, S. 295

Occhionorelli, S. 15, 16, 18

Odero, A. 200, 201, 229

Oliva, D. 397

Olmi, S. 136

Oragano, L. 480

Oreggia, B. 356

Orlandi, V. 31

Ornaghi, D. 252

Orsenigo, E. 10, 43, 44, 105, $106,116,117,118,119,268$, 270, 301, 302

Orsini, V. 68

Osman, N. 106, 279

Ottaviano, A. 61

Oven, H.V. 542

Pachera, S. 103, 104, 133

Paci, M. 165

Pacilè, M.A. 235

Pacilé, V. 93, 363

Pacilè, V. 94, 95, 96, 97, 98, 99, $285,286,287,326,327,396$, $397,398,399,400,475,476$

Pacini, D. 260

Padoan, G. 514

Pagan, V. 157, 161

Paganelli, M. 378

Pagani, M. 241

Pagiataki, J. 25

Pagliari, A.V. 314, 338, 357, $392,439,447,448$ 
Paino, R. 146

Pala, C. 41

Palaia, R. 365

Palazzo, A. 58, 274, 483

Palazzo, F.F. 486, 487

Palermo, S. 114, 115

Palladino, A. 84

Palleschi, A. 163, 172

Palma, P. 513, 514, 515

Palmieri, P.N. 200

Palombo, D. 217, 218, 219, 220, 221

Paludetti, G. 510

Palumbo, A. 1

Panebianco, V. 373, 374

Panetta, A. 113

Panico, S. 51

Panicucci, S. 383, 384

Panizzo, N. 466

Pansoni, C. 4

Paolini, G. 256, 257, 468, 469

Papagno, P. 57, 246

Papalia, R. 412, 413, 414, 415, 420

Papandrea, N. 341, 443

Papapietro, N. 417

Paradisi, A. 460

Pariali, M. 326, 327, 328

Pariscenti, G.L. 35, 166, 167, 358

Parisi, V. 365, 366

Parmeggiani, D. $85,86,87,88$, $89,90,91,477$

Parmeggiani, U. 88, 89, 91

Parodi, O. 254

Parodi, P.C. 466, 470

Partelli, S. 451

Pasquini, E. 502, 511, 518, 519

Passari, G. 192, 194

Pastena, F. 59, 60, 382

Pastore, P. 81, 82

Pastore, S. 262

Pastorino, U. 184

Pata, F. 28, 29, 30, 68

Pata, G. 54

Patella, S. 423

Patella, V. 423

Patriti, A. 378

Patrizi, I. 107

Paulli, S. 433, 434

Pavanello, P.M. 76, 77

Peca, C. 497

Pecchi, A. 293, 294

Pecori, B. 365

Pederzoli, A. 226

Pedrazzani, C. 346, 347, 348, 349

Pedrazzini, G. 288, 289
Pedrini, L. 230

Pellegrini, D. 15, 16, 17, 18

Pellegrino, T. 1, 2

Pelosi, E. 353

Pepino, P. 148

Perdonà, S. 366

Perego, E. 92

Perigli, F. 61

Perilli, L. 224

Perrone, A. 154

Perrone, G. 71, 336

Perrone, V. 350

Perrotta, M. 85, 86, 87, 90

Perrotta, M.E. 347, 348, 349

Persiani, R. 359, 360, 361

Persico, F. 242, 243

Persico Stella, L.P. 339, 393, 394

Pertosa, A.M. 328

Peruffo, A. 329

Peruzzi, S. 395

Pesce, V. 423

Pesso, M. 253

Petito, L. 123

Petrassi, C. 224

Petruzzelli, F. 123

Petteruti, F. 148

Pettinato, M. 400

Pezzatini, M. 377

Pezzoli, F. 291, 295, 296, 298, 300

Piancastelli, L. 52, 539

Piardi, T. 3, 362

Piatto, A. 88, 89, 91

Piazzese, E. 93, 94, 95, 96, 97 , 98, 99, 363, 396, 397, 398, 399, 400, 475, 476

Piazzini, M. 311

Piccin, O. 484, 504, 505, 506, 511

Piccione, E. 405

Piccolo, D. 228

Piervittori, M. 309

Pigna, A. 542

Pilato, A. 230

Pilato, E. 260, 261, 262

Pinna, A. 293, 294, 327, 328

Pinna, A.D. 134, 282, 291, 295, 296, 297, 298, 300, 335, 379, 395

Pinto, E. 346

Piraino, A. 182, 183

Pironi, L. 291, 295, 296

Pirrelli, S. 200, 201

Pirro, F. 253

Pisaniello, D. $88,89,91$

Pisciaroli, A. 378

Pistan, V. 108

Pistochini, A. 514
Pistritto, A. 373, 374

Pittarello, F. 321, 323, 324

Pivi, S. 52

Pizzio, M. 34

Podetta, M. 334

Poggi, P. 333

Pola, E. 421

Pola, L. 317, 318, 323, 324

Poletto, G. 229

Polimeno, E. 154

Polistena, A. 11, 331

Ponsetto, M. 50

Popovich, A. 229

Porta, F. 257

Portincasa, P. 67

Possamai, L. 18

Potalivo, A. 52, 538, 539, 541, 542, 543

Pozzato, G. 176

Pozzi, R. 47

Prandi, M. 381

Pratesi, C. 209, 210, 211, 232

Pratesi, G. 209, 210, 232

Premoli, P. 173

Presepi, S. 407

Prezzi, D. 285, 286, 287, 326, 327, 328

Princi, P. 479, 481, 482, 491, 492

Procaccini, E. 56

Procaccini, F. 56

Proietti, I. 460

Proietti, L. 421

Prouse, G. 225

Provenzano, R. 536, 537

Puccini, M. 33

Puddu, A. 455

Puglisi, A. 156

Pulcini, A. 213

Puletti, G. 47

Pulli, R. 209, 210, 232

Puma, F. 159, 177, 178

Puppio, B. 343, 344, 345

Puviani, L. 285, 286, 287, 326, 327, 328

Puzzo, L. 373, 374

Quarantotto, F. 162

Quartierini, G. 71

Quercia, R. 150

Rached, A. 227

Raffaelli, M. 478, 479, 480, 481, 482, 491, 492

Rago, R.R. 19, 21, 22

Raimondi, M.C. 484

Rampinelli, L. 336

Randazzo, C. 342

Randone, B. 331
Ranieri, A. 503

Ranocchi, F. 263

Rapicetta, C. 155, 168

Rastrelli, M. 340

Rausei, S. 360, 361

Ravaioli, M. 297, 298

Ravanello, P.M. 75

Raveglia, F. 169, 170

Ravera, A. 50

Ravini, M. 146

Raviola, P. 50

Rea, F. 171, 176

Reggiani, L.M. 409

Regina, G. 189, 310

Reguzzi, C. 278

Renzi, A. 36, 65, 369

Renzi, L. 469

Resta, G. 16, 488

Resti, A.G. 533

Restuccia, G. 411

Revelli, L. 491, 492

Ribechini, A. 185

Riberti, C. 466

Riccardo, M. 100

Ricchiuti, C. 234, 278, 364

Ricci, S. 470

Ricciardello, A. 373, 374

Riccio, P.A. 75, 76, 77

Ricella, C. 141

Ricucci, C. 223

Ridolfi, C. 337, 385

Righi, I. 53

Righini, P. 229

Rimondini, A. 108

Rinaldi, A. 62, 101, 102, 109, 132

Risaliti, A. 284, 305, 306, 307

Ristagno, S. 373, 374

Rivellini, C. 233

Rizzardi, G. 171

Rizzello, G. 412, 413, 414, 415, 417, 418, 419, 420

Rizzo, G. 308

Rizzuti, F. 41

Robaldo, A. 217, 218, 219, 220, 221

Rocca, G.L. 145

Rocchetta, D. 441

Rocco, B. 320

Rocha, C. 356

Romano, A. 74, 281, 282, 292, 367, 368

Romano, F. 92

Romario, U.F. 273

Romeo, B.G. 390

Romeo, R. 234

Rompianesi, G. 281

Rompianesi, G.L. 74

Roncoroni, L. 84 
Rondoletti, S. 78, 352

Rosada, A. 538

Rosati, R. 273

Roscitano, A. 259

Roscitano, G. 192, 193, 194

Roseano, M. 55

Rosetti, P. 37

Rossetti, M. W. 503

Rossetti, O. 303, 304

Rossi, C. 231

Rossi, V. 522, 523, 524, 525, 526, 527, 528, 529, 530, 531

Rossit, L. 5, 6, 7, 8, 9

Rosso, L. 163, 172

Rota, E. 173

Rousas, N. 217, 218, 219, 220, 221

Roviaro, G.C. 241

Roviello, F. 346, 347, 348, 349

Rubba, F. 51

Rubino, C. 455, 467

Ruffino, S.F. 190, 191

Ruffolo, F. 365, 366

Rufini, V. 478

Ruggeri, E. 280

Ruggiero, C. 174

Ruggiero, R. 56, 57

Rugiero, G. 86, 87

Rulli, F. 264, 385, 485

Ruperto, M. 11

Rusca, M. 140

Russo, C. 254, 255

Russo, M.L. 276, 377

Rutolo, F. 228

Ruzzenente, A. 103, 104, 133

Ruzzini, L. 412, 413, 414, 415, 417

Sabino, G. 235

Saccenti, P. 178

Sacco, R. 28, 29, 30, 68

Saccomano, E. 7, 9

Saccone, A. 47

Sadighi, A. 12

Saggese, D. 511

Sainz-Barriga, M. 283

Salerni, B. 54

Salesi, G. 188

Salgarello, M. 460

Salis, P. 299

Saltarel, A. 436

Salvatori, M. 482

Salvestrini, F. 322, 325

Salvi, R. 157, 158, 161

Samele, F. 39, 40, 121, 122, 123, 124

Samolsky-Dekel, B.G. 543

Sampietro, R. 105, 301, 302

Sangalli, F. 256
Sani, F. 139

Sanna, S. 142, 164, 175, 181

Sansalone, C.V. 289, 303, 304

Santacroce, C. 39, 40, 121, 122, $123,124,125$

Santambrogio, L. 163, 172

Santanelli, F. 468, 469

Santini, D. 134, 162, 375, 376

Santini, M. 17, 153, 154

Santoni, B. 321, 323, 324

Santoro, A. 509, 512, 516, 517

Saragoni, L. 37

Sardi, I. 311

Sarli, L. 84

Sarmati, M. 504

Sartelli, M. 107

Sartori, A. 108, 524, 525, 526, $527,528,529,530,531$

Sartori, P. 477

Sattin, E. 186,187

Savini, C. 261

Savino, M. 322, 325

Sberze, F. 514, 515

Scaburri, A. 320

Scafarto, M.V. 51

Scaglioni, M. 333, 334

Scala, D. 365, 366

Scalinci, S.Z. 534, 535, 536, 537

Scaramellini, G. 520

Scaramellini , G. 507

Scaramuzzino, G. 505

Scassa, F. 383, 401

Schettino, M. 36, 369

Schiavo, F.L. 56

Schiavon, M. 176

Schiavone, A. 62, 101, 102, 109, 132

Schiraldi, A. 163

Schirosi, M. 457, 458

Sciamannini, M. 159, 177, 178

Sciarretta, V. 518, 519

Sciaudone, G. 110, 111, 112

Scibé, R. 107

Scieri, F. 320

Sciuchetti, J.F. 160, 256, 257

Scognamillo, F. 41

Scomersi, S. 108

Scorolli, L. 534, 535, 536, 537

Scuderi, N. 456

Scuro, A. 190, 191

Sebag, F. 486, 487

Seccia, A. 457, 458, 460

Seccia, V. 509, 512, 516, 517

Sedda, V. 254

Selleri, S. 64

Selva, S. 113

Selvaggi, F. 110, 111, 112

Selvaggi, G. 290
Semeraro, A. 159, 177, 178

Seneca, V. 500

Sensi, L. 230

Sercia, F. 317, 318

Seren, P. 317, 318

Sergiampietri, S. 384, 401

Serra, R. 235

Sessa, L. 478

Setacci, C. 206, 207

Setacci, F. 239, 240

Settembrini, A.M. 225

Settepani, F. 252

Severini, D. 45

Sforza, C. 430

Sfuncia, G. 94

Sgambelluri, A. 308

Sgambelluri, F. 350

Sgarbi, G. 165

Sgro, E. 313

Sguinzi, R. 169, 170

Sibilio, F. 85

Sica, G.S. 128

Siciliano, M. 85

Sicuro, O. 313

Sierra, M. 487

Signori, S. 308, 350

Sileri, P. 126, 127, 128

Silva, P. 288

Silvi, M.B. 337

Simmini, A. 340

Simoncello, F. 513

Simoncini, G. 542

Simonelli, I. 81, 82

Simonetti, G. 485

Sinatra, R. 259

Sirignano, P. 206, 207

Sivero, L. 472, 473

Slim, A.O. 289

Sloffer, C.A. 502

Socci, C. 105, 301, 302

Soldano, S. 303, 304

Solfanelli, E. 73

Solfrini, G. 38, 402

Sollitto, F. 149, 150

Sordelli, I. 88, 89, 91

Sorrenti, G. 505

Sorrenti, S. 114, 115

Sottimano, S. 50

Sozzi, D. 329

Spada, E. 149

Spada, M. 299, 474

Spada, M.L. 192, 193, 202, 236

Spaggiari, M. 74, 281, 282, 292, 367, 368

Spampinato, M. 299, 474

Sparavigna, L. 57, 246

Spartera, C. 224

Spaziani, A. 267, 272
Spaziani, E. 339, 393, 394

Spelzini, F. 406

Spelzini, P.A. 4

Spera, G. 389, 390, 391

Sperlongano, P. 88, 89, 91, 477

Sperti, E. 355

Spiezia, S. 147

Spina, G. 278

Spinadin, R. 321, 323, 324

Spinarelli, A. 423

Spinelli, F. 192, 193, 194, 202, 236

Spini, S. 72, 73

Spivach, A. 108

Spotti, S. 440, 441, 442

Spreafico, F. 533

Squillante, S. 116

Stagnitti, F. 339, 393, 394

Stano, R. 15, 16, 17, 18, 488

Stanzi, A. 172

Stanzione, F. 58, 274, 483

Staudacher, C. 10, 43, 44, 105 , $106,116,117,118,119,266$, $268,270,279,301,302$

Stavroulias, D. 140

Stefani, A. 179,180

Stefanini, P. 173

Steffano, G.B. 47

Stefoni, S. 287

Stegher, S. 198

Stella, A. 203, 215, 216, 231

Stener, S. 320

Stilo, F. 202, 236

Stio, F. 213

Stio, R.E. 213

Stolfi, V.M. 126, 127, 128

Sturla, M. 460

Suarez, S.M. 262

Sunseri, A. 143, 342

Suprani, A. 407

Susanna, F. 126, 127, 128

Svegliati-Baroni, G. 306

Tacchino, R. 244, 245, 459, 461, 462

Taffurelli, M. 52, 134, 375, 376, 379

Talarico, C. 341, 443, 477

Taliente, P. 4

Tallarico, F. 64

Tamburini, A. 43, 116, 117, $118,268,270$

Tampieri, A. 421

Tanzini, G. 24, 25, 26, 27, 48, 49, 63

Tarantino, E. 389

Tartaglia, G.M. 430

Tartaglia, N. 39, 40, 121, 122, 123,124 
Taruscia, D. 305

Tasca, A. 315, 316

Tasciotti, C. 339, 393, 394

Tasciotti, M. 339, 393, 394

Tauceri, F. 37, 38, 108, 120, 402, 403

Taurchini, M. 142, 164, 175, 181

Tealdi, D.G. 198

Tenconi, S. 155, 168

Tenti, G. 518, 519

Terenzi, V. 424

Tessitore, A. 182, 183

Testi, G. 203, 215, 216

Testini, M. 67, 386, 387, 388

Testoni, P.A. 118

Thsomba, Y. 240

Tiberio, G.A.M. 3, 35, 362

Titta, M. 315, 316

Toccaceli, S. 339, 393, 394

Todaro, M. 381

Tomajer, V. 118, 119

Tomasello, G. 342

Tomasi, I. 21

Tomassetti, P. 134

Tonelli, E. 259

Topatino, A. 246

Torelli, F. 36, 369

Torre, G.L. 343, 344

Torre, G.L. 345

Torre, M. 146

Torricelli, M. 524, 525, 526, $527,528,529,530,531$

Torricelli, P. 139, 293, 294

Torrini, R. 137, 309

Torroni, A. 438, 444, 445, 446

Tosi, D. 184

Tossani, E. 298

Tota, F. 150

Traficante, A. 344

Traini, E. 478,480
Trazza, F. 288

Treglia, G. 478

Trevisan, D. 195, 196, 197

Triassi, M. 51

Trignano, M. 41

Trinca, E. 4

Troisi, N. 210, 211

Trompetto, M. 23, 32

Trunfio, S. 289

Tshomba, Y. 237, 238, 239

Tsivian, M. 285, 286, 287, 326, 327,328

Tuci, C. 76

Turazza, F. 289

Turci, S. 260

Turoldo, A. 55

Turra, G. 173

Tzakis, A. 290

Uggeri, F. 92, 477

Ugolini, A. 430

Urbano, D. 277

Usò, T.D. 101

Uzzau, A. 5, 6, 8, 9

Vadalà, G. 412, 417

Vagliasindi, A. 37, 38, 402

Vaira, M. 370, 371, 372

Valadè, A. 523

Valbonetti, M. 538

Valdegamberi, A. 104, 133

Valente, M. 176

Valentini, V. 437, 438, 445, 446

Valentino, T.P. 39, 40, 121, 122, $123,124,125$

Valeri, G. 306

Valieri, L. 162

Valitutti, P. 204

Valloncini, E. 54

Valota, M. 362

Valsecchi, S. 431
Vanni, B. 114, 115

Varotti, G. 285, 286, 298

Varroni, A. 227

Vasta, F. 373, 374

Vecchi, A. 305, 306, 307

Veneroni, L. 380, 381

Veneziano, D. 313

Venosa, M. 421, 422

Ventucci, E. 436

Ventura, D. 338, 357, 392, 439, 447, 448

Ventura, M. 222

Venuta, F. 141

Venza, M. 126, 127, 128, 247

Verde, I. 57, 246

Verdecchia, G.M. 37, 38, 120, 402, 403

Verdecchia, M. 306

Vergara, P. 499, 500

Veronesi, P. 47

Verrusio, C. 343, 344, 345

Verzaro, R. 299, 474

Vesce, G. 228

Vescia, G. 68

Vespa, A. 390

Vetrano, E. 503

Vettoretto, N. 129

Vicenzo, L. 36, 369

Vicidomini, G. 153, 154

Vicini, E. 334

Viganò, J. 332, 333, 334

Viggiani, P. 97

Vignali, A. 116

Vignati, A. 47

Vigorita, V. 361

Villa, M. 264, 337, 385, 485, 541

Villani, F. 338, 357, 392, 447, 448

Vindigni, C. 48

Viotti, F. 205
Virzì, S. 113

Visci, F. 421, 422

Vistoli, F. 308, 350

Vit, A. 186, 187

Vita, M.L. 183

Vitali, E. 254, 255, 288, 289

Viti, A. 185

Vittimberga, G. 31

Vivarelli, M. 297, 298

Vizzini, G. 474

Voci, D. 68

Volpes, G. 468

Voltolini, L. 155

Weber, M. 330

Xidas, A. 41

Zabatta, F. 382

Zaccaria, A. 157, 158, 161

Zaccaroni, A. 31

Zago, M. 273

Zanfi, C. 291, 295, 296, 298, 300

Zangheri, E. 542

Zannetti, G. 471

Zannini, P. 156

Zanzi, F. 488

Zappa, M.A. 241

Zattoni, F. 319

Zennaro, R. 321, 323, 324

Zoccali, M. 359

Zolin, A. 197

Zompicchiatti, A. 5, 6, 8

Zonta, S. 332, 333, 334

Zorzi, D. 355

Zuber, V. 301

Zucchini, S. 504, 505, 506

Zuellig, R. 330

Zuin, A. 171

Zuppi, C. 479,480 